\title{
Stereoselective Synthesis of Oxacycles via Ruthenium Catalyzed Atom-Economic Coupling of Propargyl Alcohols and Michael Acceptors
}

Nabakumar Bera, Shantanu Samanta and Debayan Sarkar*

Department of Chemistry, National Institute of Technology, Rourkela, Rourkela-769008, India.

E-mail: sarkard@nitrkl.ac.in 


\section{Table of Contents}

1. Large Scale synthesis of 1-phenylhex-3-yne-1,5-diol (1a)...........................S3

2. Large Scale synthesis of $(E)$-5-ethylidene-8-hydroxy-1,8-diphenyloctane-2,6-dione

(4a)...................S3

3. Large Scale synthesis of $(2 R, 3 R, 6 R)-2$-methyl-3-(3-oxo-4-phenylbutyl)-6-phenyl tetrahydro4H-pyran-4-one(5a)...................S4

4. Large Scale synthesis of $(1 R, 3 R, 8 a S)$-1-methyl-3,5-diphenyl-1,3,4,7,8,8a-hexahydro-6Hisochromen-6-one (7b).................S4

5. Large Scale synthesis of $(1 R, 3 R)-1$-methyl-3,5-diphenylisochroman-6-ol (8a).........S5

6. ${ }^{1} \mathrm{H}$ and ${ }^{13} \mathrm{C}\left\{{ }^{1} \mathrm{H}\right\}$ NMR Spectrum of pent-2-yne-1,5-diols (1).........................S6 - S33

7. ${ }^{1} \mathrm{H}$ and ${ }^{13} \mathrm{C}\left\{{ }^{1} \mathrm{H}\right\}$ NMR Spectrum of Michael Acceptors (2).............................S34 - S42

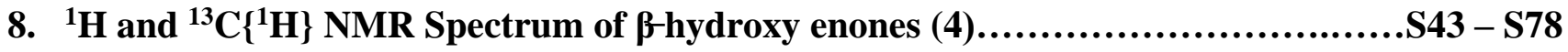

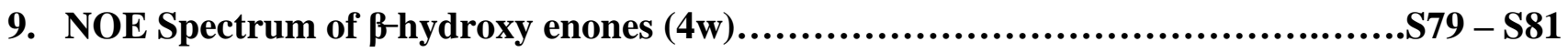

10. ${ }^{1} \mathrm{H}$ and ${ }^{13} \mathrm{C}\left\{{ }^{1} \mathrm{H}\right\}$ NMR Spectrum of tetrahydro-4H-pyran-4-one (5)...................S82 - S105

11. DEPT 135 and 90 Spectrum of tetrahydro-4H-pyran-4-one (5)......................S106 - S110

12. NOE Spectrum of tetrahydro-4H-pyran-4-one (5)..................................S111 - S126

13. COSY Spectrum of tetrahydro-4H-pyran-4-one (5h).................................2127

14. ${ }^{1} \mathrm{H}$ and ${ }^{13} \mathrm{C}\left\{{ }^{1} \mathrm{H}\right\}$ NMR Spectrum of isochromenone (6)..................................2128

15. DEPT 135 Spectrum of isochromenone (6).........................................S129

16. NOE Spectrum of isochromenone (6).........................................S130 - S132

17. COSY Spectrum of isochromenone (6).............................................S133

18. ${ }^{1} \mathrm{H}$ and ${ }^{13} \mathrm{C}\left\{{ }^{1} \mathrm{H}\right\}$ NMR Spectrum of $1,3,4,7,8,8 \mathrm{a}-h e x a h y d r o-6 H$-isochromen-6-one (7)...

19. DEPT 135 and 90 Spectrum of 1,3,4,7,8,8a-hexahydro- $6 H$-isochromen-6-one (7)...S155 - S159

20. NOE Spectrum of 1,3,4,7,8,8a-hexahydro-6H-isochromen-6-one (7)..................S160 - S169

21. ${ }^{1} \mathrm{H}$ and ${ }^{13} \mathrm{C}\left\{{ }^{1} \mathrm{H}\right\}$ NMR Spectrum of isochroman-6-ol (8)..............................S170 - S174 


\section{Large Scale synthesis of 1-phenylhex-3-yne-1,5-diol (1a)}

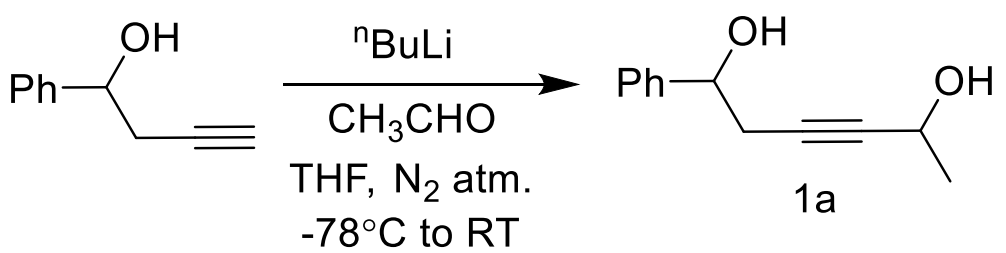

In a flame dried $100 \mathrm{~mL}$ r.b. flask, kept under $\mathrm{N}_{2}$ atm., 1-phenylbut-3-yn-1-ol [1 gm, 6.84 mmol, 1 equiv.] was dissolved in THF $[15 \mathrm{~mL}]$ and cooled down to $-78^{\circ} \mathrm{C}$. To this solution, $\mathrm{n}$-BuLi [0.963 $\mathrm{gm}(6.02 \mathrm{ml}), 15.05 \mathrm{mmol}, 2.2$ equiv., 2.5 $\mathrm{M}$ in hexanes] was added dropwise and stirring was continued at the same temperature for 1 hour. At this point, the acetaldehyde [0.3612 $\mathrm{gm}(0.46 \mathrm{ml}), 8.21 \mathrm{mmol}, 1.2$ equiv.] was added neat and the mixture was allowed to attain room temperature. The reaction mixture was quenched by adding saturated $\mathrm{NH}_{4} \mathrm{Cl}$ solution. Then it was extracted with EtOAc and dried over anhydrous $\mathrm{Na}_{2} \mathrm{SO}_{4}$. Evaporation of the organic solvent followed by flash chromatography with silica gel and eluted with 30\% EtOAc in Petroleum ether afforded 1-phenylhex-3-yne-1,5-diol (1a) $[1.043 \mathrm{gm}, 5.4825 \mathrm{mmol}, 80 \%]$.

\section{Large Scale synthesis of $(E)$-5-ethylidene-8-hydroxy-1,8-diphenyloctane-2,6-dione (4a)}

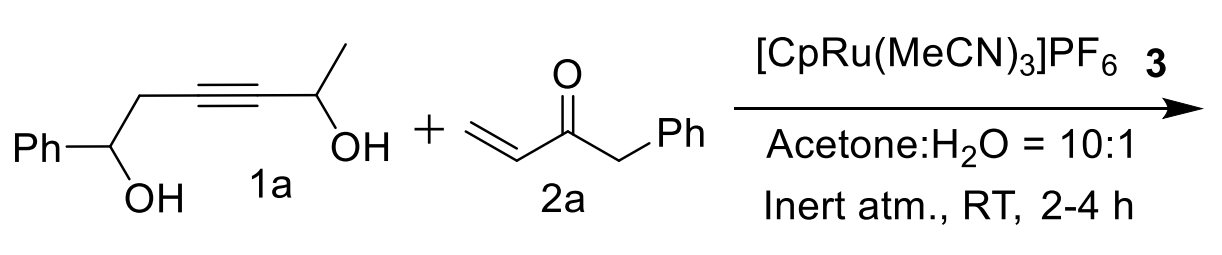<smiles>C/C=C(/CCC(=O)Cc1ccccc1)C(=O)CC(O)c1ccccc1</smiles>

$4 a$

To a well-stirred solution of 1-phenylhex-3-yne-1,5-diol (1a) [700 mg., $3.68 \mathrm{mmol}, 1$ equiv.] in acetone (10 ml), 1phenylbut-3-en-2-one (2a) [538 mg, $3.68 \mathrm{mmol}, 1$ equiv.] was added in a $25 \mathrm{ml}$ r.b. flask under $\mathrm{N}_{2}$ atmosphere. To this solution $1.0 \mathrm{ml}$ water was added. Then the catalyst $\mathrm{CpRu}\left(\mathrm{CH}_{3} \mathrm{CN}\right)_{3} \mathrm{PF}_{6}$ (3) [160 mg, $0.368 \mathrm{mmol}, 0.1$ equiv.] was added and stirred the reaction mixture at room temperature for 2 hours. After 2 hours, the acetone was evaporated out under vacuum. The reaction mixture was extracted with ether $(3 \mathrm{X} 15 \mathrm{~mL})$ and concentrated under vacuum to give a yellow liquid. The crude yellow liquid was chromatographed with silica gel and eluted with Petroleum ether: EtOAc (9:1) afforded (E)-5-ethylidene-8-hydroxy-1,8-diphenyloctane-2,6-dione (4a) [1.052 gm, $3.127 \mathrm{mmol}, 85 \%$ ]. 


\section{Large Scale synthesis of $(2 R, 3 R, 6 R)-2$-methyl-3-(3-oxo-4-phenylbutyl)-6-phenyl tetrahydro- $4 H$ - pyran-4-one(5a)}<smiles>C/C=C(/CCC(=O)Cc1ccccc1)C(=O)CC(O)c1ccccc1</smiles>

$4 \mathrm{a}$

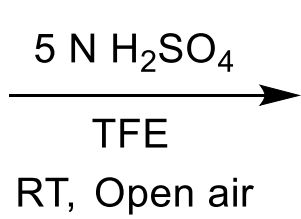

RT, Open air

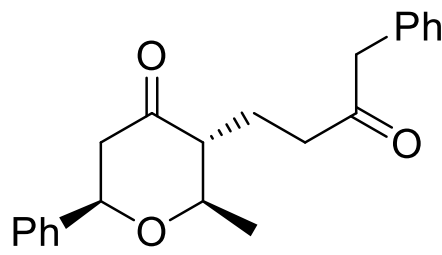

$5 a$

In a $25 \mathrm{~mL}$ r.b. flask, (E)-5-ethylidene-8-hydroxy-1,8-diphenyloctane-2,6-dione (4a) [700 mg, $2.081 \mathrm{mmol}, 1$ equiv.] were dissolved in $15 \mathrm{ml}$ 2,2,2-trifluoroethanol (TFE). To this solution, catalytic amount of $5(\mathrm{~N}) \mathrm{H}_{2} \mathrm{SO}_{4}$ was added at room temperature and stirring the reaction mixture for several hours. The completion of the reaction was confirmed by thin layer chromatography and quenched by adding saturated $\mathrm{NaHCO}_{3}$ solution. Then it was extracted with EtOAc and dried over anhydrous $\mathrm{Na}_{2} \mathrm{SO}_{4}$. Evaporation of the organic solvent followed by flash chromatography with silica gel and eluted with 7\% EtOAc in Petroleum ether afforded (2R,3R,6R)-2-methyl-3-(3-oxo-4-phenylbutyl)-6phenyl tetrahydro- 4H-pyran-4-one(5a) [646 mg, $1.92 \mathrm{mmol}, 92 \%$ ].

\section{Large Scale synthesis of $(1 R, 3 R, 8 a S)-1$-methyl-3,5-diphenyl-1,3,4,7,8,8a-hexahydro-6H-isochromen- 6-one (7b)}

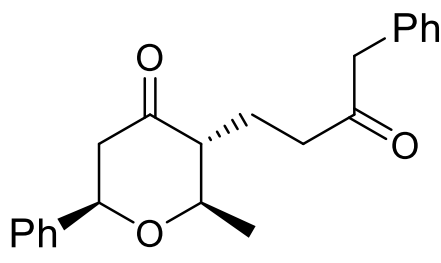

$5 a$

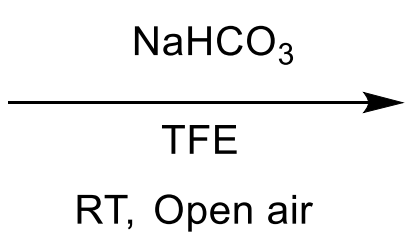

RT, Open air<smiles>C[C@H]1O[C@@H](c2ccccc2)CC2=C(c3ccccc3)C(=O)CC[C@H]21</smiles>

$7 b$

In a $25 \mathrm{~mL}$ r.b. flask, $(2 R, 3 R, 6 R)$-2-methyl-3-(3-oxo-4-phenylbutyl)-6-phenyl tetrahydro- 4H-pyran-4-one (5a) [400 $\mathrm{mg}, 1.19 \mathrm{mmol}, 1$ equiv.] was dissolved in $10 \mathrm{~mL}$ TFE. To this solution, $1 \mathrm{~mL}$ saturated $\mathrm{NaHCO}_{3}$ solution was added. Then the reaction mixture was stirred for 24 hours and completion of the reaction was comnfirmed by thin layer chromatgaphy. The reaction mixture was extracted with EtOAc $(3 \mathrm{X} 10 \mathrm{~mL})$, the organic extract was separated and concentrated under vacuum to give a yellow liquid. The crude yellow liquid was chromatographed with silica gel and eluted with $10 \%$ EtOAc in petroleum ether afforded $(1 R, 3 R, 8 a S)$-1-methyl-3,5-diphenyl-1,3,4,7,8,8a-hexahydro6H-isochromen-6-one (7b) [374 mg, $1.175 \mathrm{mmol}, 99 \%$ ]. 


\section{Large Scale synthesis of $(1 R, 3 R)$-1-methyl-3,5-diphenylisochroman-6-ol (8a)}

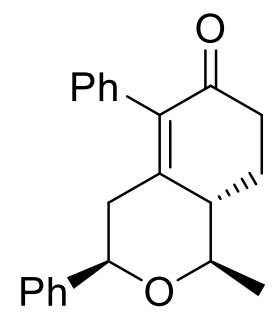

$7 b$

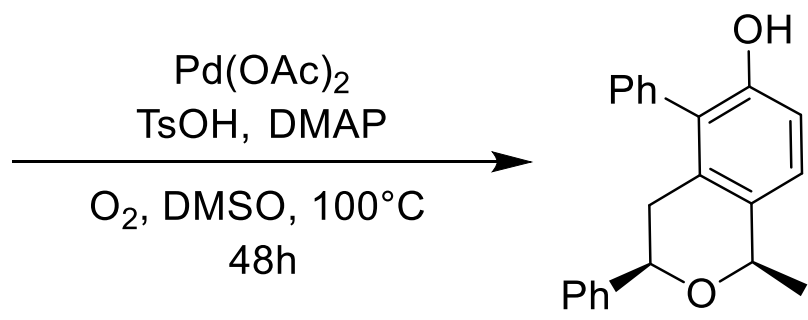

$8 a$

In a flame dried $25 \mathrm{~mL}$ two neck r.b. flask, $(1 R, 3 R, 8 a S)$-1-methyl-3,5-diphenyl-1,3,4,7,8,8a-hexahydro-6Hisochromen-6-one (7b) [200 mg, $0.6281 \mathrm{mmol}, 1$ equiv.] was dissolved in DMSO [5 mL] under oxygen atmosphere. To this solution, $\mathrm{Pd}(\mathrm{OAc})_{2}[14 \mathrm{mg}, 0.0628 \mathrm{mmol}, 0.1 \mathrm{eq}$ ], TsOH [11 mg, $0.0628 \mathrm{mmol}, 0.1 \mathrm{eq}$ ], DMAP [8 mg, 0.0628 mmol, 0.1 eq] was added and the reaction mixture was heated in an oil bath at $100^{\circ} \mathrm{C}$ for 48 hours. Then the reaction mixture was cooled down to room temperature and quenched with water. The organic part was extracted with EtOAc and dried over anhydrous $\mathrm{Na}_{2} \mathrm{SO}_{4}$. Evaporation of the organic solvent followed by flash chromatography with silica gel and eluted with 5\% EtOAc in Petroleum ether afforded (1R,3R)-1-methyl-3,5-diphenylisochroman-6-ol (8a) [137 $\mathrm{mg}, 0.433 \mathrm{mmol}, 69 \%$ ] 
1-phenylhex-3-yne-1,5-diol

$1 \mathrm{a}$

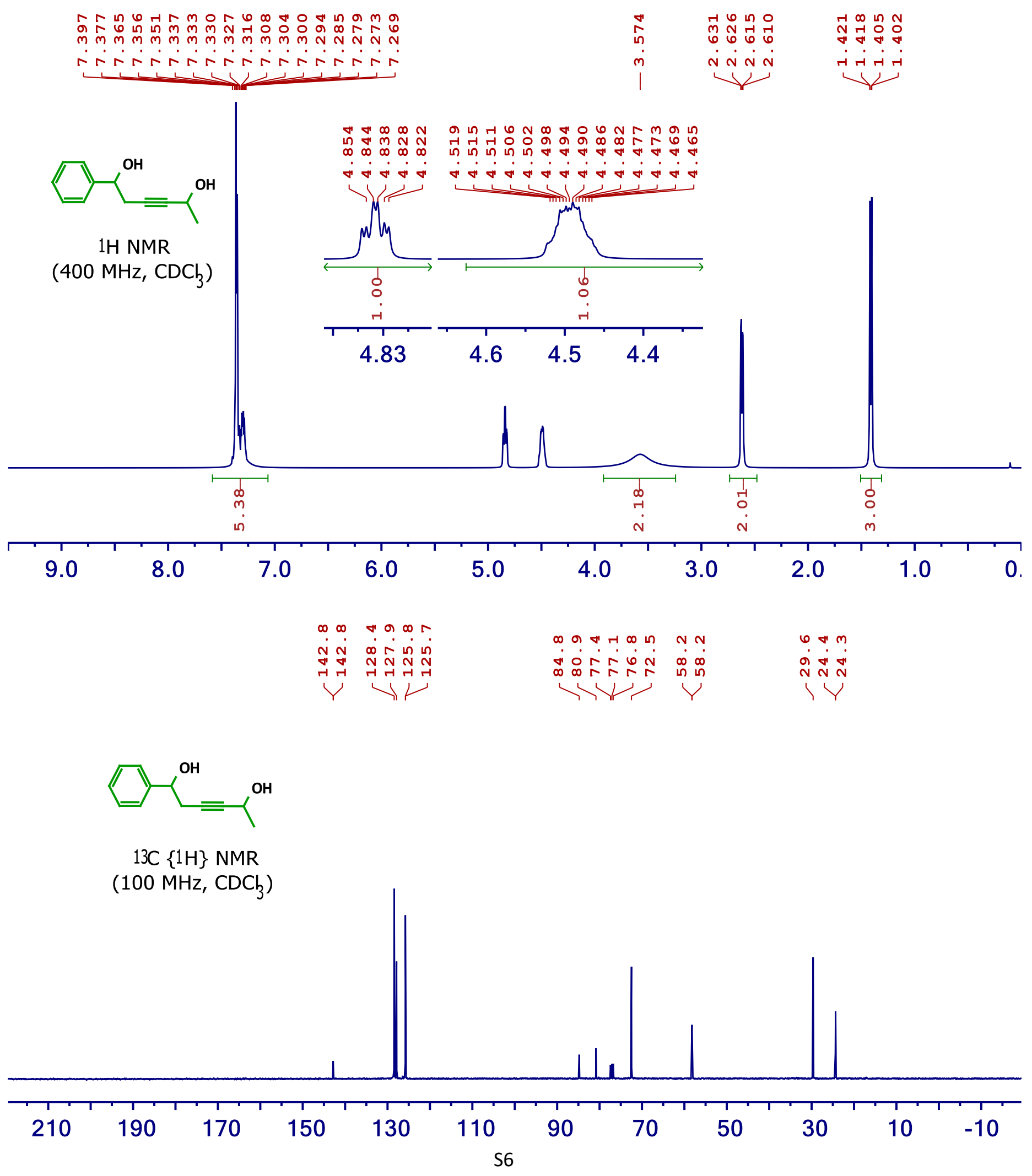


1-phenylhept-3-yne-1,5-diol

$1 \mathrm{~b}$

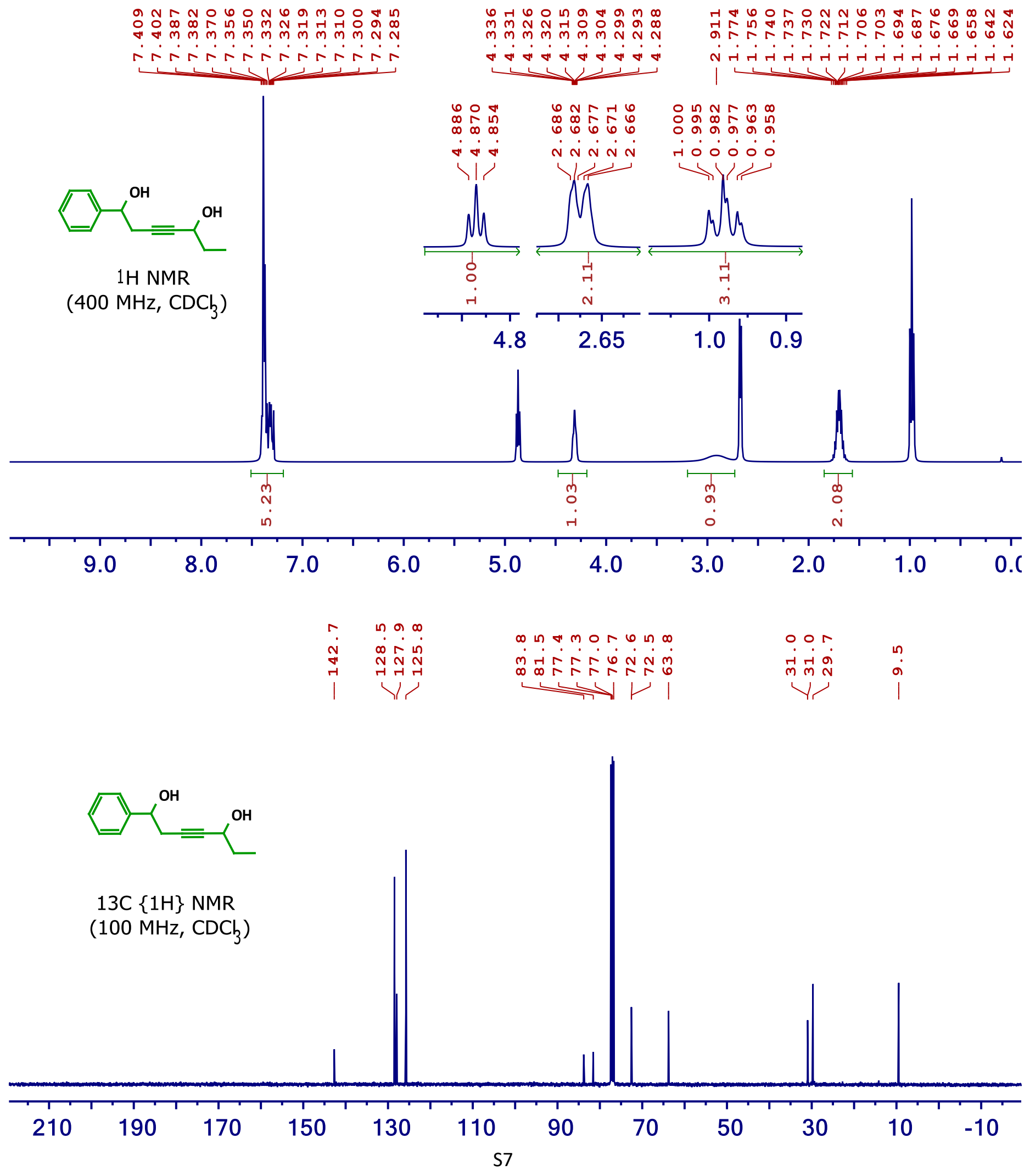




\section{1,6-diphenylhex-3-yne-1,5-diol}

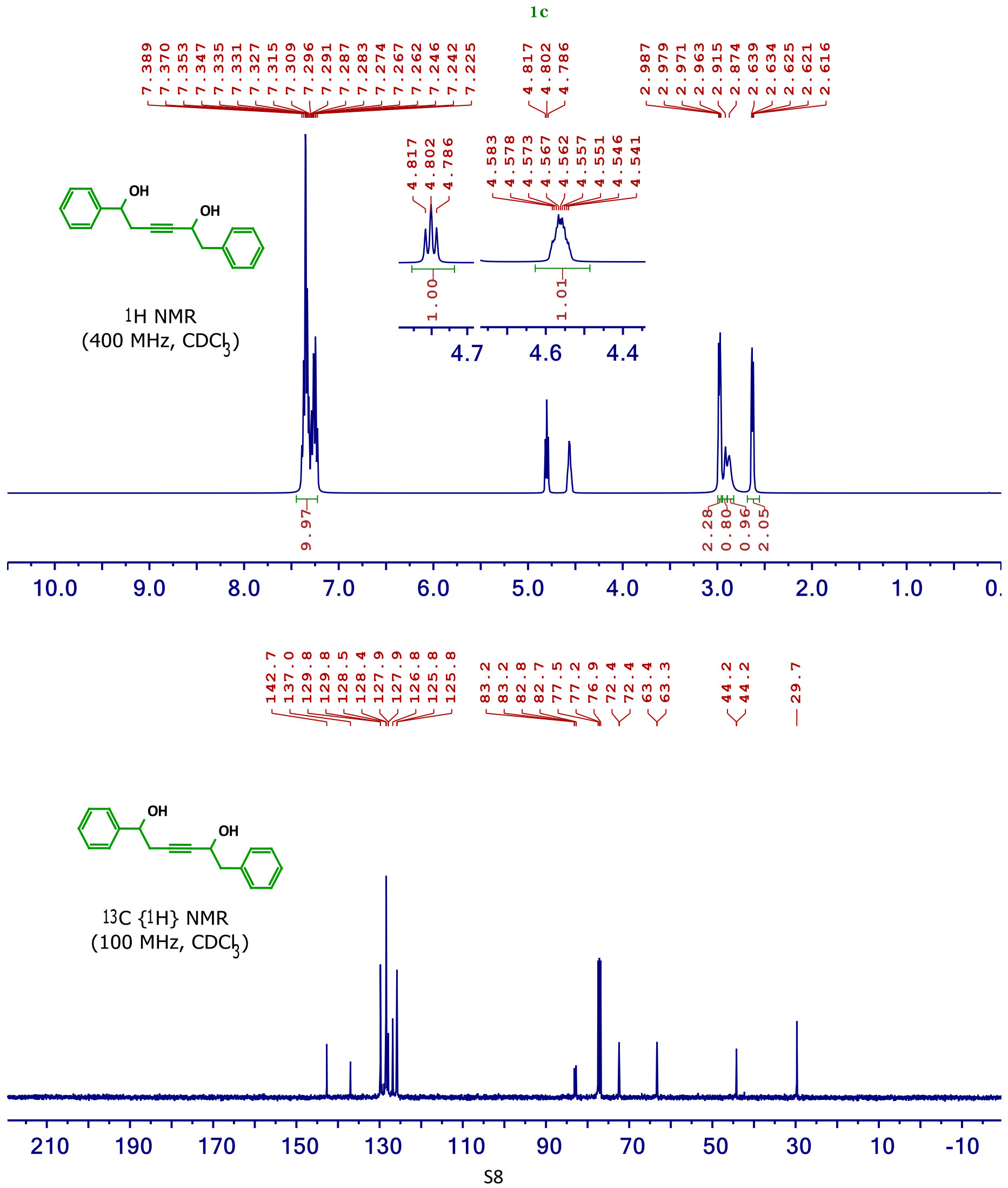


1-phenylnon-3-yne-1,5-diol

$1 d$

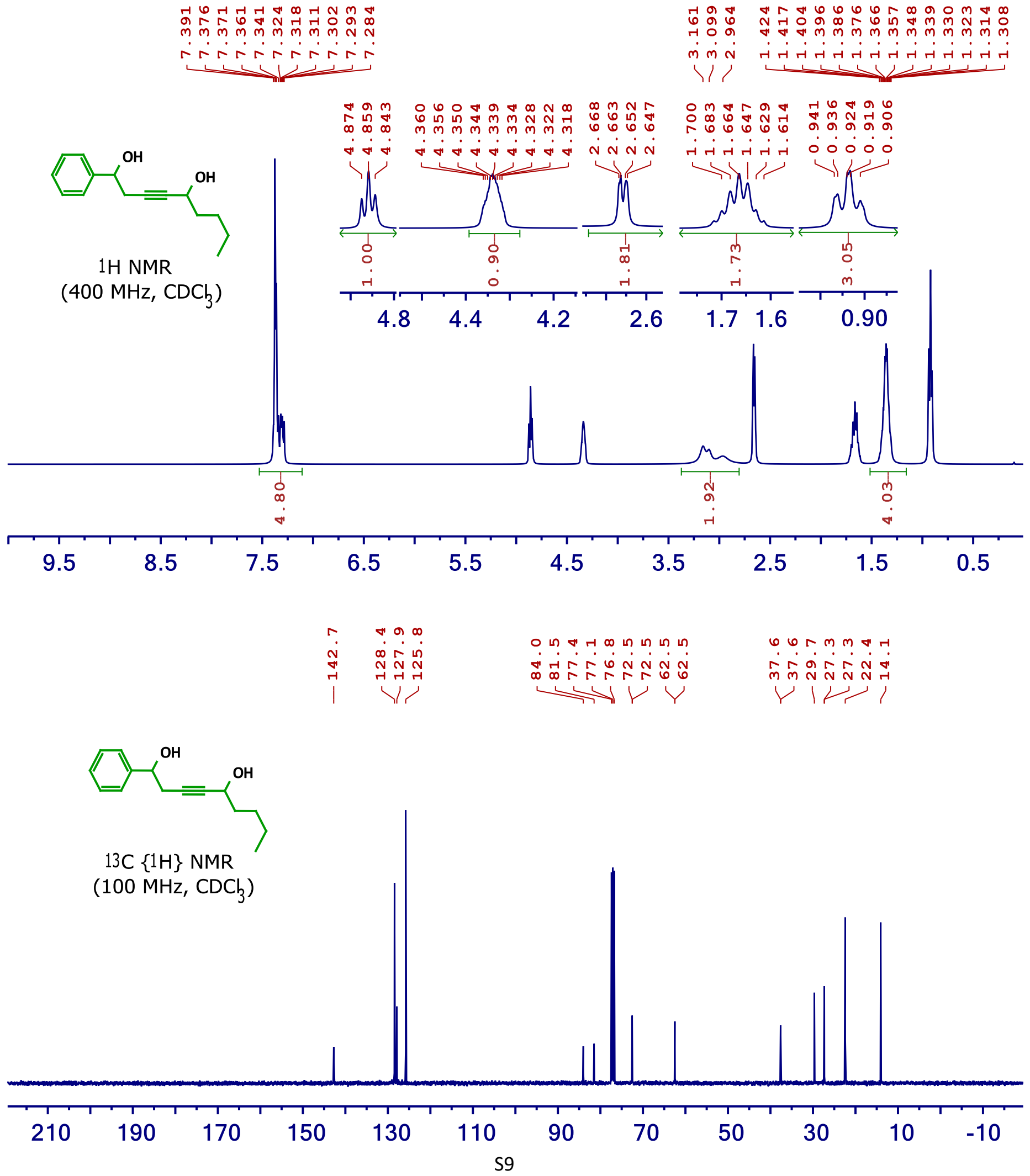


7-methyl-1-phenyloct-3-yne-1,5-diol

$1 \mathrm{e}$
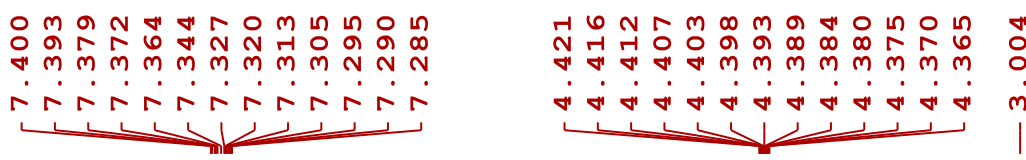

N

नै ตै नू नू ब

00000

Y
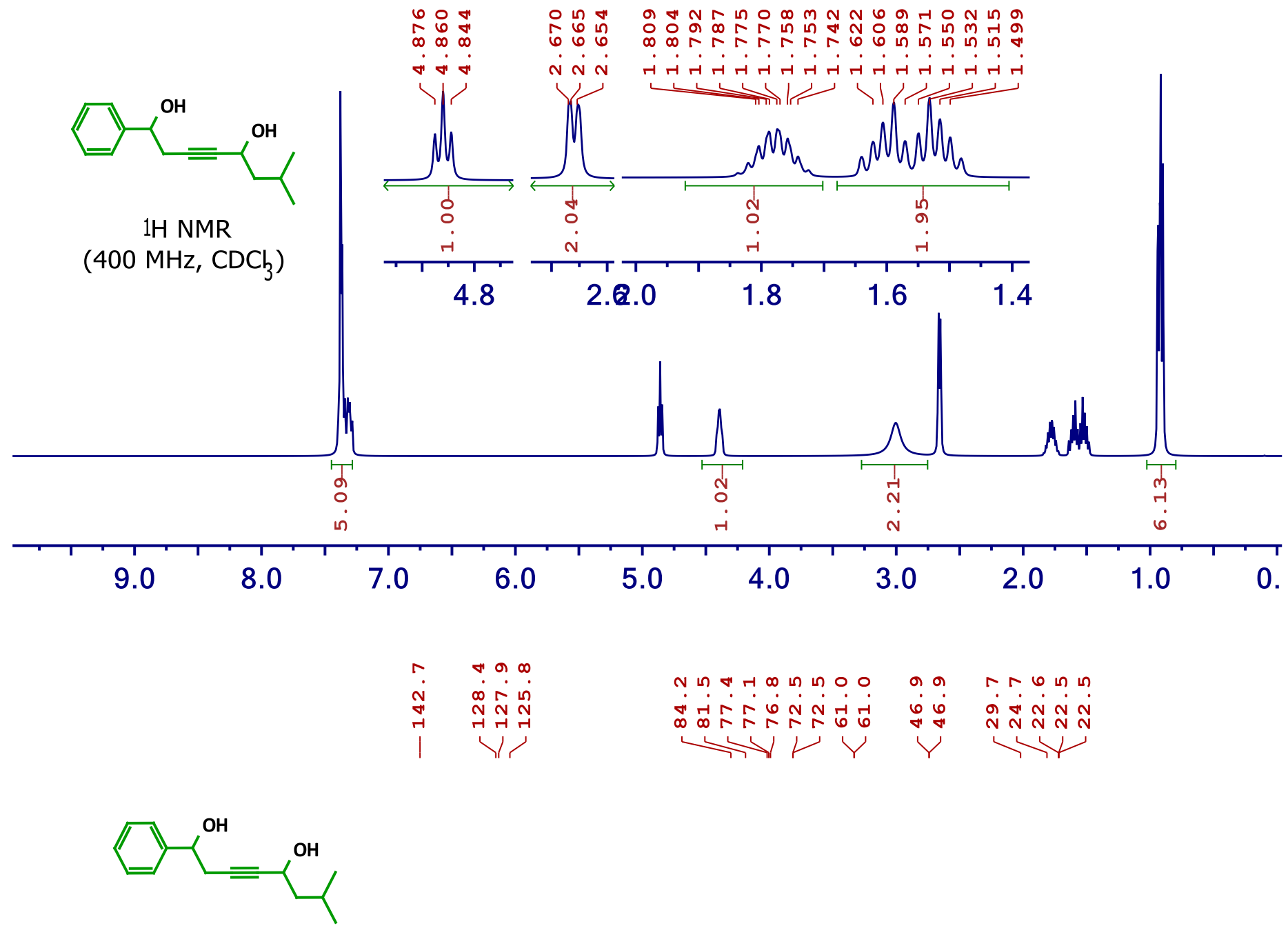

$13 \mathrm{C}\{1 \mathrm{H}\} \mathrm{NMR}$

(100 MHz, CDCl $)$

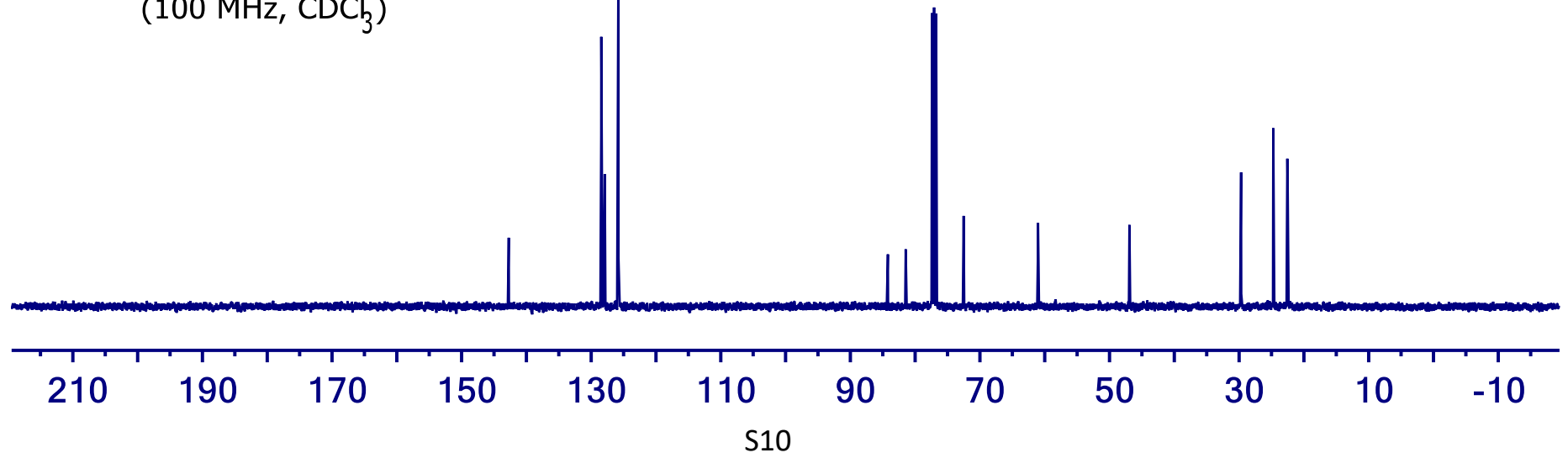


1-cyclohexyl-5-phenylpent-2-yne-1,5-diol

1f

क

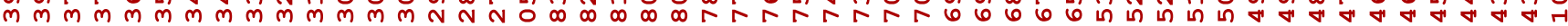

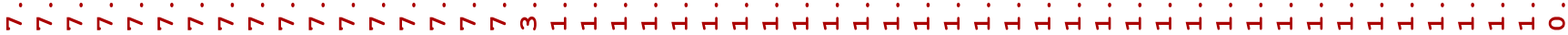
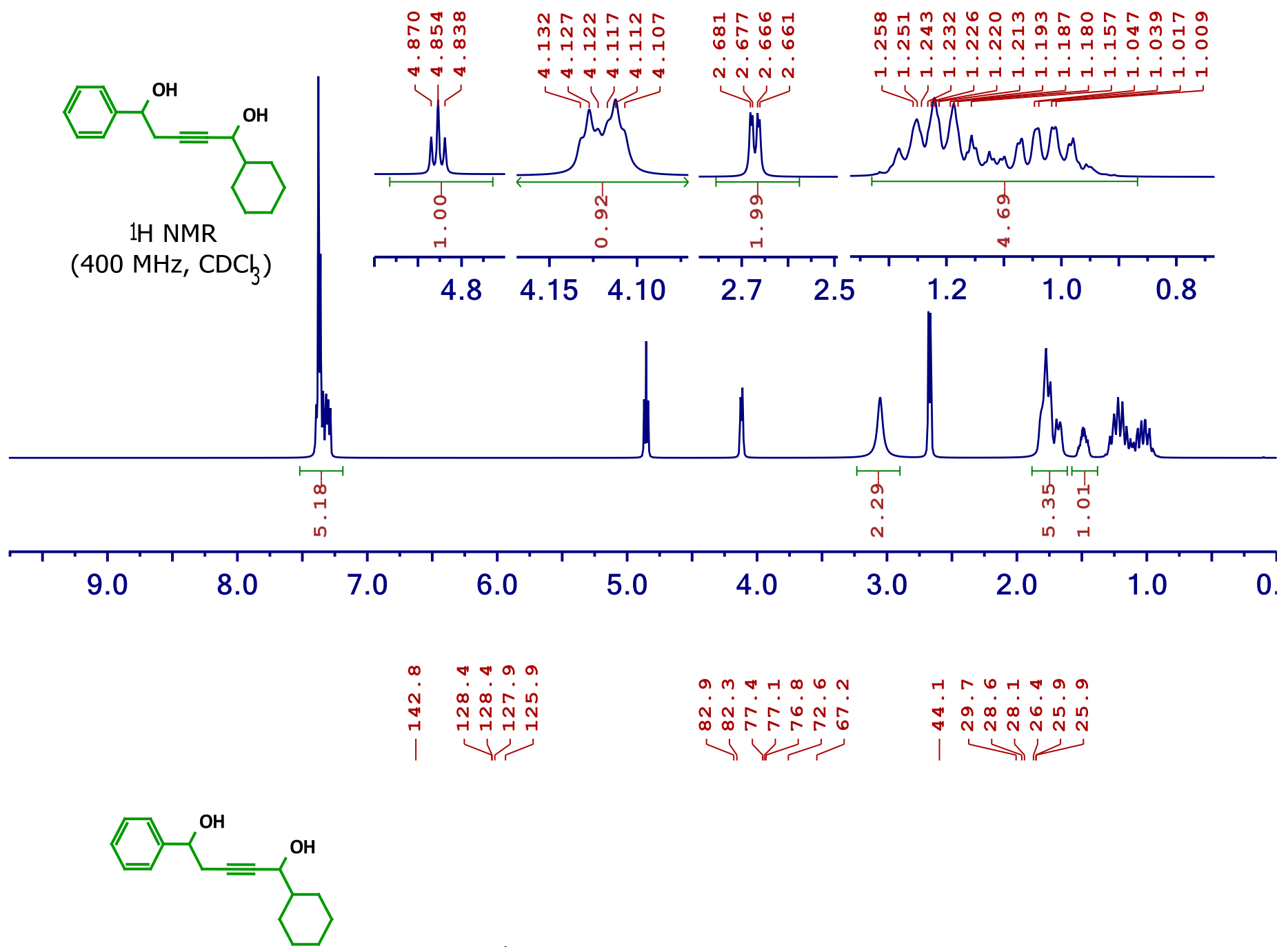

$13 \mathrm{C}\{1 \mathrm{H}\} \mathrm{NMR}$

(100 MHz, CDCh)

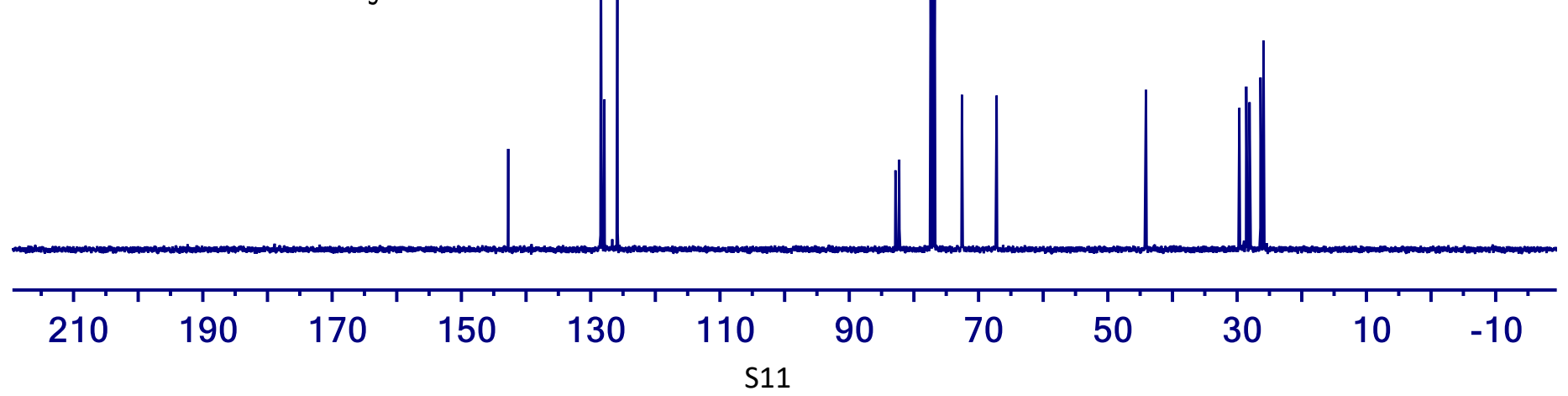




\section{1-(furan-2-yl)-5-phenylpent-2-yne-1,5-diol}

$6 \mathrm{~g}$

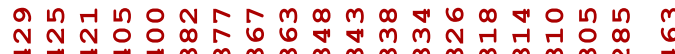

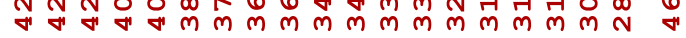

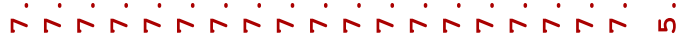

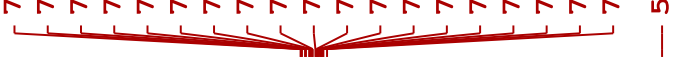

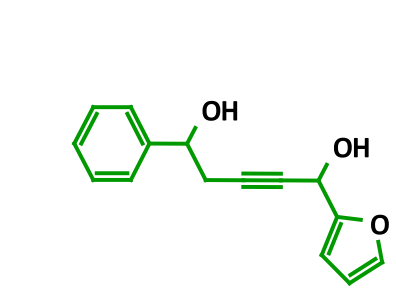

$1 \mathrm{H}$ NMR

(400 MHz, $\left.\mathrm{CDC}_{3}\right)$

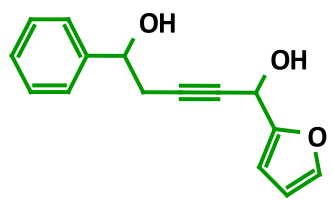

$13 \mathrm{C}\{1 \mathrm{H}\} \mathrm{NMR}$

(100 $\mathrm{MHz}, \mathrm{CDCl}_{3}$ )
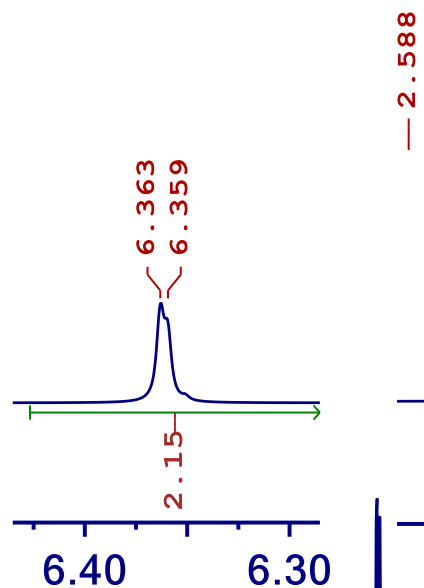

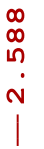

$\forall n$

ด้ ถู ดั

वं वं

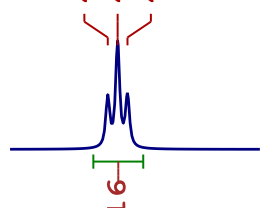

4
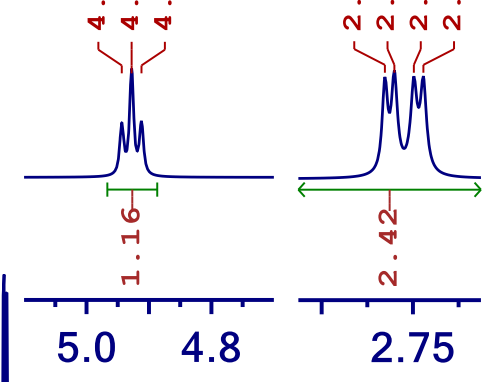

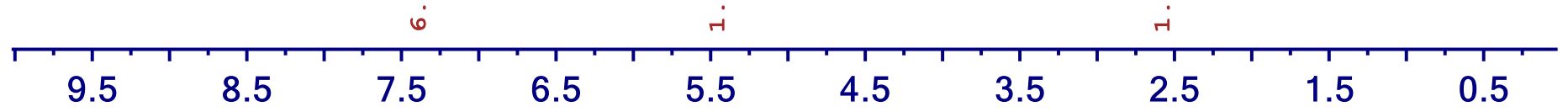

\section{5}

웅

I1

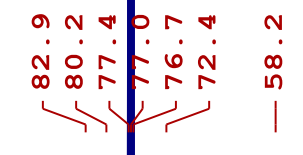

1
a
1 
6-phenylhept-3-yne-2,6-diol

$1 \mathrm{~h}$

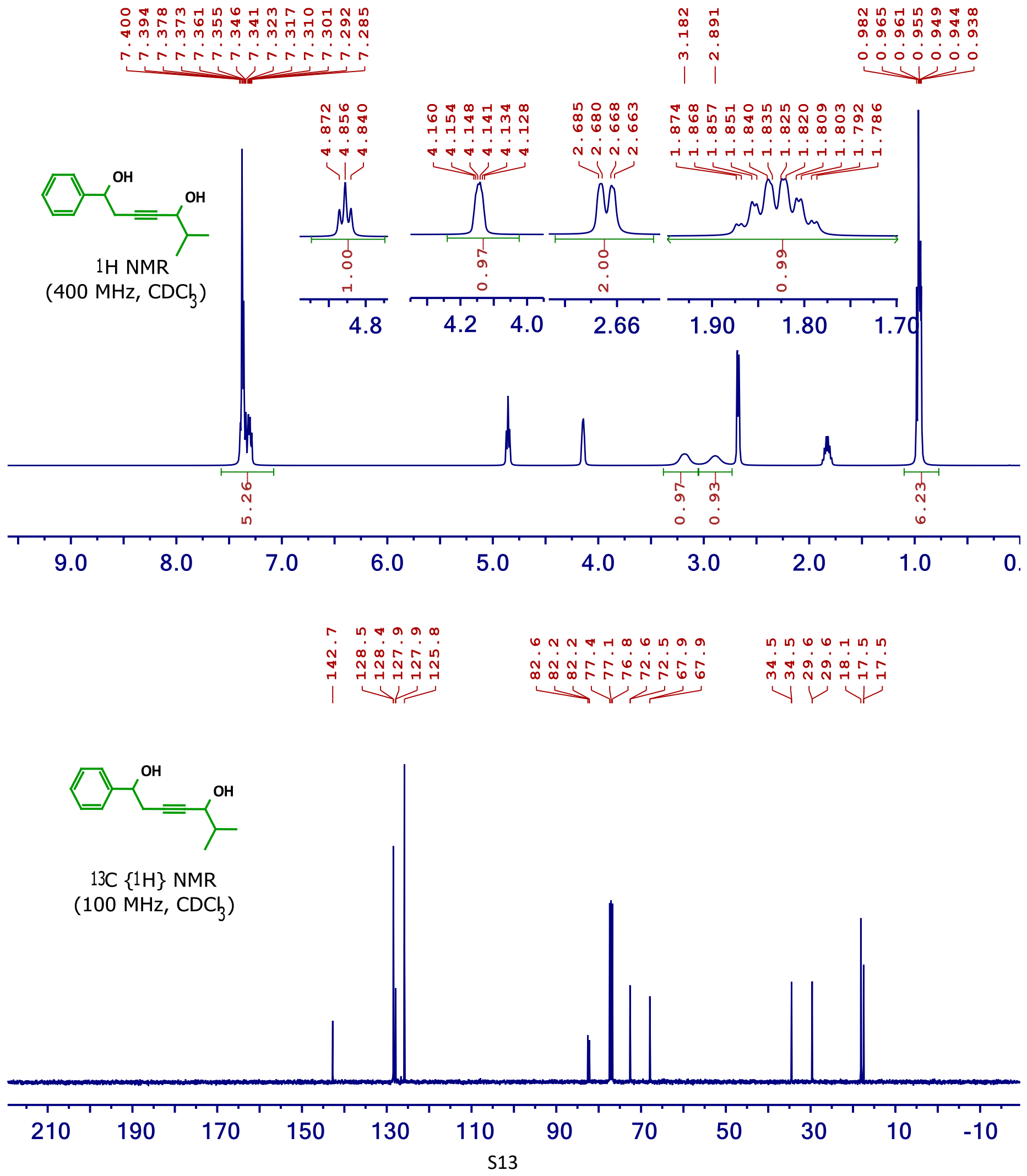


(E)-1,7-diphenylhept-6-en-3-yne-1,5-diol

$1 \mathrm{i}$

-1

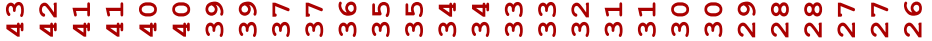

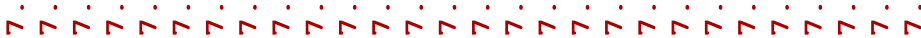

$\min _{0}$

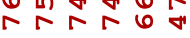

$\sin \sin$
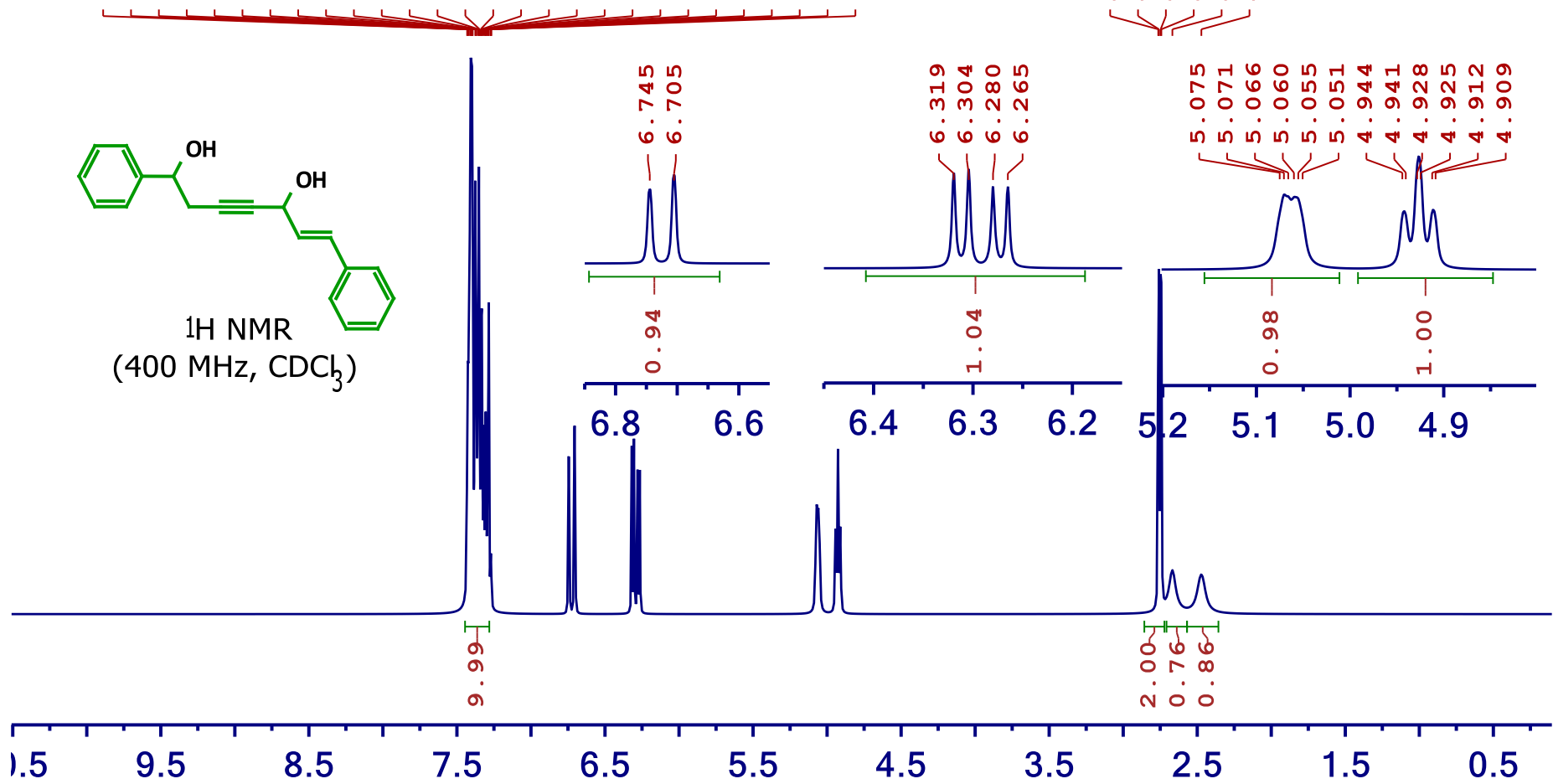

$\omega+\infty 6$ in $m+10 \infty \infty$

$\dot{\sim} \dot{0} \dot{r} \dot{\infty} \dot{\infty} \dot{\infty} \dot{\infty} \dot{\infty} \dot{\omega}$ i

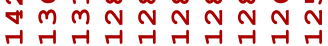

$\underbrace{\sim}$

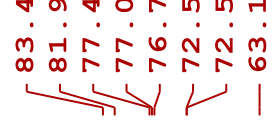

$\infty$

$\stackrel{\infty}{N}$

$\longrightarrow$
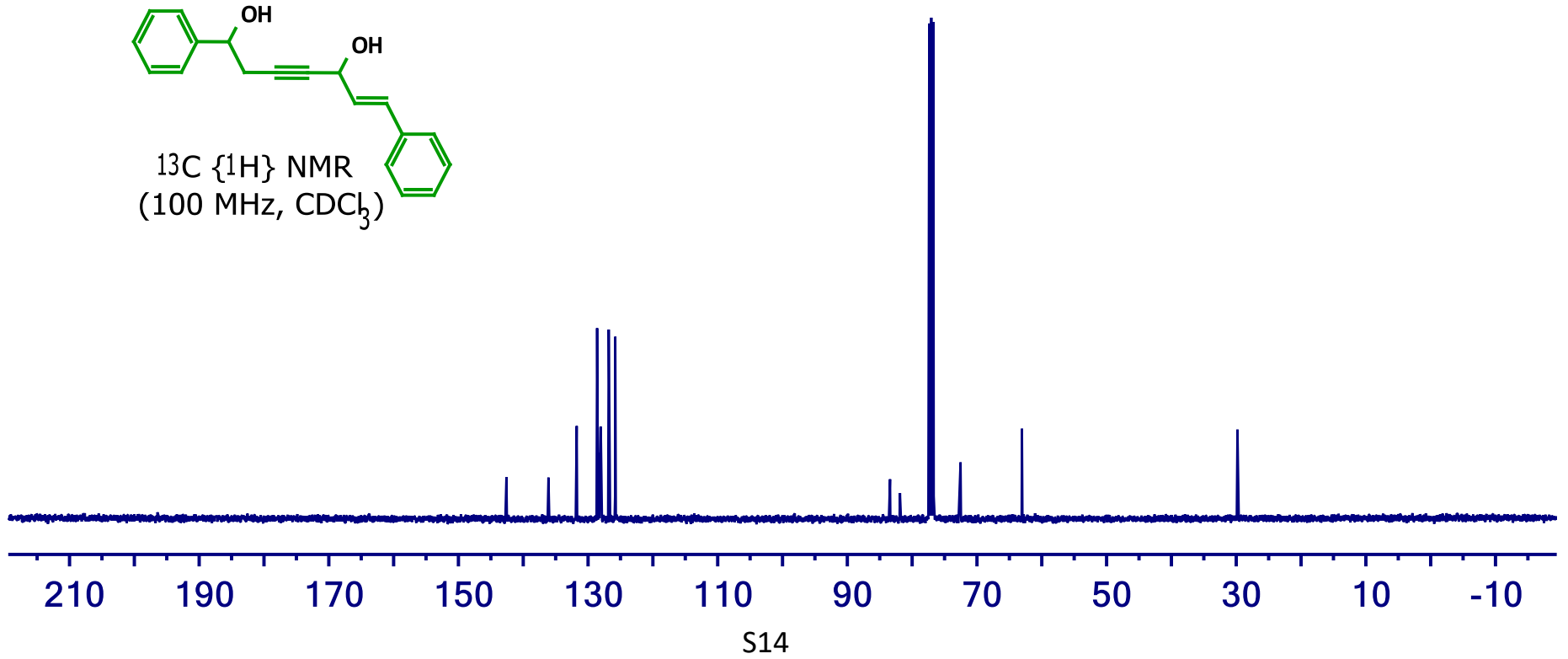
1-cyclopropyl-5-phenylpent-2-yne-1,5-diol

$1 \mathrm{j}$

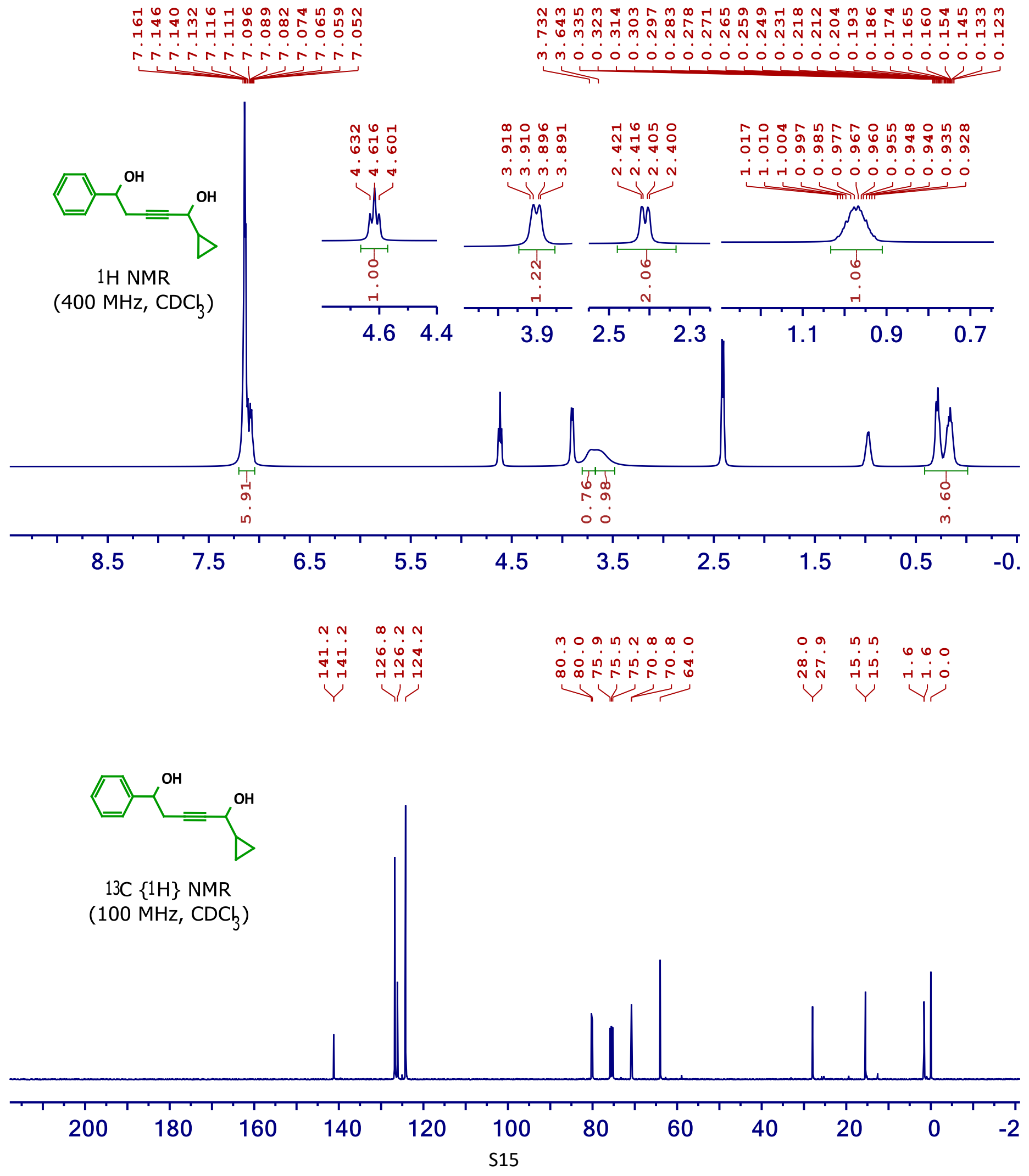


5-phenyl-1-(p-tolyl)pent-2-yne-1,5-diol

$1 \mathrm{k}$
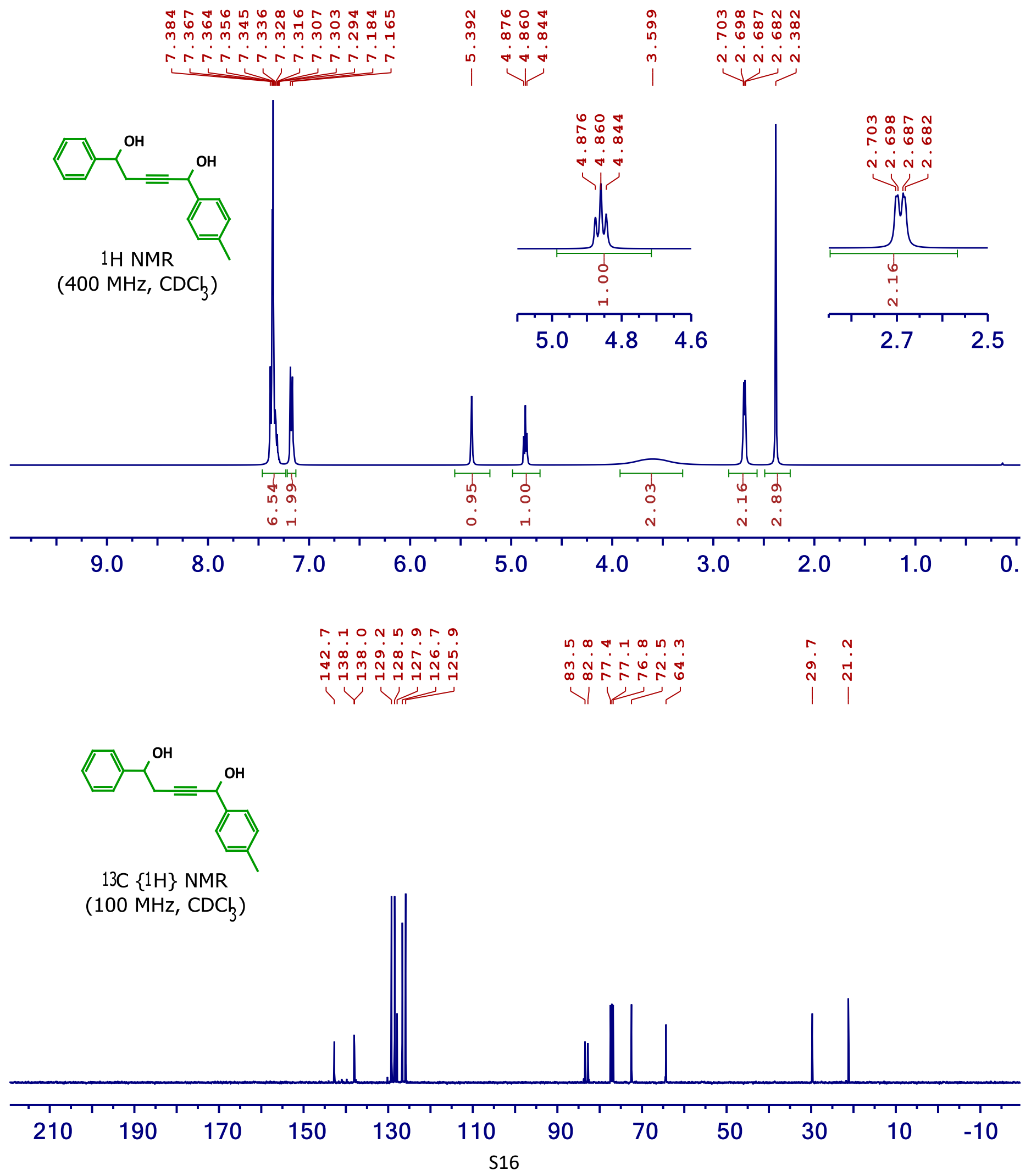
1-(4-methoxyphenyl)-5-phenylpent-2-yne-1,5-diol

11

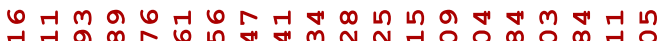

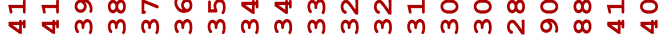

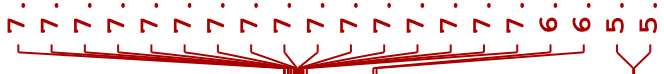

$m_{m}^{m}$

m

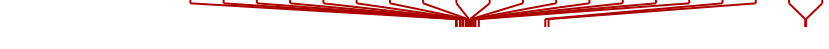

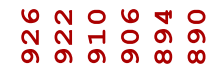

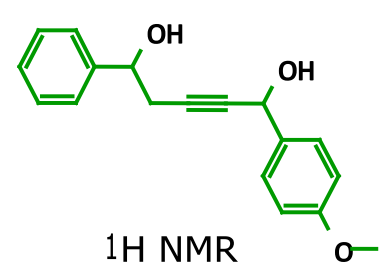

จா
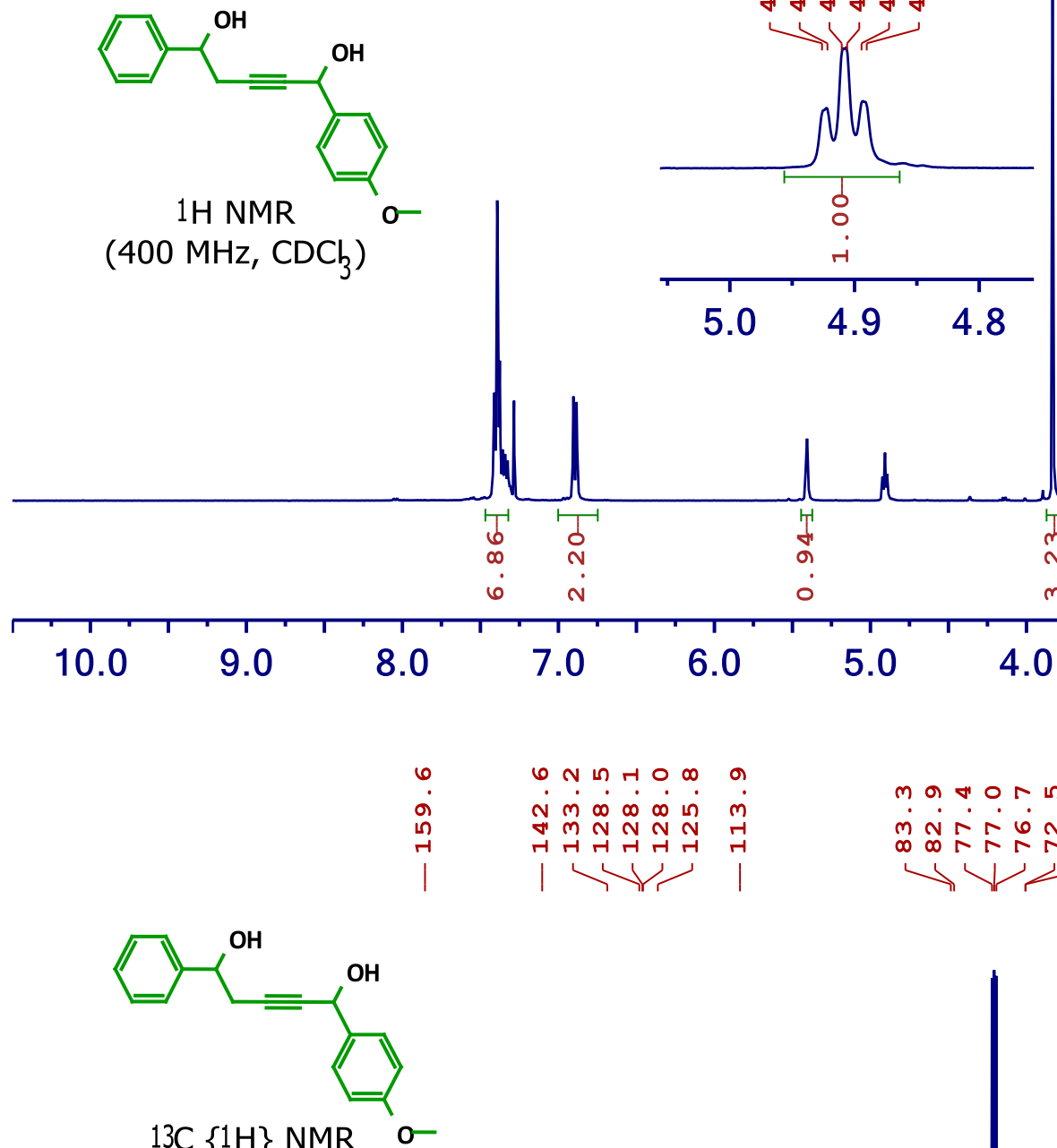

(100 MHz, CDCh)

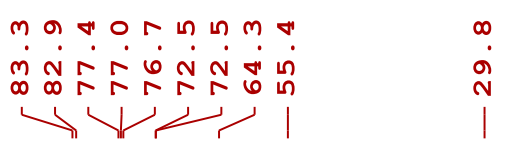

$\infty$
a
1
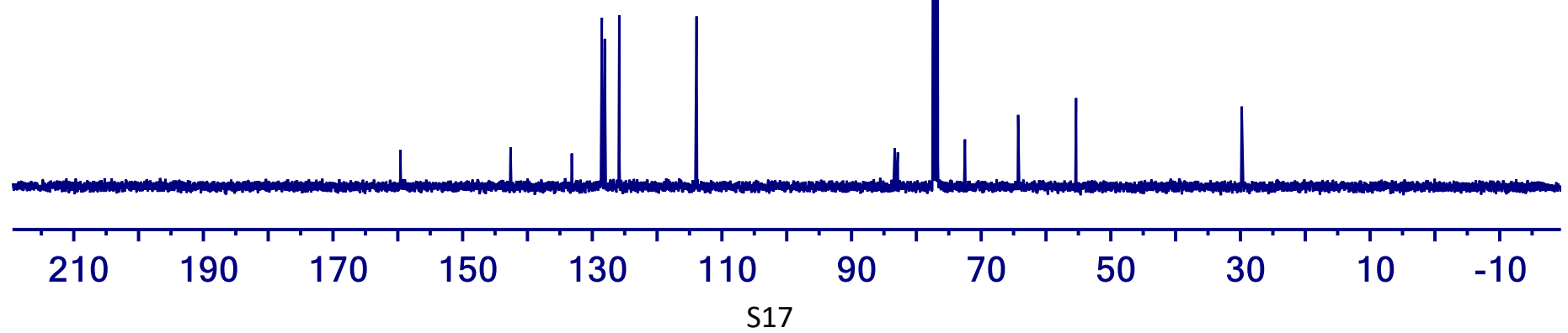
5-phenyl-1-(thiophen-2-yl)pent-2-yne-1,5-diol

$1 \mathrm{~m}$

-

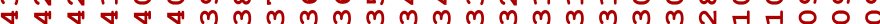

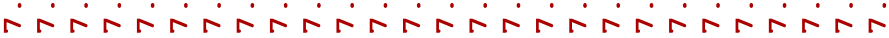

은요요

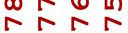

in $\cos$

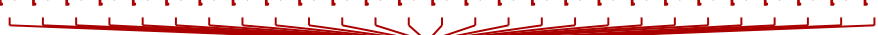

N
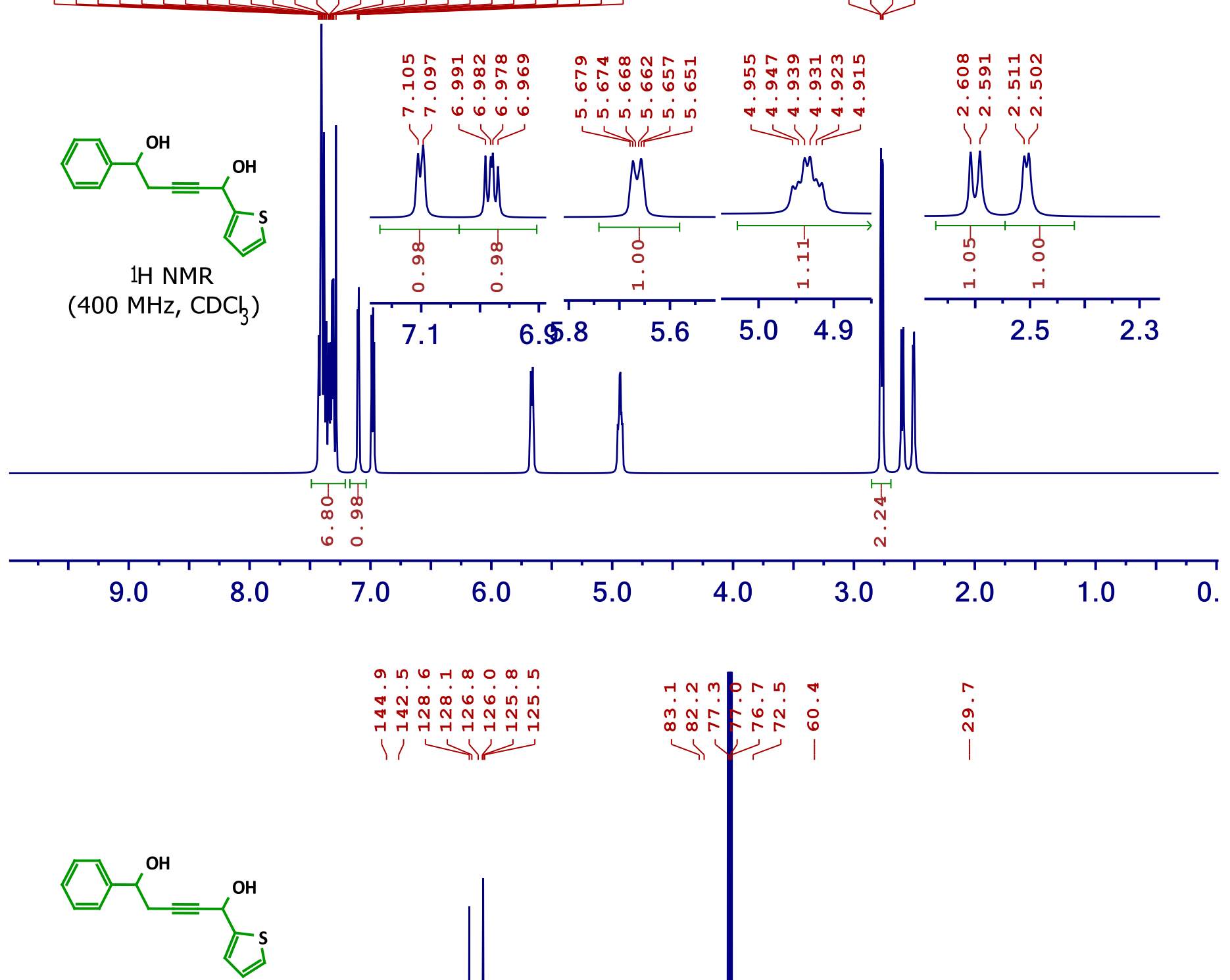

0 in 6 - $\infty \circ \infty$ in

$\dot{\sim} \dot{N} \dot{\infty} \dot{\infty} \dot{0} \dot{0}$ in

$\begin{array}{llll}4 & 4 & 4 & 4 \\ A & 4 & 4\end{array}$

$13 \mathrm{C}\{1 \mathrm{H}\} \mathrm{NMR}$

(100 $\left.\mathrm{MHz}, \mathrm{CDC}_{3}\right)$

$1 / 1$

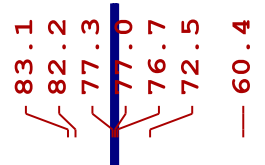

$r$ 
1-(naphthalen-1-yl)hex-3-yne-1,5-diol

$1 \mathrm{n}$

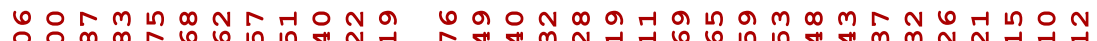
只 เก brisirisiris

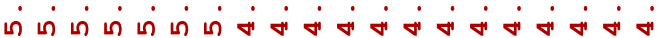

$\underset{m}{\operatorname{m}} \underset{\sim}{m} \underset{\sim}{m} \underset{\sim}{N} \underset{\forall}{\mathbb{N}}$

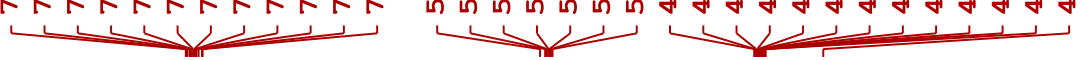

4. 4 i 4 i

4
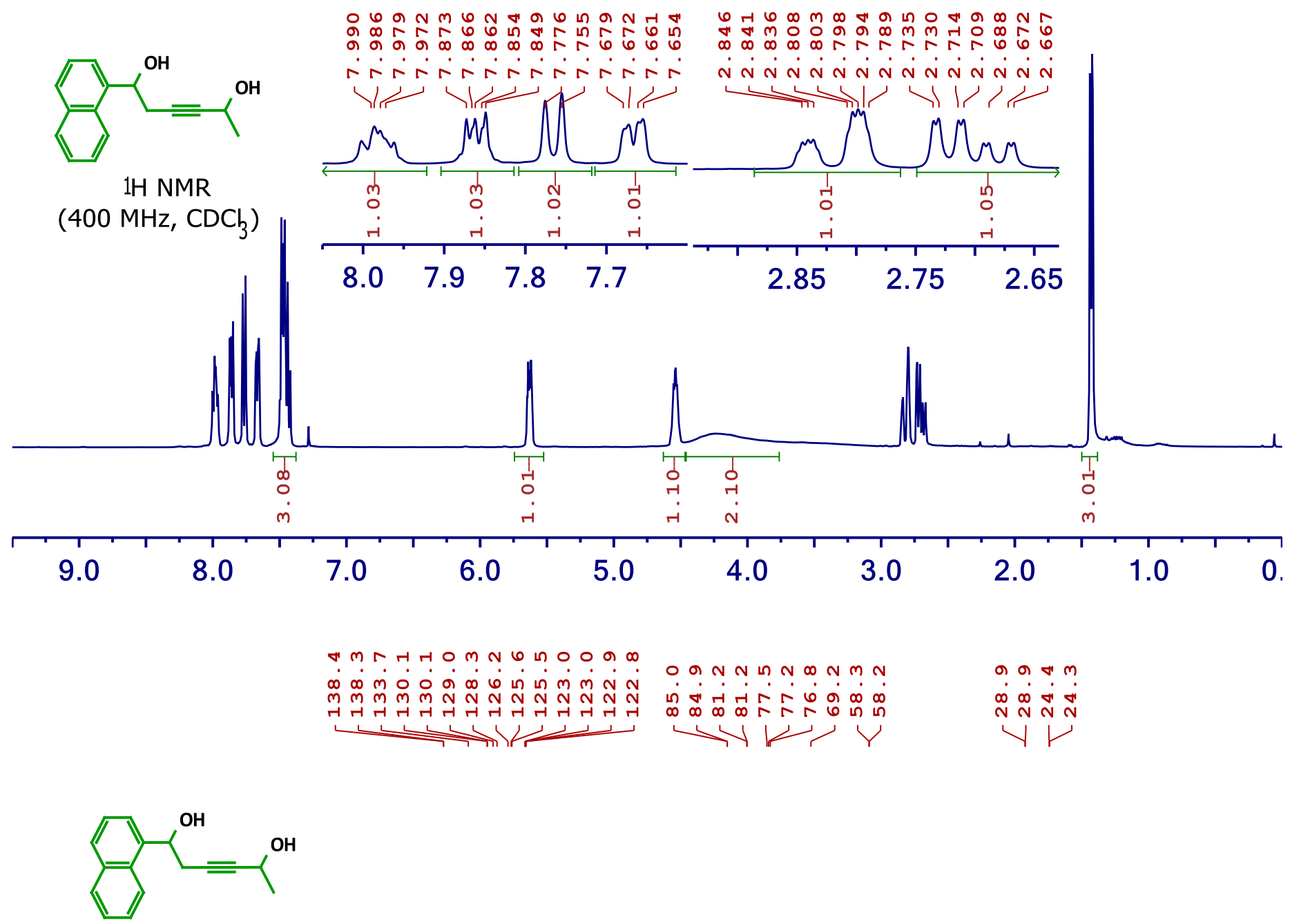

$13 \mathrm{C}\{1 \mathrm{H}\} \mathrm{NMR}$

$\left(100 \mathrm{MHz}, \mathrm{CDCl}_{3}\right)$

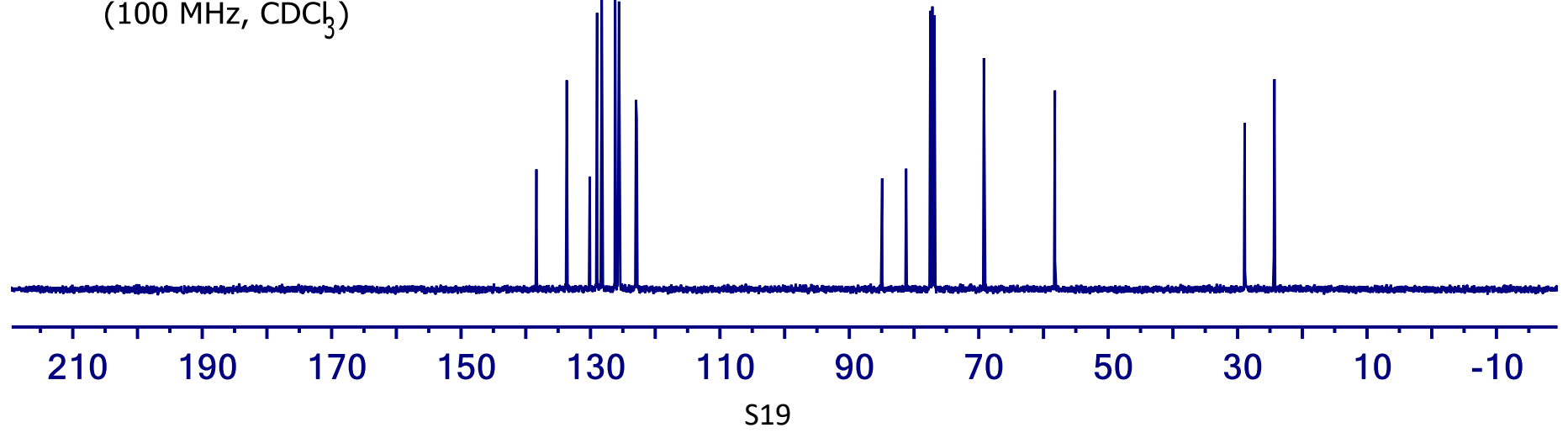




\section{1-(o-tolyl)hex-3-yne-1,5-diol}

10

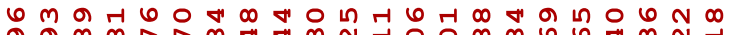
* $*$ "

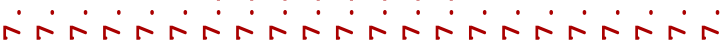

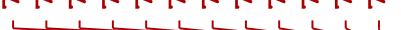

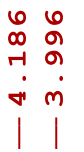

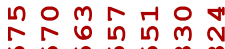
in in in in in $m \mathrm{~m}$

nin

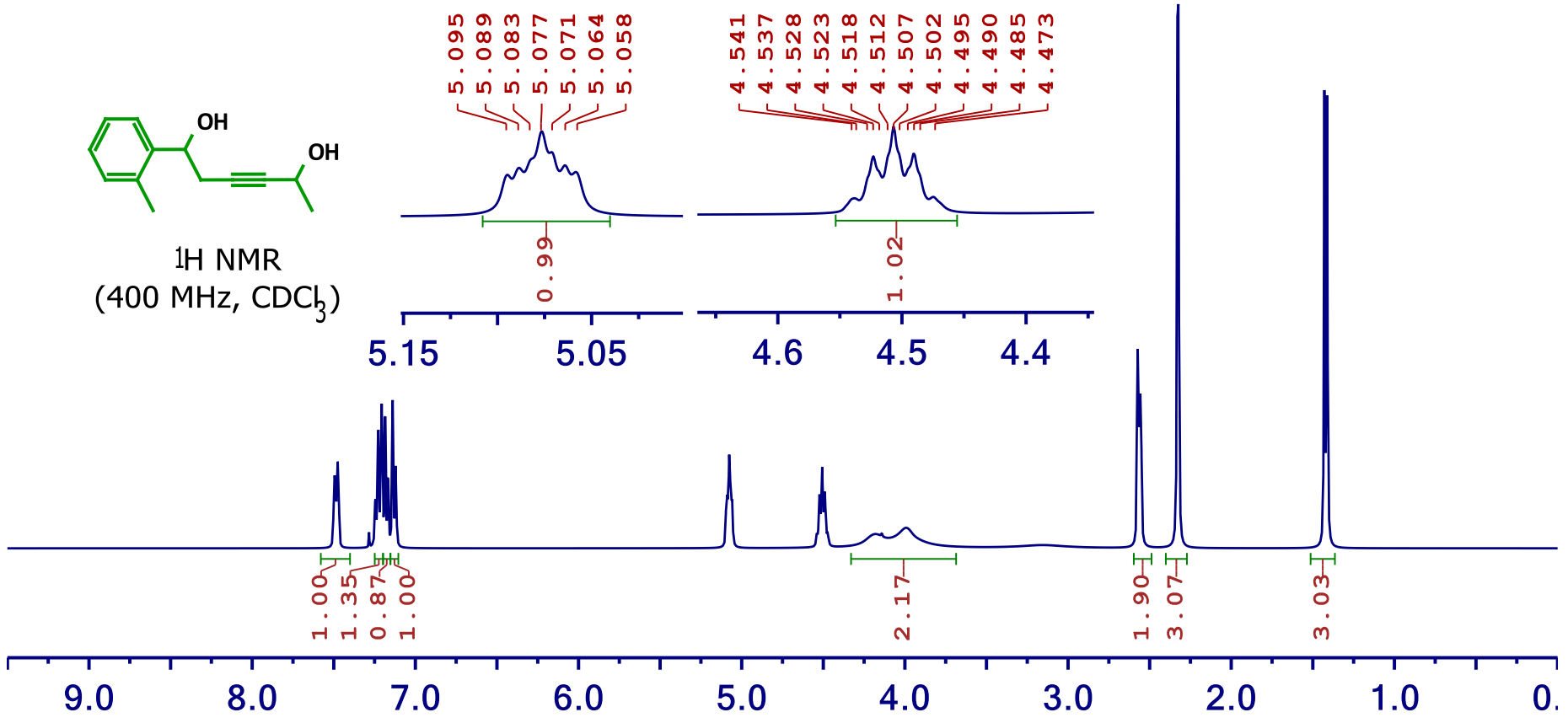

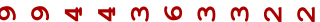
$\dot{\circ} \circ \dot{\nabla} \dot{0} \dot{\sim} \dot{0} \dot{0}$ in

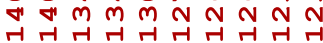

ก $\mathrm{H}$ ก $\mathrm{Na}$ a $\mathrm{N}$ $\dot{\infty}$
$\infty$

$\longrightarrow$ $\nabla n m+r$

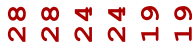

$\rightarrow Y$

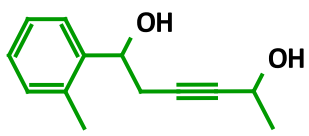

$13 \mathrm{C}\{1 \mathrm{H}\} \mathrm{NMR}$

(100 $\mathrm{MHz}, \mathrm{CDC}_{3}$ )

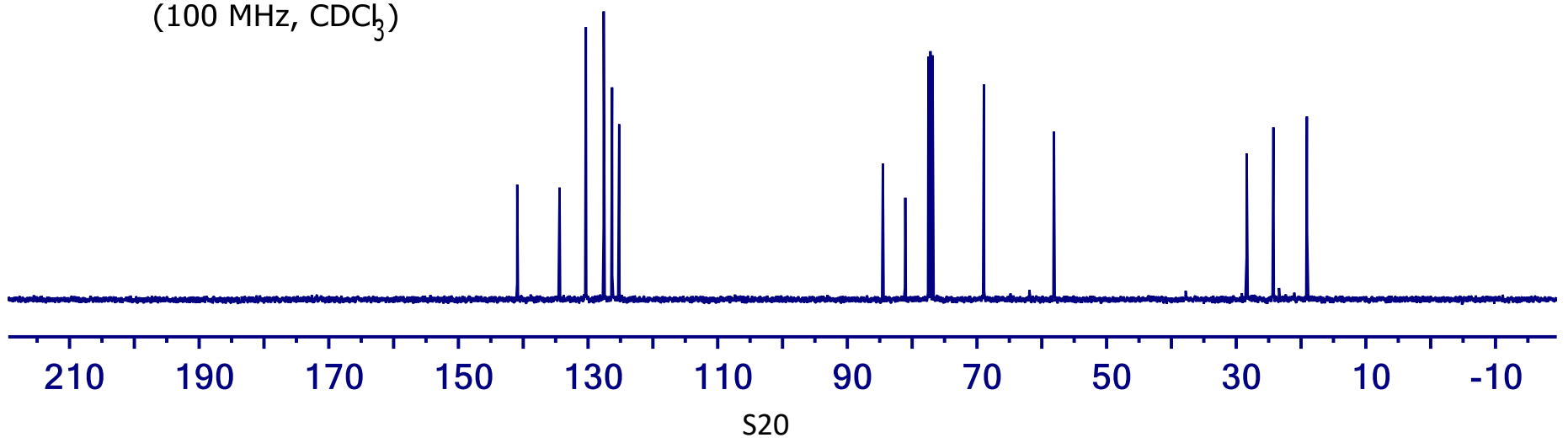


1-(4-methoxyphenyl)hex-3-yne-1,5-diol

$1 p$

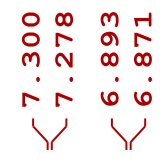

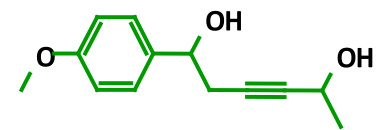

$1 \mathrm{H}$ NMR

(400 MHz, $\mathrm{CDC}_{3}$ )

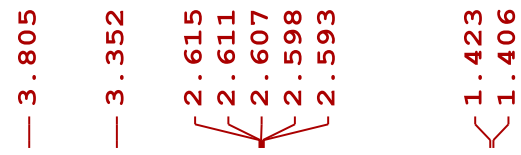

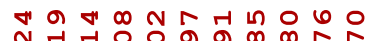
กำ

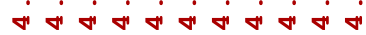
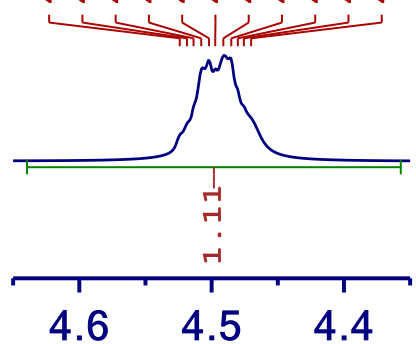

ن'

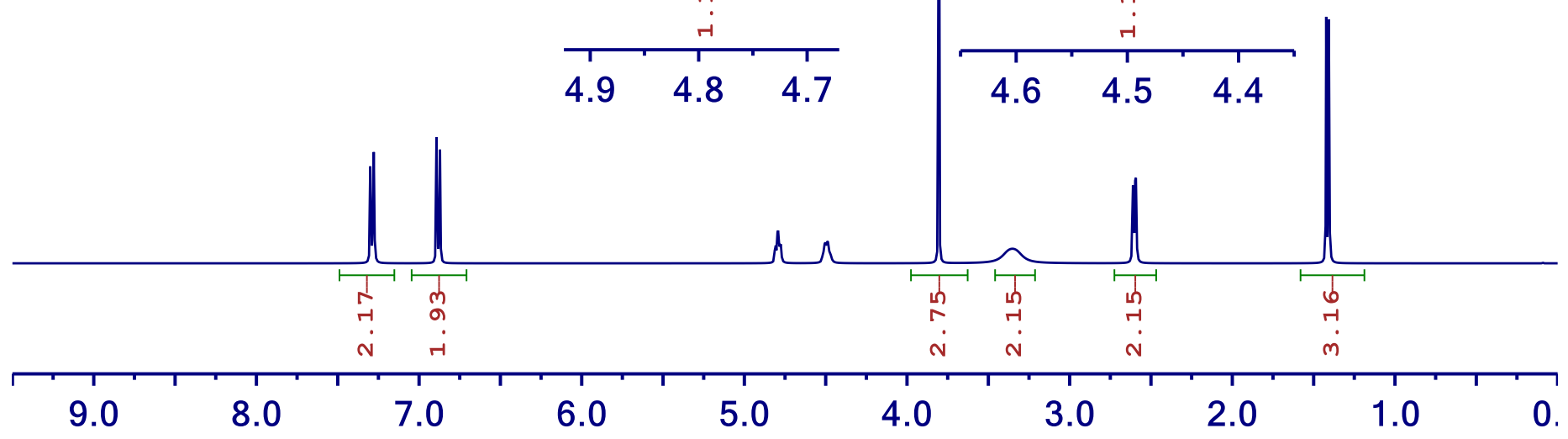

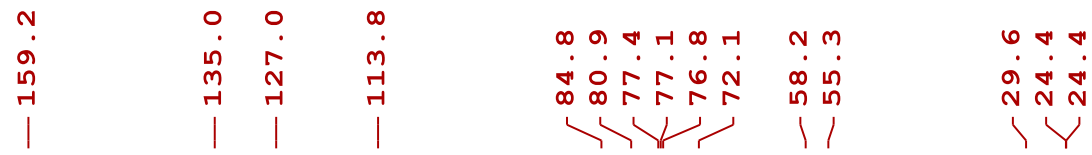

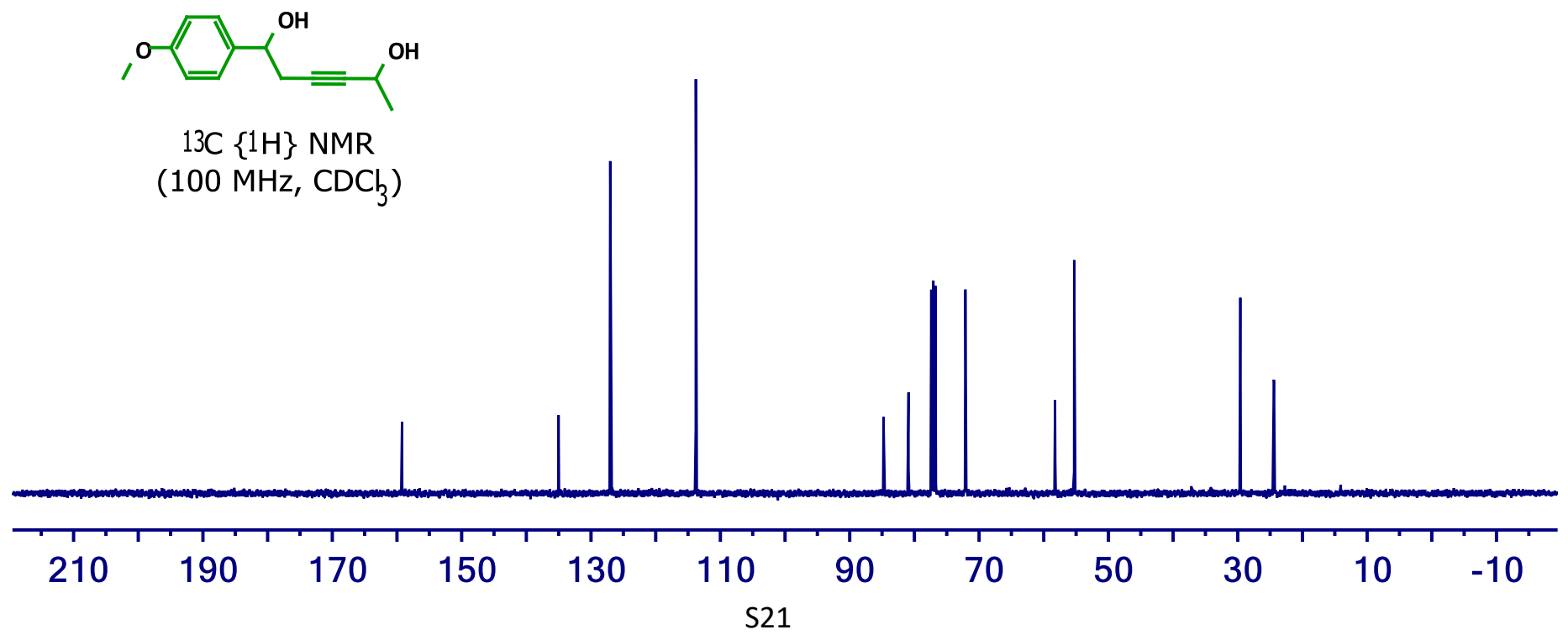

$13 \mathrm{C}\{1 \mathrm{H}\} \mathrm{NMR}$

(100 MHz, CDCh)

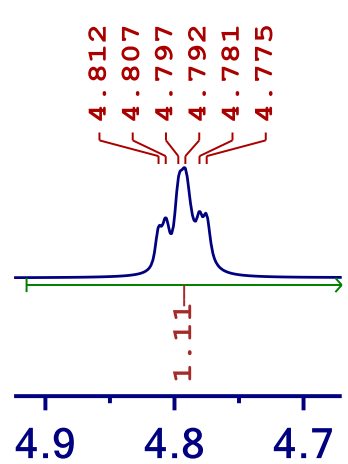


7-phenylhept-3-yne-2,6-diol

$1 \mathrm{q}$

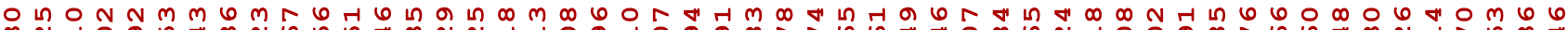

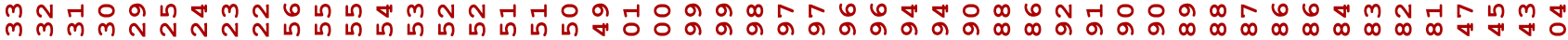

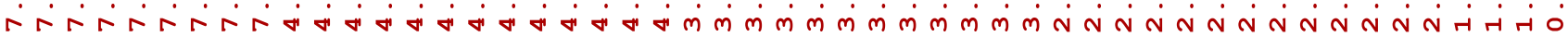
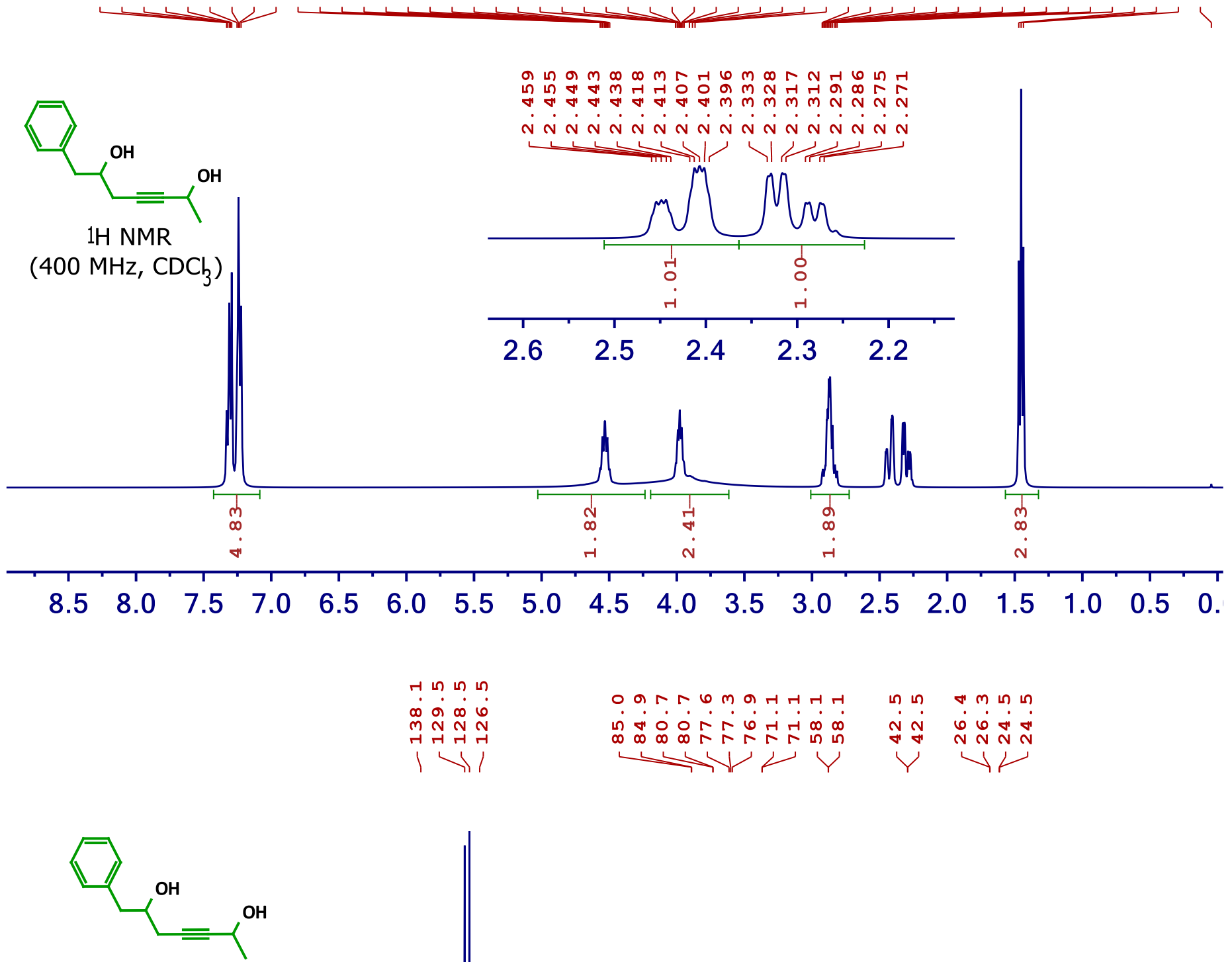

$13 \mathrm{C}\{1 \mathrm{H}\} \mathrm{NMR}$ $\left(100 \mathrm{MHz}, \mathrm{CDCh}_{3}\right)$

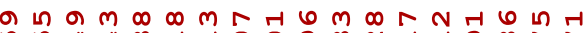

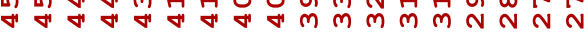

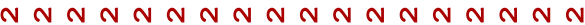


(E)-8-phenyloct-7-en-3-yne-2,6-diol

$1 \mathbf{r}$

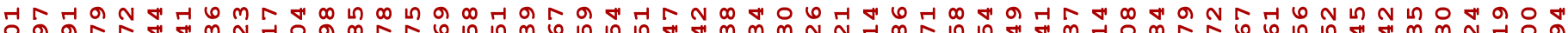

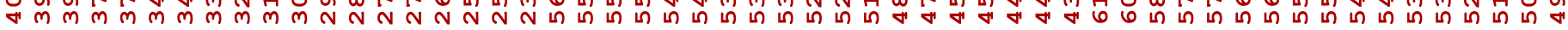

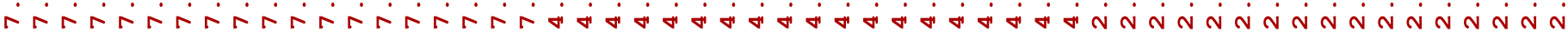
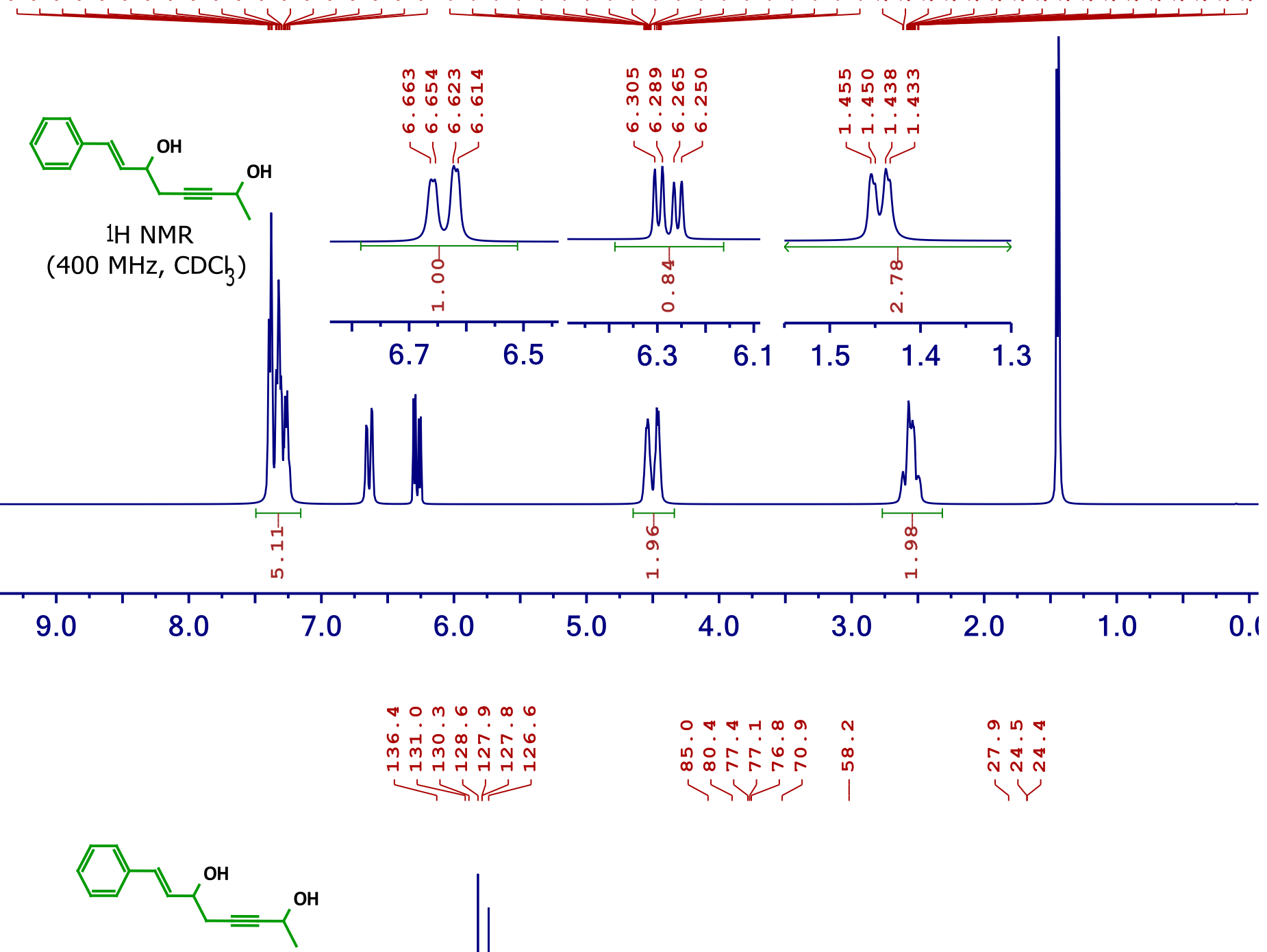

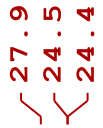

$13 \mathrm{C}\{1 \mathrm{H}\} \mathrm{NMR}$

(100 $\mathrm{MHz}, \mathrm{CDC}_{3}$ )
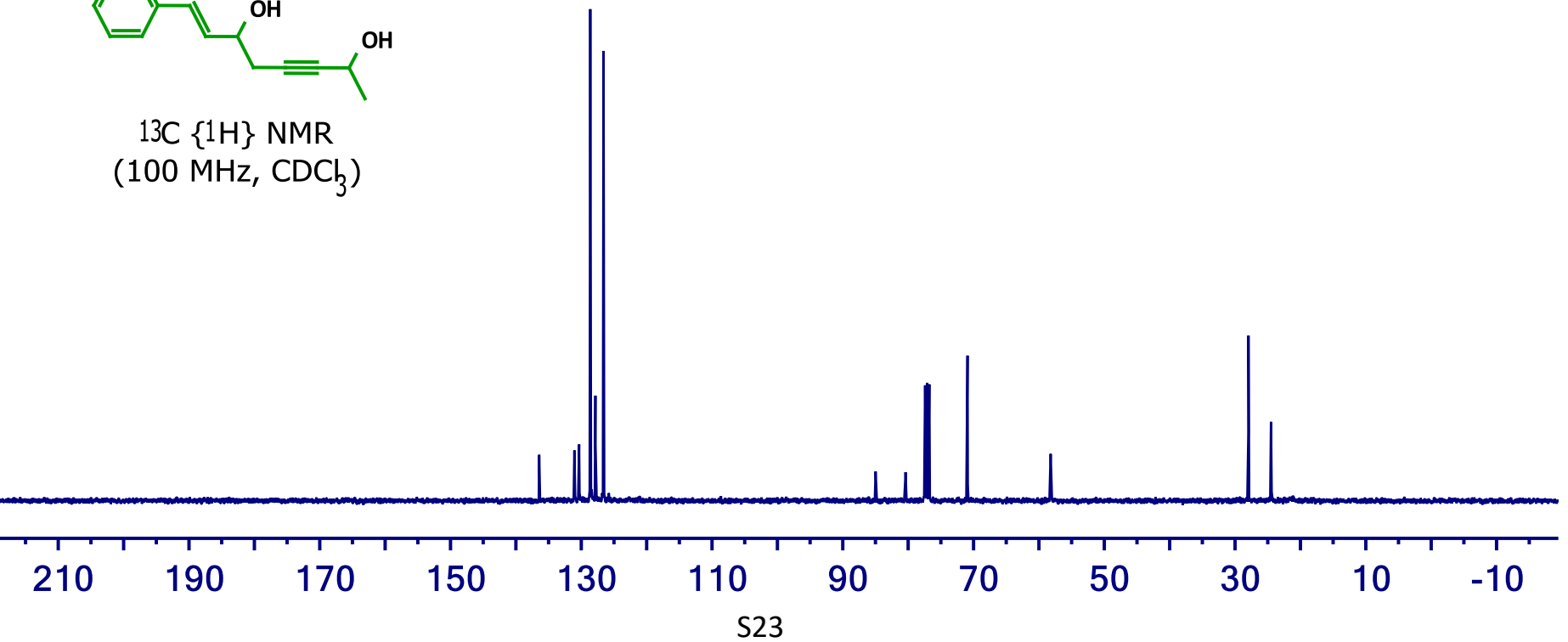
dec-3-yne-2,6-diol

$1 \mathrm{~s}$

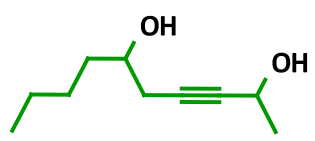

- $m$ ก m

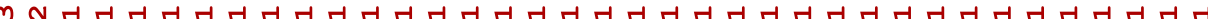

$1 \mathrm{H} N \mathrm{NMR}$ (400 MHz, $\left.\mathrm{CDC}_{3}\right)$

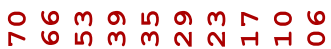

rroristris

$\dot{m} \dot{m} \dot{m} \dot{m} \dot{m} \dot{m} \dot{m} \dot{m}$

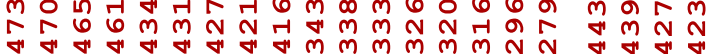

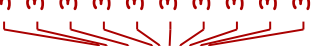

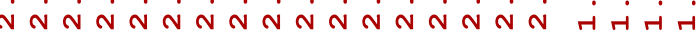

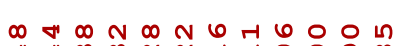

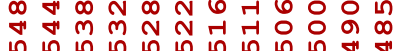

वं वं वं वं वं वं वं वं
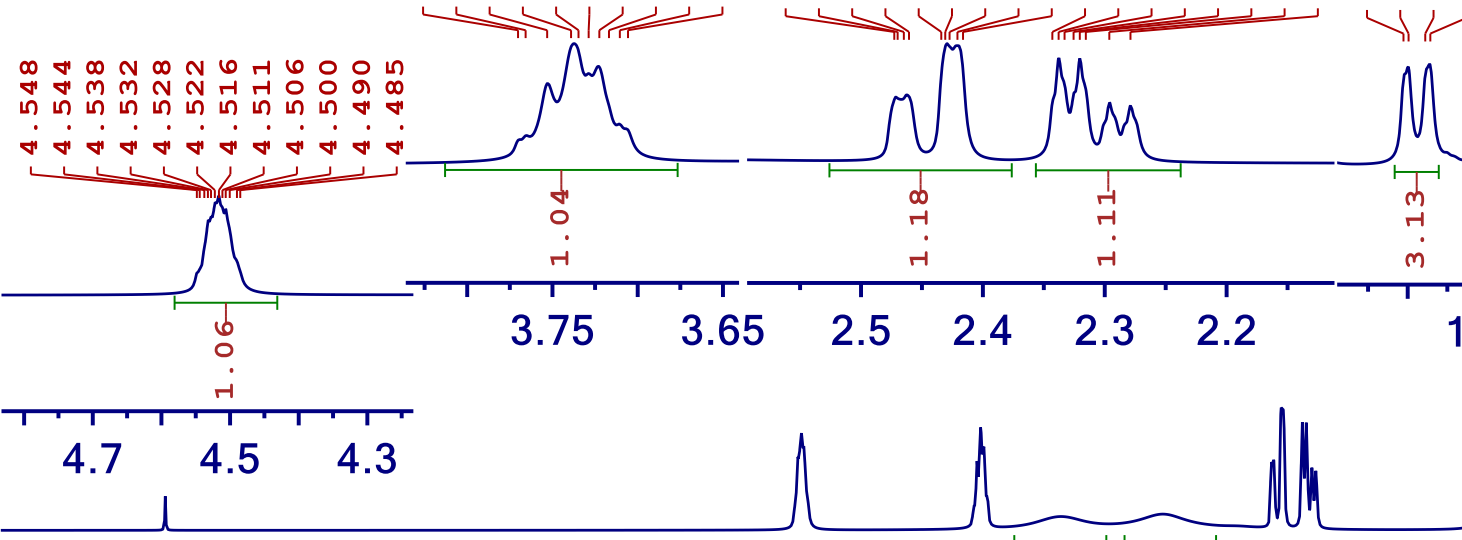

4

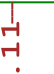

4

$\begin{array}{llll}2.5 & 2.4 & 2.3 & 2.2\end{array}$
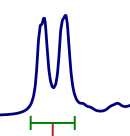

r.

m
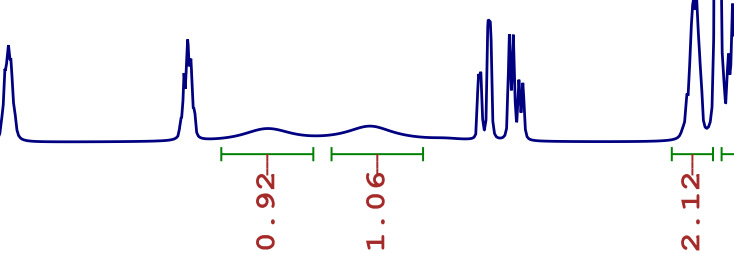

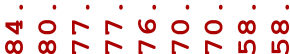

a $a \infty m m a n \ln 00$

Y மூ

$\rightarrow$

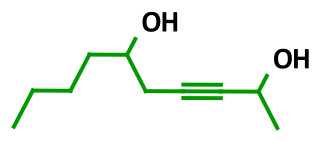

$13 \mathrm{C}\{1 \mathrm{H}\} \mathrm{NMR}$

(100 MHz, CDCh)

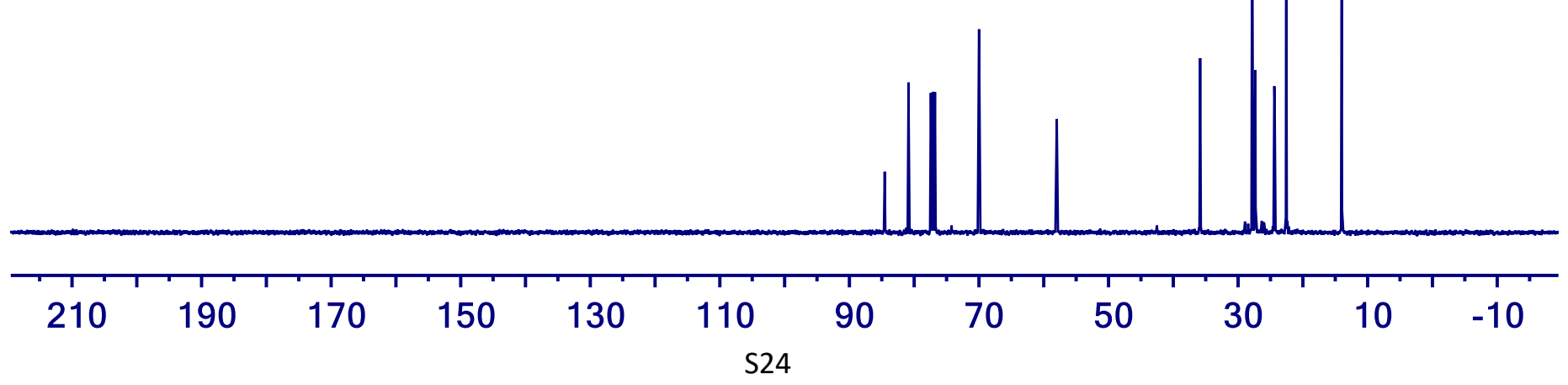


m 崩

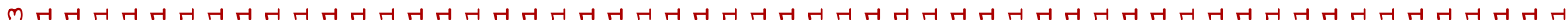
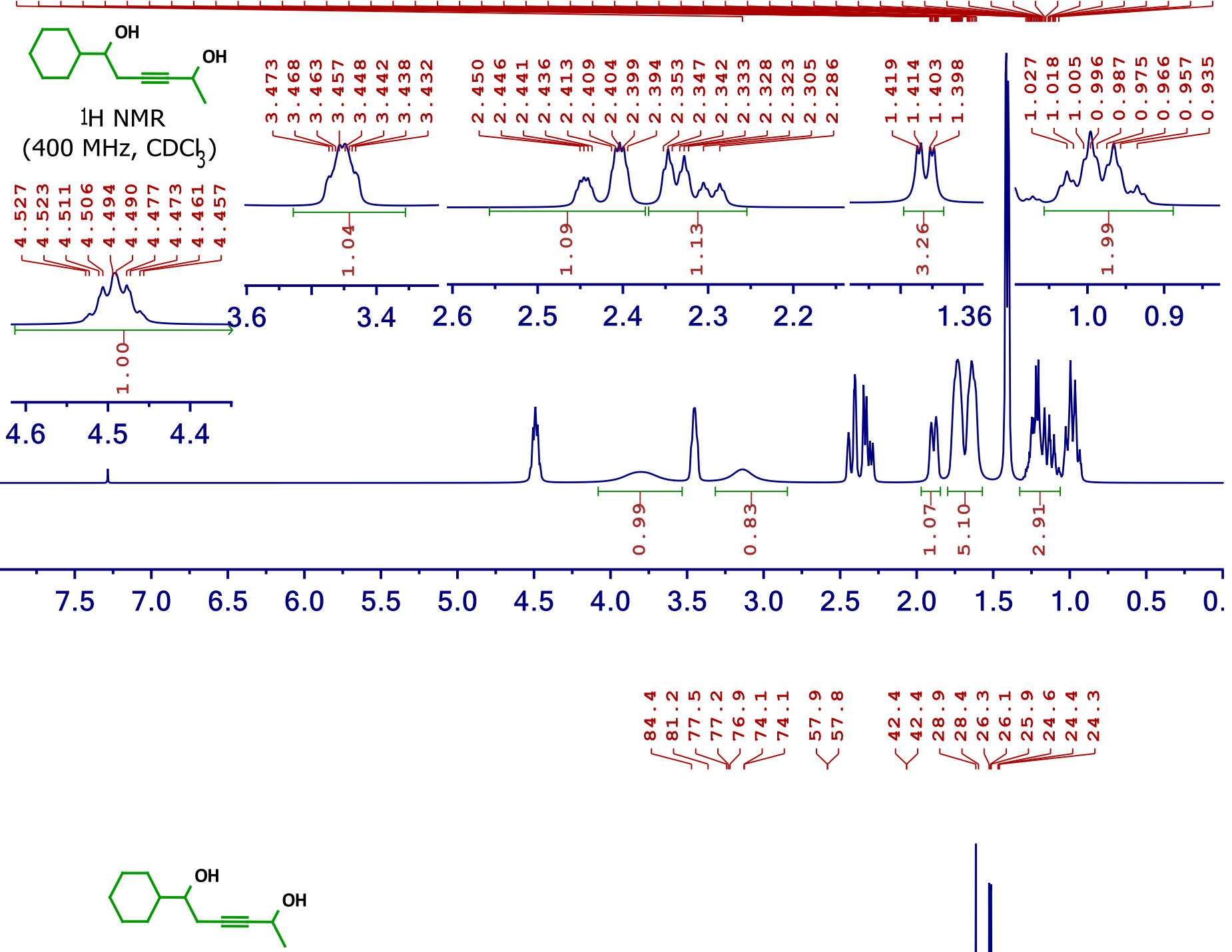

Rn 2 เ 2 a -1 a $\infty$ 悉

*

$13 \mathrm{C}\{1 \mathrm{H}\} \mathrm{NMR}$ (100 $\left.\mathrm{MHz}, \mathrm{CDC}_{3}\right)$

\begin{tabular}{llllllllllll}
\hline 210 & 190 & 170 & 150 & 130 & 110 & 90 & 70 & 50 & 30 & 10 & -10
\end{tabular}




\section{1-(2-chlorophenyl)hex-3-yne-1,5-diol}

* $66 \mathrm{mmMMN} N \mathrm{~N} N \mathrm{~N} N$

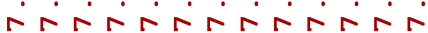
$\longrightarrow$

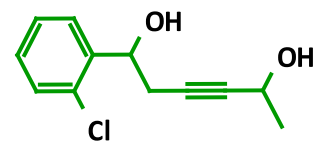

$1 \mathrm{H}$ NMR (400 MHz, CDCb)
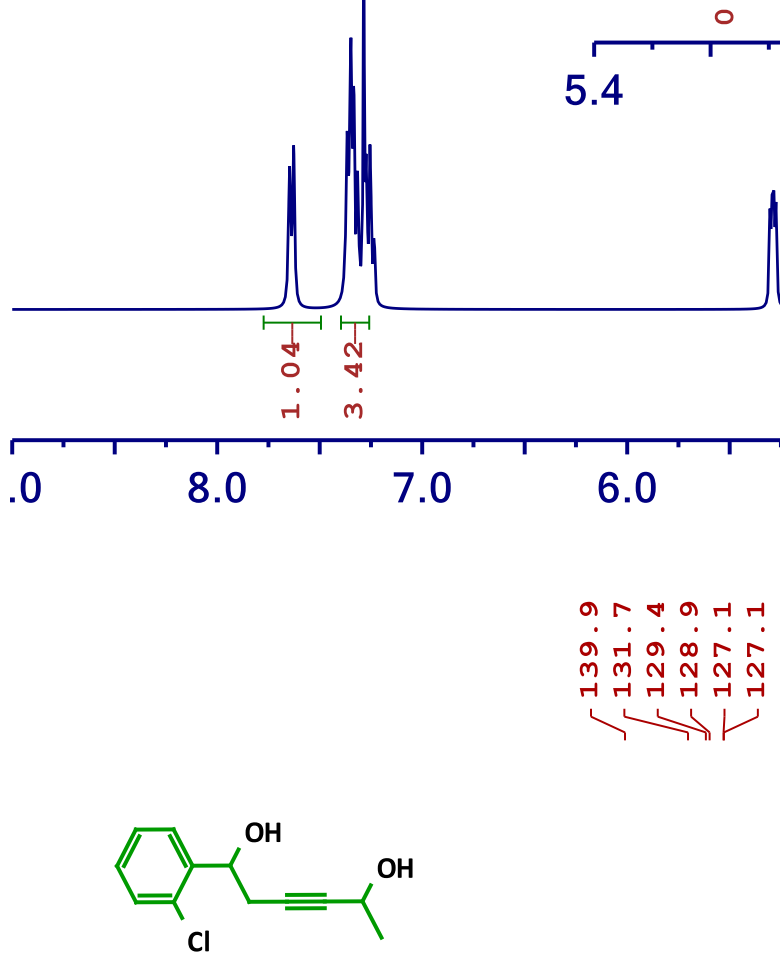

$13 \mathrm{C}\{1 \mathrm{H}\} \mathrm{NMR}$ (100 MHz, $\mathrm{CDC}_{3}$ )

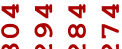

M N N

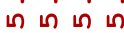

)!
$1 \mathrm{u}$

ด ด

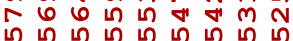

هั
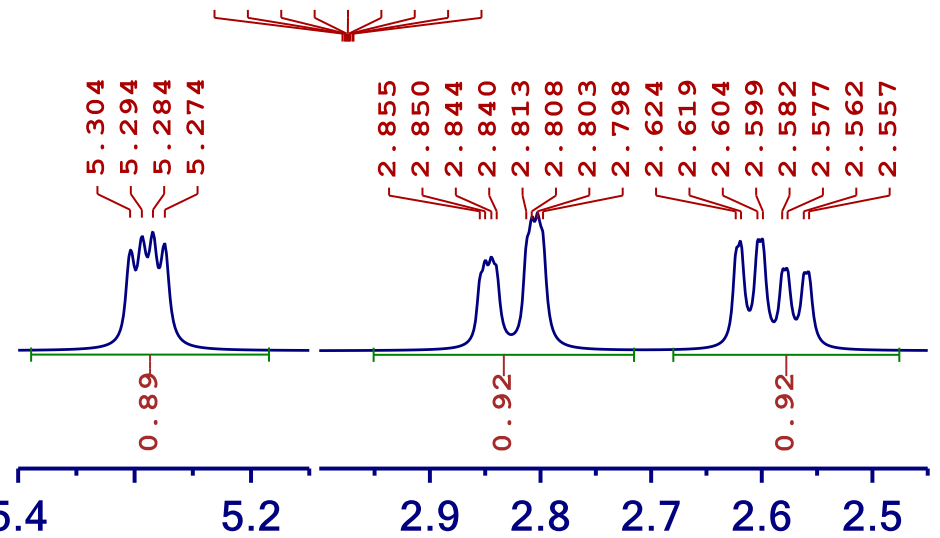

5.2

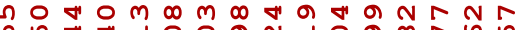

ก ก

a v

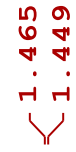

से

Y

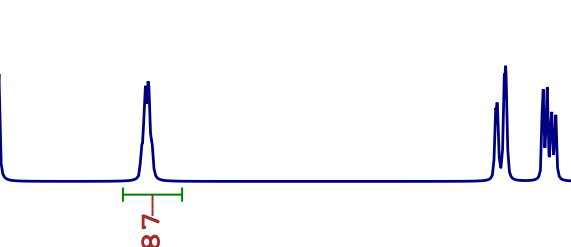

:

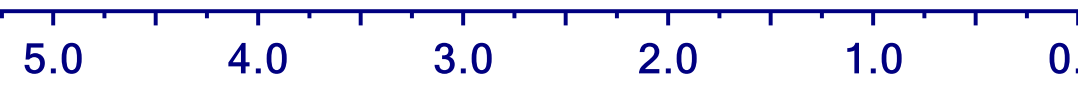

0 in

$\stackrel{\infty}{\sim} \stackrel{\sim}{N}$

$\infty \int_{\infty}=0$

$\rightarrow$

11

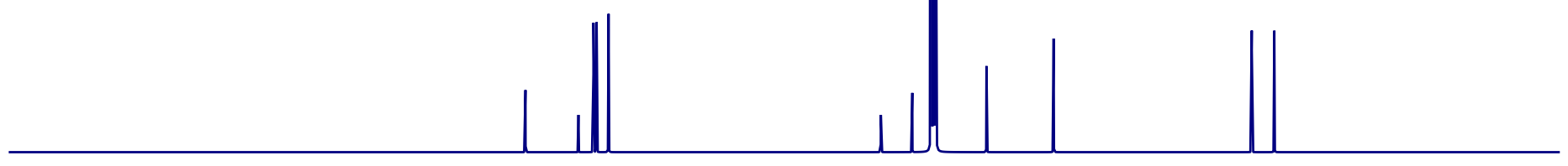

\begin{tabular}{llllllllllll}
\hline 210 & 190 & 170 & 150 & 130 & 110 & 90 & 70 & 50 & 30 & 10 & -10
\end{tabular}


1-(4-isopropylphenyl)hex-3-yne-1,5-diol

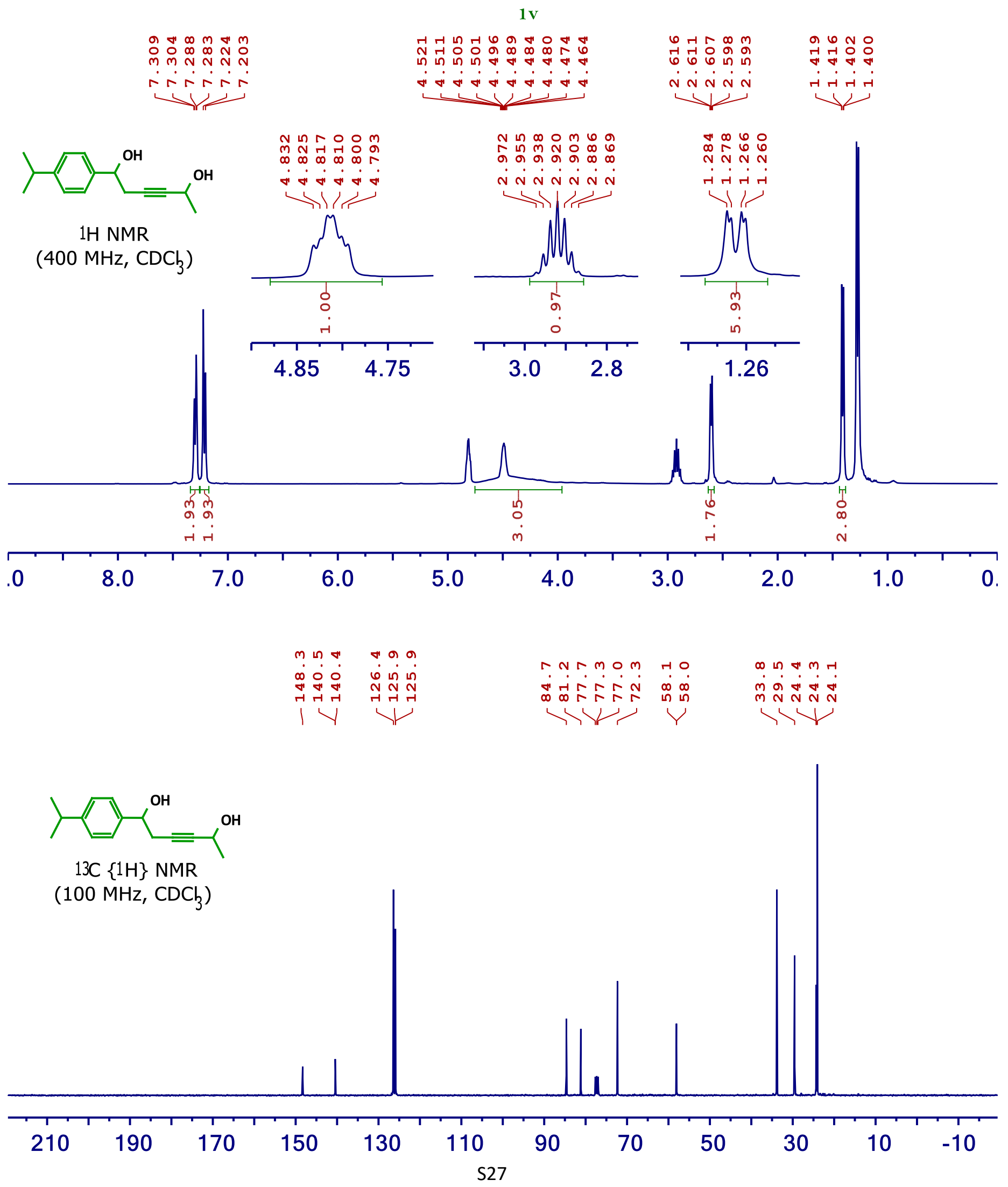


1-(4-methoxyphenyl)-6-phenylhex-2-yne-1,5-diol

$1 \mathrm{~W}$

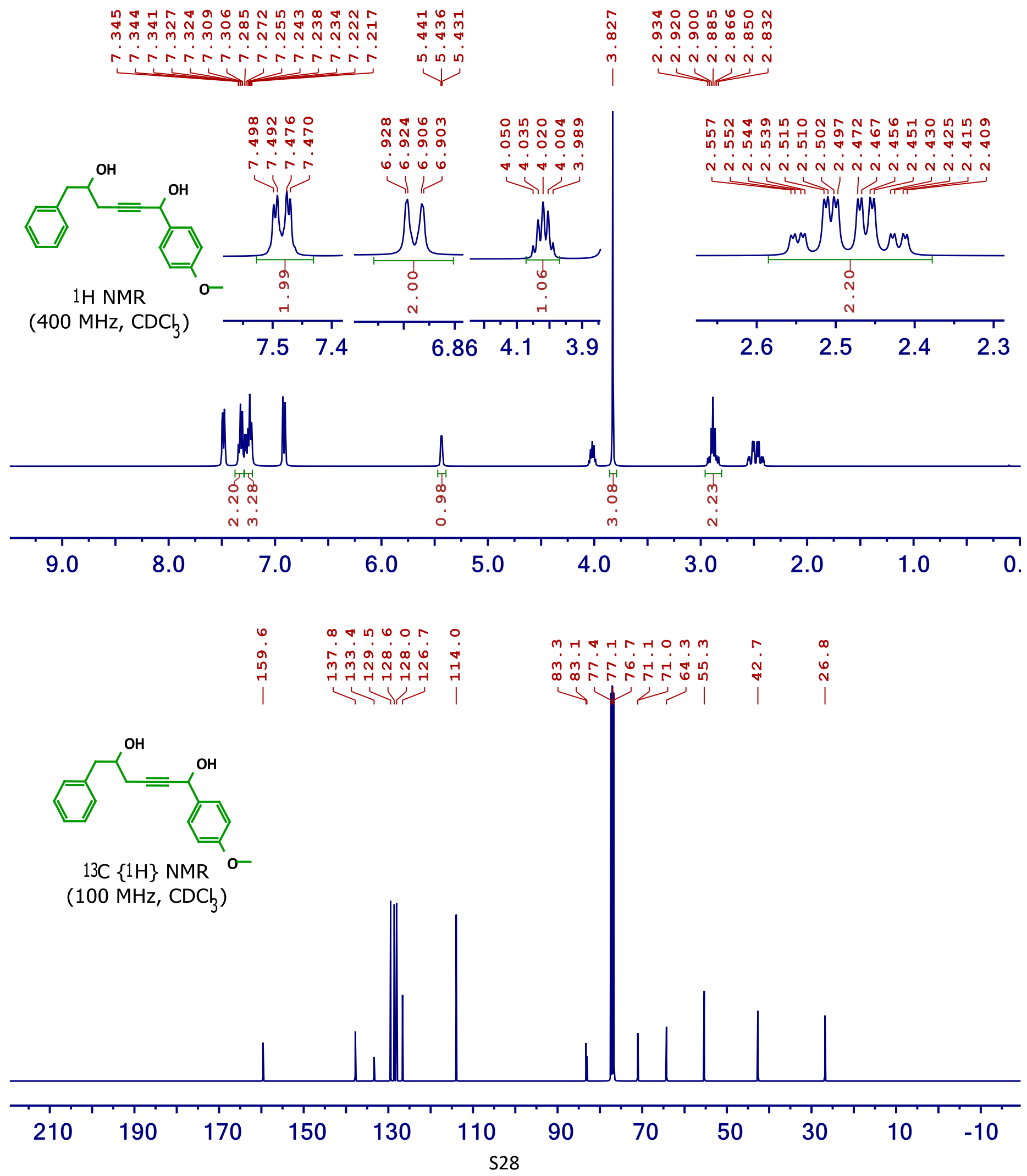




\section{1-(4-hydroxypent-2-yn-1-yl)cyclopentan-1-ol}

$1 \mathrm{x}$

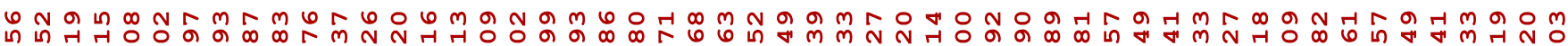

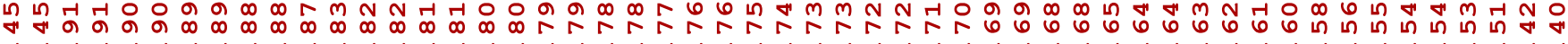

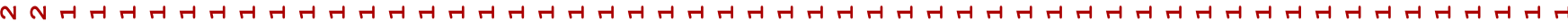

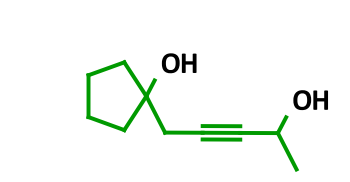

$1 \mathrm{H} N M R$

$\left(400 \mathrm{MHz}, \mathrm{CDC}_{3}\right)$

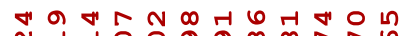

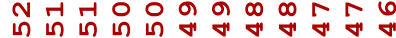

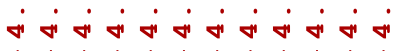
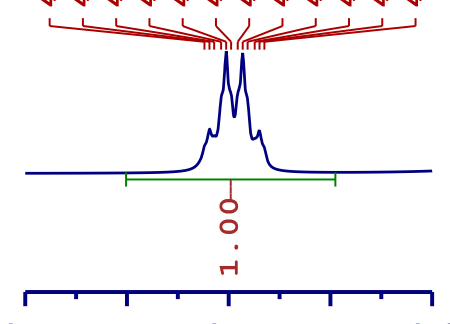

4.7

4.5

4.3

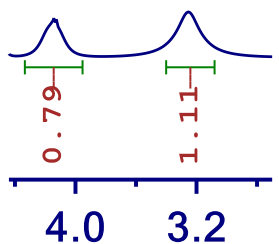

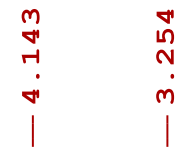

1

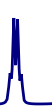

NMn $\mathrm{N}$ -

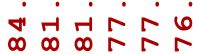

$\longrightarrow$

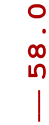

H 6 in 0

बे में

$1<1$

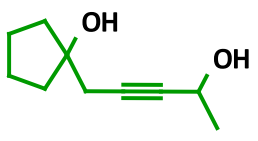

$13 \mathrm{C}\{1 \mathrm{H}\} \mathrm{NMR}$

(100 MHz, $\mathrm{CDC}_{3}$ )

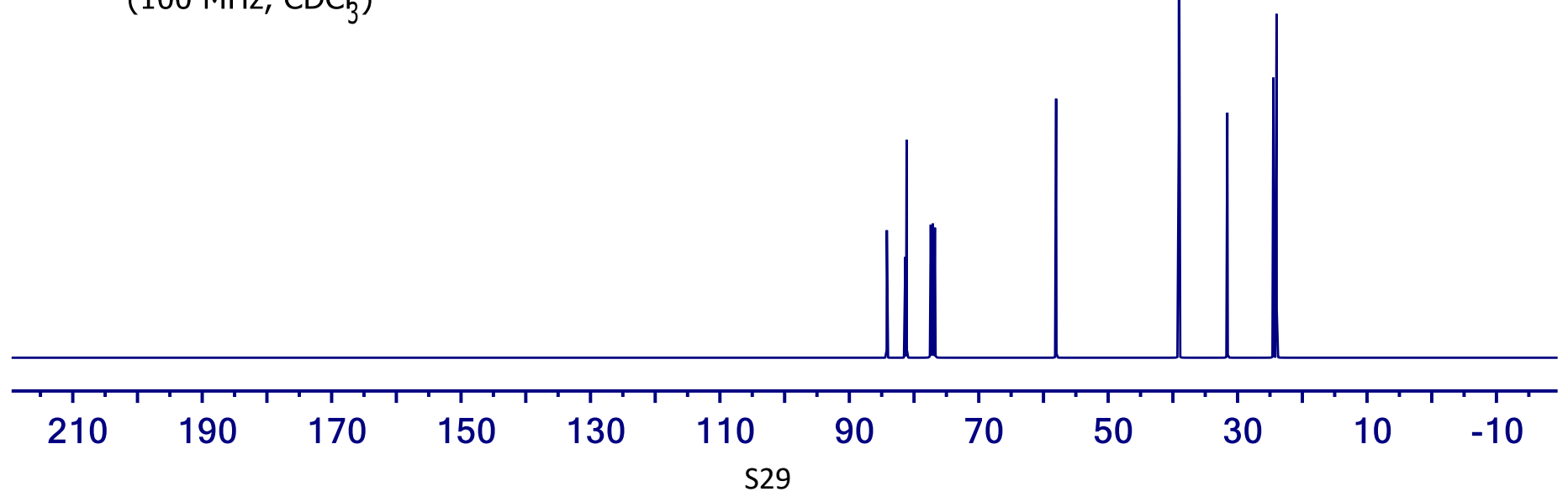


6-phenylhept-3-yne-2,6-diol

$1 \mathrm{y}$

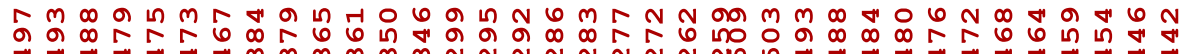

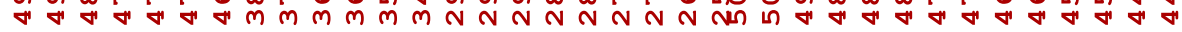

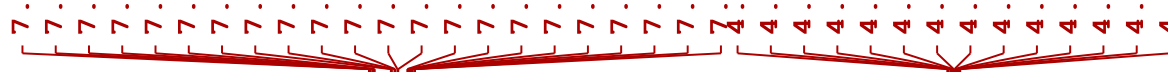
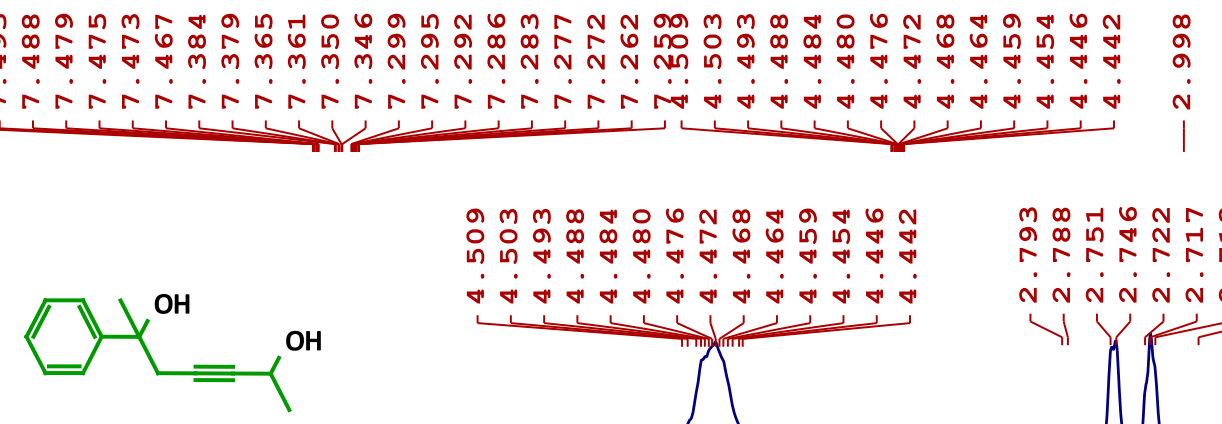

$1 \mathrm{H} N M R$

(400 MHz, $\mathrm{CDCb}_{3}$ )

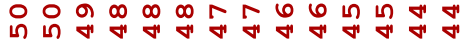
ன் ன் ன் ன் ன் ன் ன் ன் ன்

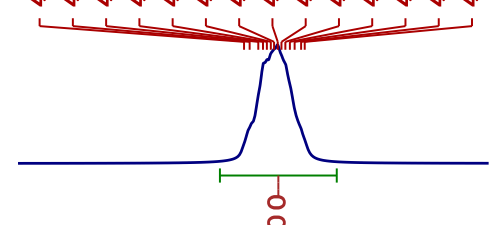

웅

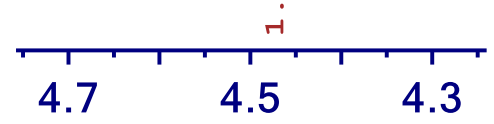

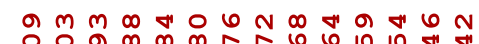
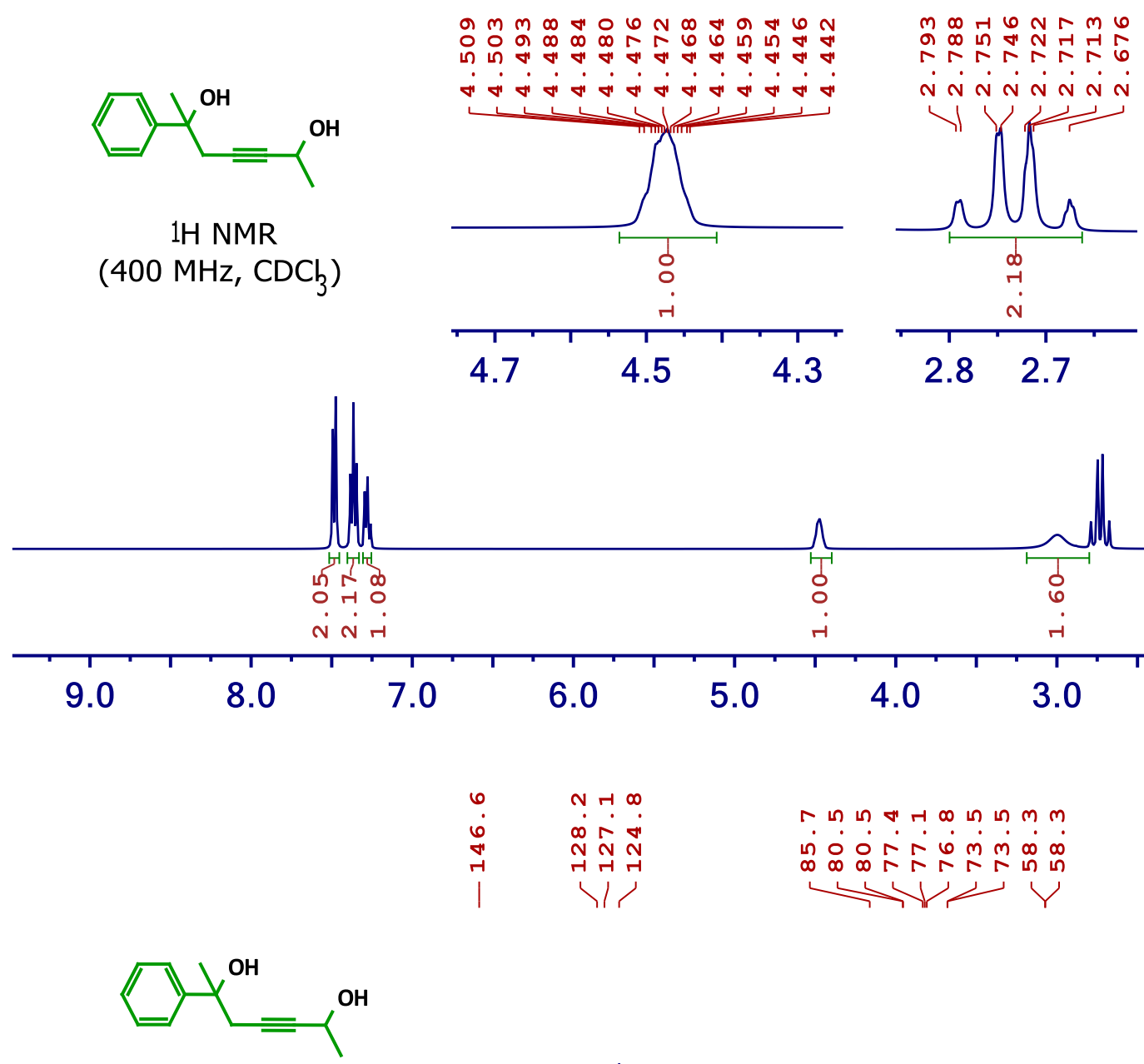

$13 \mathrm{C}\{1 \mathrm{H}\} \mathrm{NMR}$ (100 $\mathrm{MHz}, \mathrm{CDC}_{3}$ )
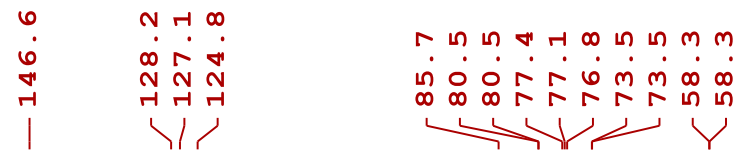

$\infty$ H mำ

$|Y|$ $\forall \infty$

$\forall m m m$

तं

)?
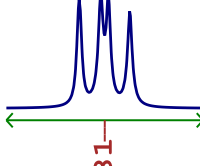

-

N

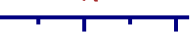

1.33

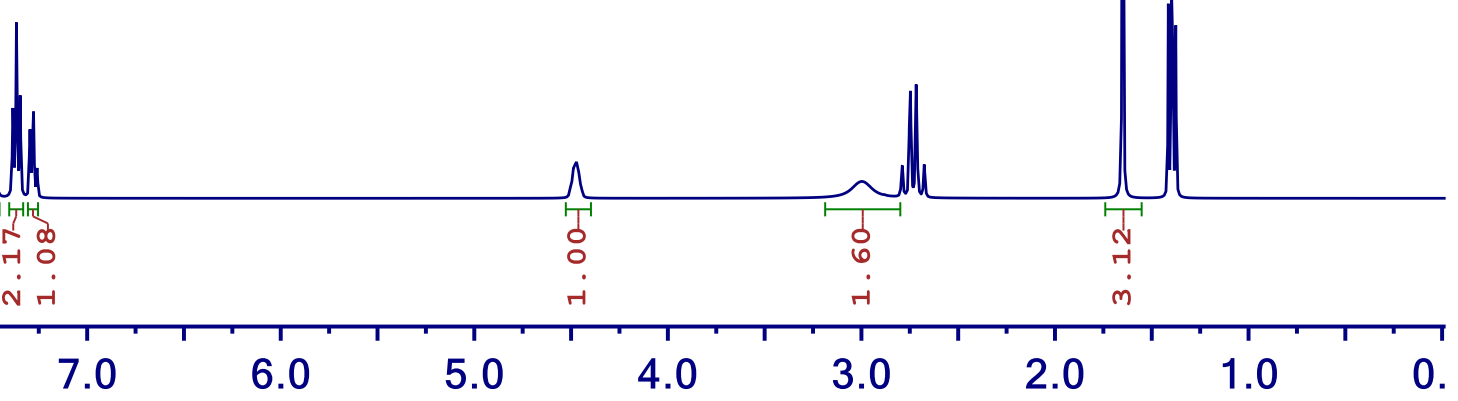


1-(furan-2-yl)hex-3-yne-1,5-diol

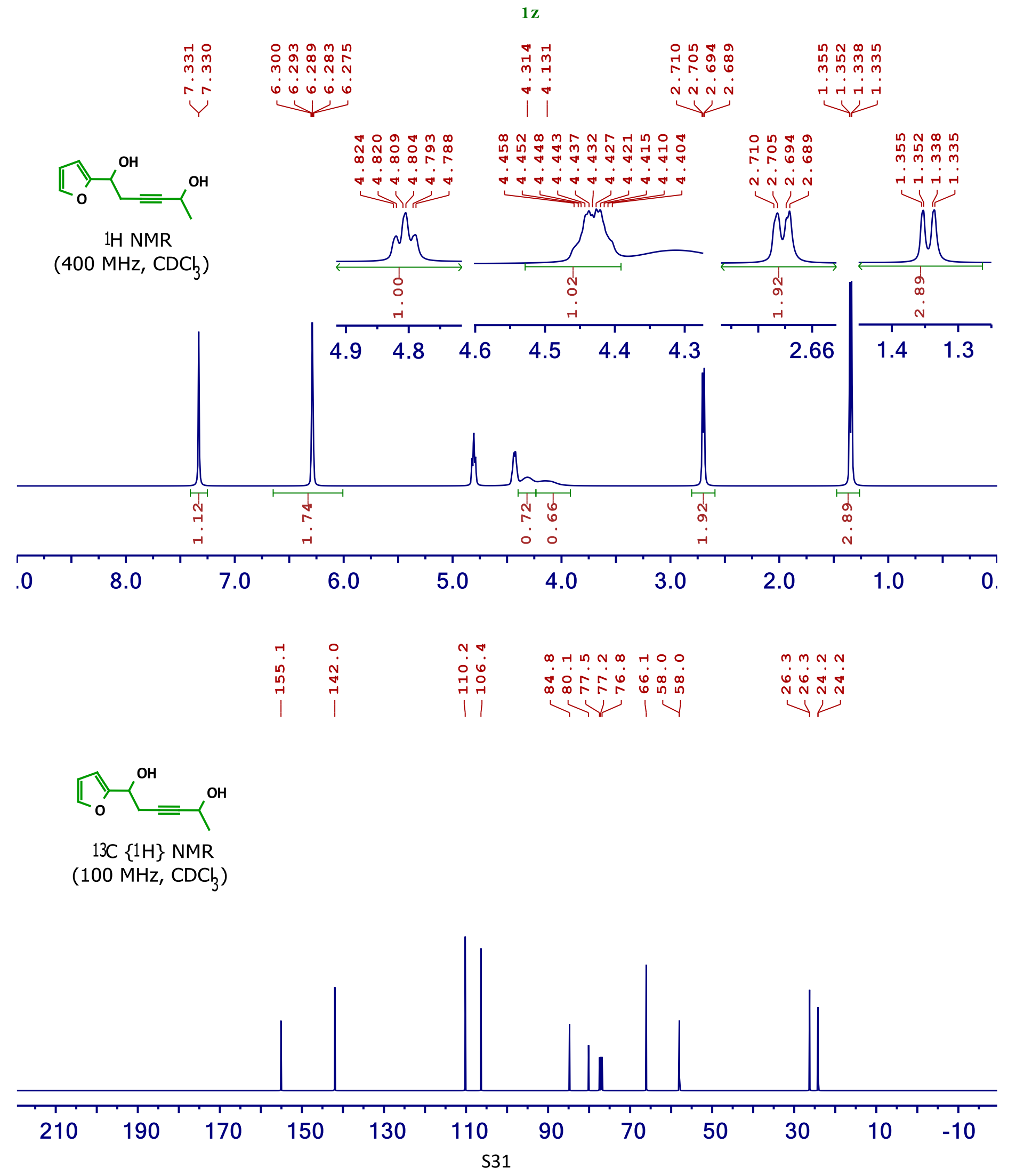


1-(thiophen-2-yl)hex-3-yne-1,5-diol

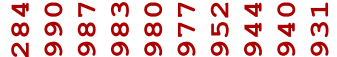

$\therefore \dot{0} \dot{0} \dot{0} \dot{0} \dot{0} \dot{0} \dot{0}$

$\longrightarrow$

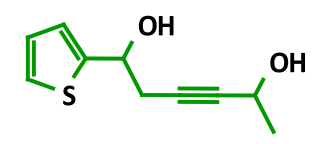

1H NMR

(400 MHz, $\mathrm{CDC}_{3}$ )
1 aa

$\infty \begin{array}{lllll}m & \infty & 0 & \cdots & 0\end{array}$

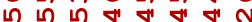

वं वं वं वं

$\longrightarrow$

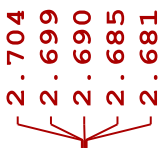

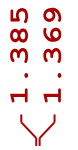

$m \pi n$

N N $\mathrm{N}$

anin

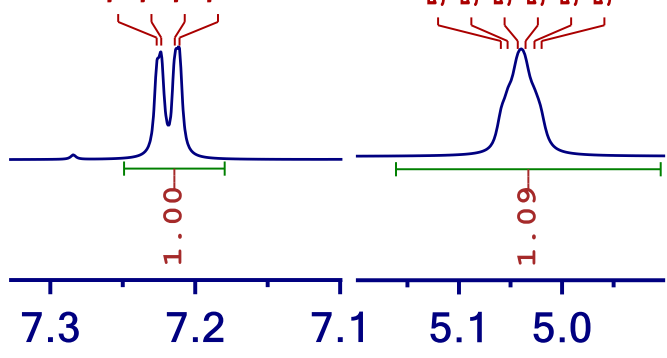

a $m m$ In

กำ ำกำ ำ ำ

in 10 in
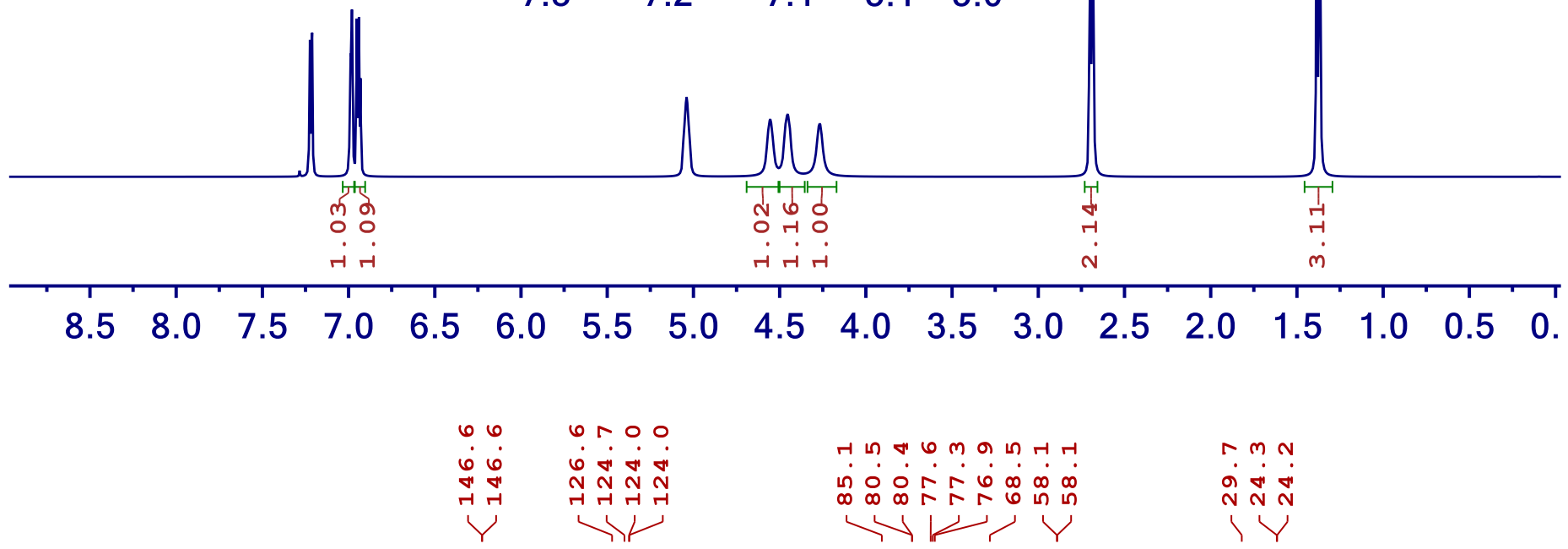

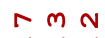

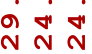

$\backslash Y$

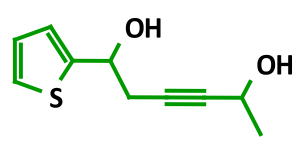

13C $\{1 \mathrm{H}\}$ NMR

(100 MHz, CDCb)

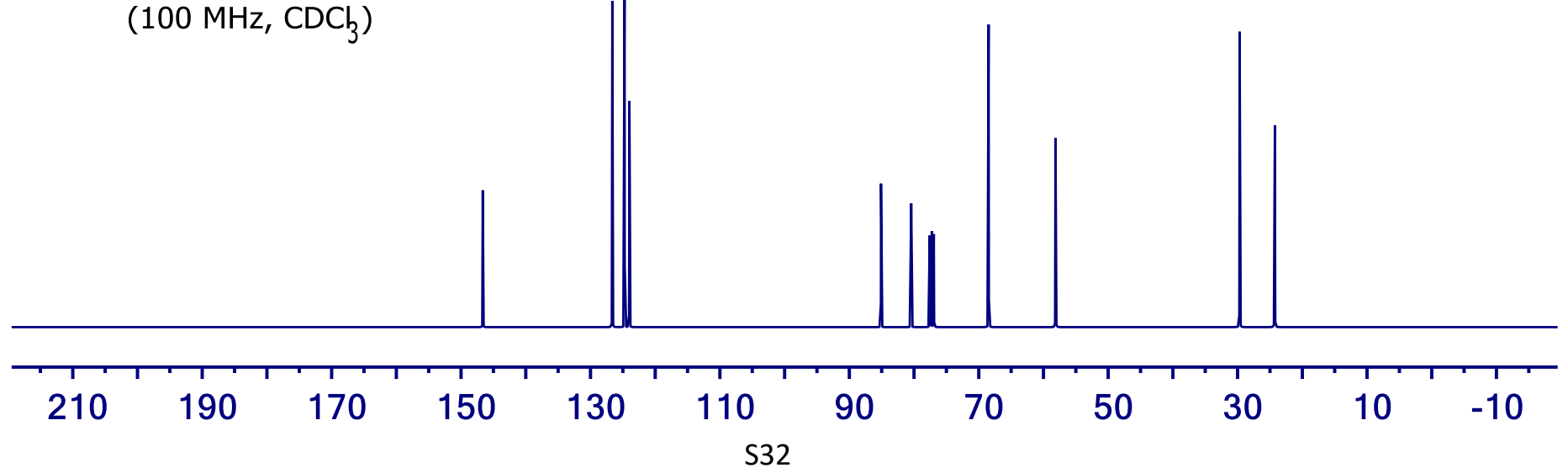


5-(furan-2-yl)-1-(thiophen-2-yl)pent-2-yne-1,5-diol

$1 \mathbf{a b}$

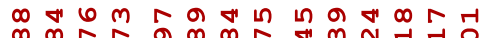

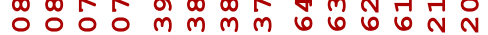

rrín

W

N.

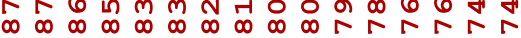

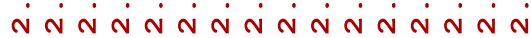

$\sum_{0}^{\mathrm{OH}}=\sum_{-\mathrm{OH}}^{\mathrm{OH}}$

1H NMR

(400 MHz, Acetone-q)
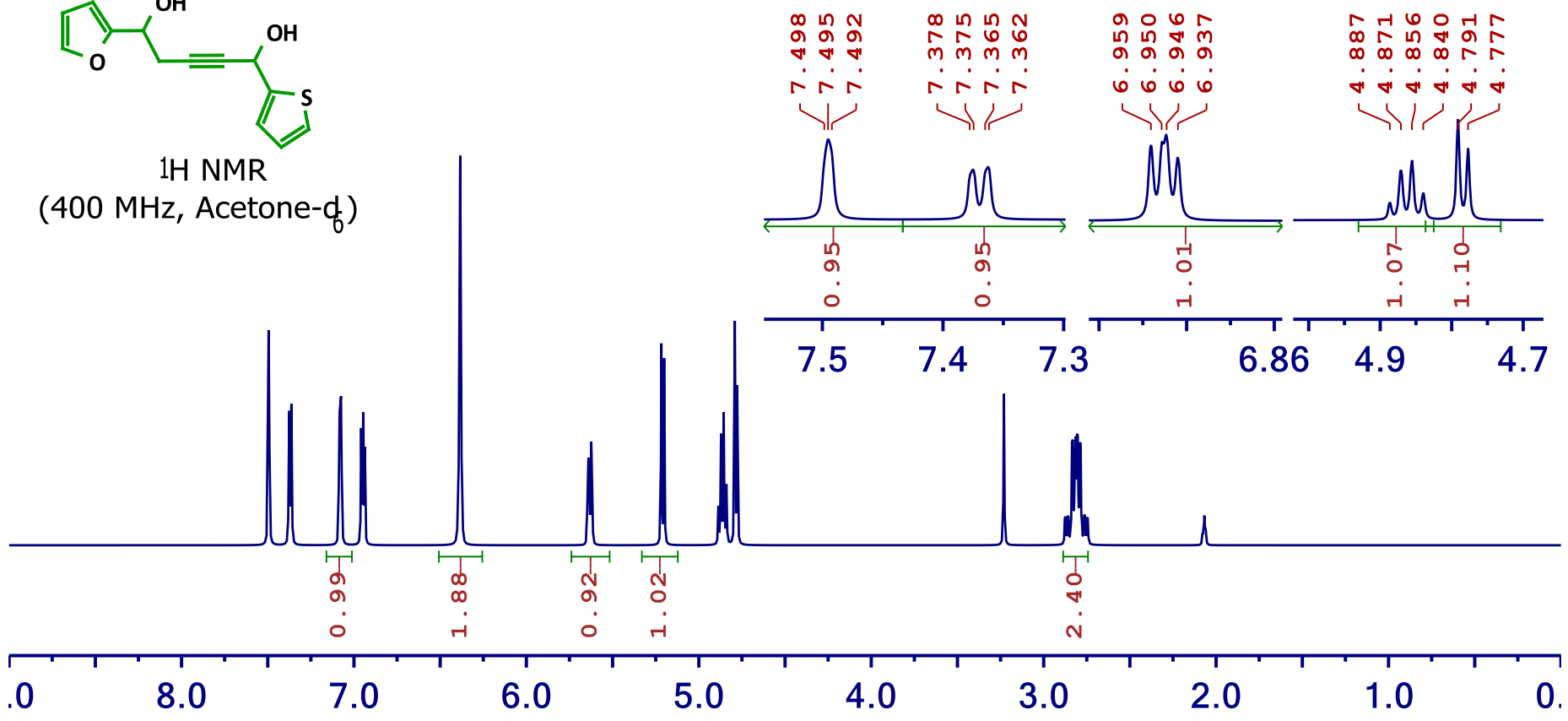

1/1/

1
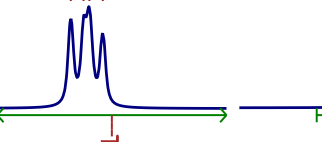

둥ำ

यं वं वं वं

)1/
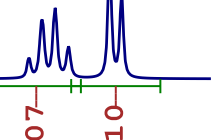

\section{7}

$N \infty \quad 0$ n

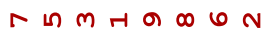

* $N a \quad m m a \quad N$

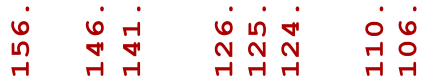

l 1

Ir

11

$\underset{\infty}{\infty} \underset{\infty}{ }$

$\begin{array}{ll}6 & 0 \\ 6 & \text { 1 }\end{array}$

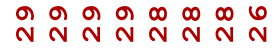

,<smiles>OC(C#CCC(O)c1ccco1)c1cccs1</smiles>

$13 \mathrm{C}\{1 \mathrm{H}\} \mathrm{NMR}$

(100 MHz, Acetone-dq)
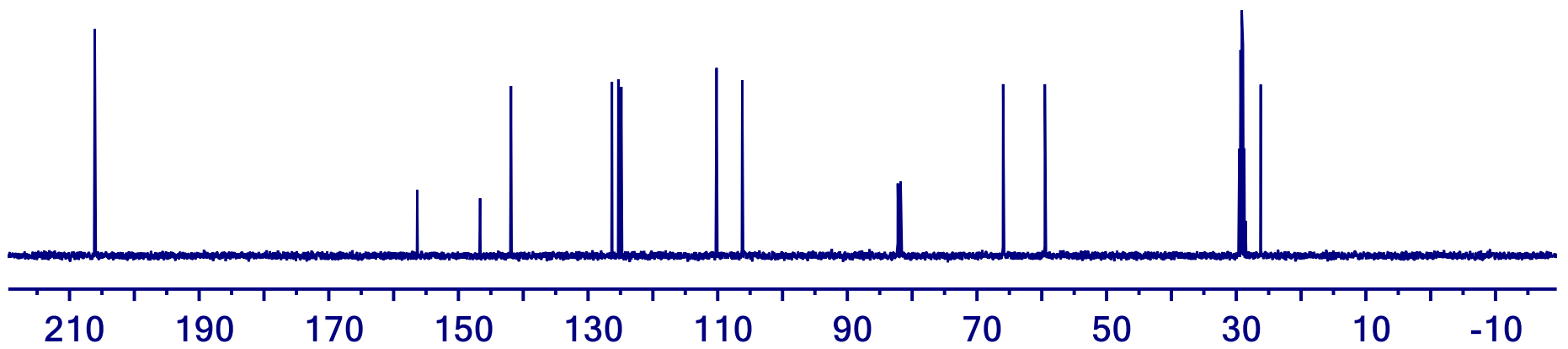
1-phenylbut-3-en-2-one

$2 a$

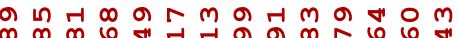

$m m$ m m m $m \stackrel{N}{N} \stackrel{N}{N} N \mathbb{N}$

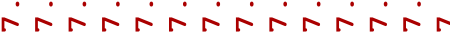
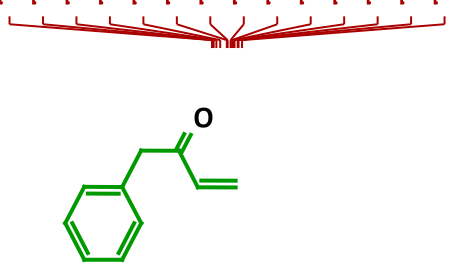

1H NMR

(400 MHz, CDCl)
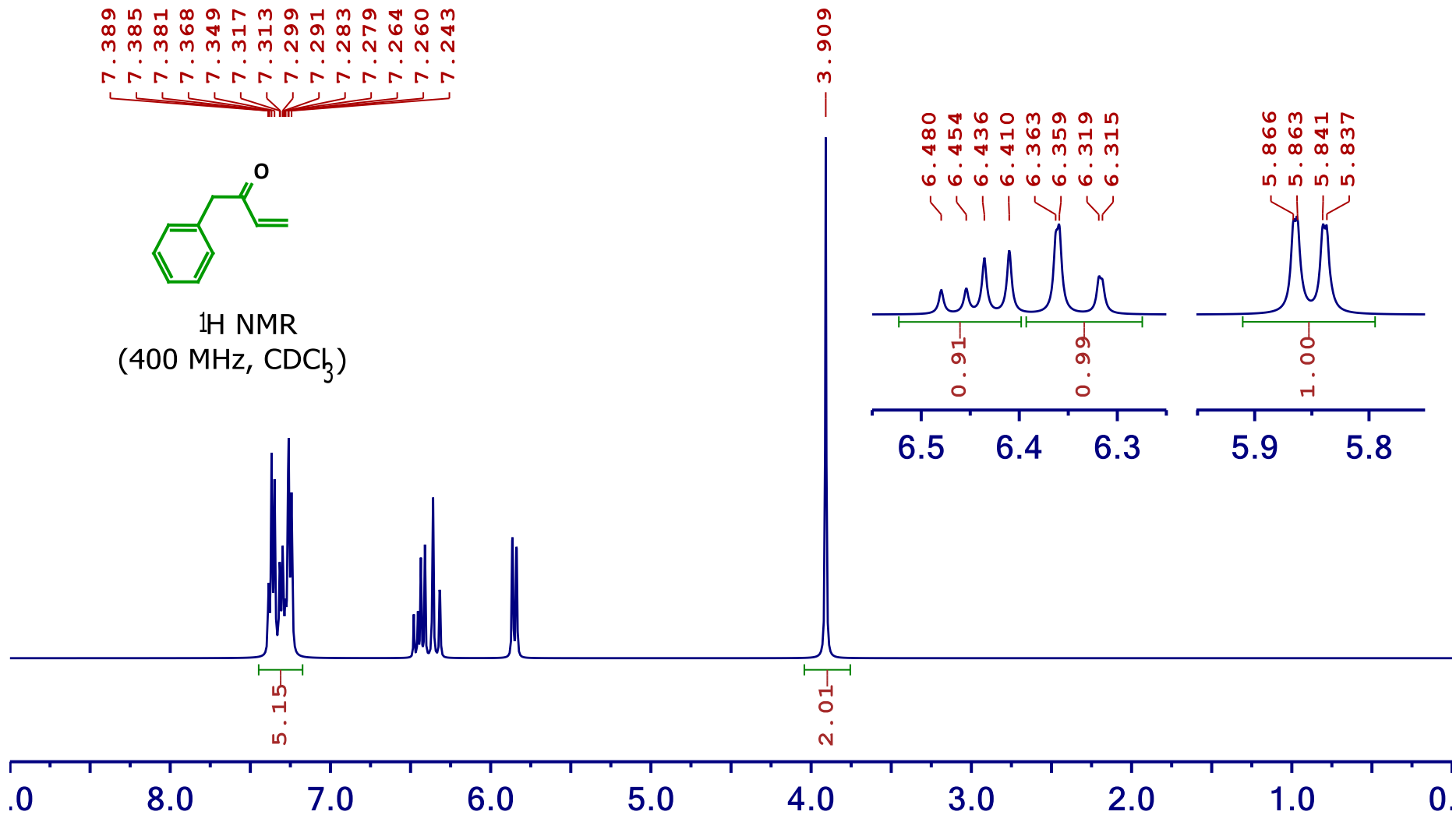

$\infty$
0
0
$r$
1
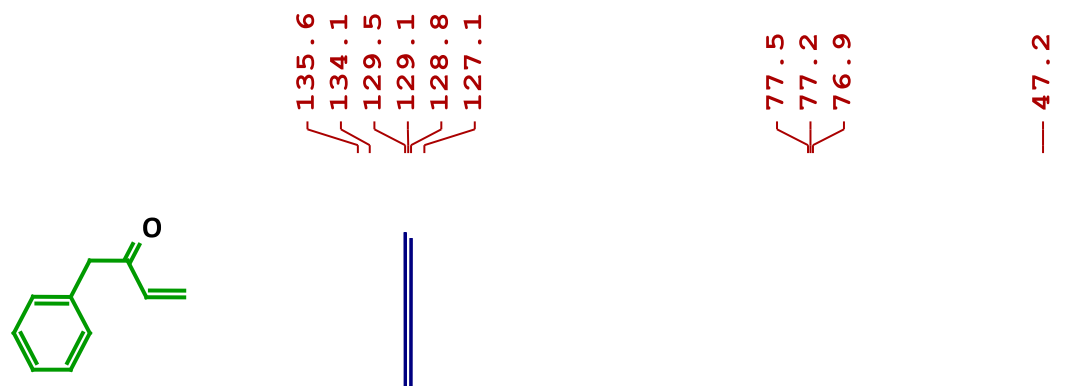

$13 \mathrm{C}\{1 \mathrm{H}\} \mathrm{NMR}$ (100 MHz, $\mathrm{CDC}_{3}$ )
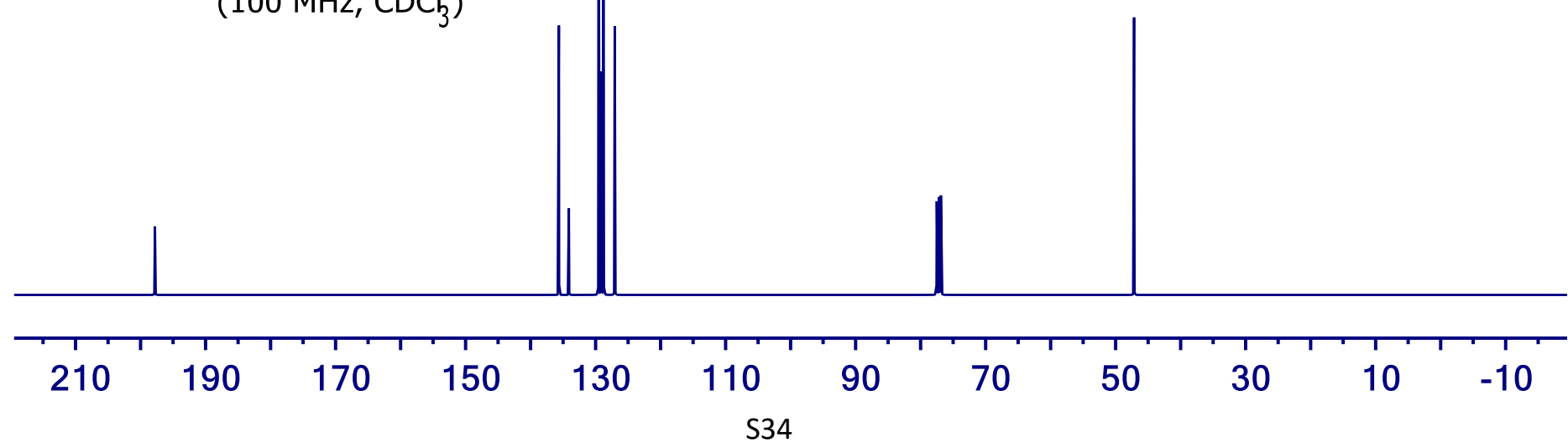

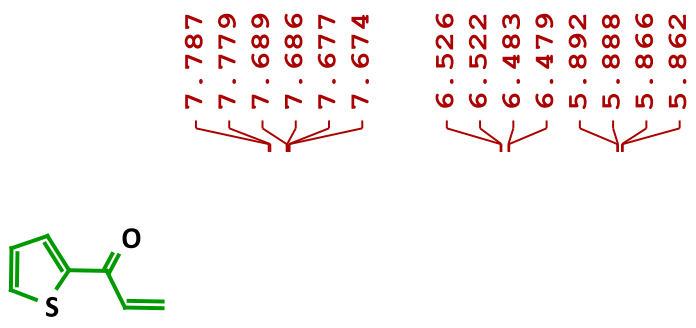

ก ก

H H H 4000

1H NMR

(400 MHz, $\mathrm{CDC}_{3}$ )
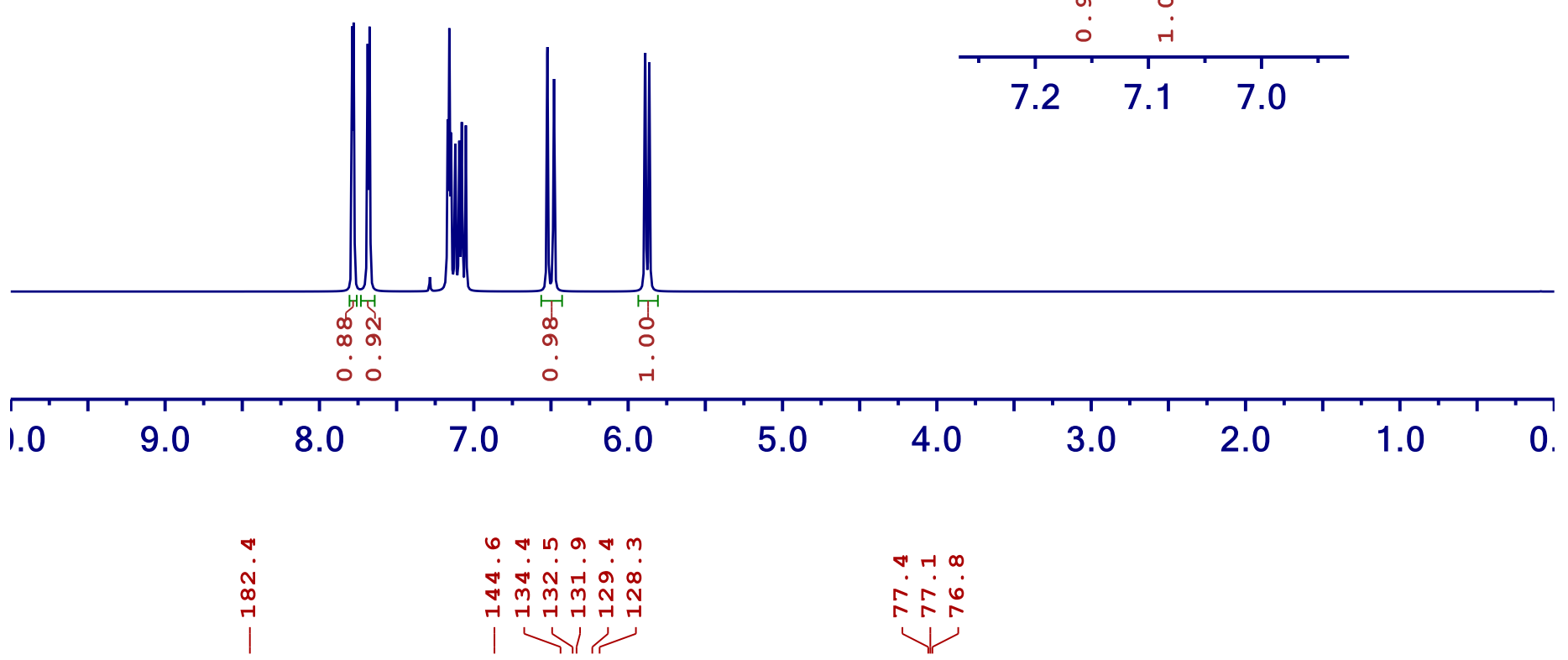

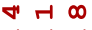

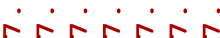

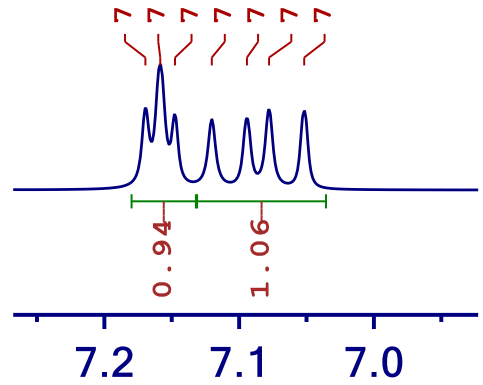

$1.0 \quad 9.0 \quad 8.0$

पा।

5下去

$\checkmark$

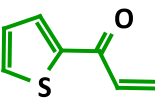

$13 \mathrm{C}\{1 \mathrm{H}\} \mathrm{NMR}$

(100 MHz, CDCb)
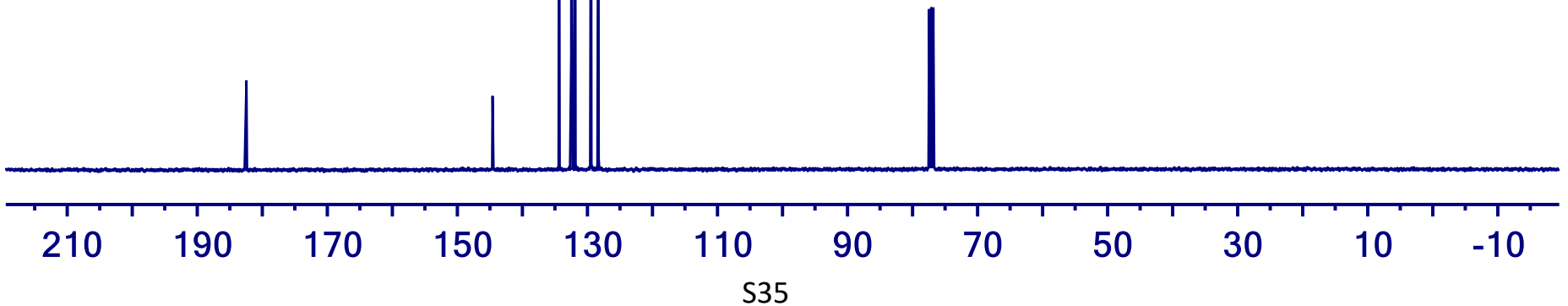


\section{1-(furan-2-yl)prop-2-en-1-one}

2c

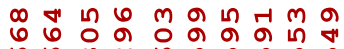

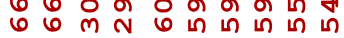

ririo

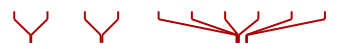

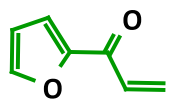

$1 \mathrm{H} N M R$

(400 MHz, CDCh)

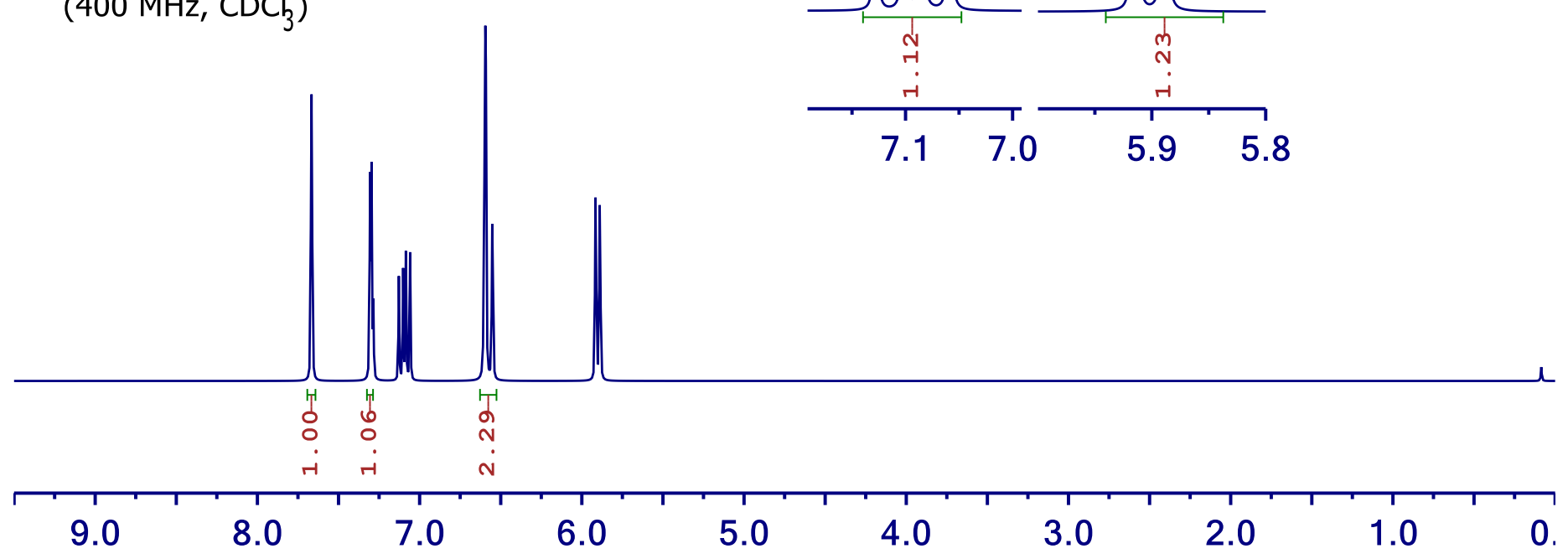

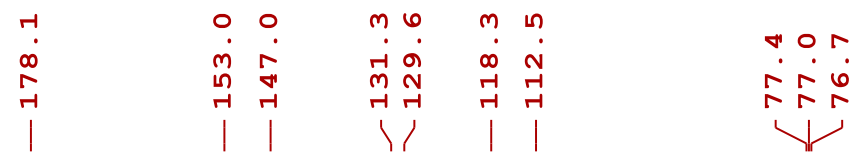

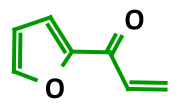

$13 \mathrm{C}\{1 \mathrm{H}\} \mathrm{NMR}$

(100 MHz, CDCh)

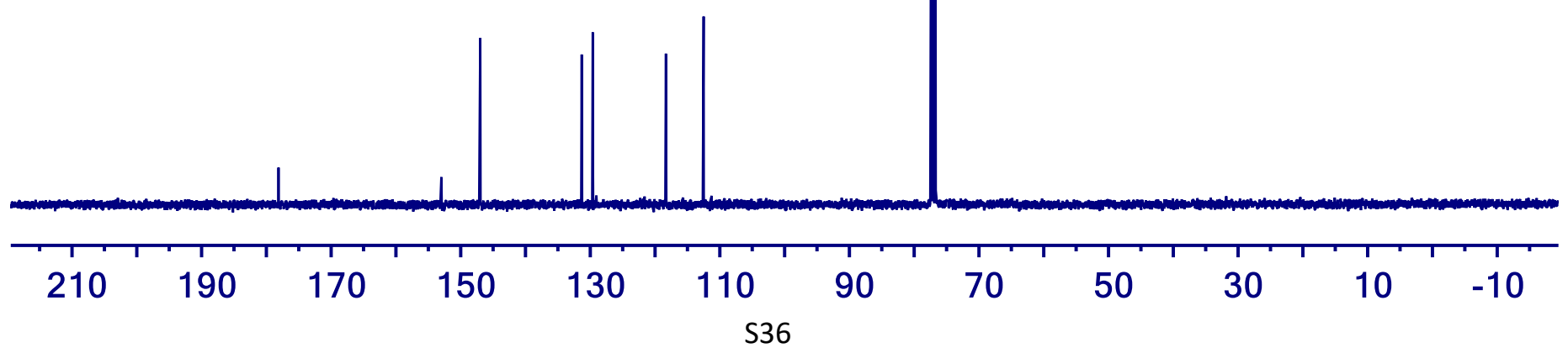


5-phenylpent-1-en-3-one

2d

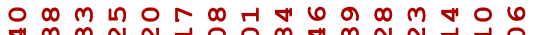

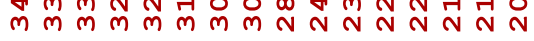

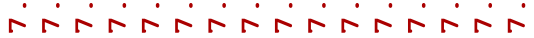

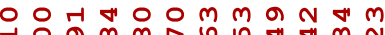

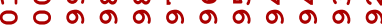

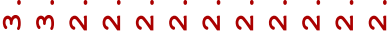

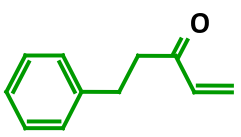

$1 \mathrm{H} N \mathrm{NMR}$

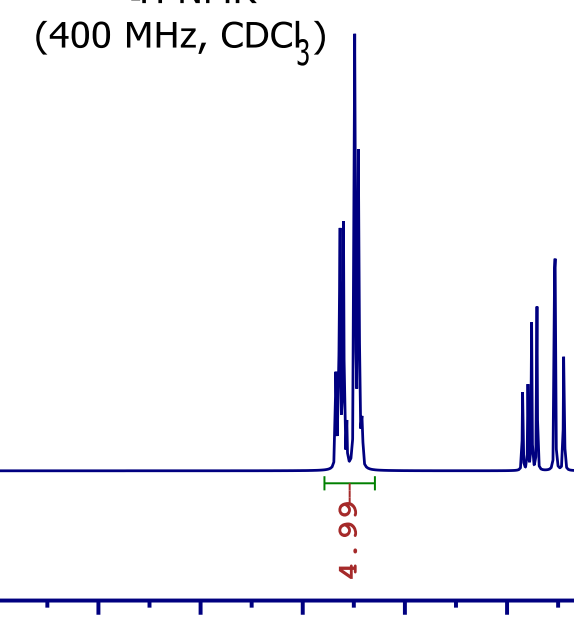

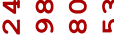

\& $m m m$

$\dot{0} \dot{0} 6$

1111

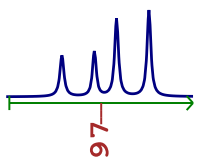

60

N N

$\stackrel{m}{N} \stackrel{0}{N}$

$\dot{0}$

11

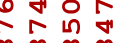

$\infty \begin{array}{lll}\infty & \infty & \infty\end{array}$

เก เก เก เก
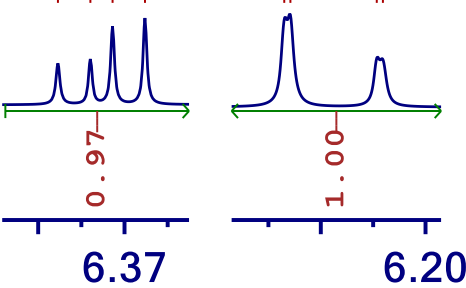

II
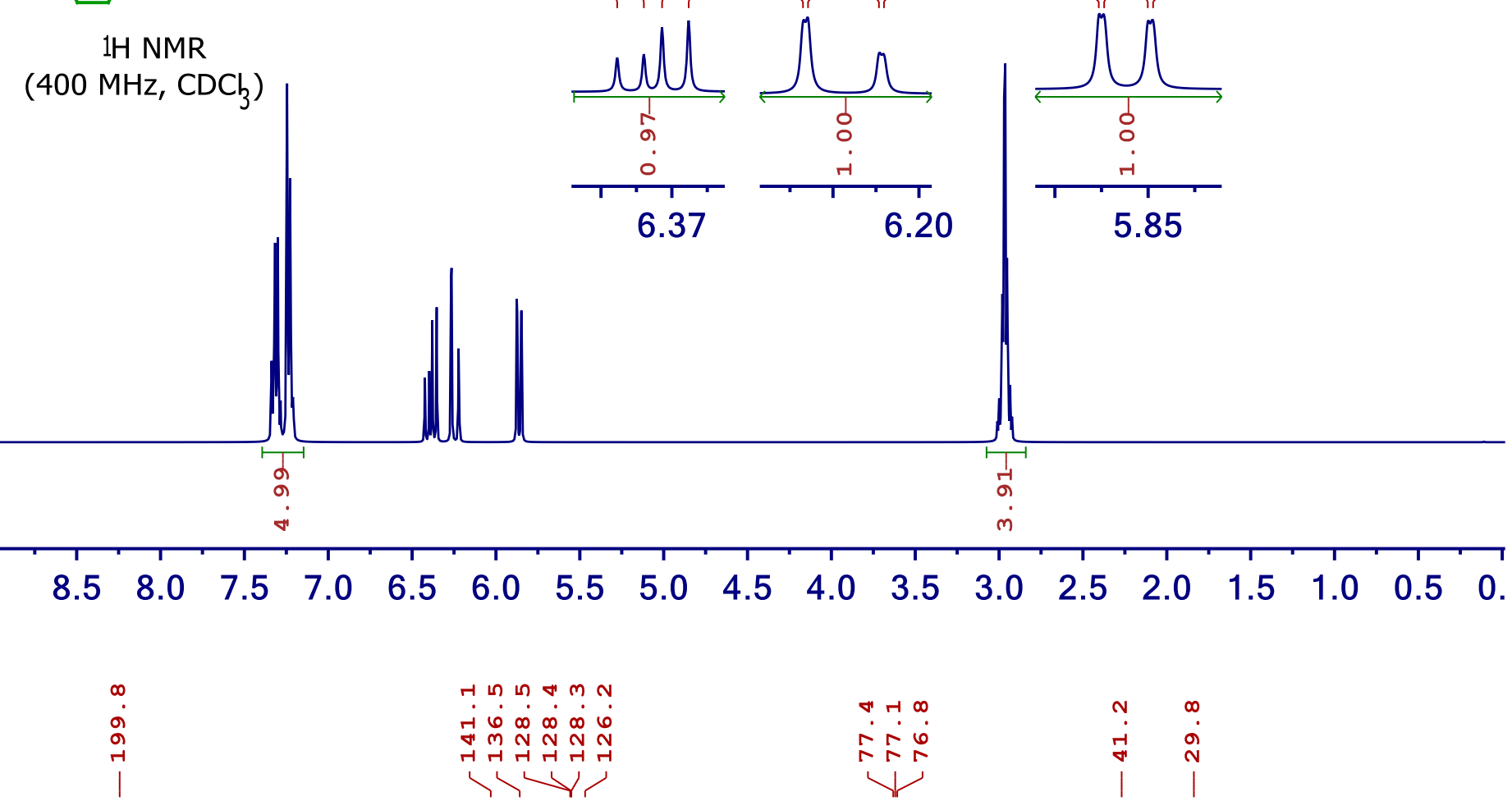

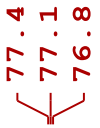
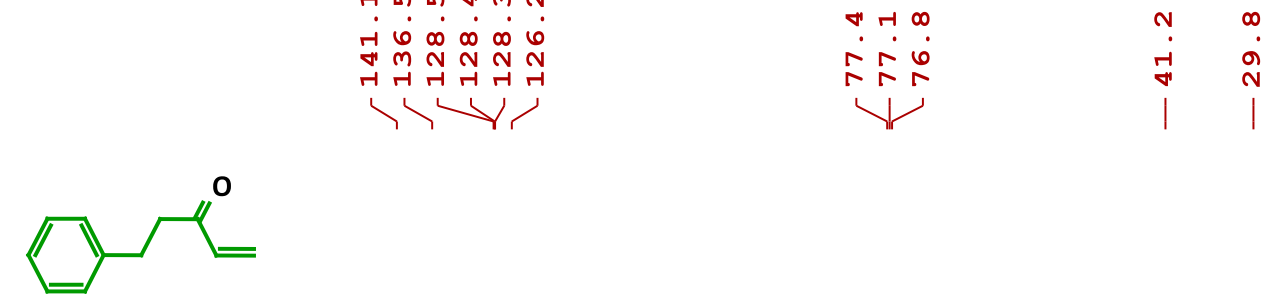

$13 \mathrm{C}\{1 \mathrm{H}\} \mathrm{NMR}$

(100 MHz, CDCb $)$

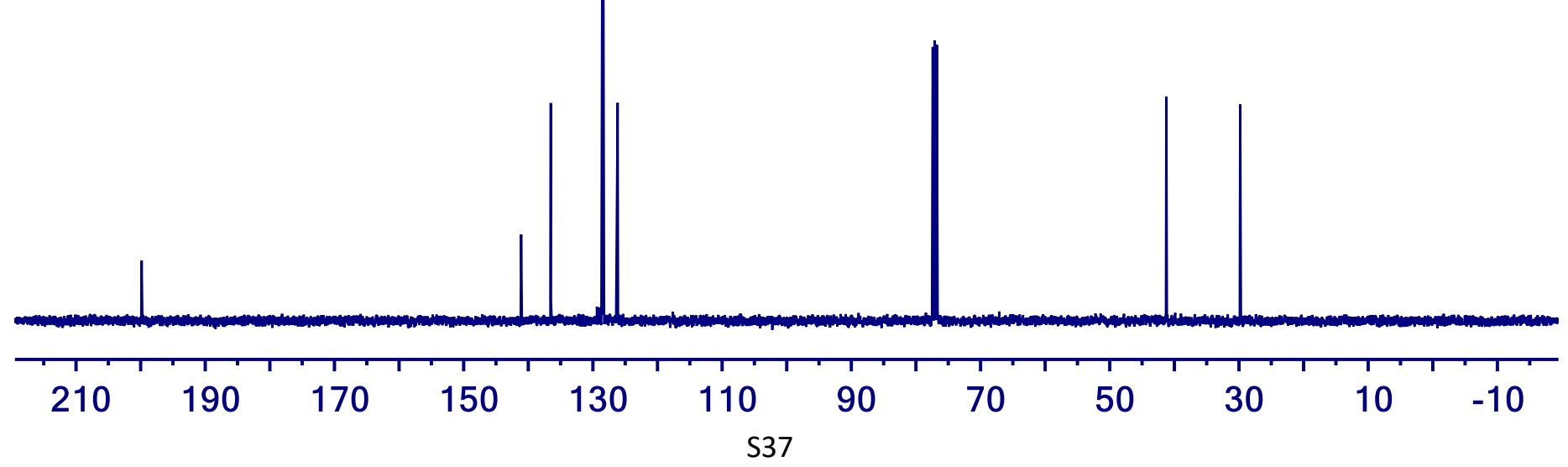


1-phenylprop-2-en-1-one

2e

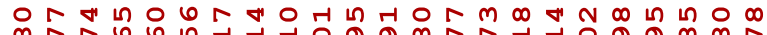

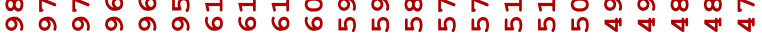

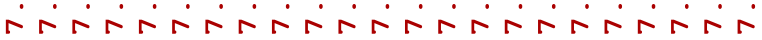

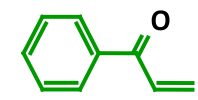

$1 \mathrm{H}$ NMR
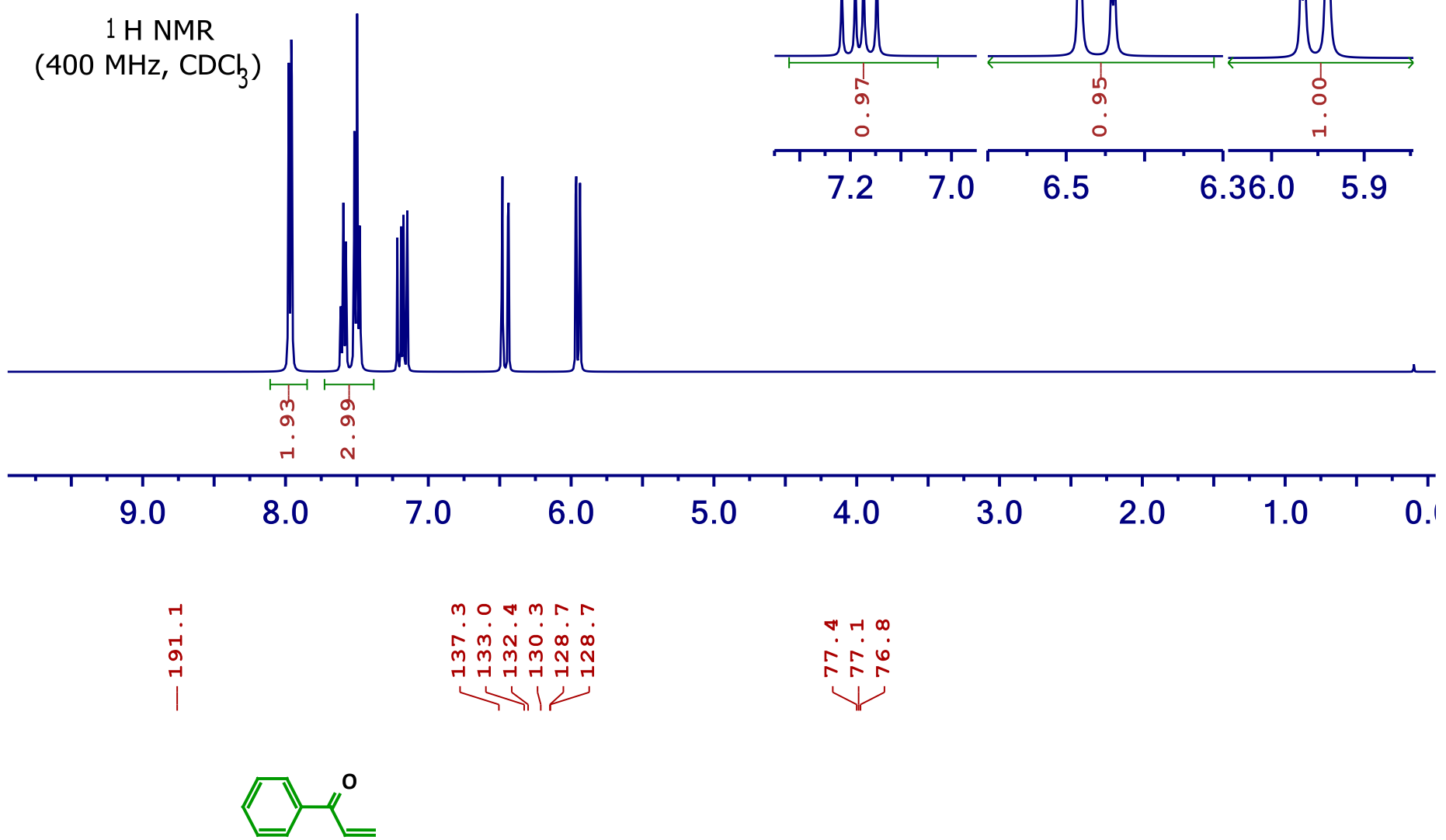

13C $\{1 \mathrm{H}\}$ NMR

(100 $\mathrm{MHz}, \mathrm{CDC}_{3}$ )

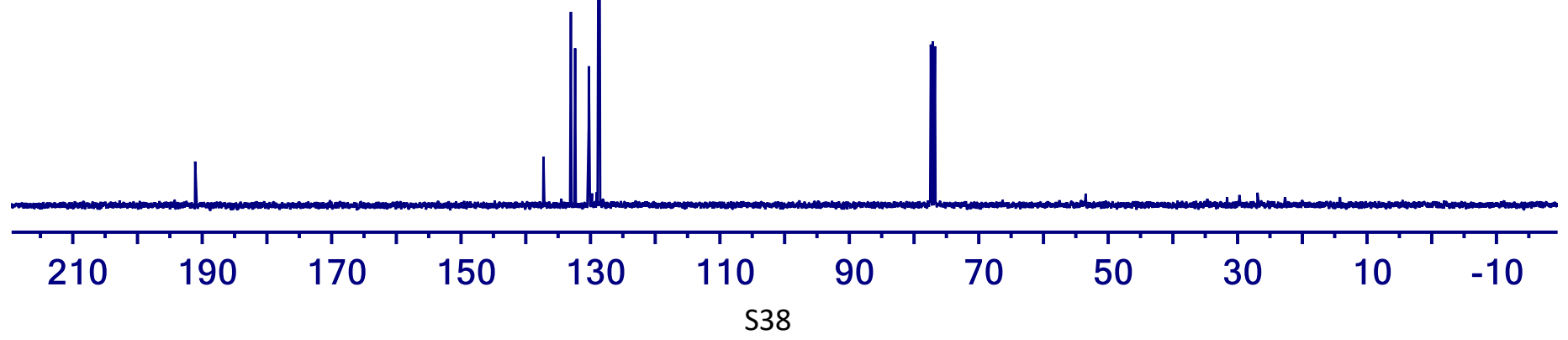




\section{1-cyclohexylprop-2-en-1-one}

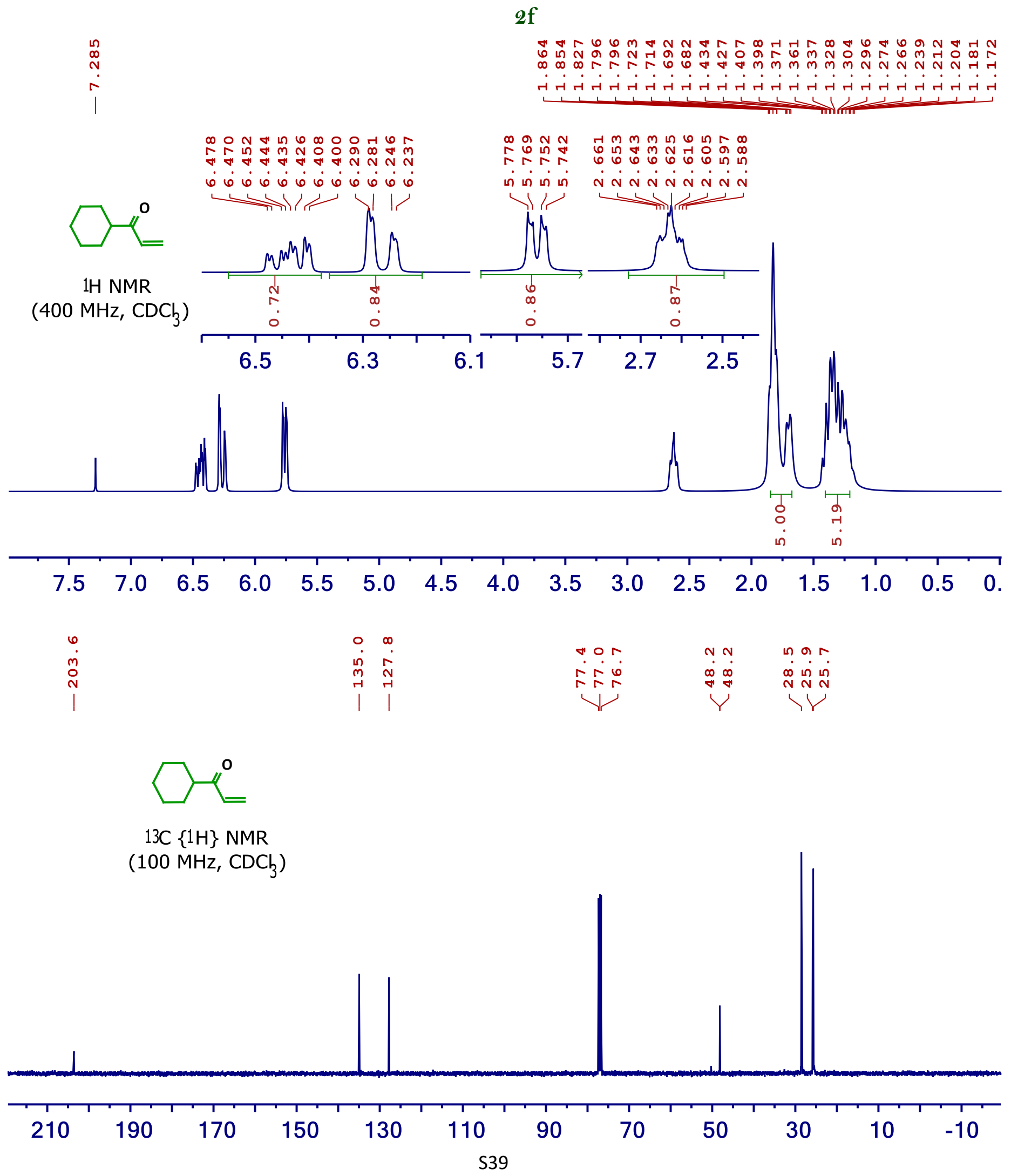


(E)-1-phenylpenta-1,4-dien-3-one

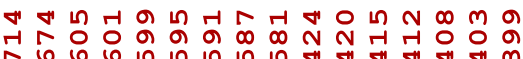

$2 \mathrm{~g}$

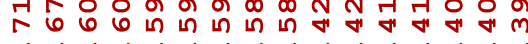

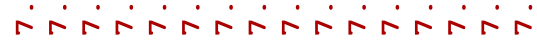

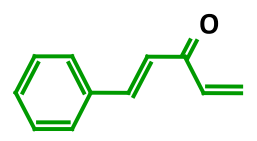

1H NMR (400 MHz, CDCl )

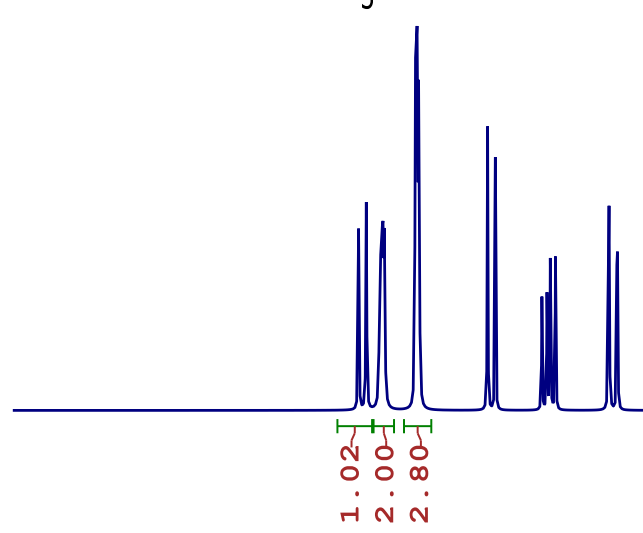

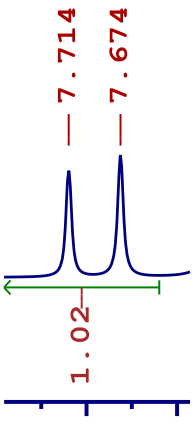

กู

rir

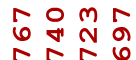

$\therefore 60$.

$\stackrel{m}{N}$ 운은

स $\rightarrow$ m

๑ं

>1/

Y
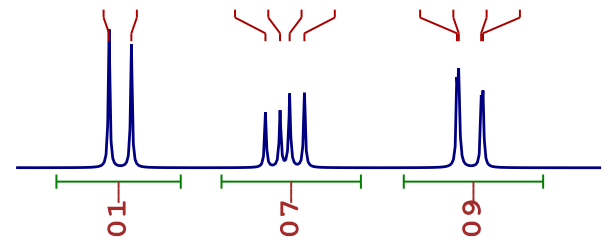

7.63

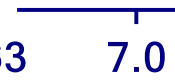

7.0

6.5

6.0
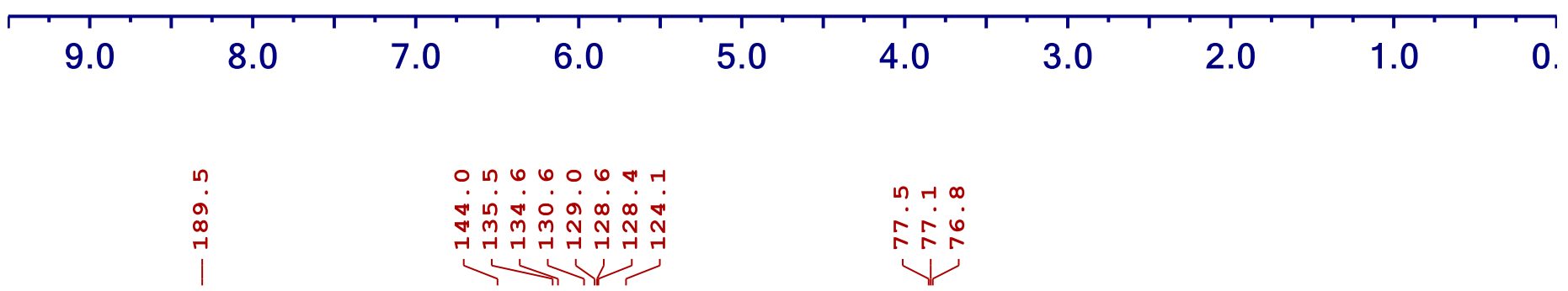

เ $-\infty$

ลำ

Yr

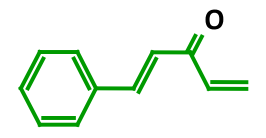

$13 \mathrm{C}\{1 \mathrm{H}\} \mathrm{NMR}$

(100 MHz, $\mathrm{CDC}_{3}$ )
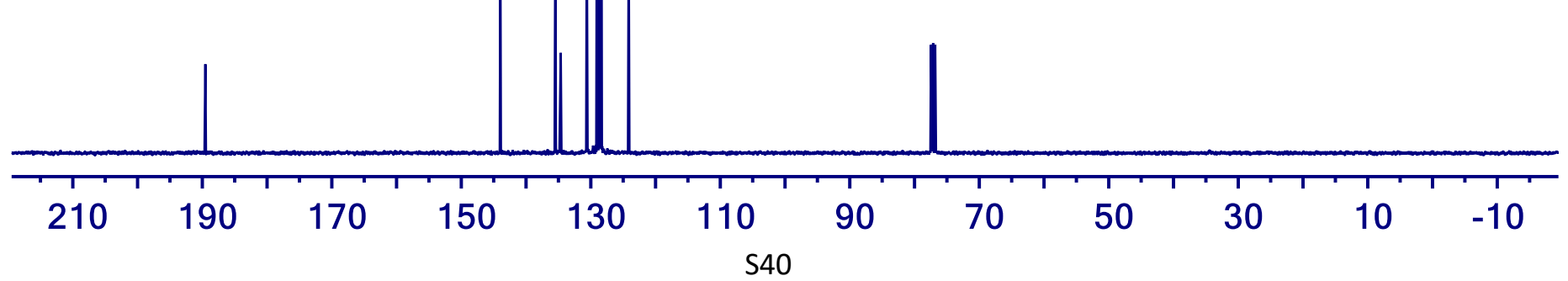


\section{1-(2,6-dichlorophenyl)prop-2-en-1-one}

$2 \mathrm{~h}$

ดิ

m m m m m m m m N m m

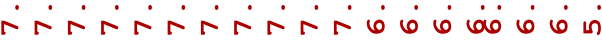

LII

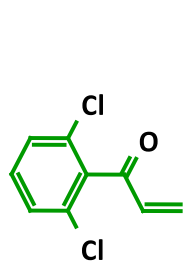

$1 \mathrm{H} N M R$

(400 MHz, CDCb)

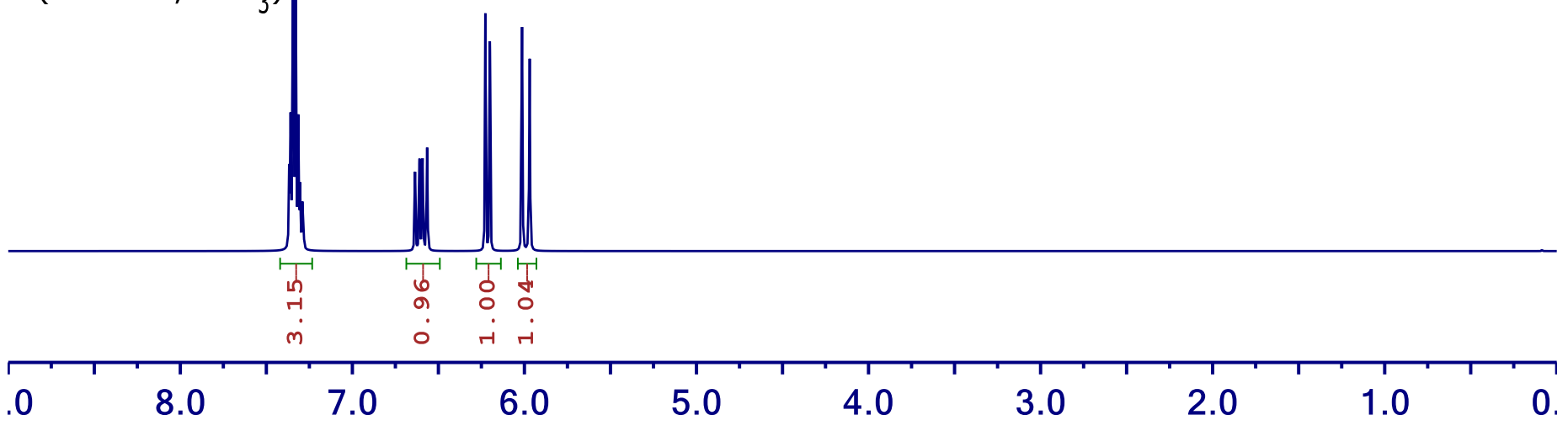

$\nabla$
ma
a
1

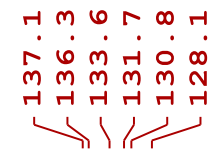

$\nabla+4 \infty$

ing

$\checkmark$

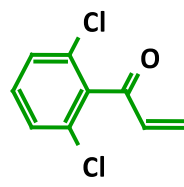

$13 \mathrm{C}\{1 \mathrm{H}\} \mathrm{NMR}$

(100 MHz, CDCh)
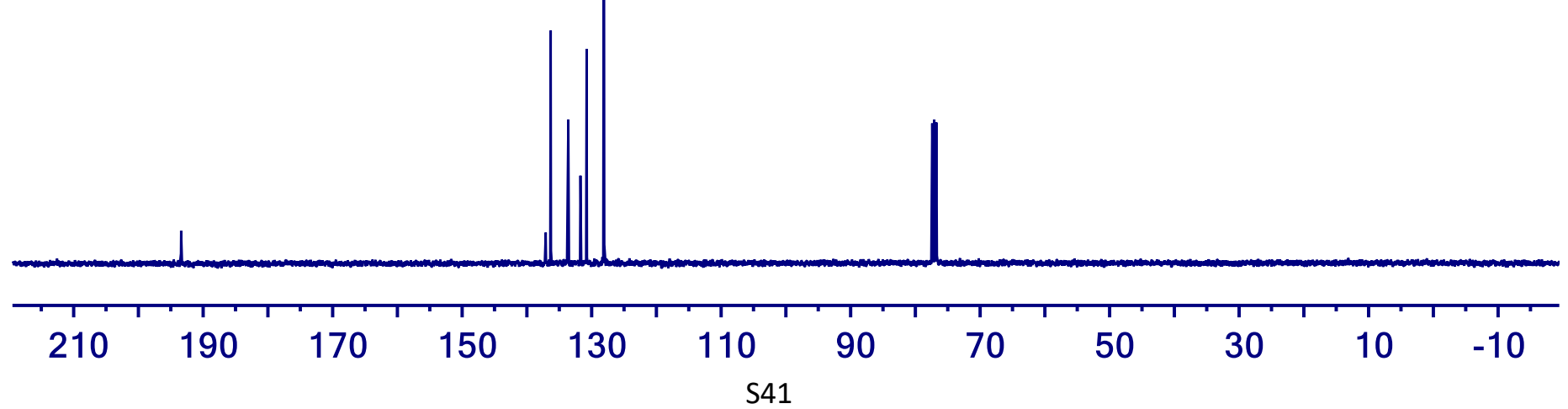
1-(naphthalen-1-yl)prop-2-en-1-one

$2 \mathrm{i}$

60 -

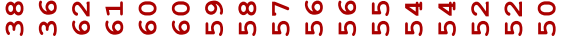

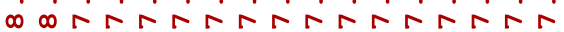

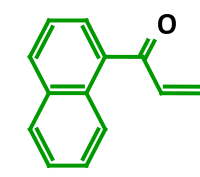

1H NMR

(400 $\mathrm{MHz}, \mathrm{CDCh}_{3}$ )
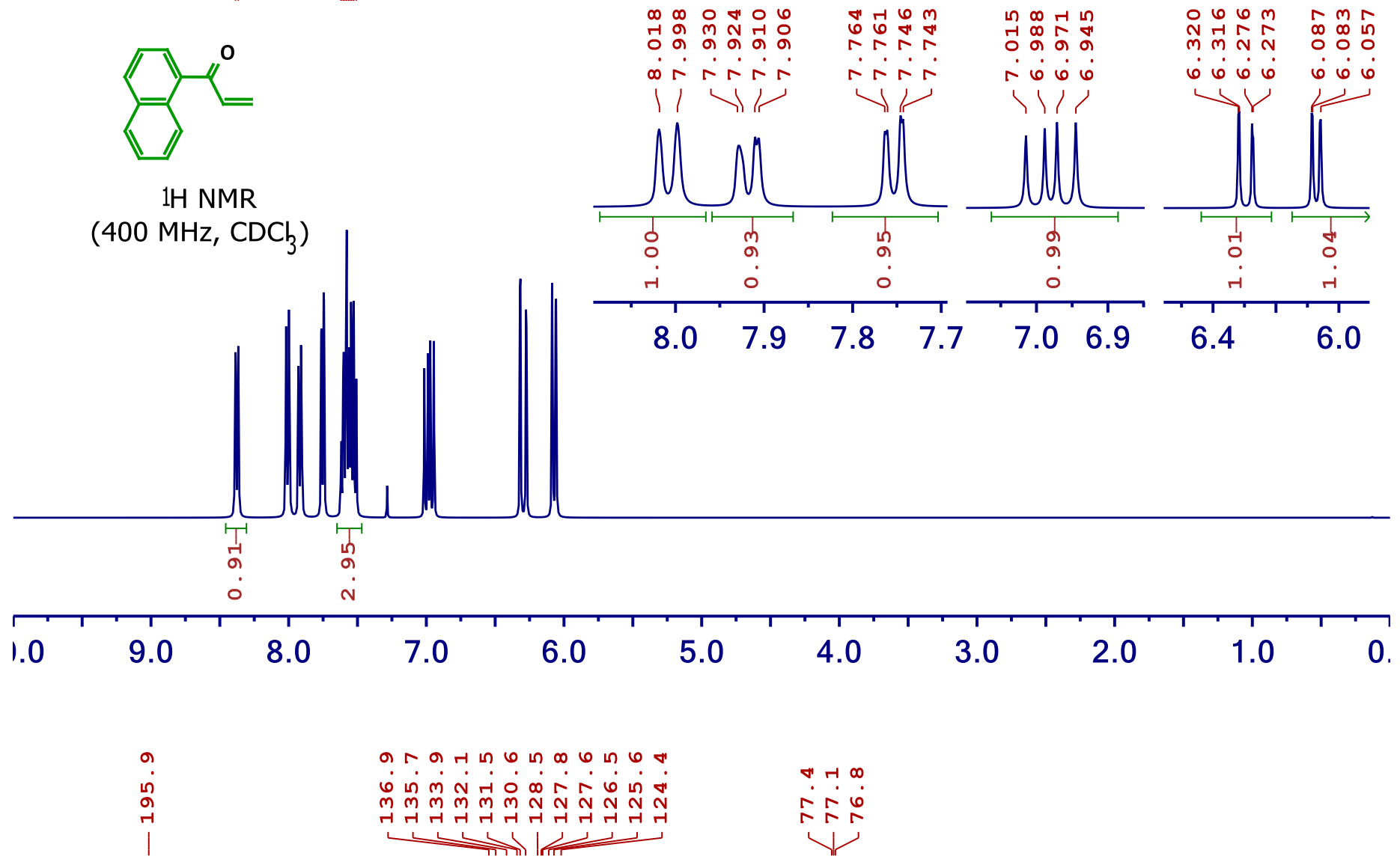

$\Delta+4 \infty$

$\operatorname{rin}$

r

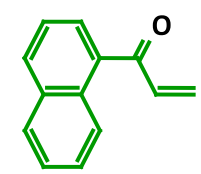

$13 \mathrm{C}\{1 \mathrm{H}\} \mathrm{NMR}$

(100 $\mathrm{MHz}, \mathrm{CDCl}_{3}$ )

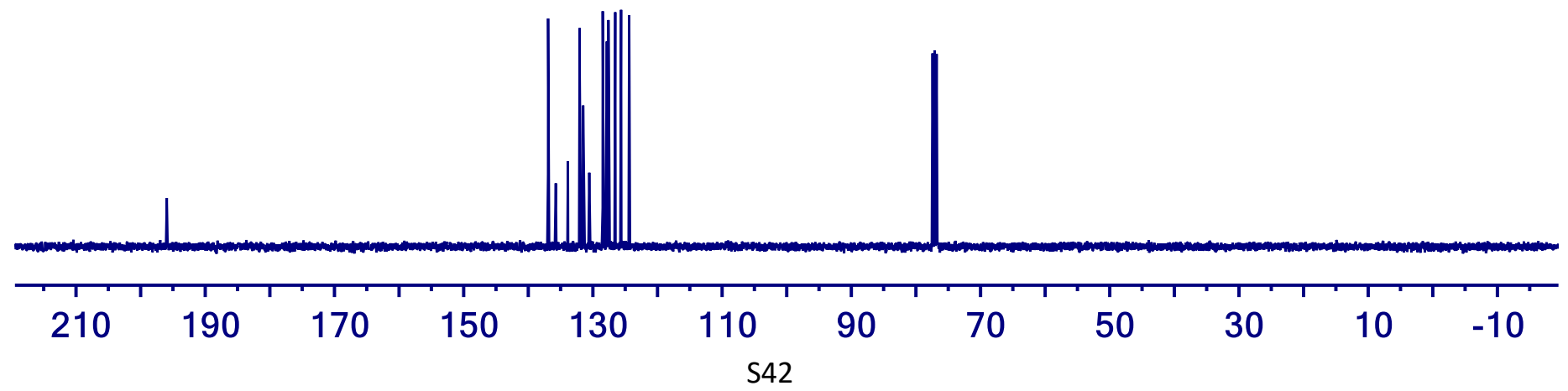


(E)-5-ethylidene-8-hydroxy-1,8-diphenyloctane-2,6-dione

$4 a$

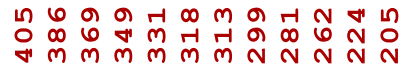

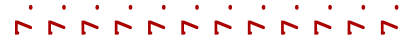

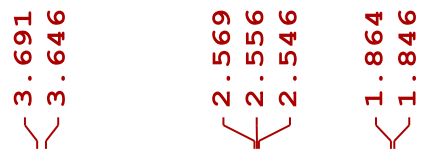

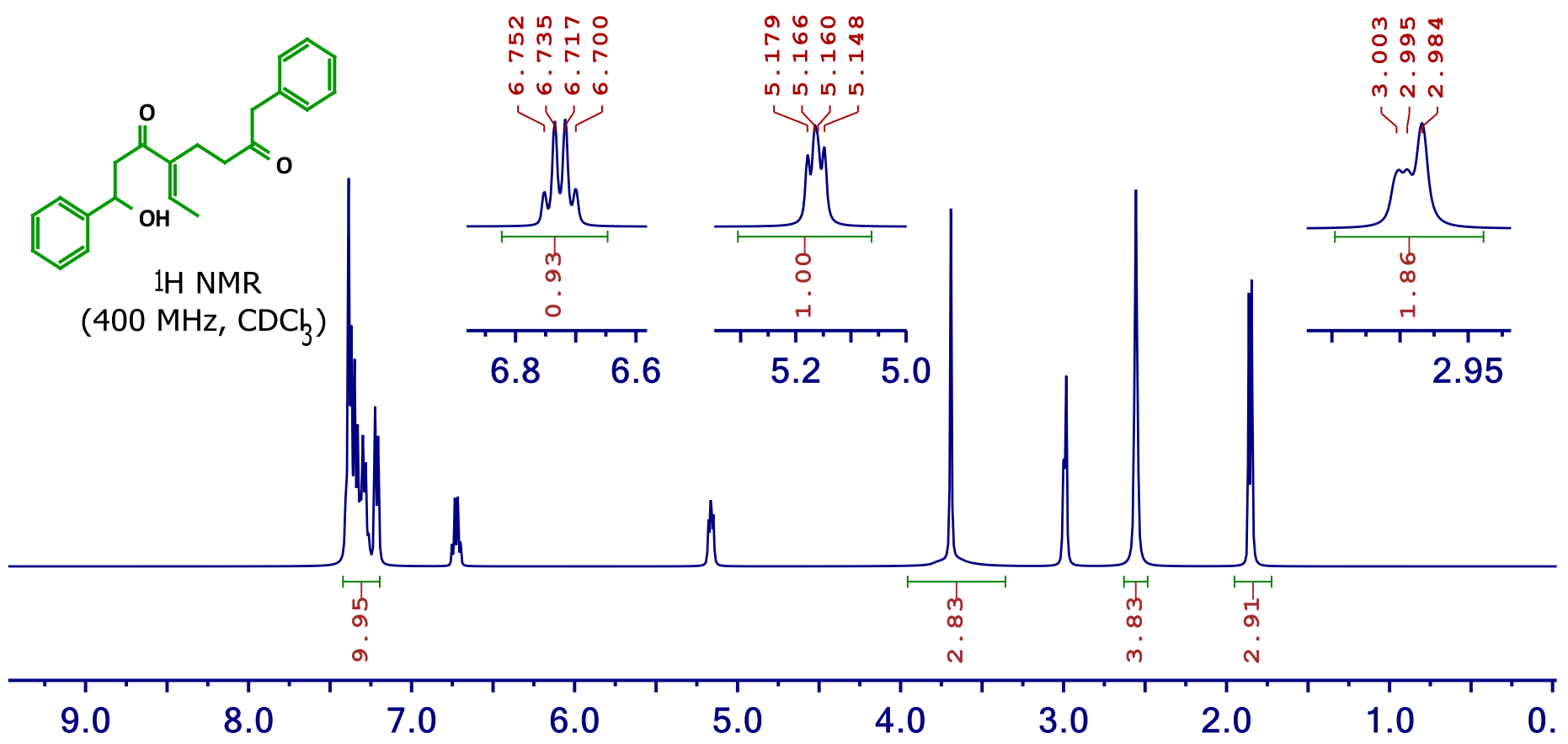

$\begin{array}{ll}r & -1 \\ i & -1 \\ \text { N } & \text { O } \\ 1 & 1\end{array}$

a n

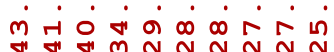

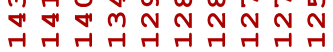

$\longrightarrow$

ᄂ $N \infty \pi$

isio

Ha 0

N?

ㅇำ

$+\infty$

111

a 2 तो<smiles>C/C=C(/CCC(=O)Cc1ccccc1)C(=O)CC(O)c1ccccc1</smiles>

$13 \mathrm{C}\{1 \mathrm{H}\} \mathrm{NMR}$

(100 MHz, CDCh)
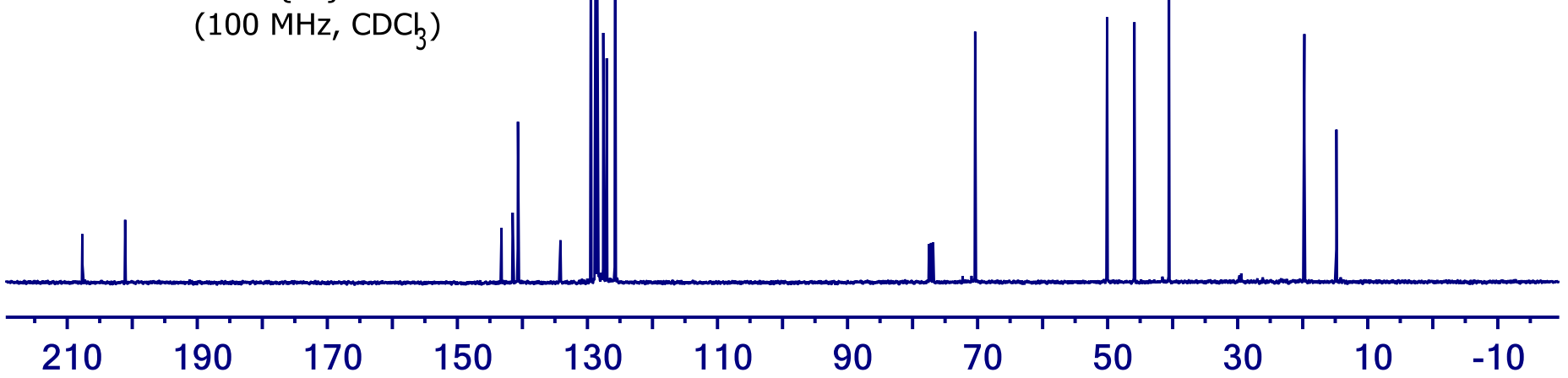
(E)-4-ethylidene-7-hydroxy-7-phenyl-1-(thiophen-2-yl)heptane-1,5-dione

$4 b$

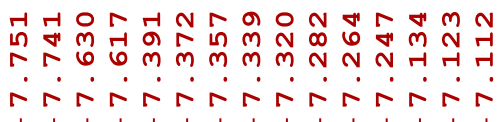

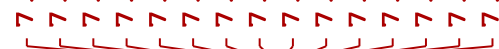<smiles>C/C=C(\CCC(=O)c1cccs1)C(=O)CC(O)c1ccccc1</smiles>

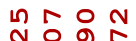

क

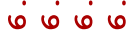

ㄷํㅇํำ

तन न न

$\underset{\substack{\infty \\ m \\ \infty}}{m}$

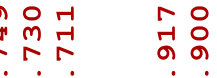

मnं

$\left(400 \mathrm{MHz}, \mathrm{CDC}_{3}\right)$

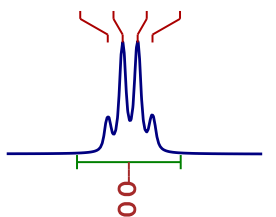

ก เก เก เก

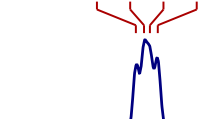

|

Y Y

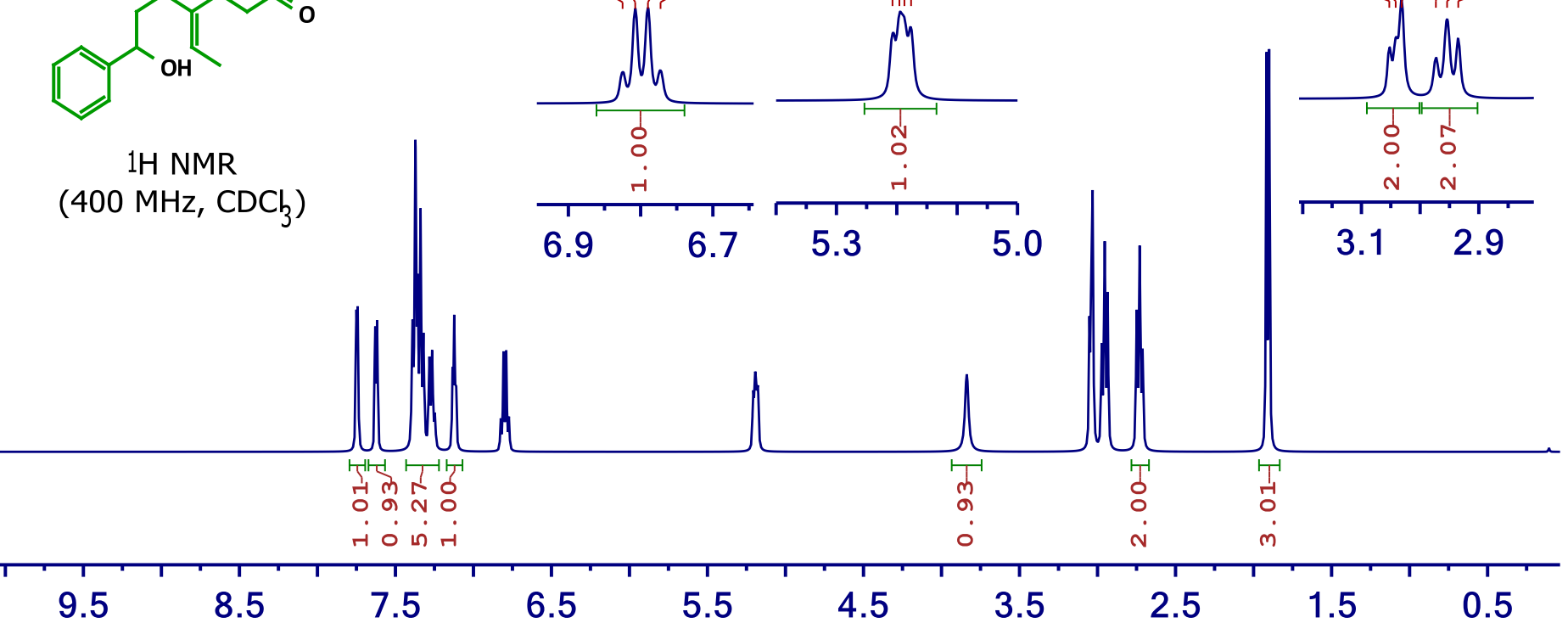

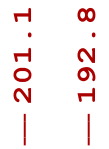

4 $2 m$ N

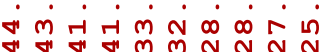

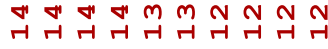

要

ก $\frac{2 \pi}{2}$

송웅

W!

$\begin{array}{cc}0 & r \\ \dot{b} & \infty \\ & 0 \\ \mid & \mid\end{array}$

a 0

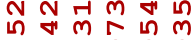

०००कबन

mm $m$ N N

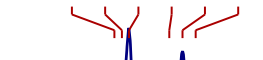

$3.1 \quad 2.9$<smiles>C/C=C(\CCC(=O)c1cccs1)C(=O)CC(O)c1ccccc1</smiles>

$13 \mathrm{C}\{1 \mathrm{H}\} \mathrm{NMR}$

(100 MHz, CDCb)
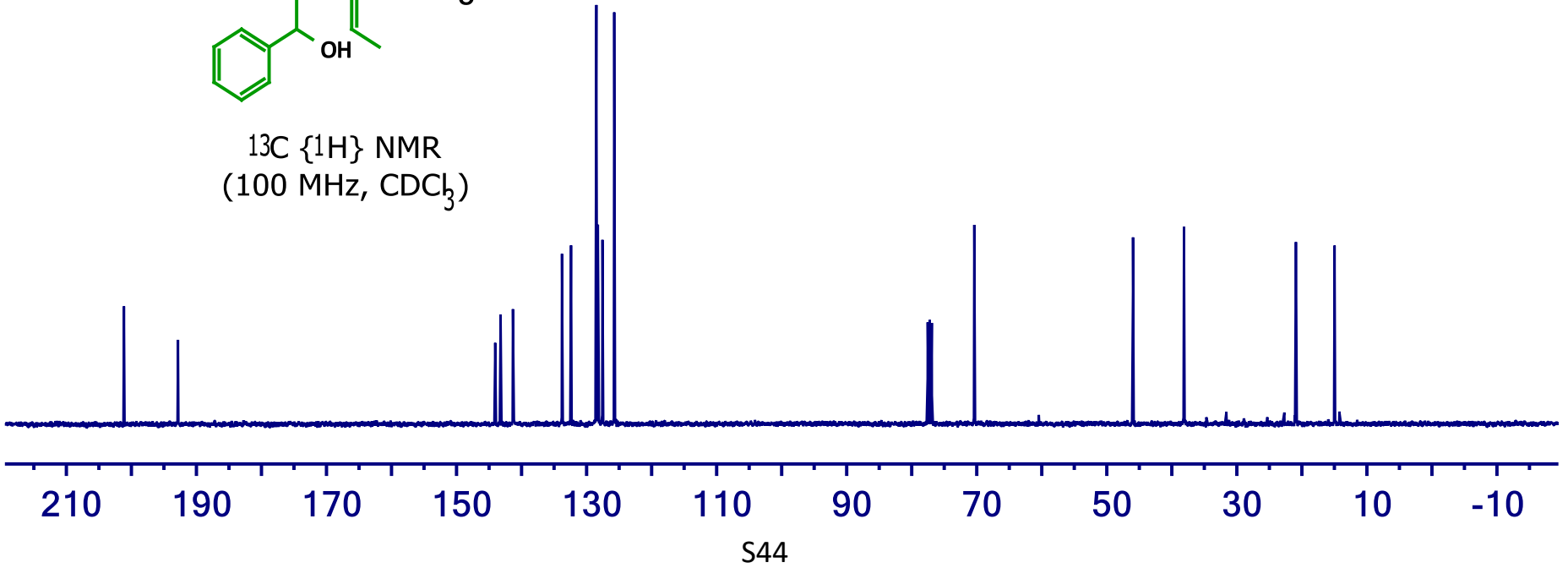
(E)-4-ethylidene-1-(furan-2-yl)-7-hydroxy-7-phenylheptane-1,5-dione

$4 c$

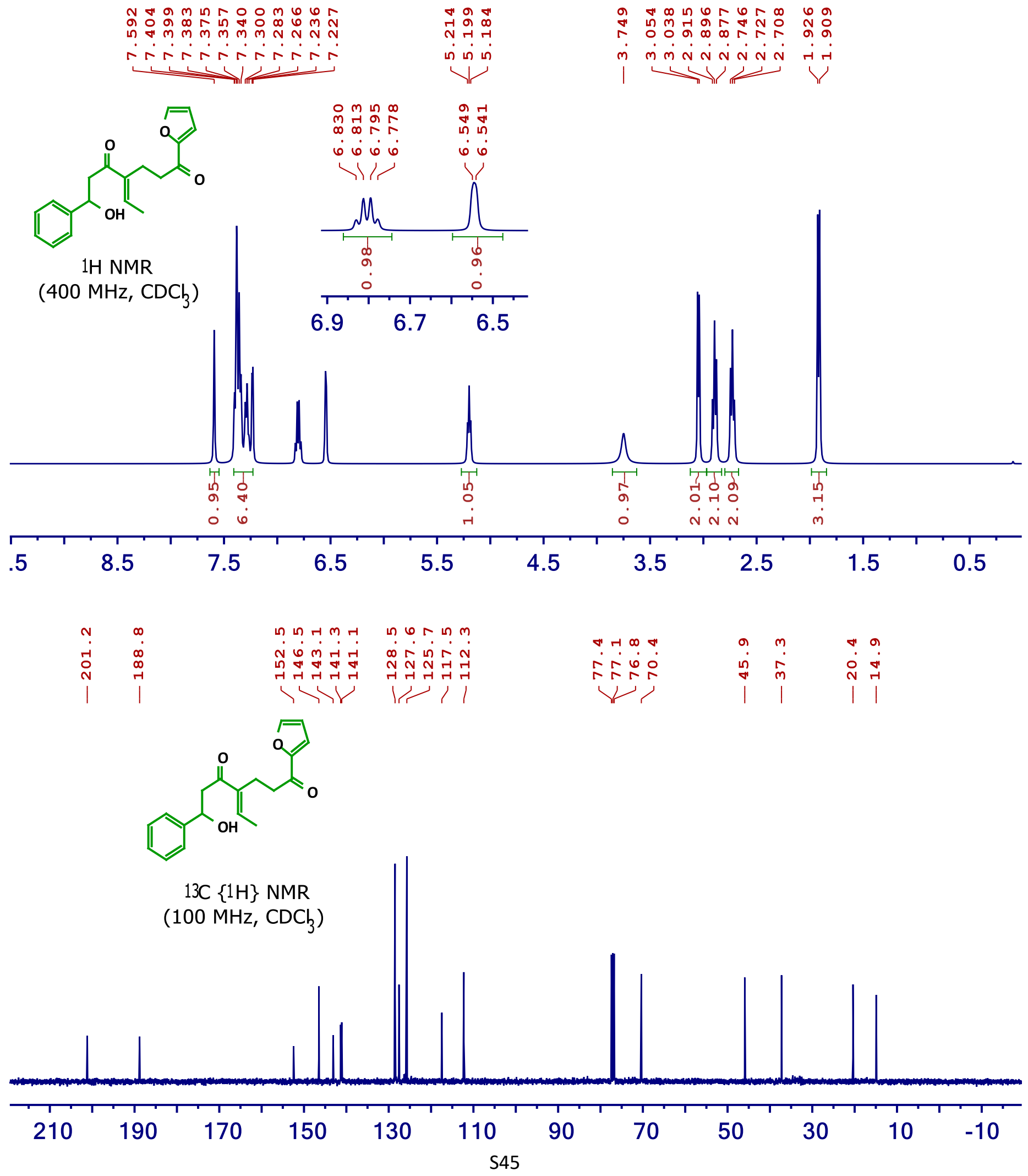


(E)-4-ethylidene-1-hydroxy-1,9-diphenylnonane-3,7-dione

$4 d$

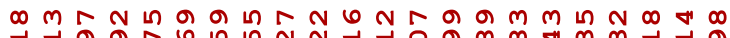
F F

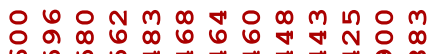

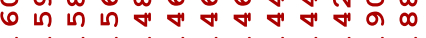

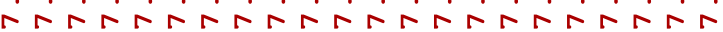

$\operatorname{con} 4 \sin n$ n 244

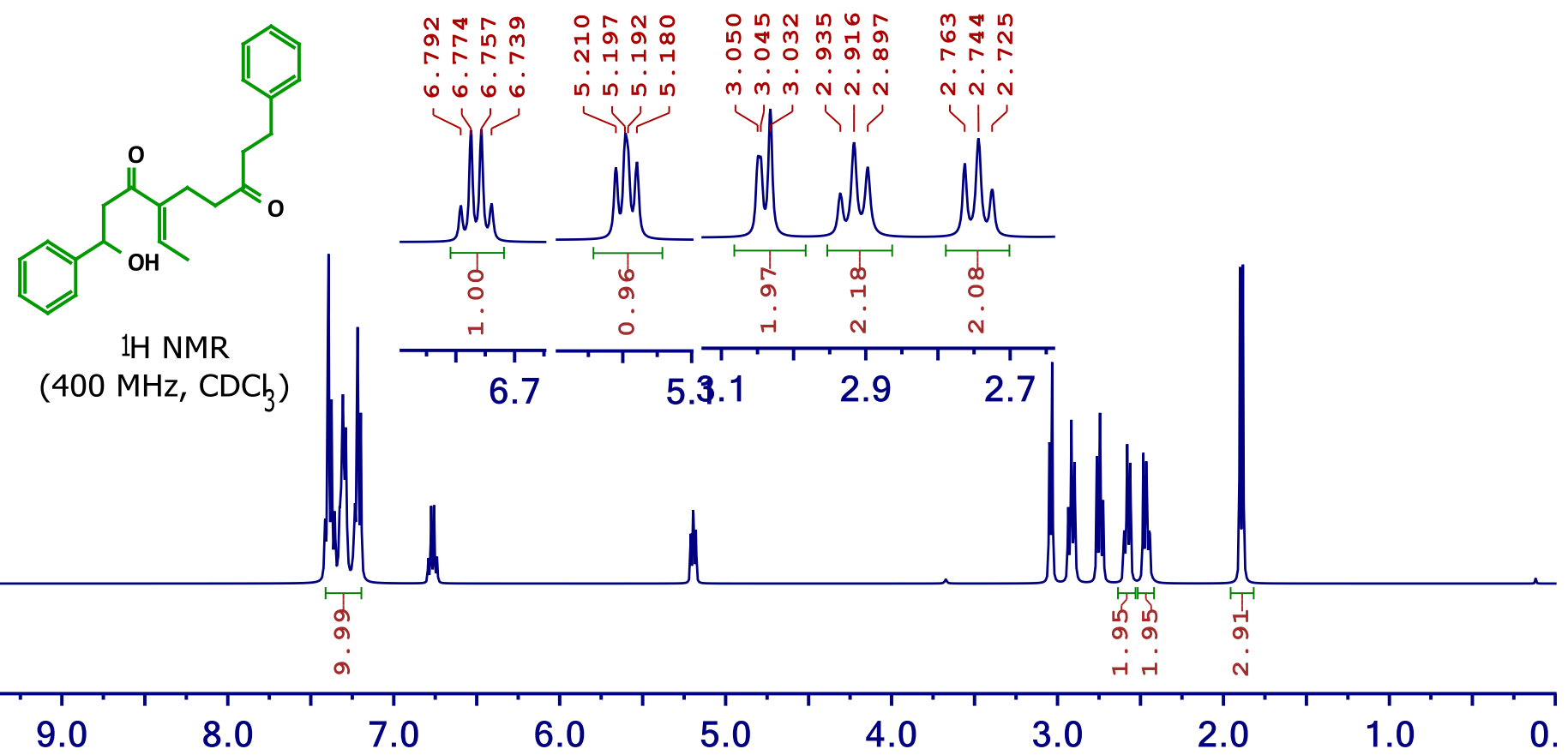

$\begin{array}{cc}\text { n) } & \text { N } \\ 0 & \text { r } \\ 0 & 0 \\ \text { N } & \text { N } \\ 1 & 1\end{array}$

$404 \pi \ln m e 4 t$

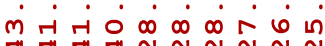

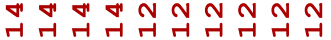

$\longrightarrow$

$\nabla ⿱ 4 \pi \infty$

is

a $N$ ᄂ $\infty \quad \sim \infty$

N/

约<smiles>C/C=C(/CCC(=O)CCc1ccccc1)C(=O)CC(O)c1ccccc1</smiles>

13C $\{1 \mathrm{H}\} \mathrm{NMR}$

(100 MHz, $\left.\mathrm{CDC}_{3}\right)$
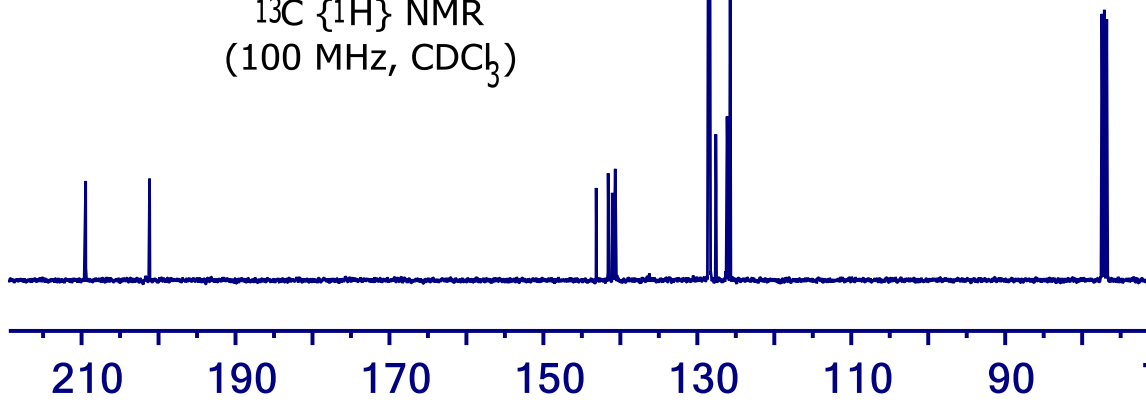

210

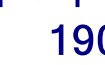

190

170

150

130

110

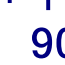


(E)-4-ethylidene-7-hydroxy-1,7-diphenylheptane-1,5-dione

$4 e$

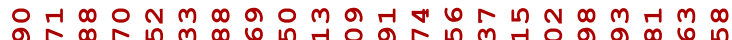

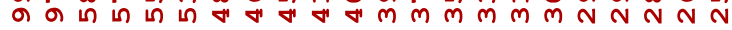

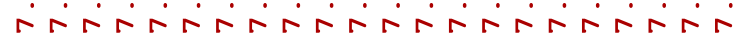

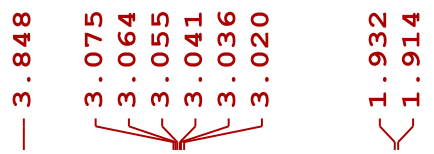<smiles>C/C=C(\CCC(=O)c1ccccc1)C(=O)CC(O)c1ccccc1</smiles>

$1 \mathrm{H} N M R$
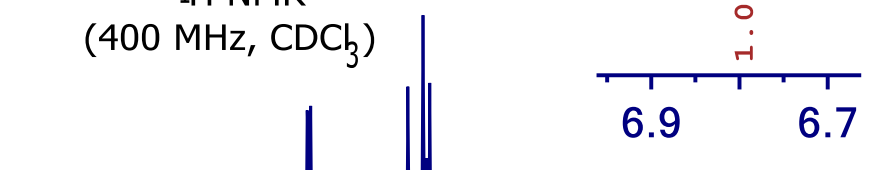

\begin{tabular}{llll}
\multirow{1}{*}{} & 0 & $\infty$ & -1 \\
$m$ & -1 & 0 & $\infty$ \\
$\infty$ & $\infty$ & 1 & 1
\end{tabular}

.060
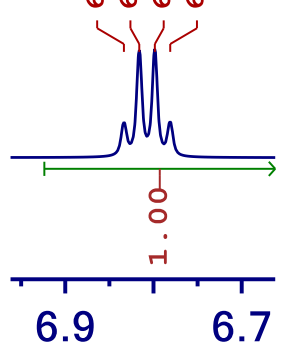

6.7

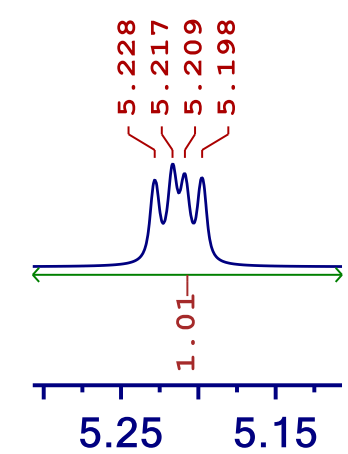

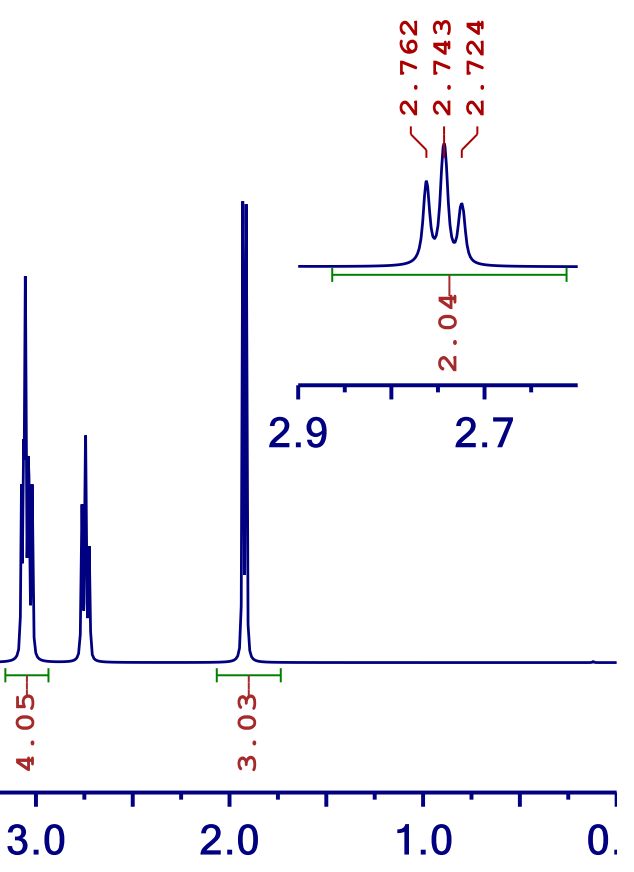

\begin{tabular}{ll}
$N$ & $\infty$ \\
\hdashline & 0 \\
0 & 9 \\
$N$ & 9 \\
$1 /$
\end{tabular}

n a n

$\dot{m} \sim \dot{\sim} \dot{0} \dot{m} \dot{m} \dot{\infty} \dot{\infty} \dot{\sim}$ in

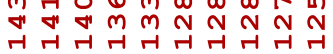

皮)

5.0

4.0

3.0

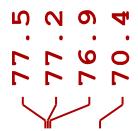

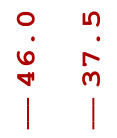

in $a$

ค.<smiles>C/C=C(\CCC(=O)c1ccccc1)C(=O)CC(O)c1ccccc1</smiles>

$13 \mathrm{C}\{1 \mathrm{H}\} \mathrm{NMR}$

$\left(100 \mathrm{MHz}, \mathrm{CDC}_{3}\right)$

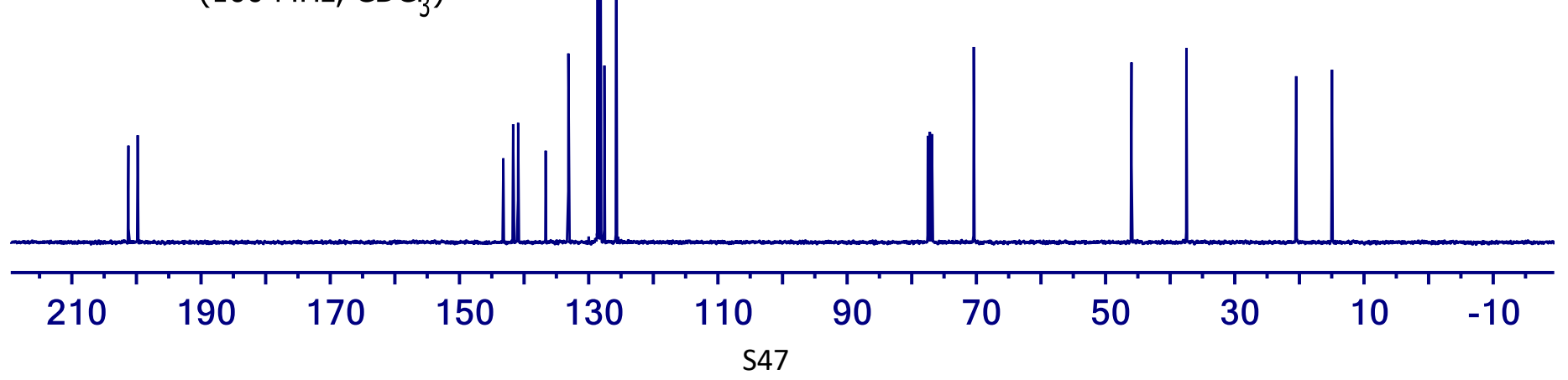


(E)-1-cyclohexyl-4-ethylidene-7-hydroxy-7-phenylheptane-1,5-dione

4f

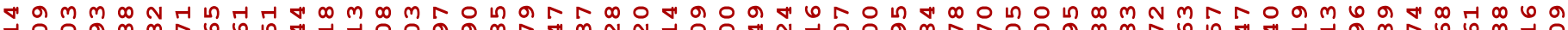
子

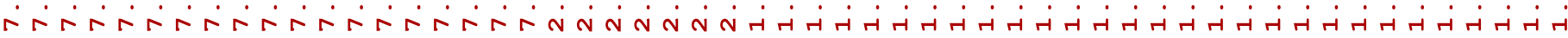
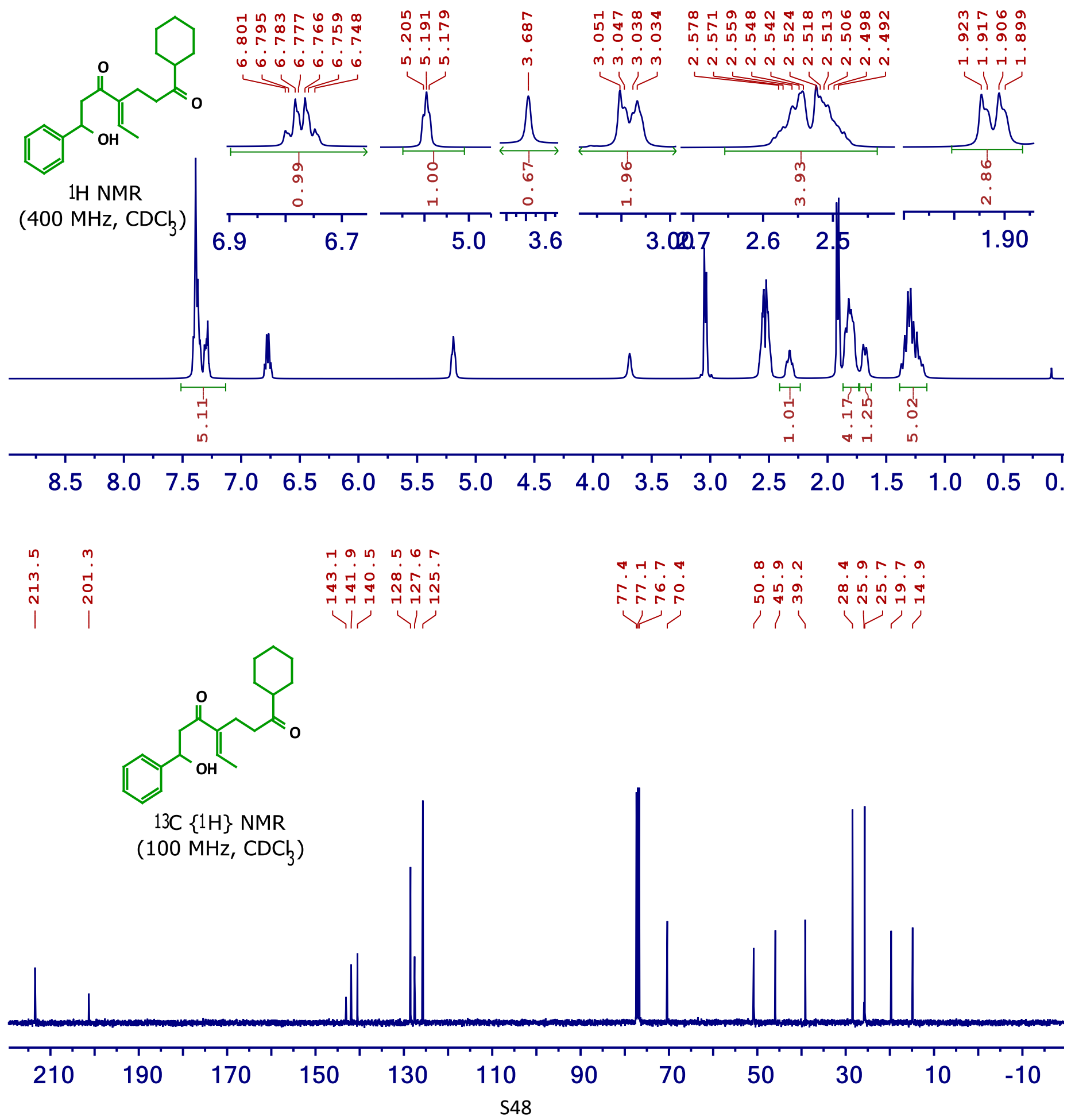
(1E,6E)-6-ethylidene-9-hydroxy-1,9-diphenylnon-1-ene-3,7-dione

$4 \mathrm{~g}$

今

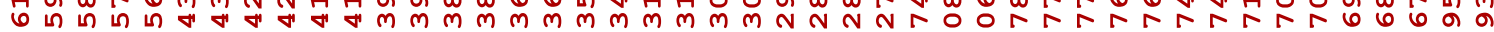

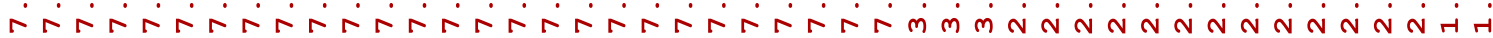
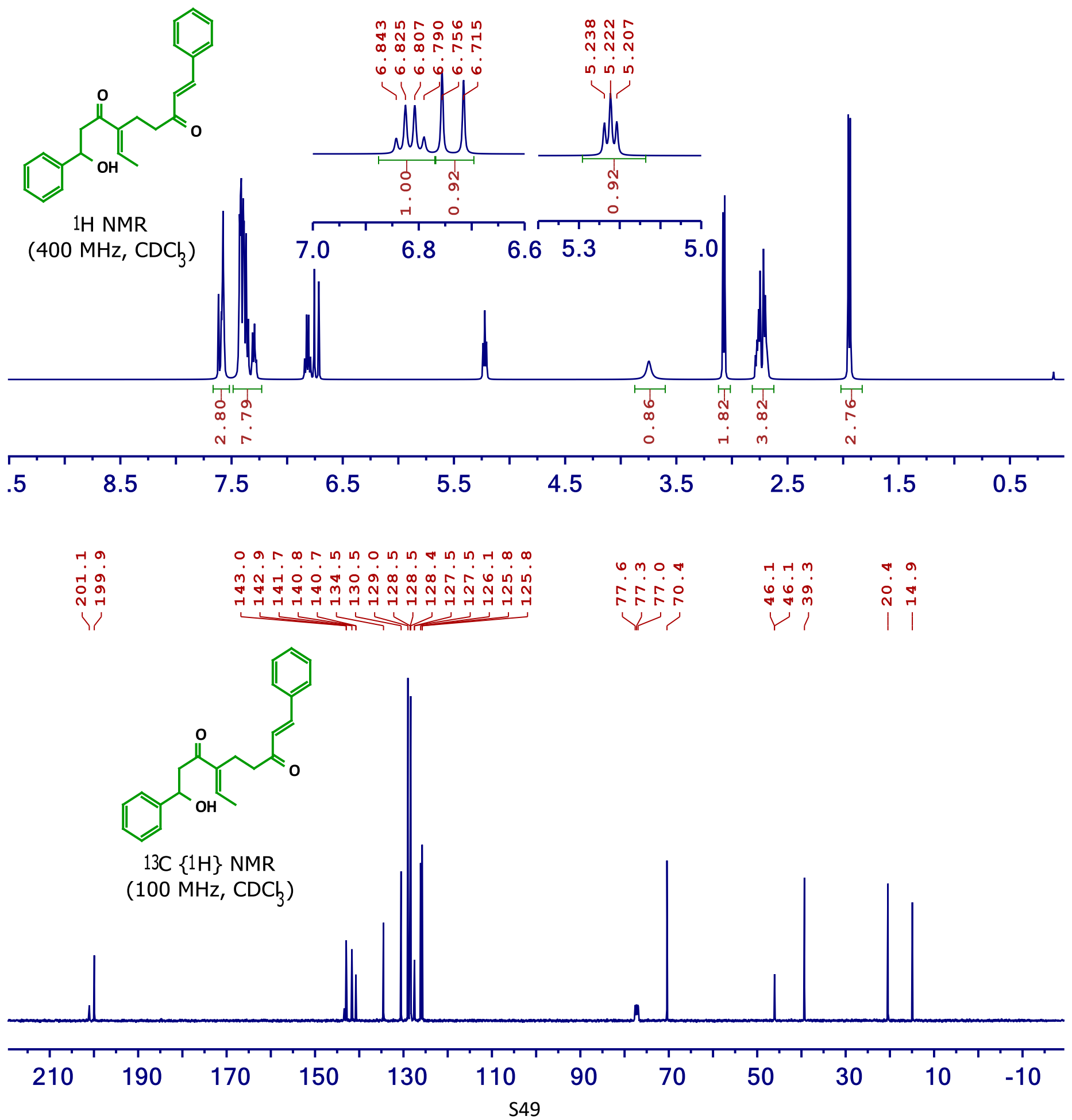
(E)-1-(2,6-dichlorophenyl)-4-ethylidene-7-hydroxy-7-phenylheptane-1,5-dione

$4 h$

N $m m m m m M m M m M N N N N N N N$

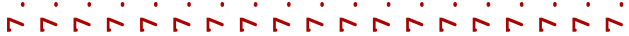

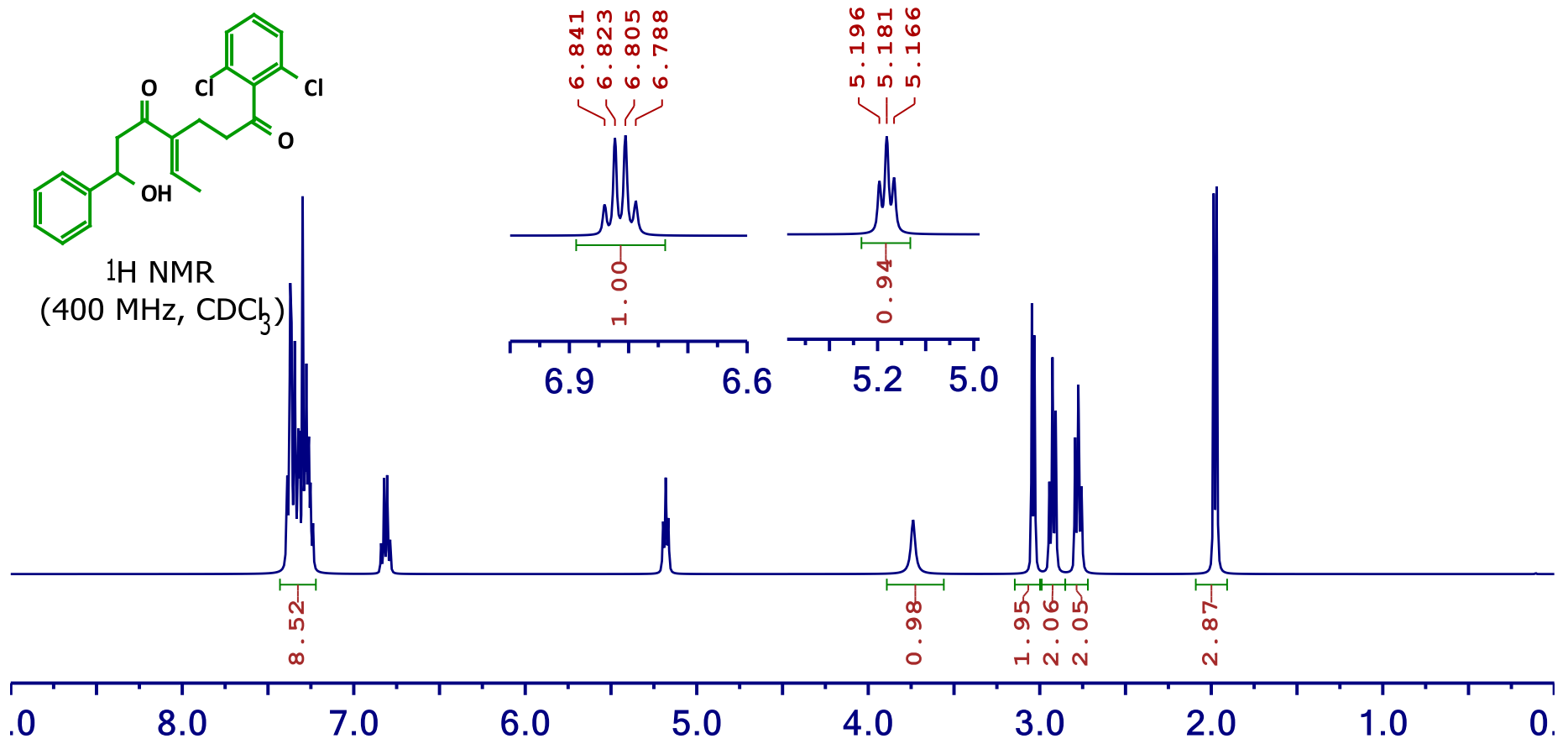

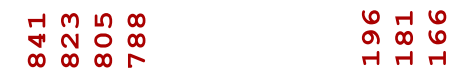

in

ம் $\dot{0} \dot{0}$

1/ 1

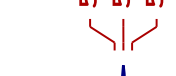

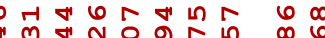

० व बबतr a ब

m $\dot{m} \dot{m} \dot{N} \dot{N} \dot{N} \dot{N} \vec{r}$

W

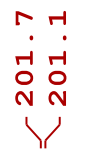

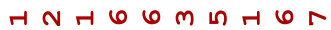

mं तं

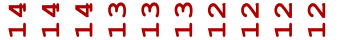

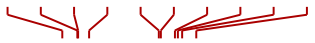

1) $N \infty \mathrm{m}$

年走是

Wl

a $m$

00

กู่

11

$\begin{array}{ll}4 & \text { 1 } \\ \text { a } & \text { 1 }\end{array}$<smiles>C/C=C(\CCC(=O)c1c(Cl)cccc1Cl)C(=O)CC(O)c1ccccc1</smiles>

$13 \mathrm{C}\{1 \mathrm{H}\} \mathrm{NMR}$

(100 MHz, CDCb)
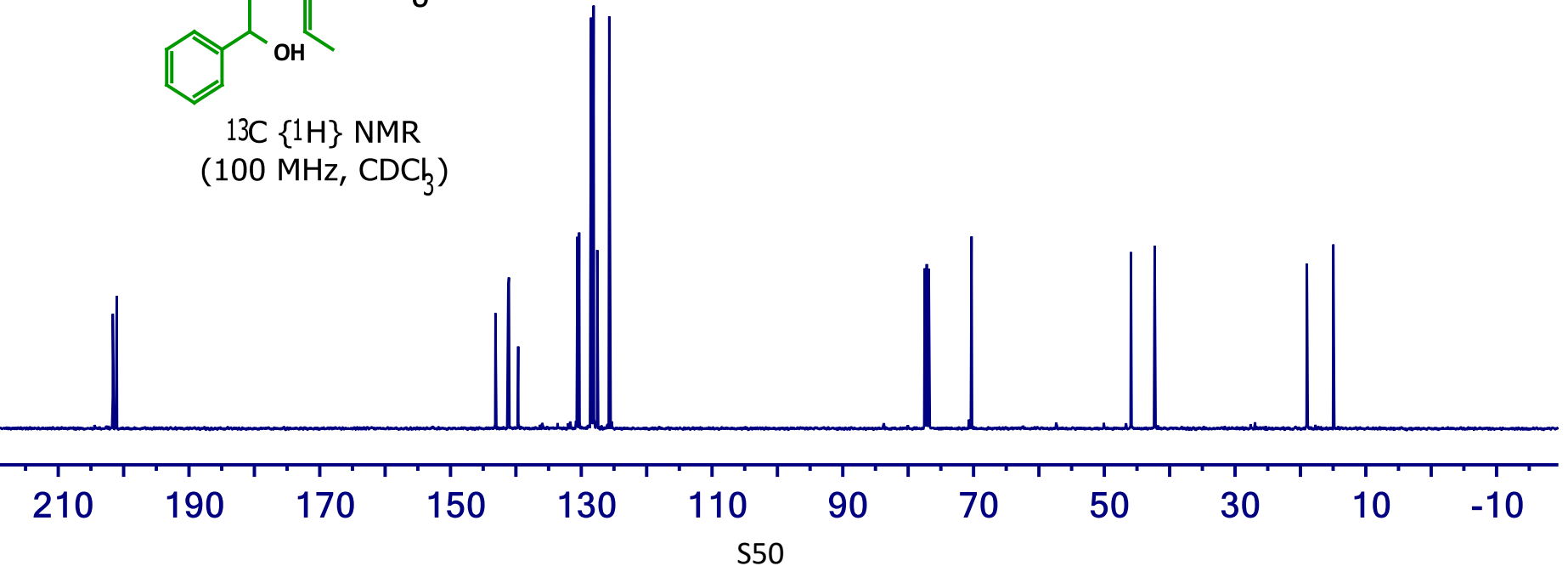
(E)-4-ethylidene-7-hydroxy-1-(naphthalen-1-yl)-7-phenylheptane-1,5-dione

$4 \mathbf{i}$

几

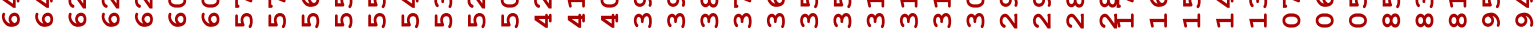

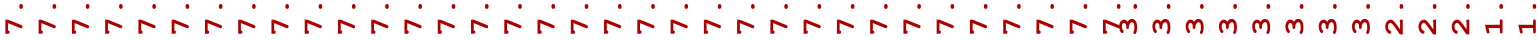<smiles>C/C=C(/CCC(=O)c1cccc2ccccc12)C(=O)CC(O)c1ccccc1</smiles>

$1 \mathrm{H} N \mathrm{NMR}$

(400 $\left.\mathrm{MHz}, \mathrm{CDC}_{3}\right)$

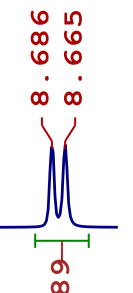

ํํำ

incisisin

$1 / 14$

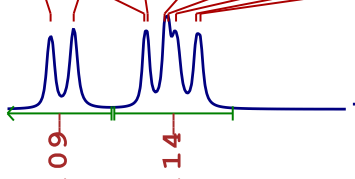

0<smiles>CCC</smiles><smiles>[3H][Te]C1CCCCC1</smiles>

8.6

8.0

4

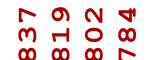

$\dot{0} \dot{0} \dot{0} \dot{0}$

$1 / 1$

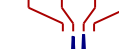

n $⿻$ ก

เก เก เก เ

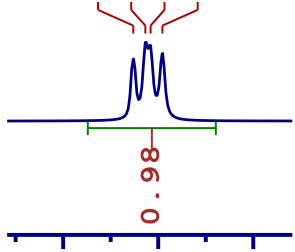

5.3

5.1

$\begin{array}{cc}\infty & m \\ m & \dot{1} \\ 0 & 0 \\ \text { w } & \text { v } \\ 1 & 1\end{array}$

Hก

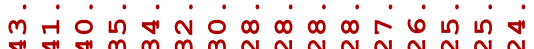

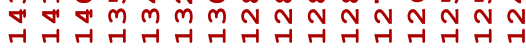

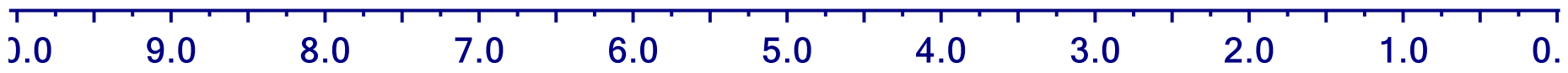

$\Delta+4 \infty$

iล잉

H?

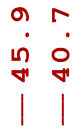

$\infty 0$

श्रा

装 $\dot{m}$<smiles>C/C=C(\CCC(=O)c1cccc2ccccc12)C(=O)CC(O)c1ccccc1</smiles>

$13 \mathrm{C}\{1 \mathrm{H}\} \mathrm{NMR}$ (100 $\left.\mathrm{MHz}, \mathrm{CDC}_{3}\right)$
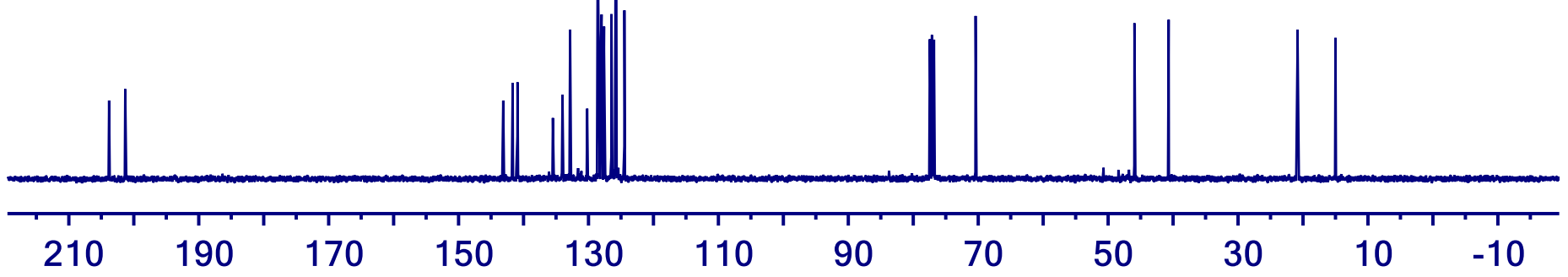
(E)-1-hydroxy-1,9-diphenyl-4-propylidenenonane-3,7-dione

$4 \mathrm{j}$

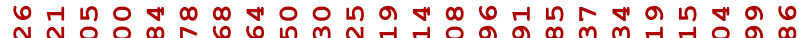

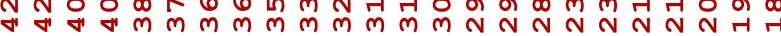

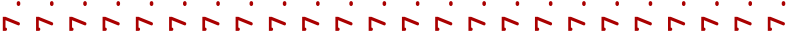
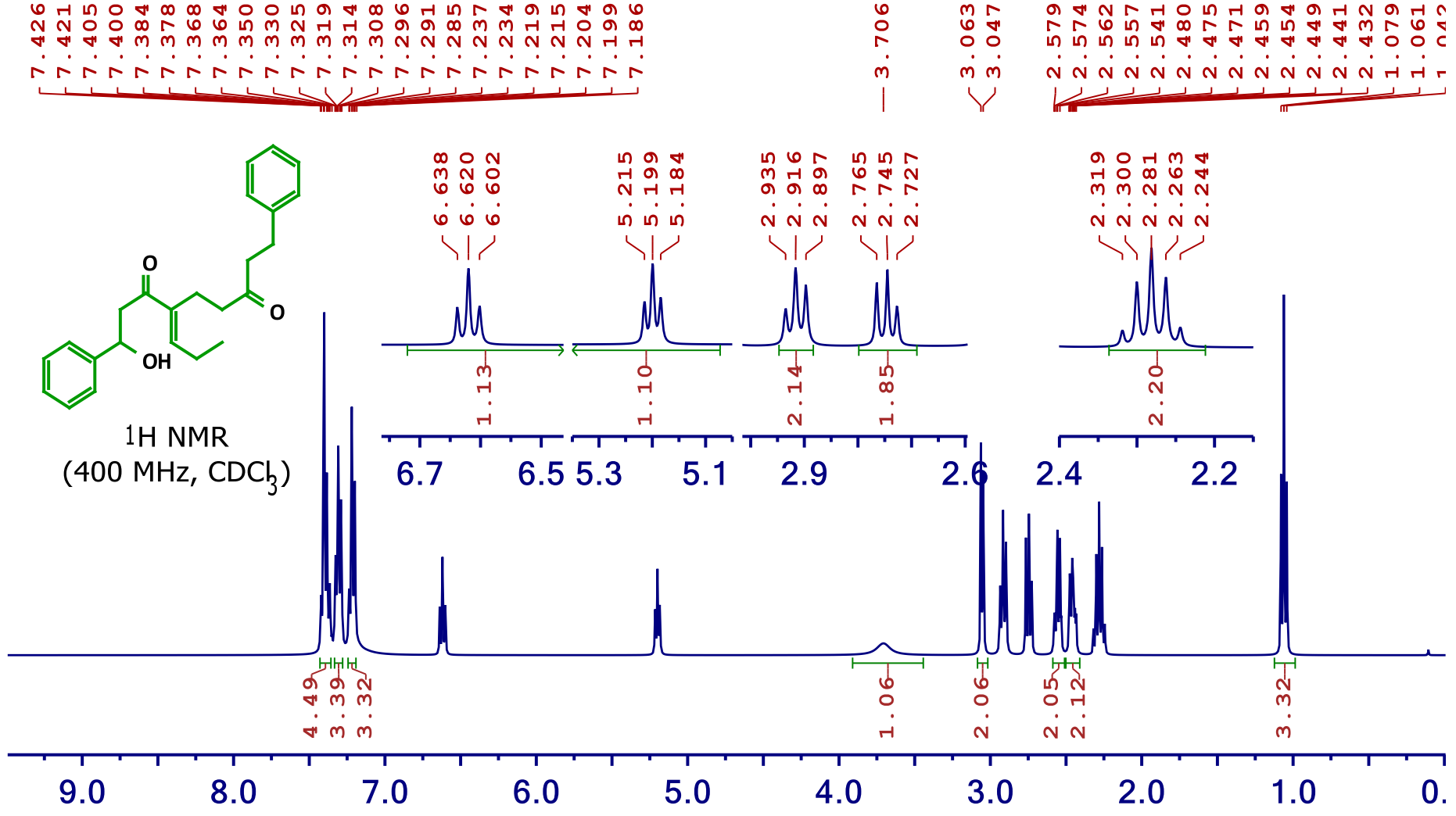

$13 \mathrm{C}\{1 \mathrm{H}\} \mathrm{NMR}$ (100 $\left.\mathrm{MHz}, \mathrm{CDC}_{3}\right)$

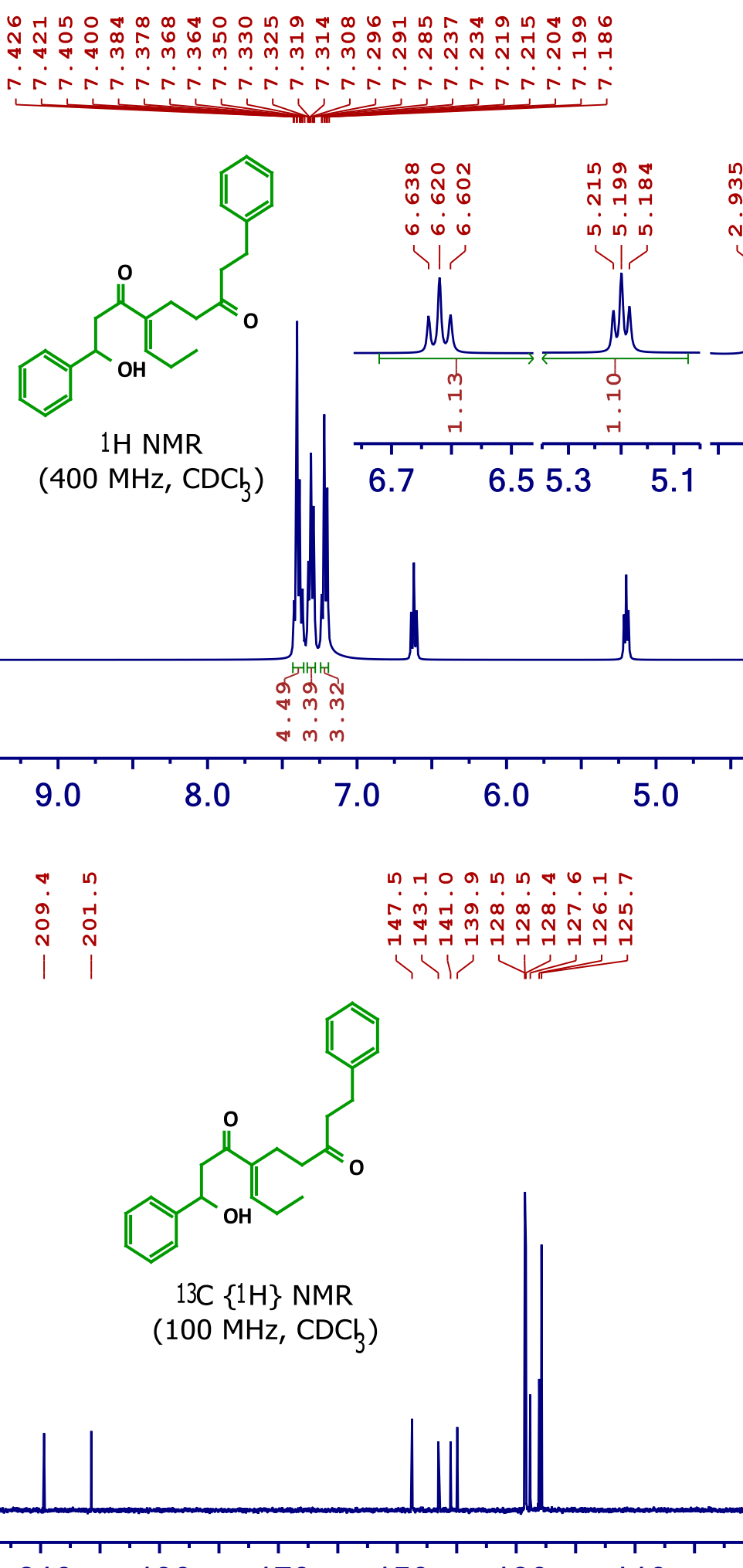

1 400 ถ

$\therefore \dot{m} 000$ Hٓ

\र।
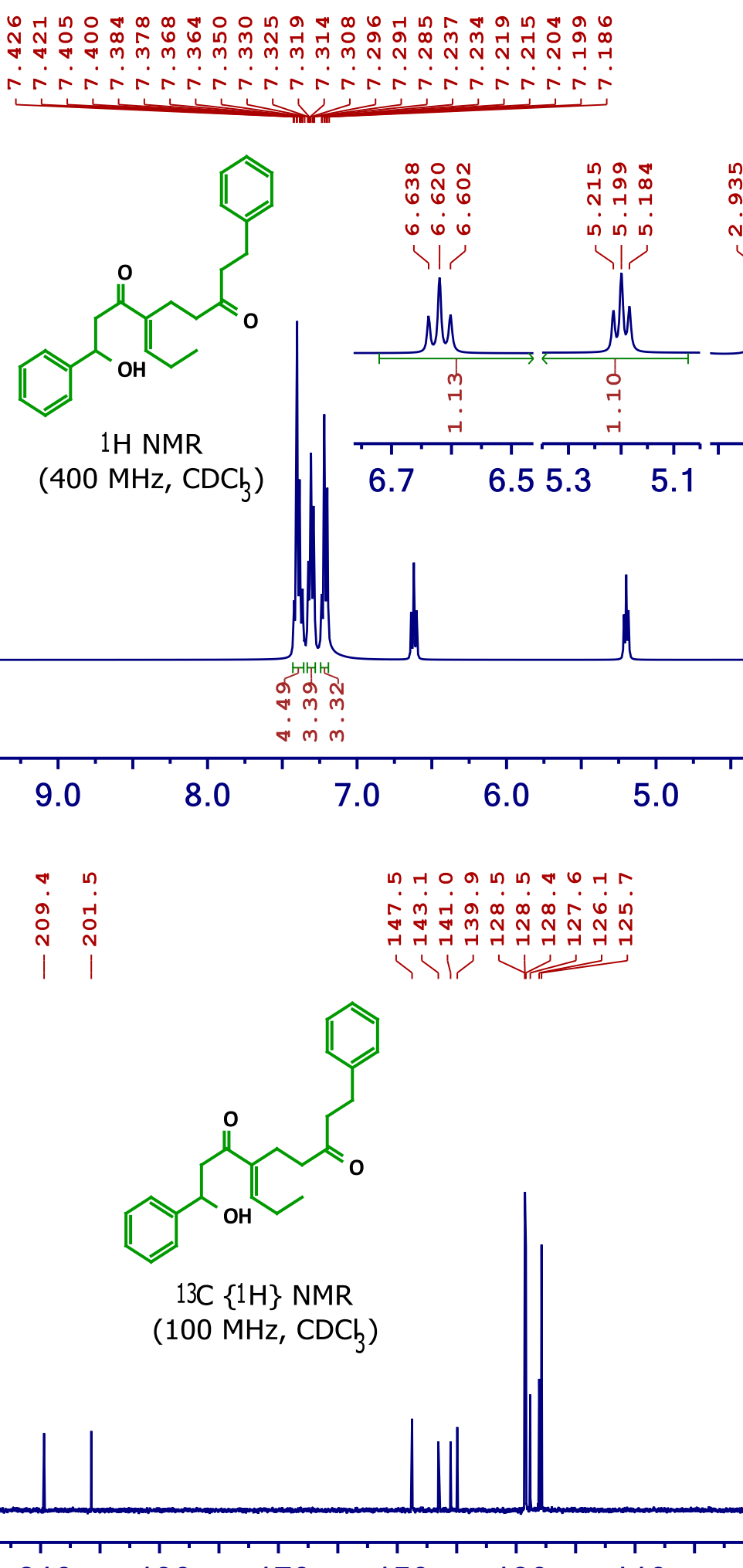
)
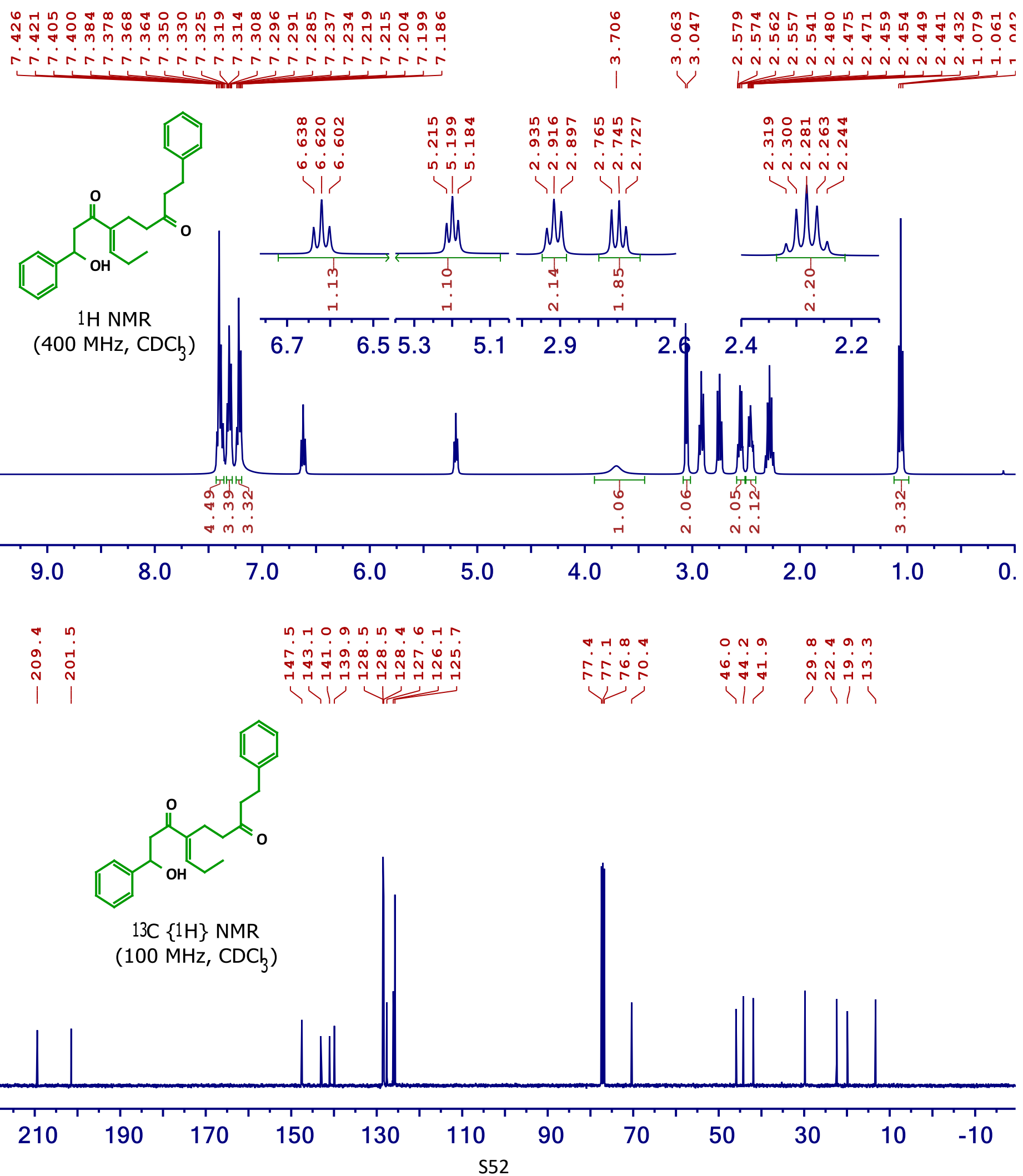
(E)-1-hydroxy-1,9-diphenyl-4-(2-phenylethylidene)nonane-3,7-dione

$4 k$

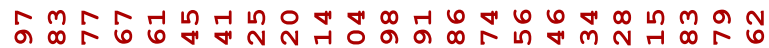

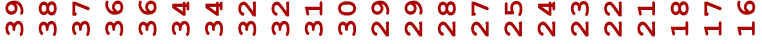

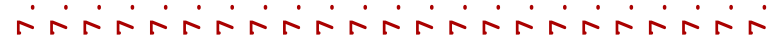

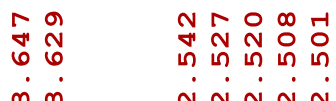

$\int^{2}$
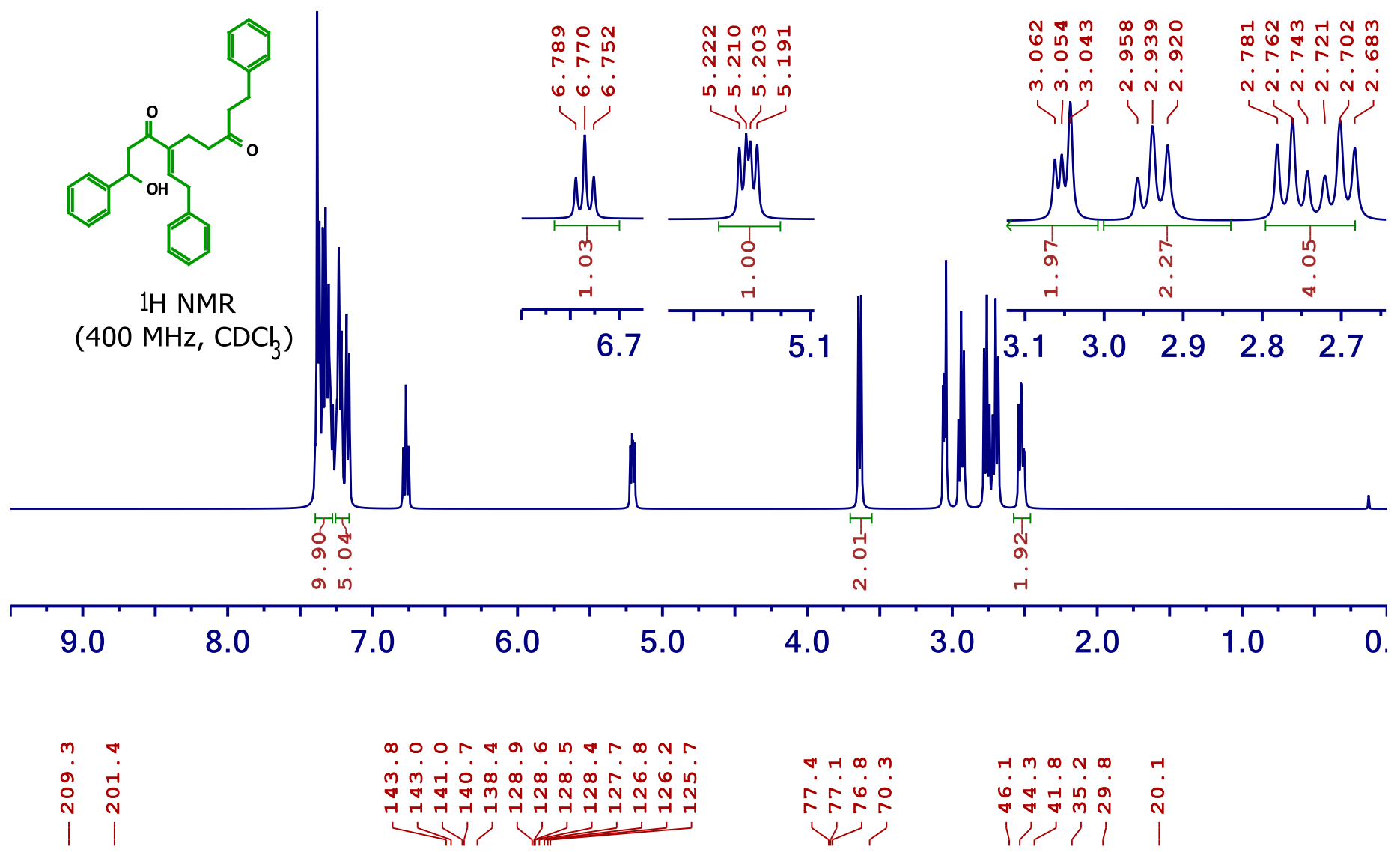<smiles>O=C(CCCc1ccccc1)CCC(=O)C(O)Cc1ccccc1</smiles>

$13 \mathrm{C}\{1 \mathrm{H}\} \mathrm{NMR}$

(100 $\mathrm{MHz}, \mathrm{CDC}_{3}$ )
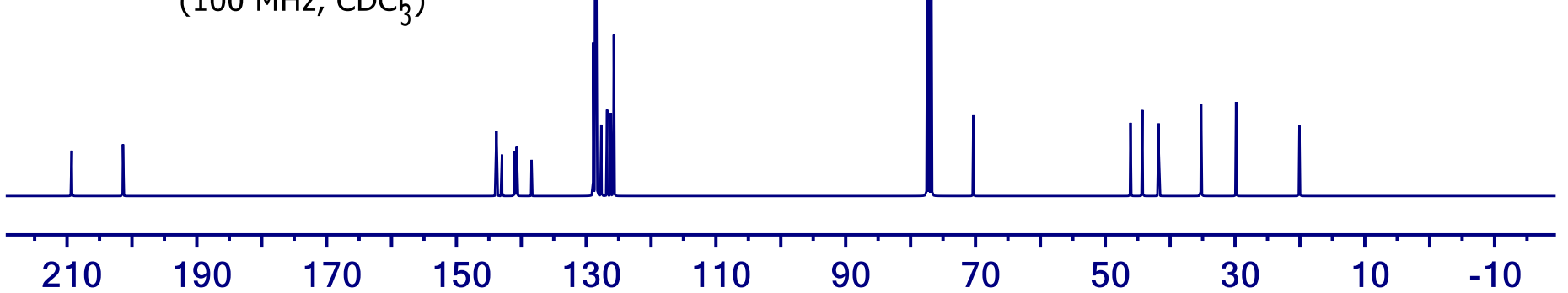
(E)-1-hydroxy-4-pentylidene-1,9-diphenylnonane-3,7-dione

41

ดิ

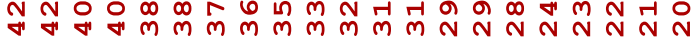

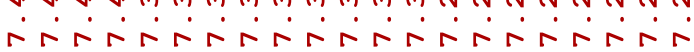

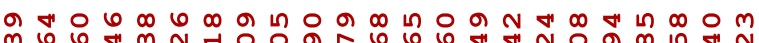

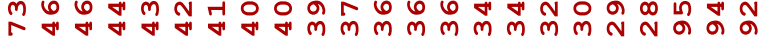

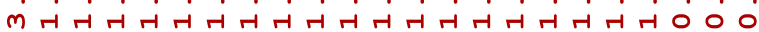

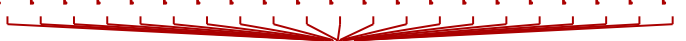

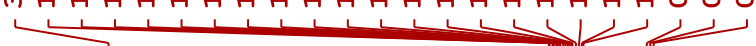

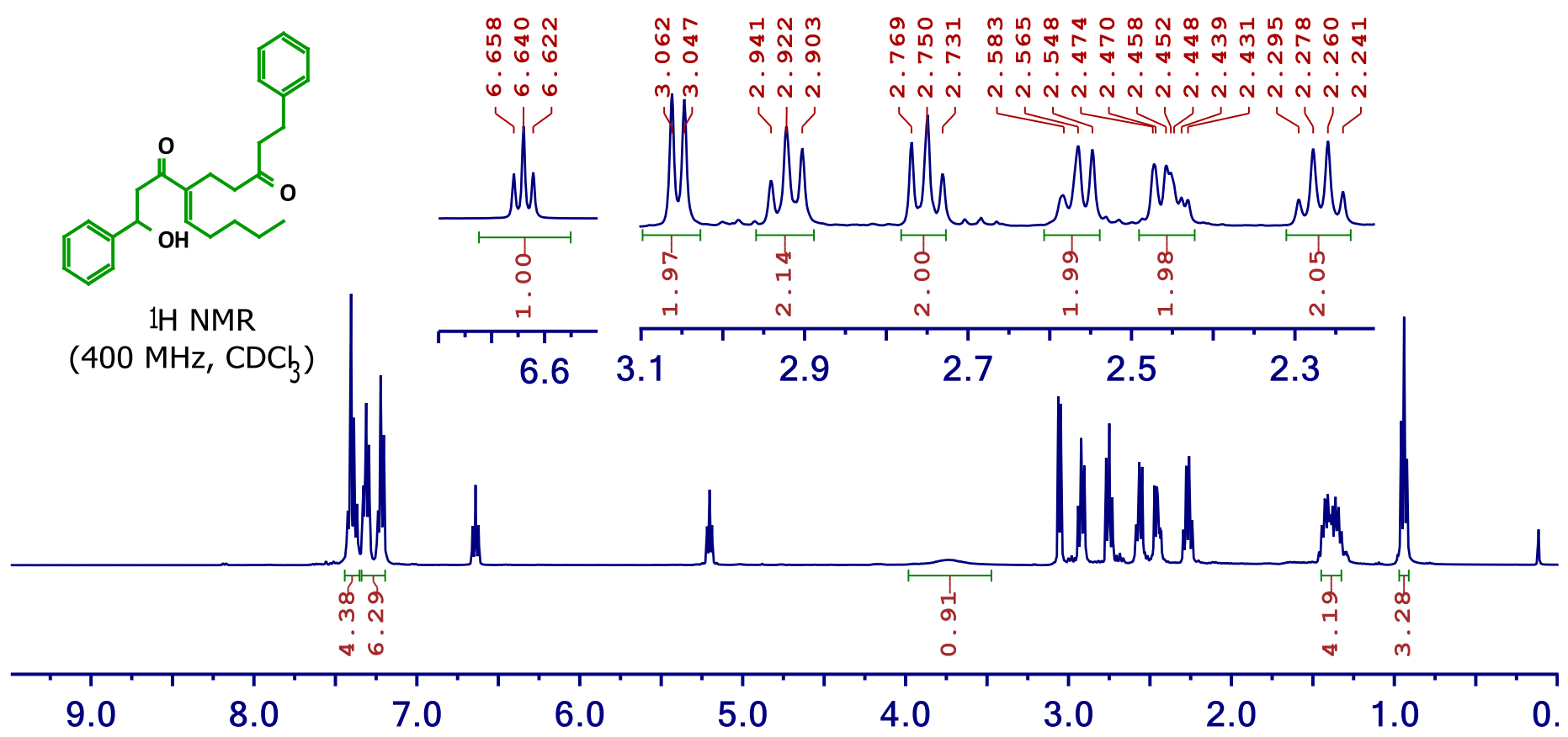

$\begin{array}{cc}\pi & + \\ 0 & -1 \\ 0 & 0 \\ \text { N } & \text { N } \\ 1 & 1\end{array}$

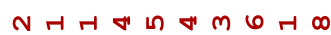
$\dot{m} \dot{m} \dot{0} \dot{\infty} \dot{\infty} \dot{\infty} \dot{\sim} \dot{\varphi}$ in

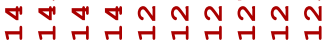

$>1 /$

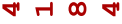

○ $\operatorname{ar} a \infty$ n 0 a

ingo

ט

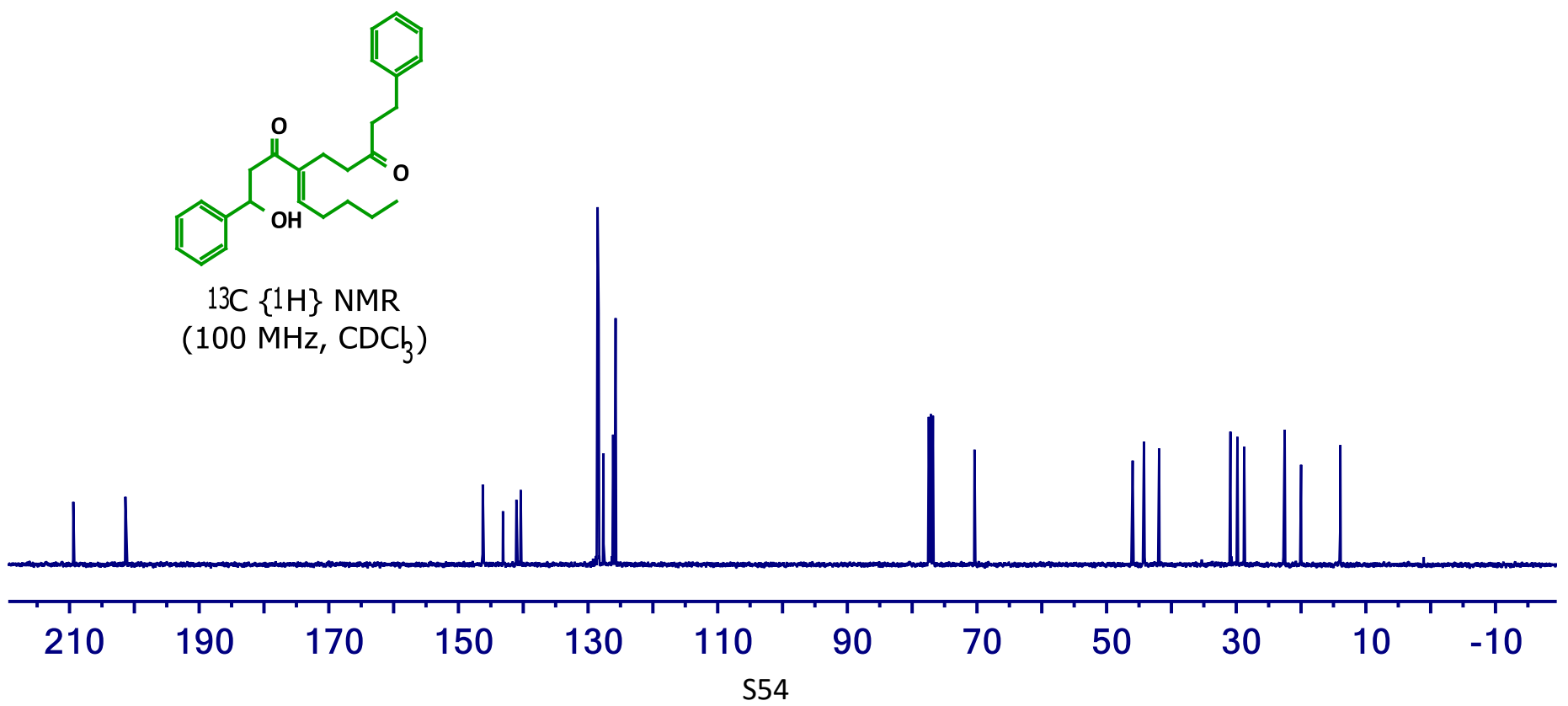

N?

II l1? 
(E)-1-hydroxy-4-(3-methylbutylidene)-1,9-diphenylnonane-3,7-dione

$4 \mathrm{~m}$

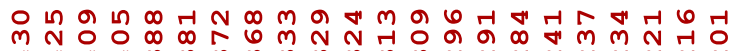

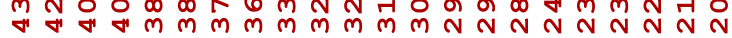

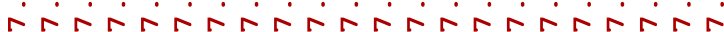

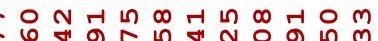

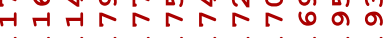

N 24444444400

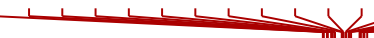
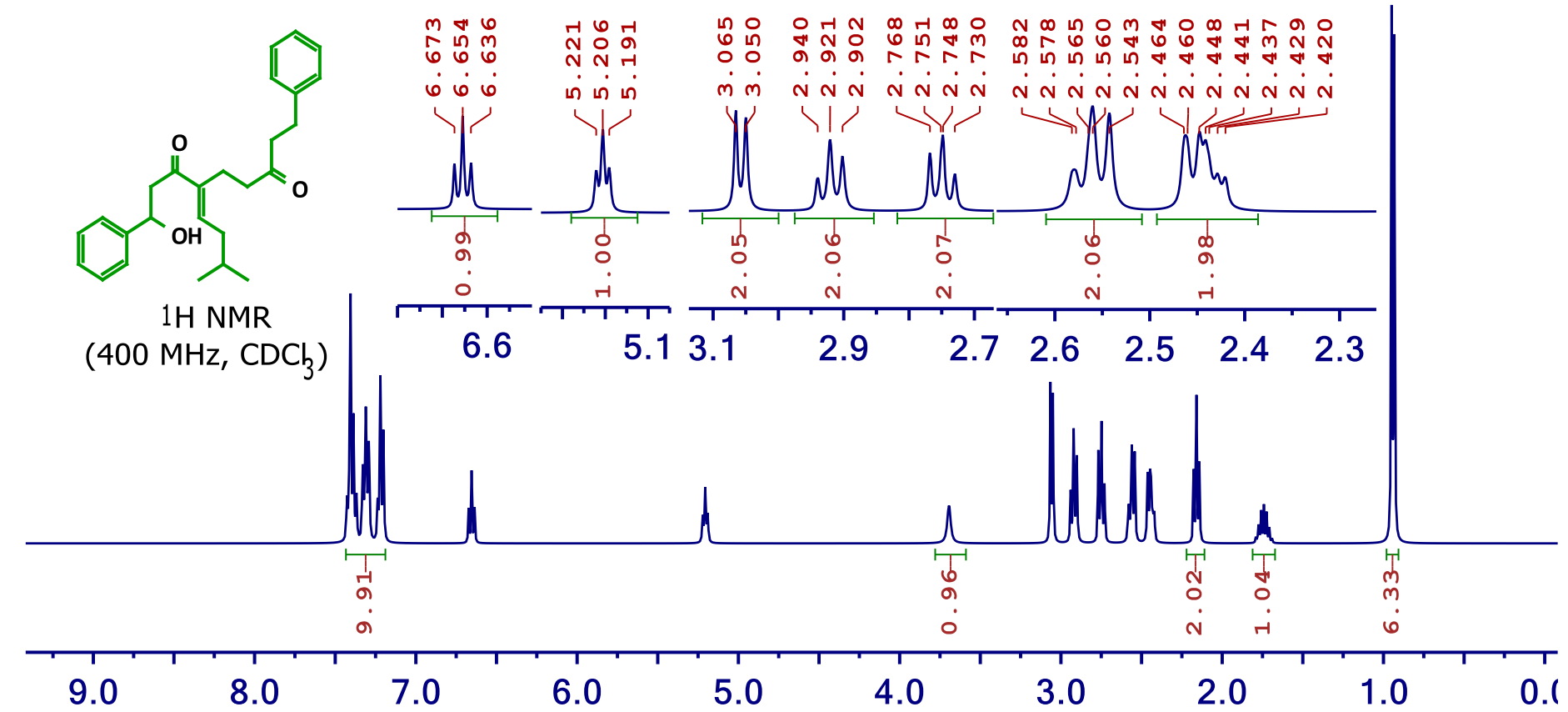

(1)
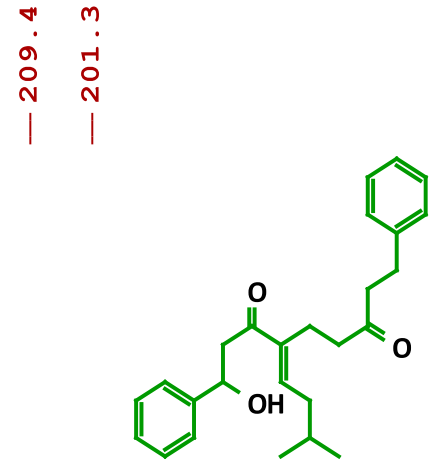

13C $\{1 \mathrm{H}\}$ NMR $\left(100 \mathrm{MHz}, \mathrm{CDC}_{3}\right)$
4 400 in

iि $\dot{m} \dot{-i} \dot{\infty} \dot{\infty} \dot{\sim} \dot{0}$

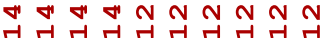

)

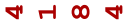

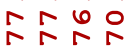

W/
$0 \leqslant \infty 0 \infty \pi n$

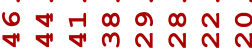

\/।/1/1/

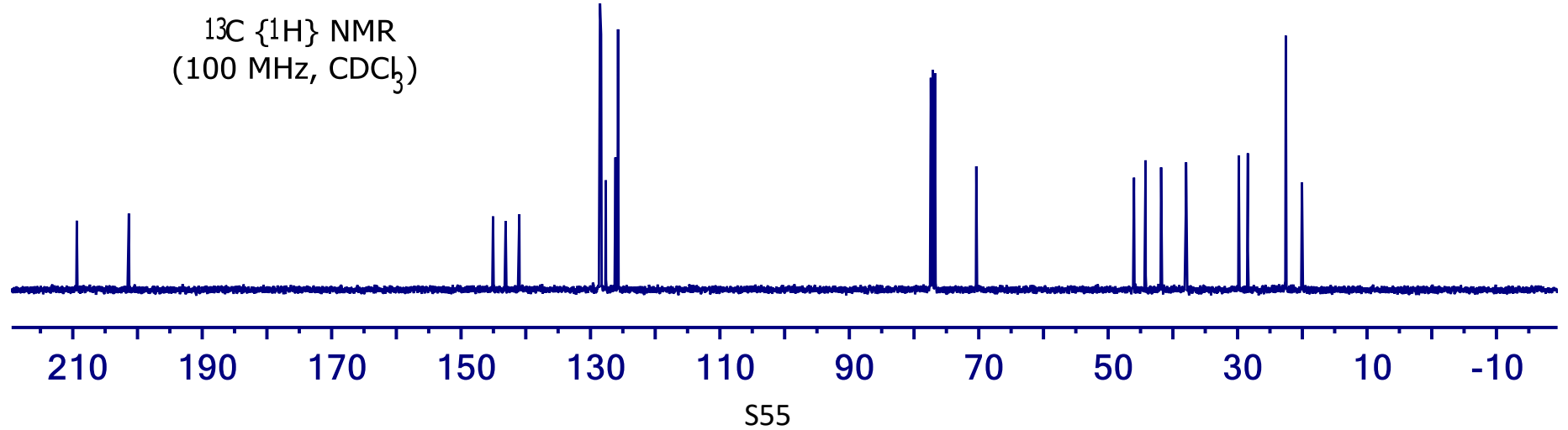


(E)-4-(cyclohexylmethylene)-1-hydroxy-1,9-diphenylnonane-3,7-dione

4n

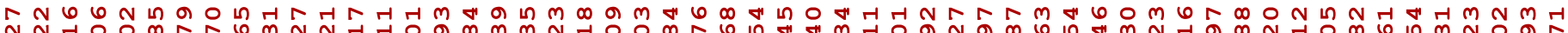

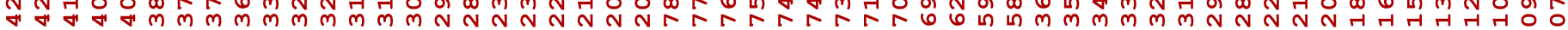

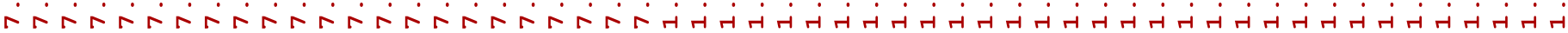
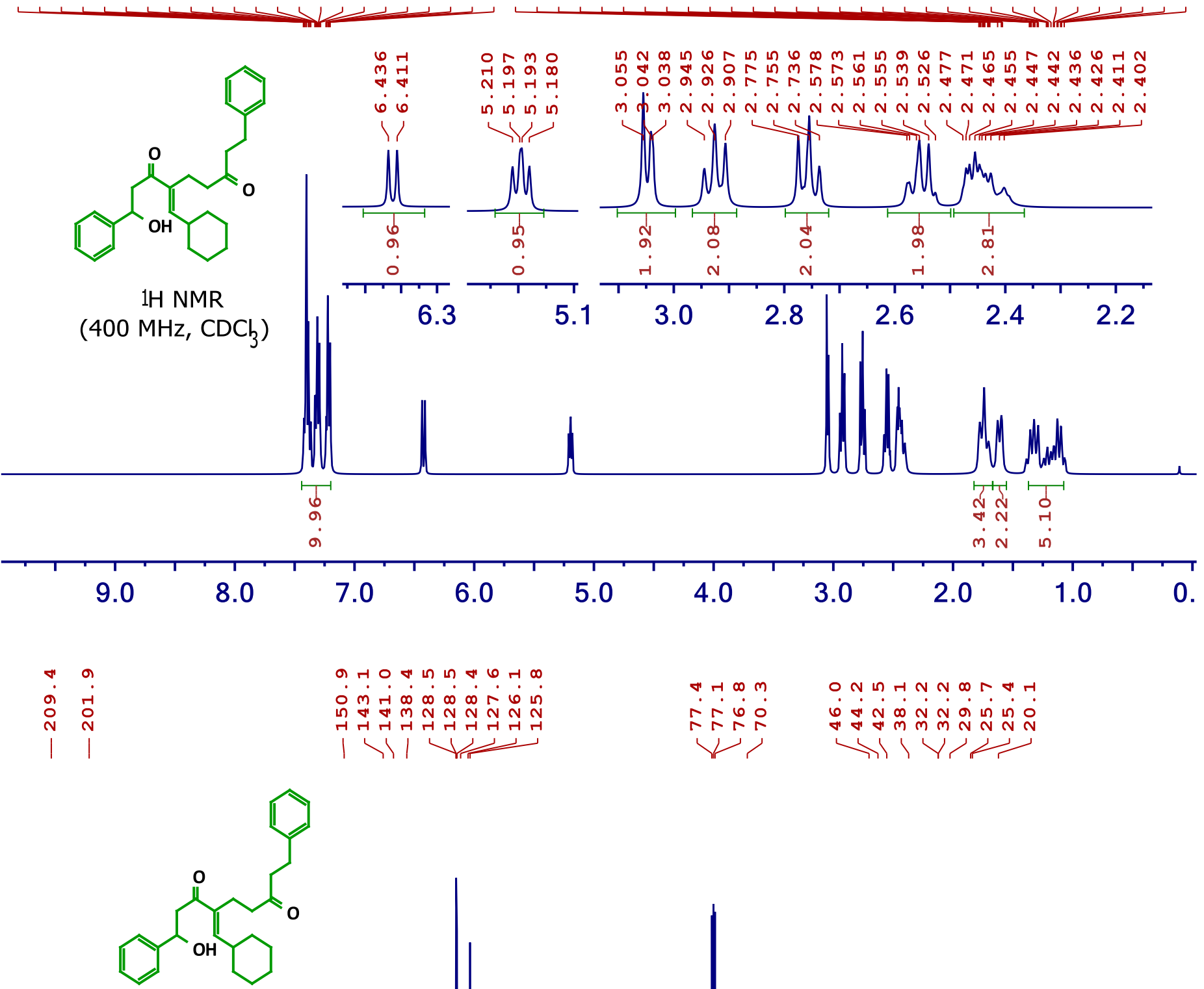

$13 \mathrm{C}\{1 \mathrm{H}\} \mathrm{NMR}$

(100 $\left.\mathrm{MHz}, \mathrm{CDC}_{3}\right)$
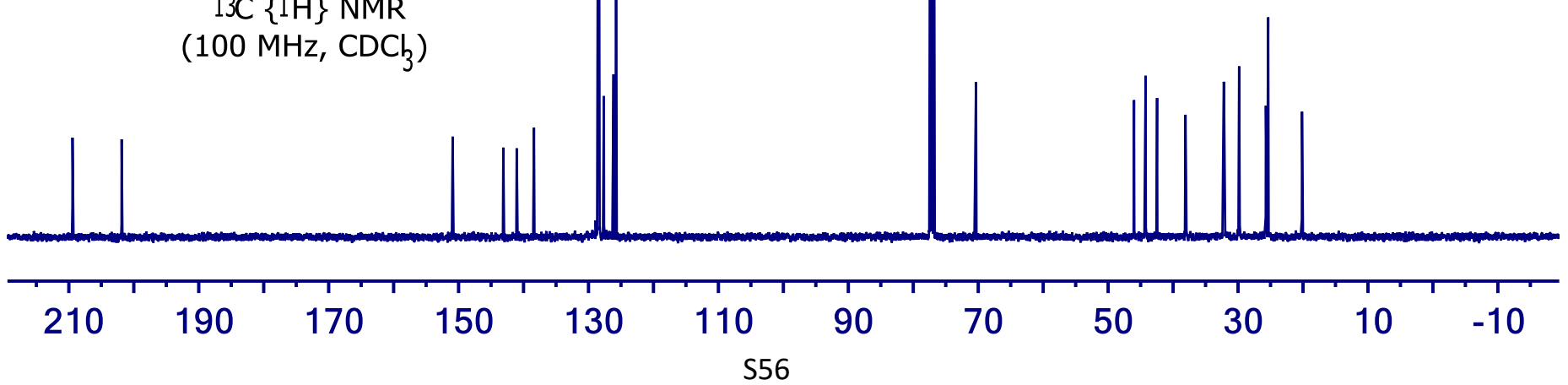
(E)-4-(furan-2-ylmethylene)-1-hydroxy-1,9-diphenylnonane-3,7-dione

40

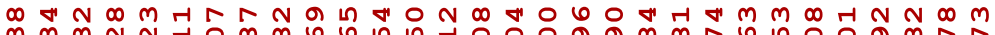

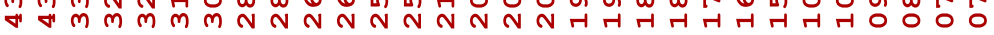

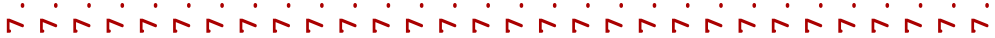

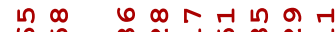

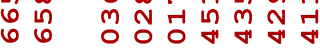

mं mंmंर्Nivi

L ل

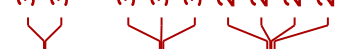

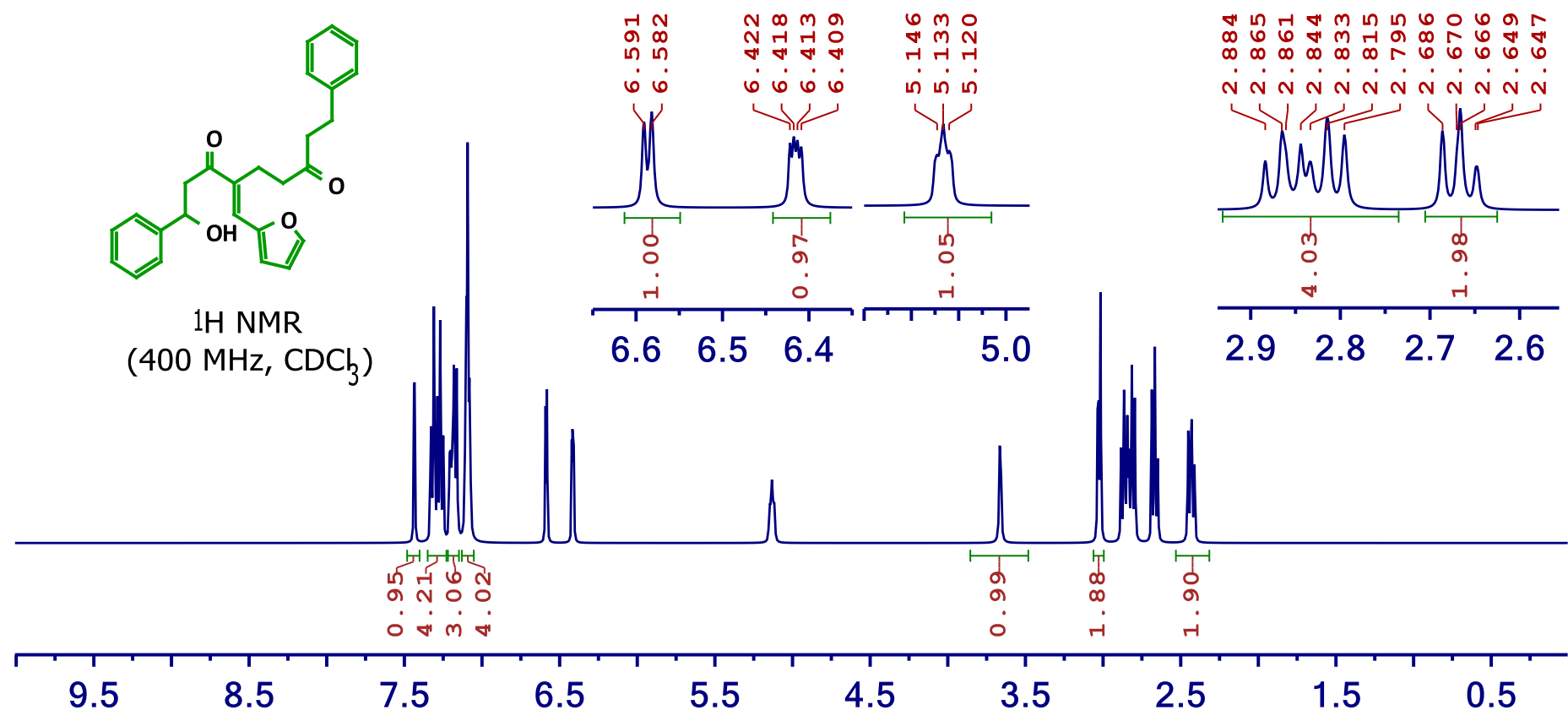

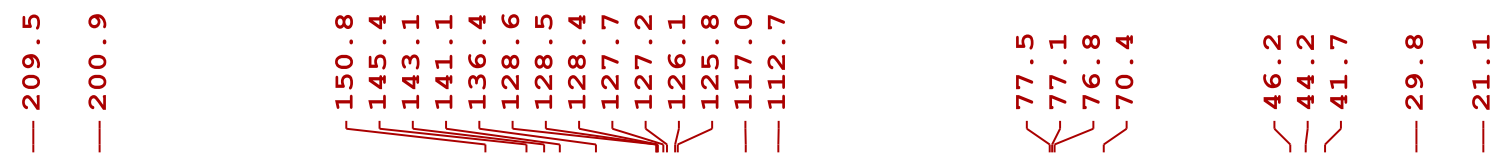<smiles>O=C(CCC(=O)C(O)Cc1ccco1)CCc1ccccc1</smiles>

$13 \mathrm{C}\{1 \mathrm{H}\} \mathrm{NMR}$ $\left(100 \mathrm{MHz}, \mathrm{CDC}_{3}\right)$

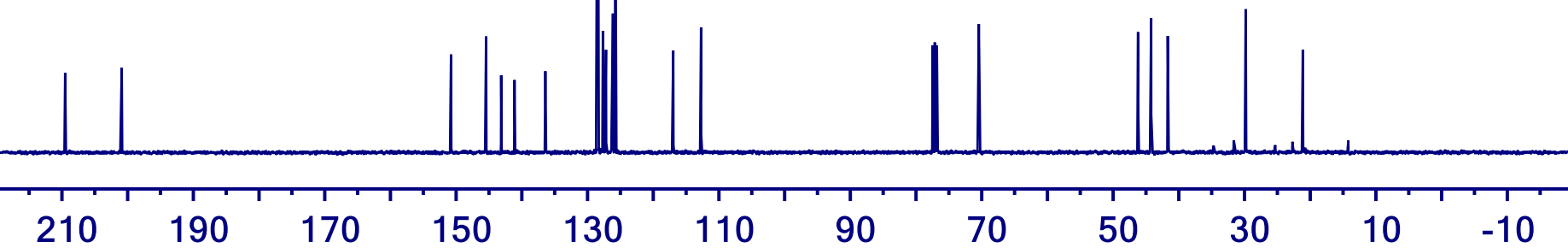


(E)-1-hydroxy-4-(2-methylpropylidene)-1,9-diphenylnonane-3,7-dione

$4 p$

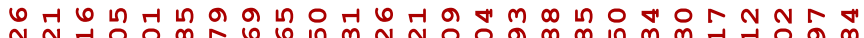
$\forall \forall \rightarrow m m m m m m m m N \sim N N \sim N N$ N $N$ N $N$

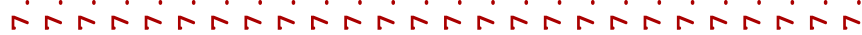
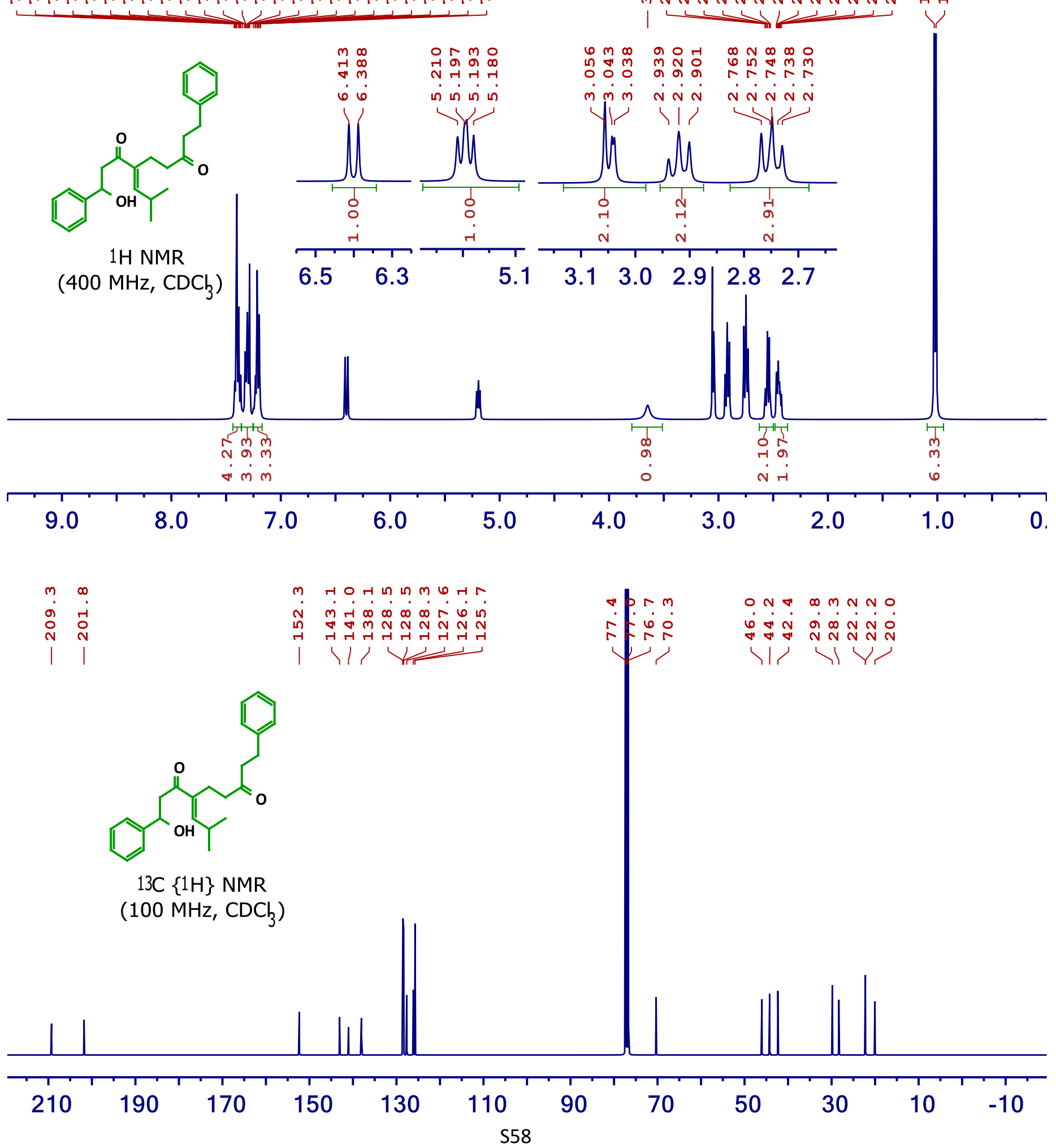
(E)-1-hydroxy-1,9-diphenyl-4-((E)-3-phenylallylidene)nonane-3,7-dione

$4 q$

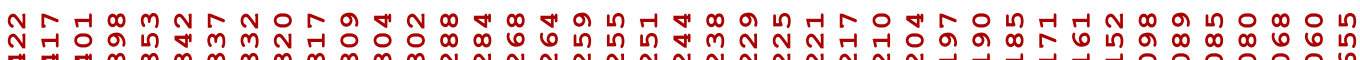

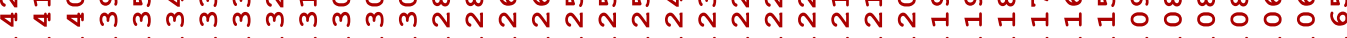

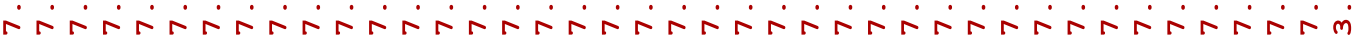

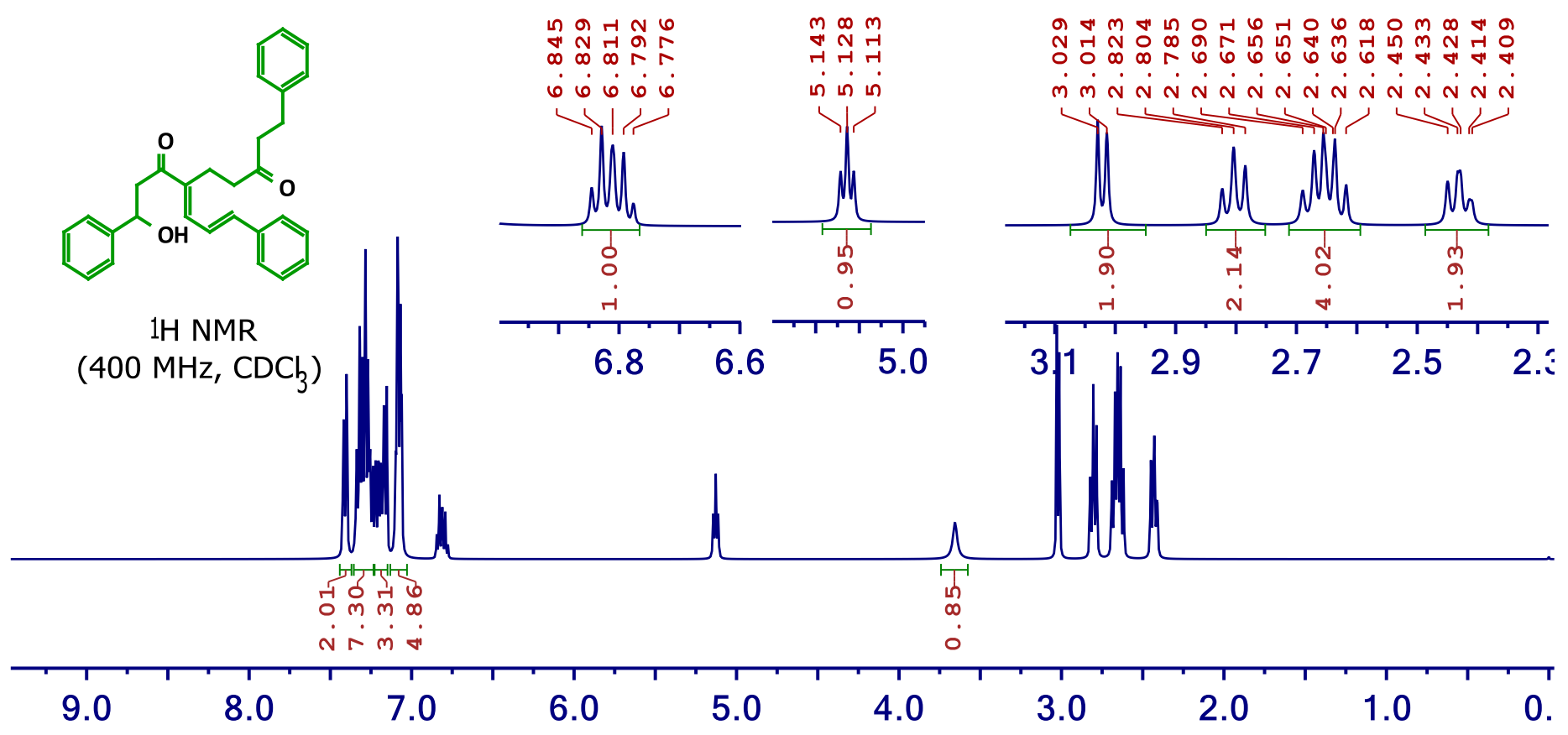

\begin{tabular}{|c|c|c|c|c|}
\hline $\begin{array}{ll}\nabla & 0 \\
\dot{0} & \dot{1} \\
\circ & 0 \\
N & N \\
1 & 1\end{array}$ & 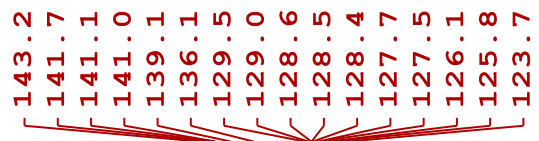 & 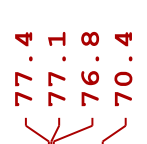 & 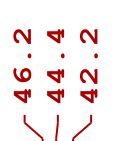 & $\begin{array}{l}\infty \\
\stackrel{\circ}{N}\end{array}$ \\
\hline
\end{tabular}<smiles>O=C(CCC(=O)C(O)CC(=O)CCc1ccccc1)CCc1ccccc1</smiles>

$13 \mathrm{C}\{1 \mathrm{H}\} \mathrm{NMR}$ (100 $\left.\mathrm{MHz}, \mathrm{CDC}_{3}\right)$

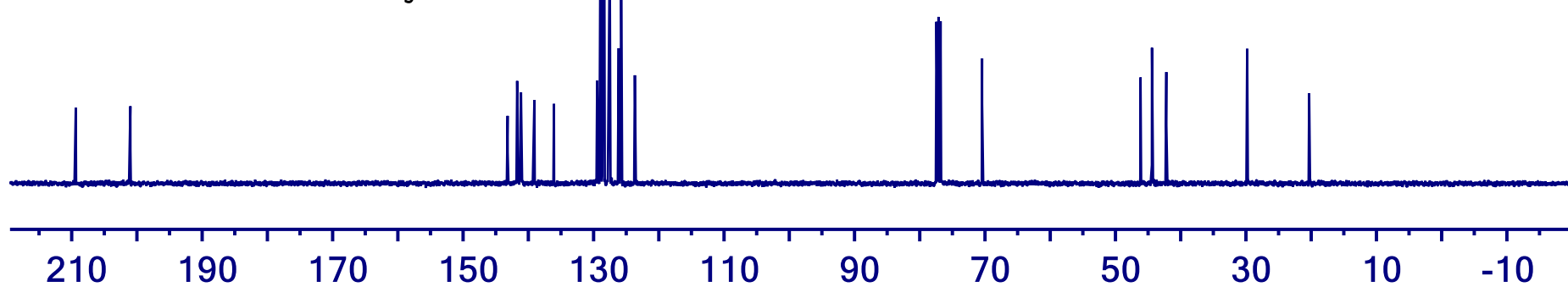


(E)-4-(cyclopropylmethylene)-1-hydroxy-1,9-diphenylnonane-3,7-dione

$4 r$

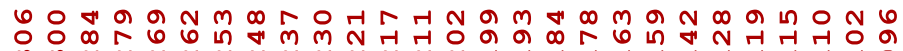

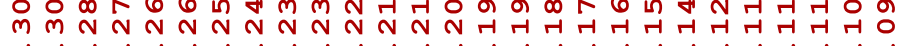

TH 0 ma

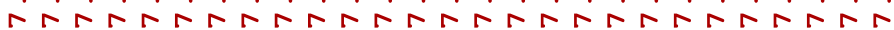

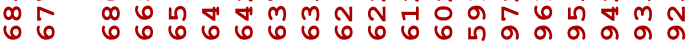

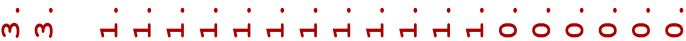

$\lambda \sqrt{14}$<smiles>O=C(CCC(=CC1CC1)C(=O)CC(O)c1ccccc1)CCc1ccccc1</smiles>

이료

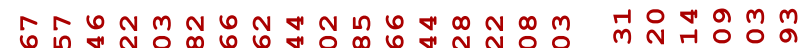

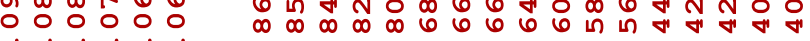

in $\operatorname{\text {in}}$ in in in in

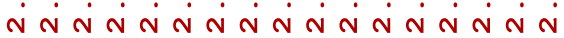

in in

年

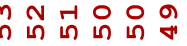

000000

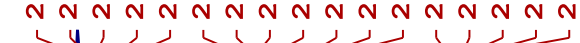

皮

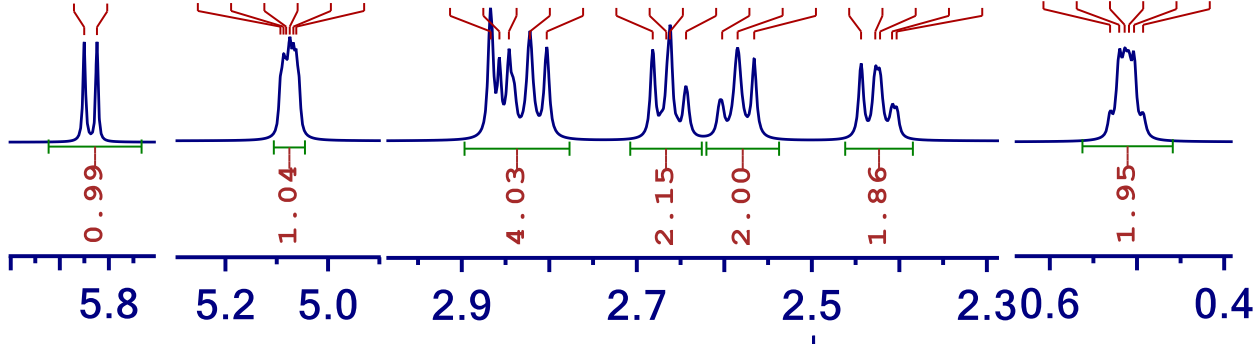

(400 $\left.\mathrm{MHz}, \mathrm{CDC}_{3}\right)$

$\begin{array}{lll}5.2 & 5.0 & 2.9\end{array}$

2.7

2.5

2.30 .6

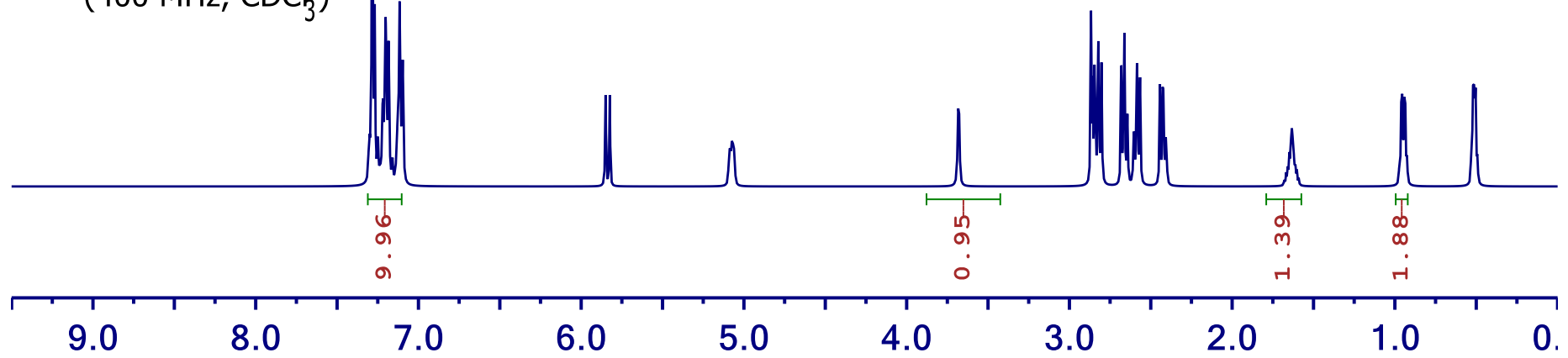

$\ln 24 m \ln 2 \pi, 45$

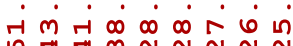
ก)

। ।

$13 \mathrm{C}\{1 \mathrm{H}\} \mathrm{NMR}$ (100 MHz, CDCh)

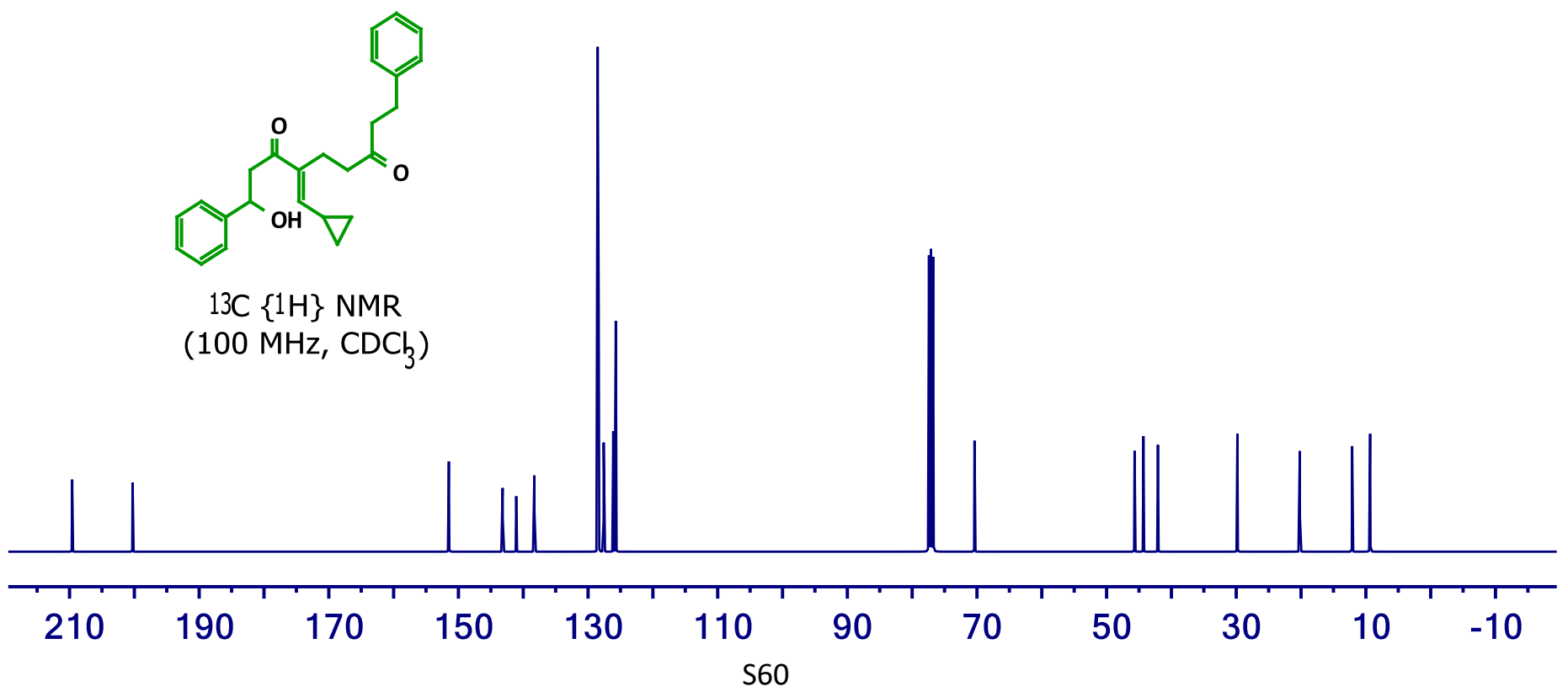

$\Delta A+\infty \pi$

रำ응

W 
(E)-1-hydroxy-4-(4-methylbenzylidene)-1,9-diphenylnonane-3,7-dione

45

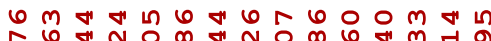

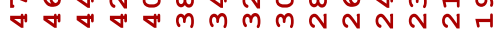

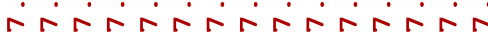

$\infty m \infty$

$\stackrel{n}{N} \stackrel{n}{\sim} \stackrel{n}{\sim}$

เท เก เก

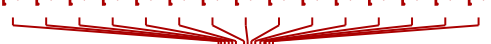

1

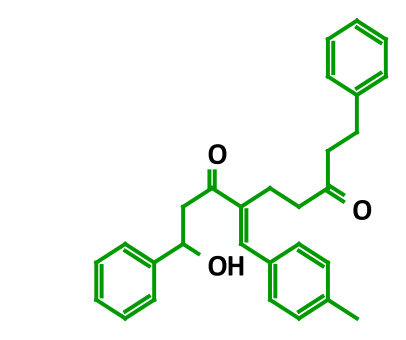

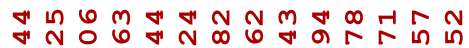

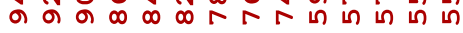

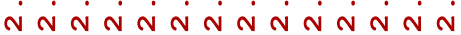
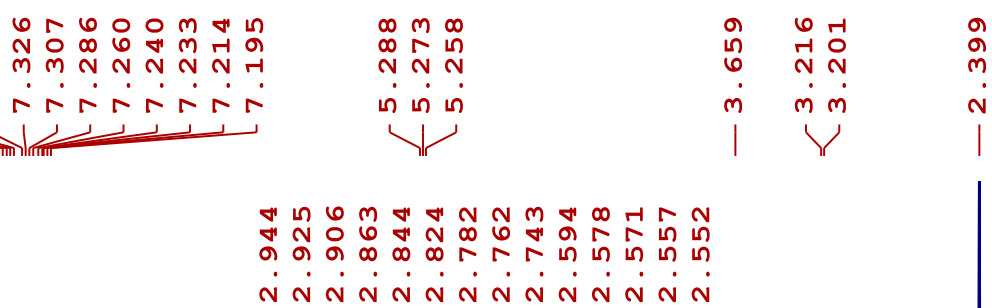

H NMR

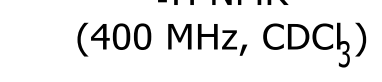

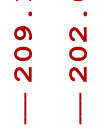

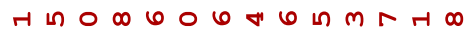

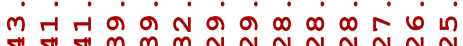

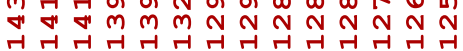
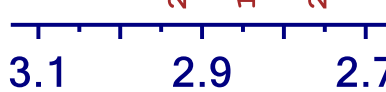

사
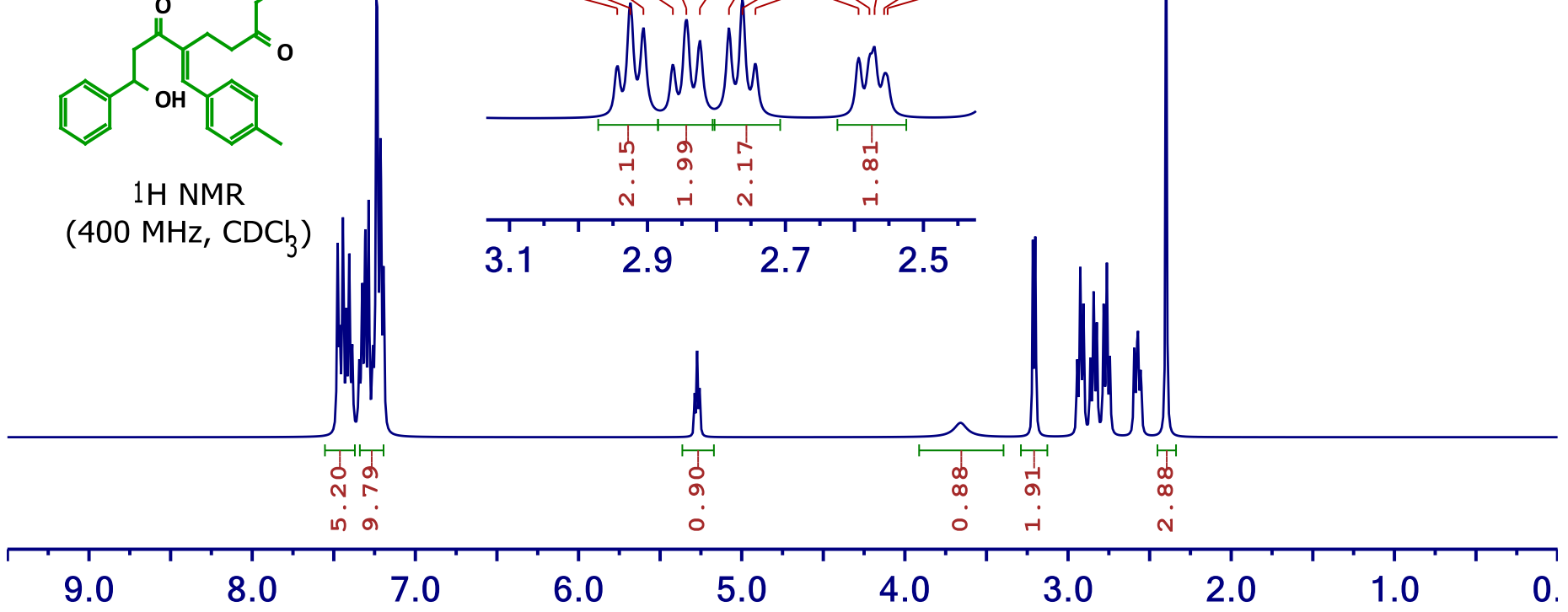<smiles>Cc1ccc(CC(=CCC(=O)CCc2ccccc2)C(=O)CC(O)c2ccccc2)cc1</smiles>

$13 \mathrm{C}\{1 \mathrm{H}\} \mathrm{NMR}$ (100 MHz, CDCh)

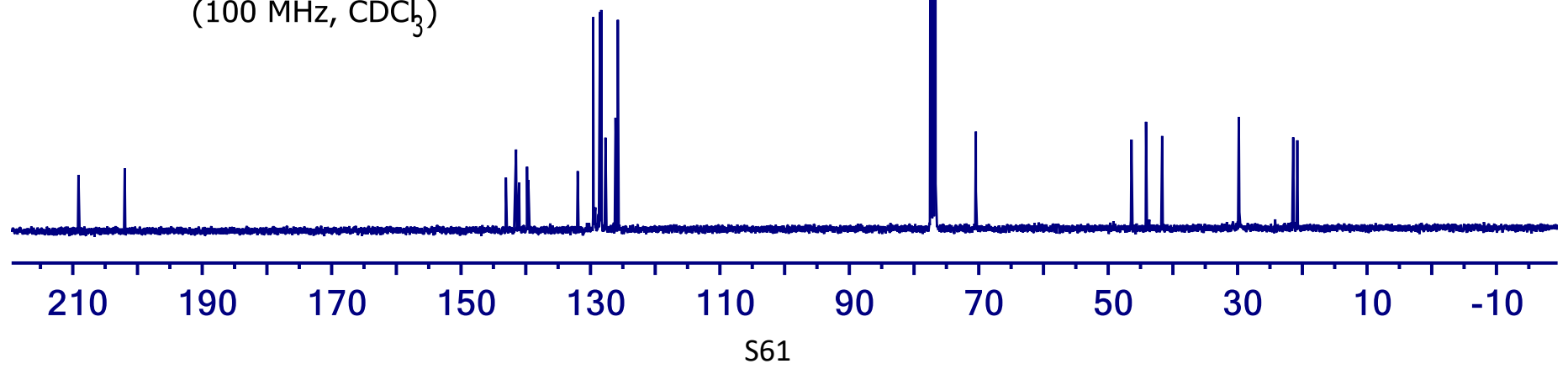


(E)-1-hydroxy-4-(4-methoxybenzylidene)-1,9-diphenylnonane-3,7-dione

$4 t$

ธก

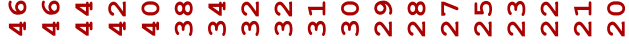

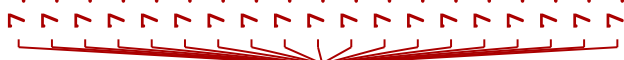

mํำ

$\infty \pi$ h

m $m$ m $m \dot{N}$ N $N$ N

1) 110

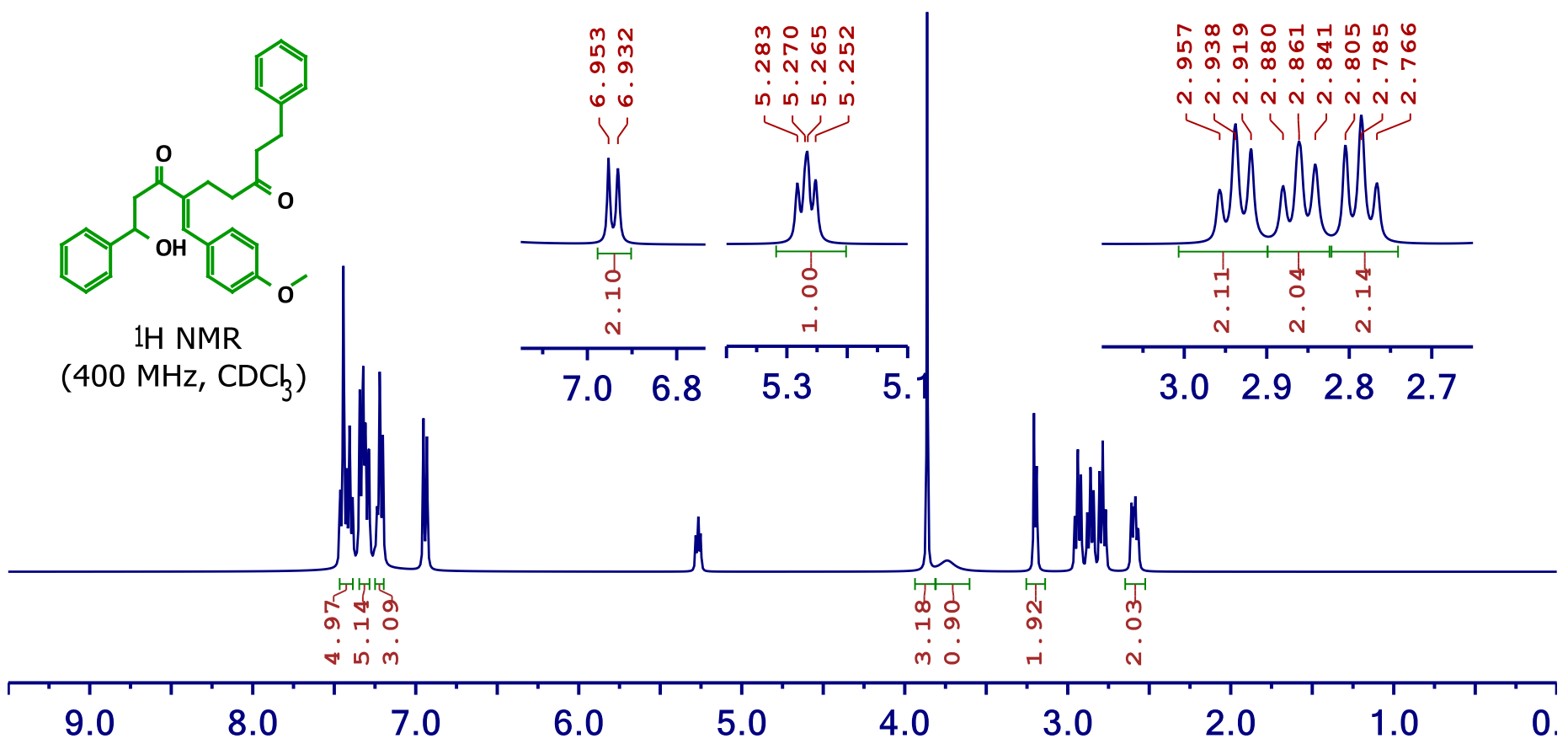

$\begin{array}{cc}m & 0 \\ 0 & \text { - } \\ 0 & 0 \\ \text { N } & \text { N } \\ 1 & 1\end{array}$

n4 20 L

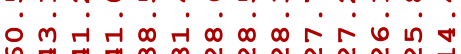

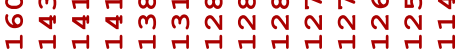

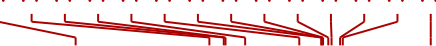

An

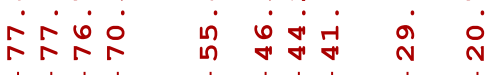<smiles>COc1ccc(C=C(CCC(=O)CCc2ccccc2)C(=O)CC(O)c2ccccc2)cc1</smiles>

13C $\{1 \mathrm{H}\}$ NMR

(100 $\mathrm{MHz}, \mathrm{CDC}_{3}$ )

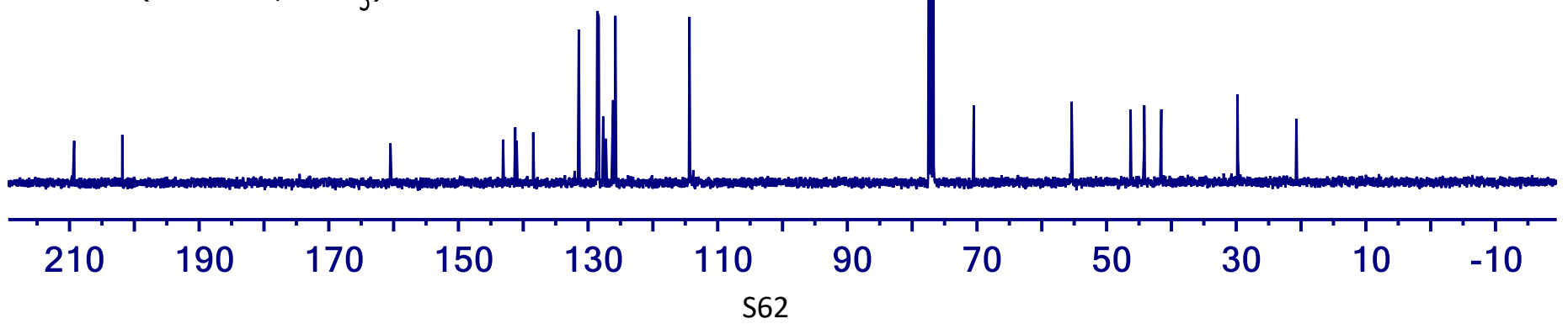


(E)-1-hydroxy-1,9-diphenyl-4-(thiophen-2-ylmethylene)nonane-3,7-dione

$4 \mathrm{u}$

유ㅁㅠㅠ 6 ก ก

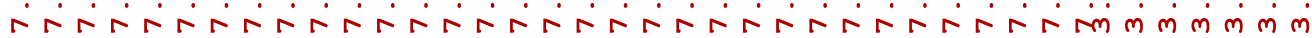

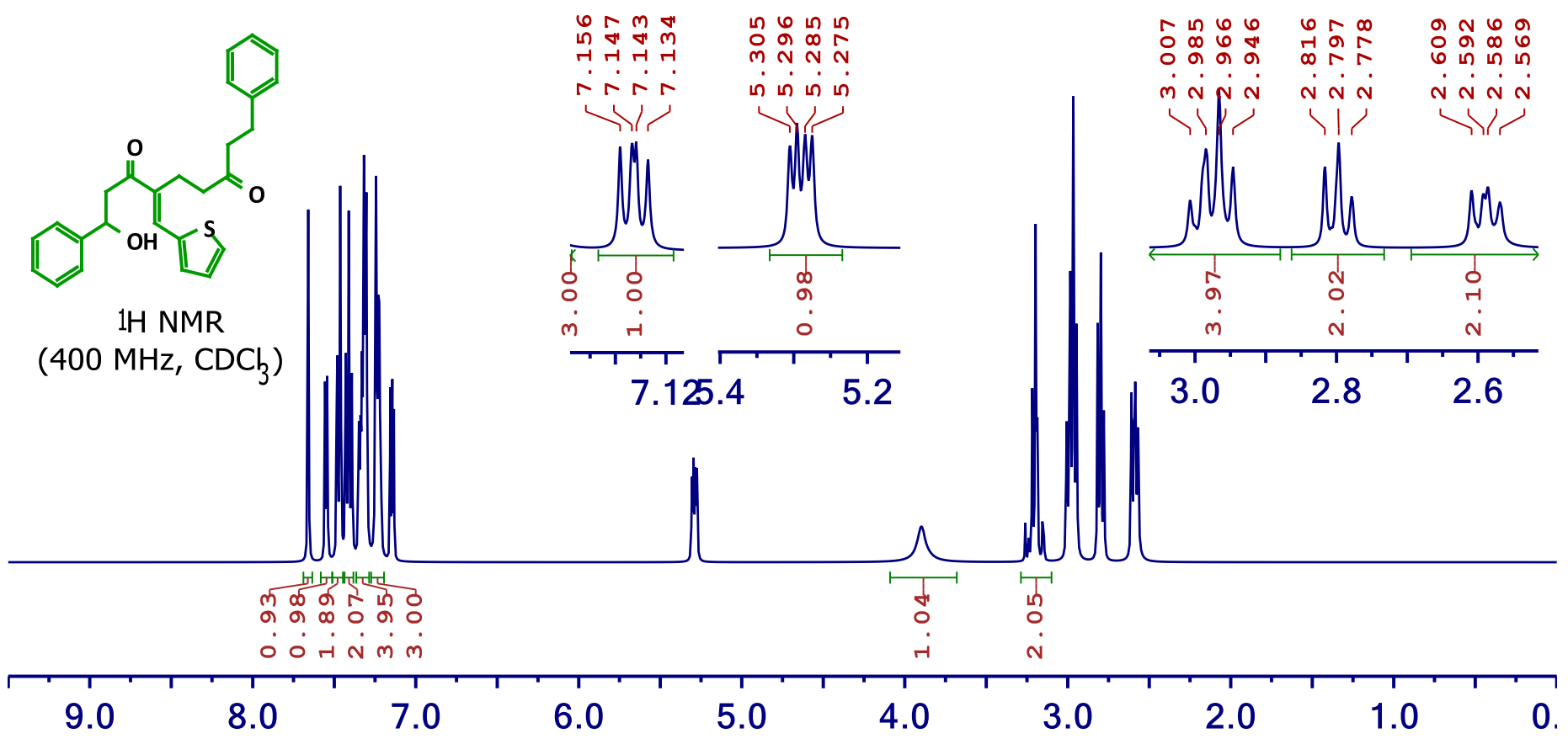
$\begin{array}{ll}\mathfrak{N} & \infty \\ 0 & 0 \\ 0 & 0 \\ \text { N } & \stackrel{0}{N} \\ 1 & 1\end{array}$

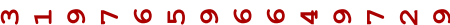

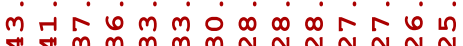

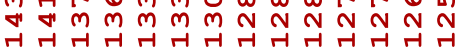
$6 \mathrm{~m} \circ \mathrm{in}$
$m \sim \infty \quad a-m$
inis
Y

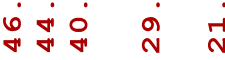
11

(<smiles>O=C(CCC(=Cc1cccs1)C(=O)CC(O)c1ccccc1)CCc1ccccc1</smiles>

13C $\{1 \mathrm{H}\}$ NMR

$\left(100 \mathrm{MHz}, \mathrm{CDC}_{3}\right)$
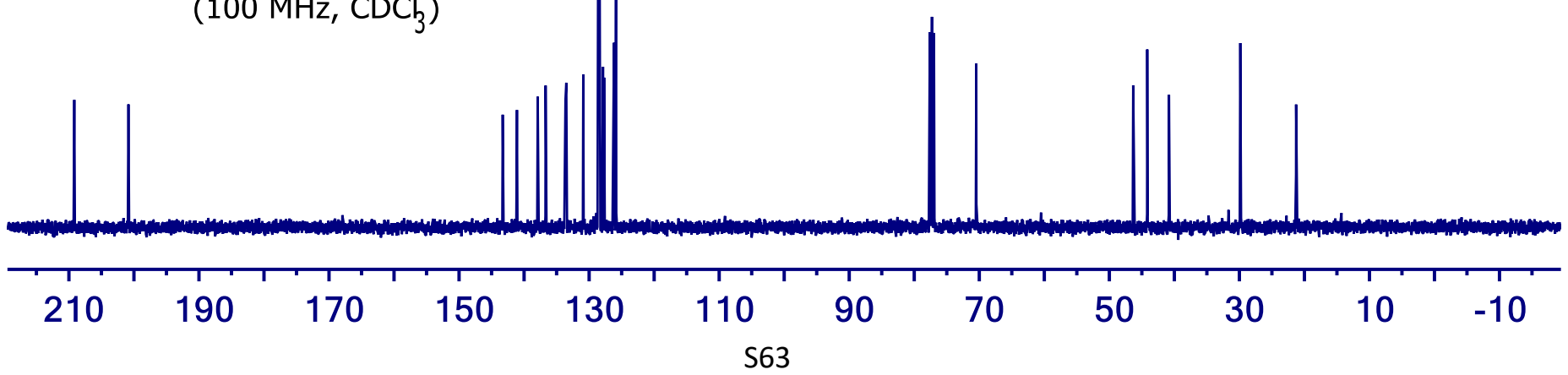
(E)-5-ethylidene-8-hydroxy-8-(naphthalen-1-yl)-1-phenyloctane-2,6-dione

4V

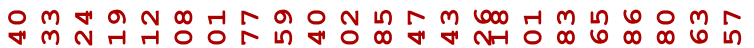

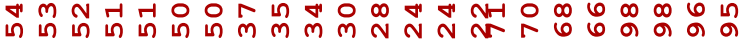

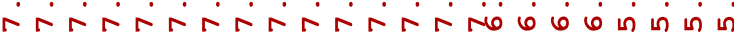
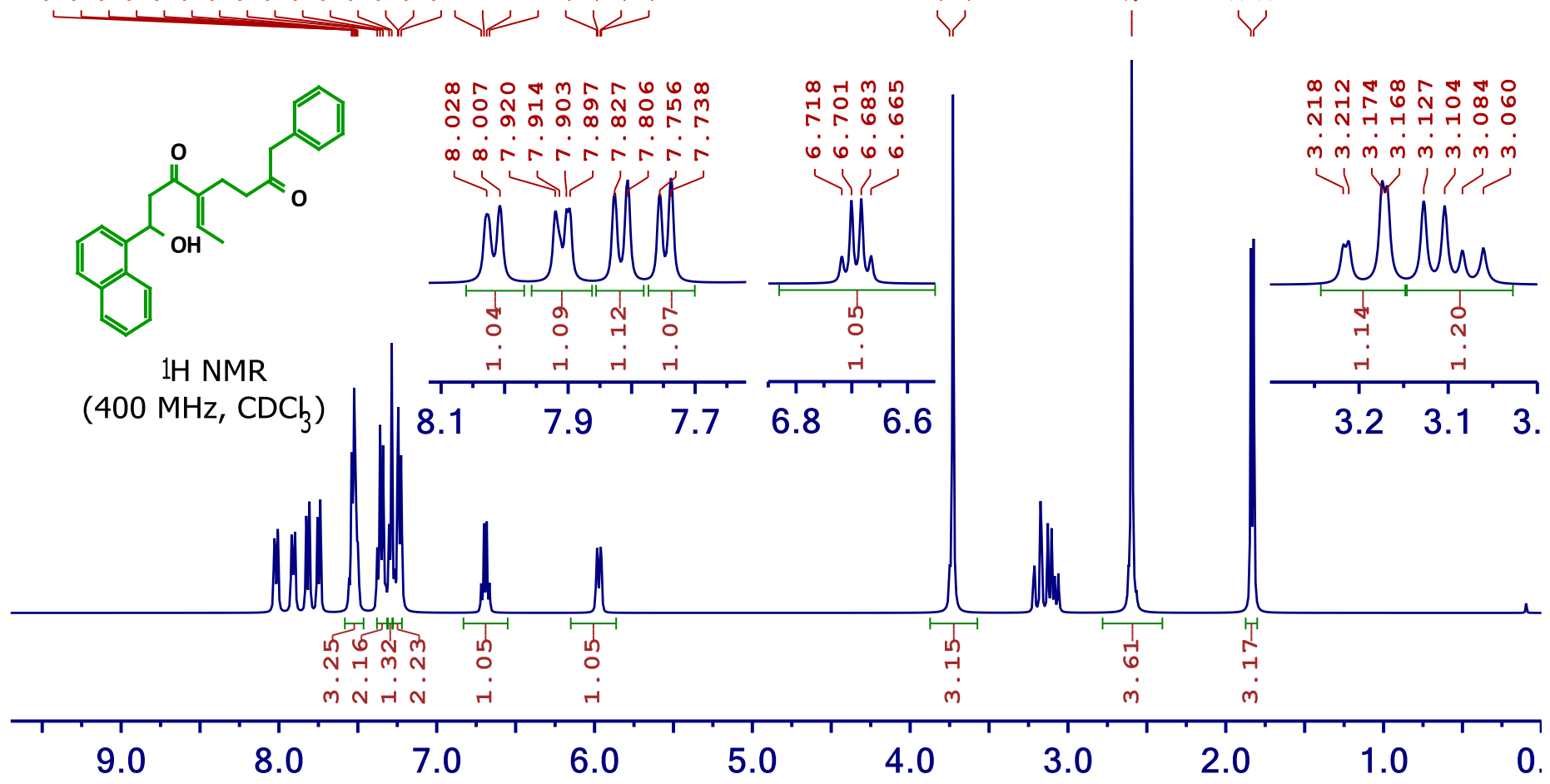

$\begin{array}{cc}r & m \\ i & -1 \\ \text { N } & \text { N } \\ 1 & \mid\end{array}$

n $\infty 6$ H

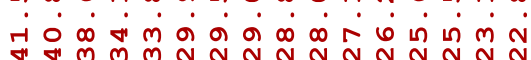
H.

啇

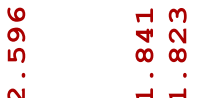

\)

8.0

ב<smiles>C/C=C(\CCC(=O)Cc1ccccc1)C(=O)CC(O)c1cccc2ccccc12</smiles>

$13 \mathrm{C}\{1 \mathrm{H}\} \mathrm{NMR}$ (100 MHz, CDCb)
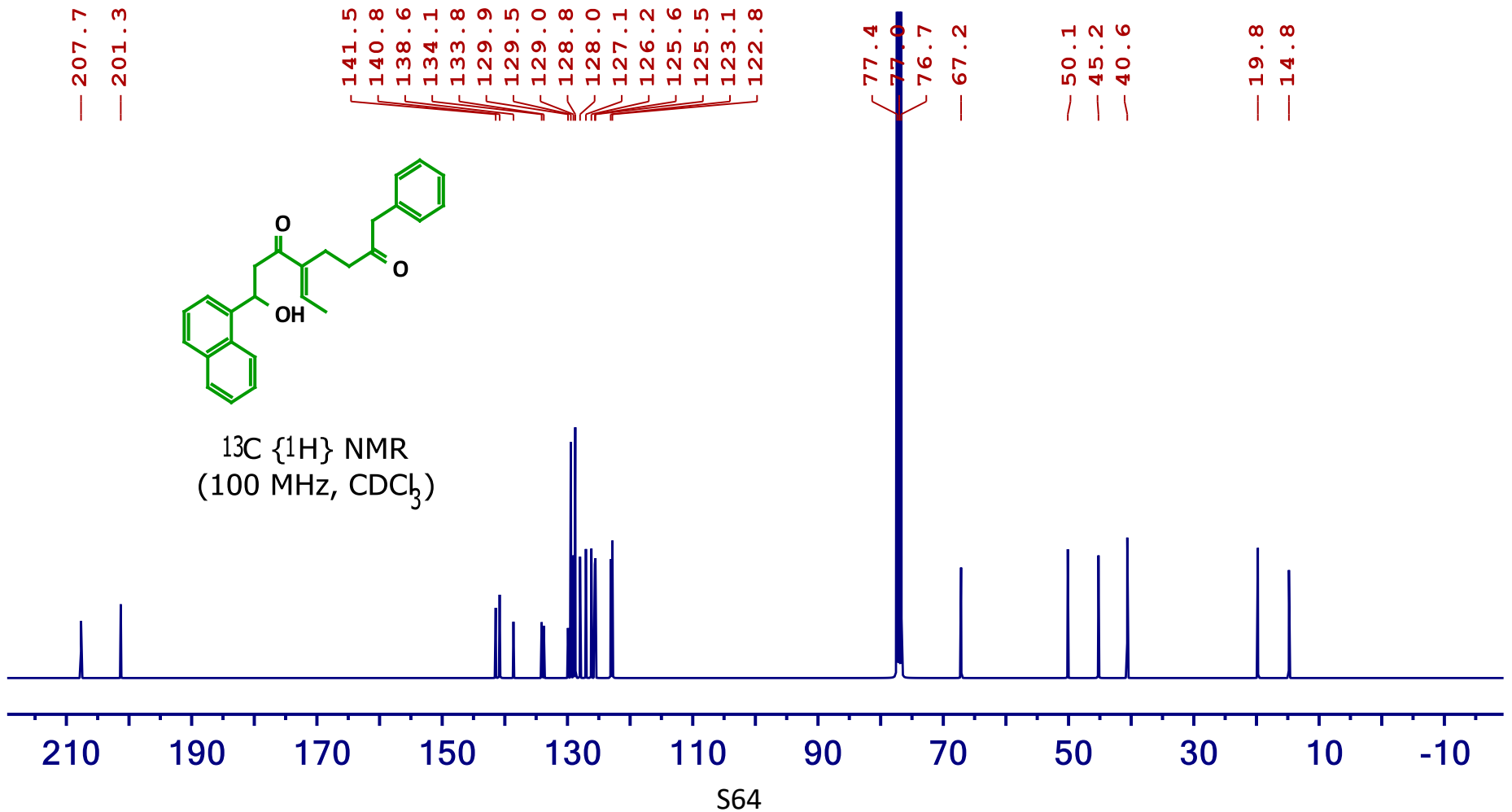
(E)-5-ethylidene-8-hydroxy-1-phenyl-8-(o-tolyl)octane-2,6-dione

4w

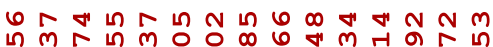

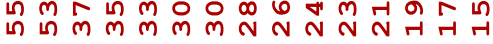

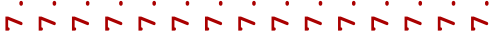

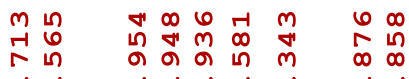

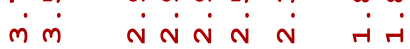

L m

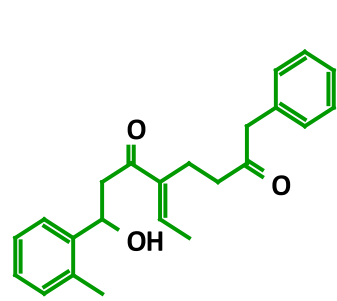

1H NMR

(400 $\left.\mathrm{MHz}, \mathrm{CDCl}_{3}\right)$
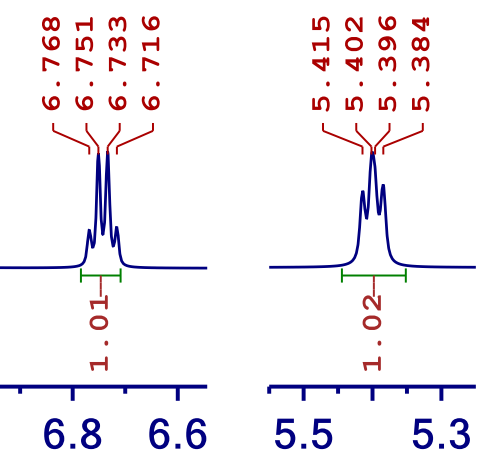

$1 / 2$

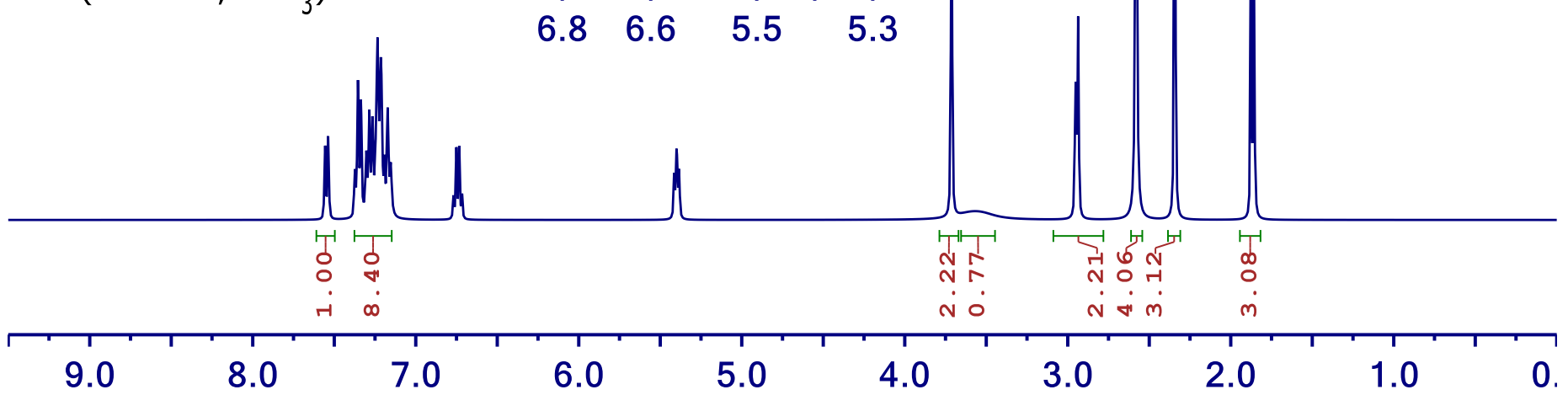

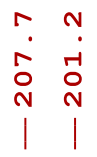

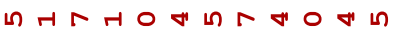

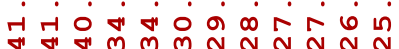
F

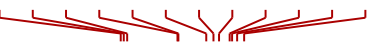

$\begin{array}{lll}1 & 0 & 9 \\ 1 & 0 & 0 \\ 5 & 0\end{array}$

40

궁요

$\checkmark$

111

$\infty \sim \infty$

बने

\!<smiles>CC=C(CCC(=O)Cc1ccccc1)C(=O)CC(O)c1ccccc1C</smiles>

$13 \mathrm{C}\{1 \mathrm{H}\} \mathrm{NMR}$ (100 MHz, CDCb)
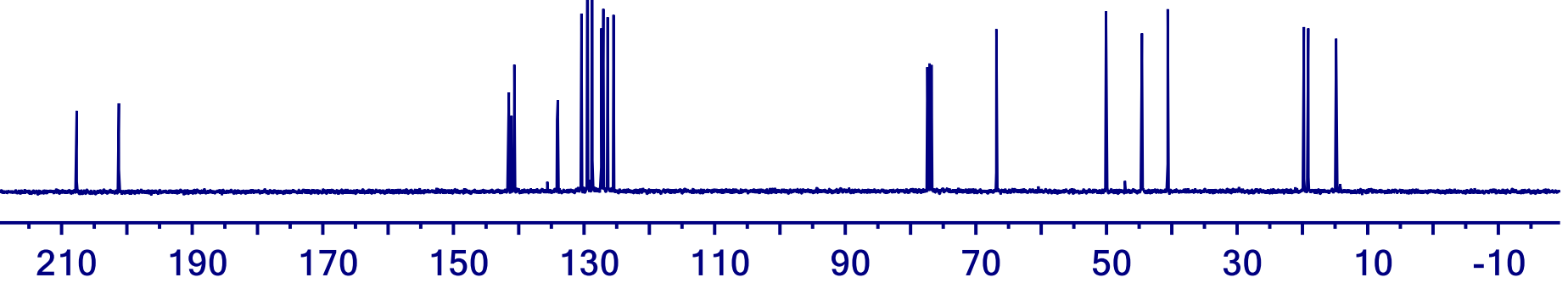
(E)-5-ethylidene-8-hydroxy-8-(4-methoxyphenyl)-1-phenyloctane-2,6-dione

$4 x$

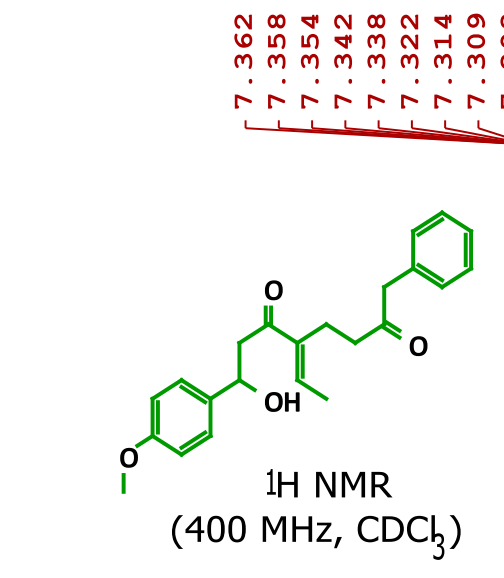

N $\infty$ \#

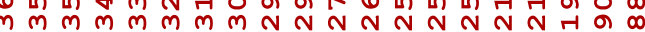

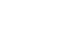

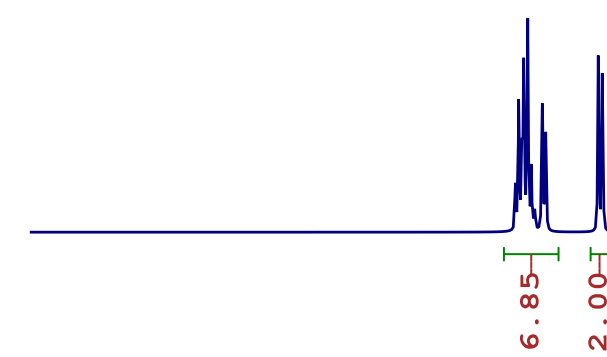

ํํㅇํㄴำ

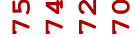

ம் $\dot{0} \dot{0} \dot{0}$

) I
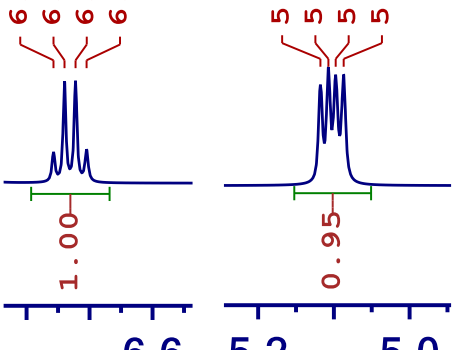

$\begin{array}{lll}\infty & 1 \\ 0 & \infty & \infty \\ \infty & 0\end{array}$

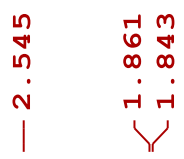
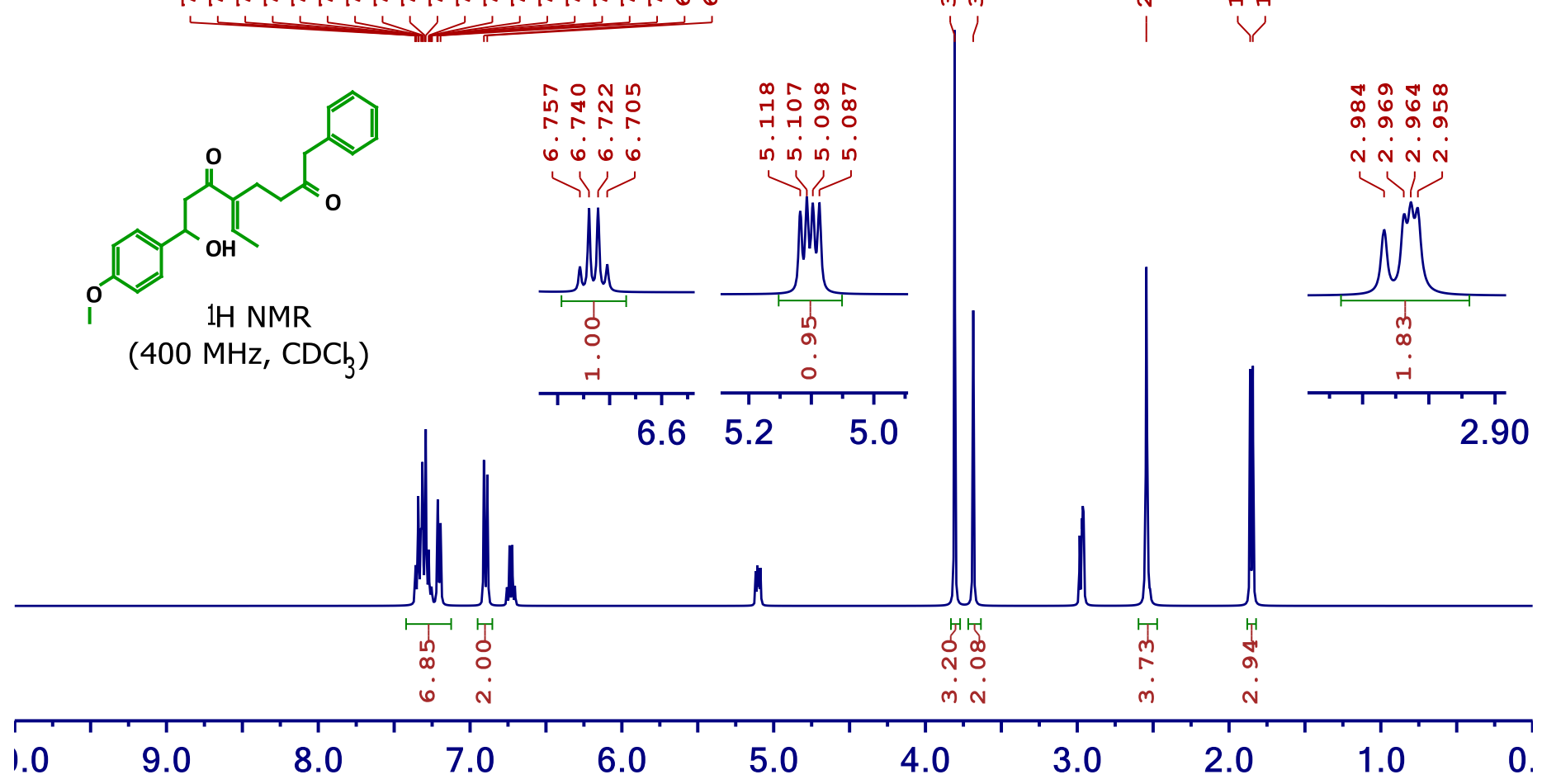

$\begin{array}{cc}\infty & N \\ i & - \\ 0 & \circ \\ N & N \\ 1 & \mid\end{array}$

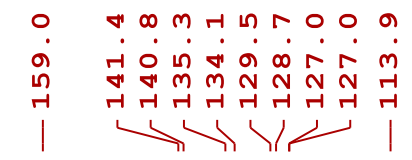

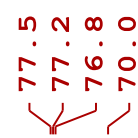

$m+\infty 6$

in 0 in

1111

$\begin{array}{ll}1 & 0 \\ \text { वे } & \text { ते }\end{array}$<smiles>C/C=C(/CCC(=O)Cc1ccccc1)C(=O)CC(O)c1ccc(OC)cc1</smiles>

13C $\{1 \mathrm{H}\}$ NMR

(100 MHz, CDCb)

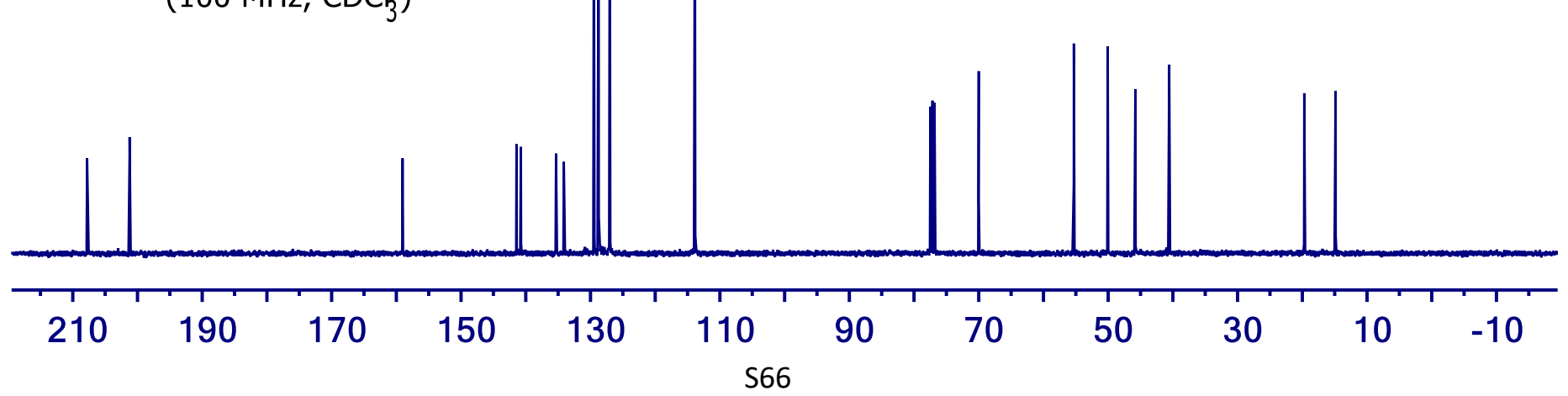


(E)-6-ethylidene-9-hydroxy-1,10-diphenyldecane-3,7-dione

$4 y$

ดี

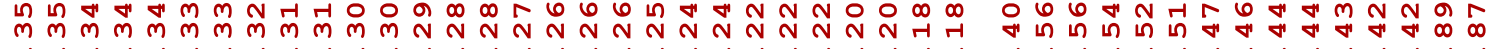

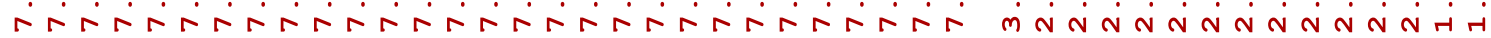

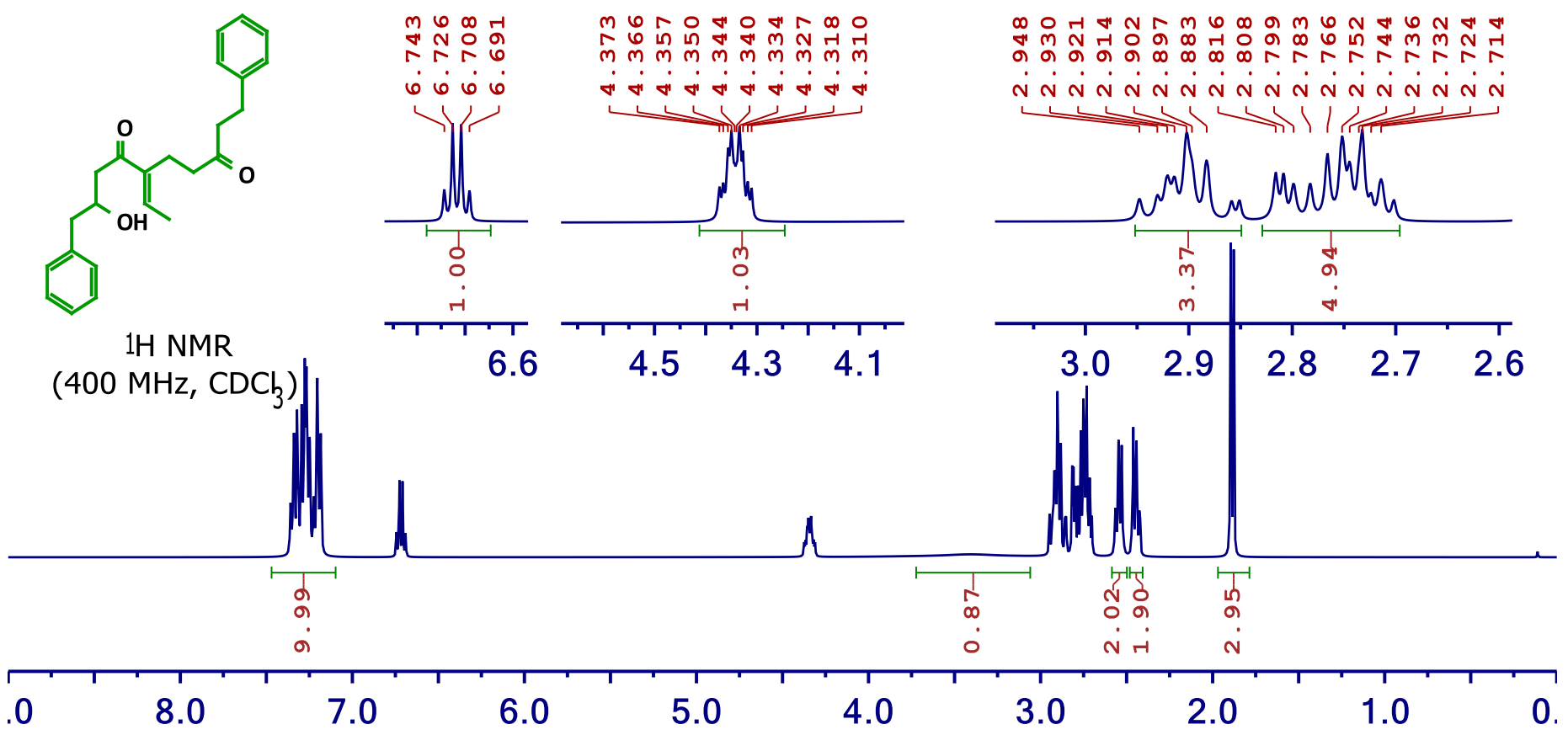

$\begin{array}{cc}\text { n } & \text { ? } \\ 0 & \dot{H} \\ 0 & 0 \\ \text { N } & \text { N } \\ 1 & \mid\end{array}$

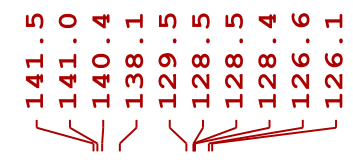

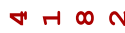

sरำ

N $060 \quad 0 \quad \infty \quad 6 \infty$

ind

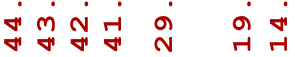<smiles>CC=C(CCC(=O)CCc1ccccc1)C(=O)CC(O)Cc1ccccc1</smiles>

$13 \mathrm{C}\{1 \mathrm{H}\} \mathrm{NMR}$ (100 $\mathrm{MHz}, \mathrm{CDCl}_{3}$ )
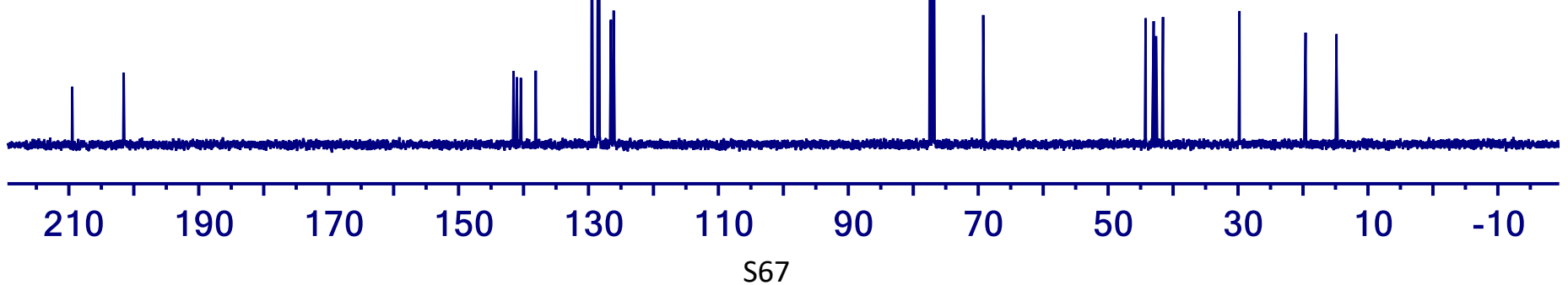
(6E,10E)-6-ethylidene-9-hydroxy-1,11-diphenylundec-10-ene-3,7-dione

$4 z$

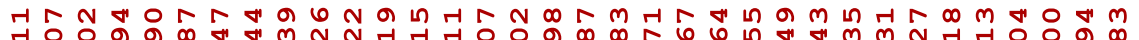

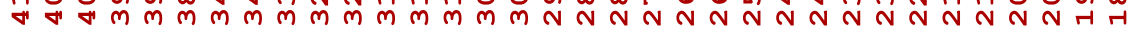

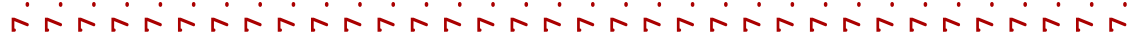

a $m$ in 6

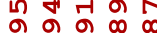
ก $\rightarrow$

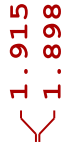

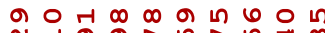
Nลำ

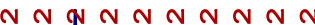

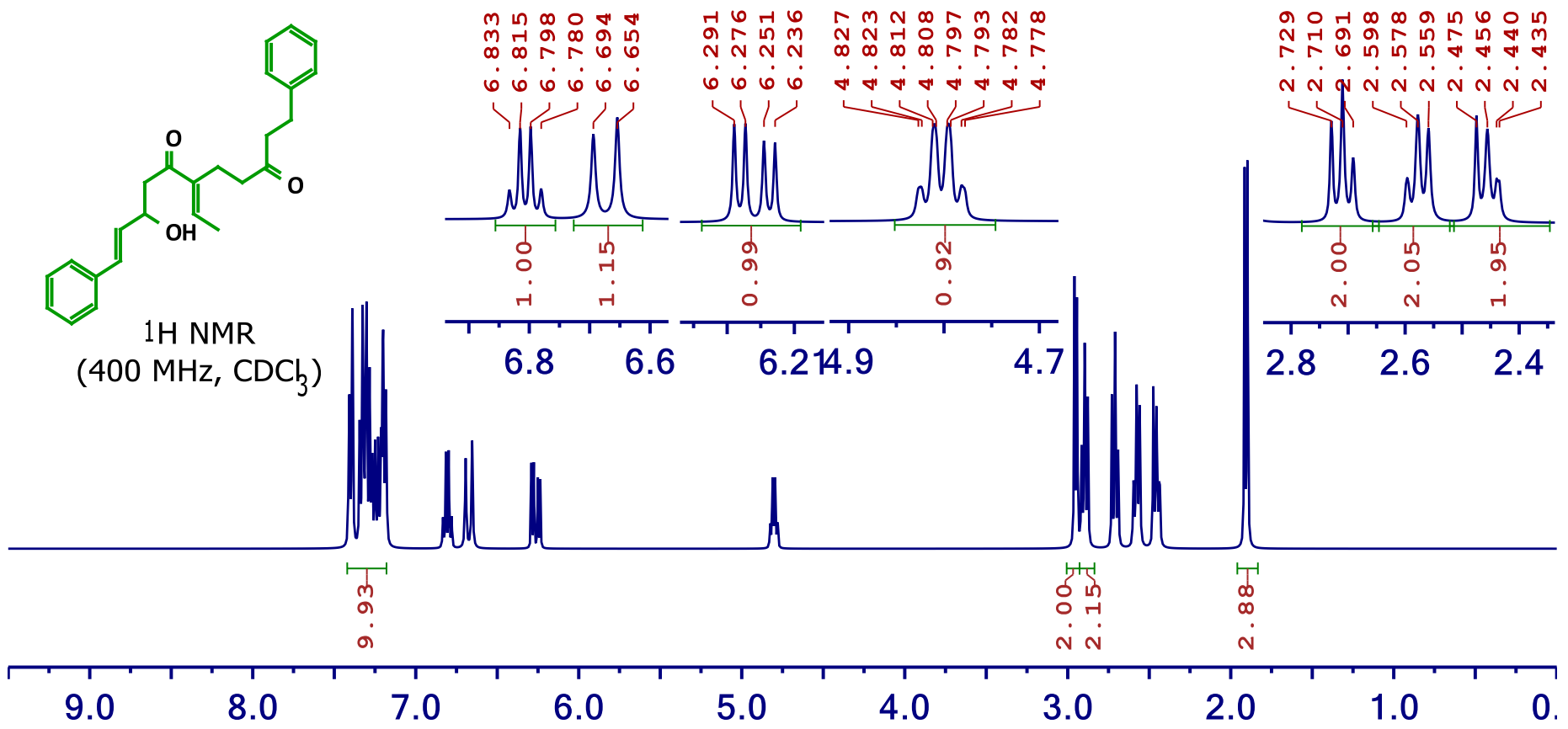

ה4 H + O H

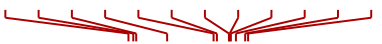

$\checkmark$<smiles>C/C=C(/CCC(=O)CCc1ccccc1)C(=O)CC(O)/C=C/c1ccccc1</smiles>

$13 \mathrm{C}\{1 \mathrm{H}\}$ NMR (100 MHz, CDCh)

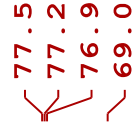

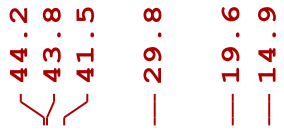
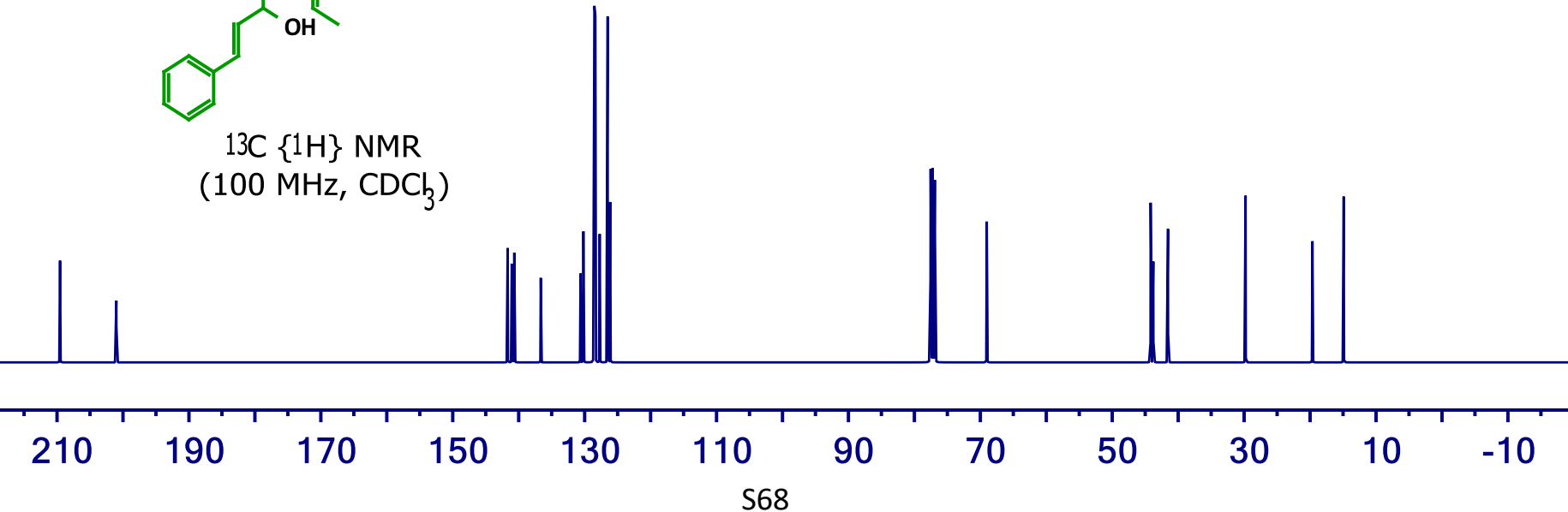
(E)-6-ethylidene-9-hydroxy-1-phenyltridecane-3,7-dione

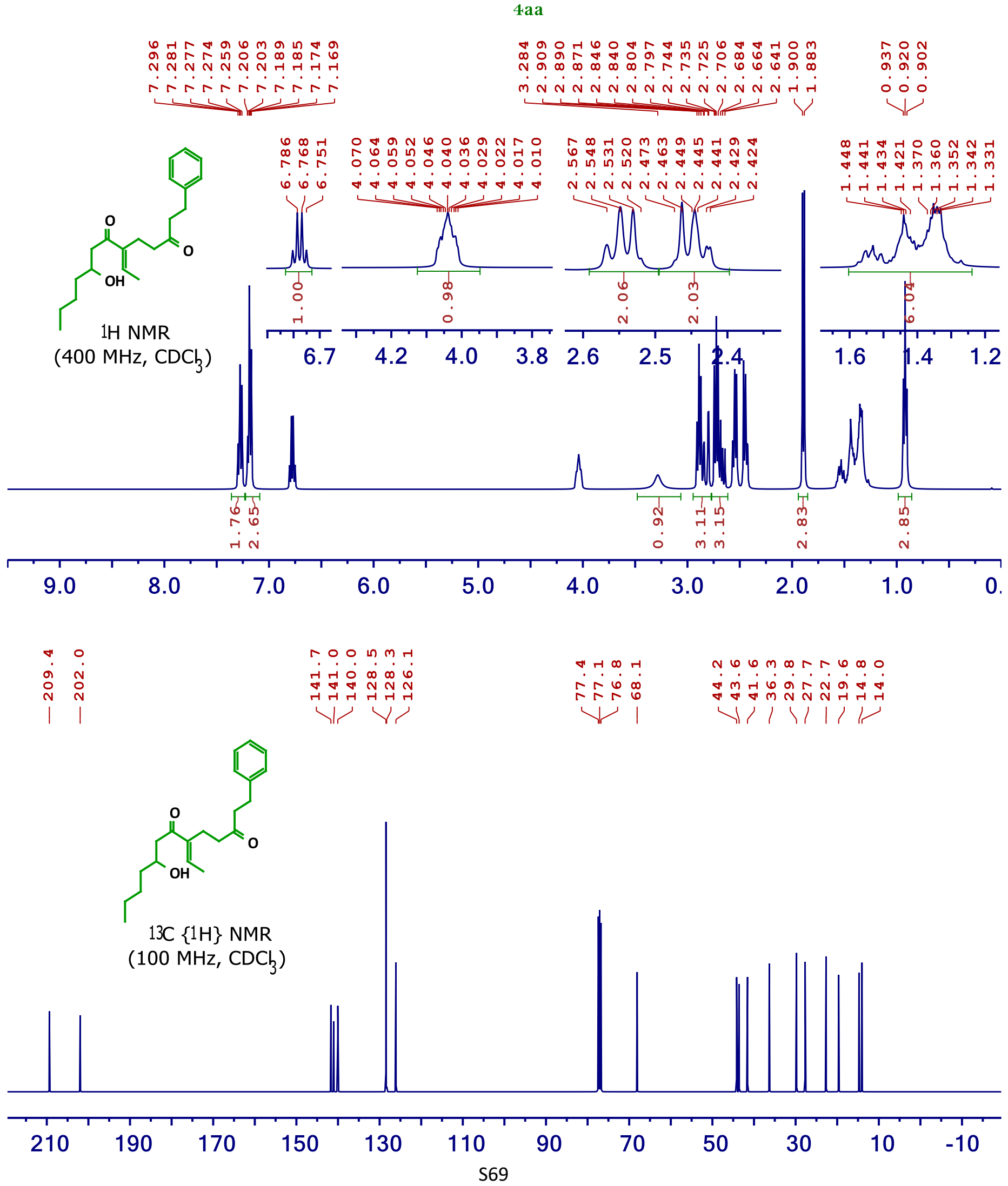


(E)-1-cyclohexyl-4-ethylidene-1-hydroxy-9-phenylnonane-3,7-dione

$4 a b$

-

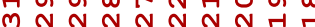

irisirisin

$\longrightarrow$

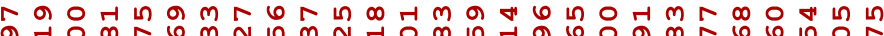
त

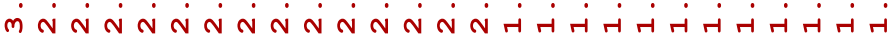

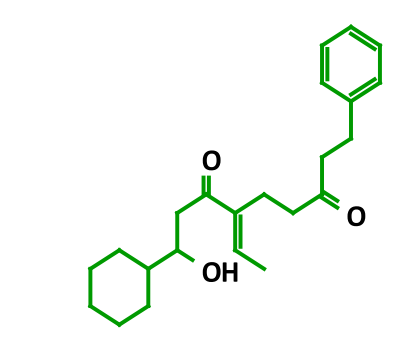

$1 \mathrm{H} N M R$

(400 $\left.\mathrm{MHz}, \mathrm{CDC}_{3}\right)$
กำㅇำ

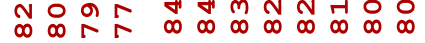

$\dot{0} \dot{0} \dot{0} \mathrm{~mm} m \mathrm{mmmm}$

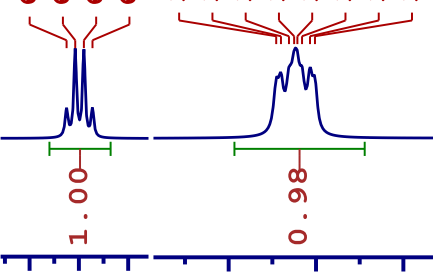

$6.7 \quad 3.9$

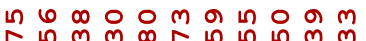

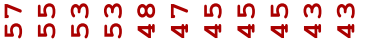

isicising

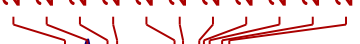

Tnm $m$ m N N N N N N N N O O

$4 \operatorname{tr} 4$

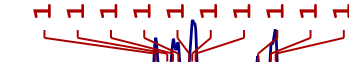

$$
\text { ( }
$$
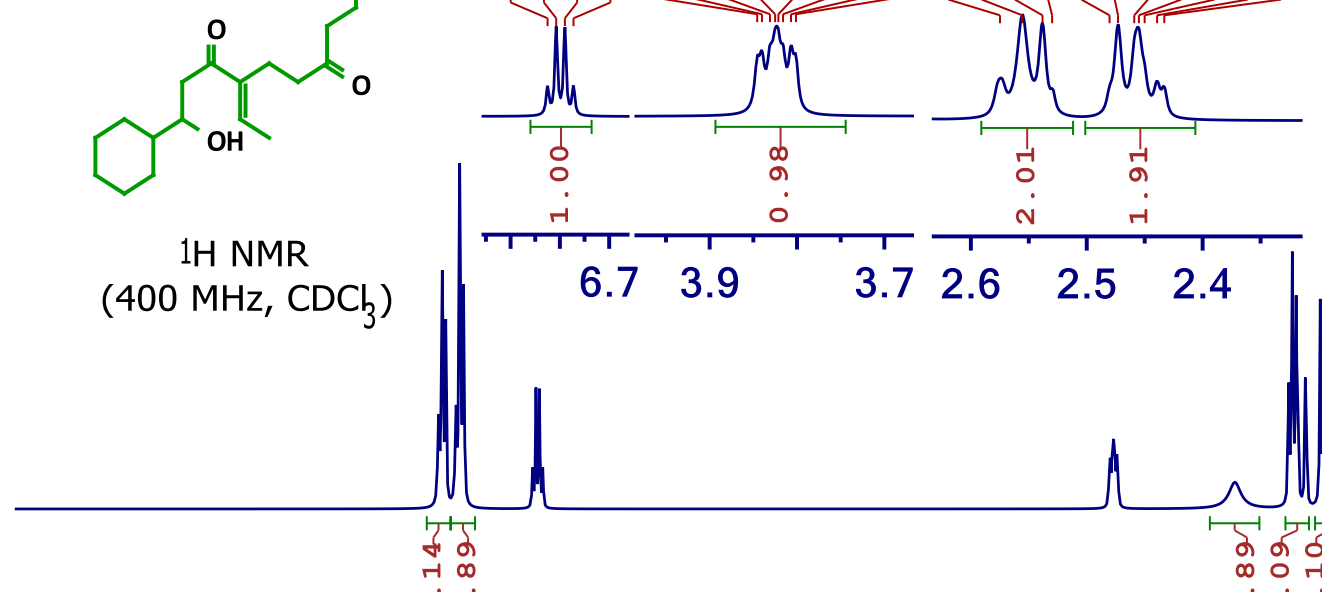

$\dot{N} \dot{N}$

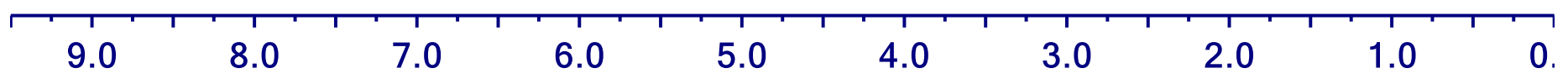

$\begin{array}{cc}\text { ก } & \text { r } \\ 0 & \text { N } \\ 0 & 0 \\ \text { N } & \text { N } \\ \mid & \mid\end{array}$
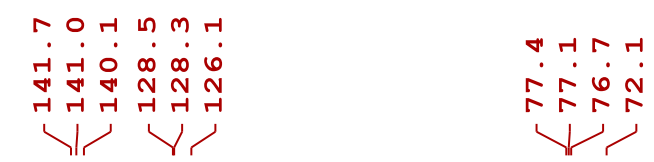

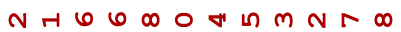

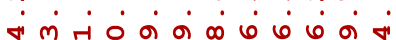

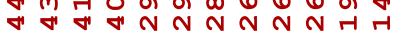<smiles>C/C=C(\CCC(=O)CCc1ccccc1)C(=O)CC(O)C1CCCCC1</smiles>

$13 \mathrm{C}\{1 \mathrm{H}\} \mathrm{NMR}$

(100 $\left.\mathrm{MHz}, \mathrm{CDC}_{3}\right)$
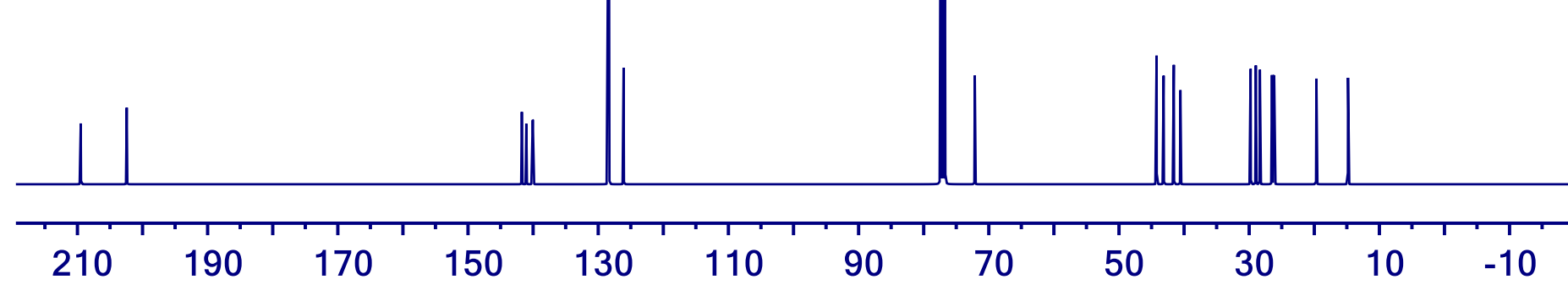
(E)-1-(2-chlorophenyl)-4-ethylidene-1-hydroxy-9-phenylnonane-3,7-dione

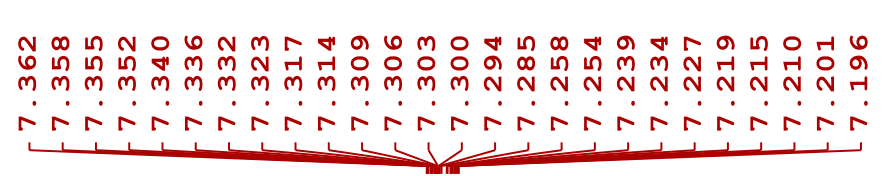

4ac

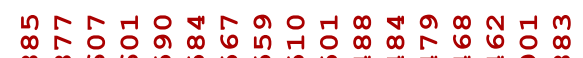

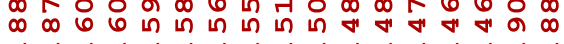

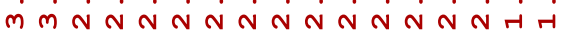

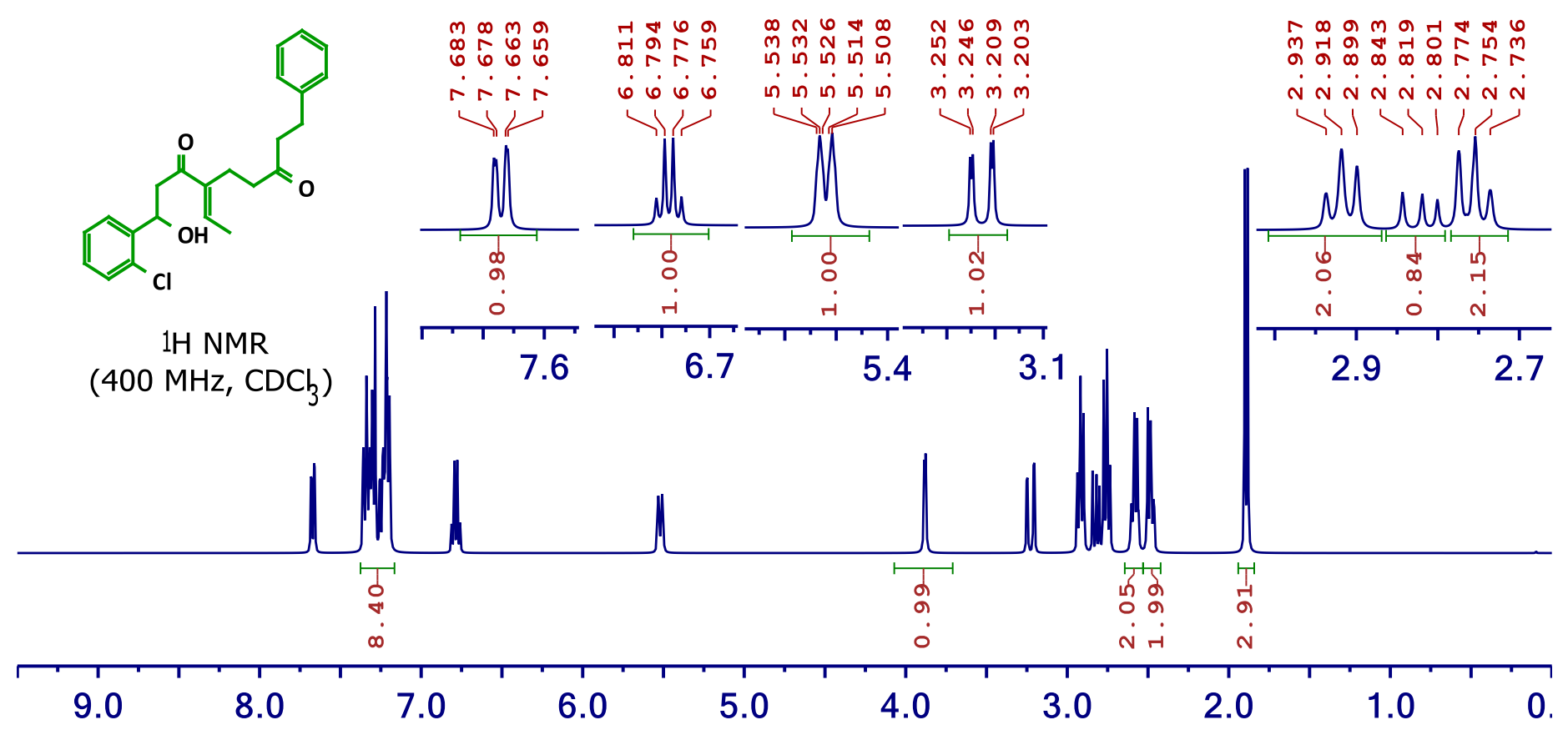

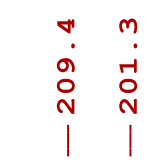

a. 00 Dh

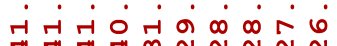

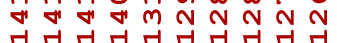

身)

5
750
750

$m a$ in $\infty a$

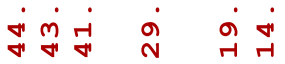

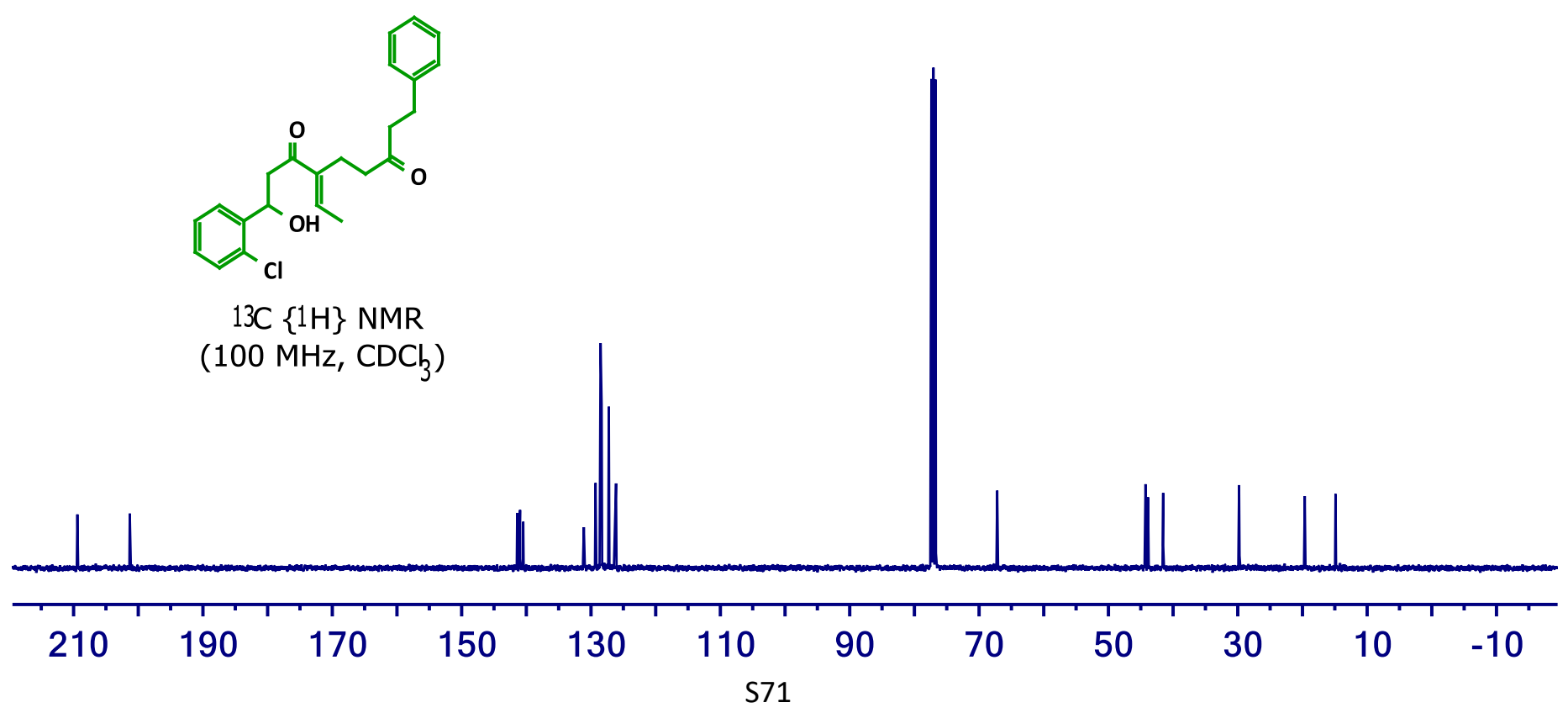

। 11 
(E)-4-ethylidene-1-hydroxy-1-(4-isopropylphenyl)-9-phenylnonane-3,7-dione

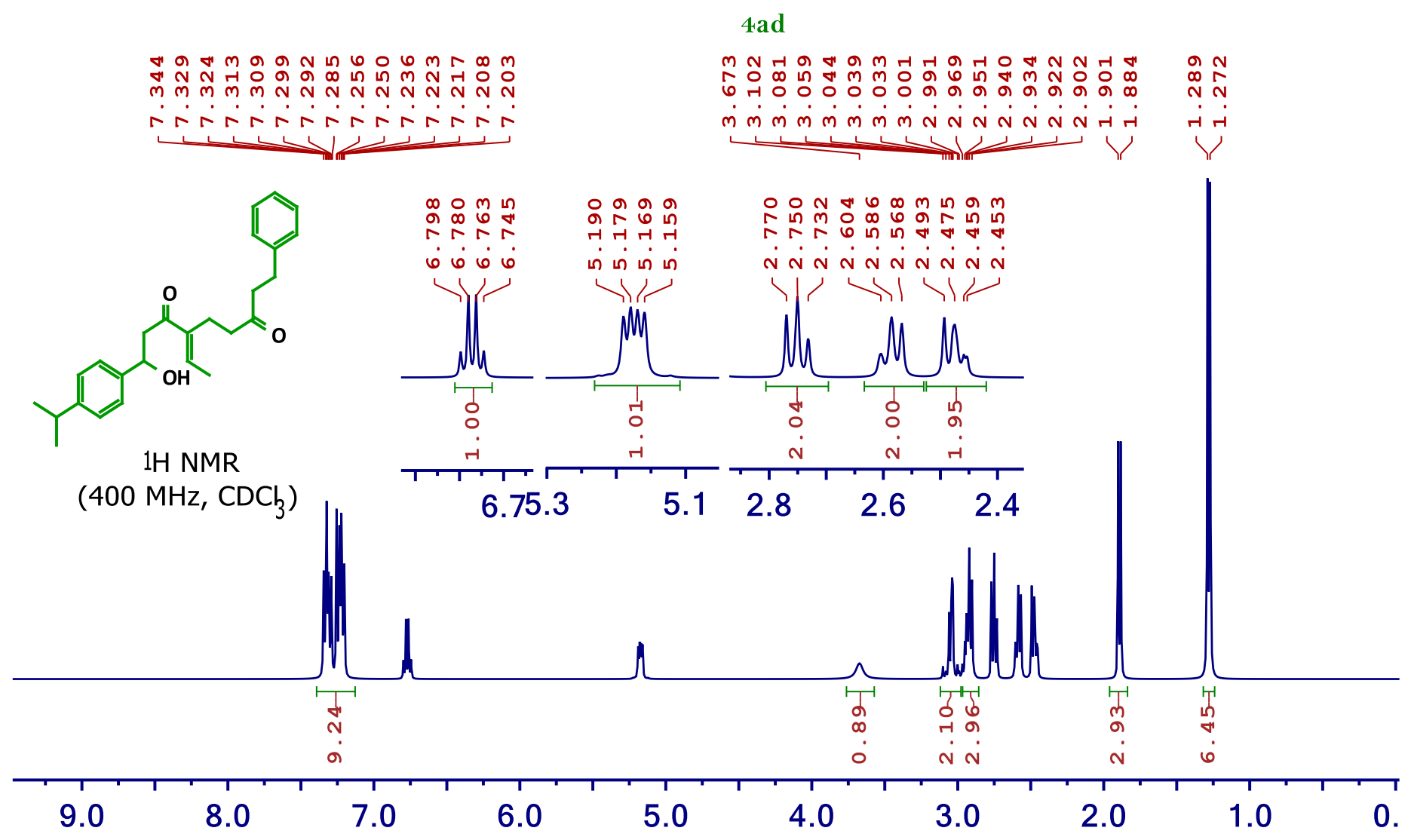

$m 6$ m 6 เ

$\dot{\infty} \dot{r} \dot{r} \dot{0} \dot{\infty} \dot{\infty} \dot{0} \dot{0}$ in

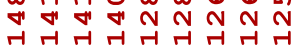

(1)
1) $\mathrm{Nam}$

年走是

W/ a $\mathrm{N}$ ब $\infty$ ता

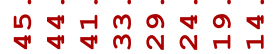
II, 1, 1

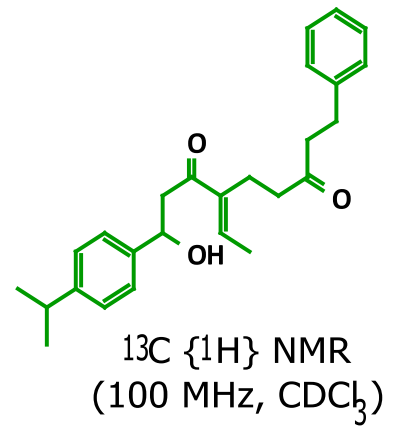

$13 \mathrm{C}\{1 \mathrm{H}\} \mathrm{NMR}$

(100 $\mathrm{MHz}_{3} \mathrm{CDC}_{3}$

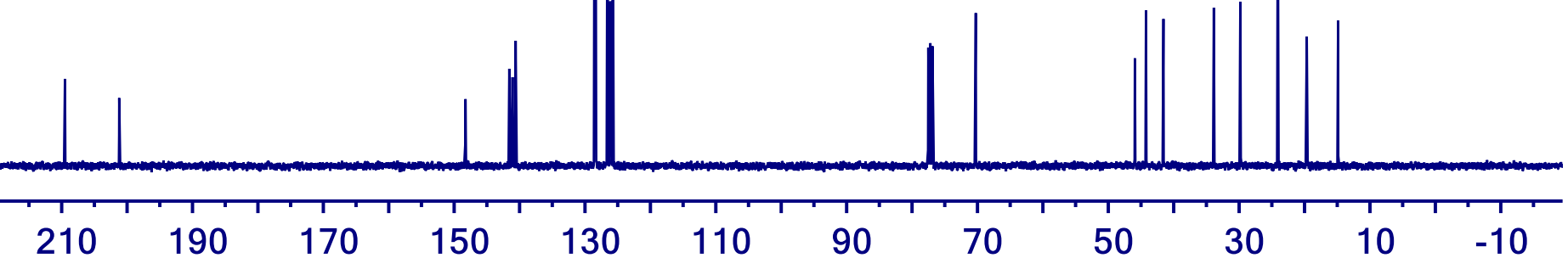


(E)-8-hydroxy-5-(4-methoxybenzylidene)-1,9-diphenylnonane-2,6-dione

4ae

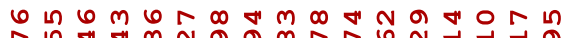

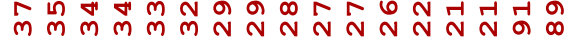

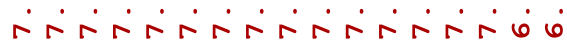<smiles>COc1ccc(/C=C(\CCC(=O)Cc2ccccc2)C(=O)CC(O)Cc2ccccc2)cc1</smiles>

$1 \mathrm{H}$ NMR

(400 $\mathrm{MHz}, \mathrm{CDCl}_{3}$ )

$\begin{array}{cc}0 & -1 \\ 0 & 0 \\ \text { N } & 0 \\ \text { N } & \text { N } \\ \mid & \mid\end{array}$

m ฟै Fु F वं वं वं वं वं
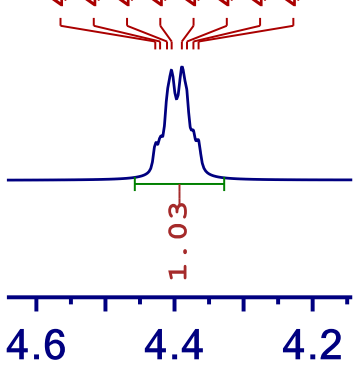

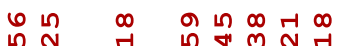

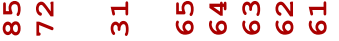

$\dot{m} \dot{m} \dot{N} \dot{N} \dot{N}$

111

กด น

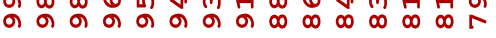

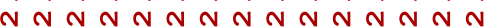

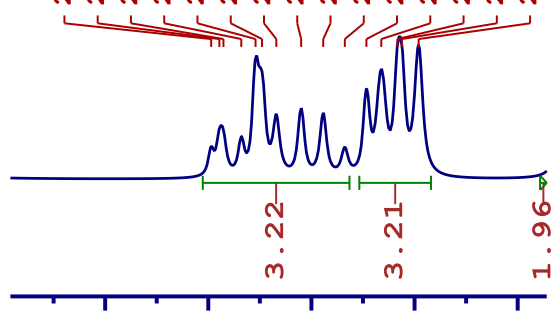

$\begin{array}{lllll}3.1 & 3.0 & 2.9 & 2.8 & 2.7\end{array}$
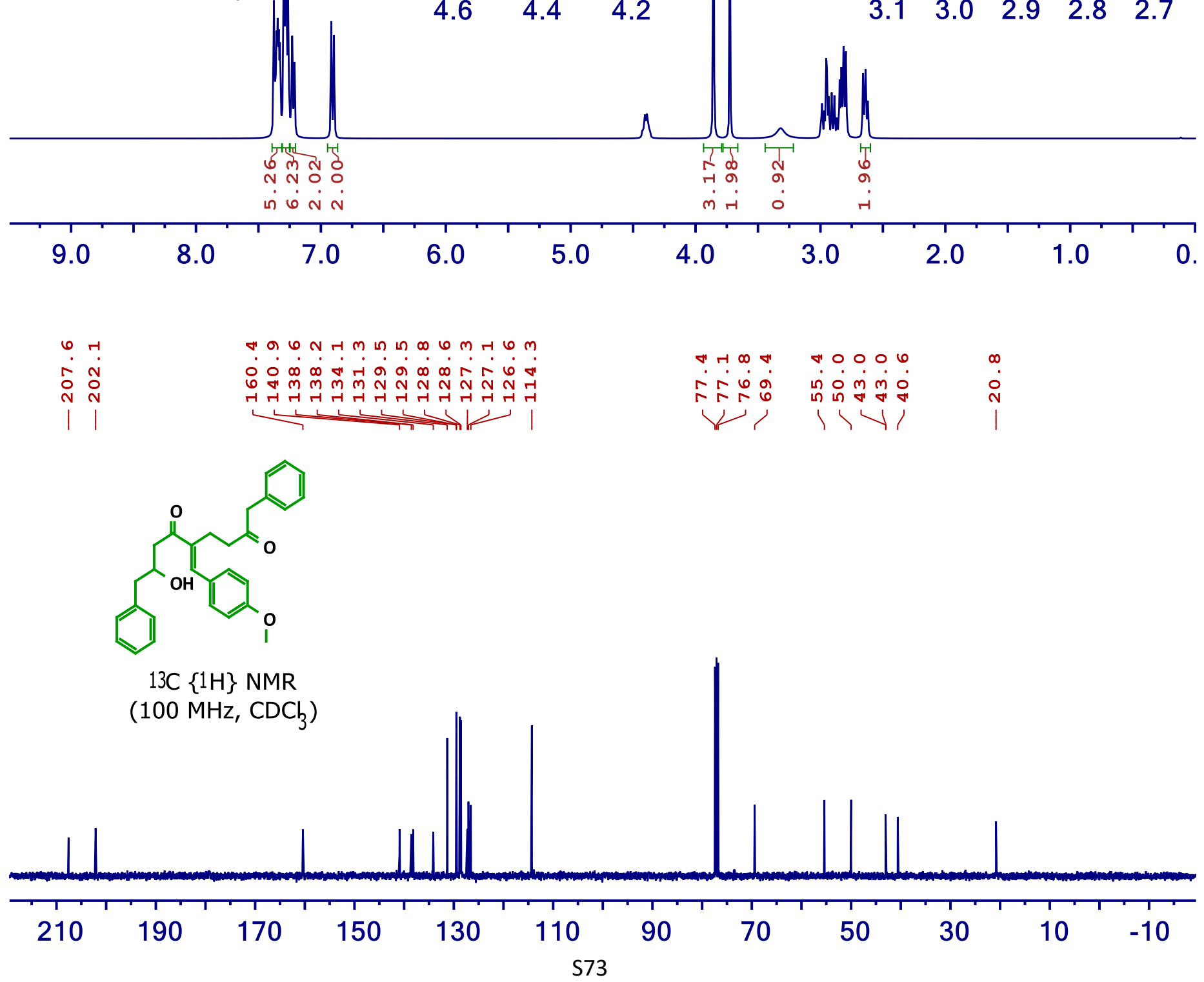

$13 \mathrm{C}\{1 \mathrm{H}\} \mathrm{NMR}$ (100 $\mathrm{MHz}, \mathrm{CDC}_{3}$ )

सी

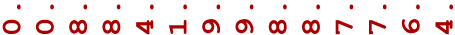
G

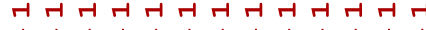

$\pi 4 \pi \pi t \pi 000$

송ํำ

I

114

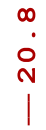


(E)-3-ethylidene-1-(1-hydroxycyclopentyl)-8-phenyloctane-2,6-dione

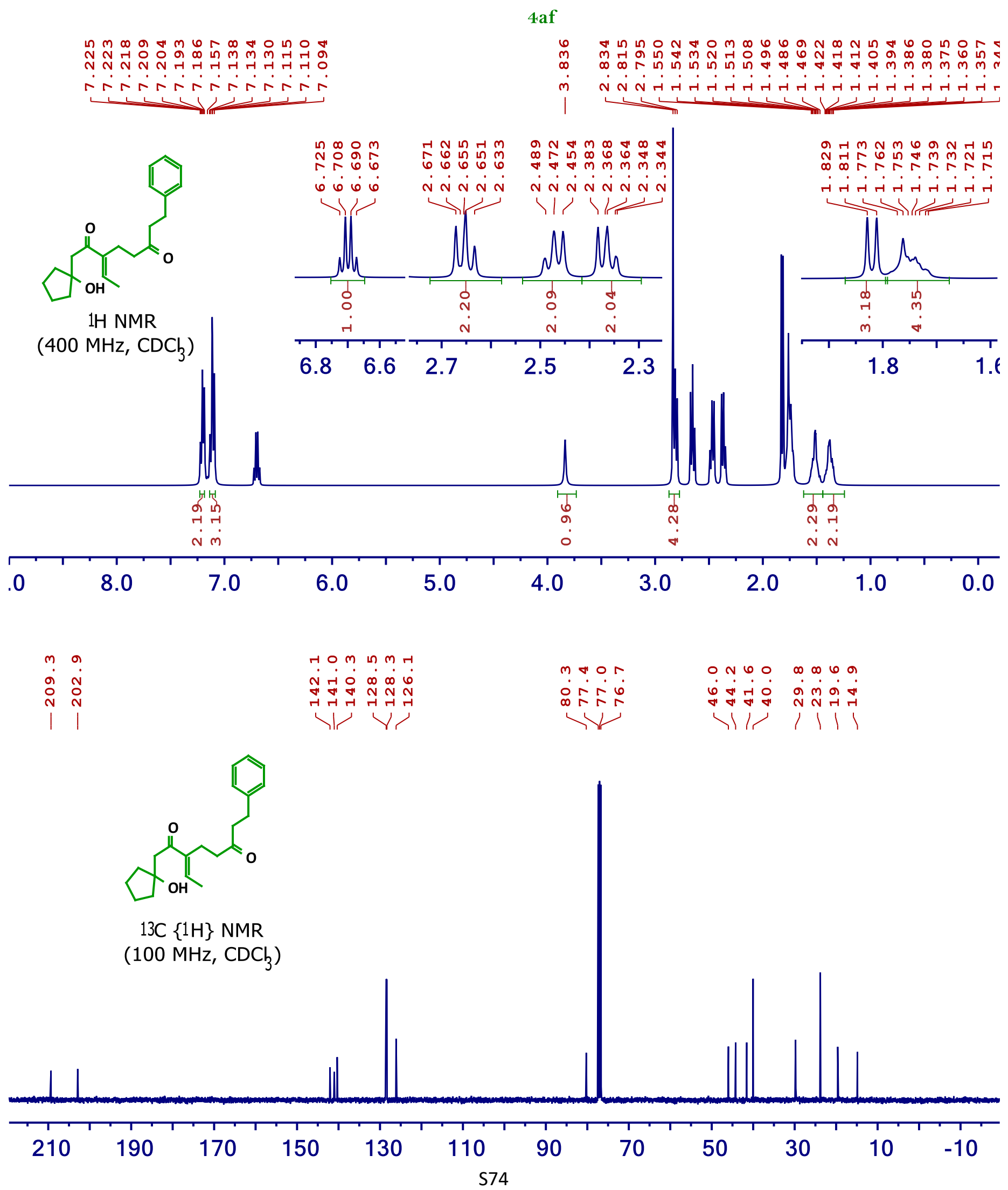


(E)-6-ethylidene-9-hydroxy-1,9-diphenyldecane-3,7-dione

4ag

울 m

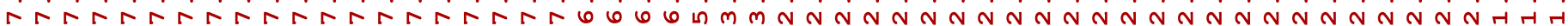

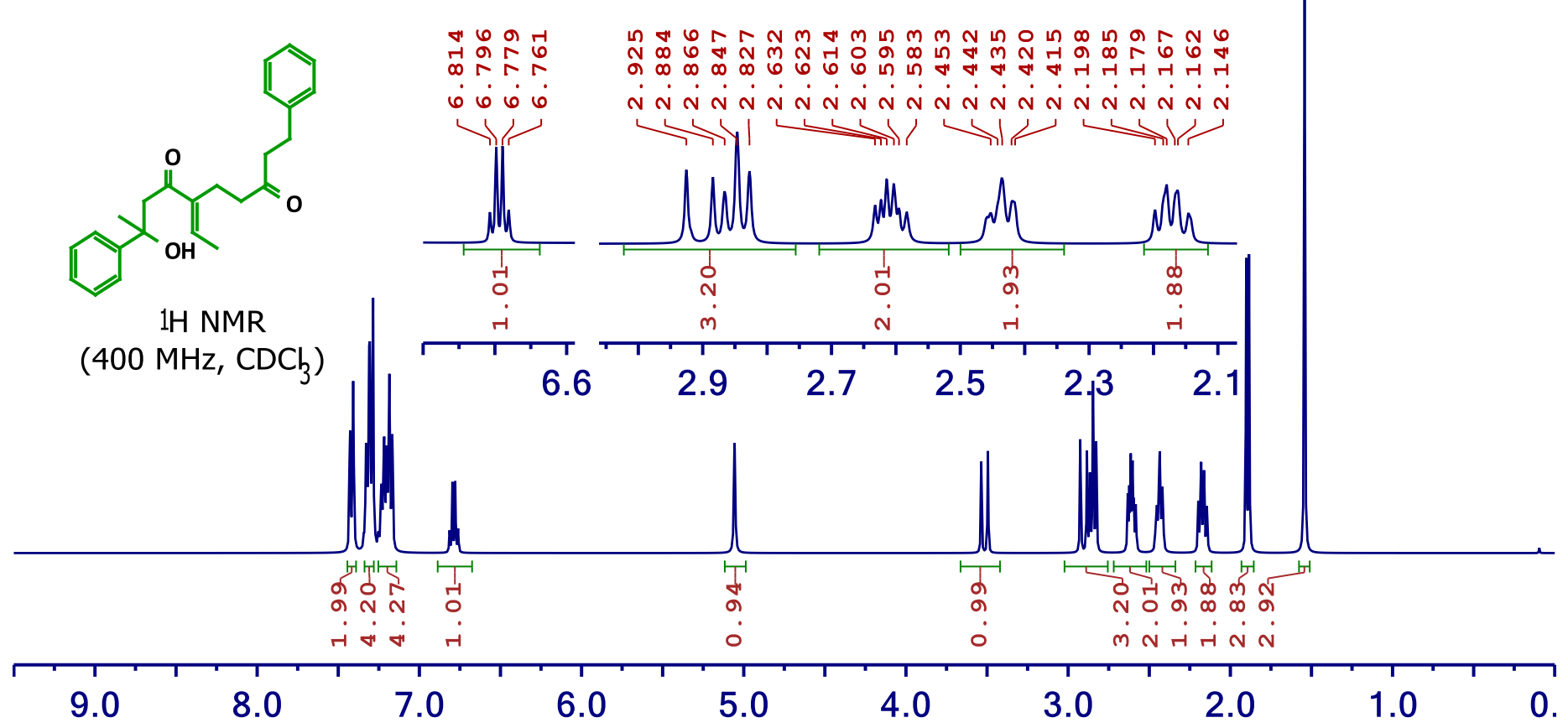<smiles>CCC(CCC(=O)CCc1ccccc1)C(=O)CC(C)(O)c1ccccc1</smiles>

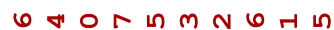

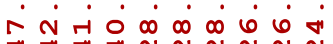

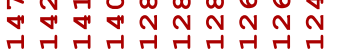

$>1$

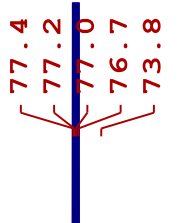

O $N H$ Hr ma

\&

$13 \mathrm{C}\{1 \mathrm{H}\} \mathrm{NMR}$ $\left(100 \mathrm{MHz}, \mathrm{CDCl}_{3}\right)$

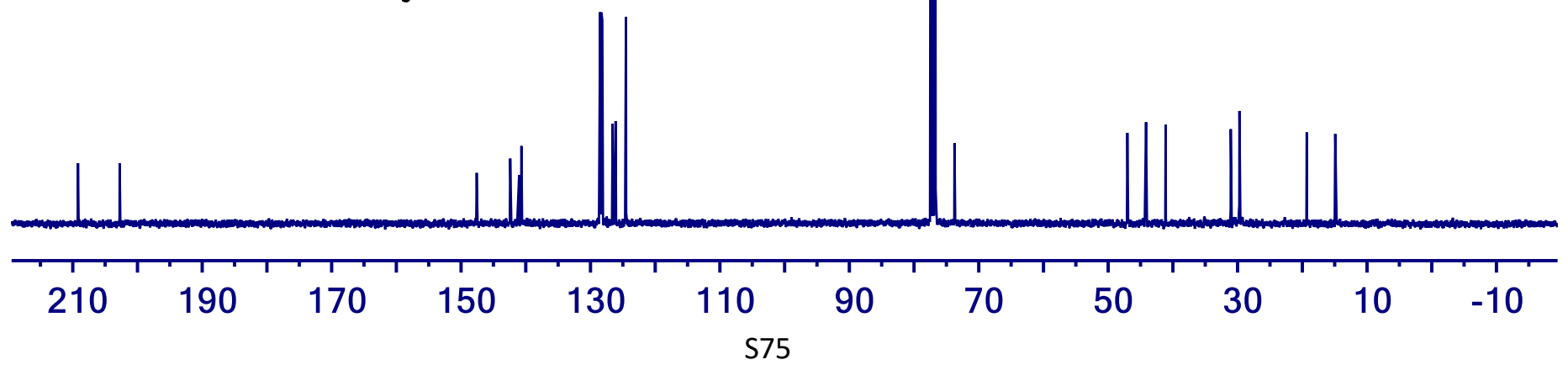


(E)-4-ethylidene-1-(furan-2-yl)-1-hydroxy-9-phenylnonane-3,7-dione

4ah

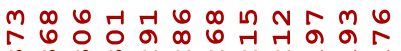

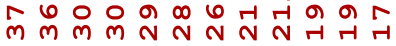

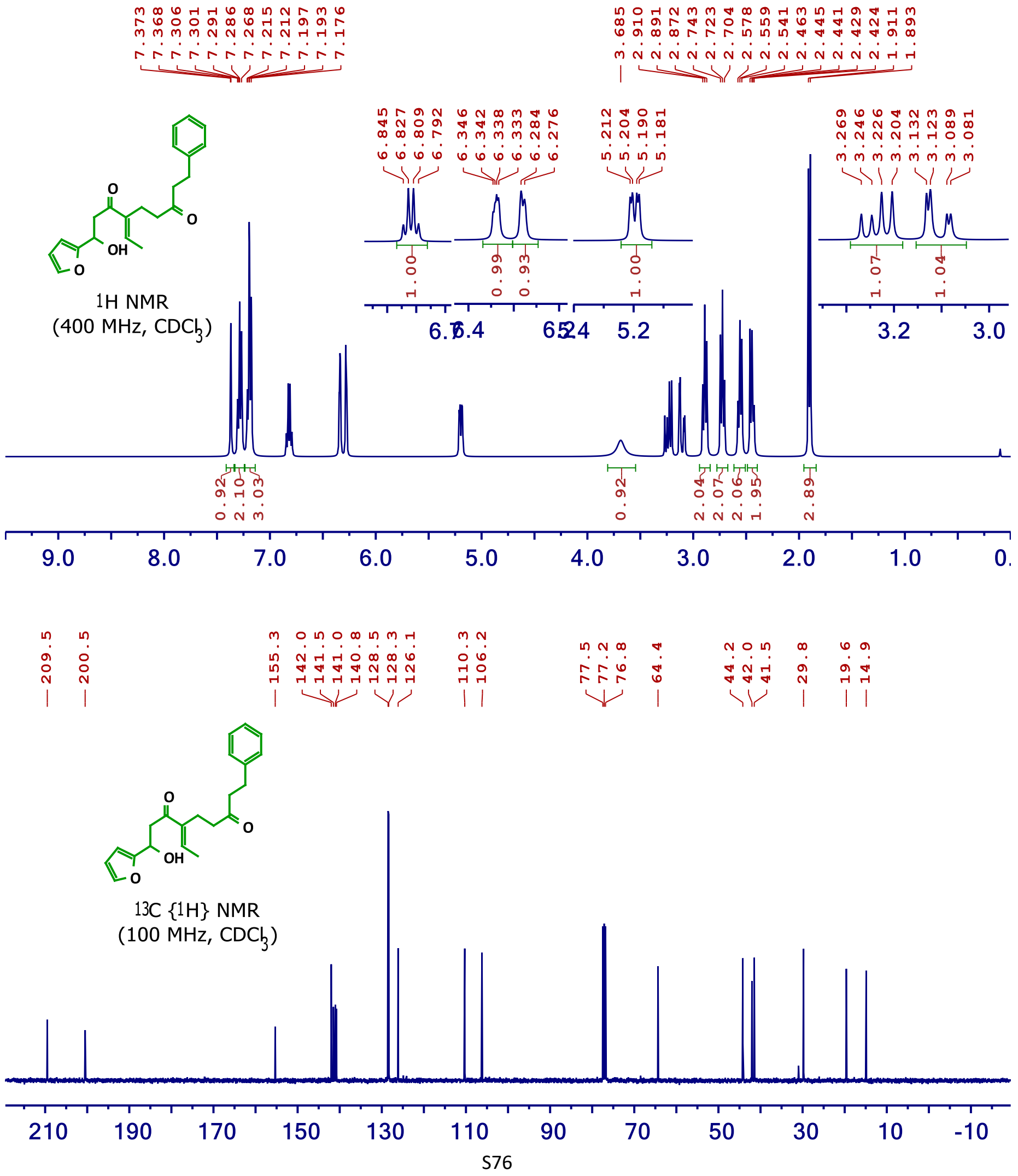


(E)-4-ethylidene-1-hydroxy-9-phenyl-1-(thiophen-2-yl)nonane-3,7-dione

4ai

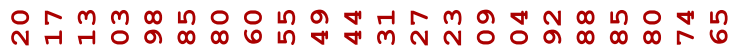

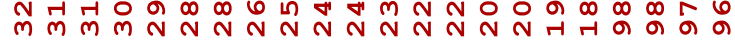

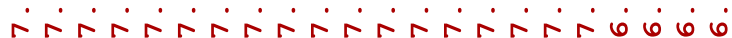

เ

a

.

$m m m m m m m m$

P

$r a$
5
$\sigma$
0
$\dot{1}$

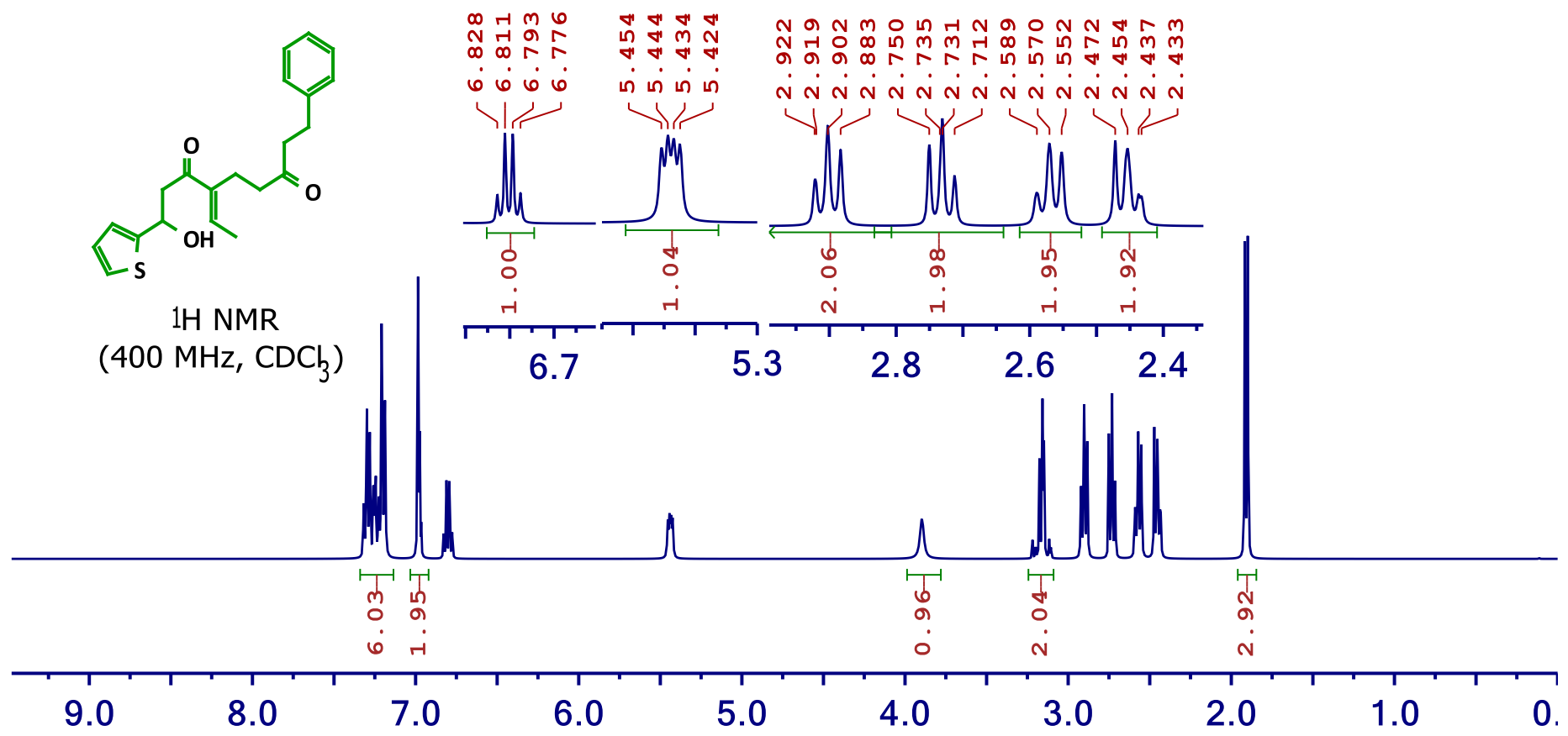

○ เ 0 の เ

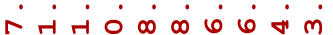

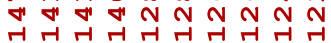

(1)<smiles>C/C=C(/CCC(=O)CCc1ccccc1)C(=O)CC(O)c1cccs1</smiles>

$13 \mathrm{C}\{1 \mathrm{H}\} \mathrm{NMR}$

$\left(100 \mathrm{MHz}, \mathrm{CDC}_{3}\right)$

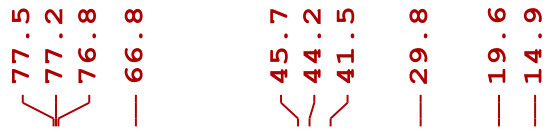

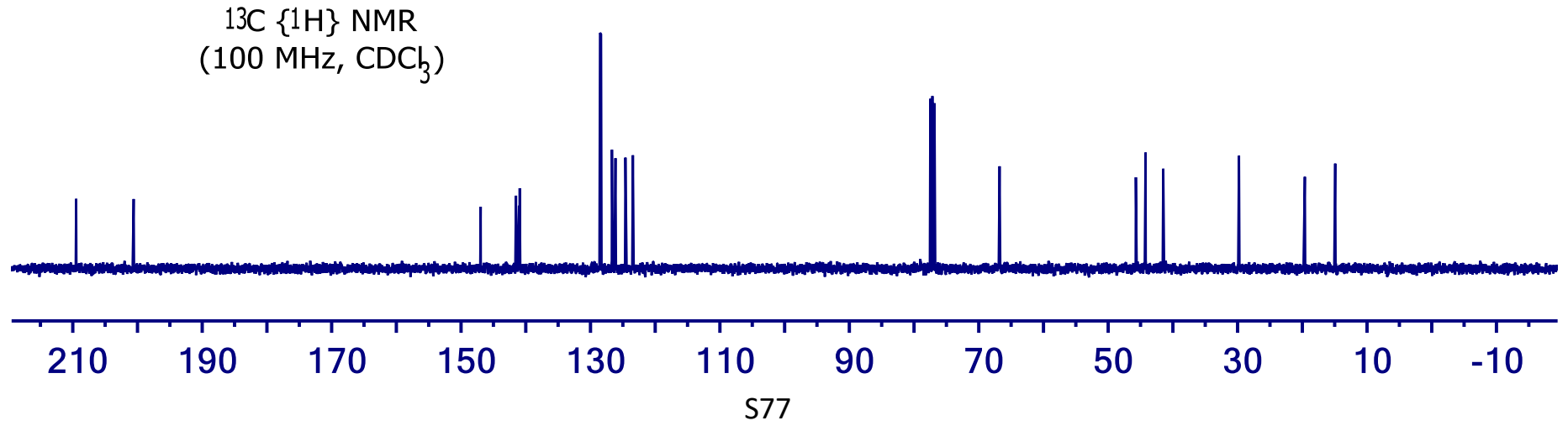


(E)-8-(furan-2-yl)-8-hydroxy-1-phenyl-5-(thiophen-2-ylmethylene)octane-2,6-dione

$4 a j$

- $060 \%$

6 m m mmmmmmmmm N N N N N N N N N

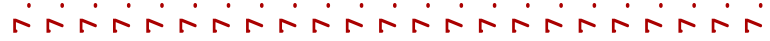

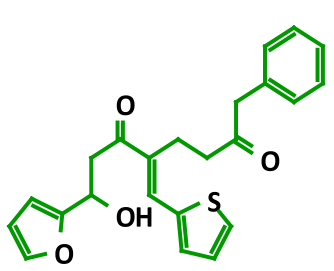

$1 \mathrm{H} N M R$

(400 $\left.\mathrm{MHz}, \mathrm{CDC}_{3}\right)$

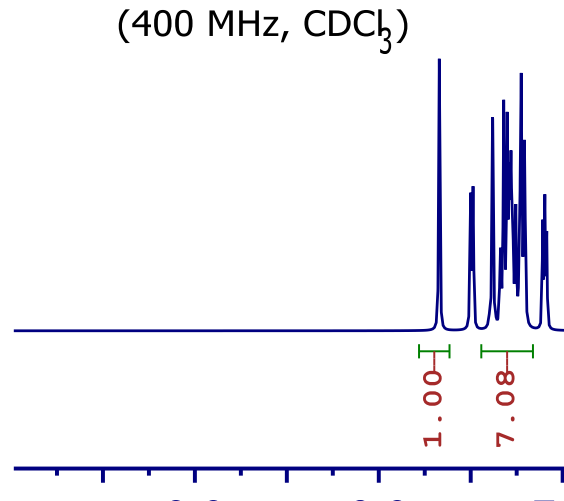

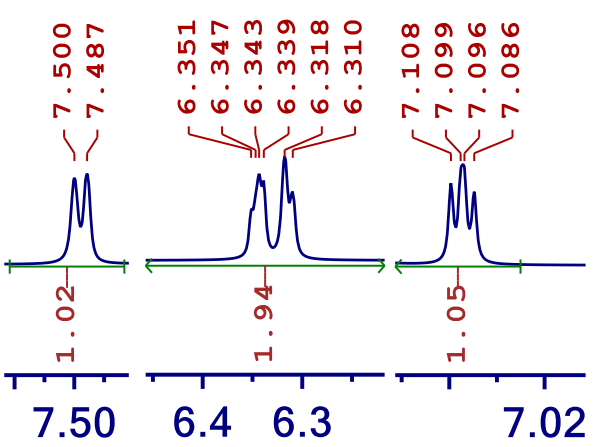

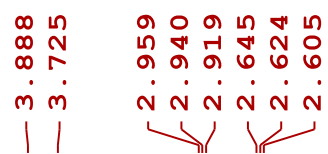

9.0

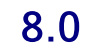

7.0

6.0

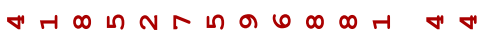

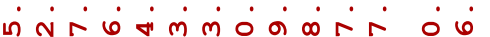

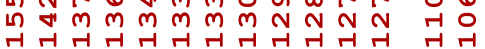

$\begin{array}{cc}n & -1 \\ ن & 0 \\ 0 & 0 \\ \text { N } & \text { N } \\ \mid & \mid\end{array}$

$\underbrace{.45}$

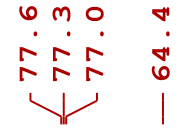

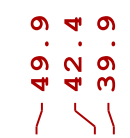

2.0

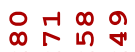

n $N$ N

เก เก เก เก

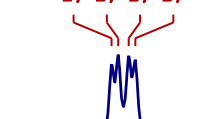

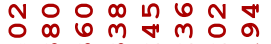

$\forall m m m N N$ N

$m m m m m m m m$

$111<1$
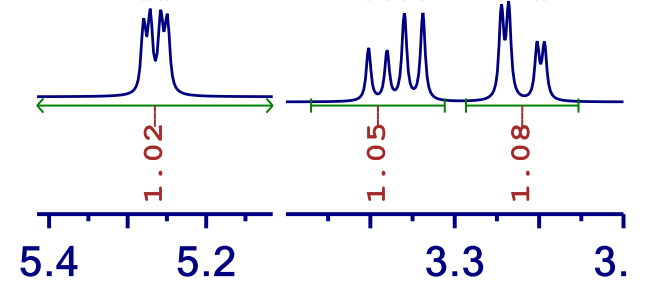

3.<smiles>C=C(CCC(=O)CCC(=O)Cc1ccccc1)C(O)Cc1ccco1</smiles>

$13 \mathrm{C}\{1 \mathrm{H}\} \mathrm{NMR}$

(100 $\mathrm{MHz}, \mathrm{CDC}_{3}$ )
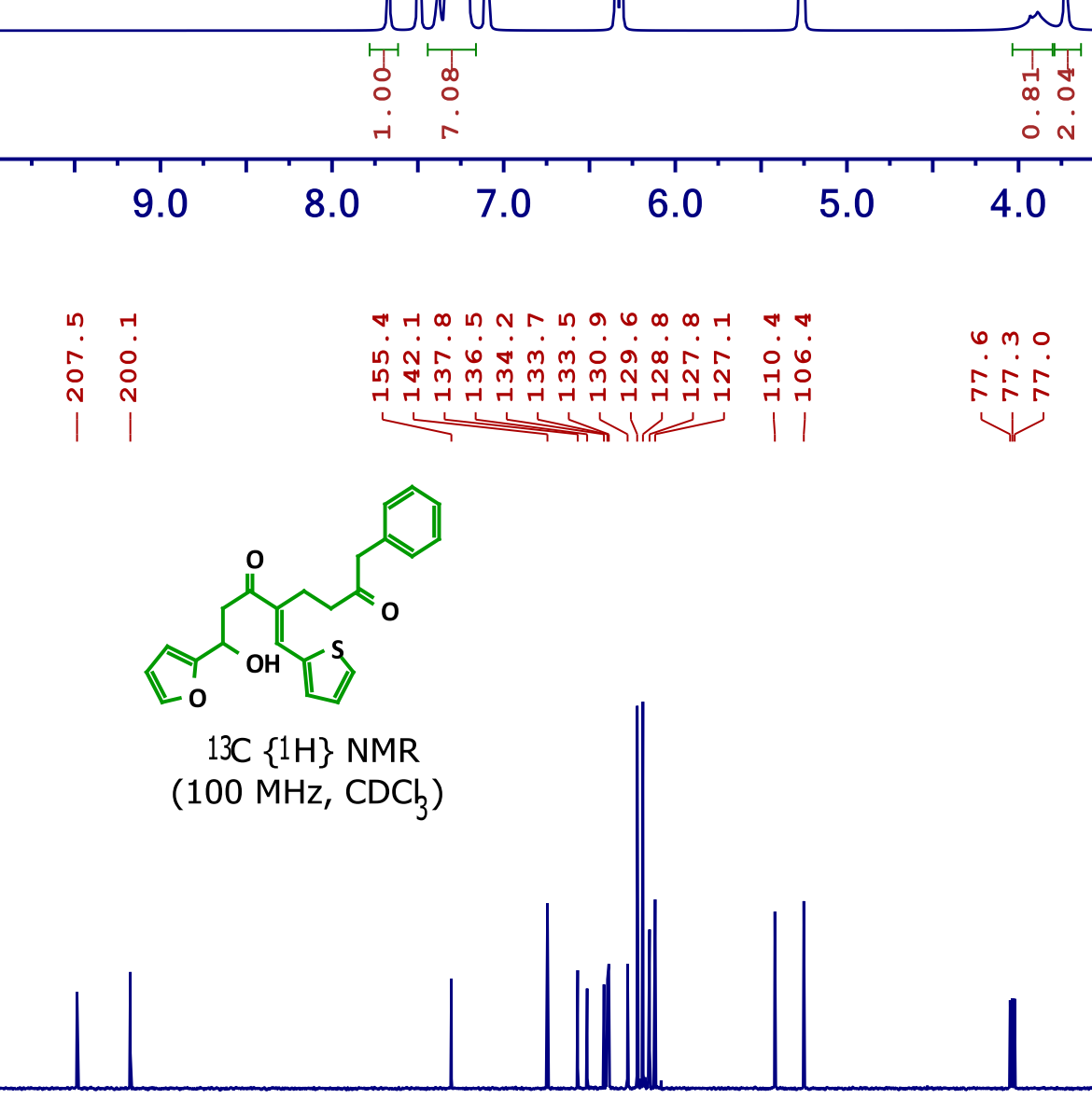

न

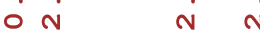

4.0

3.0

1.0

0.

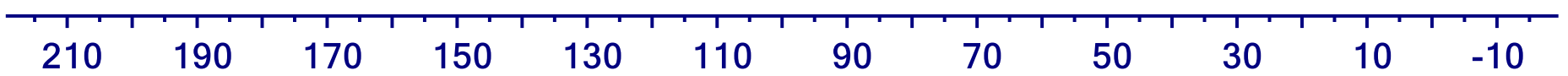


(E)-5-ethylidene-8-hydroxy-1-phenyl-8-(o-tolyl)octane-2,6-dione

4w

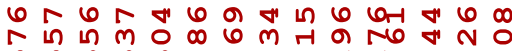
i

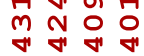

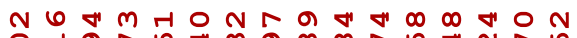

ก.

in 10

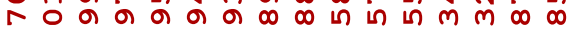

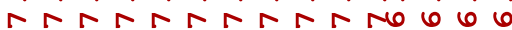

in

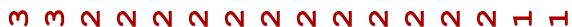
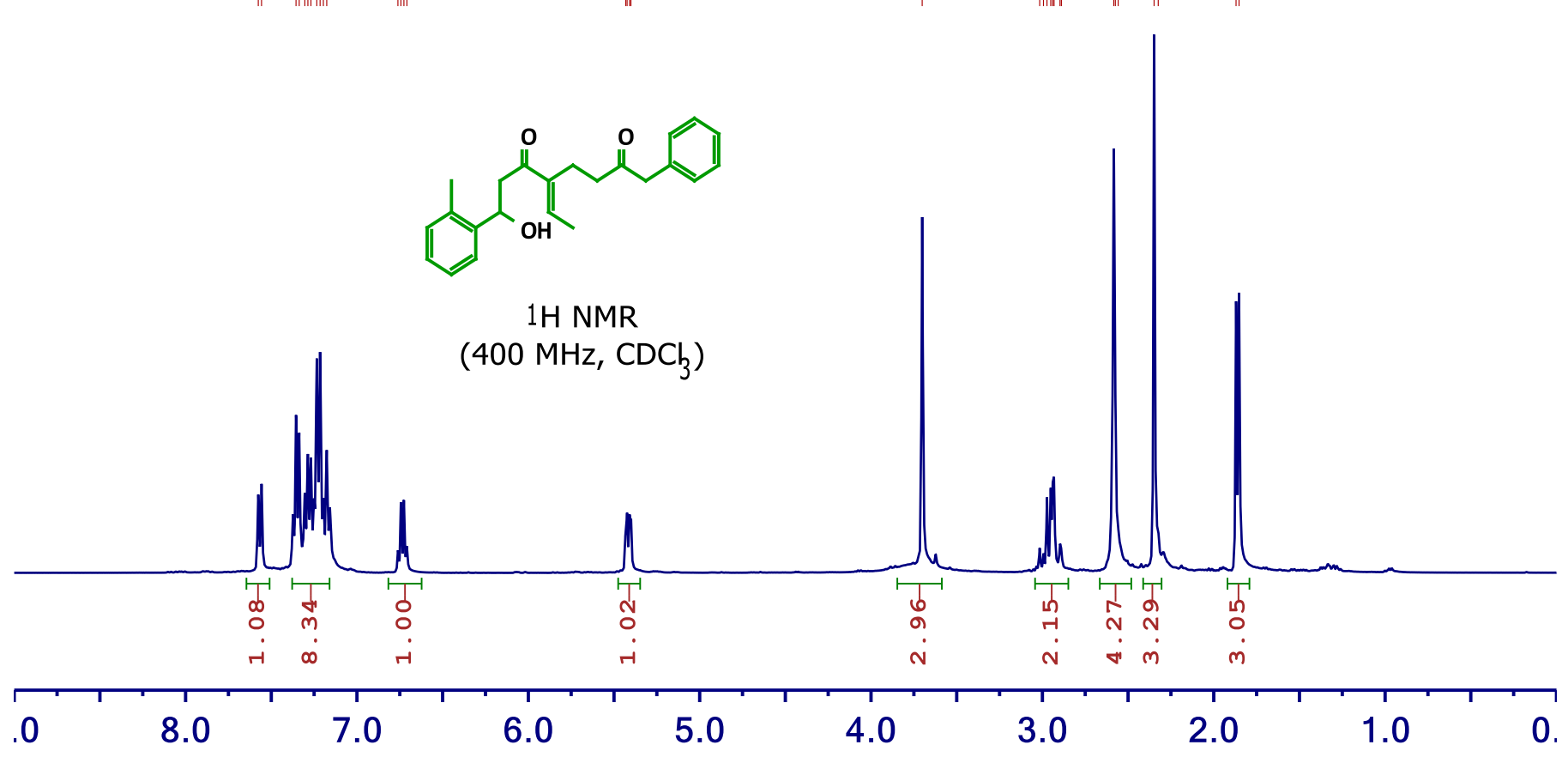

\begin{tabular}{llllllllllll}
\hline .0 & 8.0 & 7.0 & 6.0 & 5.0 & 4.0 & 3.0 & 2.0 & 1.0 & 0.0
\end{tabular}

N n

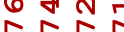

$\dot{0} \dot{0} \dot{0}$

ก ก

Hâ

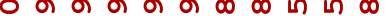

4

$\operatorname{m} N$ N $N$ N $N$ N $N-4-1$

(400 MHz, $\left.\mathrm{CDCb}_{3}\right)$
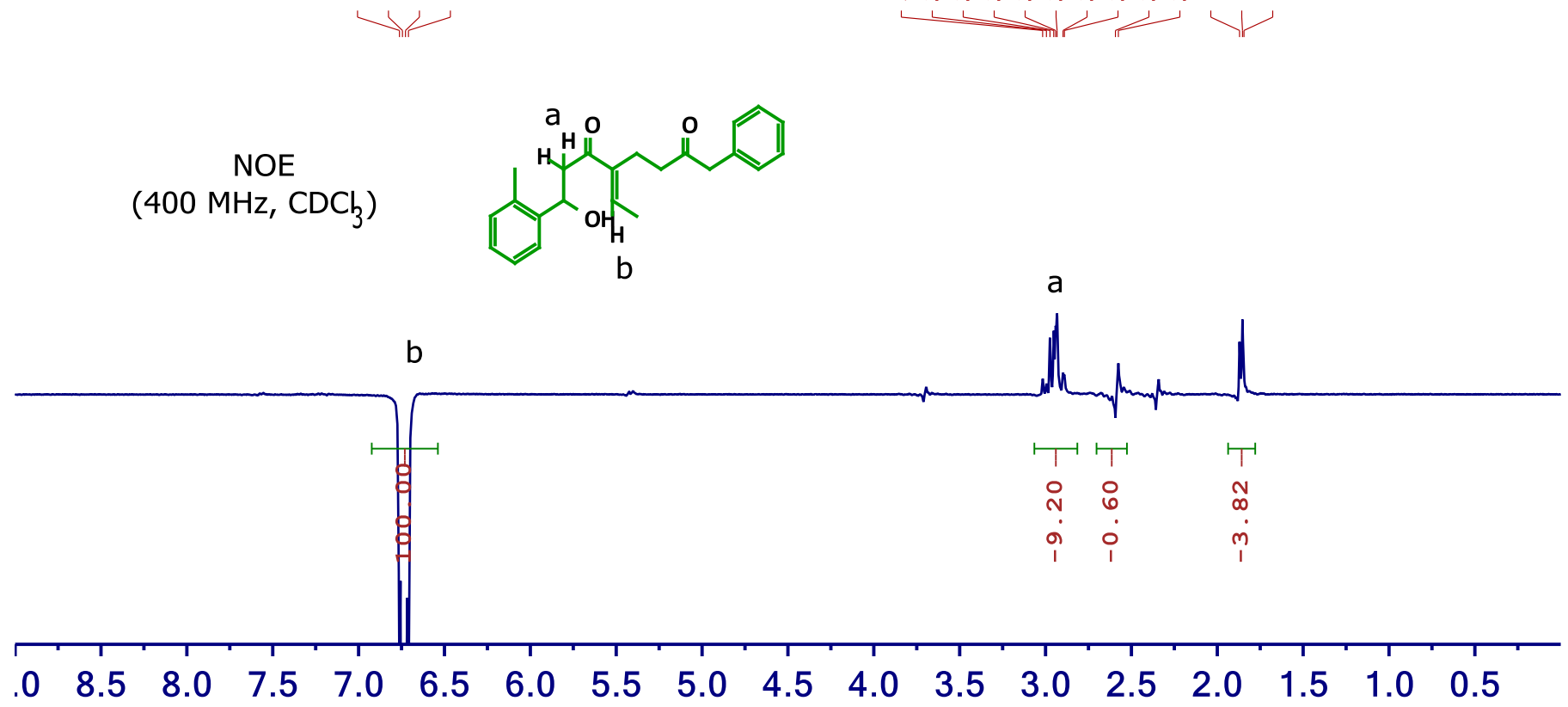
(E)-5-ethylidene-8-hydroxy-1-phenyl-8-(o-tolyl)octane-2,6-dione

4w

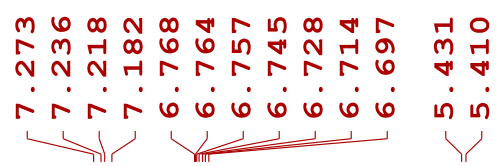

$m$ in

○ $N$ ה

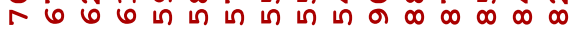

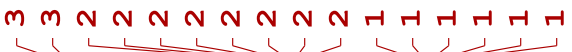

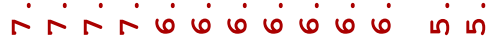

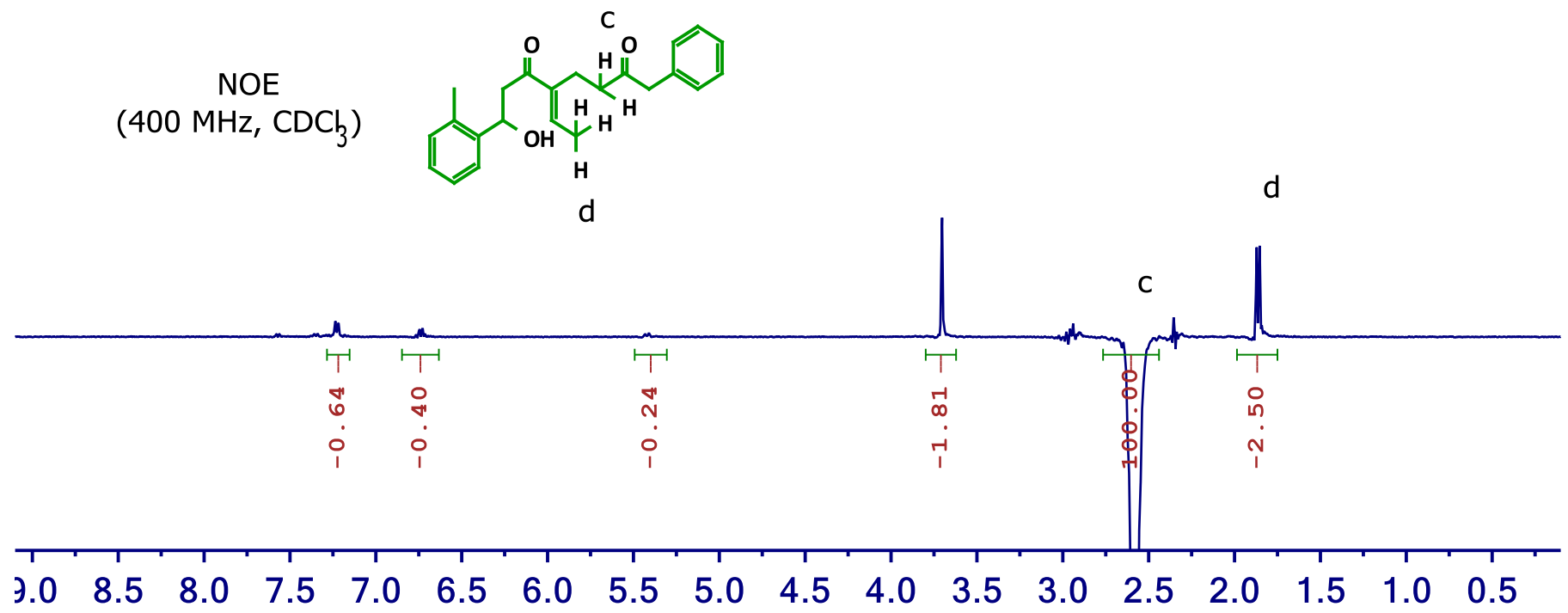

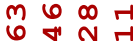

$\operatorname{rin} 12$

ம்

rif

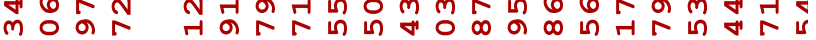

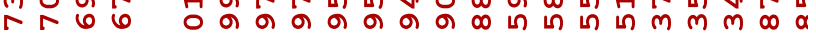
$\dot{m} \dot{m} \dot{m}$ $\dot{M} \dot{N} \dot{N} \dot{N} \dot{N} \dot{N} \dot{N} \dot{N} \dot{N} \dot{N} \dot{N} \dot{H}$
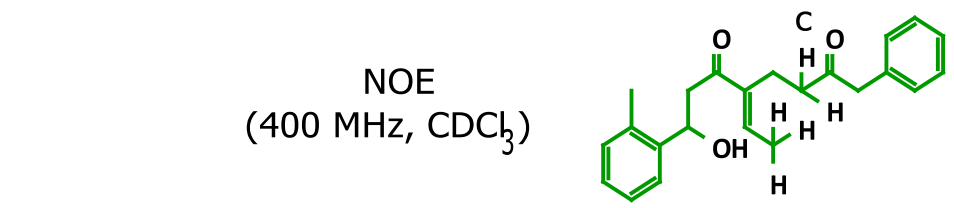

rif
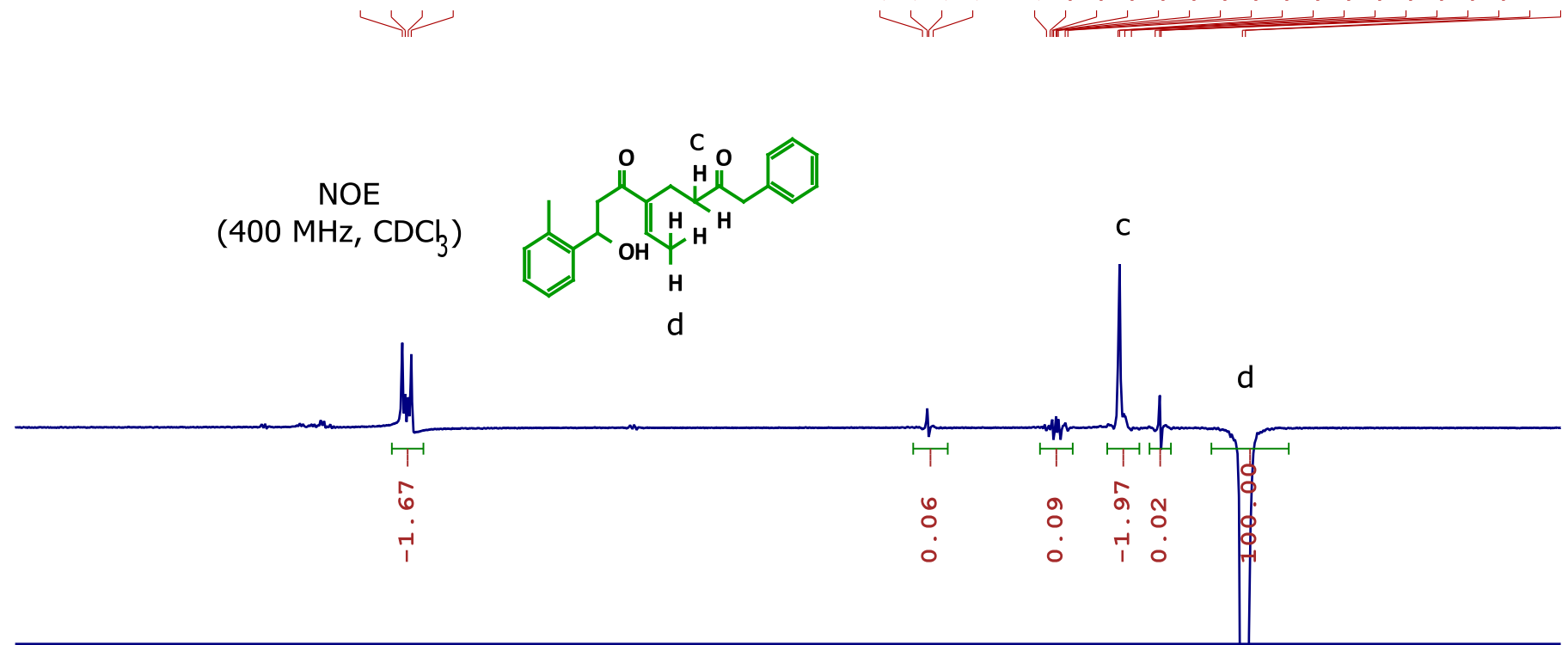

$\begin{array}{llllllllllllllllll}.0 & 8.5 & 8.0 & 7.5 & 7.0 & 6.5 & 6.0 & 5.5 & 5.0 & 4.5 & 4.0 & 3.5 & 3.0 & 2.5 & 2.0 & 1.5 & 1.0 & 0.5\end{array}$ 
(E)-5-ethylidene-8-hydroxy-1-phenyl-8-(o-tolyl)octane-2,6-dione

4w

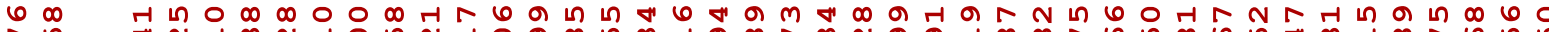
In $N$ N $N$ N

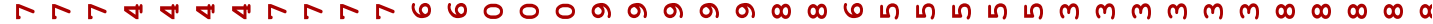

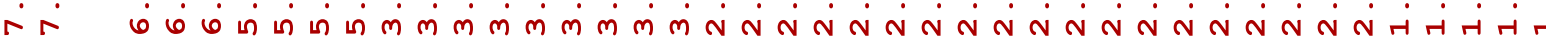
I 666
1

NOE $\left(400 \mathrm{MHz}, \mathrm{CDC}_{3}\right)$<smiles>CC(CCC(=O)c1ccccc1)C(O)C(O)C(C)c1ccccc1</smiles>

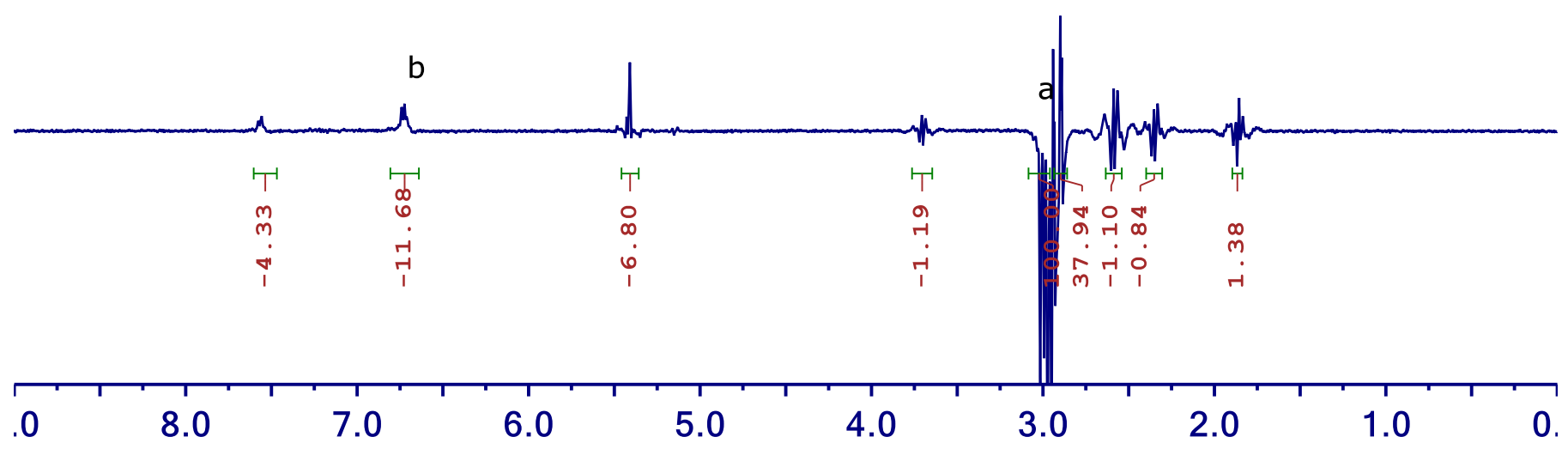


(2R,3R,6R)-2-methyl-3-(3-oxo-4-phenylbutyl)-6-phenyltetrahydro-4H-pyran-4-one

$5 \mathbf{a}$

今 m 유류 * F

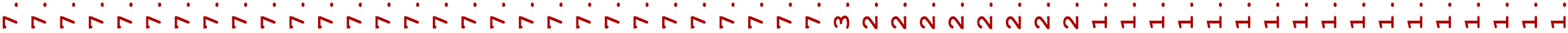

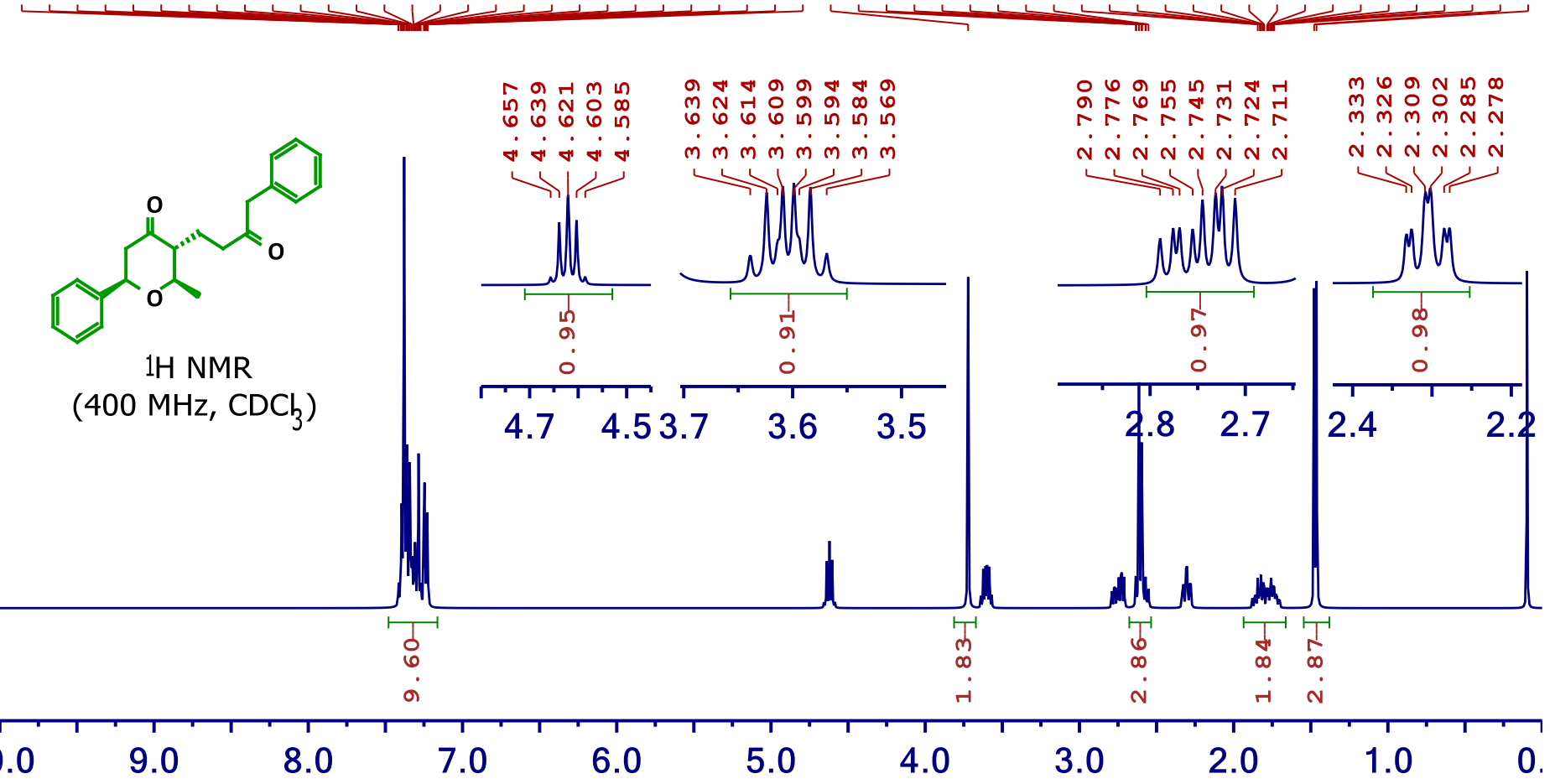

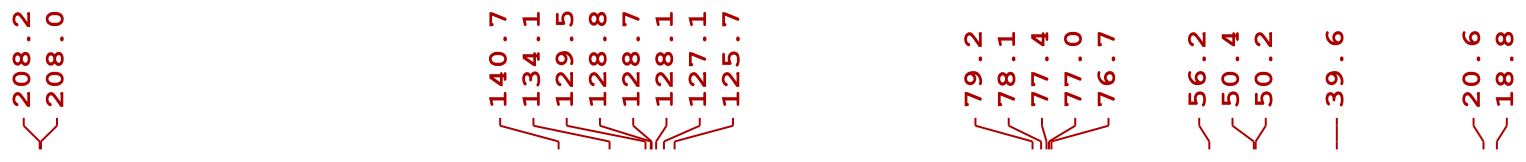<smiles>C[C@H]1OC(c2ccccc2)CC(=O)[C@H]1CCC(=O)Cc1ccccc1</smiles>

$13 \mathrm{C}\{1 \mathrm{H}\} \mathrm{NMR}$

(100 $\left.\mathrm{MHz}, \mathrm{CDCl}_{3}\right)$

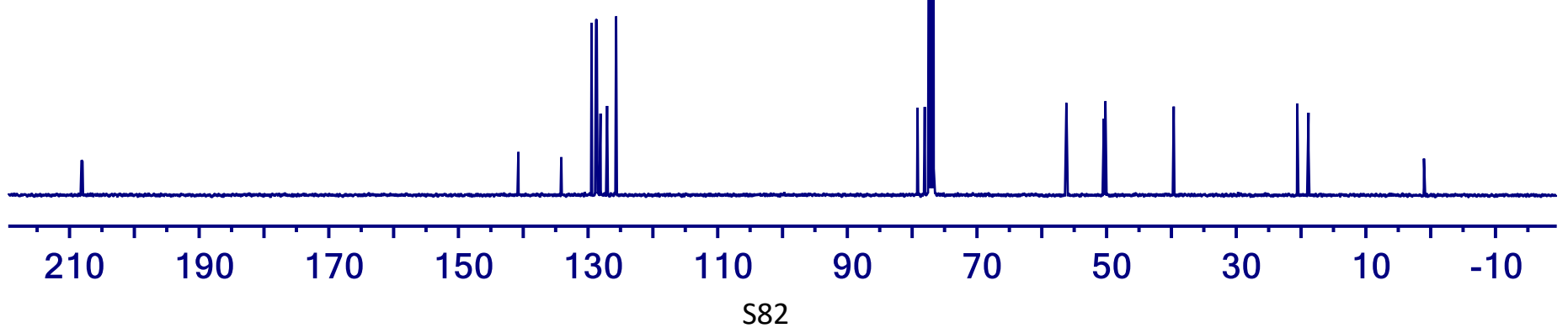


(2R,3R,6R)-2-methyl-3-(3-oxo-3-(thiophen-2-yl)propyl)-6-phenyltetrahydro-4H-pyran-4-one

$5 \mathrm{~b}$

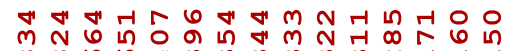

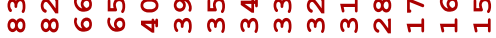

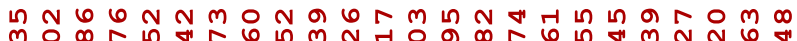

0

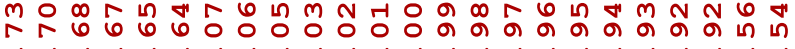

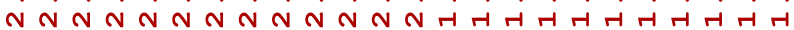<smiles>CC1OC(c2ccccc2)CC(=O)C1CCC(=O)c1cccs1</smiles>

$1 \mathrm{H}$ NMR

$\left(400 \mathrm{MHz}, \mathrm{CDC}_{3}\right)$

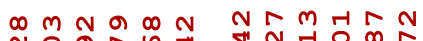

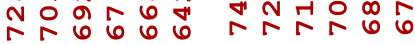
$\forall \forall \forall \dot{\nabla} \dot{m} \dot{m} \dot{m} \dot{m}$
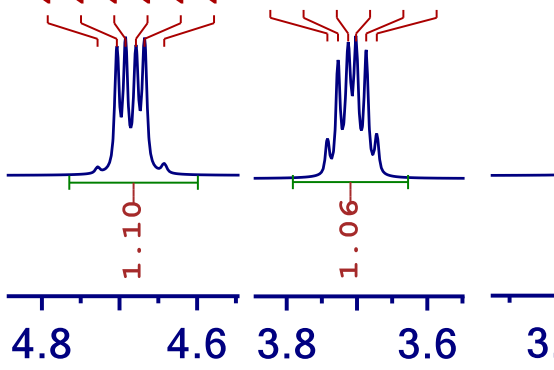

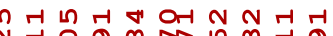
N

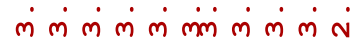
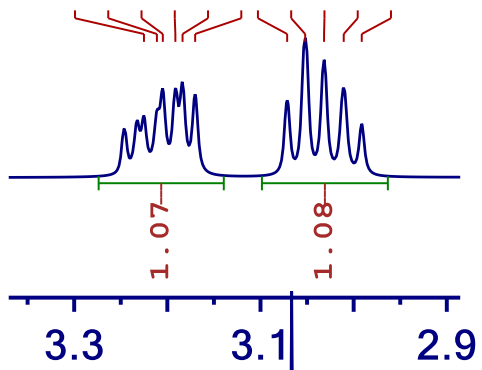
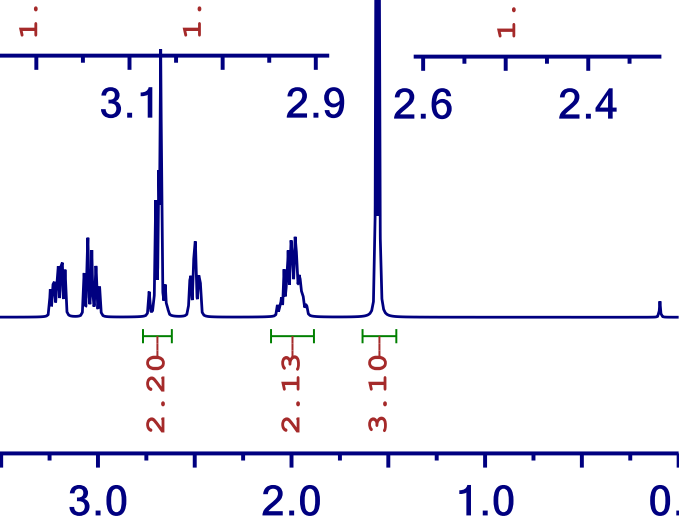

\begin{tabular}{llllllllllll}
\hline 9.0 & 8.0 & 7.0 & 6.0 & 5.0 & 4.0 & 3.0 & 2.0 & 1.0 & 0
\end{tabular}

$\begin{array}{cc}m & 0 \\ \infty & m \\ 0 & a \\ N & r \\ 1 & 1\end{array}$

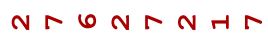

$\dot{0} \dot{0} \dot{N} \dot{\infty} \dot{\infty} \dot{\infty}$ in

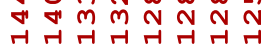

>

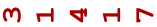

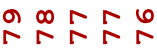

番

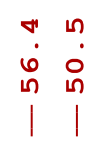

$\begin{array}{cc}0 & \text { n } \\ 0 & 0 \\ \text { in } & \text { in } \\ 1 & \mid\end{array}$
- 0 m 6 a กำ ำ ㅇำ N $\dot{N}$ N

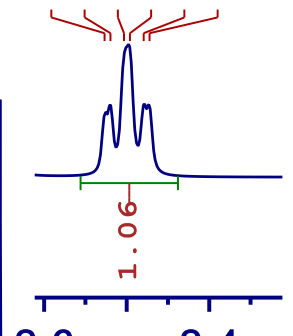


$(2 R, 3 R, 6 R)-3-(3-(f u r a n-2-y l)-3-o x o p r o p y l)-2-m e t h y l-6-p h e n y l t e t r a h y d r o-4 H-p y r a n-4-o n e$

$5 \mathrm{c}$

ᄂ 둥둥 $60 \% \mathrm{~m} m \mathrm{~m} m \mathrm{~m} m \mathrm{~m}$ m $\mathrm{N}$ N

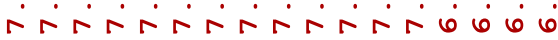
$\longrightarrow$

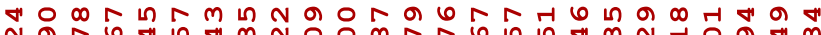

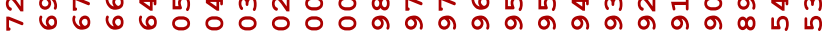

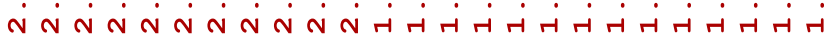<smiles>C[C@H]1OC(c2ccccc2)CC(=O)[C@H]1CCC(=O)c1ccco1</smiles>

$1 \mathrm{H} N M R$ (400 $\mathrm{MHz}, \mathrm{CDC}_{3}$ )

$\stackrel{1}{N} \begin{array}{lllll}0 & 0 & 0 & 0 & 0 \\ & 0 & 0 & 0 & 0 \\ 0 & 0 & 0 & 0\end{array}$

मं

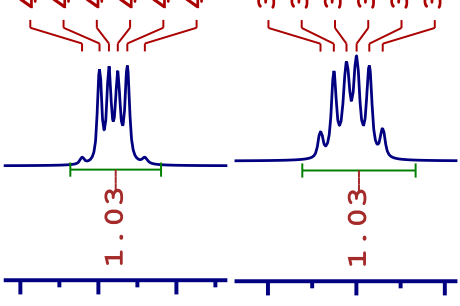

4.8

$\begin{array}{ll}4.6 & 3.8\end{array}$

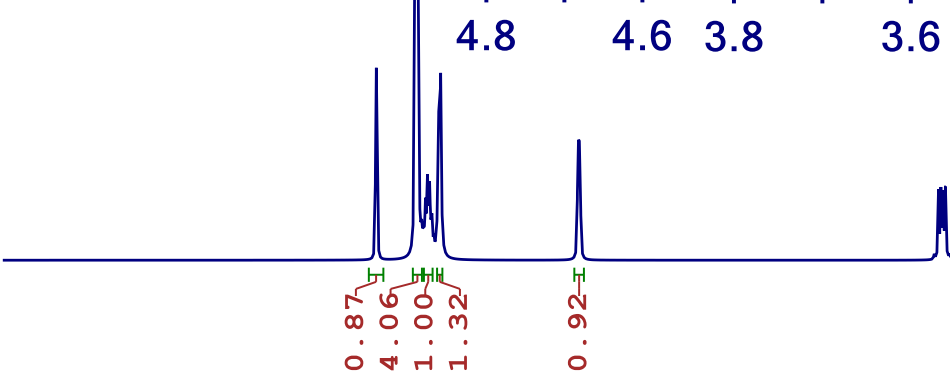

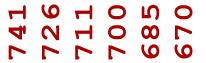

F

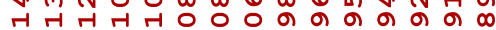

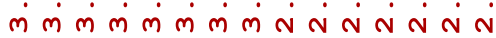
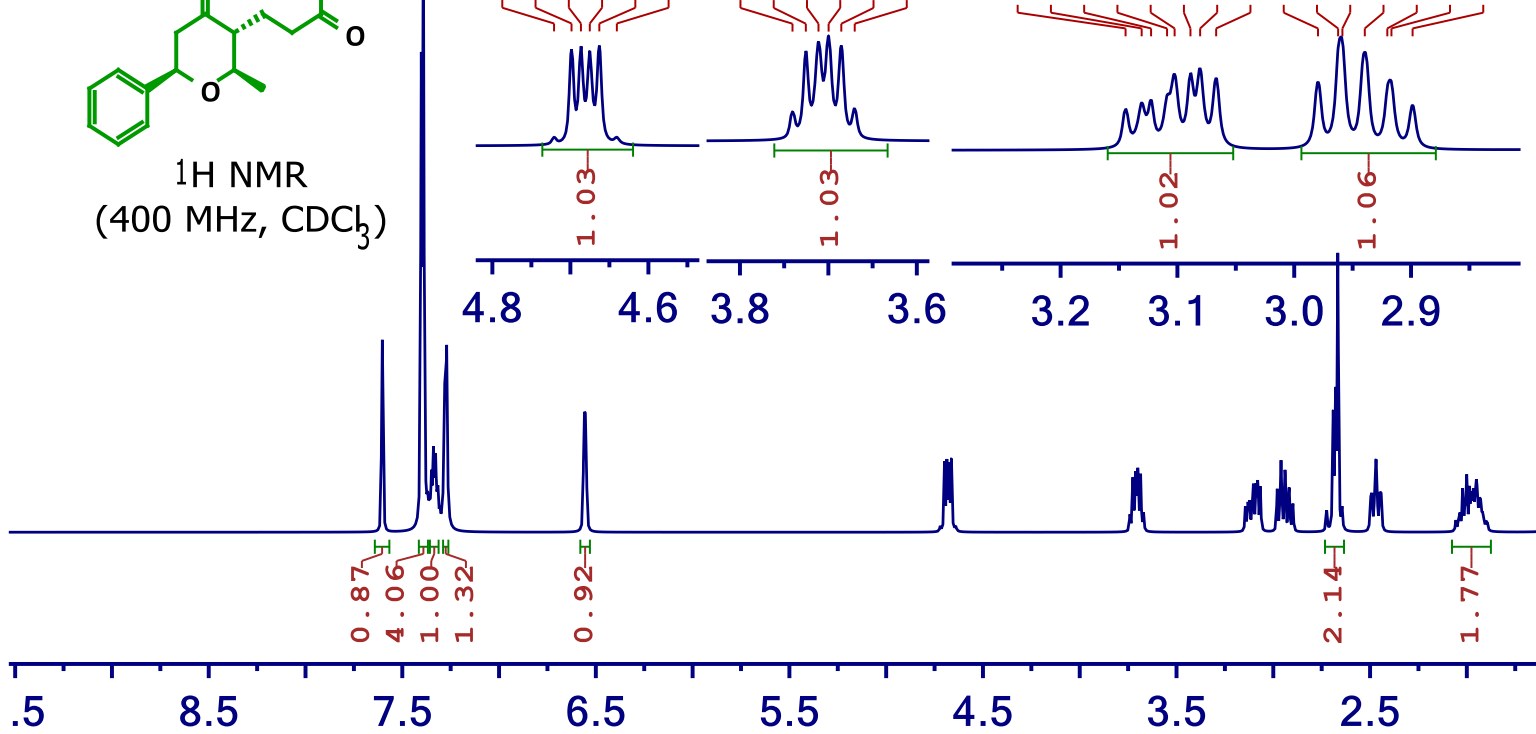

6.5

5.5

4.5

3.2
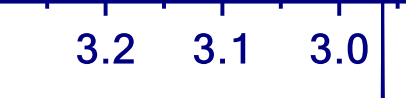

2.9
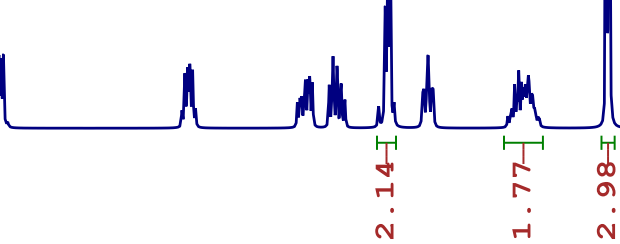
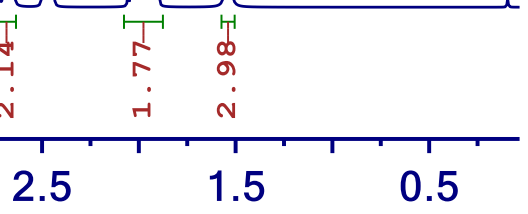

ล่ํํำㅇำ

子"

กักัก ก

$\rightarrow$

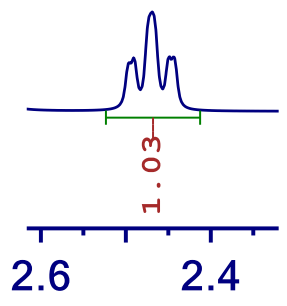


(2R,3R,6R)-2-methyl-3-(3-oxo-5-phenylpentyl)-6-phenyltetrahydro-4H-pyran-4-one

$5 \mathrm{~d}$

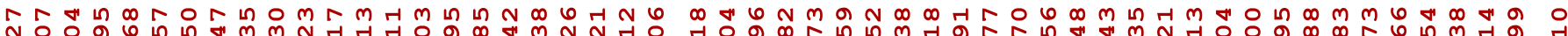

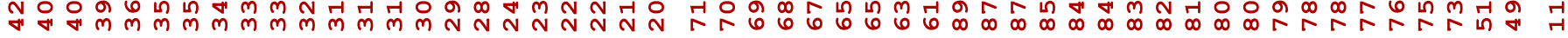

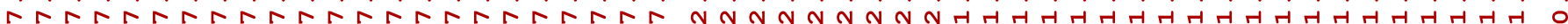
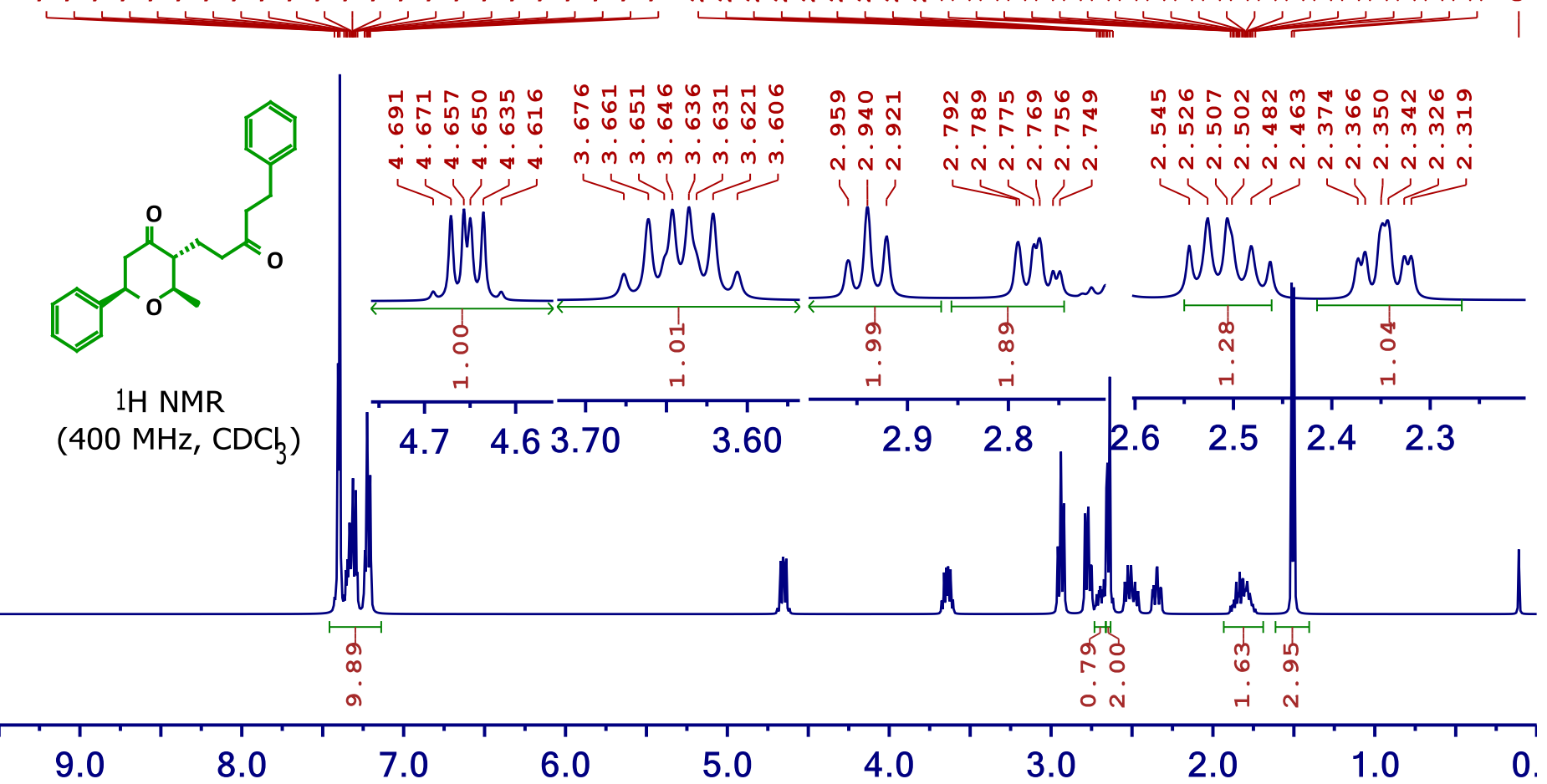

\begin{tabular}{|c|c|c|c|c|}
\hline 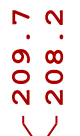 & 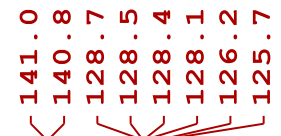 & 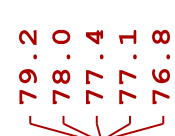 & 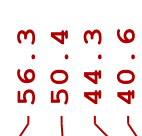 & $\begin{array}{c}a \\
\dot{\sigma} \\
N\end{array}$ \\
\hline
\end{tabular}<smiles>CC1OC(c2ccccc2)CC(=O)C1CCC(=O)CCc1ccccc1</smiles>

$13 \mathrm{C}\{1 \mathrm{H}\} \mathrm{NMR}$ $\left(100 \mathrm{MHz}, \mathrm{CDC}_{3}\right)$
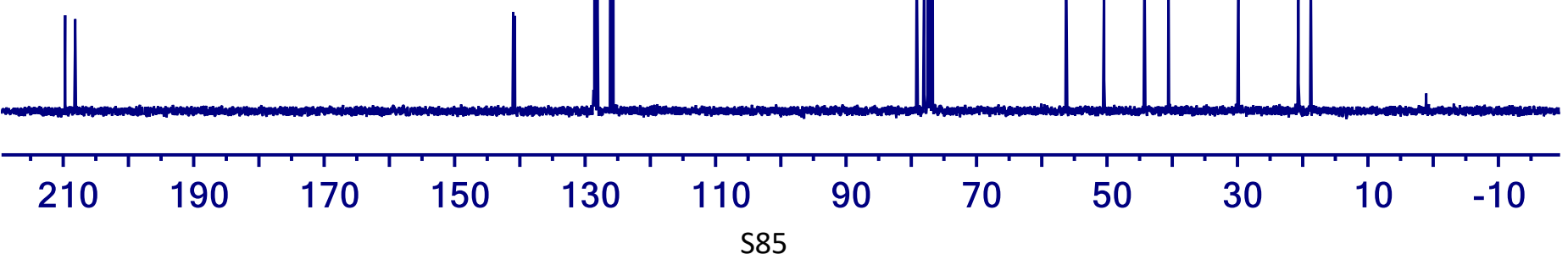
(2 R,3R,6R)-2-methyl-3-(3-oxo-3-phenylpropyl)-6-phenyltetrahydro-4H-pyran-4-one

$5 \mathrm{e}$

둔 。 m

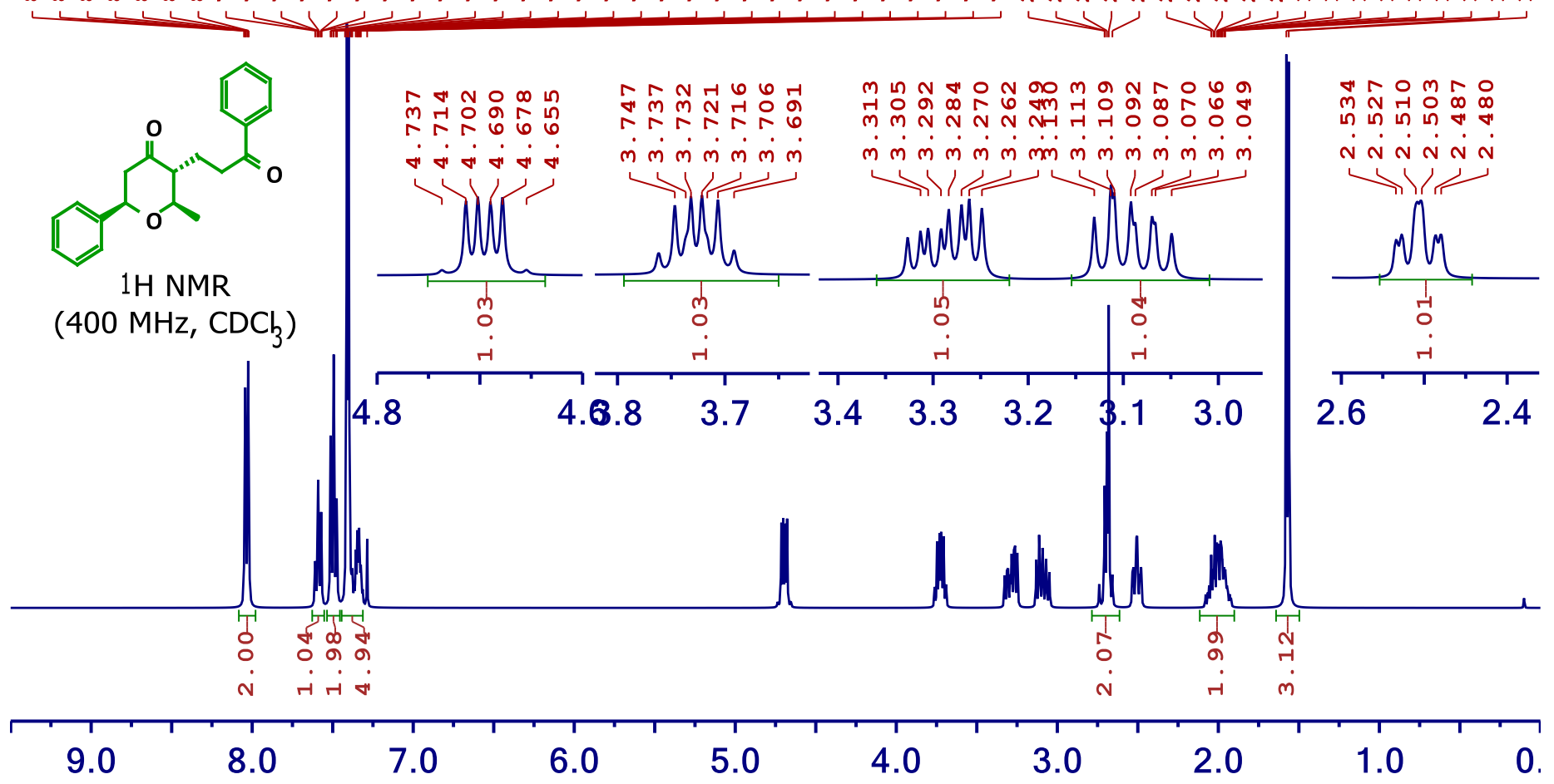

$\infty \infty \pi+\infty$ H

$\dot{0} \dot{0} \dot{m} \dot{\infty} \dot{\infty} \dot{\infty} \dot{\infty}$ in

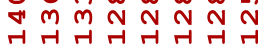

$\underbrace{2}$

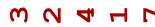

a NNR

$\rightarrow$

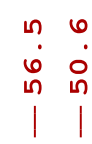

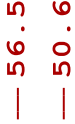

16

우

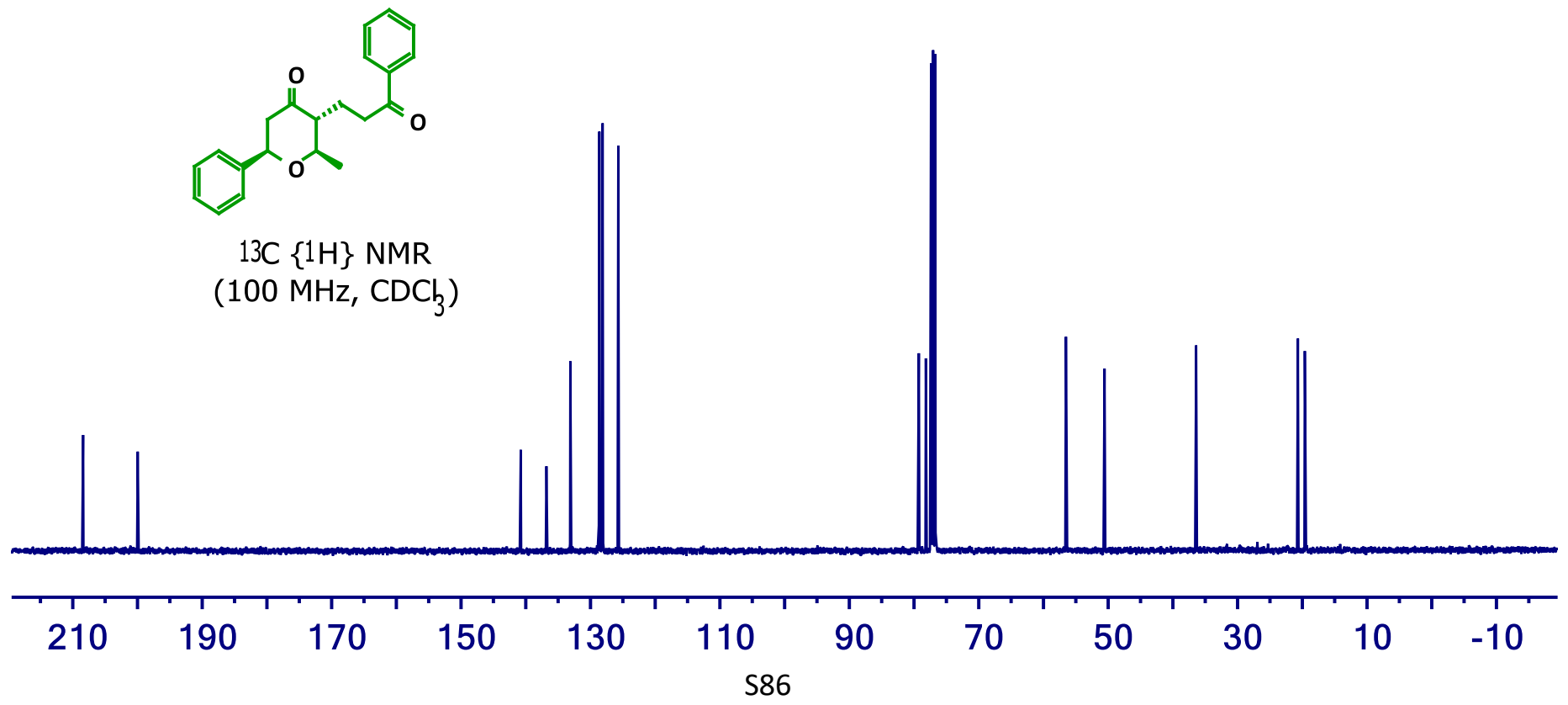


$(2 R, 3 R, 6 R)-3-(3-c y c l o h e x y l-3-o x o p r o p y l)-2-m e t h y l-6-p h e n y l t e t r a h y d r o-4 H-p y r a n-4-o n e$

$5 f$

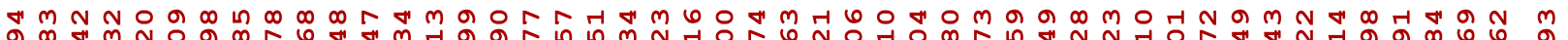

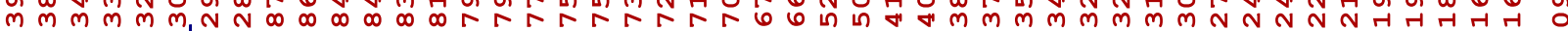

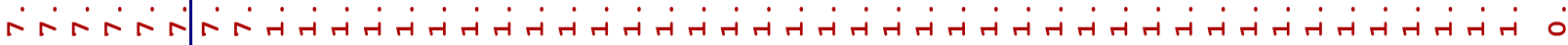

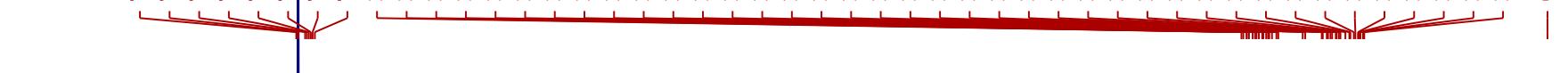

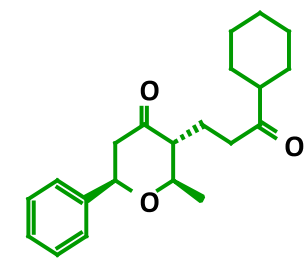

$1 \mathrm{H}$ NMR (400 $\left.\mathrm{MHz}, \mathrm{CDC}_{3}\right)$

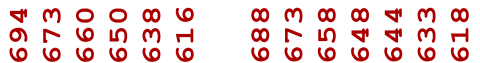

$\dot{\nabla} \nabla \dot{\nabla} \dot{\nabla} \dot{m} \dot{m} \dot{m} \dot{m} \dot{m} \dot{m}$

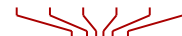

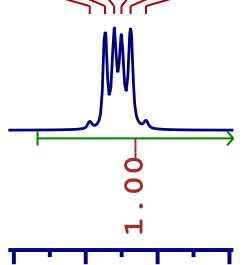

is
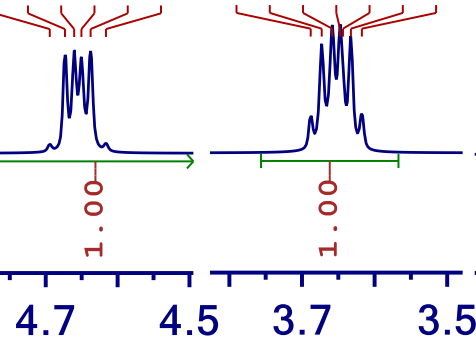

4.7
윴

4
15

4ลง
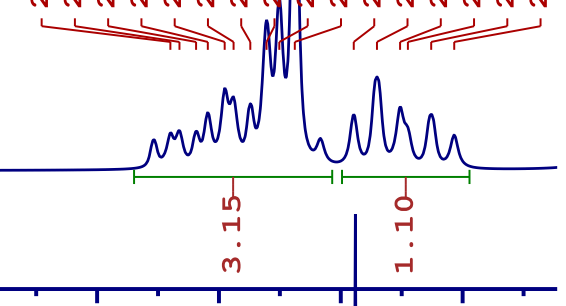

ด̆ $m m m m m m m$

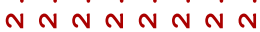
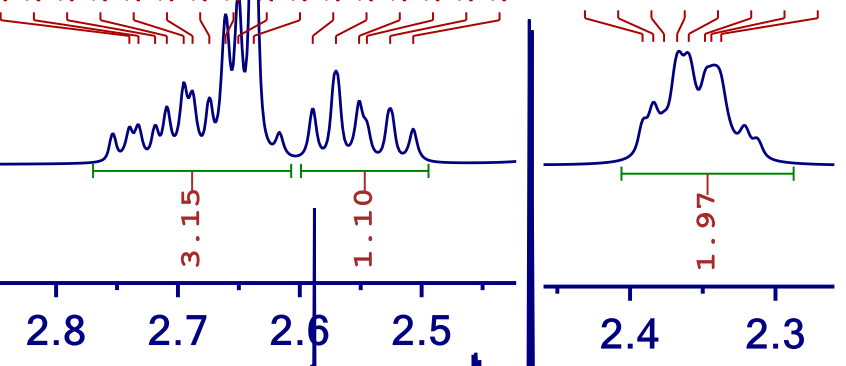

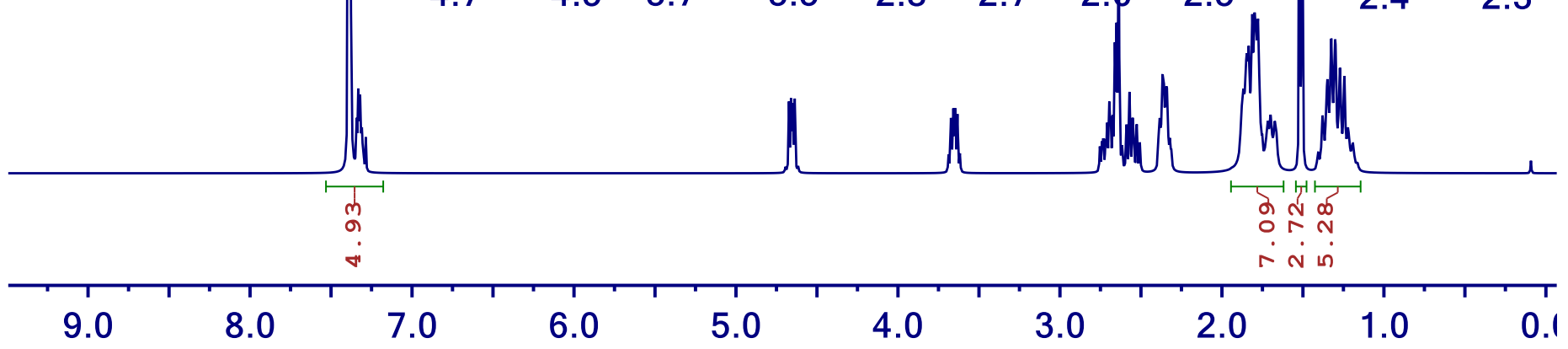<smiles>CC1OC(c2ccccc2)CC(=O)[C@H]1CCC(=O)C1CCCCC1</smiles>

$13 \mathrm{C}\{1 \mathrm{H}\} \mathrm{NMR}$ (100 MHz, CDCh)
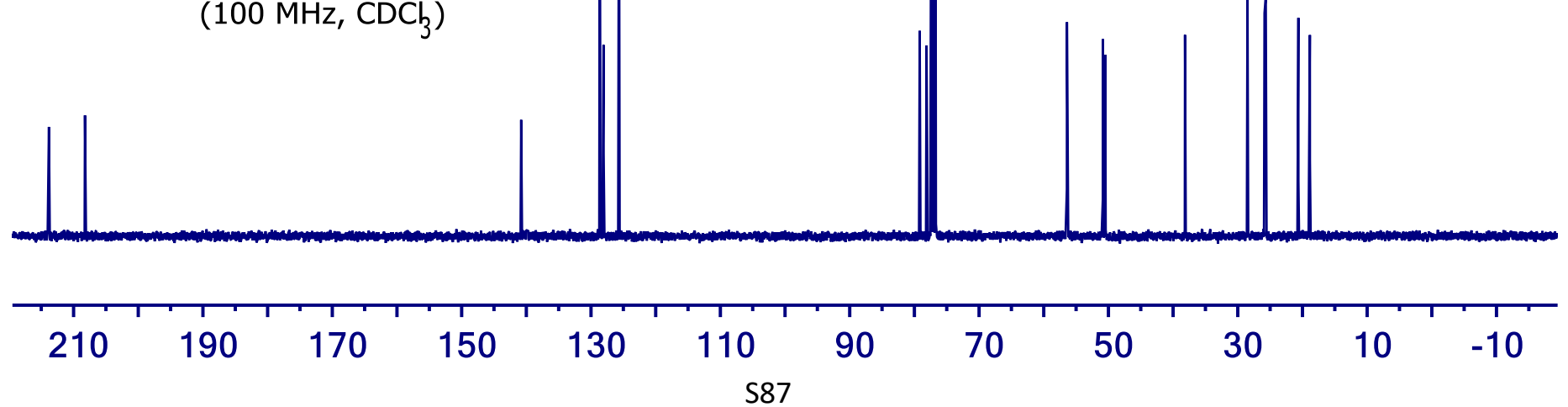
$(2 R, 3 R, 6 R)-2-m e t h y l-3-((E)-3-o x o-5-p h e n y l p e n t-4-e n-1-y l)-6-p h e n y l t e t r a h y d r o-4 H-p y r a n-4-o n e$

$5 \mathrm{~g}$

H

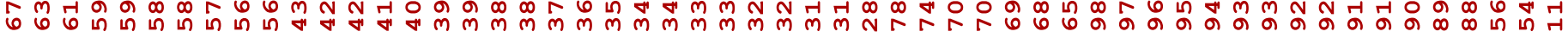

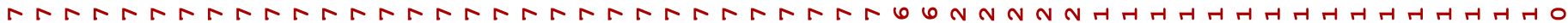
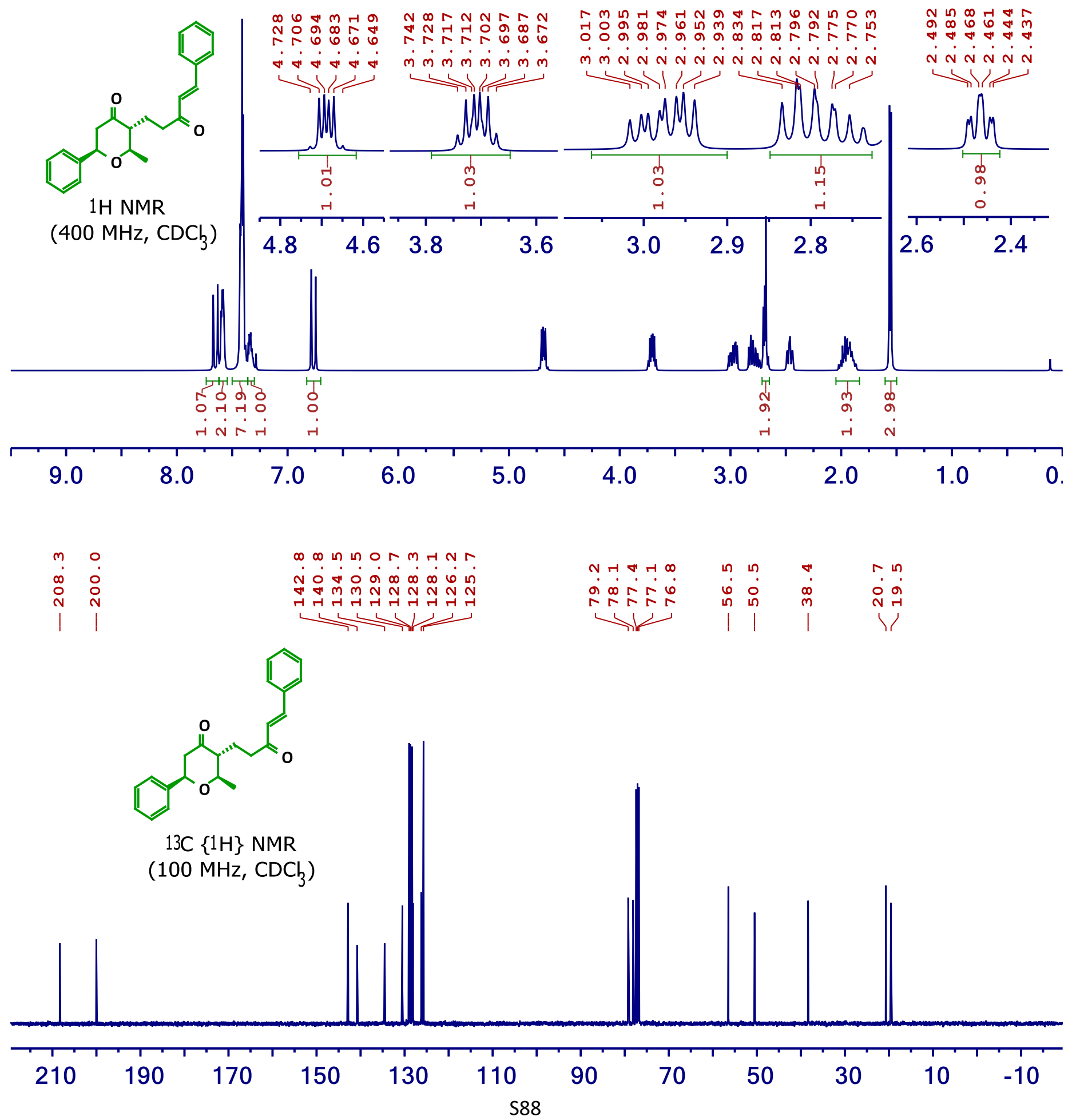
(2R,3R,6R)-3-(3-(2,6-dichlorophenyl)-3-oxopropyl)-2-methyl-6-phenyltetrahydro-4H-pyran-4-one

$5 \mathrm{~h}$

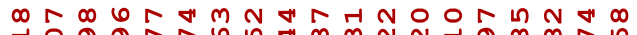
$\forall M M m m M M m M M m M N N N N N$

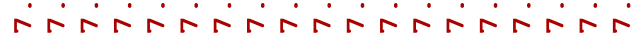

1)

$\therefore 606060$

வா

$m$ 은

$\rightarrow$

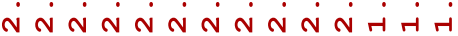
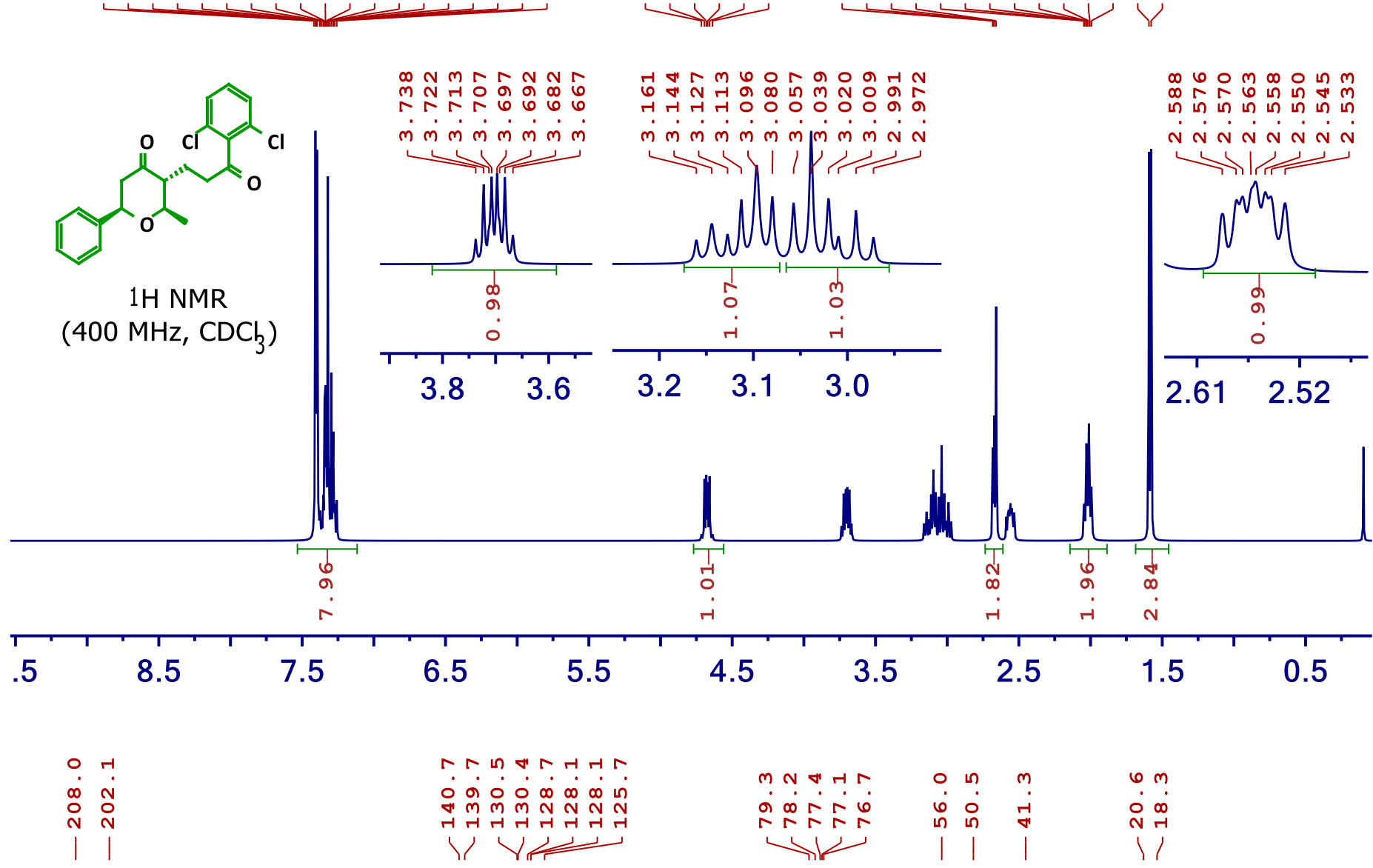<smiles>CC1OC(c2ccccc2)CC(=O)C1CC(=O)c1c(Cl)cccc1Cl</smiles>

$13 \mathrm{C}\{1 \mathrm{H}\} \mathrm{NMR}$

(100 $\mathrm{MHz}, \mathrm{CDC}_{3}$ )

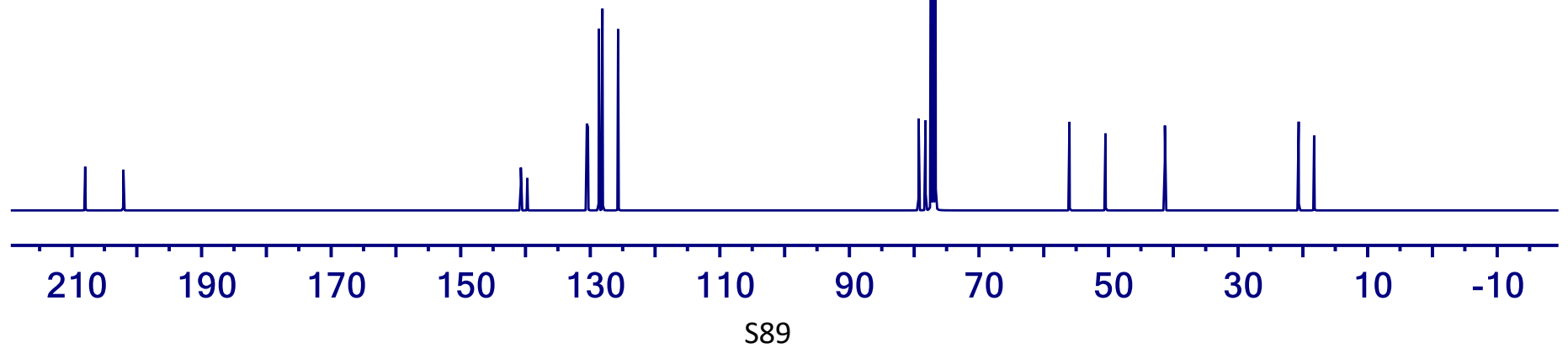


$(2 R, 3 R, 6 R)-2-m e t h y l-3-(3-(n a p h t h a l e n-1-y l)-3-o x o p r o p y l)-6-p h e n y l t e t r a h y d r o-4 H-p y r a n-4-o n e$

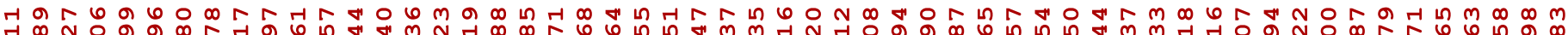
붕

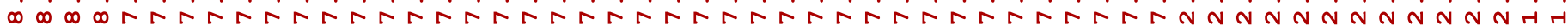

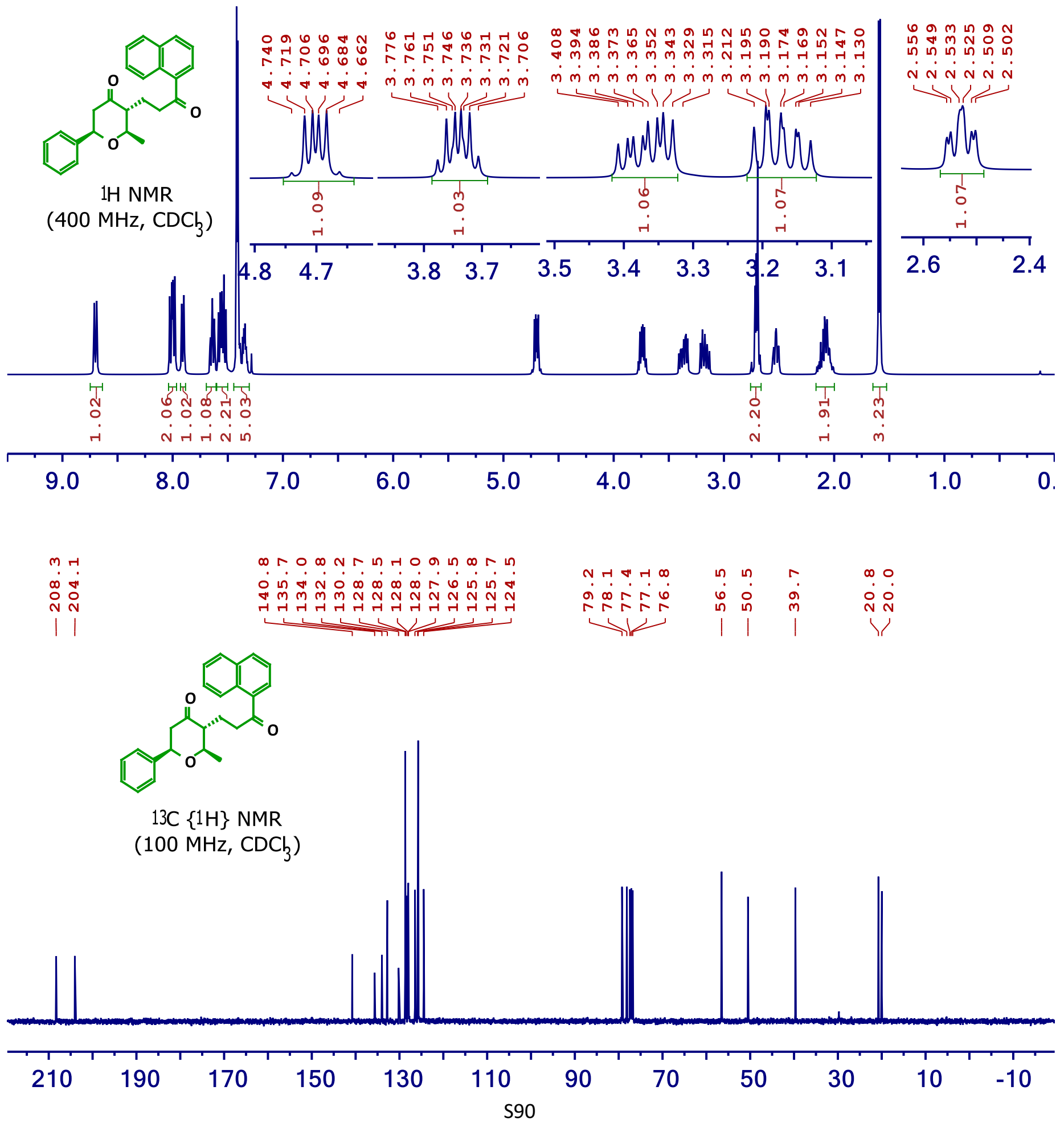


(2R,3R,6R)-2-ethyl-3-(3-oxo-5-phenylpentyl)-6-phenyltetrahydro-4H-pyran-4-one

$5 \mathbf{j}$

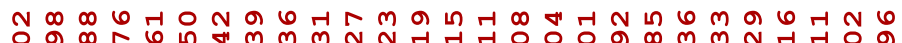
$\rightarrow m m m m m m m m m m m m m m m \stackrel{N}{N} \stackrel{N}{N} \stackrel{N}{N} \stackrel{N}{N}$

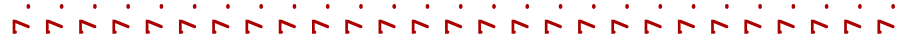
a인 ब

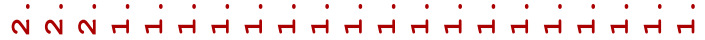

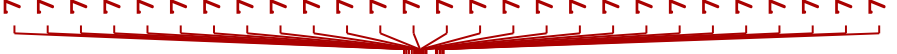

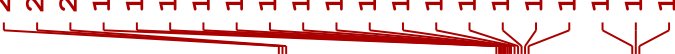<smiles>CCC1OC(c2ccccc2)CC(=O)C1CCC(=O)CCc1ccccc1</smiles>

$1 \mathrm{H} \mathrm{NMR}$ (400 $\mathrm{MHz}, \mathrm{CDC}_{3}$ )

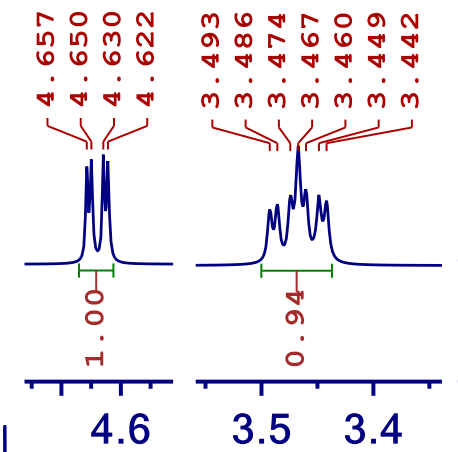

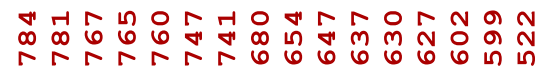

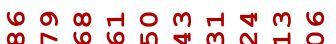

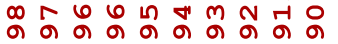
กัก 444 44 44 44

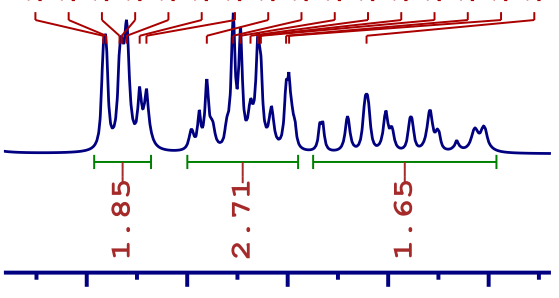

मो।/1/1
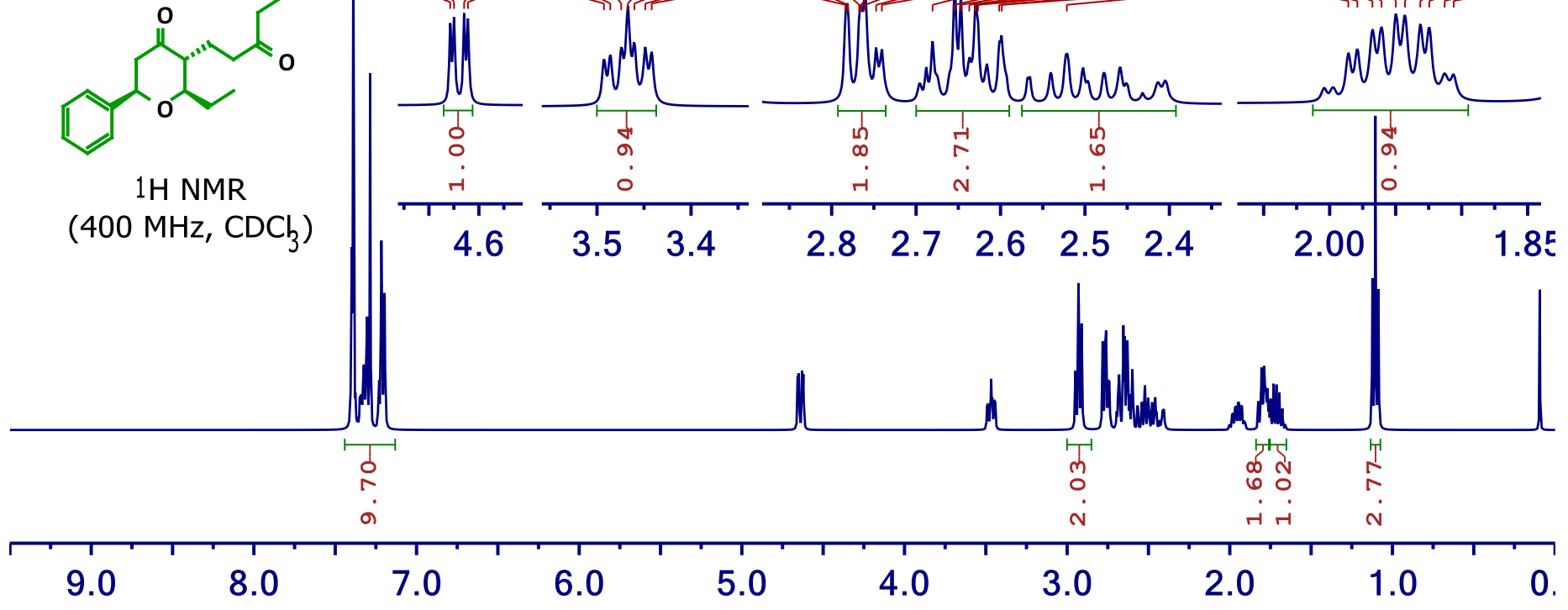

$\begin{array}{ll}\infty & 1 \\ 0 & \infty \\ 0 & \infty \\ \text { N } & \text { N } \\ \backslash & \end{array}$

4๐

त

HT

$Y$ W

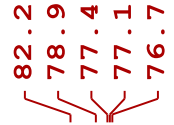

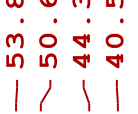

a 6 in

$\ddot{0} \dot{0}$

a 4 a<smiles>CCC1OC(c2ccccc2)CC(=O)C1CCC(=O)CCc1ccccc1</smiles>

13C $\{1 \mathrm{H}\}$ NMR (100 MHz, CDCb)

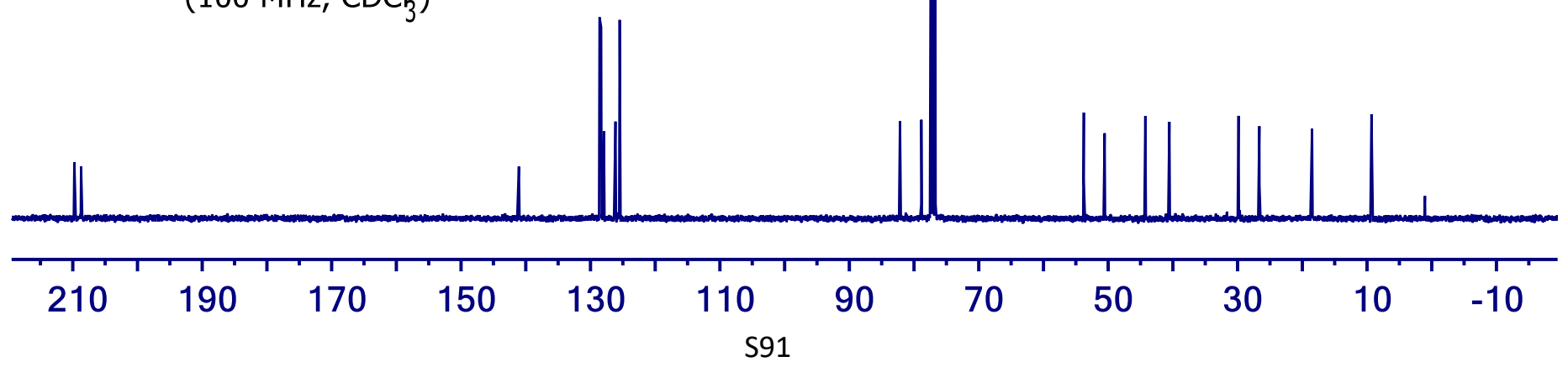


$(2 R, 3 R, 6 R)-2-$ benzyl-3-(3-oxo-5-phenylpentyl)-6-phenyltetrahydro-4H-pyran-4-one

$5 \mathbf{k}$

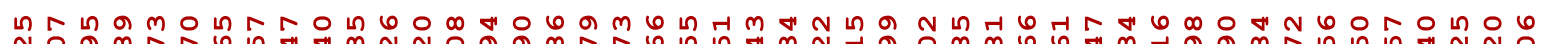
J

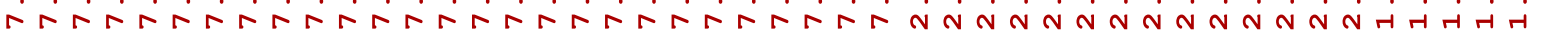
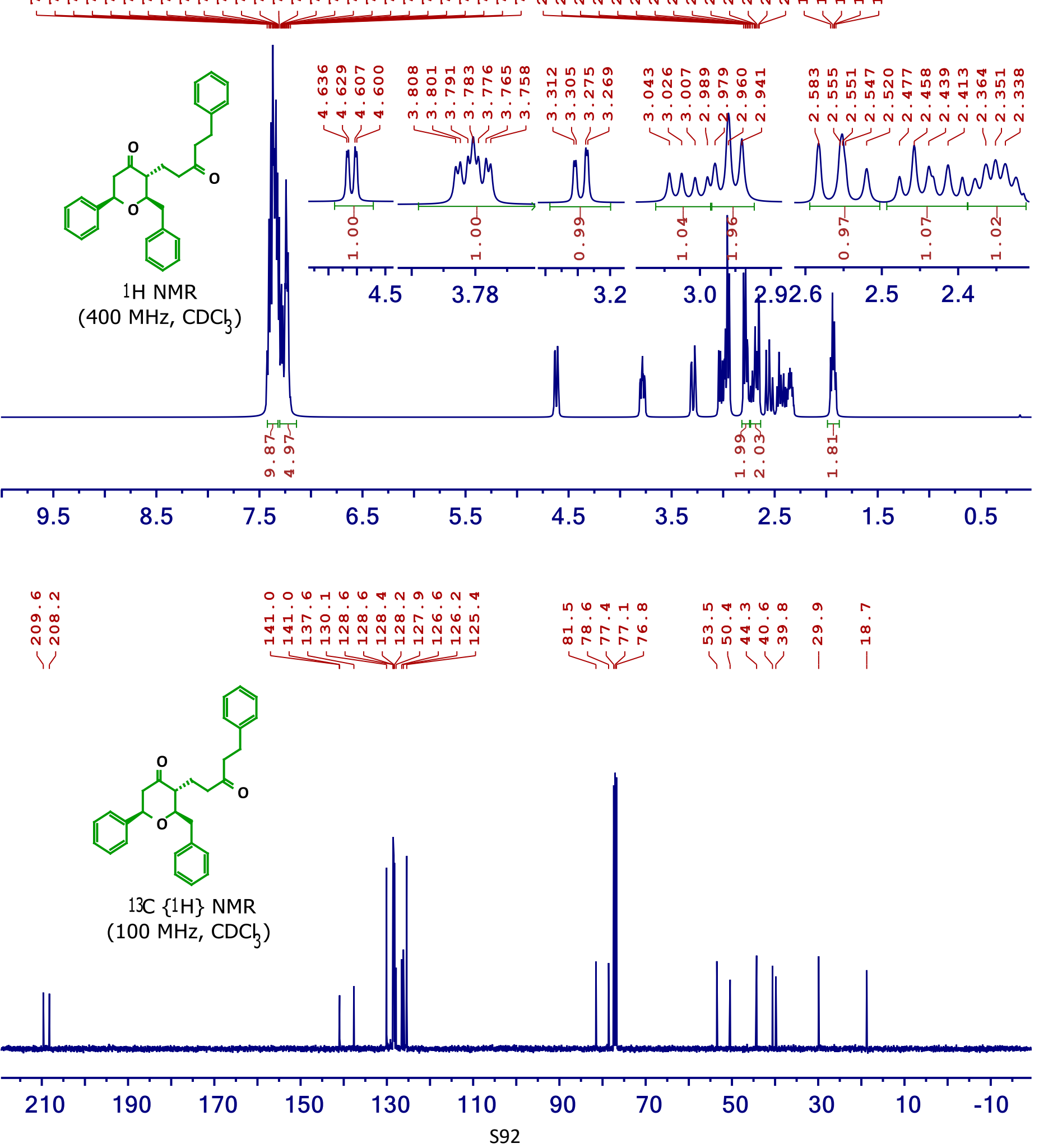
$(2 R, 3 R, 6 R)-2-b u t y l-3-(3-o x o-5-p h e n y l p e n t y l)-6-p h e n y l t e t r a h y d r o-4 H-p y r a n-4-o n e$

51

Noิ

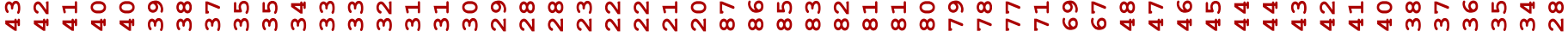

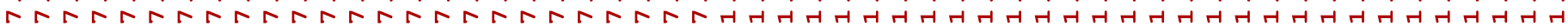

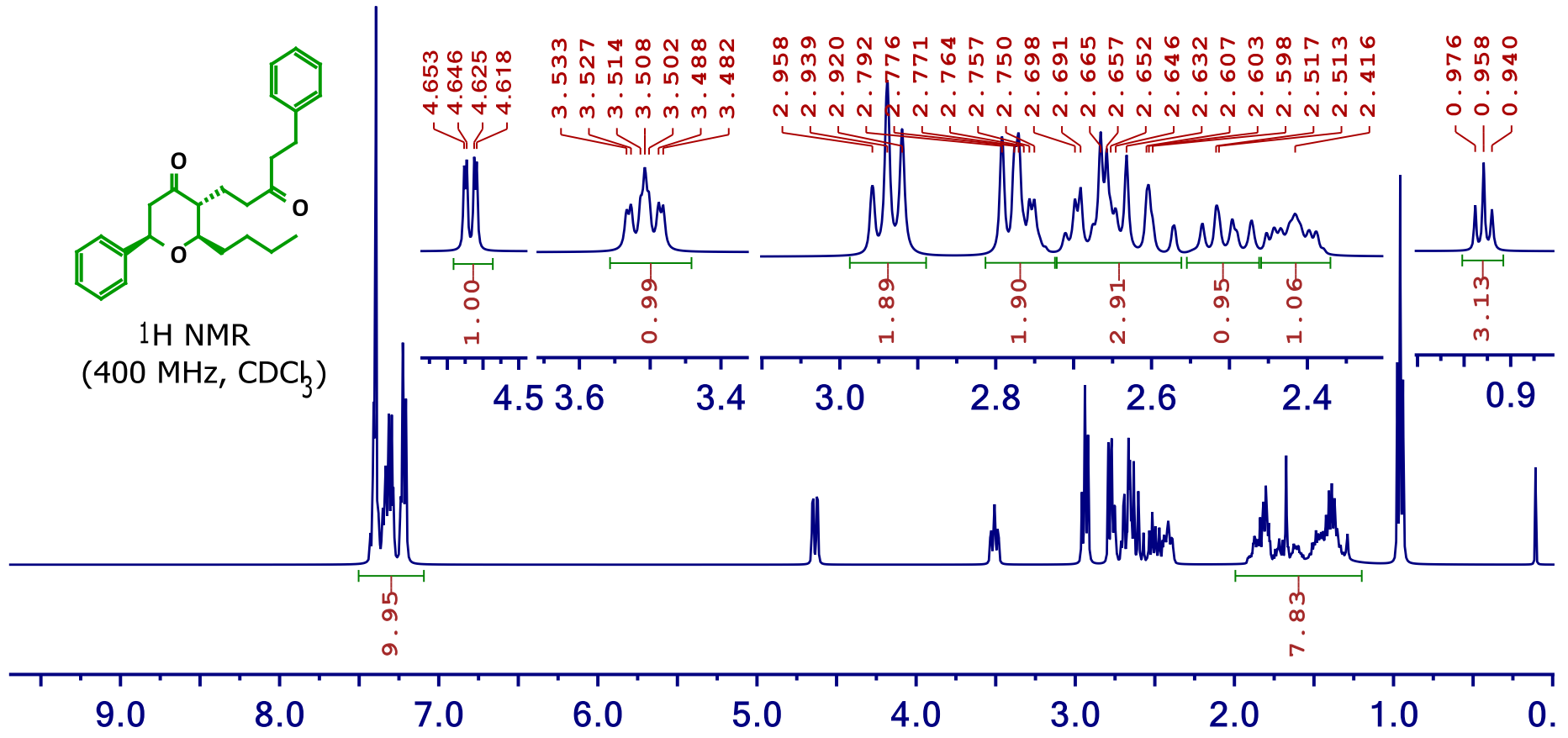

$\begin{array}{ll}\infty & 1 \\ 0 & \infty \\ 0 & 0 \\ N & 0 \\ 1\end{array}$

$40,4 \ln 0$ in

$\dot{4} \dot{0} \dot{\infty} \dot{\infty} \dot{0} \dot{0}$ in

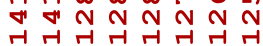

Y<smiles>CCCC[C@H]1OC(c2ccccc2)CC(=O)[C@@H]1CCC(=O)CCc1ccccc1</smiles>

$13 \mathrm{C}\{1 \mathrm{H}\} \mathrm{NMR}$

(100 MHz, CDCb)

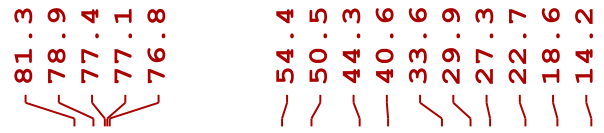

年年会

$1 / 1|>1| 1 \mid$

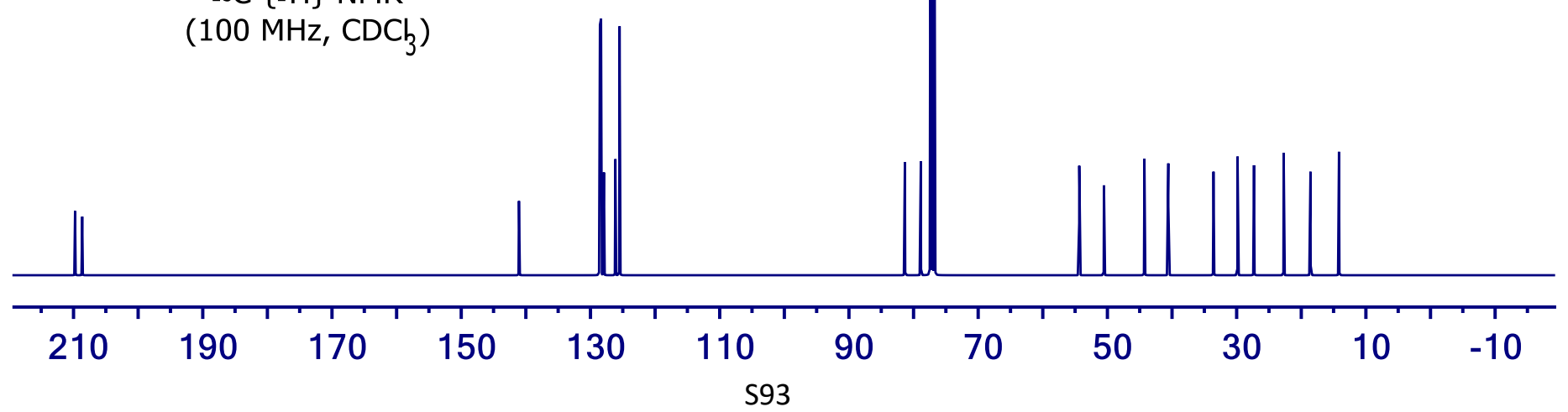


(2R,3R,6R)-2-isobutyl-3-(3-oxo-5-phenylpentyl)-6-phenyltetrahydro-4H-pyran-4-one

$5 \mathrm{~m}$

일

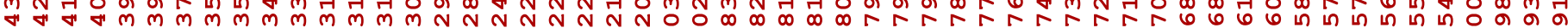

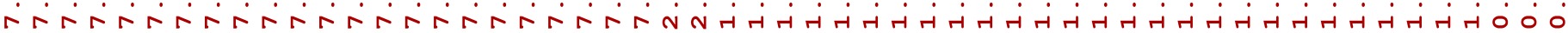<smiles>CC(C)C[C@H]1O[C@@H](c2ccccc2)CC(=O)[C@H]1CCC(=O)CCc1ccccc1</smiles>

$1 \mathrm{H} N M R$ (400 $\mathrm{MHz}_{1} \mathrm{CDC}_{3}$ )

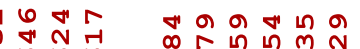

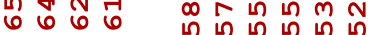

$\ddot{\nabla} \dot{\nabla} \dot{\nabla} \dot{m} \dot{m} \dot{m} \dot{m} \dot{m}$

त)

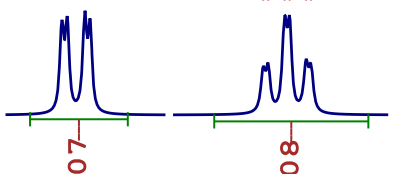

으은 ด้

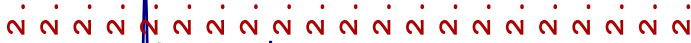
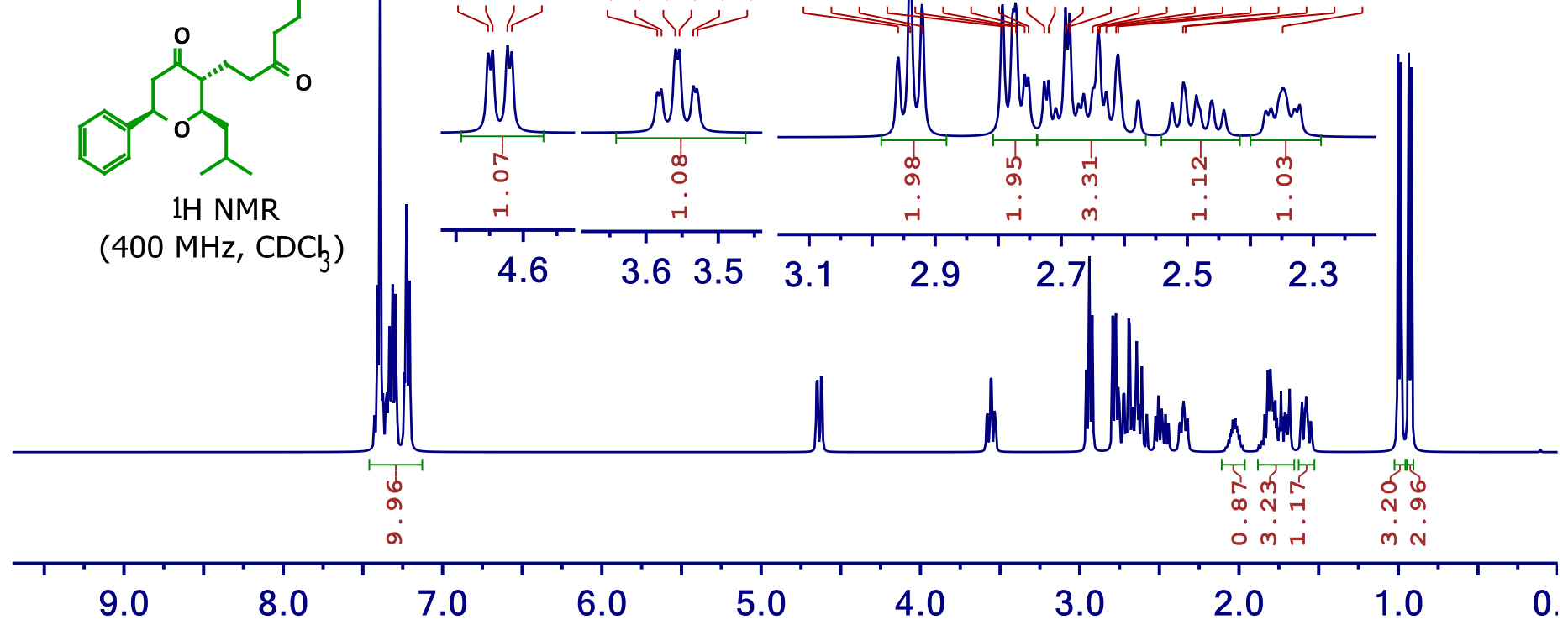

$\begin{array}{ll}N & 0 \\ 0 & \infty \\ 0 & 0 \\ \text { N } & \text { N } \\ 1\end{array}$

$000 \ln 2 \pi 4 \pi$

- $\dot{0} \dot{\infty} \dot{0} \dot{0}$

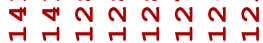

$Y$
0
0
0
0

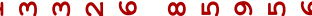

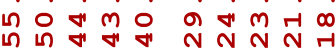

$1 \mid \backslash 1,111$<smiles>CC(C)C[C@H]1OC(c2ccccc2)CC(=O)[C@H]1CCC(=O)CCc1ccccc1</smiles>

$13 \mathrm{C}\{1 \mathrm{H}\} \mathrm{NMR}$ (100 MHz, CDCb $)$
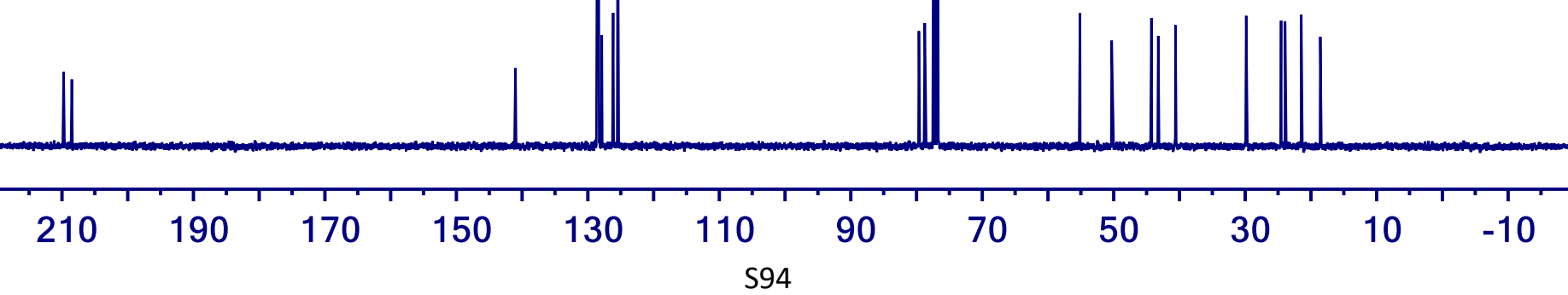
(2R,3R,6R)-2-cyclopropyl-3-(3-oxo-5-phenylpentyl)-6-phenyltetrahydro-4H-pyran-4-one

$5 q$

トm

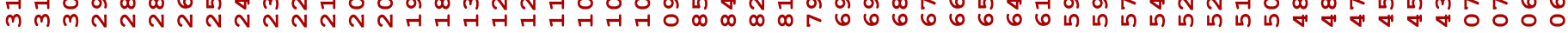

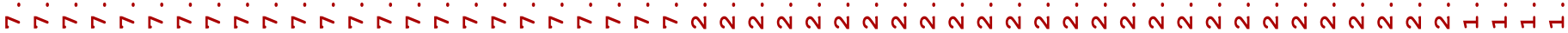<smiles></smiles>

$1 \mathrm{H} N M R$ (400 $\left.\mathrm{MHz}, \mathrm{CDC}_{3}\right)$

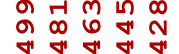

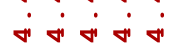

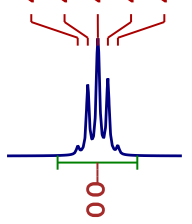

H

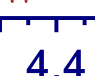

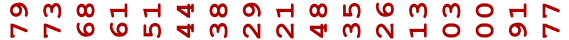

ด̆ด̆ด̆ด̆ด̆ ด̆

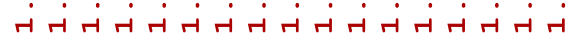
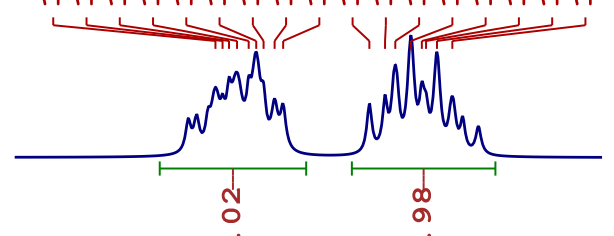

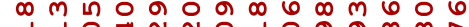

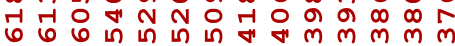
00000000000000
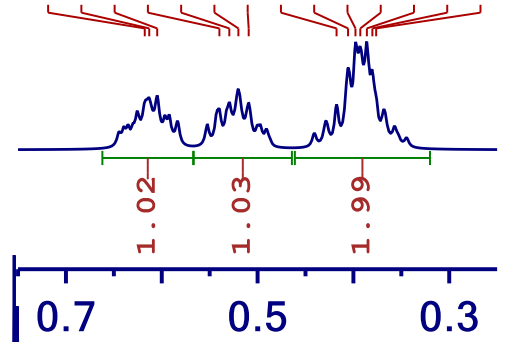

d

a)

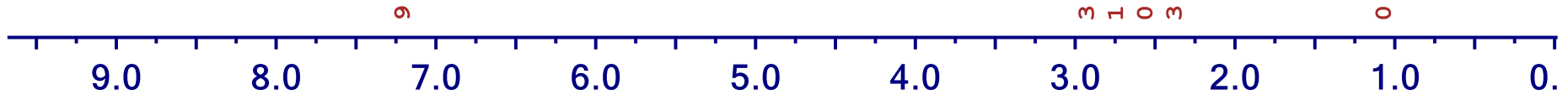

$\begin{array}{ll}\infty & m \\ 0 & \infty \\ 0 & \infty \\ \text { N } & \stackrel{1}{N} \\ 1 & \end{array}$

40,40004040

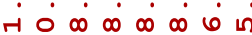
Hี

$\mathrm{Y}$

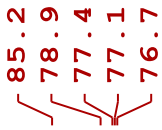

TIm $\mathrm{ma}$ ํํำ 1111

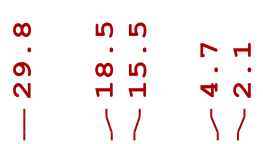<smiles>O=C(CCC1CC1)CC[C@H]1C(=O)CC(c2ccccc2)OC1C1CC1</smiles>

$13 \mathrm{C}\{1 \mathrm{H}\} \mathrm{NMR}$ (100 MHz, CDCl $)$
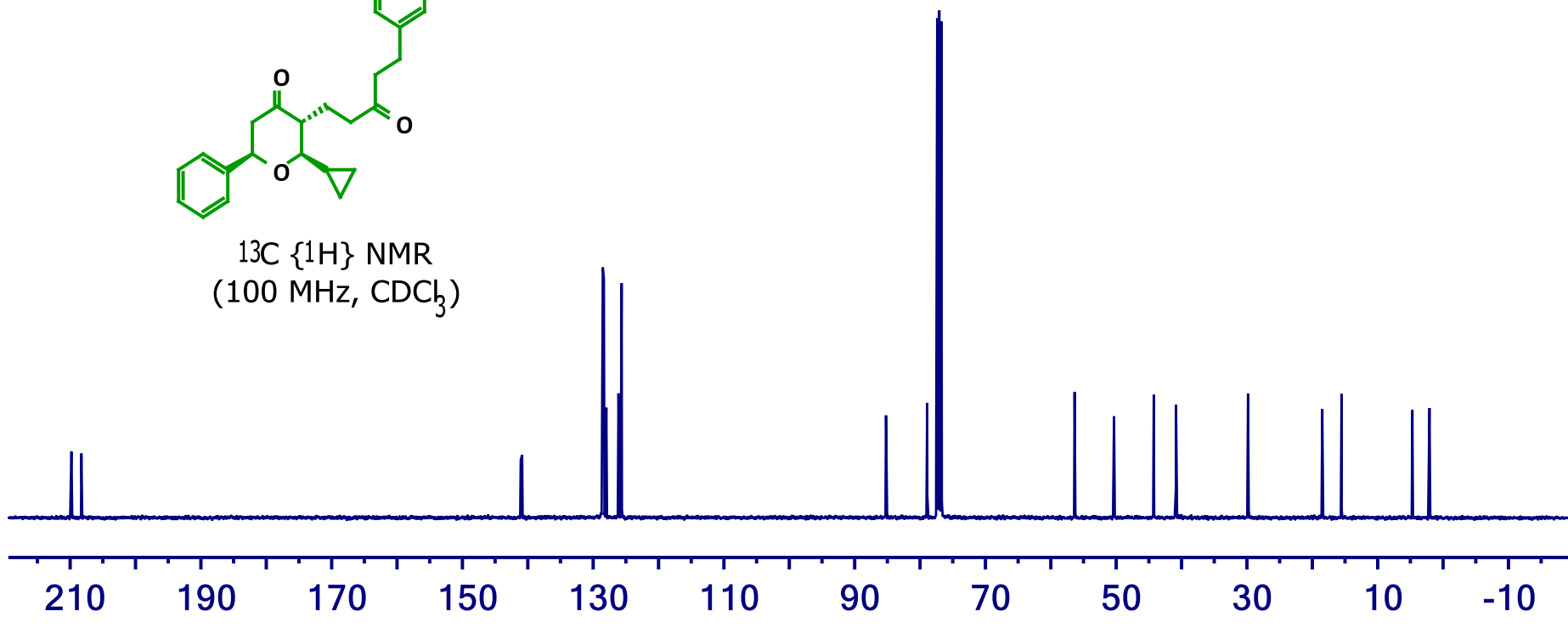


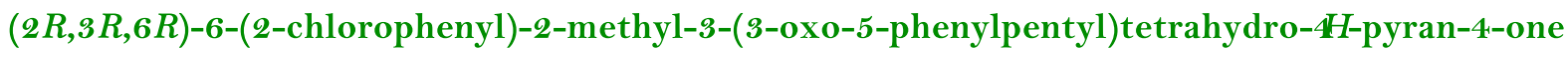

$5 \mathrm{r}$

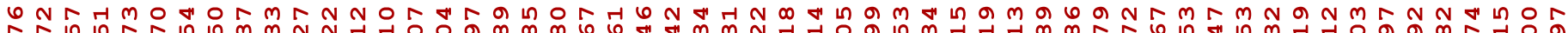

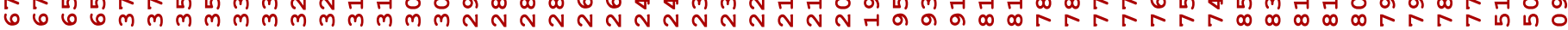

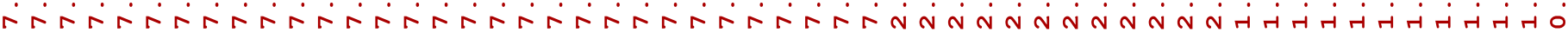

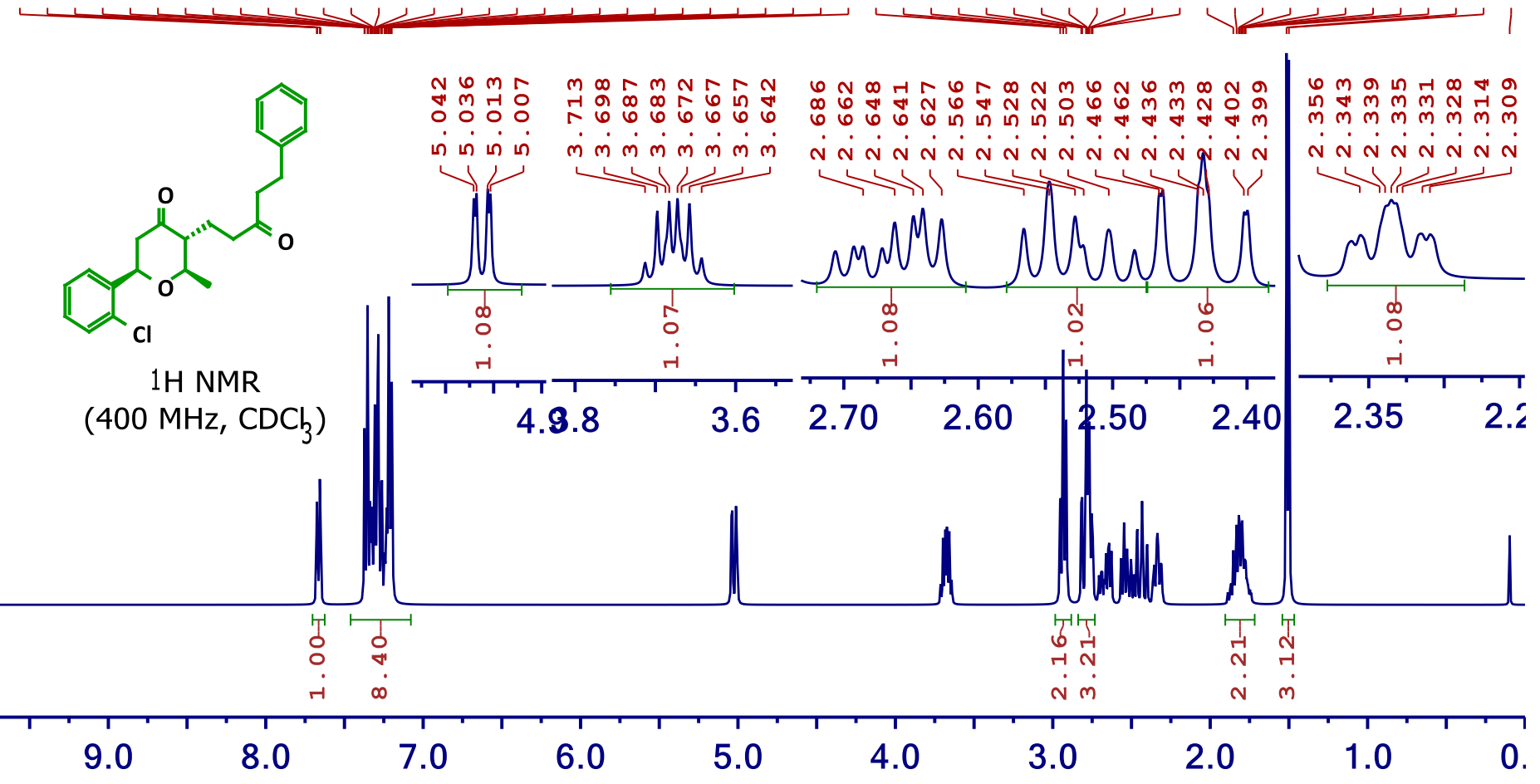

$r o$
0
in
No
11
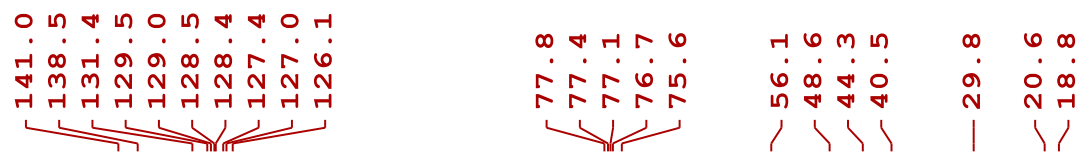<smiles>C[C@H]1OC(c2ccccc2Cl)CC(=O)[C@@H]1CCC(=O)CCc1ccccc1</smiles>

13C $\{1 \mathrm{H}\}$ NMR $\left(100 \mathrm{MHz}, \mathrm{CDCh}_{3}\right)$

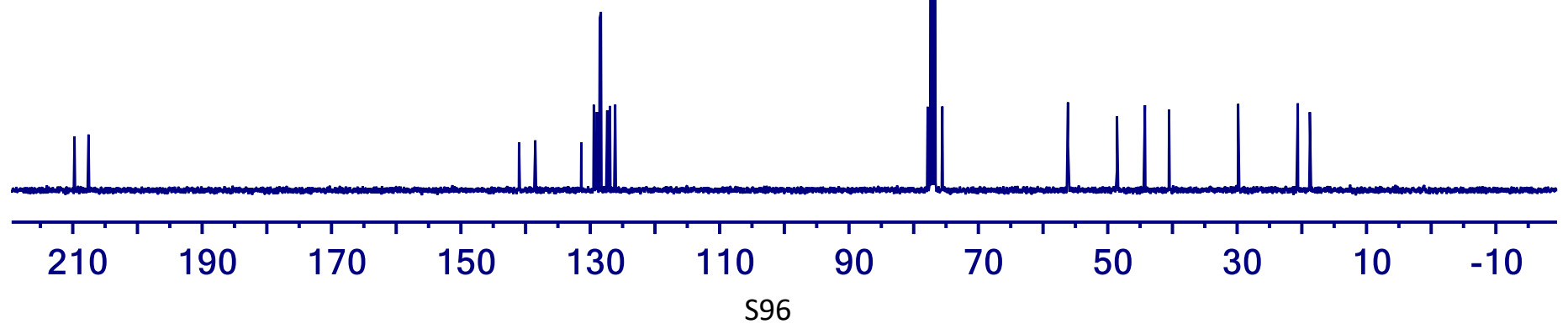


$(2 R, 3 R, 6 R)-2-m e t h y l-6-(n a p h t h a l e n-1-y l)-3-(3-0 x o-4-p h e n y l b u t y l) t e t r a h y d r o-4 H-p y r a n-4-o n e$

$5 \mathbf{s}$

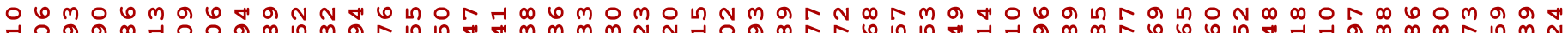

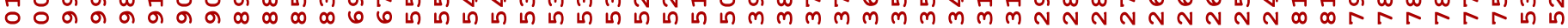

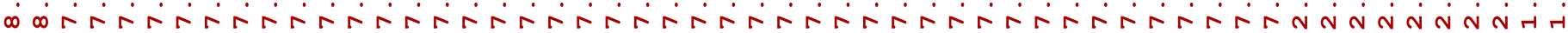
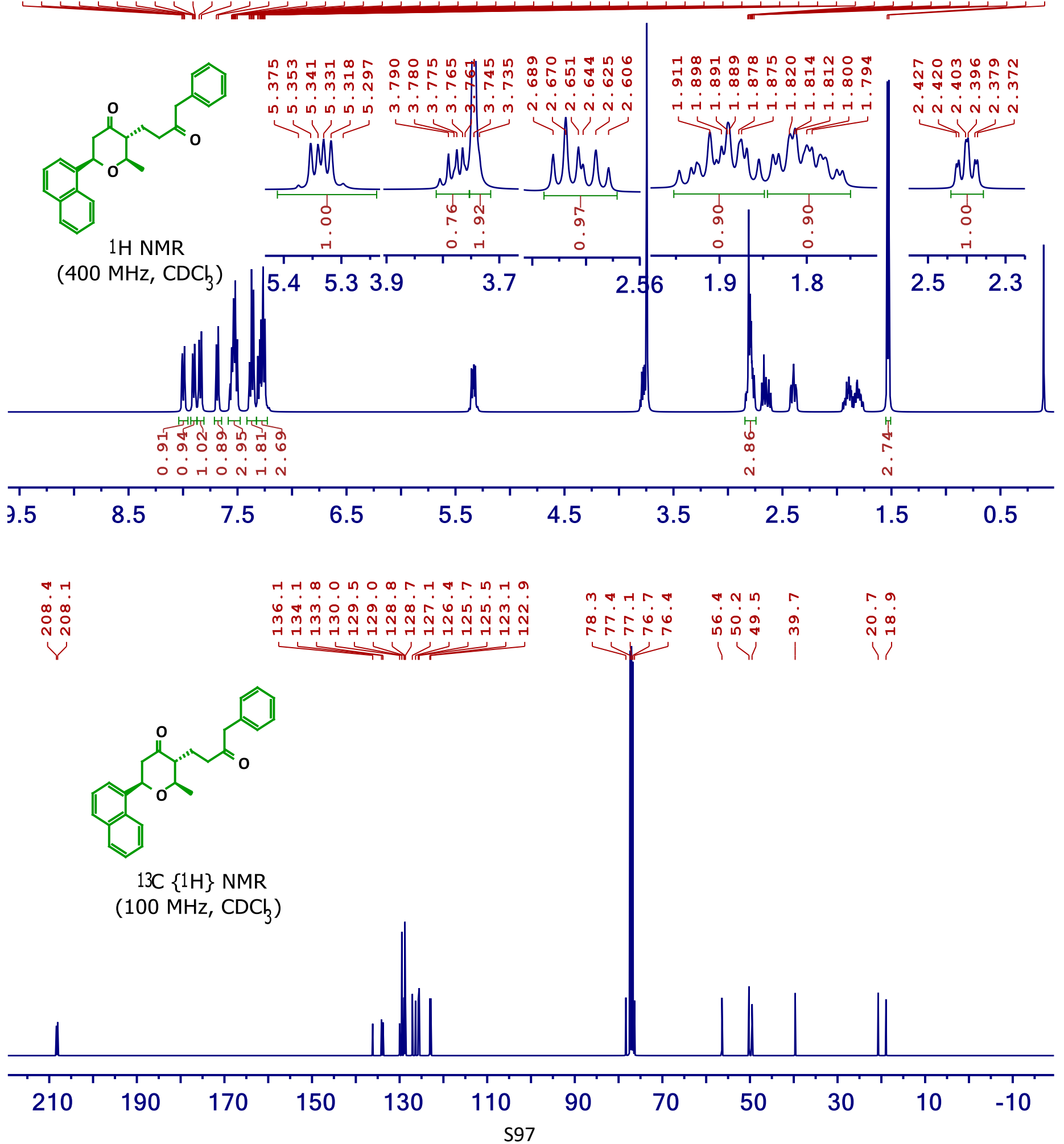
(2R,3R,6R)-2-methyl-3-(3-oxo-4-phenylbutyl)-6-(o-tolyl)tetrahydro-4H-pyran-4-one

$5 \mathrm{t}$

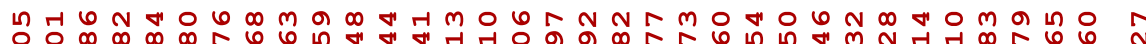

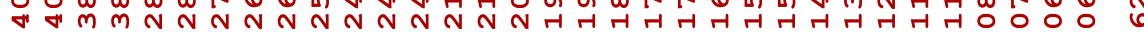

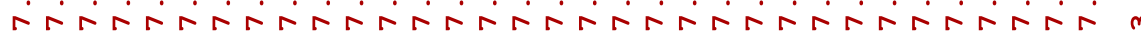

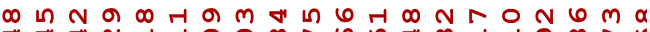

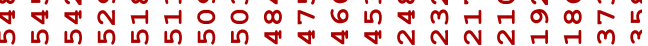

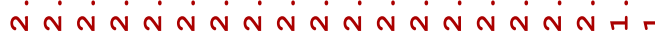

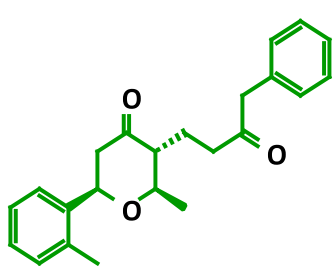

$1 \mathrm{H} N M R$ $\left(400 \mathrm{MHz}, \mathrm{CDCl}_{3}\right)$

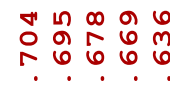

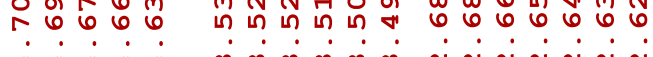

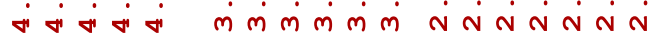
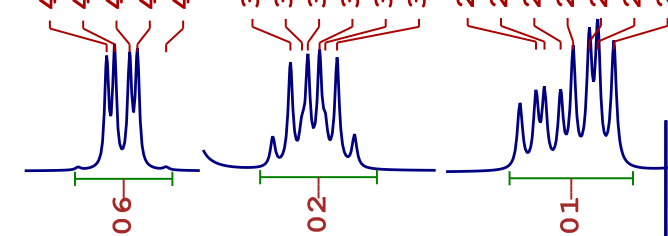

4366

(1)

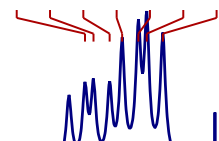

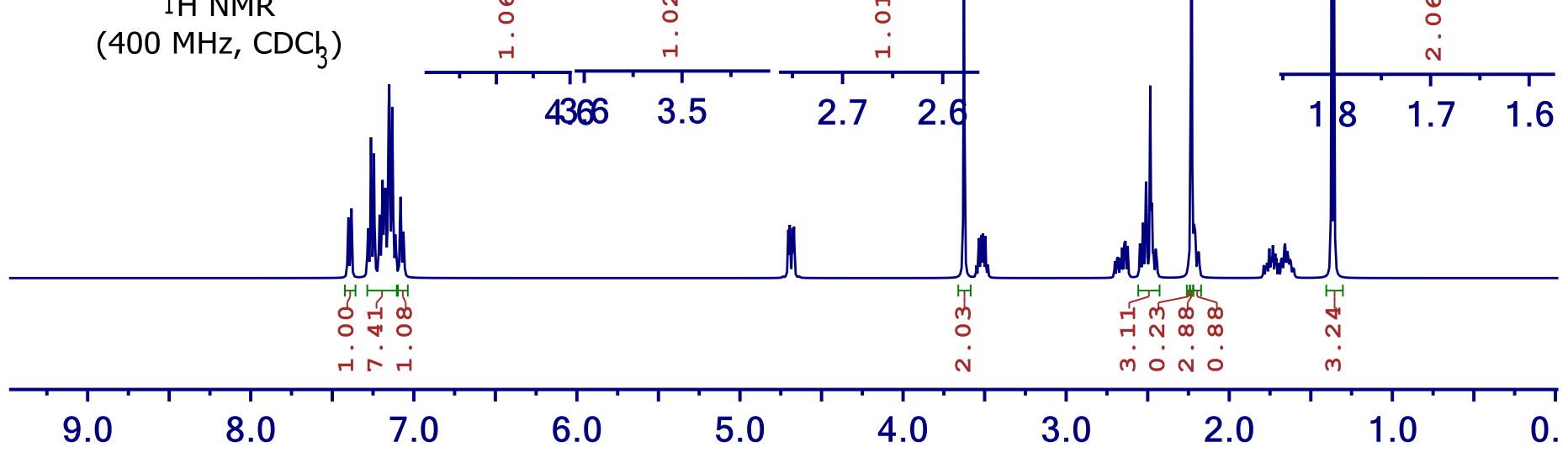
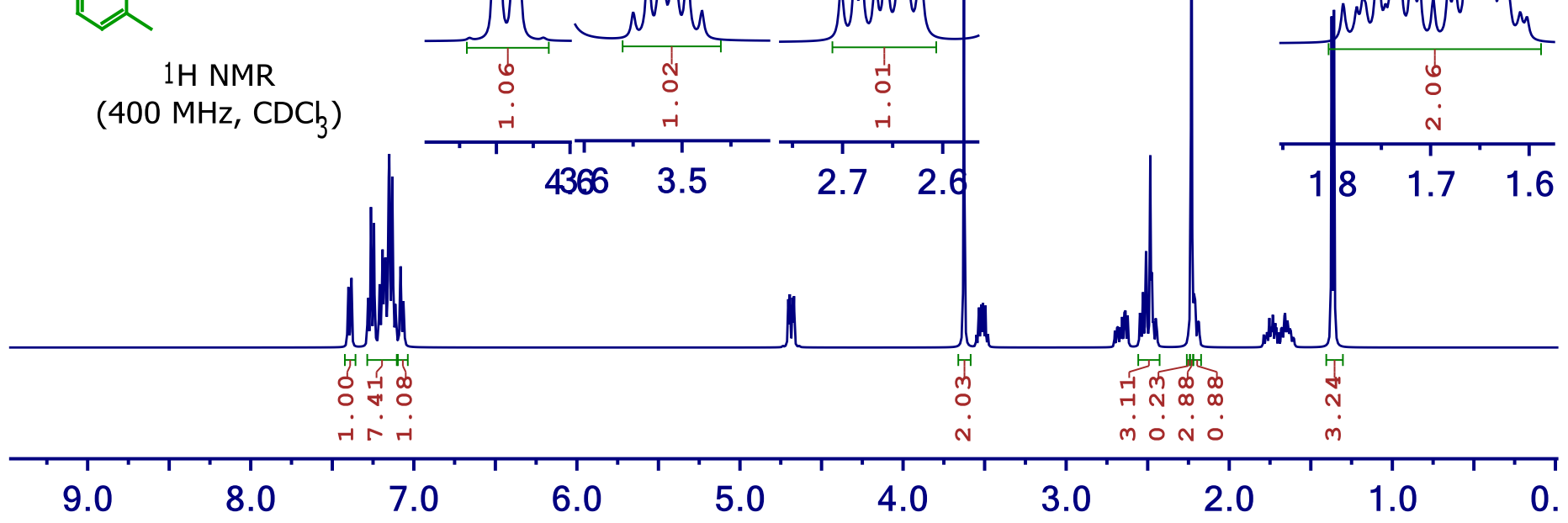

$\forall 0$

6 6

$\begin{array}{ll}\infty & \infty \\ \circ & \circ \\ N & N\end{array}$

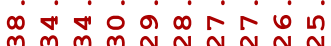

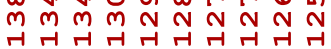

$\checkmark$

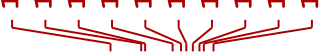

N 4 4

m N $~ r$

609

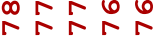

$\begin{array}{llll}6 & \circ & \circ & 9 \\ \text { เก } & \text { เ } & m\end{array}$

앗<smiles>Cc1ccccc1C1CC(=O)C(CCC(=O)Cc2ccccc2)[C@@H](C)O1</smiles>

13C $\{1 \mathrm{H}\}$ NMR (100 MHz, $\mathrm{CDCl}_{3}$ )
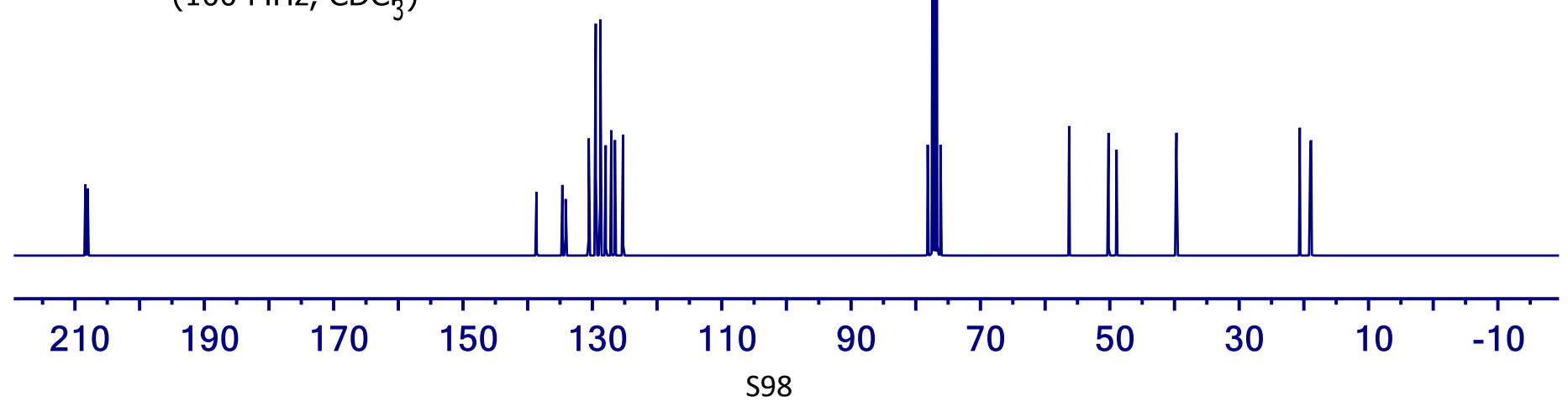
$(2 R, 3 R, 6 R)-6$-cyclohexyl-2-methyl-3-(3-oxo-5-phenylpentyl)tetrahydro-4H-pyran-4-one

$5 \mathrm{v}$

N $m$ m $m \stackrel{N}{N} \stackrel{\infty}{N} N$ N N

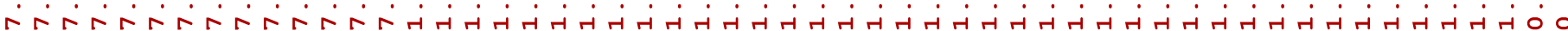<smiles>C[C@H]1OC(C2CCCCC2)CC(=O)[C@H]1CCC(=O)CCc1ccccc1</smiles>

$1 \mathrm{H} N M R$ (400 $\mathrm{MHz}, \mathrm{CDC}_{3}$ )
H

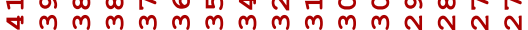
$\dot{m} \dot{m} \dot{m} \dot{m} \dot{m} \dot{m} \dot{m} \dot{m} \dot{m}$
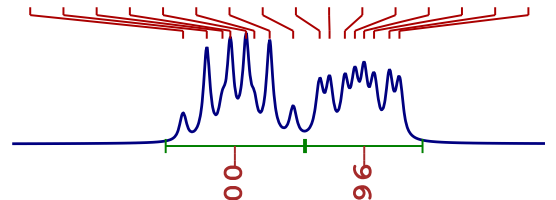

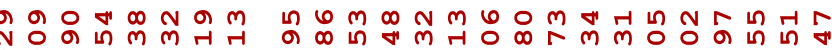

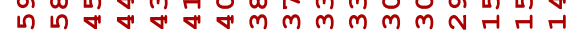

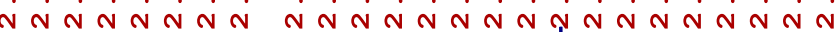
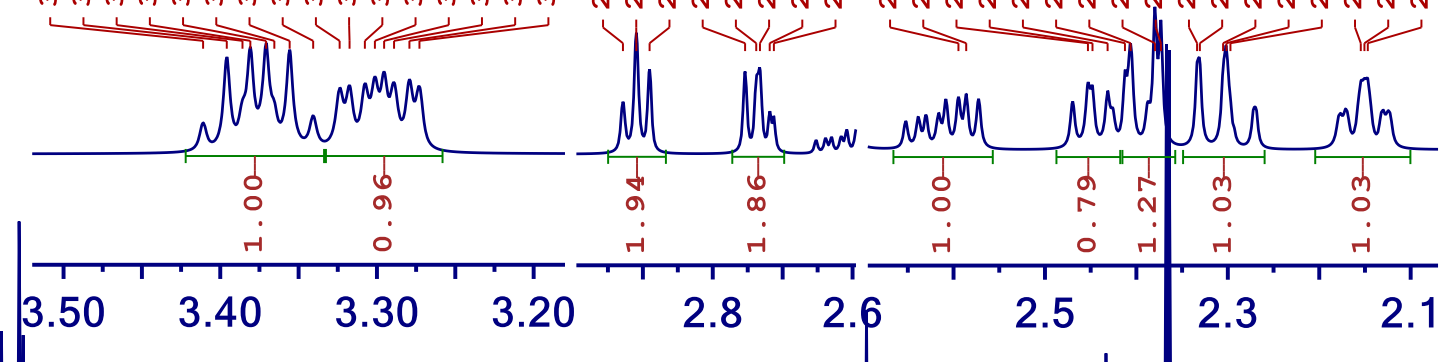
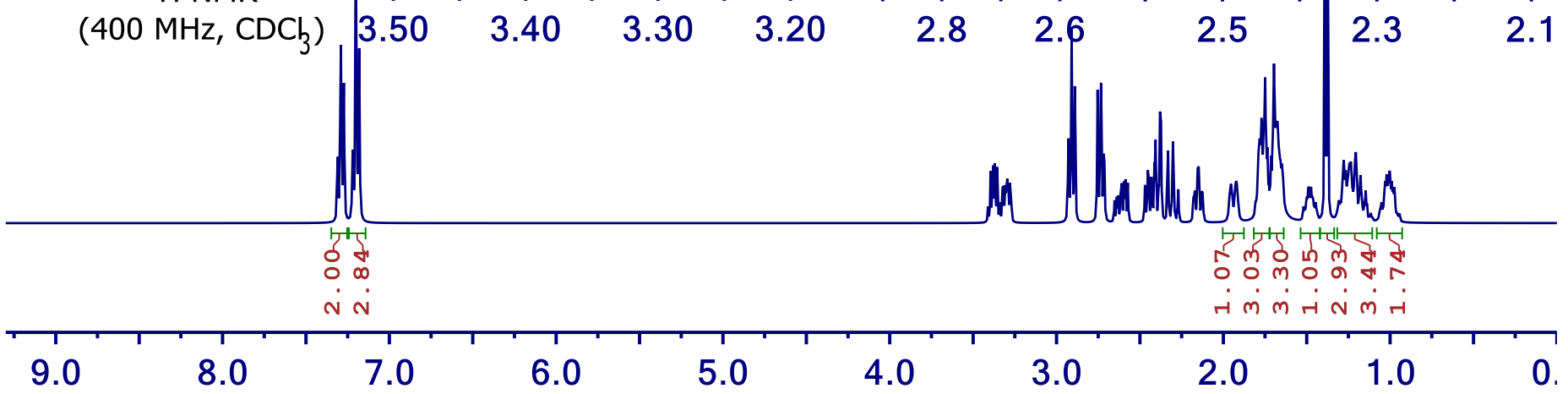

\section{r n \\ aे \\ ก $\stackrel{\text { N }}{\circ}$ \\ Y}

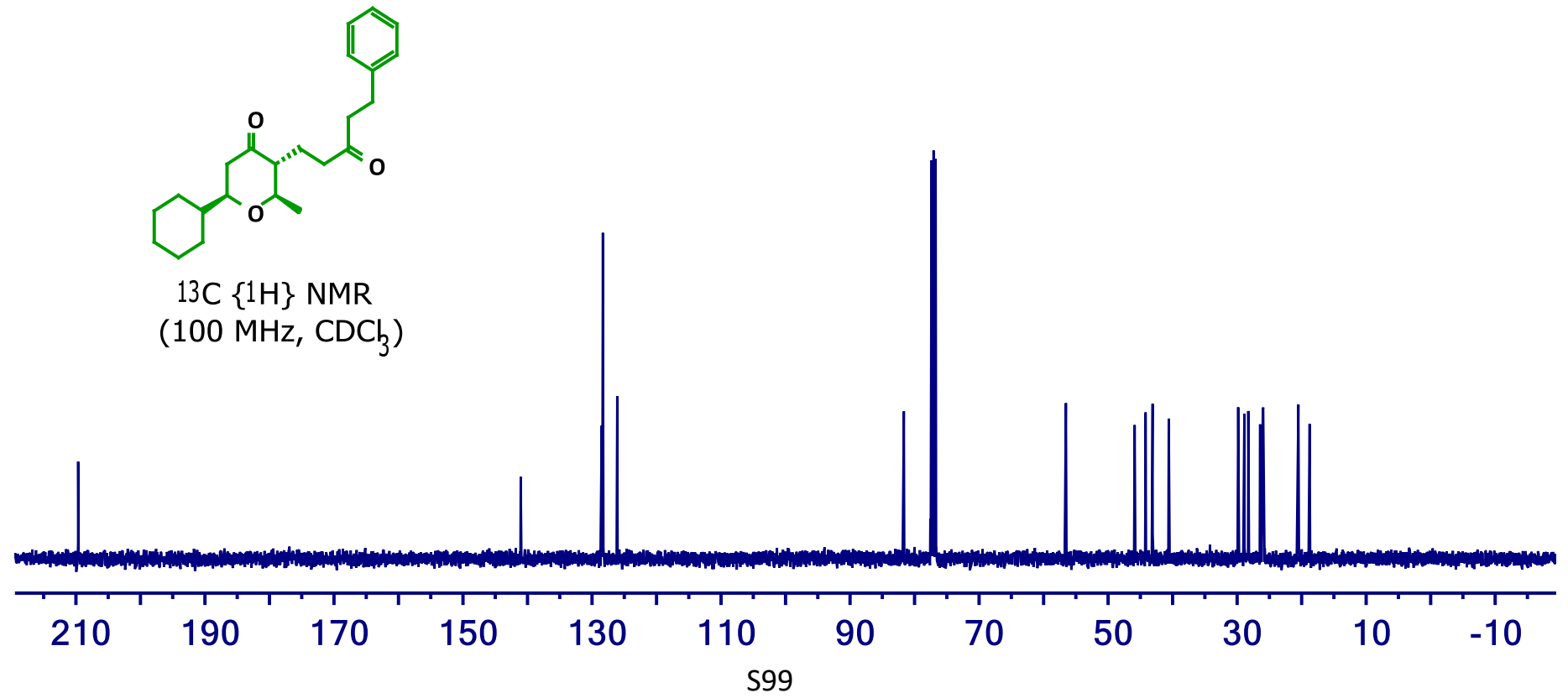

$\sin 4 \pi$

n $2 N+6 \infty a m \nabla 00$ in $\infty$ minis

Y ம்

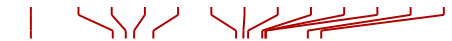


(2R,3 R,6S)-6-benzyl-2-methyl-3-(3-oxo-5-phenylpentyl)tetrahydro-4H-pyran-4-one

$5 \mathbf{w}$

일

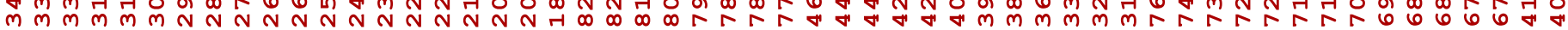

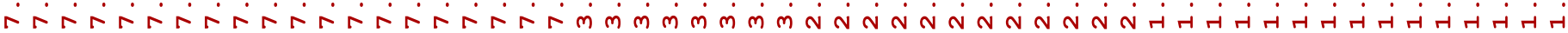
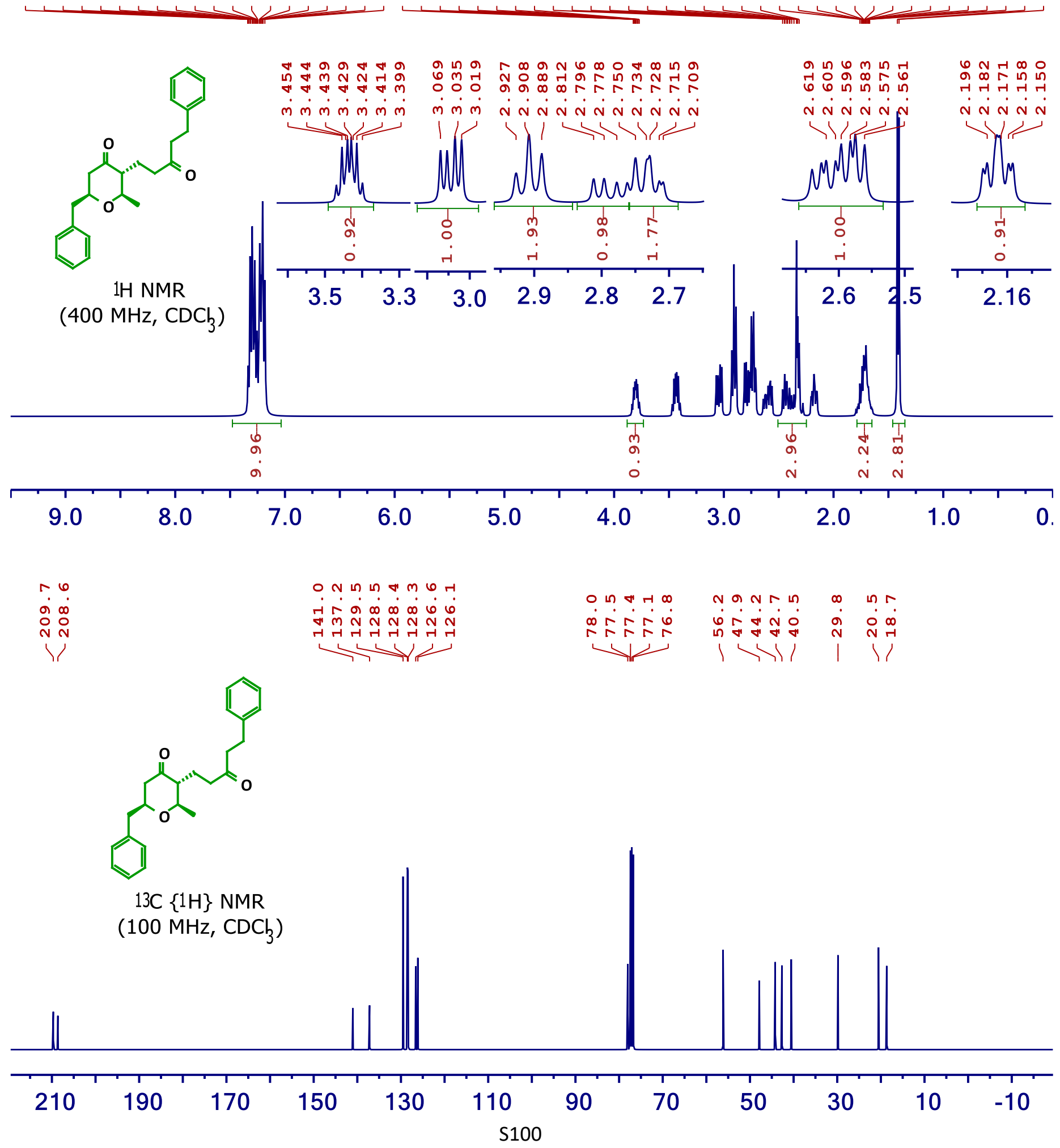
$(2 R, 3 R, 6 R)-6-(4-i s o p r o p y l p h e n y l)-2-m e t h y l-3-(3-o x o-5-p h e n y l p e n t y l) t e t r a h y d r o-4 H-p y r a n-4-o n e$

$5 \mathrm{x}$

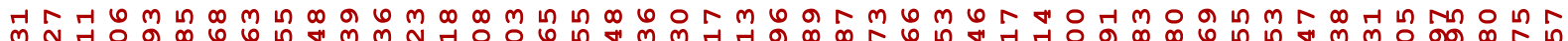
m $m$ m $m$ m

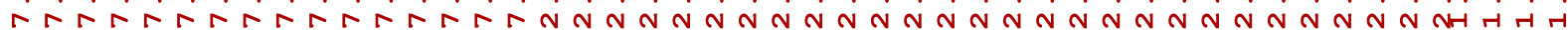

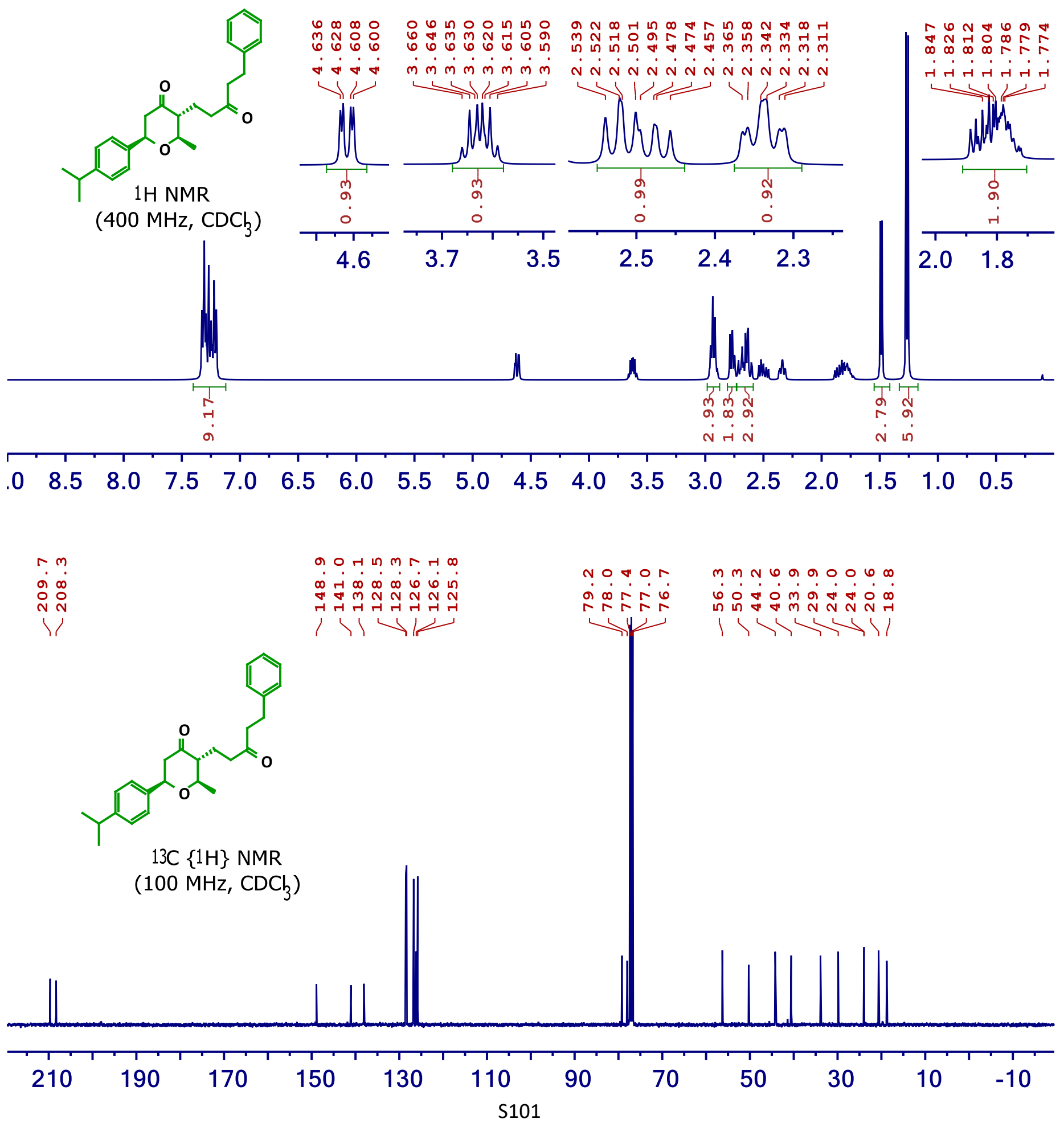


$(2 R, 5 R, 6 R)-2,6$-dimethyl-5-(3-oxo-5-phenylpentyl)-2-phenyltetrahydro-4H-pyran-4-one

$5 y 1$

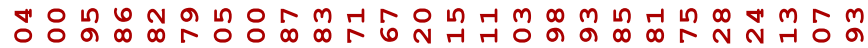

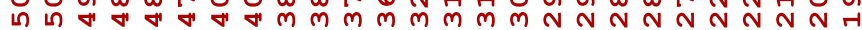

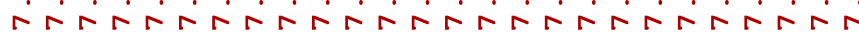

I ด้ ส้ํำ

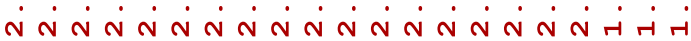

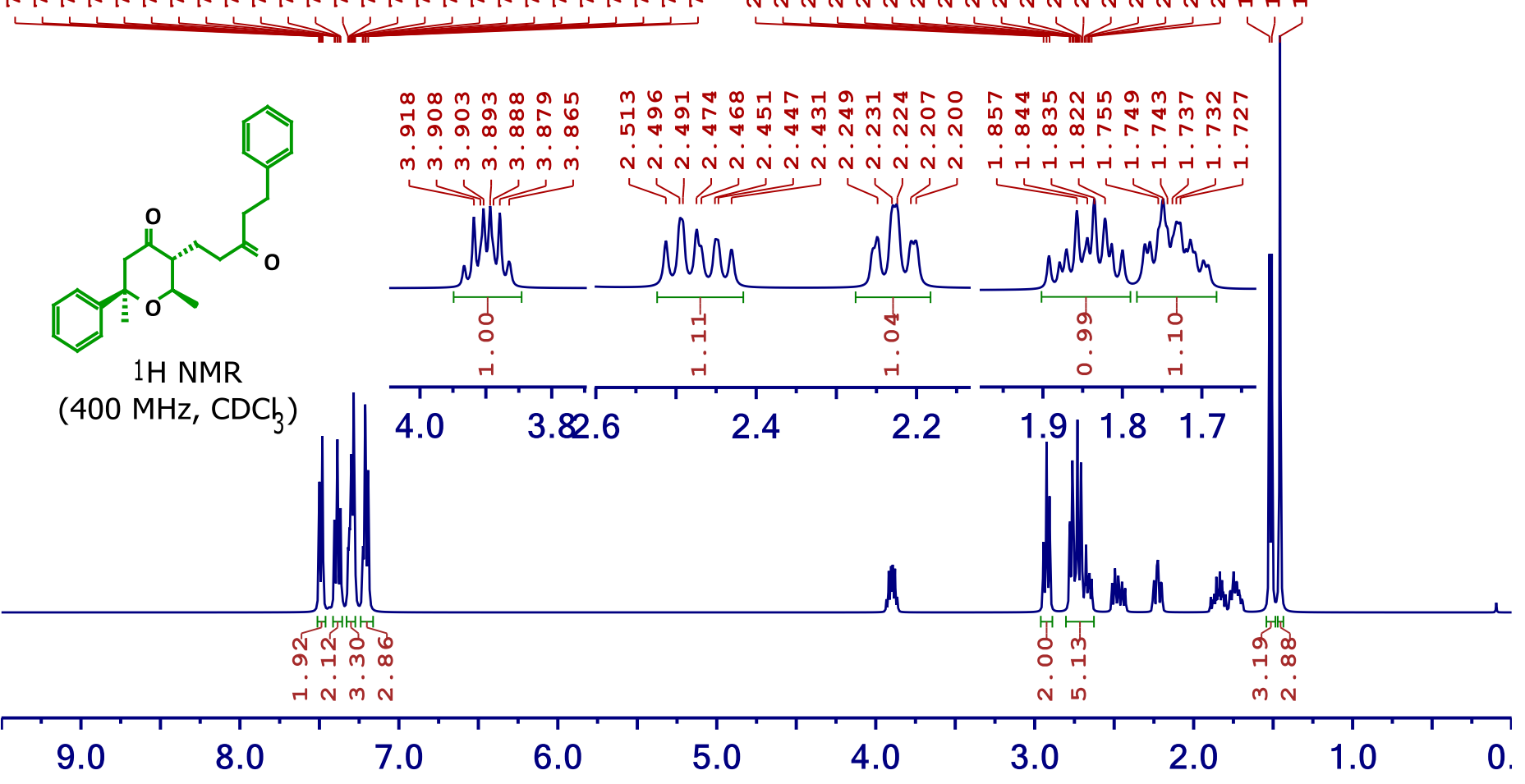

$\begin{array}{ll}N & \infty \\ 0 & \dot{0} \\ 0 & 0 \\ N & N \\ Y & \end{array}$

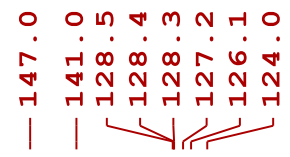

nathr ha mo nat

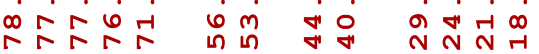

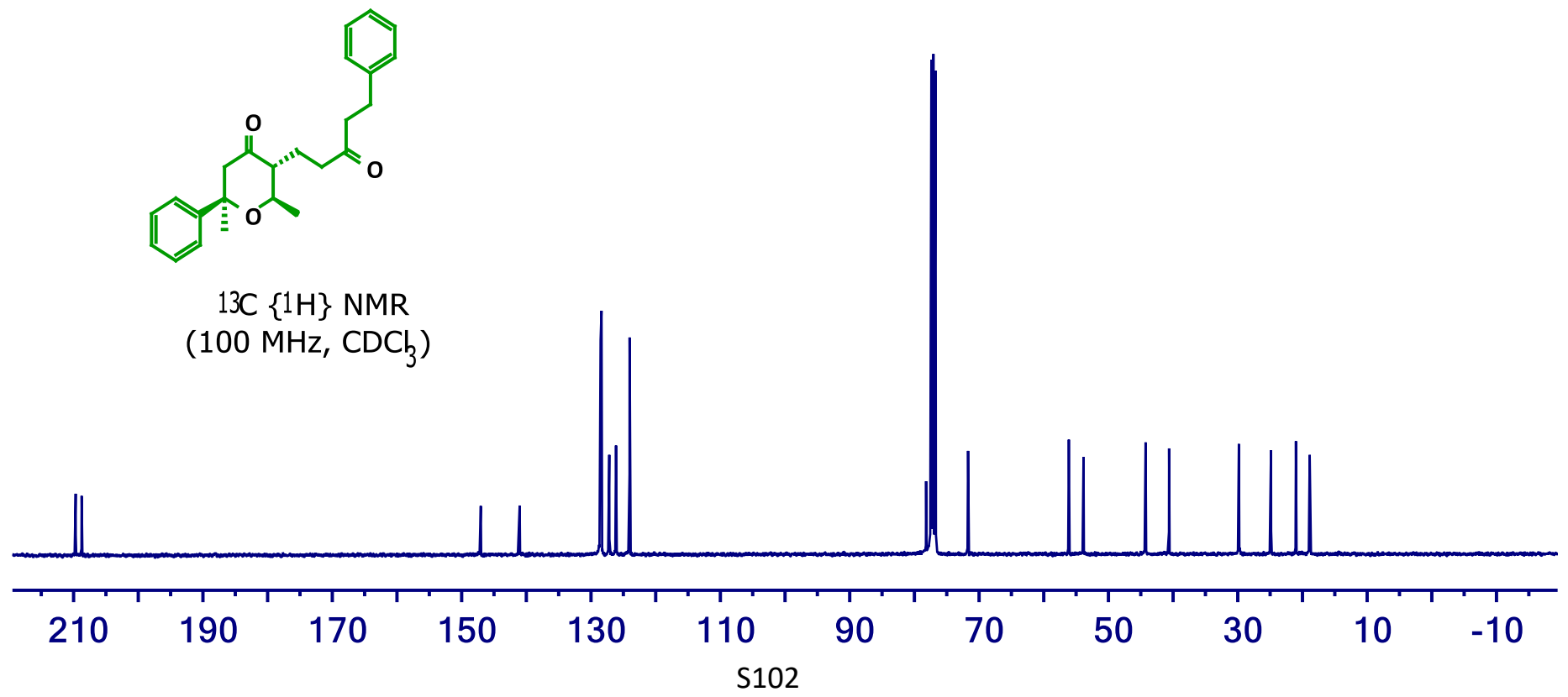

1111111 
(2S,5R,6R)-2,6-dimethyl-5-(3-oxo-5-phenylpentyl)-2-phenyltetrahydro-4H-pyran-4-one $5 y^{2}$

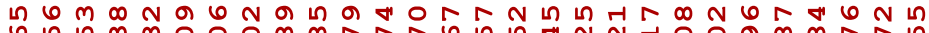

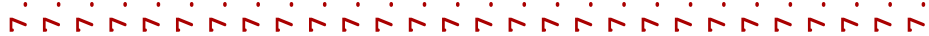

-1 0 요 $\theta$ in in $\ln$ in in in $m m$ मं

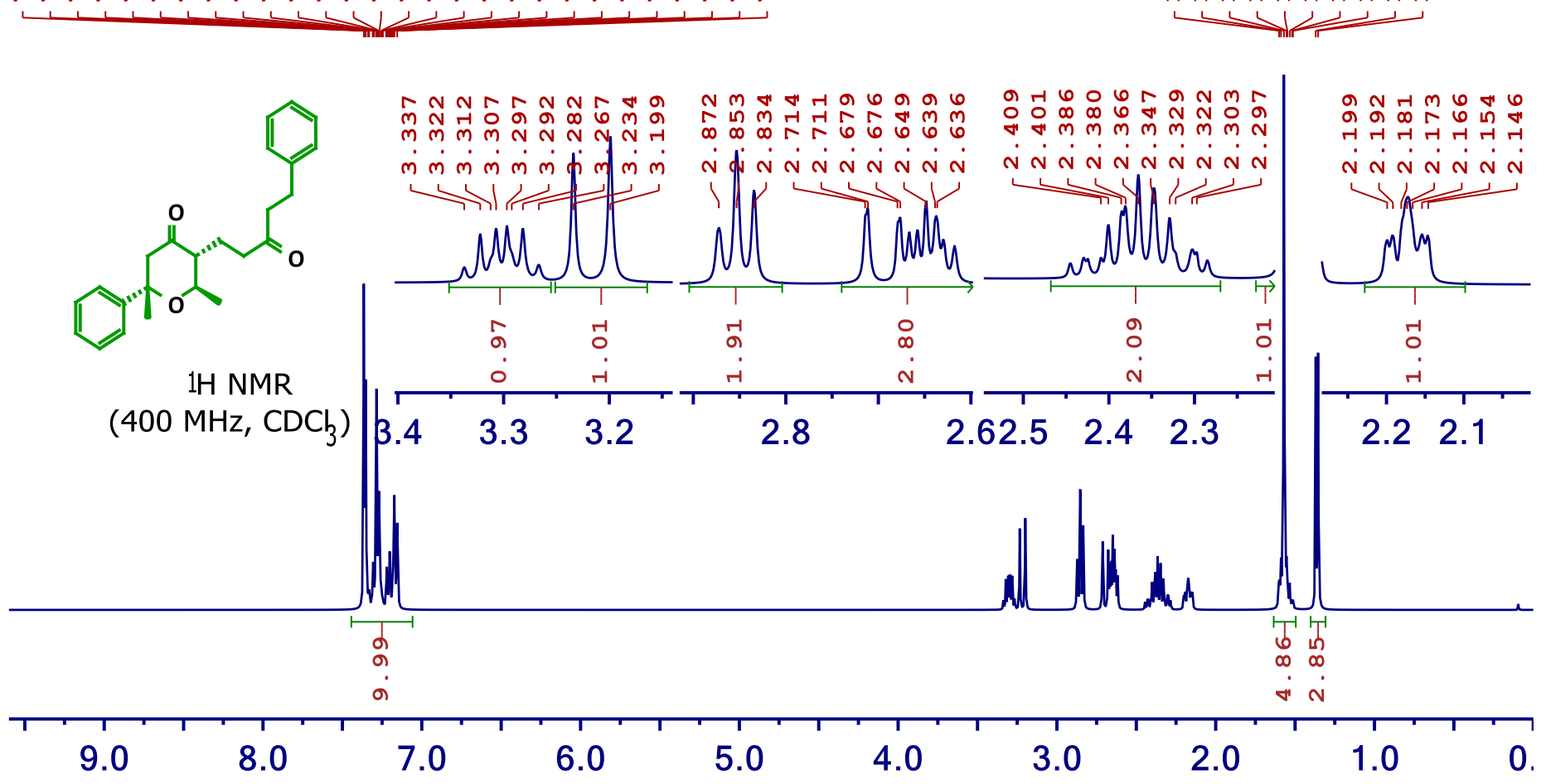

$\begin{array}{ll}0 & \nabla \\ 0 & 0 \\ 0 & 0 \\ 0 & 0 \\ N & N \\ 1 & 1\end{array}$

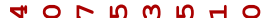

$\dot{m} \vec{i} \dot{\infty} \dot{\infty} \dot{\infty} \dot{0}$

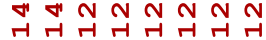

111<smiles>CC1O[C@]2(c3ccccc3)CC(=O)[C@@H](CCC(=O)CCc3ccccc3)[C@@H]1C2</smiles>

$13 \mathrm{C}\{1 \mathrm{H}\} \mathrm{NMR}$

$\left(100 \mathrm{MHz}, \mathrm{CDCb}_{3}\right)$
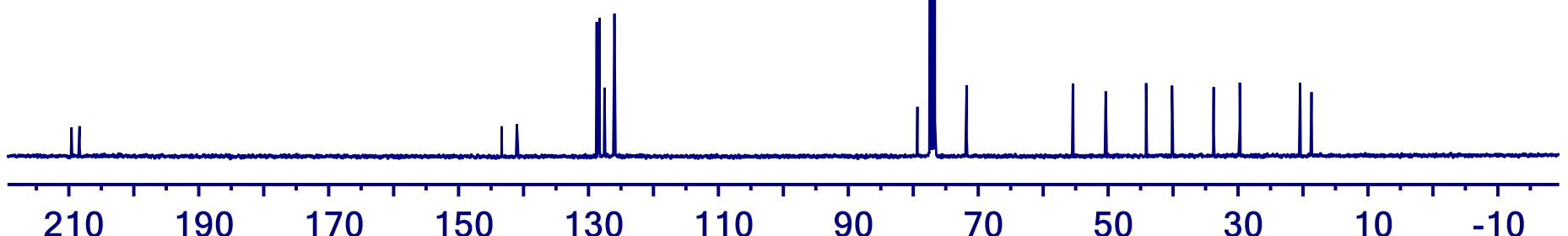
$(2 R, 3 R, 6 S)-6-b u t y l-2-m e t h y l-3-(3-0 x o-5-p h e n y l p e n t y l) t e t r a h y d r o-4 H-p y r a n-4-o n e$

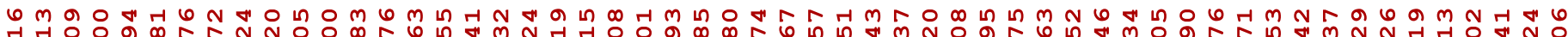
m $m$ m

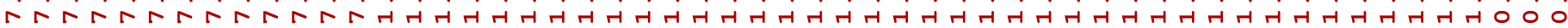

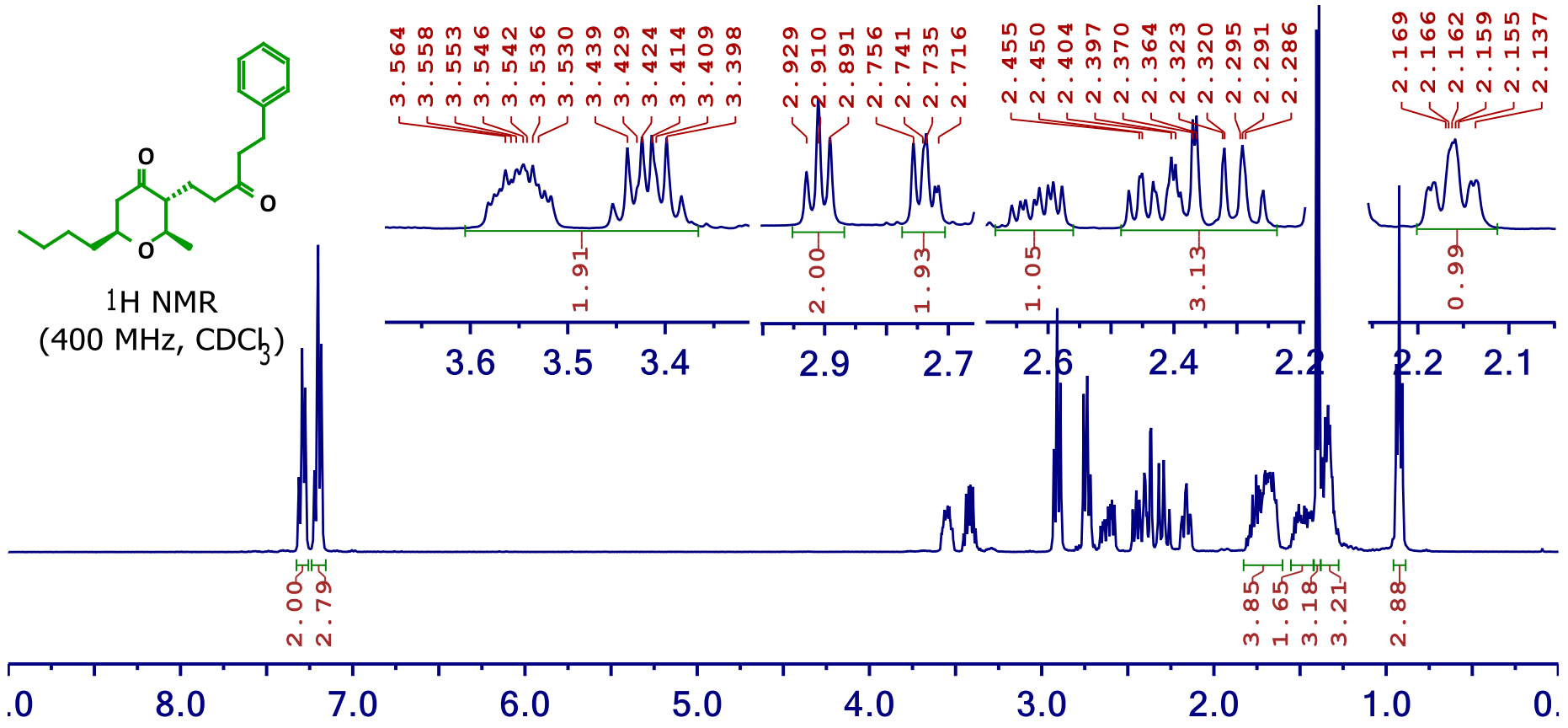

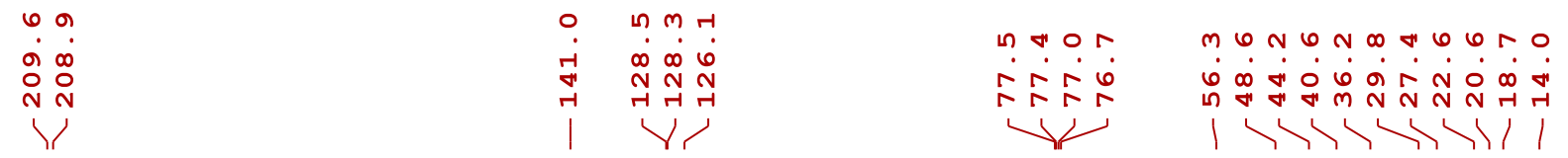

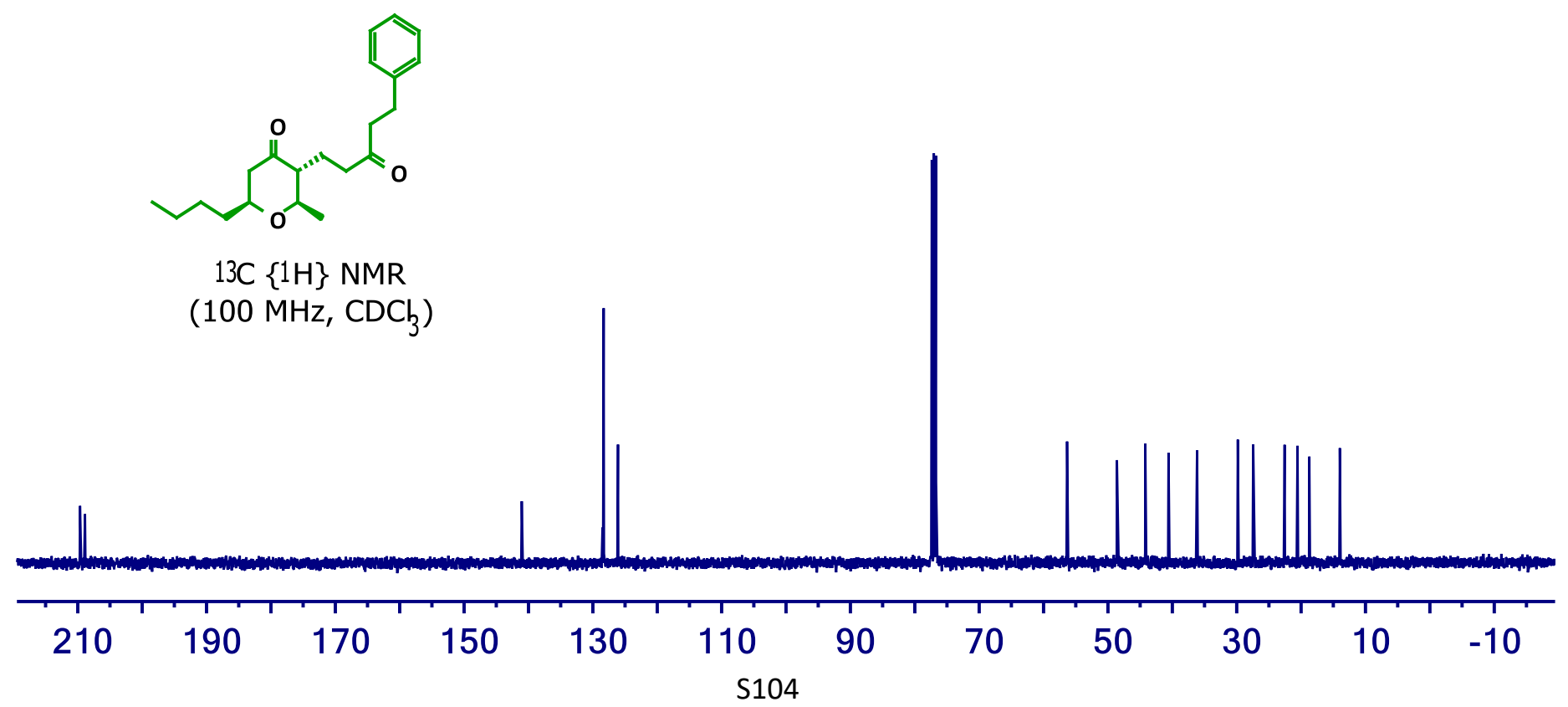


(7R,8R)-7-methyl-8-(3-oxo-5-phenylpentyl)-6-oxaspiro[4.5]decan-9-one

5 aa

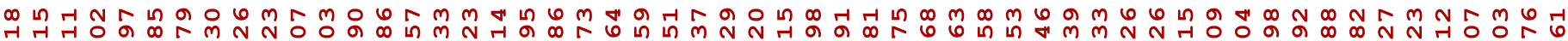
m

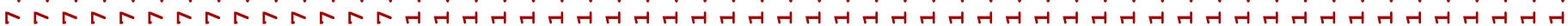

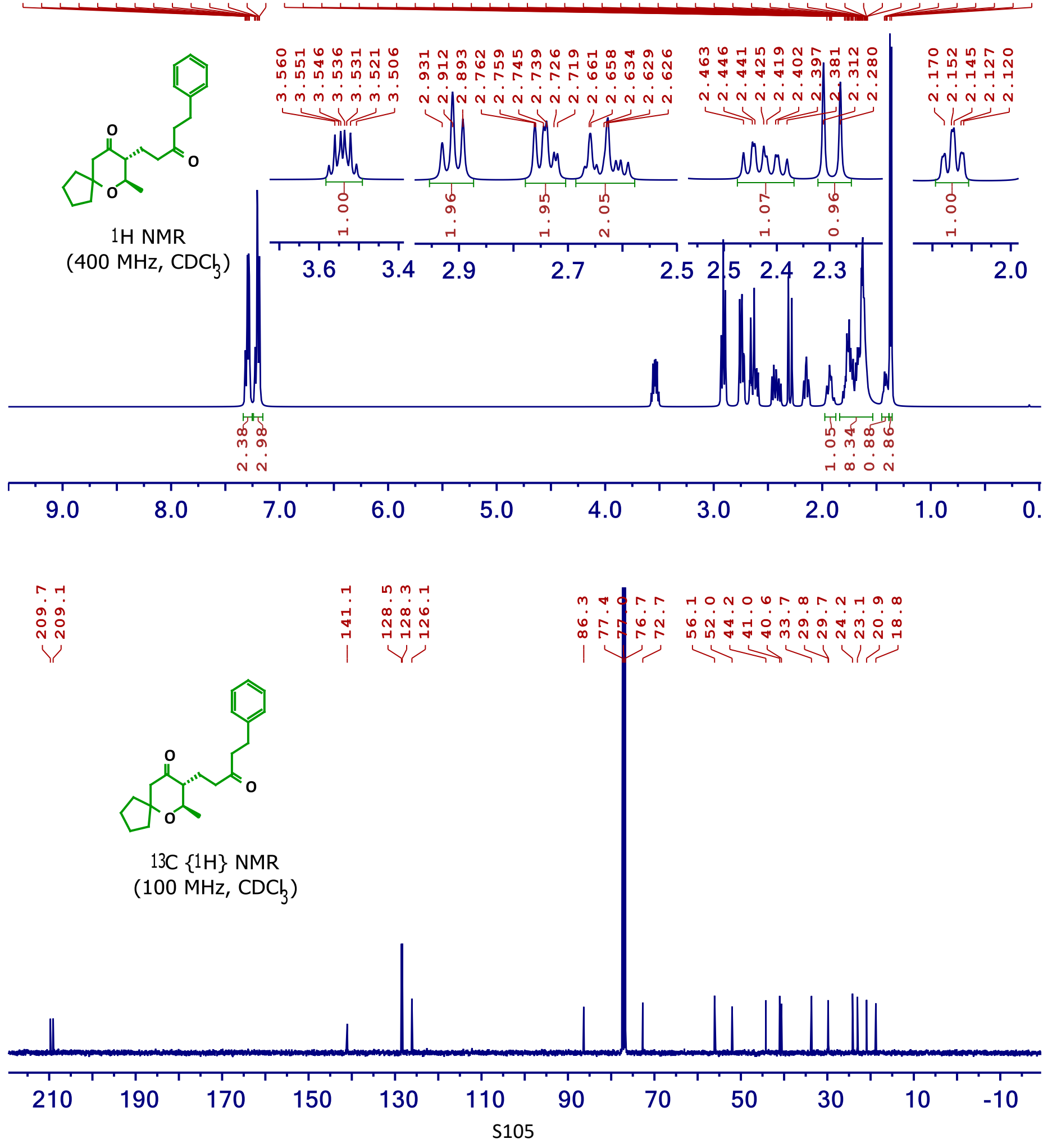


$(2 R, 3 R, 6 R)-2-m e t h y l-3-(3-o x o-4-p h e n y l b u t y l)-6-p h e n y l t e t r a h y d r o-4 H-p y r a n-4-o n e$ $5 \mathbf{a}$

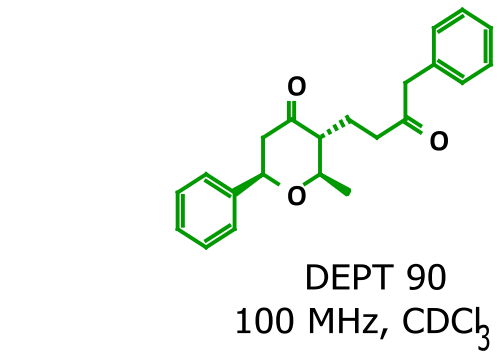

$\ln \infty r+4 r$

$\dot{0} \dot{\infty} \dot{\infty} \dot{\infty} \dot{0}$

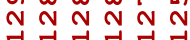

$\rightarrow$

$N+1$

$\stackrel{1}{\infty} \infty$

)

ก
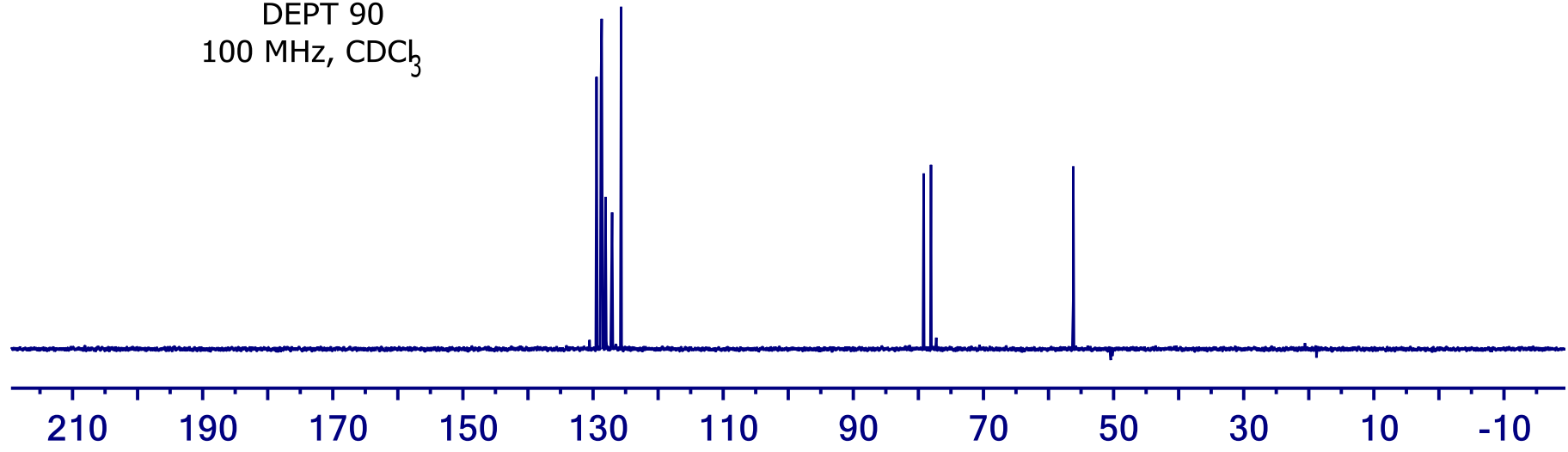

$(2 R, 3 R, 6 R)-2-m e t h y l-3-(3-o x o-4-p h e n y l b u t y l)-6-p h e n y l t e t r a h y d r o-4 H-p y r a n-4-o n e$ $5 \mathbf{a}$

$\ln \infty \pi+4 r$

a $\infty \dot{\infty} \dot{\infty} \dot{\sim}$

N $N \underset{4}{\infty} \sim$

皮

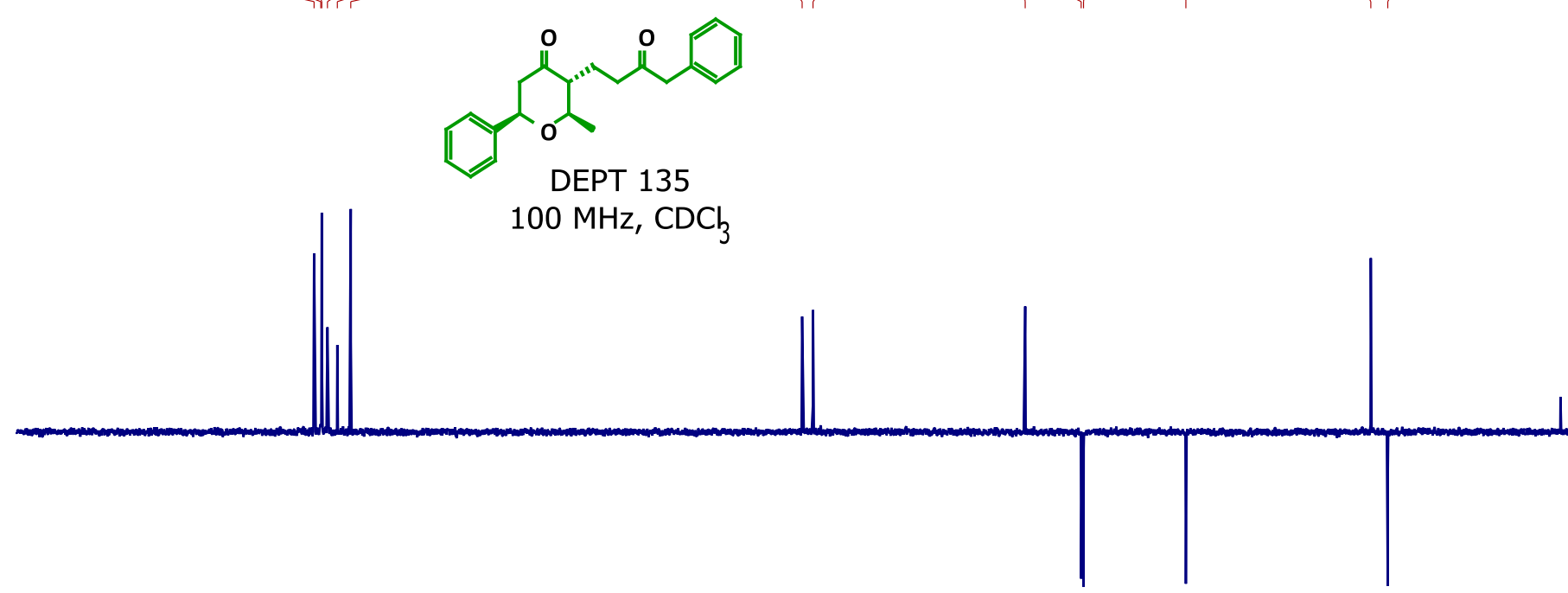

$\stackrel{1}{a}$

N

००००

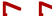

in in in

0
$\dot{m}$
1

$6 \infty$

$\circ \infty$

)

|

N

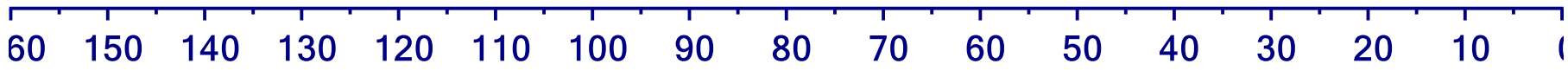


$(2 R, 3 R, 6 R)-2-m e t h y l-3-(3-o x o-3-(t h i o p h e n-2-y l) p r o p y l)-6-p h e n y l t e t r a h y d r o-44-p y r a n-4-o n e$ $5 \mathrm{~b}$

$\operatorname{sen} 64$

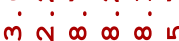

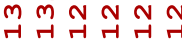

पर
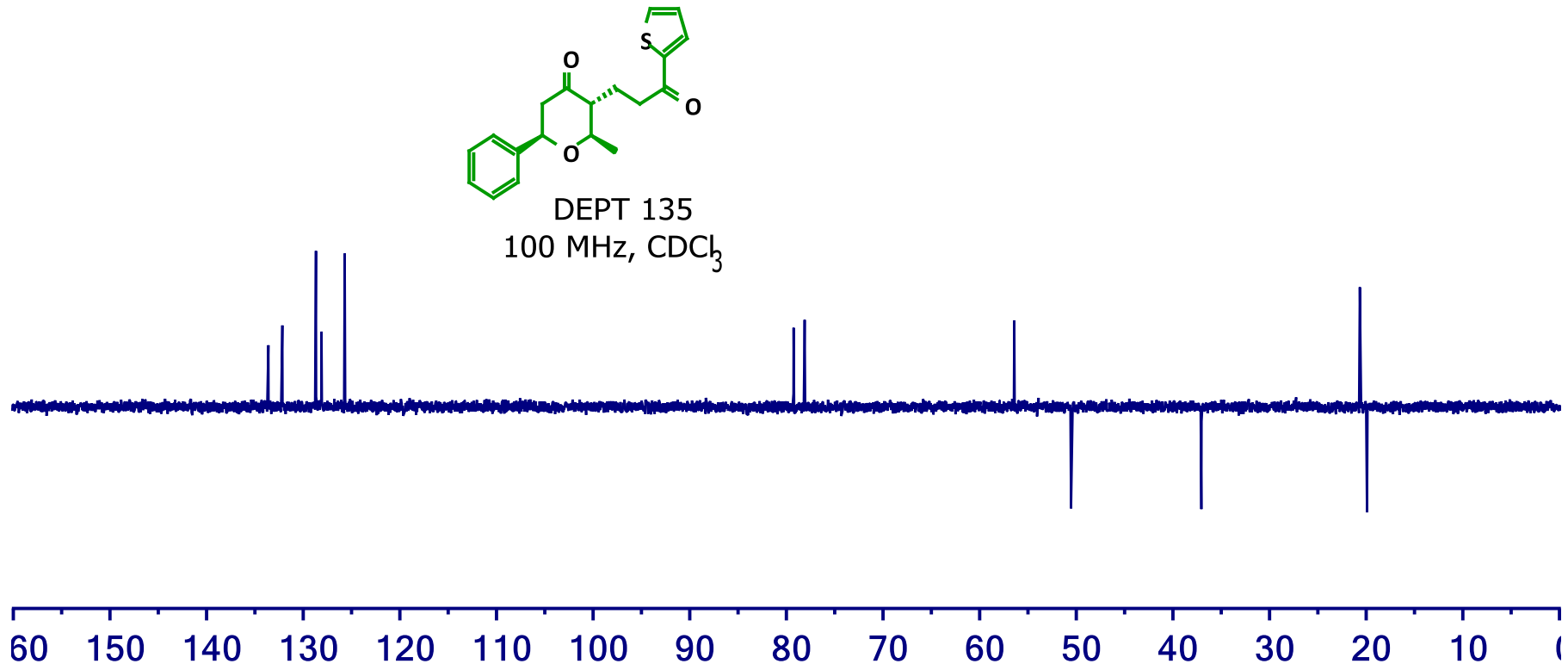

$(2 R, 3 R, 6 R)-3-(3-($ furan-2-yl)-3-oxopropyl)-2-methyl-6-phenyltetrahydro-4H-pyran-4-one $5 \mathrm{c}$

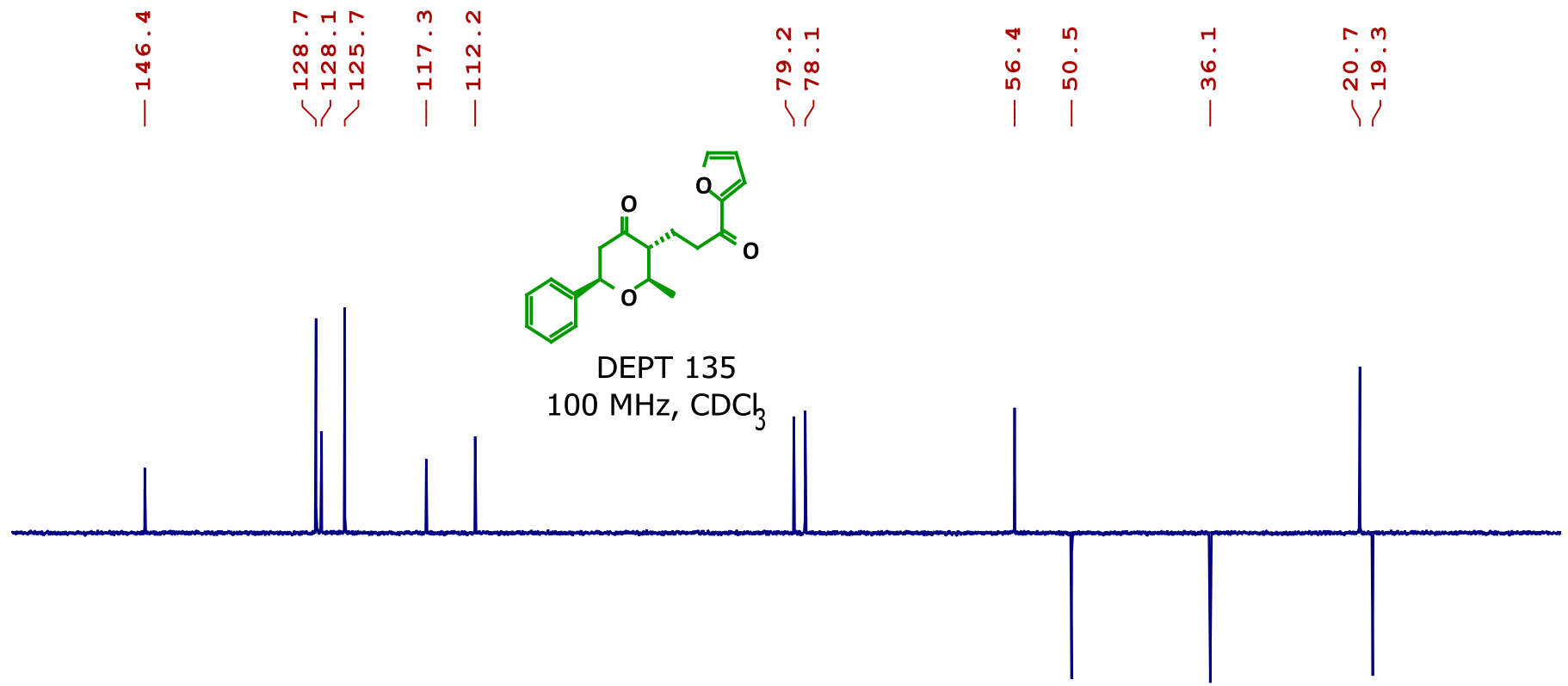

$\begin{array}{llllllllllllllll}60 & 150 & 140 & 130 & 120 & 110 & 100 & 90 & 80 & 70 & 60 & 50 & 40 & 30 & 20 & 10\end{array}$


$(2 R, 3 R, 6 R)-2$-methyl-3-(3-oxo-5-phenylpentyl)-6-phenyltetrahydro-4H-pyran-4-one $5 d$

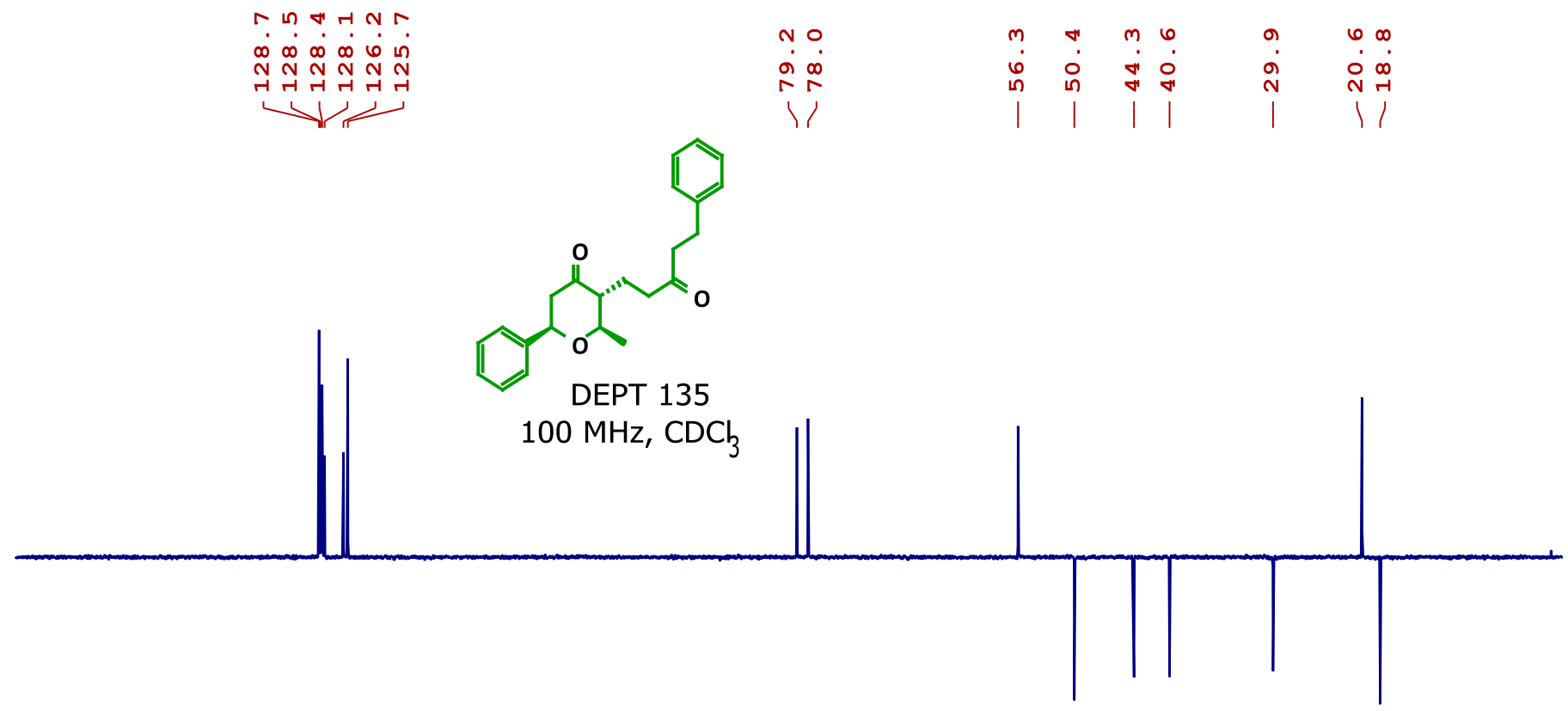

\begin{tabular}{lllllllllllllllll}
\hline 60 & 150 & 140 & 130 & 120 & 110 & 100 & 90 & 80 & 70 & 60 & 50 & 40 & 30 & 20 & 10 & 1
\end{tabular}

$(2 R, 3 R, 6 R)-2$-methyl-3-(3-oxo-3-phenylpropyl)-6-phenyltetrahydro-4H-pyran-4-one $5 \mathrm{e}$
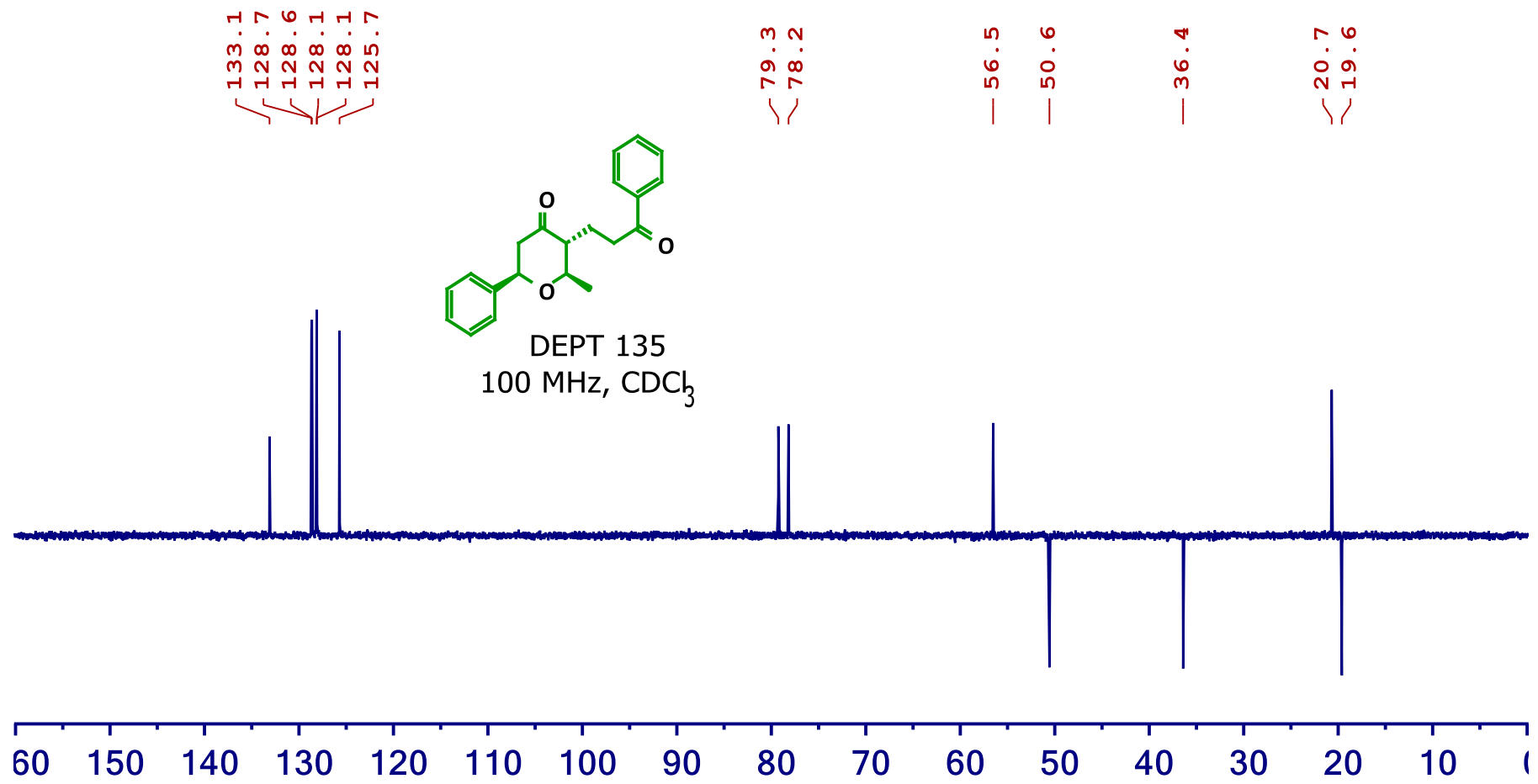
$(2 R, 3 R, 6 R)-3-(3-c y c l o h e x y l-3-o x o p r o p y l)-2-m e t h y l-6-p h e n y l t e t r a h y d r o-4 H-p y r a n-4-o n e$ 5f

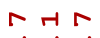

$\infty \dot{\infty}$ in

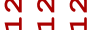

$\lambda /$
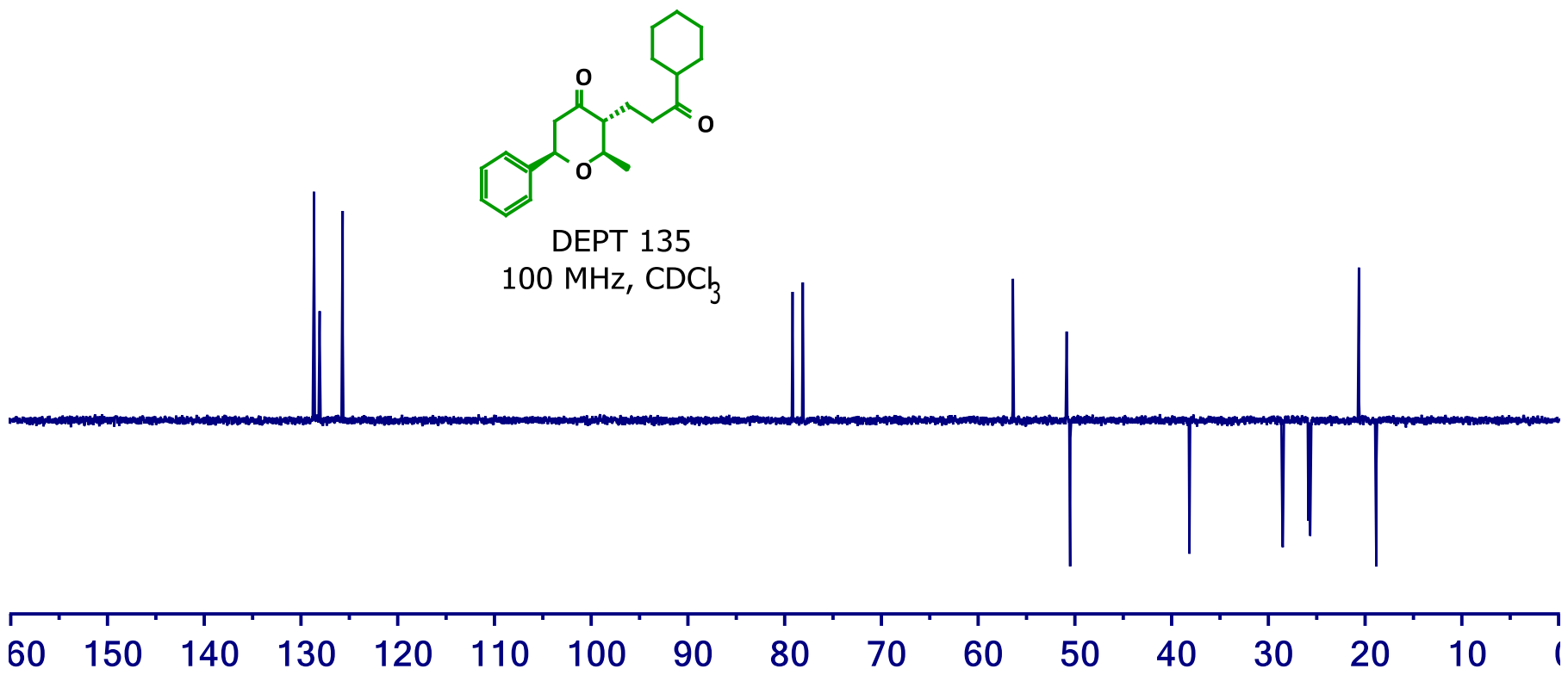

$(2 R, 3 R, 6 R)-2-m e t h y l-3-((E)-3-o x o-5-p h e n y l p e n t-4-e n-1-y l)-6-p h e n y l t e t r a h y d r o-4 H-p y r a n-4-o n e$ $5 \mathrm{~g}$

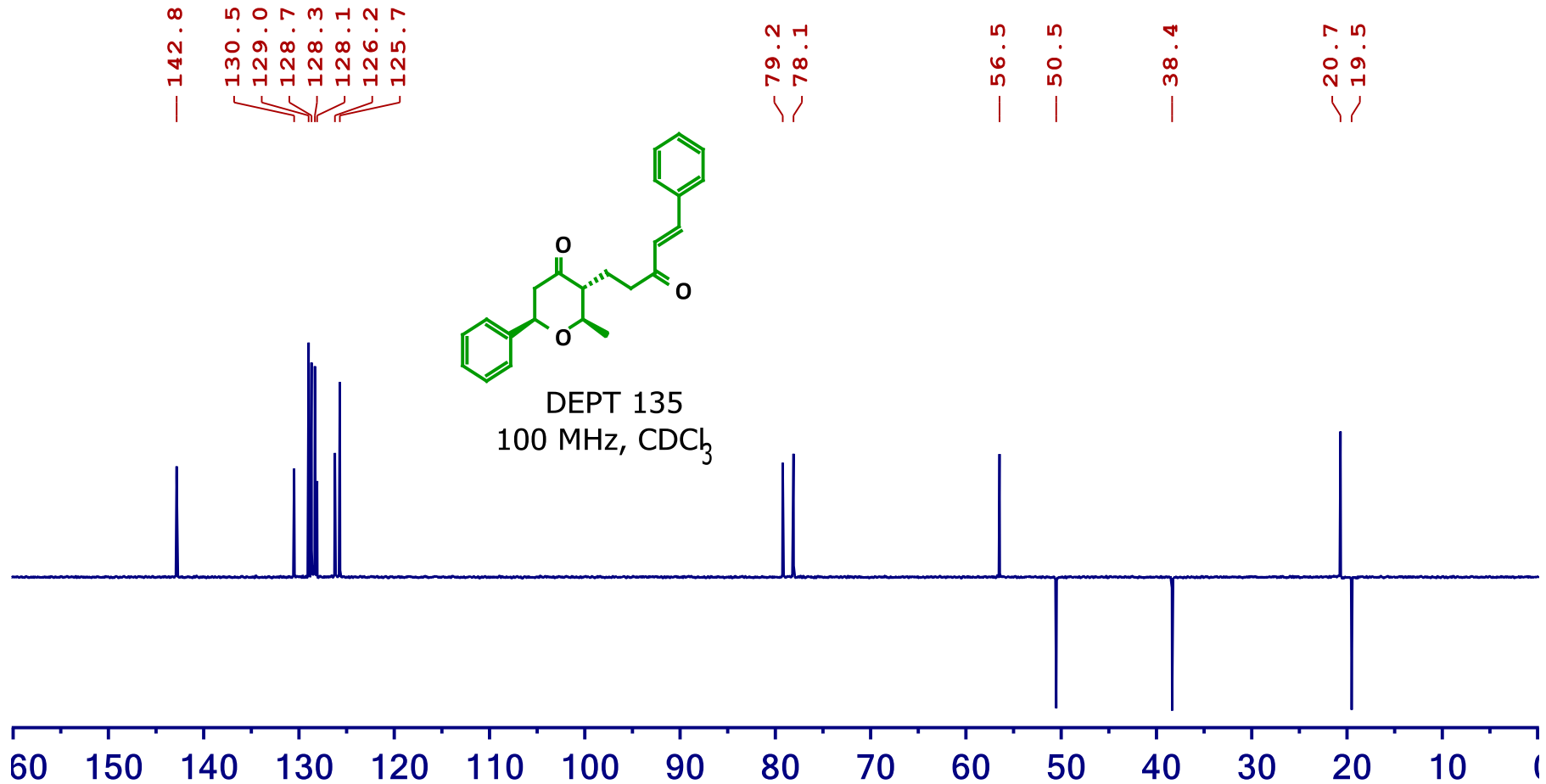


(2R,3R,6R)-3-(3-(2,6-dichlorophenyl)-3-oxopropyl)-2-methyl-6-phenyltetrahydro-4H-pyran-4-one $5 \mathrm{~h}$
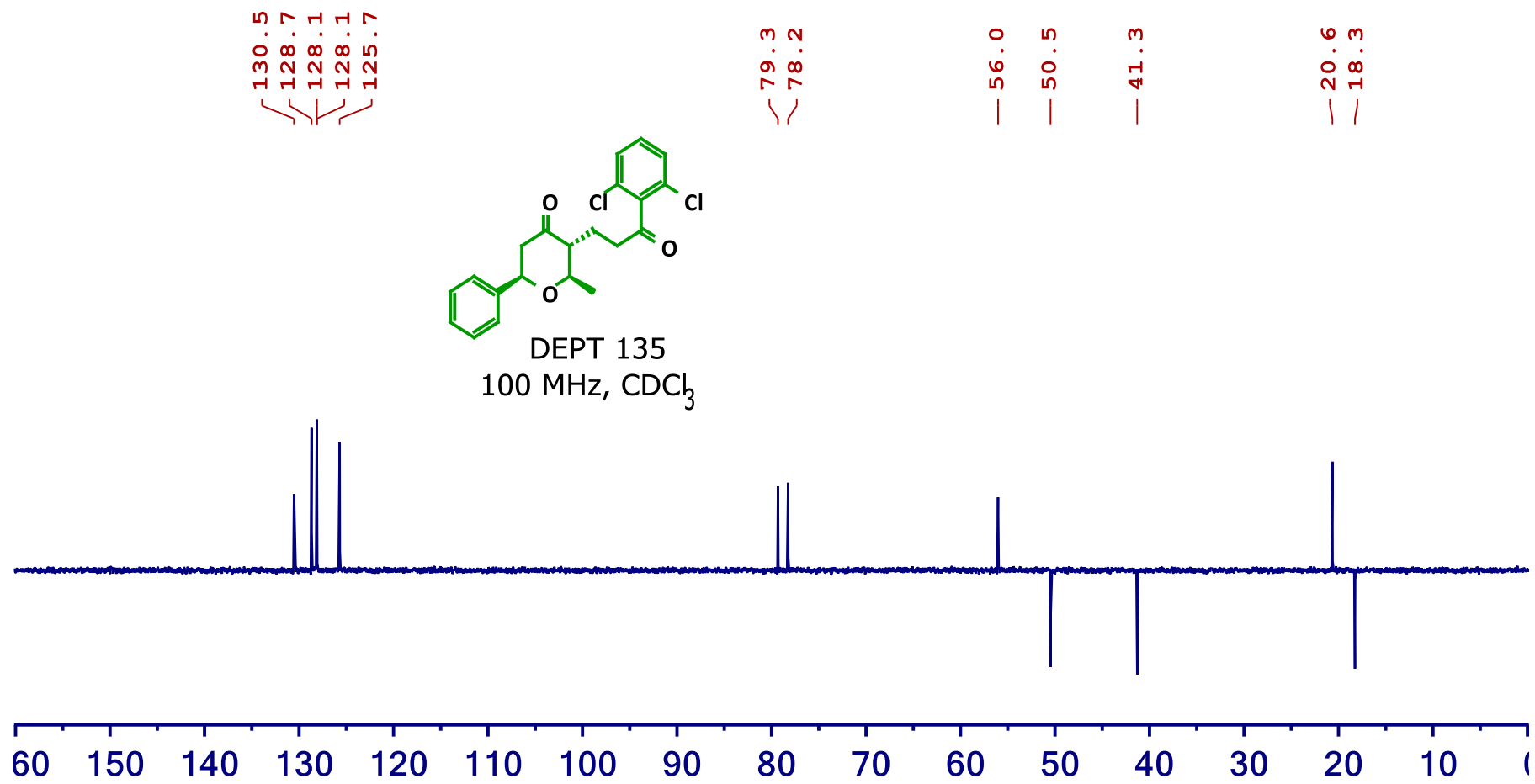

(2R,3R,6R)-2-methyl-3-(3-(naphthalen-1-yl)-3-oxopropyl)-6-phenyltetrahydro-4H-pyran-4-one $5 \mathrm{i}$

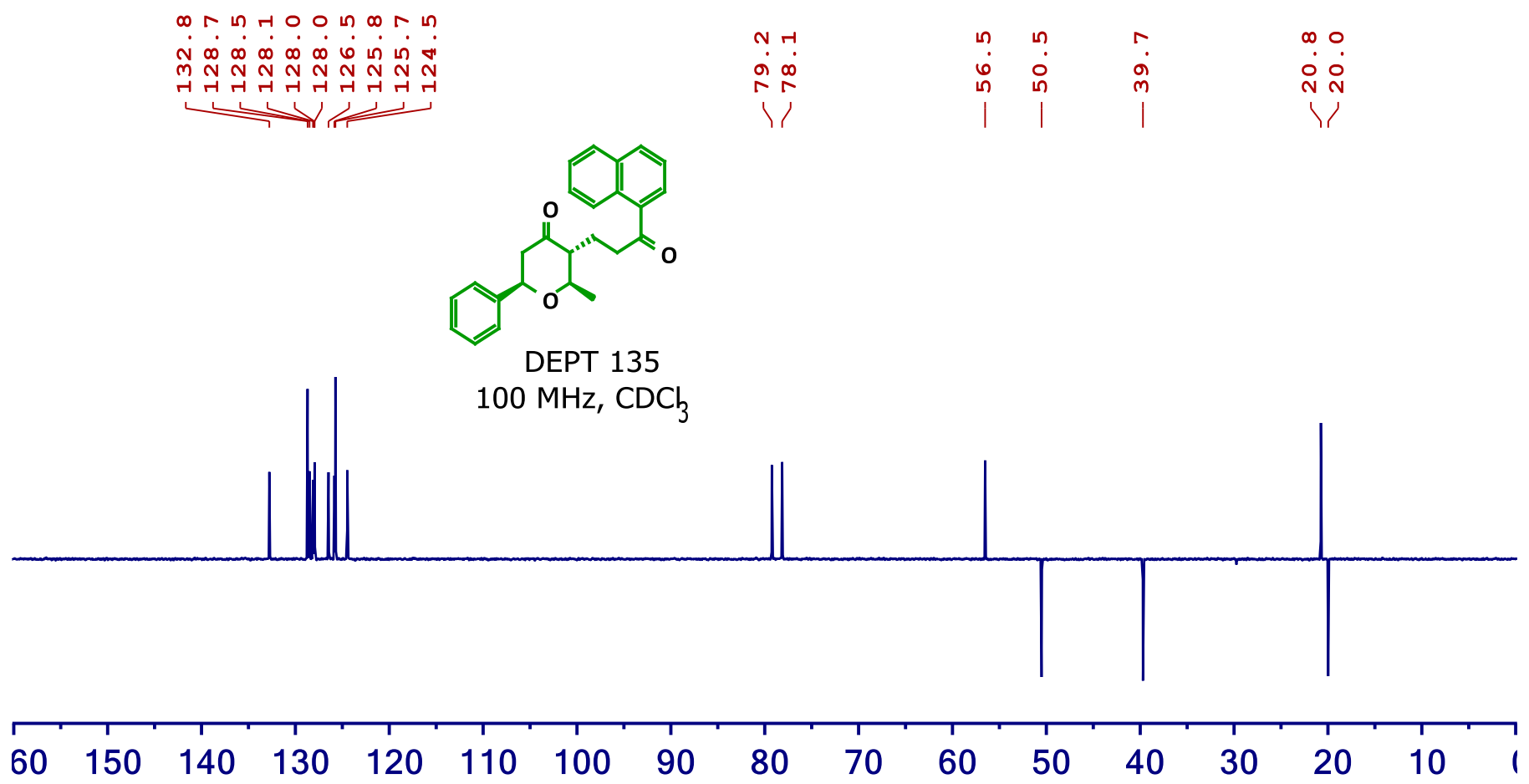


$(2 R, 3 R, 6 R)-2-m e t h y l-3-(3-0 x o-4-p h e n y l b u t y l)-6-p h e n y l t e t r a h y d r o-4 H-p y r a n-4-o n e$ $5 a$

H 6 m

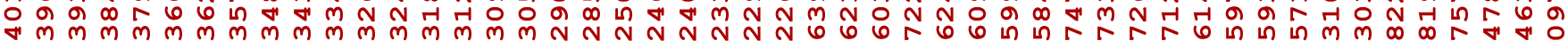

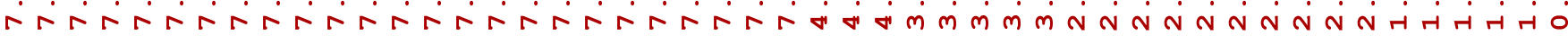

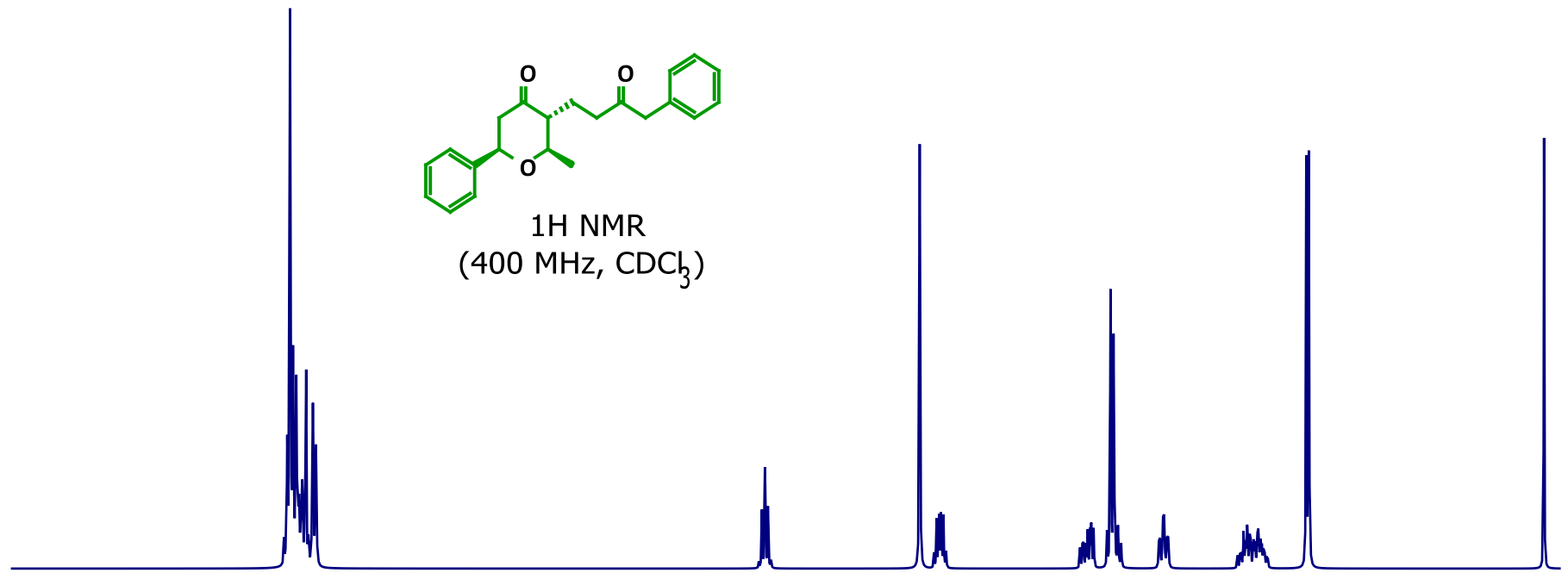

\begin{tabular}{lllllllllll}
\hline .0 & 8.0 & 7.0 & 6.0 & 5.0 & 4.0 & 3.0 & 2.0 & 1.0 & 0.
\end{tabular}
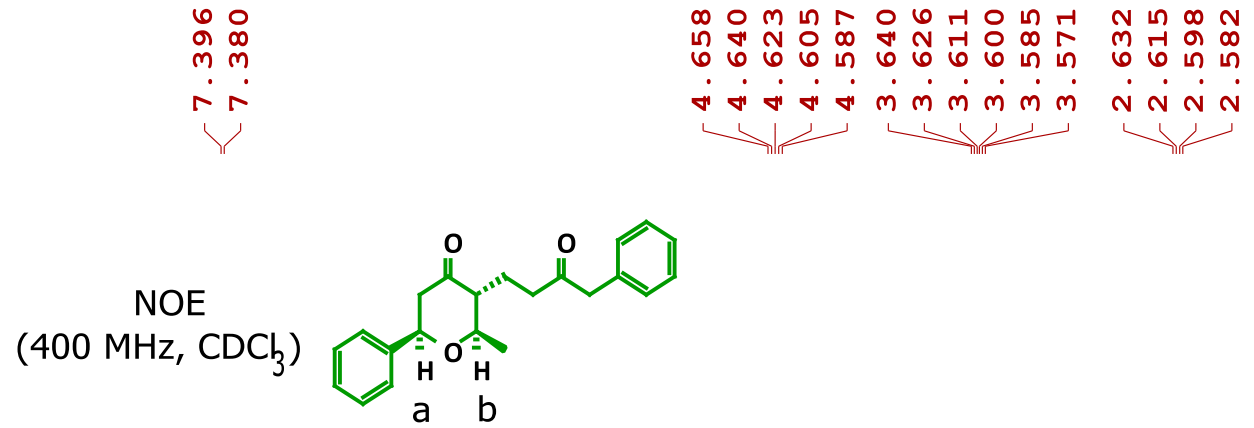

b

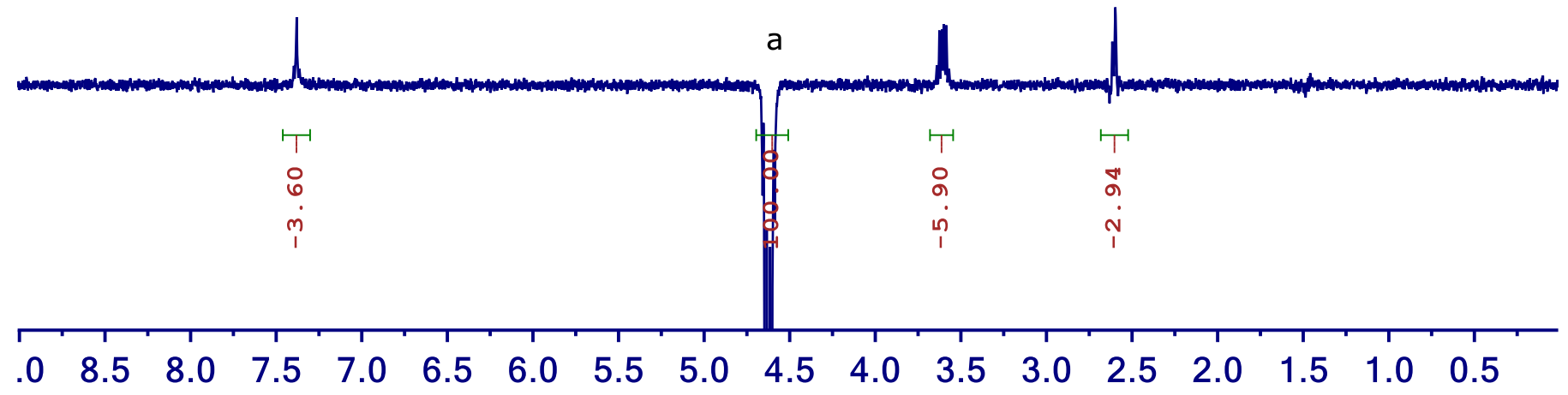


(2R,3R,6R)-2-methyl-3-(3-oxo-4-phenylbutyl)-6-phenyltetrahydro-4H-pyran-4-one $5 a$
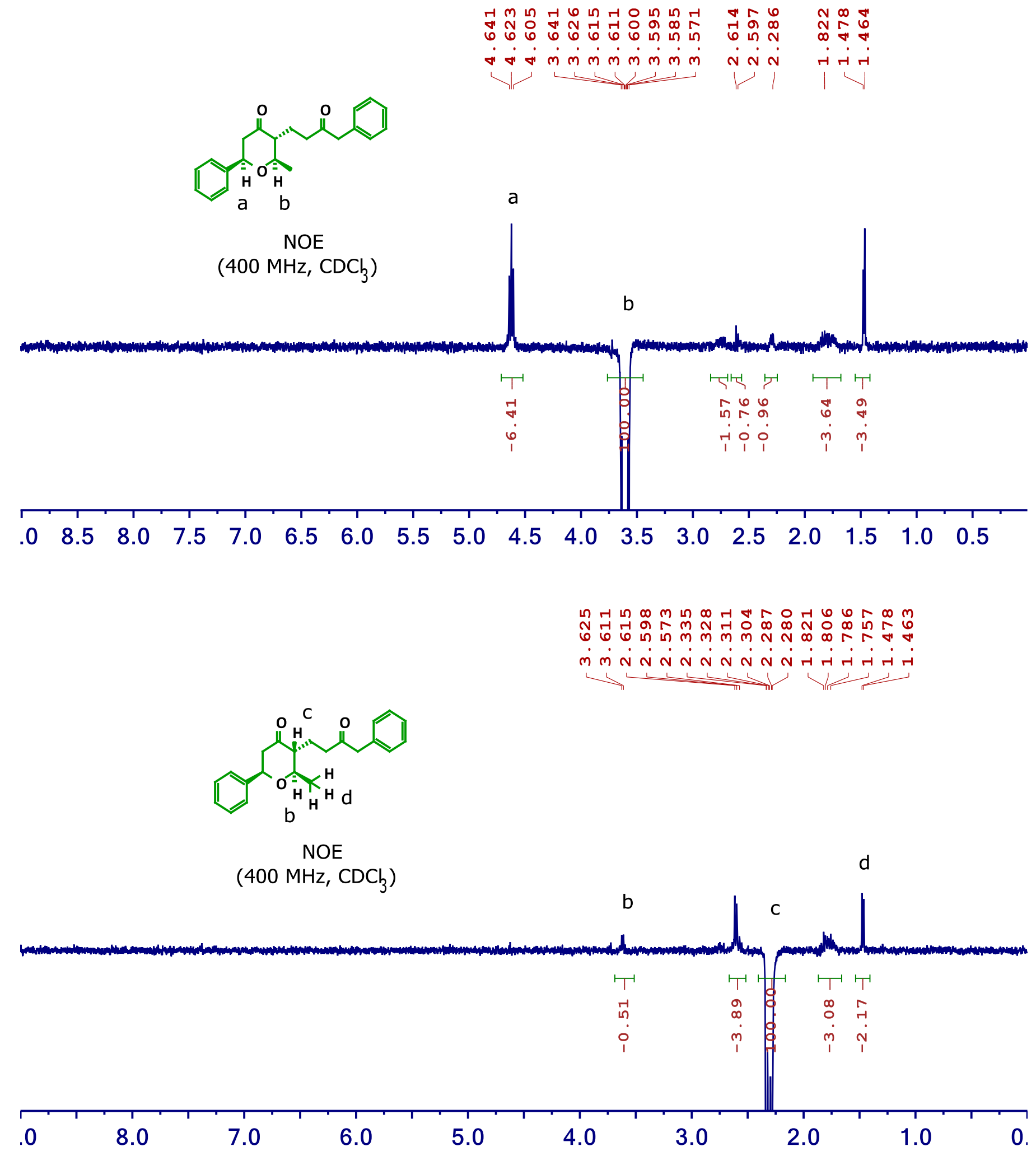
$(2 R, 3 R, 6 R)-2-m e t h y l-3-(3-o x o-3-($ thiophen-2-yl)propyl)-6-phenyltetrahydro-4H-pyran-4-one $5 \mathrm{~b}$

\#゙-1

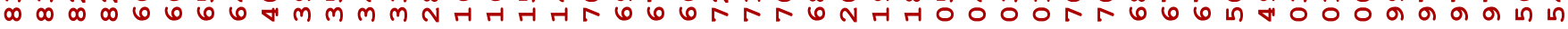

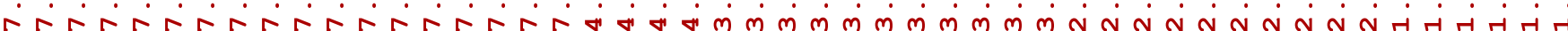
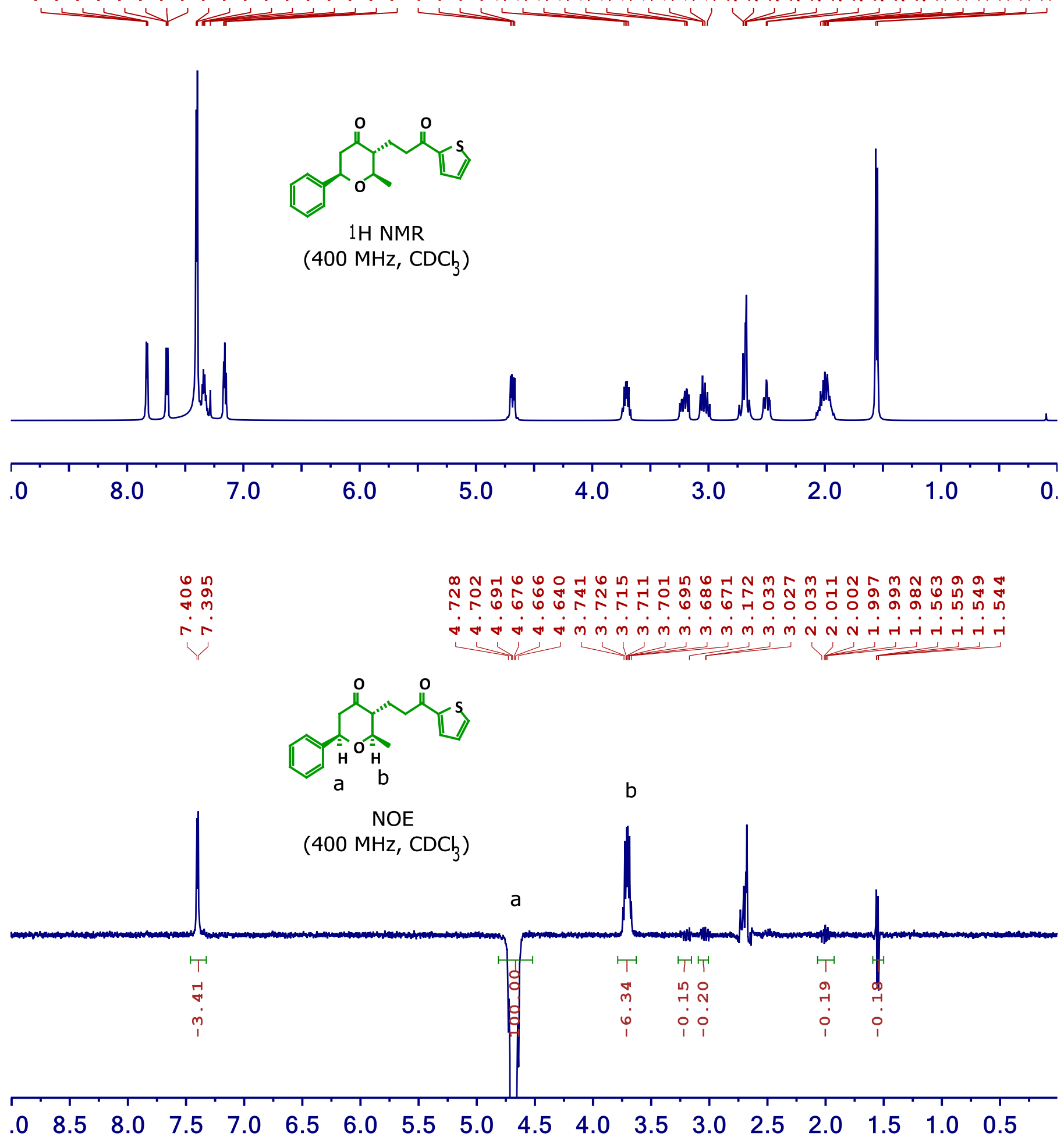
$(2 R, 3 R, 6 R)-2-m e t h y l-3-(3-o x o-3-(t h i o p h e n-2-y 1) p r o p y l)-6-p h e n y l t e t r a h y d r o-4 H-p y r a n-4-o n e$ $5 \mathbf{b}$

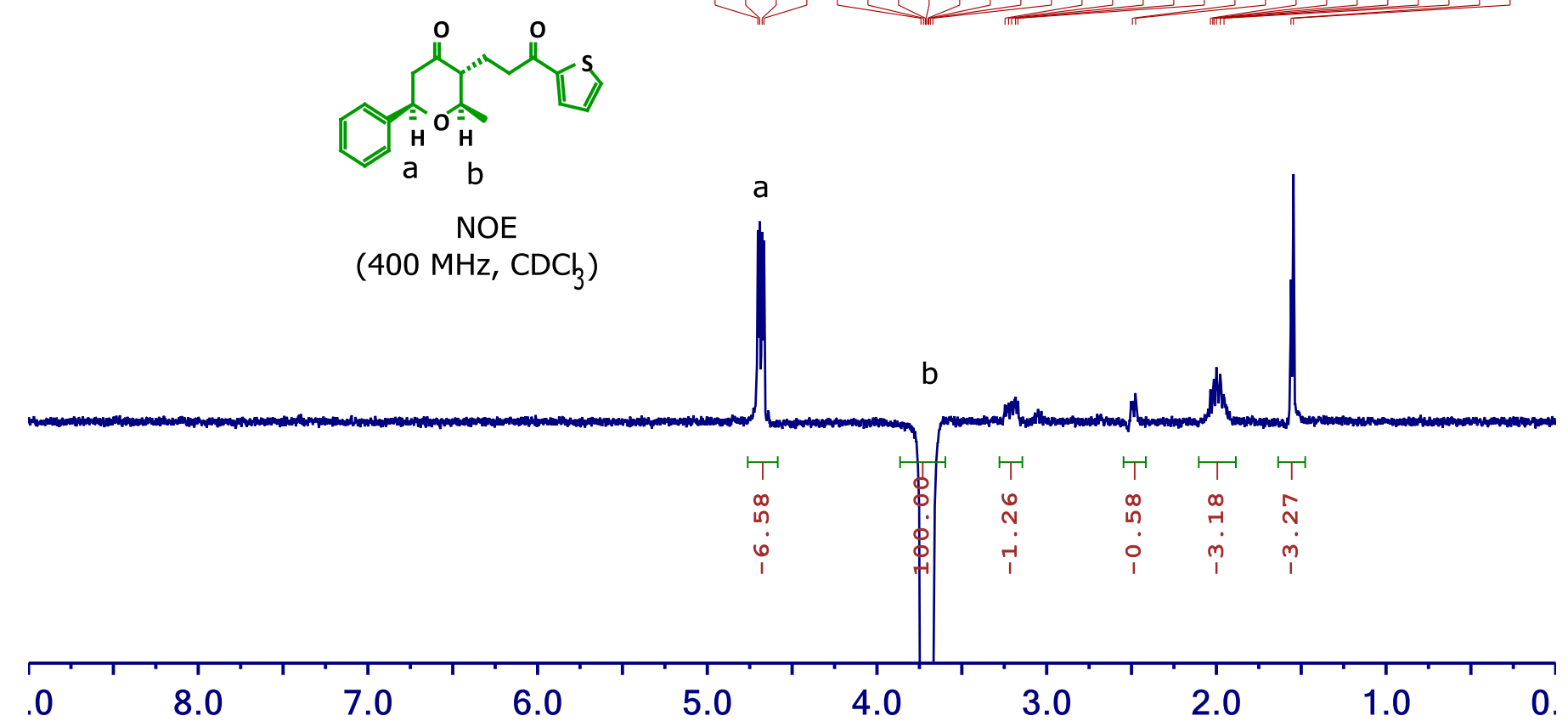

단

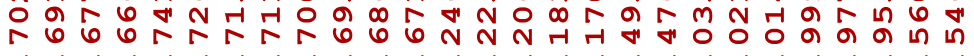

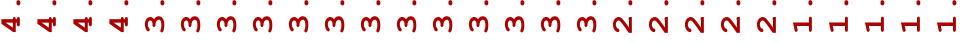

0 Inm $\quad$ N N

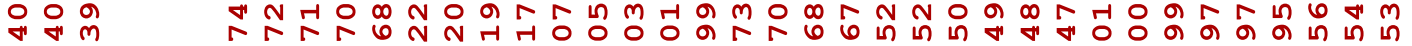

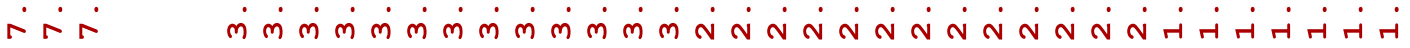
r<smiles>[2H]C([CH]Cl)[C@H]1OC(c2ccccc2)CC(=O)[C@H]1CCC(=O)c1cccs1</smiles>

NOE (400 MHz, $\mathrm{CDCl}_{3}$ )

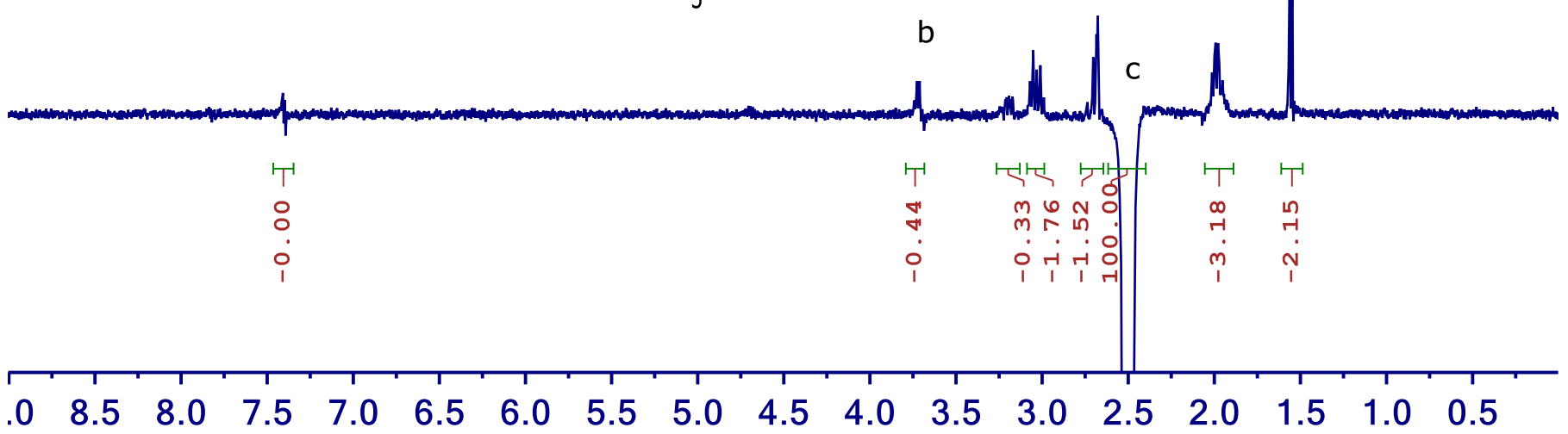


$(2 R, 3 R, 6 R)-2-m e t h y l-3-(3-0 x 0-5-p h e n y l p e n t y l)-6-p h e n y l t e t r a h y d r o-4 H-p y r a n-4-o n e$ $5 \mathrm{~d}$

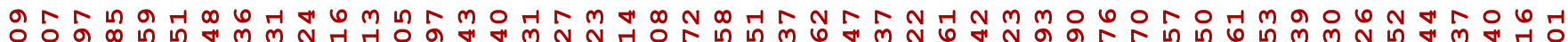

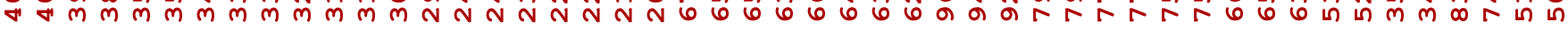

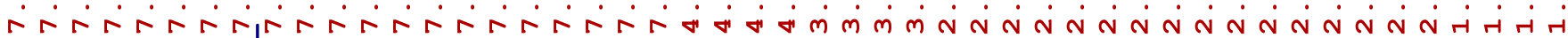

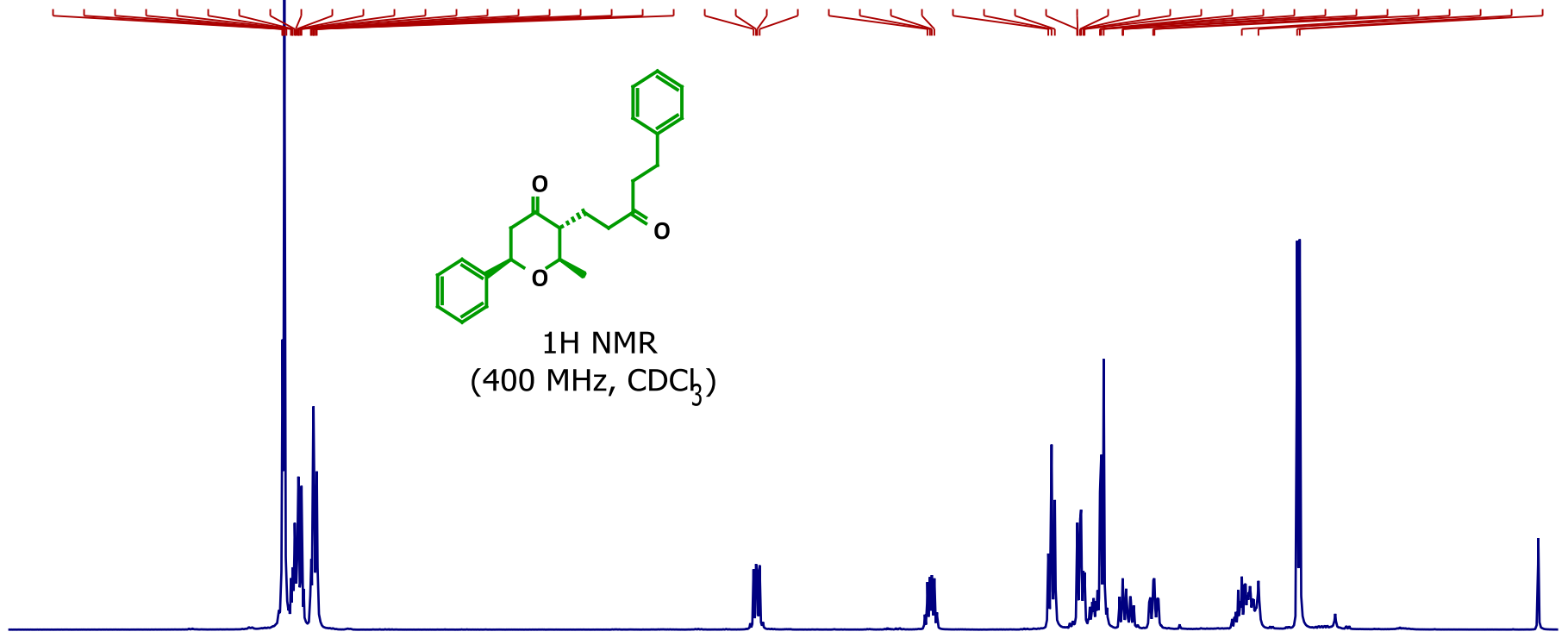

\begin{tabular}{llllllllllll}
\hline .0 & 8.0 & 7.0 & 6.0 & 5.0 & 4.0 & 3.0 & 2.0 & 1.0 & 0.0
\end{tabular}

$\infty \sim$ in

$\circ$ o $\infty$ in $m$

मn $m$

$\therefore \dot{10}$

$\rightarrow$

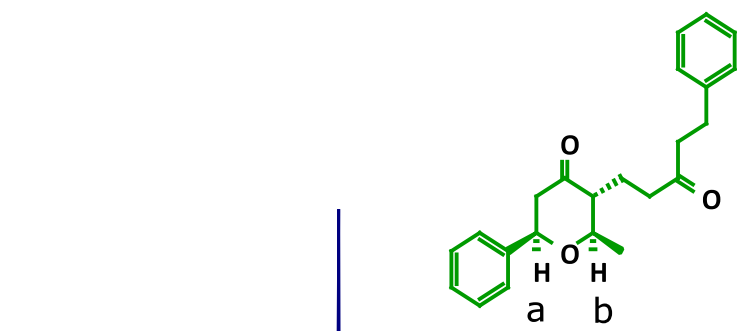

$\mathrm{a}$
$\mathrm{NOE}$
$\left.(400 \mathrm{MHz}, \mathrm{CDC})_{3}\right)$

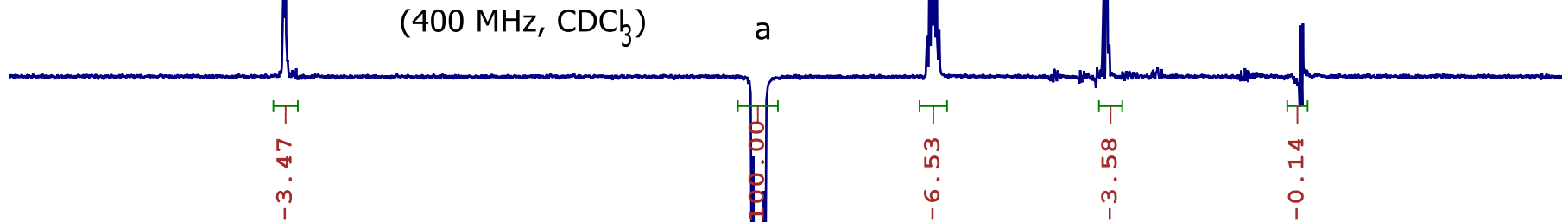

0

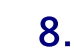

8.0

7.0

6.0

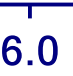

5.0

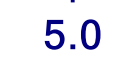

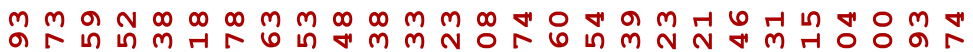

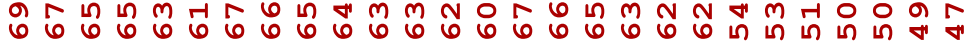

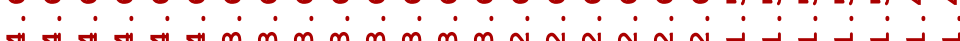

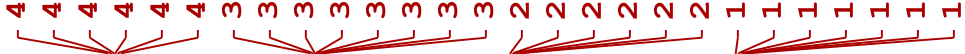
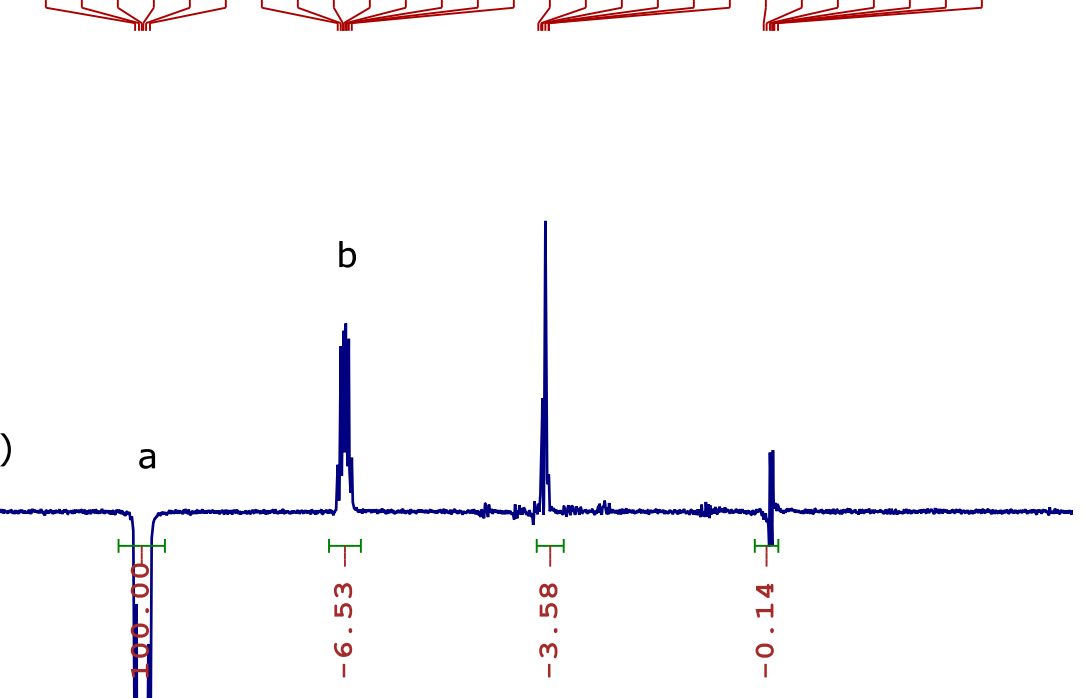
$(2 R, 3 R, 6 R)-2$-methyl-3-(3-oxo-5-phenylpentyl)-6-phenyltetrahydro-4H-pyran-4-one $5 \mathrm{~d}$

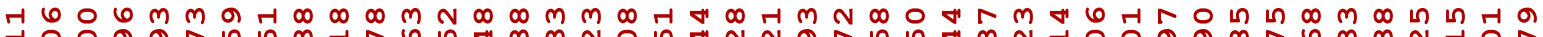

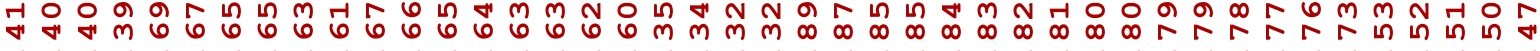

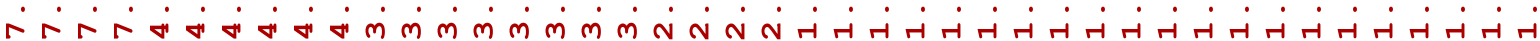

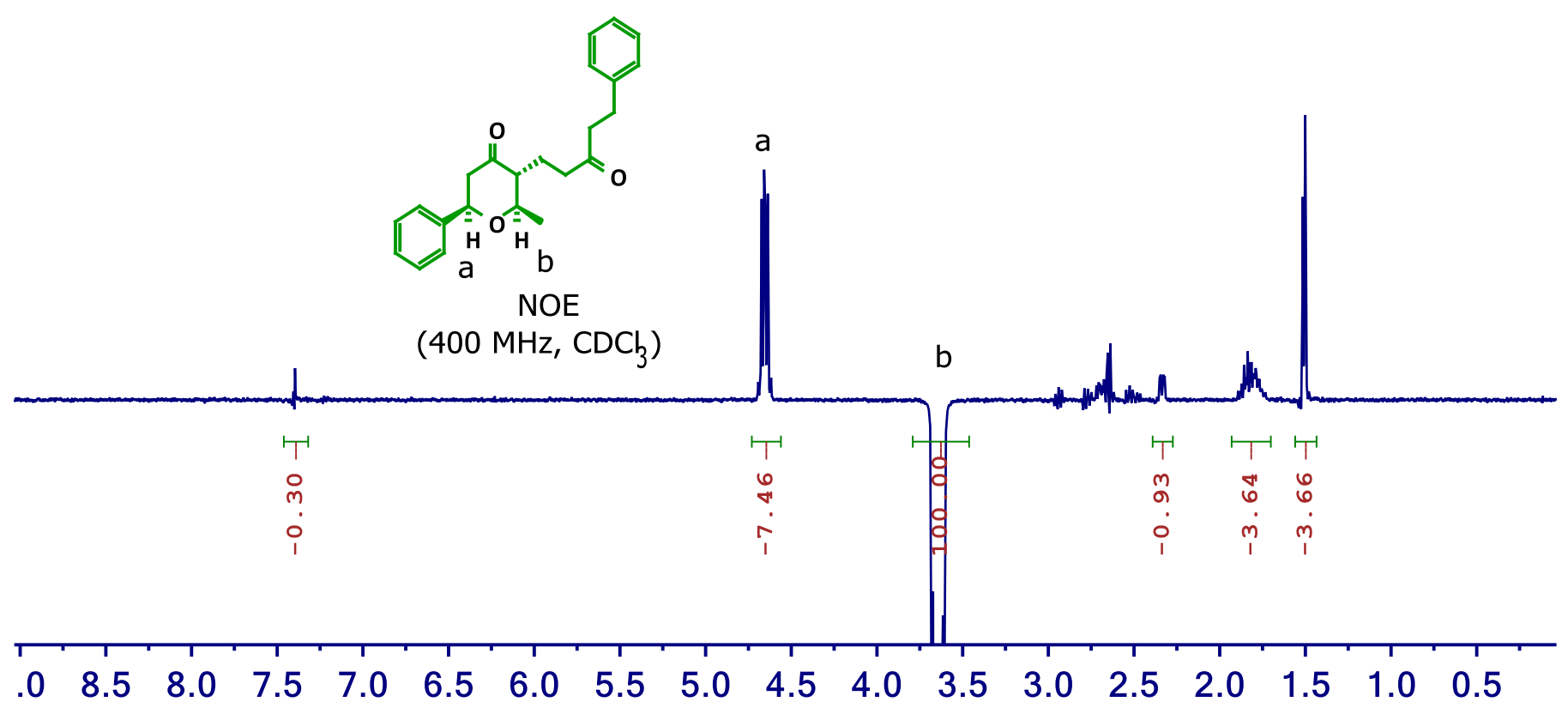

ト

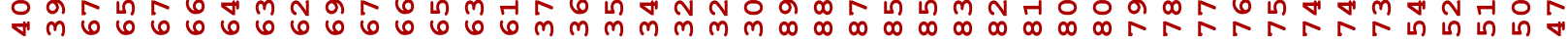

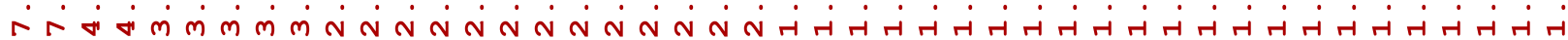
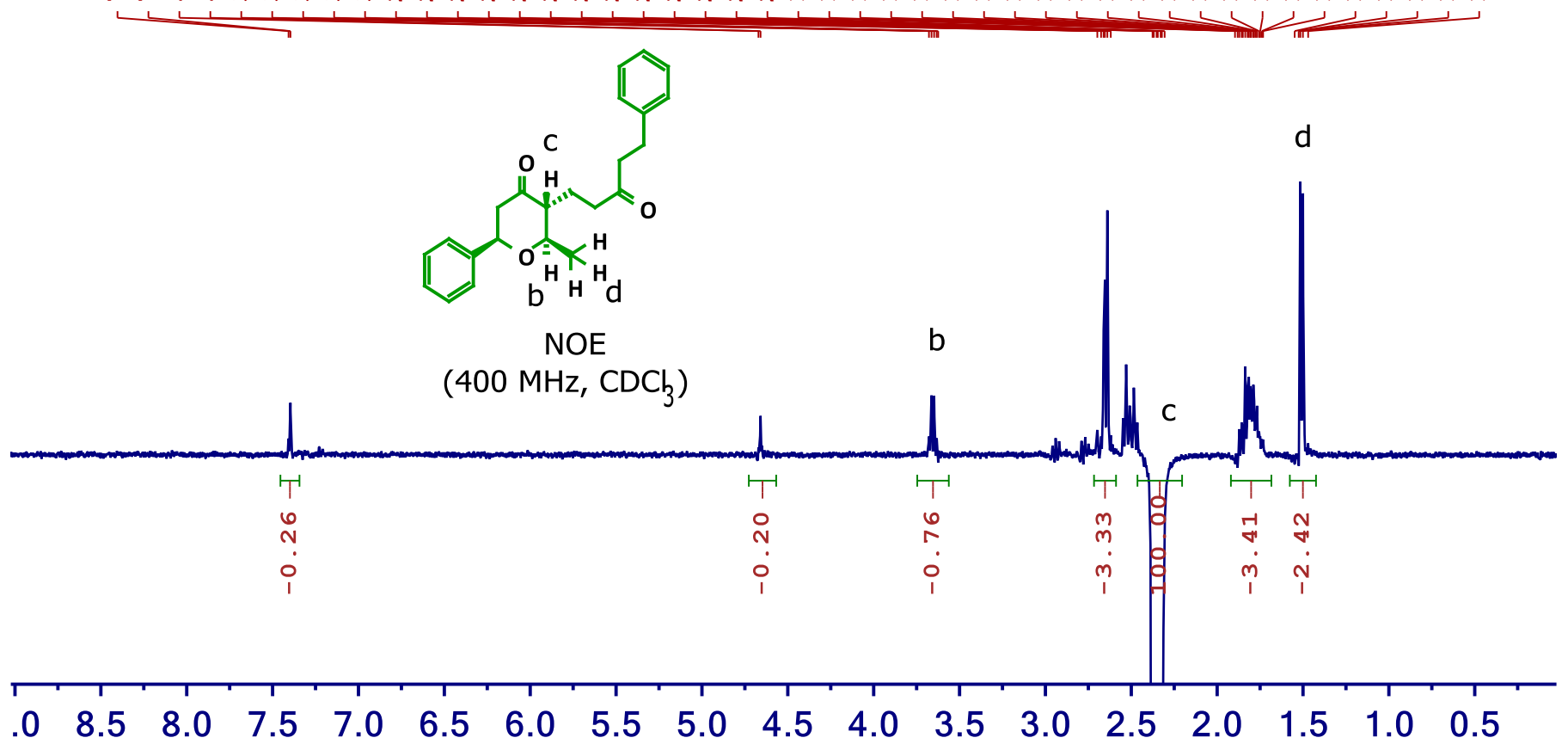
(2R,3R,6R)-2-methyl-3-(3-oxo-3-phenylpropyl)-6-phenyltetrahydro-4H-pyran-4-one

$5 \mathbf{e}$

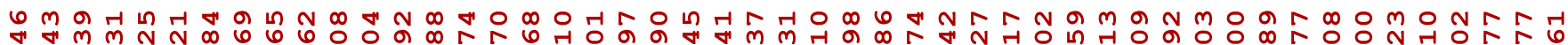

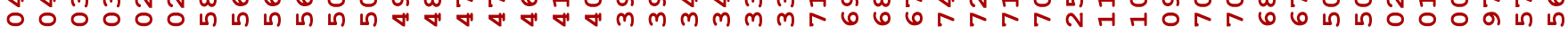

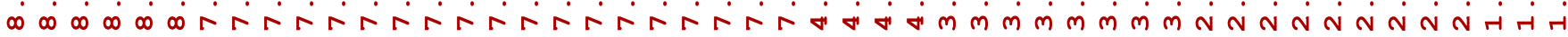
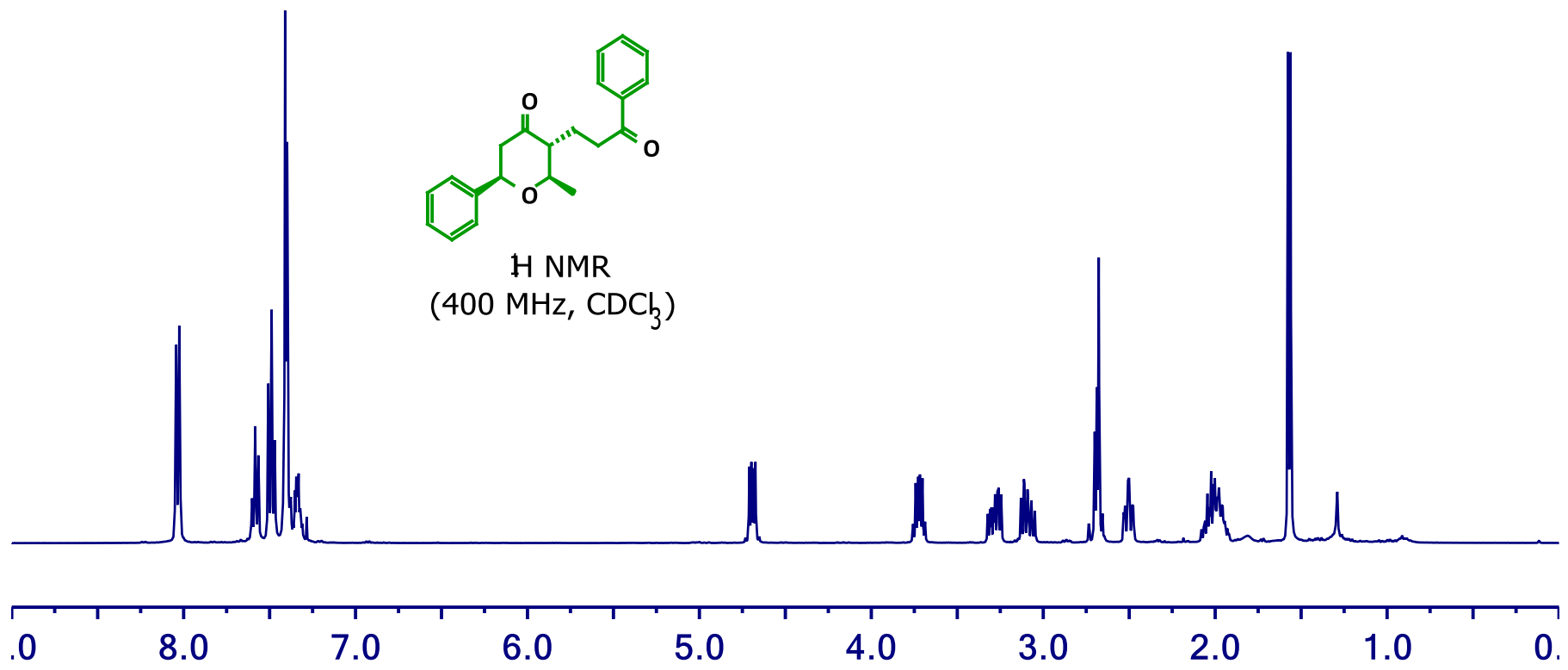

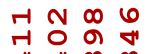

F" $m m$

$\therefore \sin$

ris

\#ت

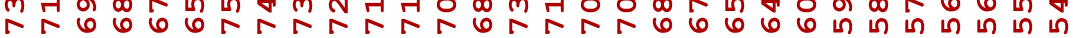

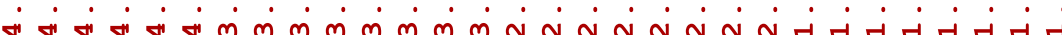

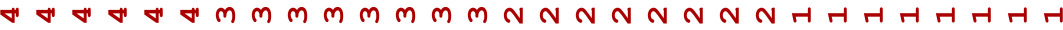

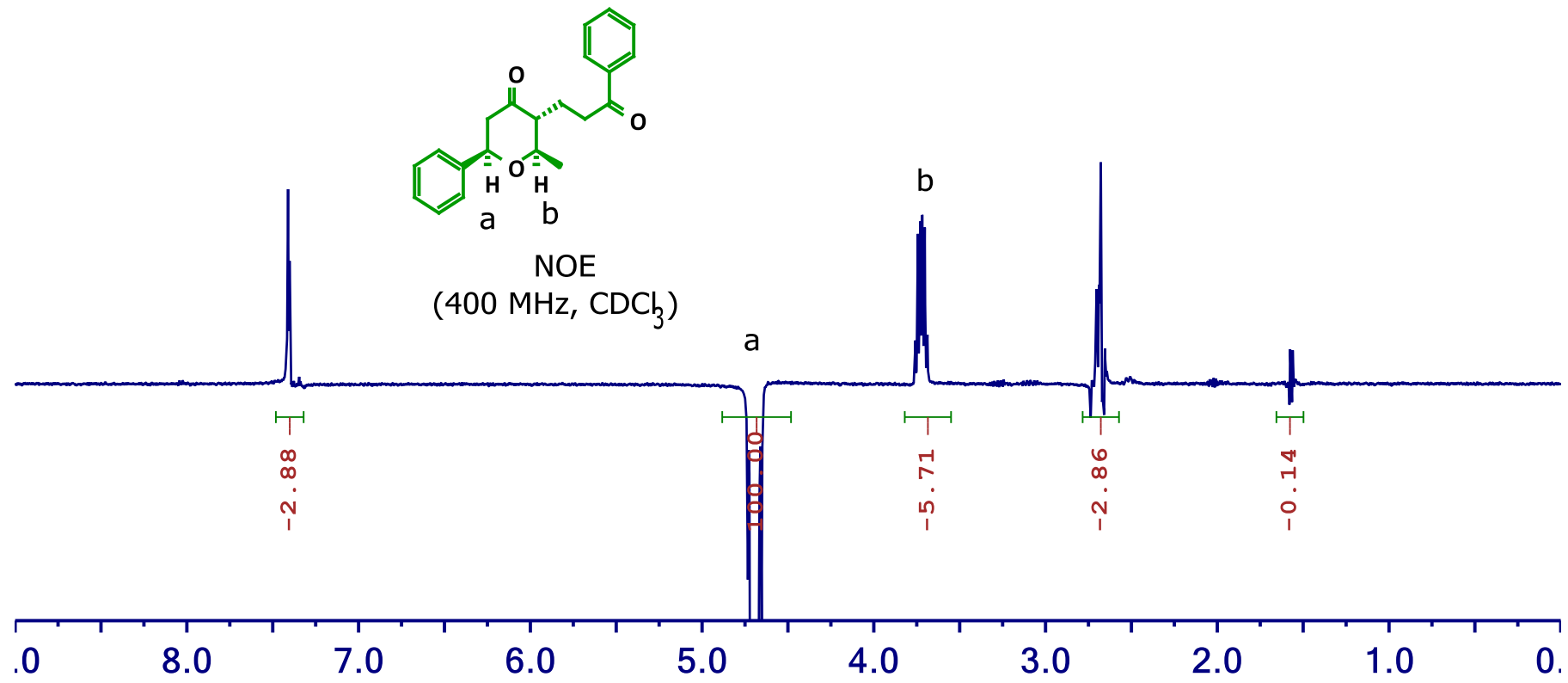


$(2 R, 3 R, 6 R)-2-m e t h y l-3-(3-o x o-3-p h e n y l p r o p y l)-6-p h e n y l t e t r a h y d r o-4 H-p y r a n-4-o n e$

$5 \mathrm{e}$

m

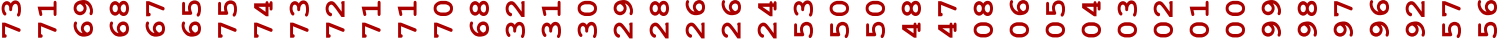

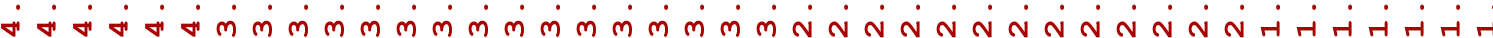

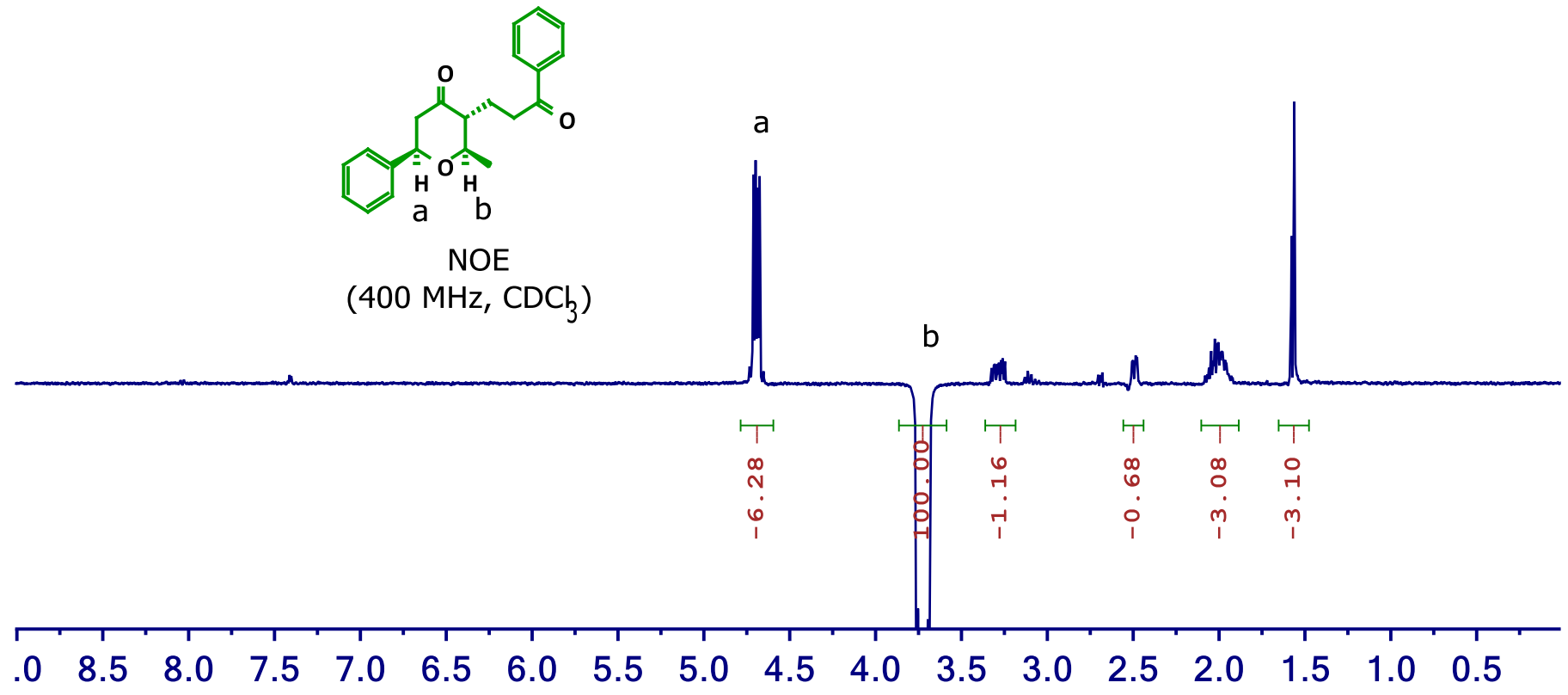

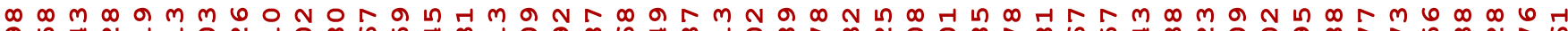

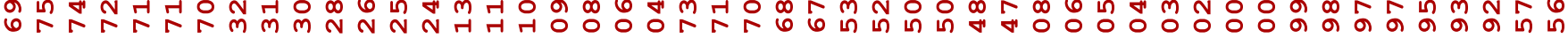

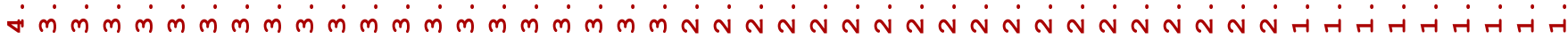
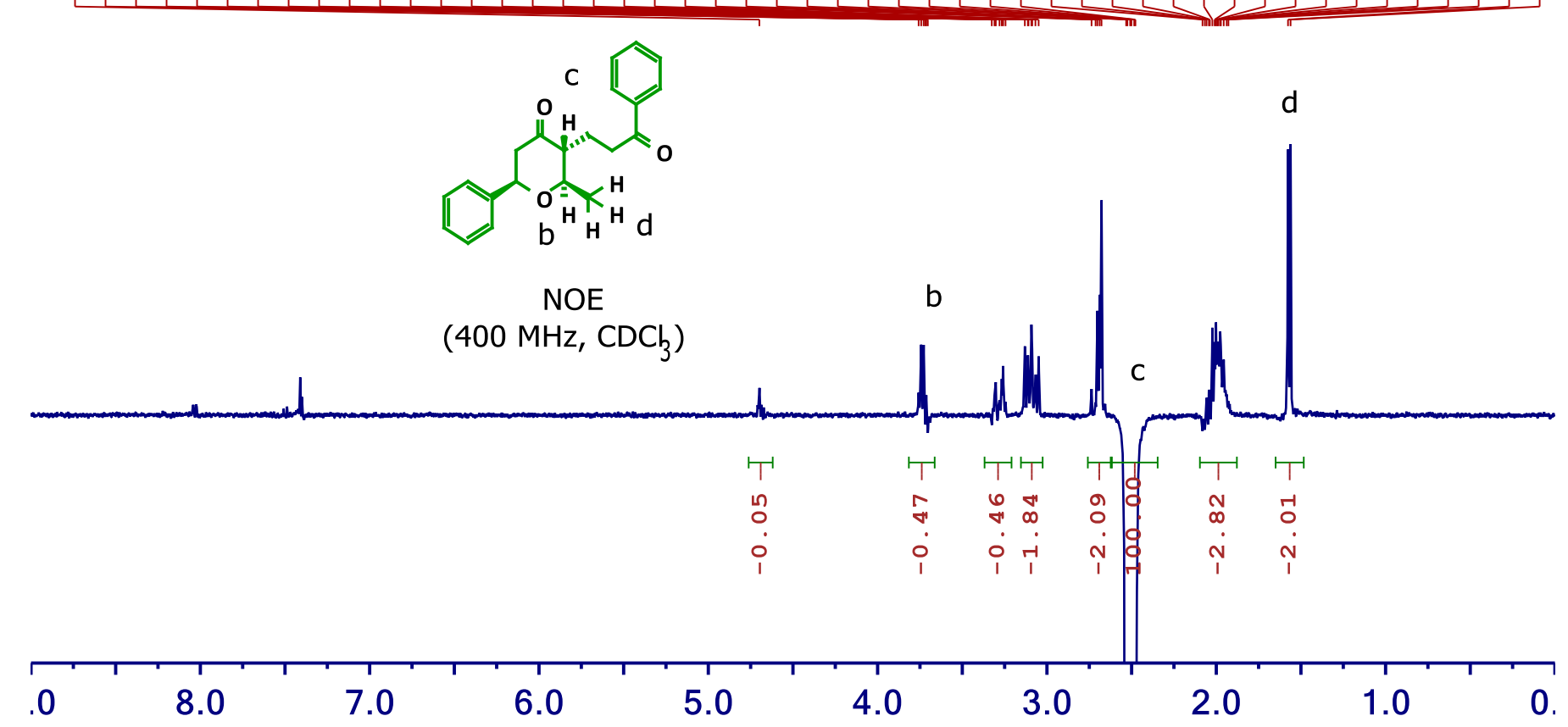


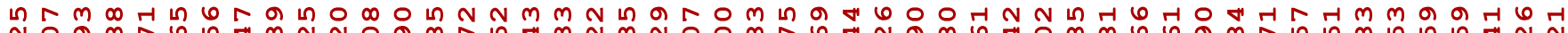
$\begin{array}{lll} & \\ & \end{array}$

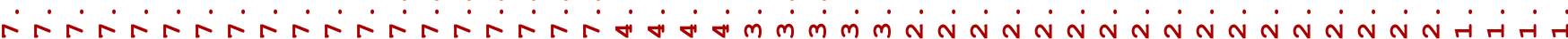

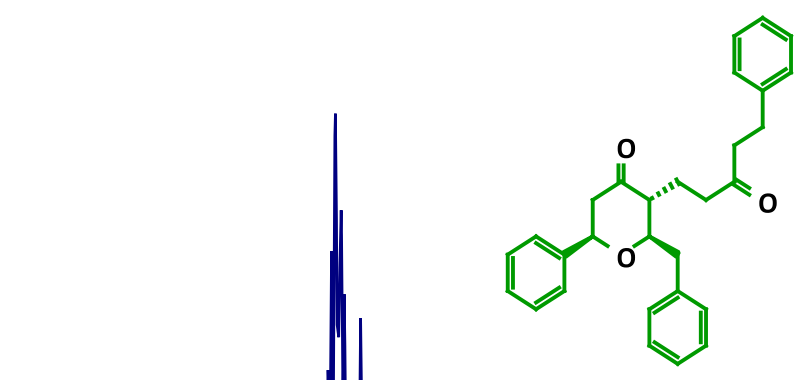

$1 \mathrm{H} N \mathrm{NMR}$

$\left(400 \mathrm{MHz}, \mathrm{CDCh}_{3}\right)$

\begin{tabular}{llllllllllll}
\hline .0 & 8.0 & 7.0 & 6.0 & 5.0 & 4.0 & 3.0 & 2.0 & 1.0 & 0.0
\end{tabular}

50 욜 i. m. m riris $\rightarrow$<smiles>O=C(CCc1ccccc1)CC[C@@H]1[C](Cc2ccccc2)OC(c2ccccc2)CC1=O</smiles>

NOE
6)

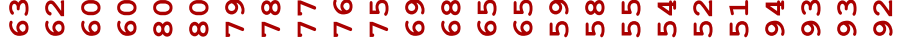
? 6 .

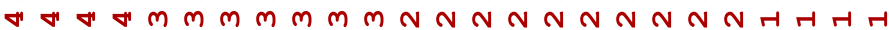

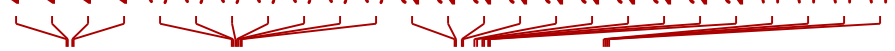

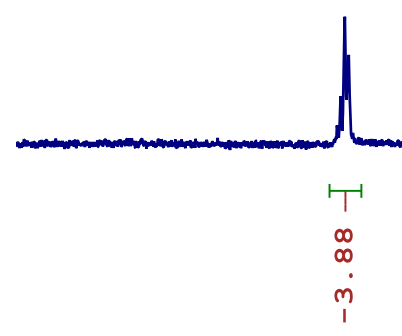

(400 $\left.\mathrm{MHz}, \mathrm{CDCl}_{3}\right)$

b
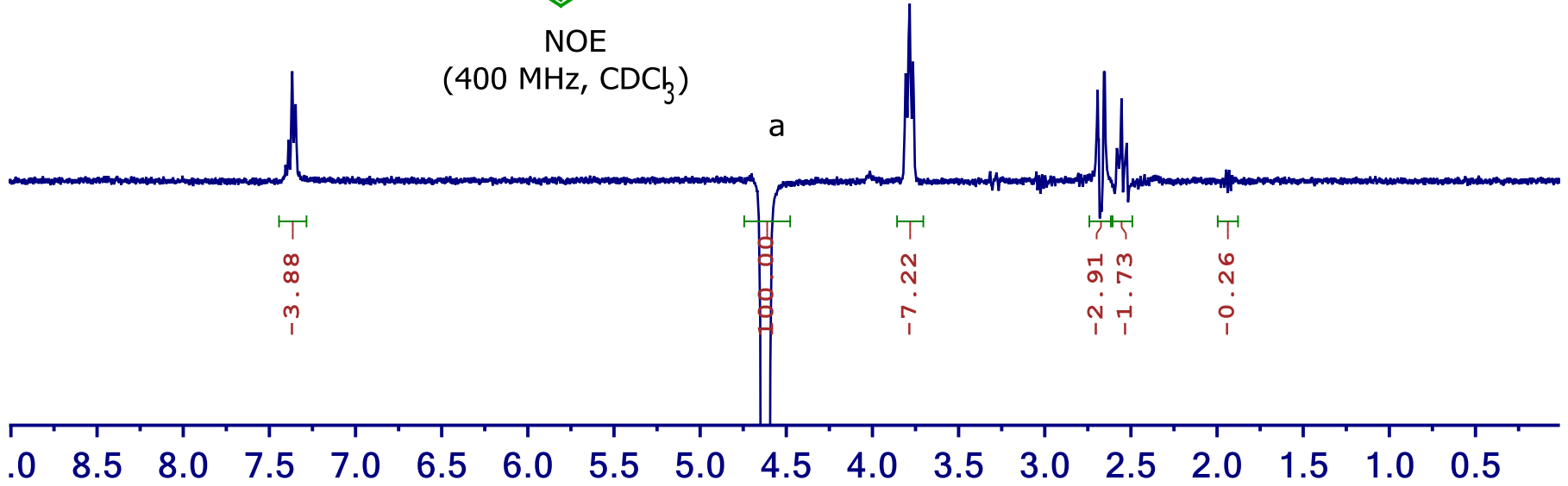
(2R,3R,6R)-2-benzyl-3-(3-oxo-5-phenylpentyl)-6-phenyltetrahydro-4H-pyran-4-one $5 \mathbf{k}$
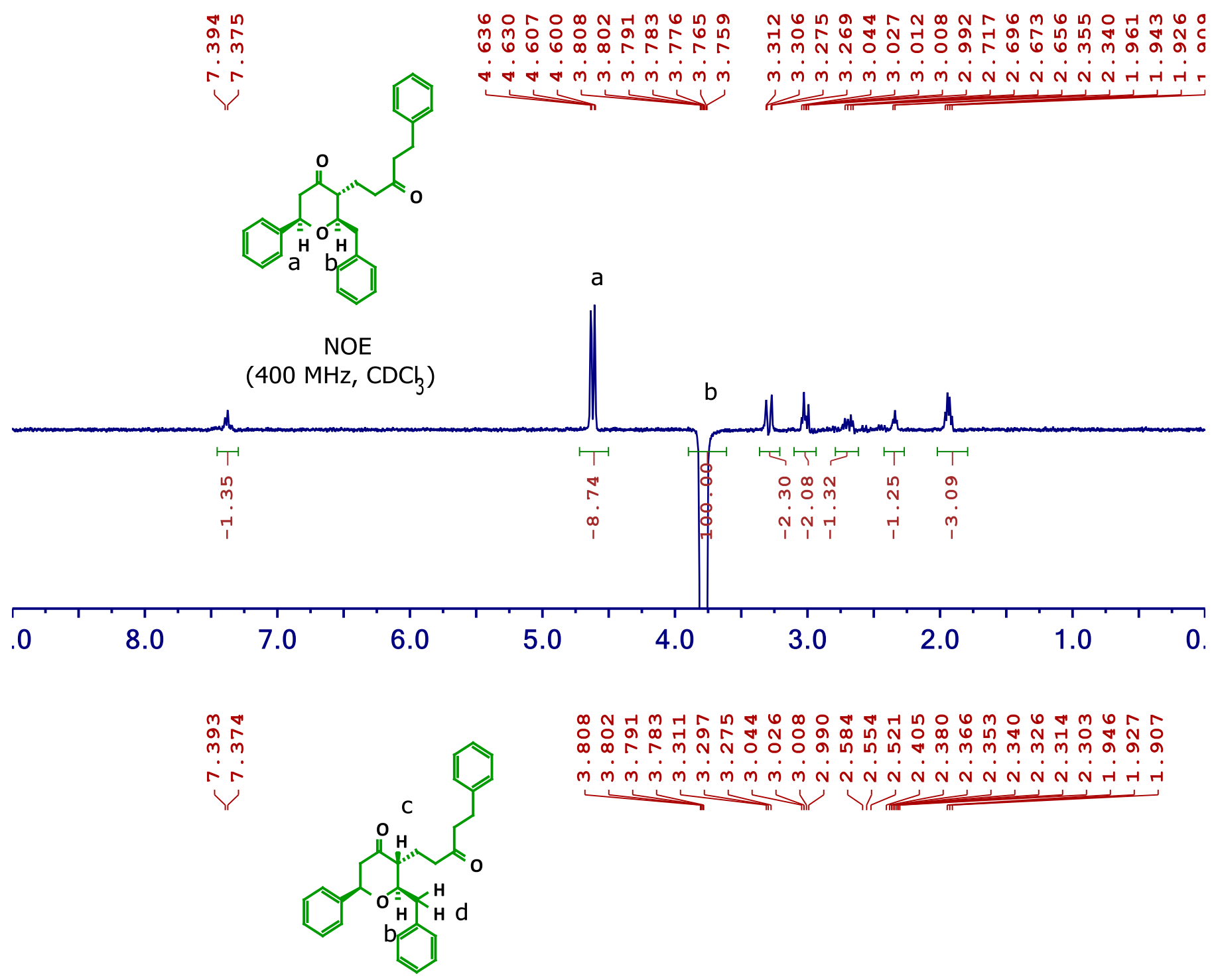

$\infty N H M H N$ M

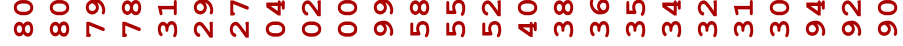

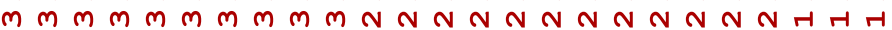

NOE

(400 $\left.\mathrm{MHz}, \mathrm{CDC}_{3}\right)$

d

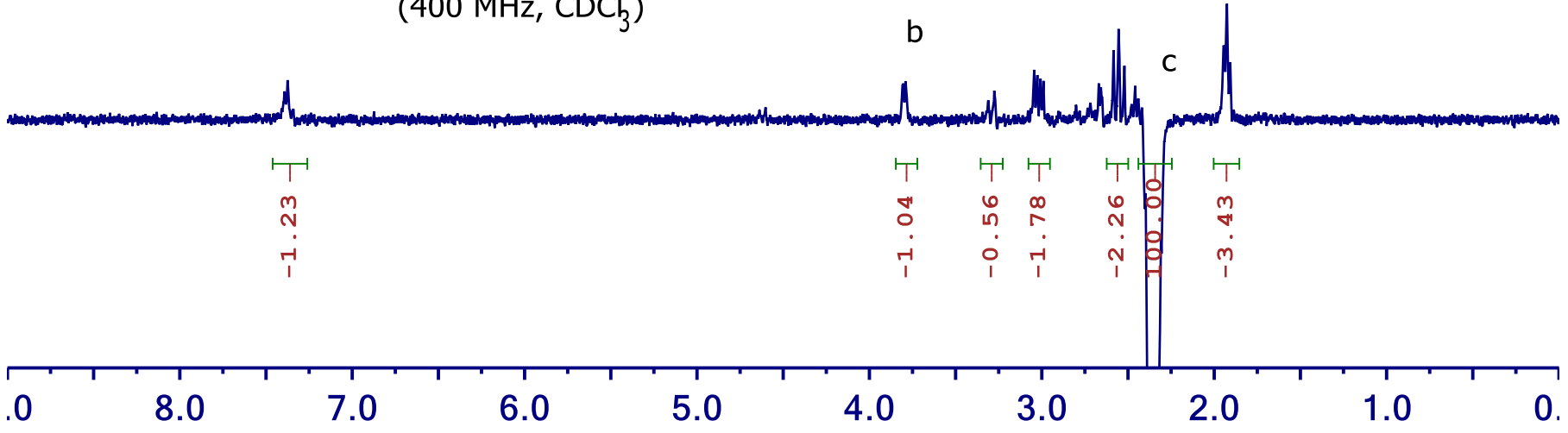


60 几

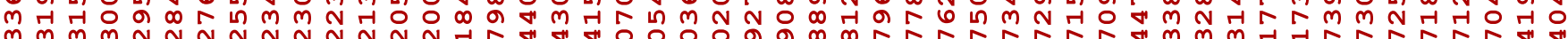

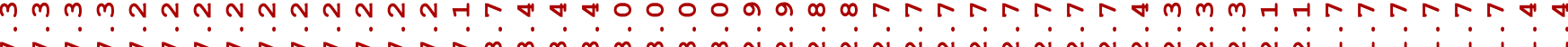

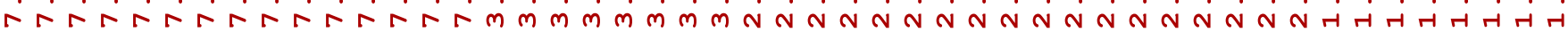

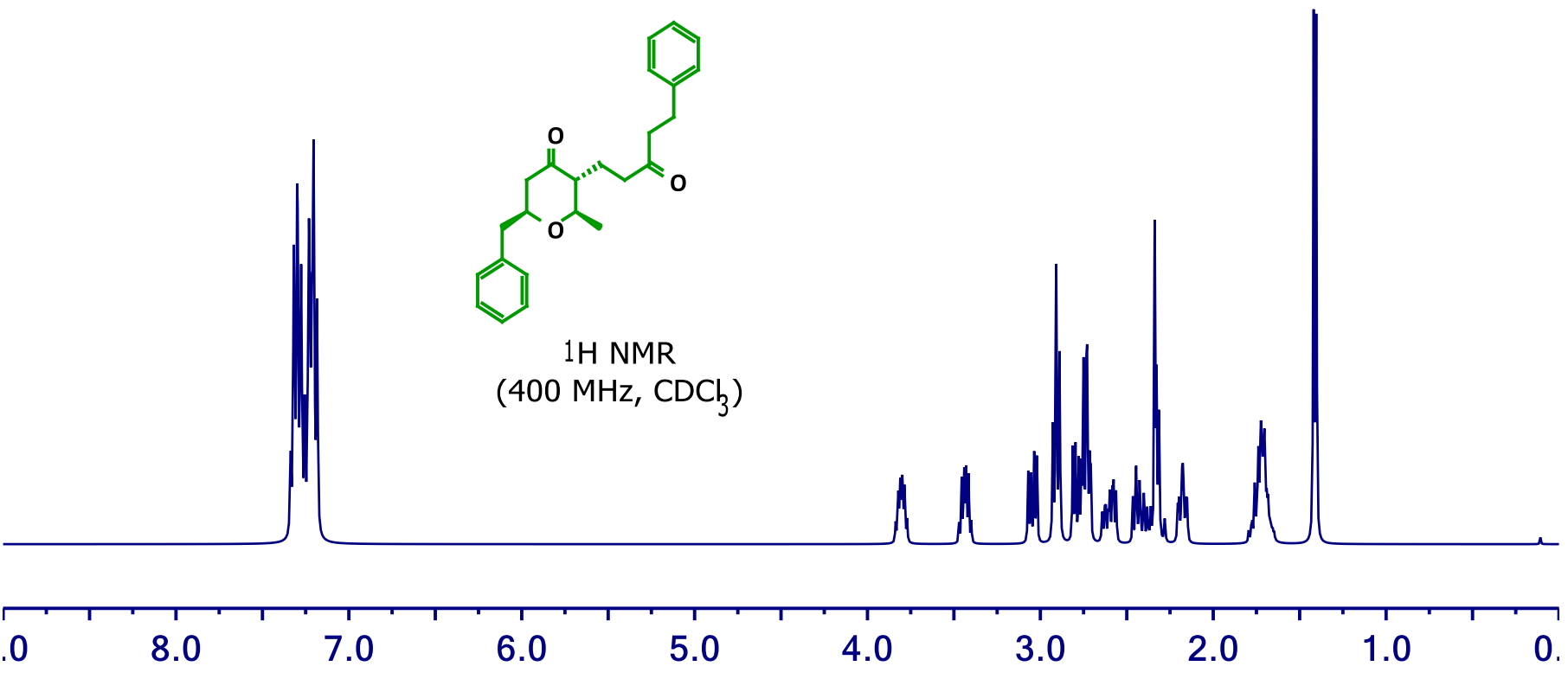

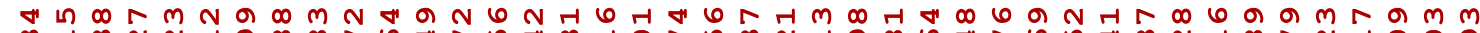

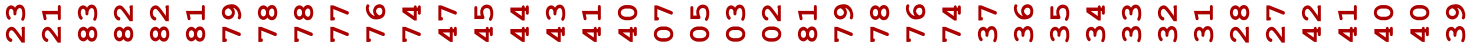

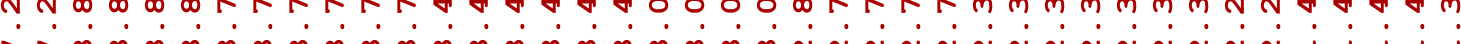

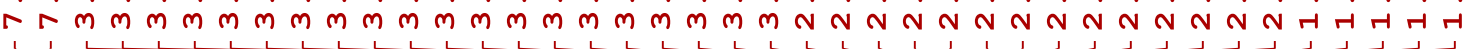

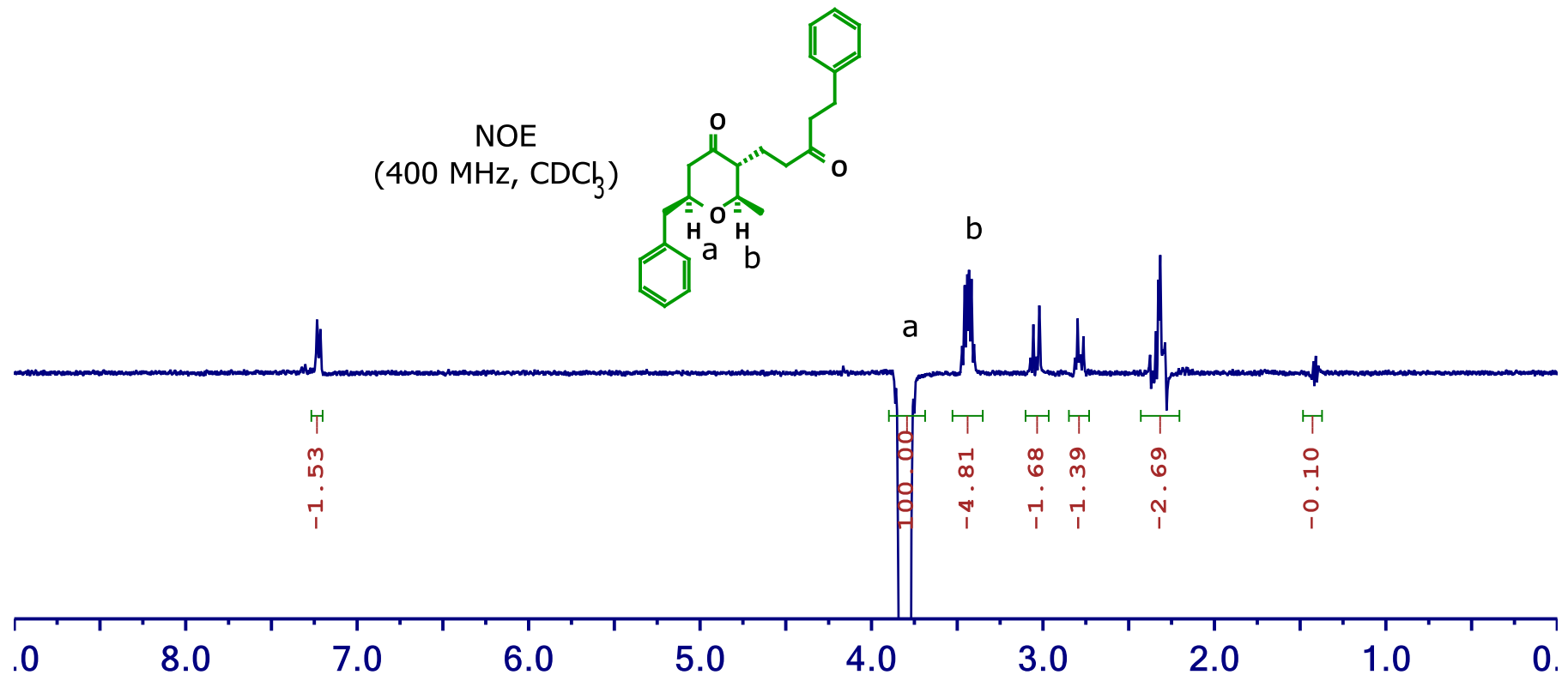


(2R,3 R,6S)-6-benzyl-2-methyl-3-(3-oxo-5-phenylpentyl)tetrahydro-4H-pyran-4-one $5 \mathrm{w}$
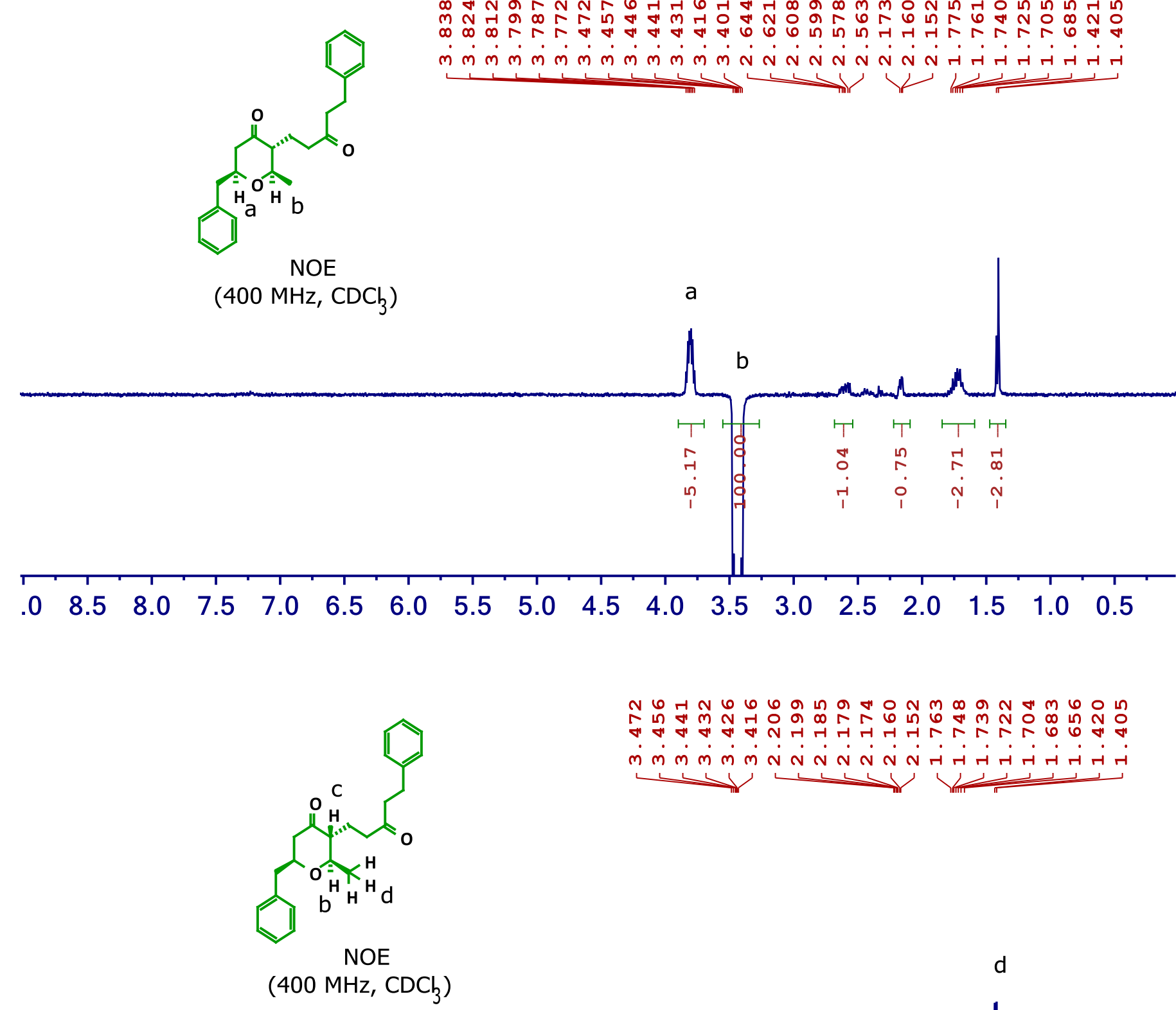

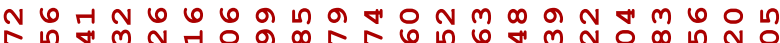

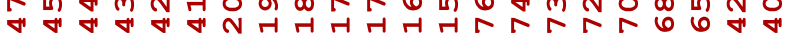
$\dot{m} \dot{m} \dot{m} \dot{m} \dot{m} \dot{N} \dot{N} \dot{N} \dot{N} \dot{N} \dot{H} \dot{H} \dot{H} \dot{H} \dot{H} \dot{H} \dot{H} \dot{H} \dot{H}$

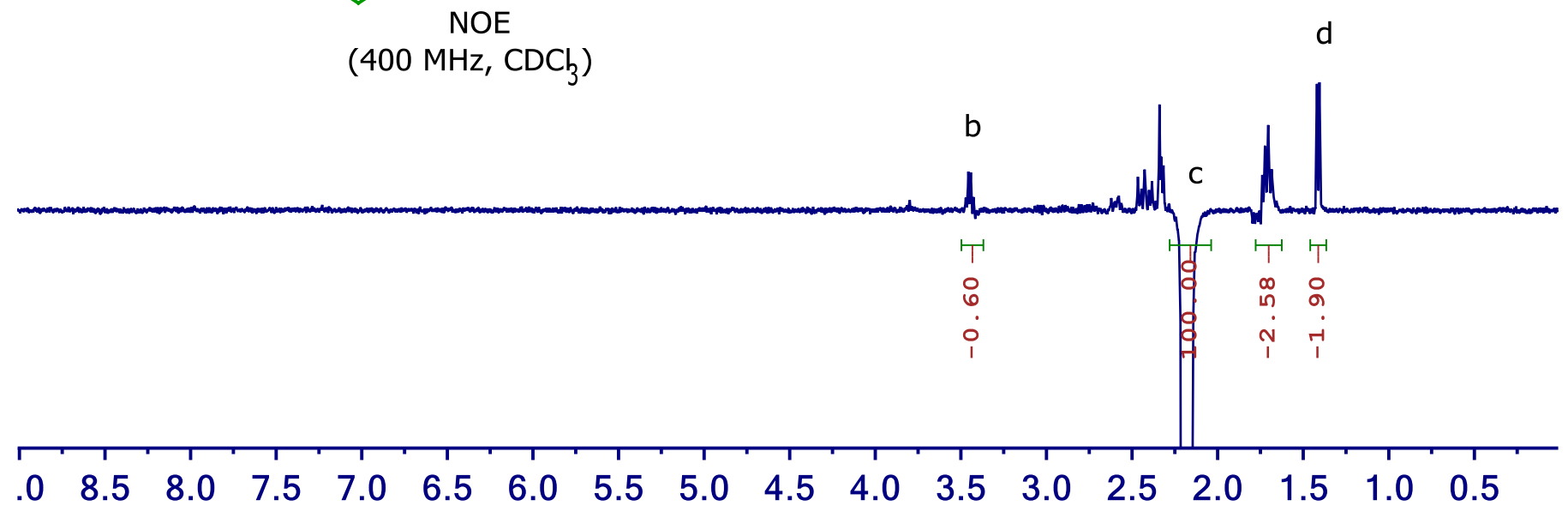


(2R,5R,6R)-2,6-dimethyl-5-(3-oxo-5-phenylpentyl)-2-phenyltetrahydro-4H-pyran-4-one $5 \mathrm{y} 1$

능웅 송 埩

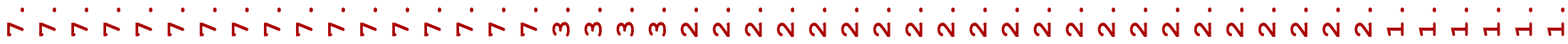<smiles></smiles>

$1 \mathrm{H} N M R$

$\left(400 \mathrm{MHz}, \mathrm{CDC}_{3}\right)$

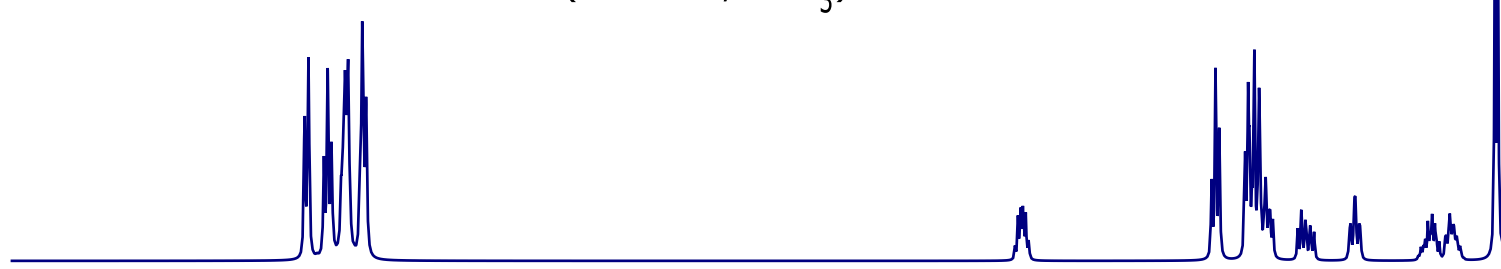

\begin{tabular}{lllllllllllllllllll}
\hline 8.5 & 8.0 & 7.5 & 7.0 & 6.5 & 6.0 & 5.5 & 5.0 & 4.5 & 4.0 & 3.5 & 3.0 & 2.5 & 2.0 & 1.5 & 1.0 & 0.5 & 0.1
\end{tabular}

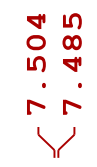<smiles>CC1OC(=O)C(CCC(=O)CCc2ccccc2)C(=O)[C@H]1c1ccccc1</smiles>

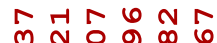
बै

$\dot{m} \dot{m} \dot{m} \dot{m}$

r m 농ํำ

$\sin$

iं

in

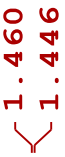

NOE

(400 MHz, $\mathrm{CDCh}_{3}$ )

b

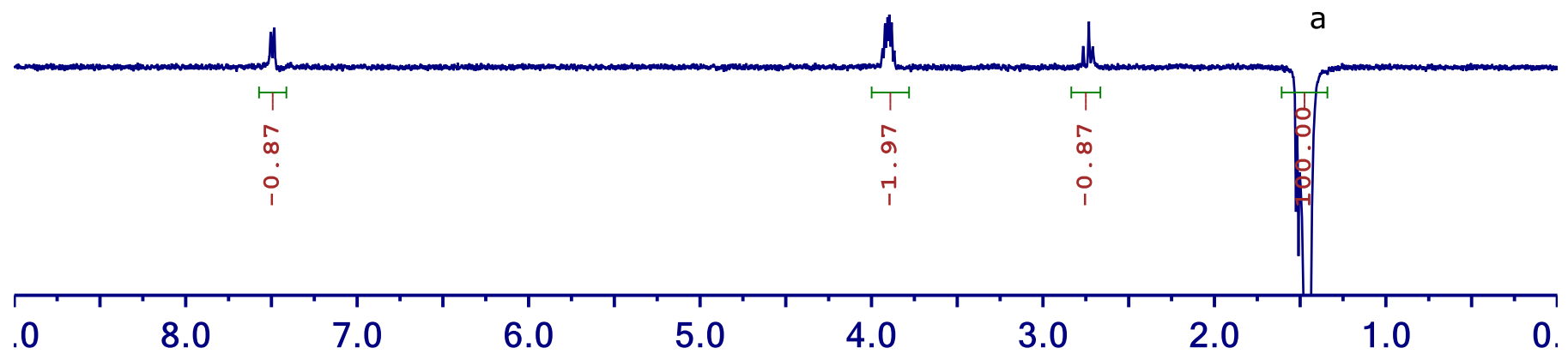


$(2 R, 5 R, 6 R)-2,6$-dimethyl-5-(3-oxo-5-phenylpentyl)-2-phenyltetrahydro-4H-pyran-4-one $5 y 1$

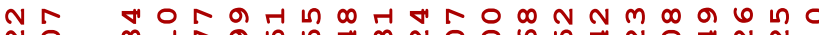
o,

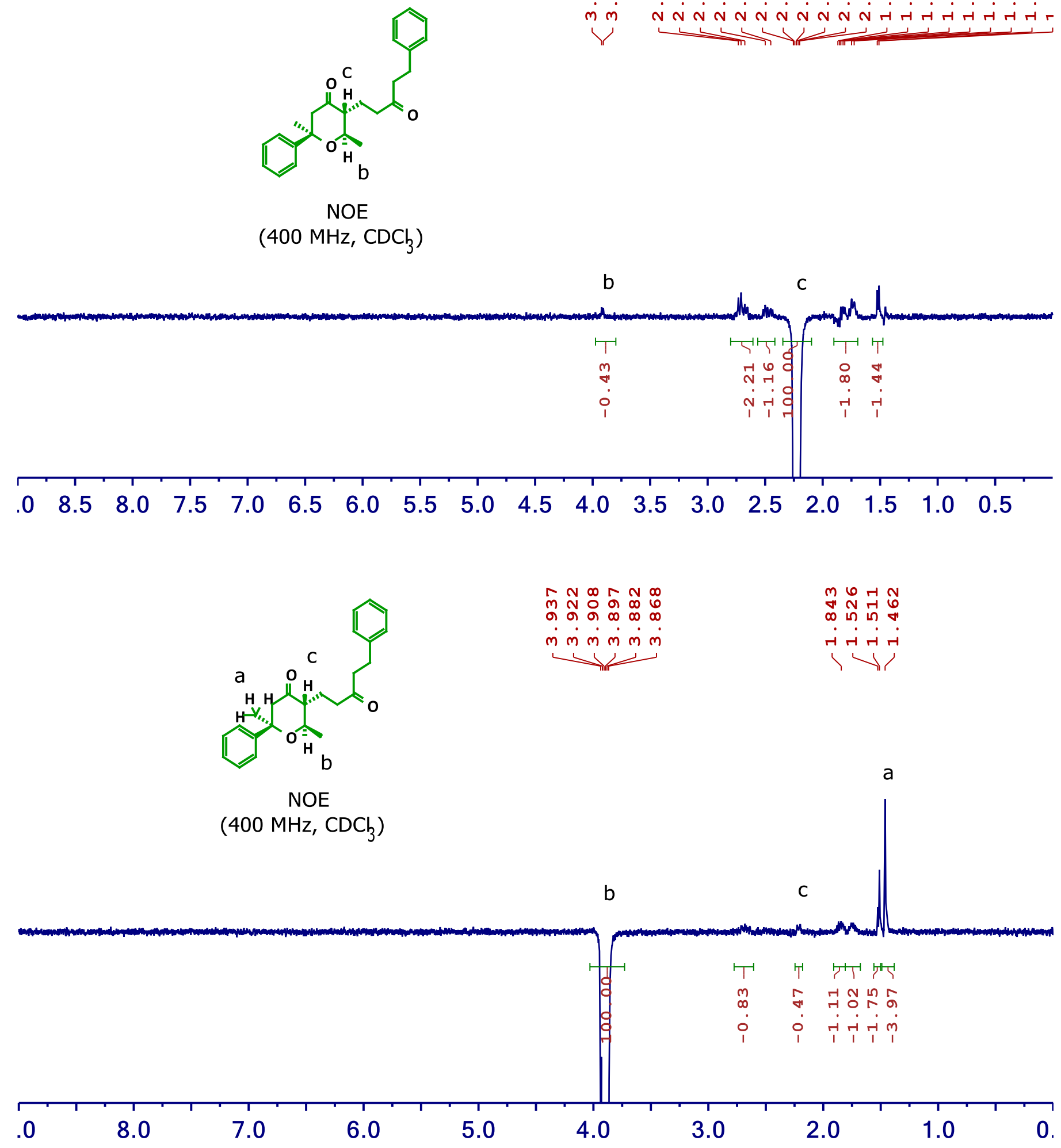

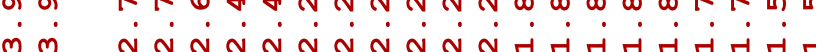


긍요

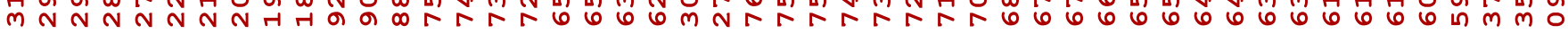

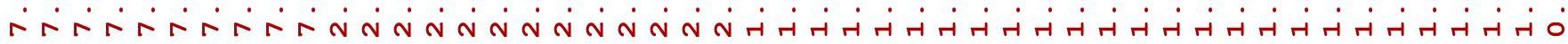
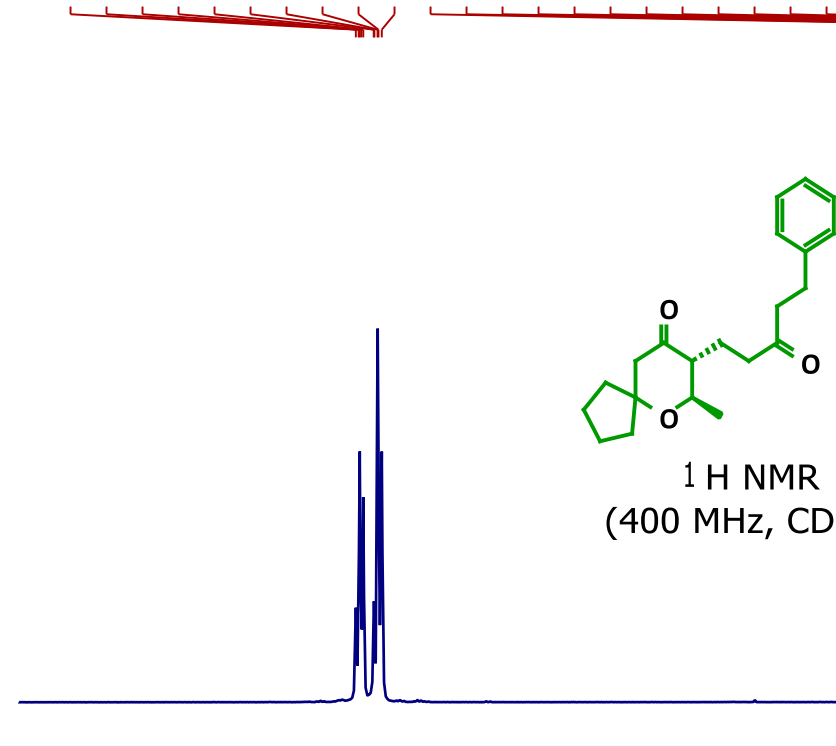

$1 \mathrm{H} N M R$

(400 $\left.\mathrm{MHz}, \mathrm{CDC}_{3}\right)$

\begin{tabular}{llllllllllll}
\hline .0 & 8.0 & 7.0 & 6.0 & 5.0 & 4.0 & 3.0 & 2.0 & 1.0 & 0.
\end{tabular}

NOE

$\left(400 \mathrm{MHz}, \mathrm{CDC}_{3}\right)$

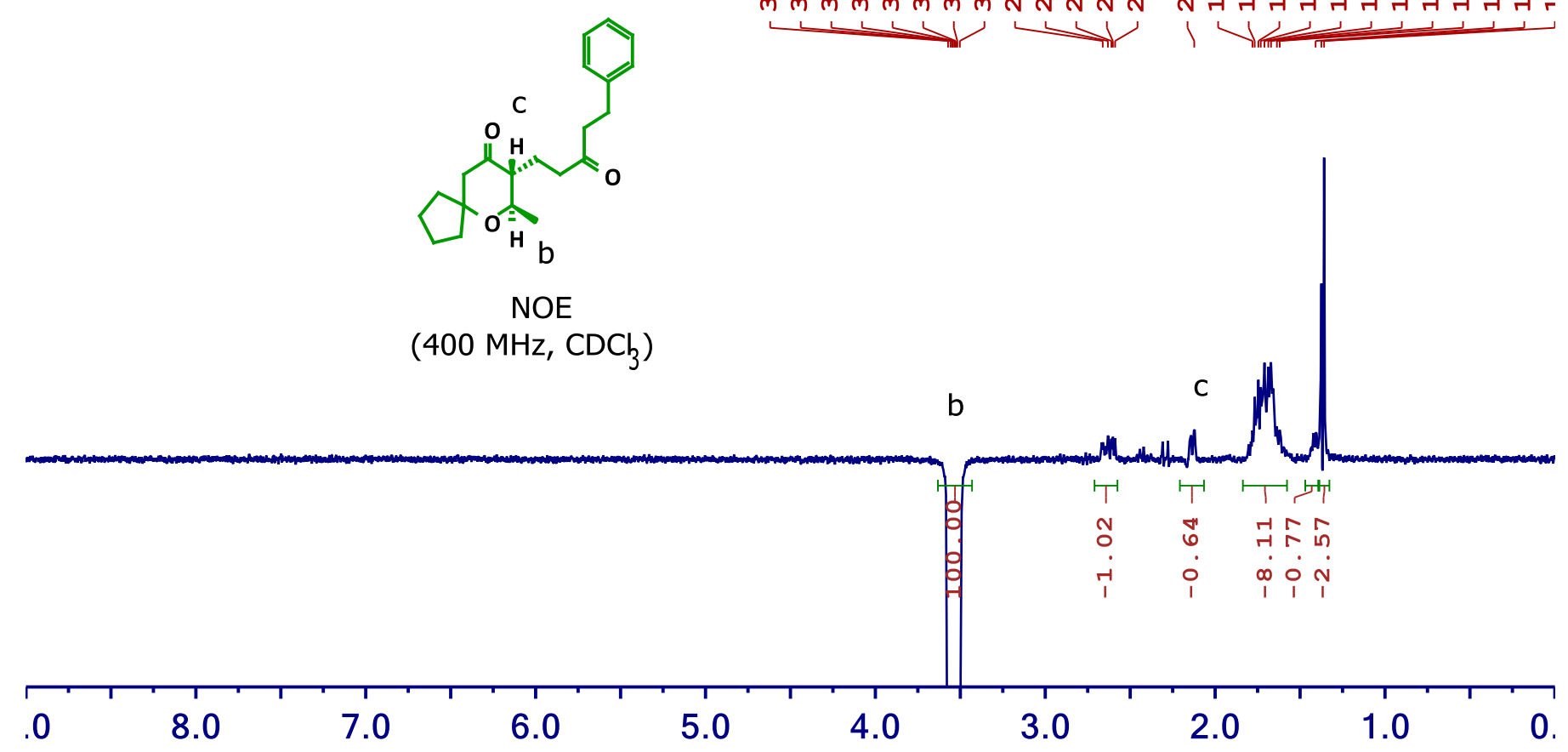

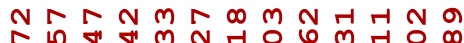
in ก ก เก $\dot{m} \dot{m} \dot{m} \dot{m} \dot{m} \dot{m} \dot{m} \dot{N} \dot{N} \dot{N} \dot{N}$

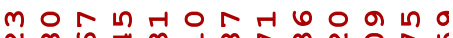

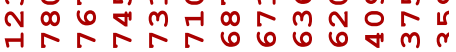

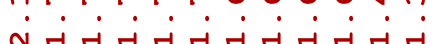
मे 
(7R,8R)-7-methyl-8-(3-oxo-5-phenylpentyl)-6-oxaspiro[4.5]decan-9-one

5aa

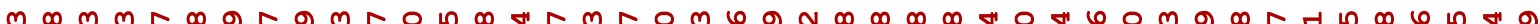
ก

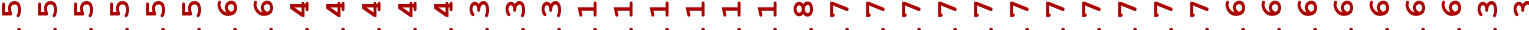

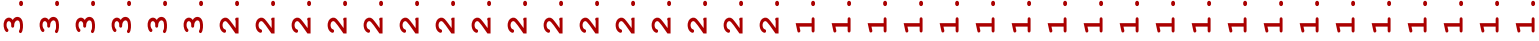
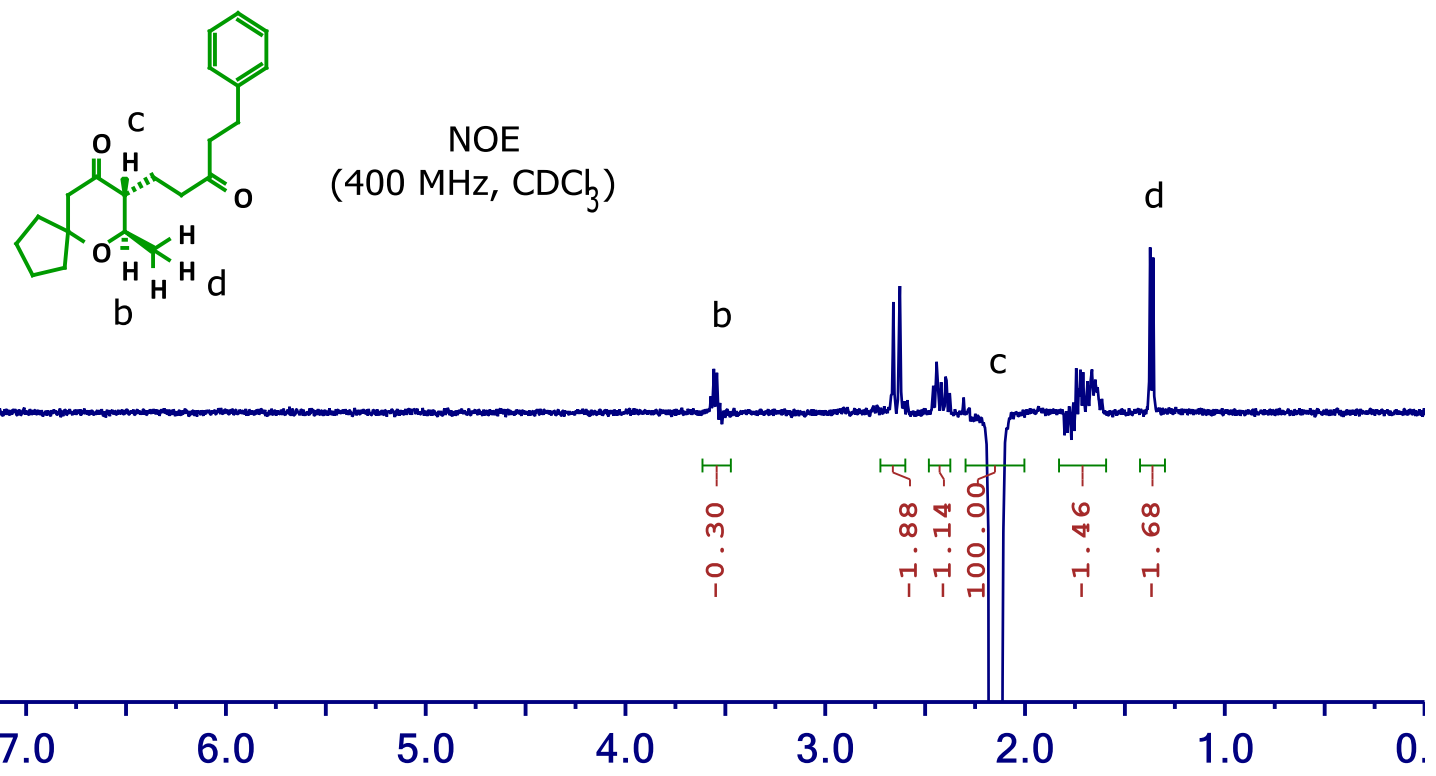
(2R,3R,6R)-3-(3-(2,6-dichlorophenyl)-3-oxopropyl)-2-methyl-6-phenyltetrahydro-4H-pyran-4-one

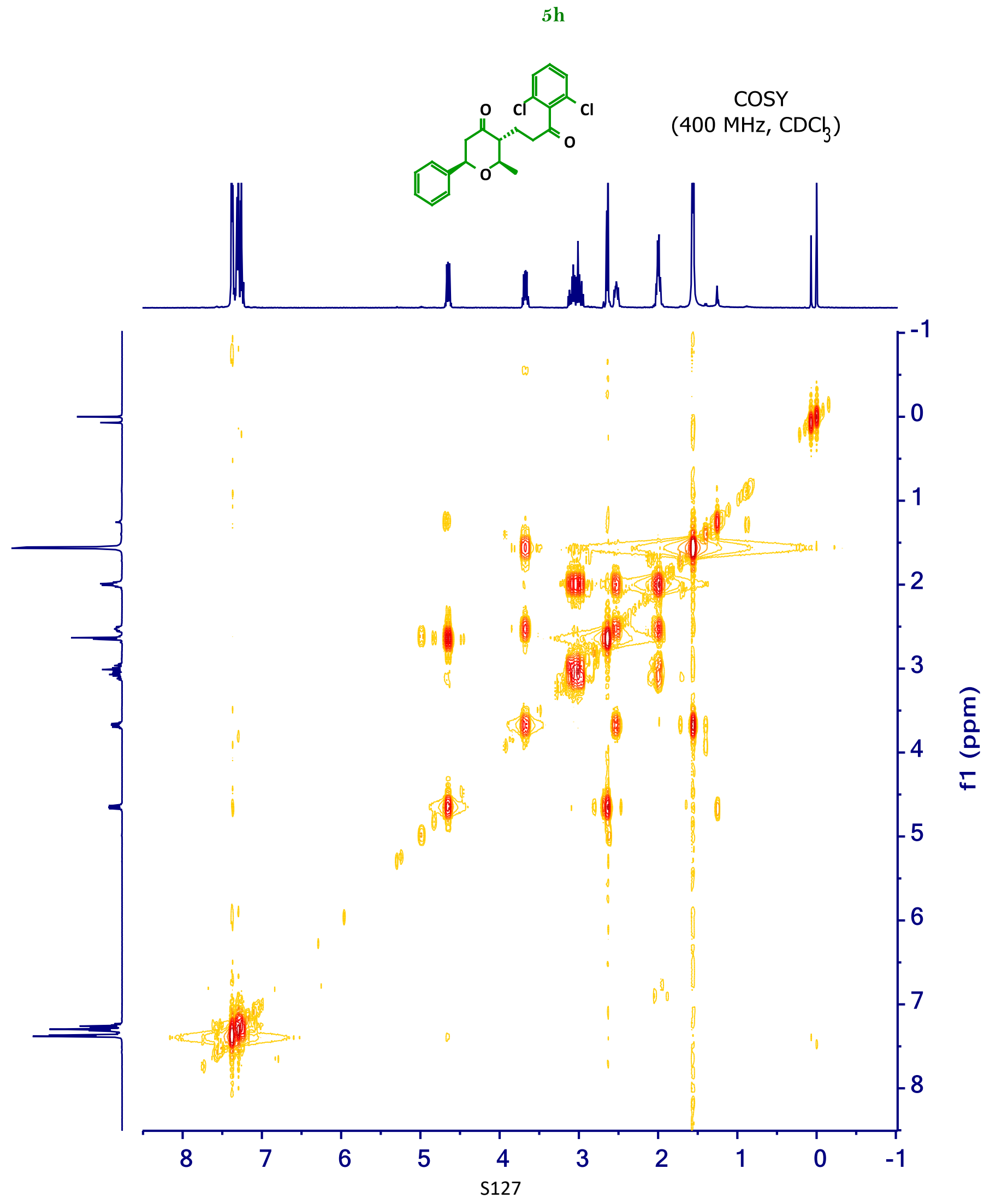


(1R,3R,4aR,5S,8aR)-4a-hydroxy-1-methyl-5-phenyl-3-(o-tolyl)octahydro-6H-isochromen-6-one

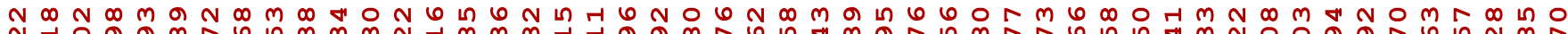

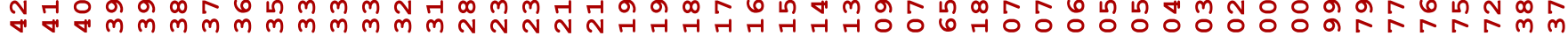

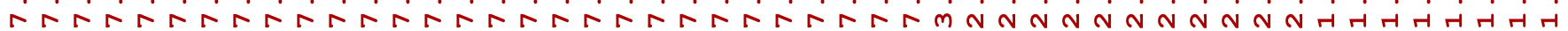

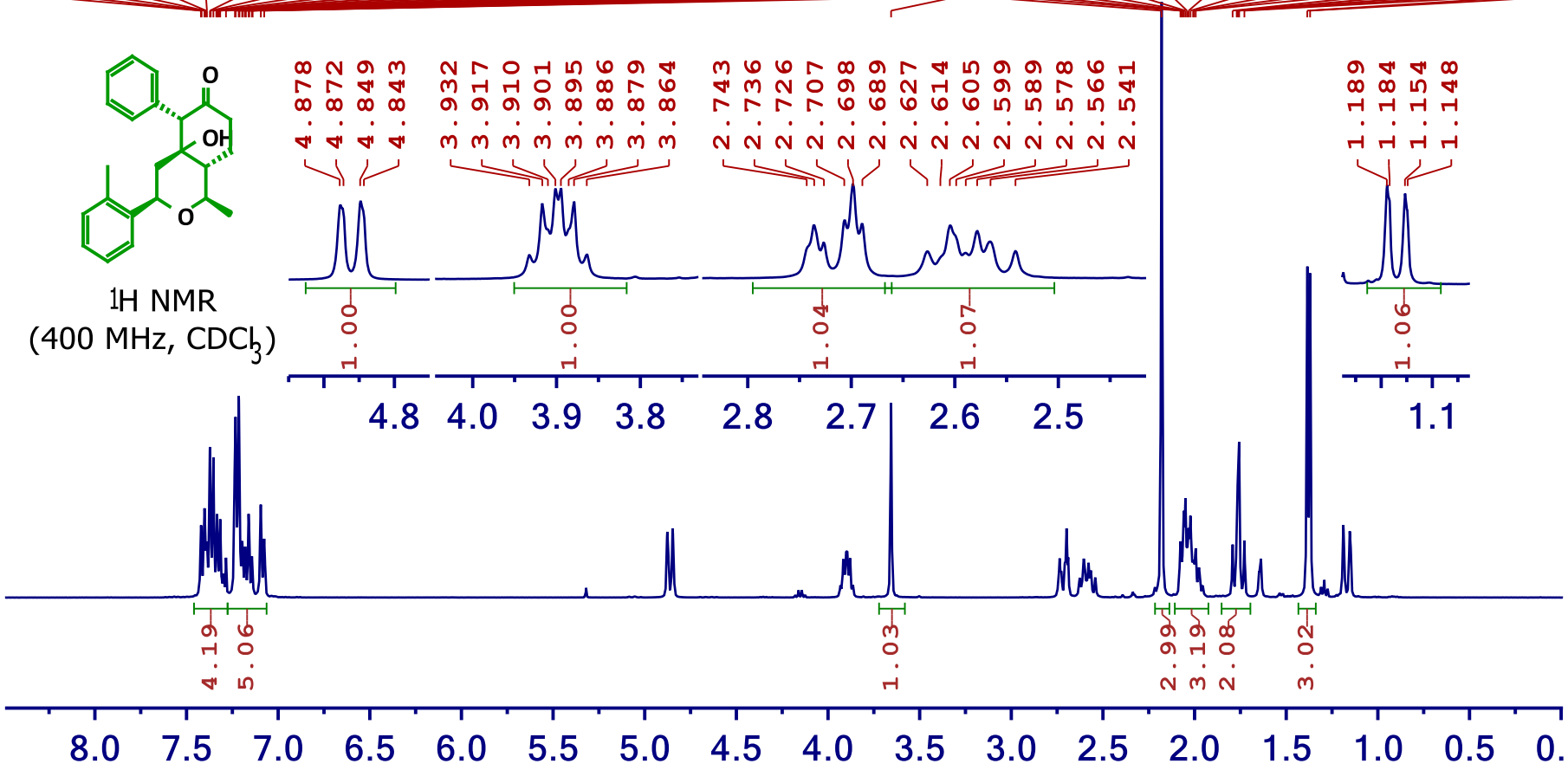

$\infty$ a $\dot{0} \dot{\nabla} \dot{m} \dot{0} \dot{0} \dot{\infty} \dot{\sim} \dot{\sim} \dot{0}$

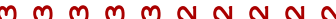

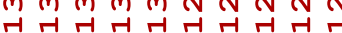

$\underbrace{2}$

$13 \mathrm{C}\{1 \mathrm{H}\} \mathrm{NMR}$ (100 $\mathrm{MHz}, \mathrm{CDC}_{3}$ )

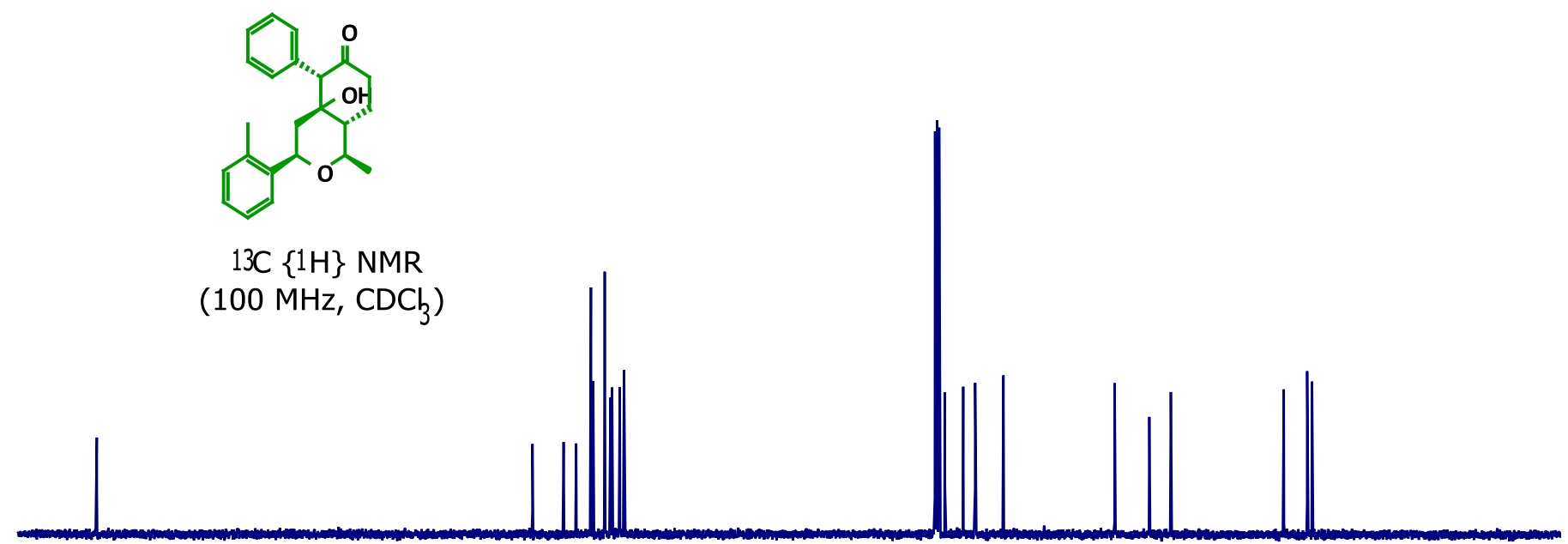

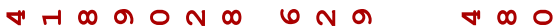

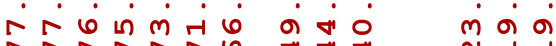

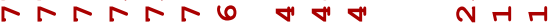

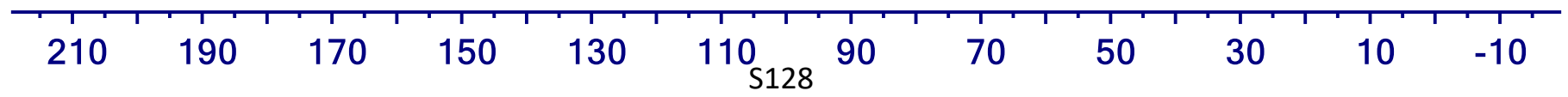


(1R,3R,4aR,5S,8aR)-4a-hydroxy-1-methyl-5-phenyl-3-(o-tolyl)octahydro-6H-isochromen-6-one

6

ก m

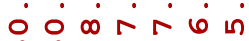

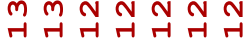

$\rightarrow$

○ $N \infty$

$\stackrel{m}{i} \dot{i} \dot{0}$

$1 / 1$

6 N 9

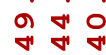

$\forall-\infty$

11

लं के

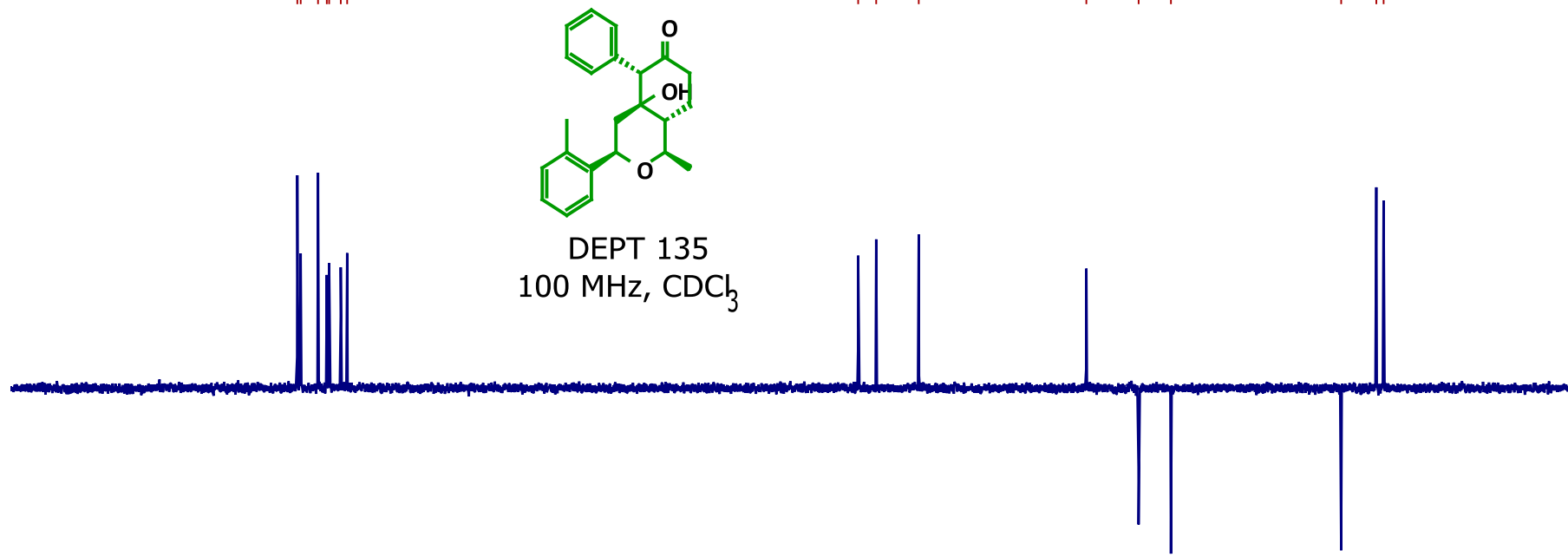

N $\mathrm{A}+\mathrm{T}$

$\backslash \backslash$

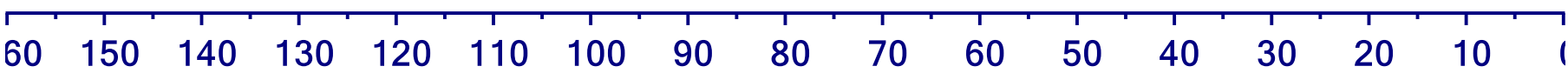


(1R,3R,4aR,5S,8aR)-4a-hydroxy-1-methyl-5-phenyl-3-(o-tolyl)octahydro-6H-isochromen-6-one

6

m

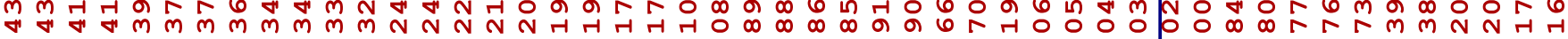

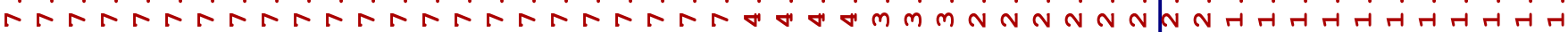<smiles>Cc1ccccc1C1CC2(c3ccccc3)OCCC(=O)[C@H]2C1C</smiles>

$1 \mathrm{H}$ NMR (400 $\mathrm{MHz}, \mathrm{CDCh}_{3}$ )
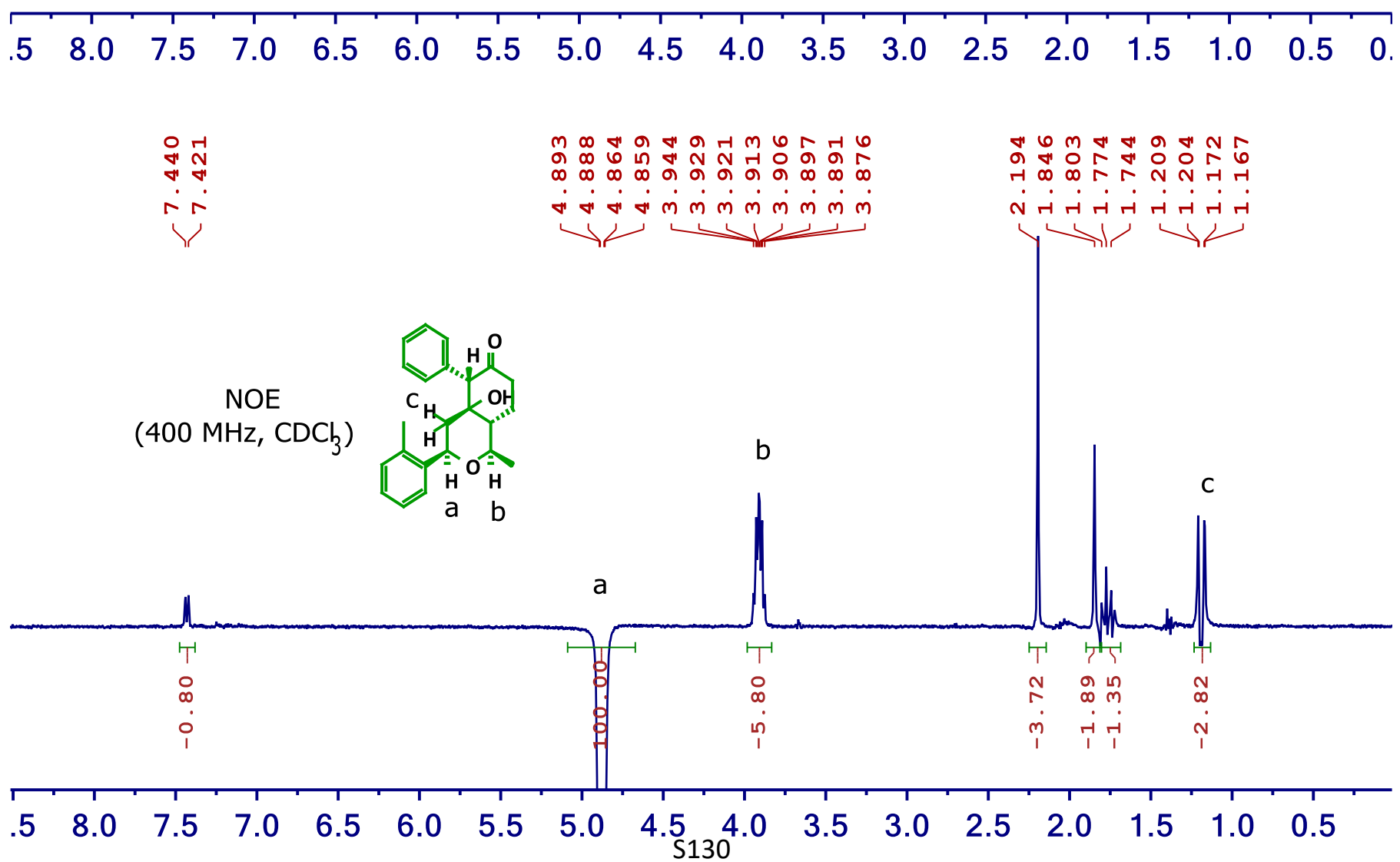
(1R,3R,4aR,5S,8aR)-4a-hydroxy-1-methyl-5-phenyl-3-(o-tolyl)octahydro-6H-isochromen-6-one

6

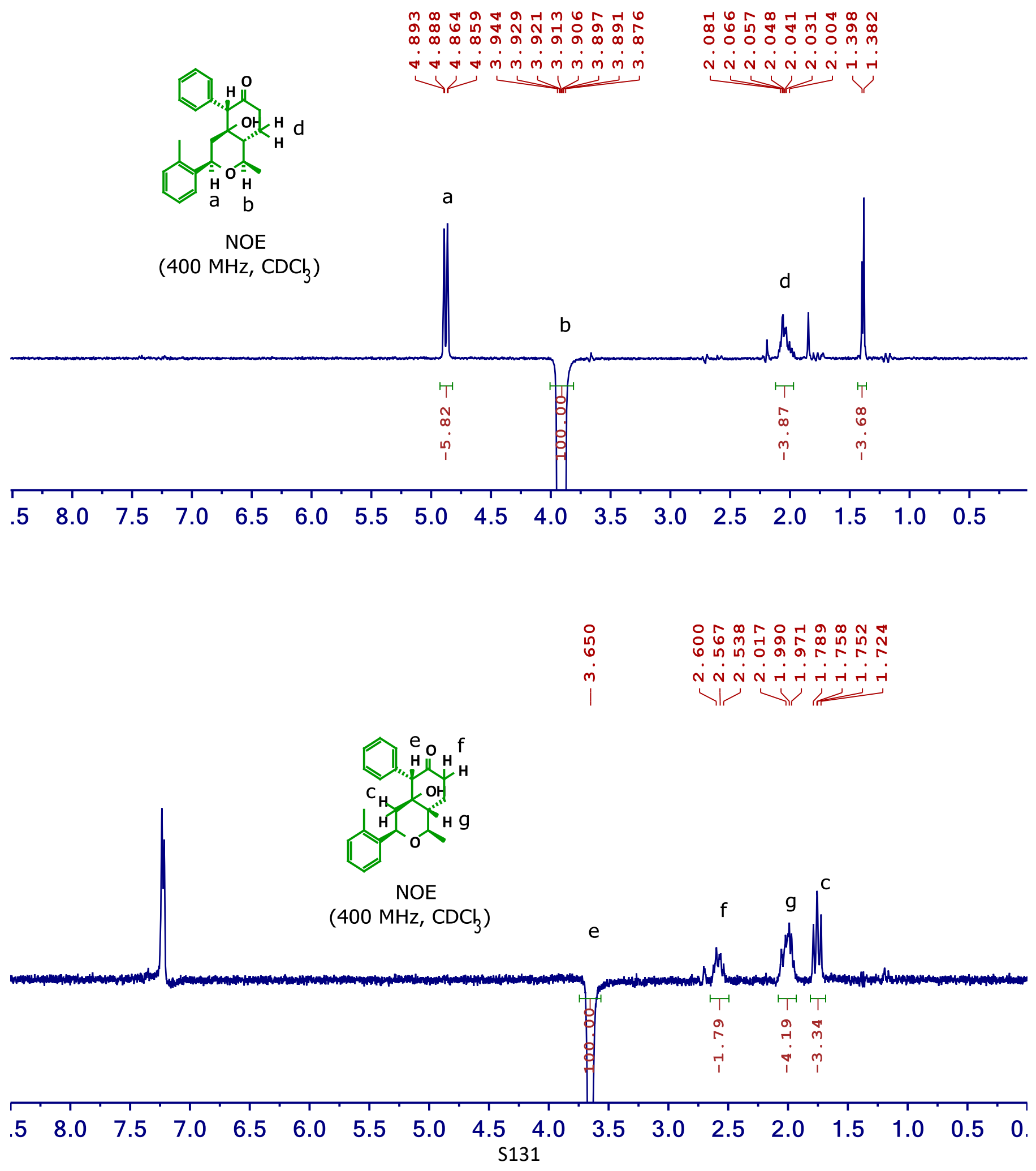


(1R,3R,4aR,5S,8aR)-4a-hydroxy-1-methyl-5-phenyl-3-(o-tolyl)octahydro-6H-isochromen-6-one

6

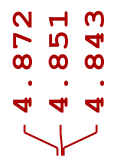

ตั

करr 1 r

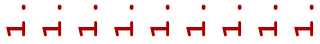

نं

N
n
$\dot{m}$
$\dot{1}$

$\longrightarrow$
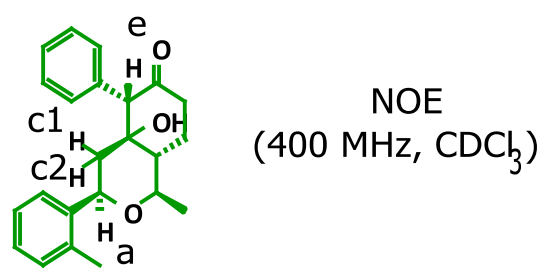

e

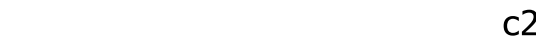

e

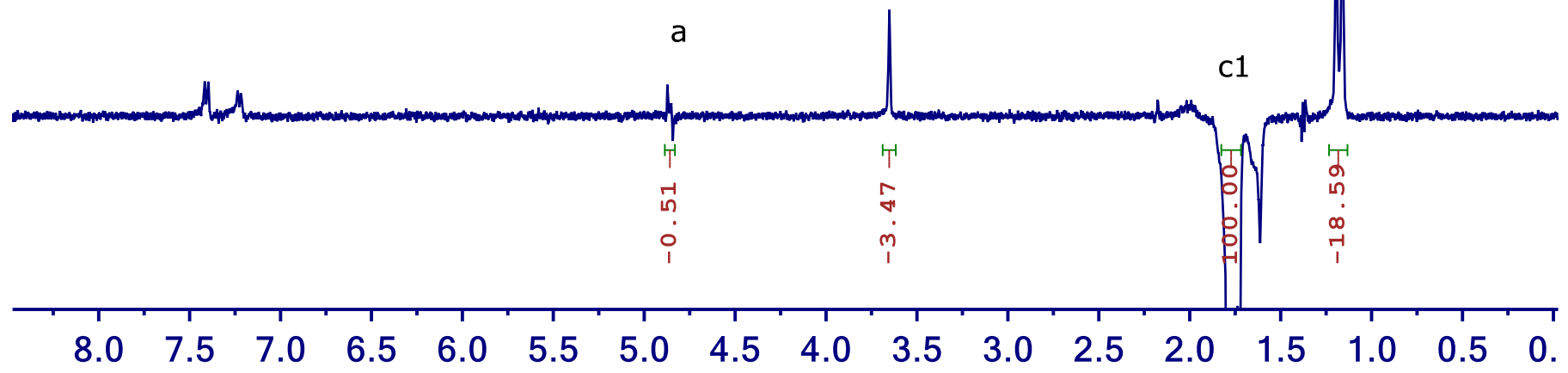

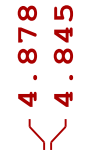

엇

N 4 H 4 $\dot{N} \dot{N} \dot{N} \dot{A} \dot{A} \dot{A} \dot{A} \dot{A} \dot{A} \dot{A} \dot{H}$

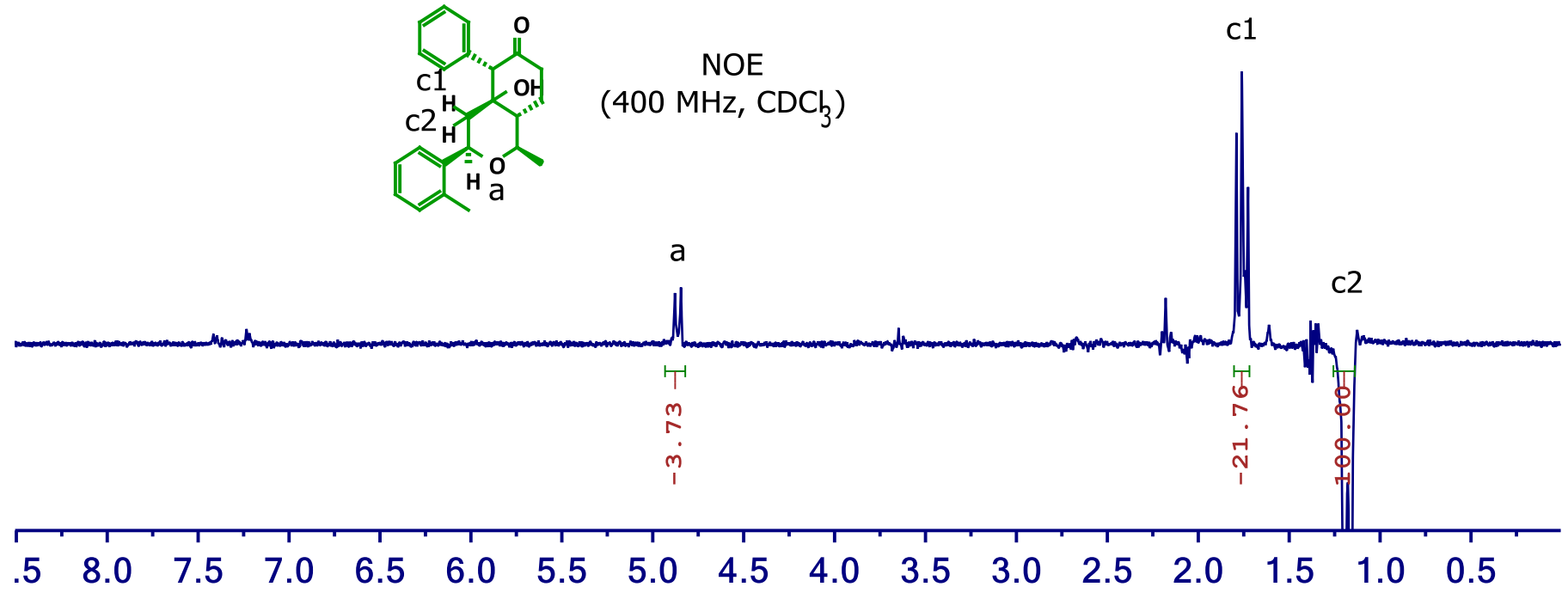

c1 
(1R,3R,4aR,5S,8aR)-4a-hydroxy-1-methyl-5-phenyl-3-(o-tolyl)octahydro-6H-isochromen-6-one<smiles>Cc1ccccc1C1CC2(O)C(C)C(=O)CC2C(=O)CC1c1ccccc1</smiles>

6

(400 MHz, CDCb)
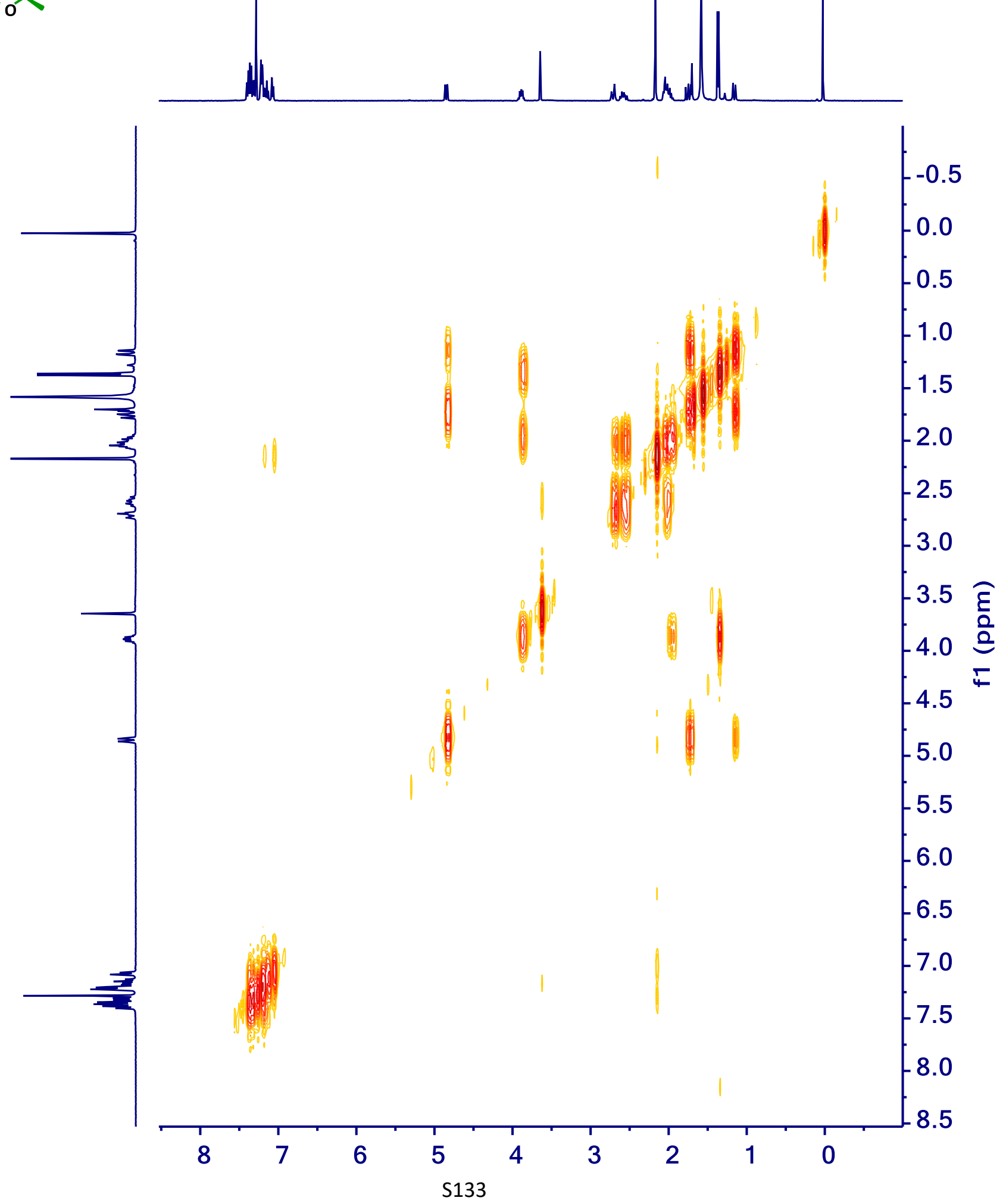
(1R,3R,8aS)-1-methyl-5-phenyl-3-(o-tolyl)-1,3,4,7,8,8a-hexahydro- 6 -isochromen-6-one

7 a

m "

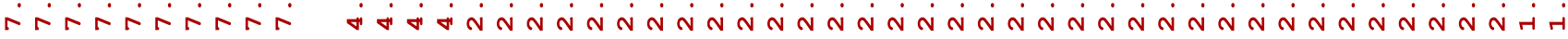

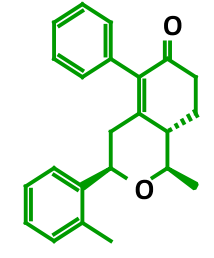

$1 \mathrm{H}$ NMR $\left(400 \mathrm{MHz}, \mathrm{CDC}_{3}\right)$

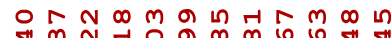

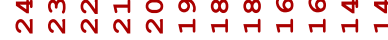

riviritivition
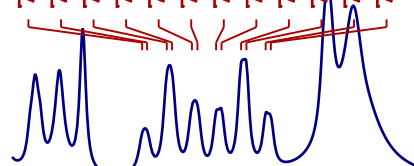

sid
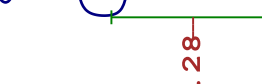

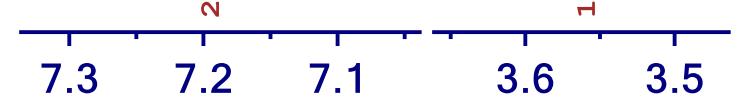

Mํ.

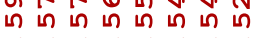

$\dot{m} \dot{m} \dot{m} \dot{m} \dot{m} \dot{m}$

$\underbrace{2}$
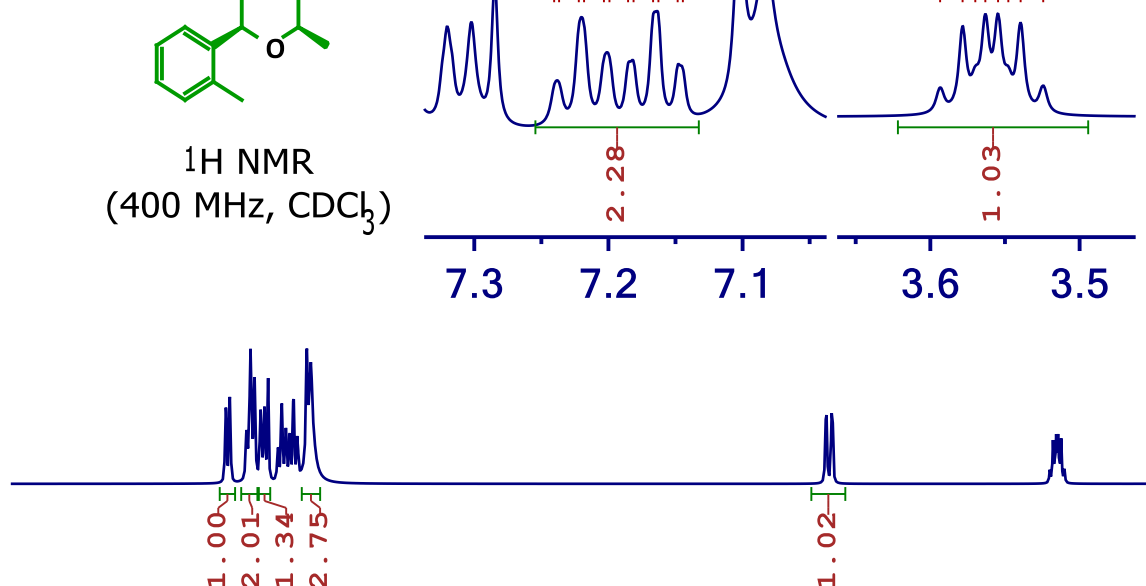

H
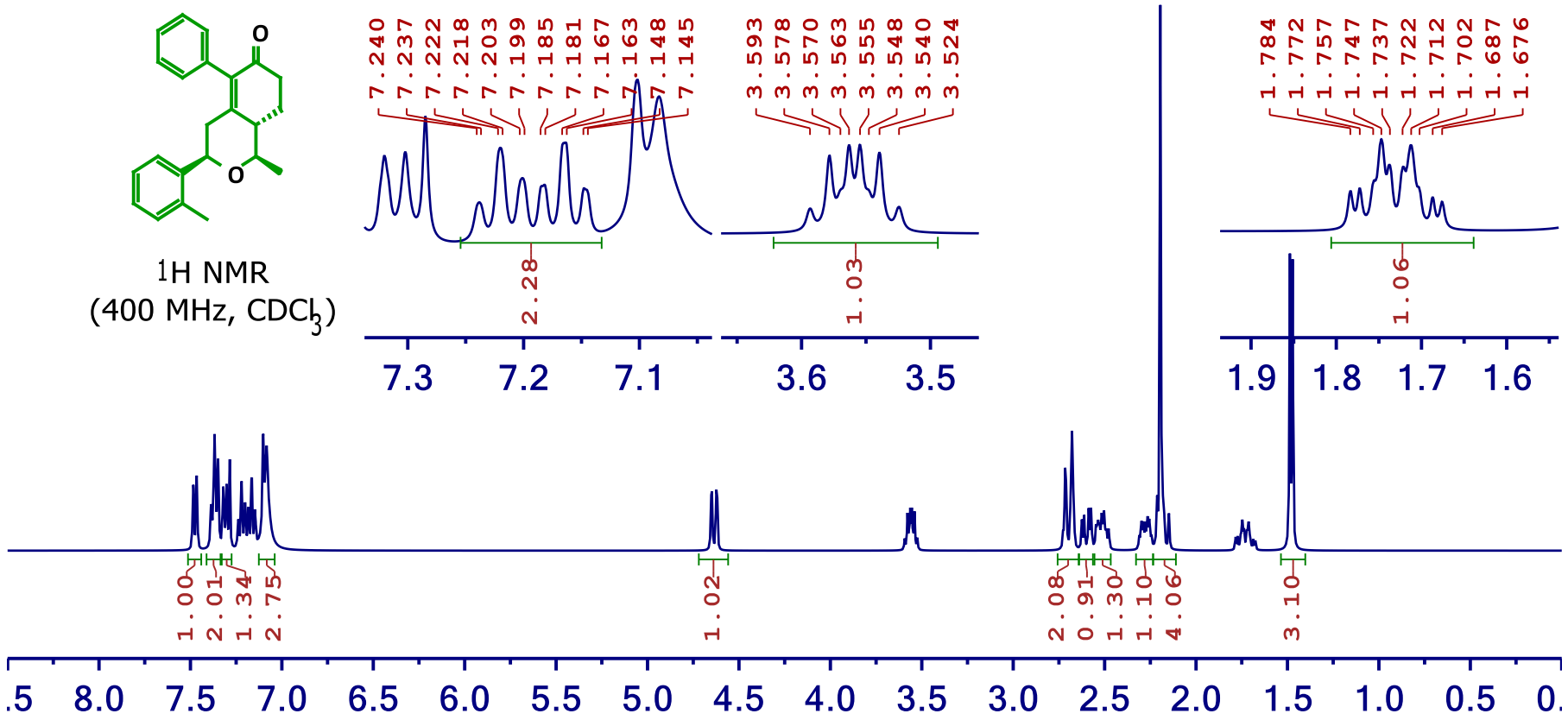

a
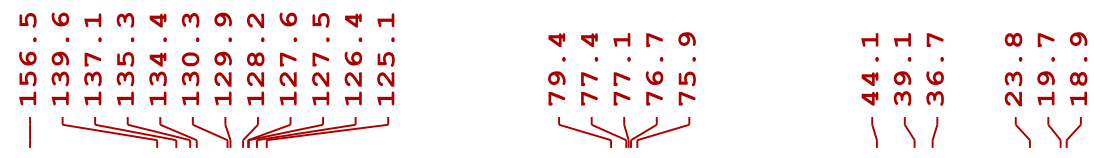<smiles>Cc1ccccc1C1CC2=C(c3ccccc3)C(=O)CC[C@H]2C(C)O1</smiles>

$13 \mathrm{C}\{1 \mathrm{H}\}$ NMR (100 $\left.\mathrm{MHz}, \mathrm{CDC}_{3}\right)$

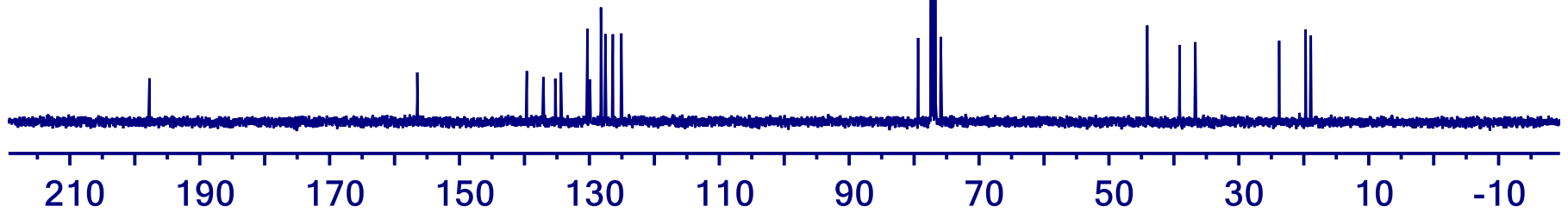


(1 $R, 3 R, 8 a S)$-1-methyl-3,5-diphenyl-1,3,4,7,8,8a-hexahydro-6H-isochromen-6-one

7b

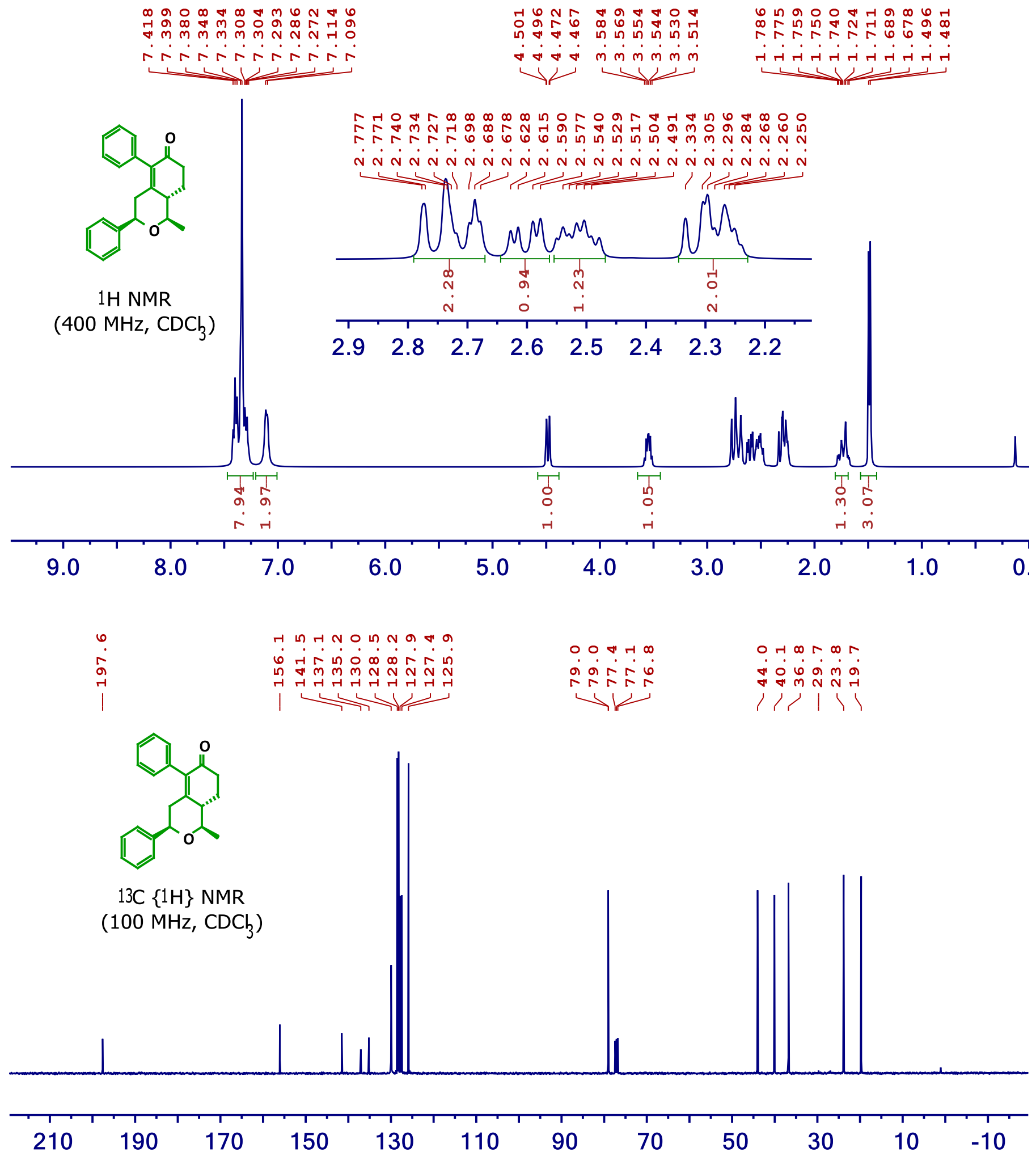


(1 $R, 3 R, 8 a S)$-1-methyl-3-(naphthalen-1-yl)-5-phenyl-1,3,4,7,8,8a-hexahydro-6H-isochromen-6-one

$7 \mathrm{c}$

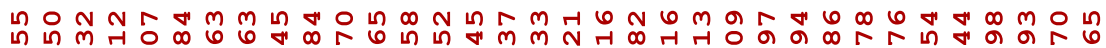

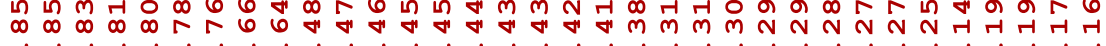

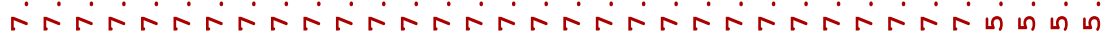

$\infty \begin{array}{llll}m & -1 & 0 \\ 0 & 0 & 0 & 0 \\ 0 & 0 & 0 & 0\end{array}$

ด้ ด้ ตे ส

4.

tri

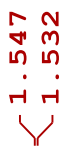<smiles>C[C@H]1OC(c2cccc3ccccc23)CC2=C(c3ccccc3)C(=O)CC[C@H]21</smiles>

$1 \mathrm{H} N M R$

(400 $\left.\mathrm{MHz}, \mathrm{CDCl}_{3}\right)$

$\stackrel{1}{i}$

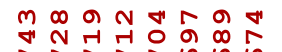

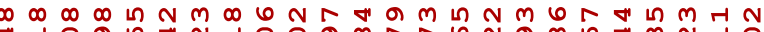

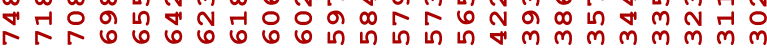

$0,0, m+4 N$ mंmmmmm

事

4 4 4 4 -4

$\longrightarrow$
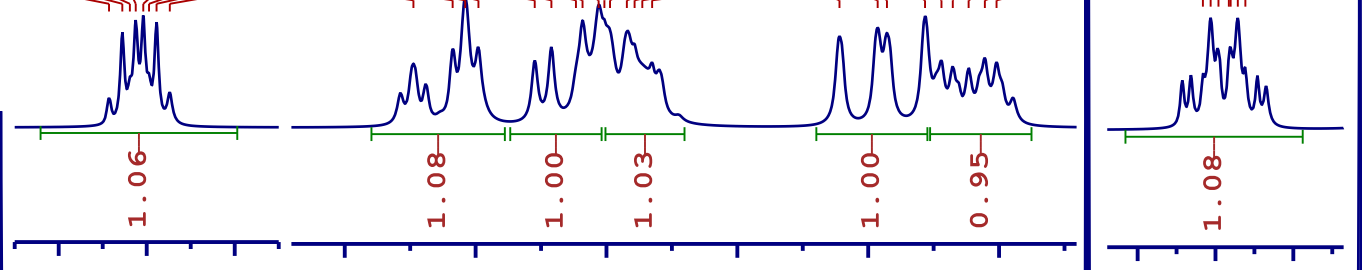

3.8

3.6

2.8

2.7

2.6

2.5

2.4

2.3

1.9

1.7

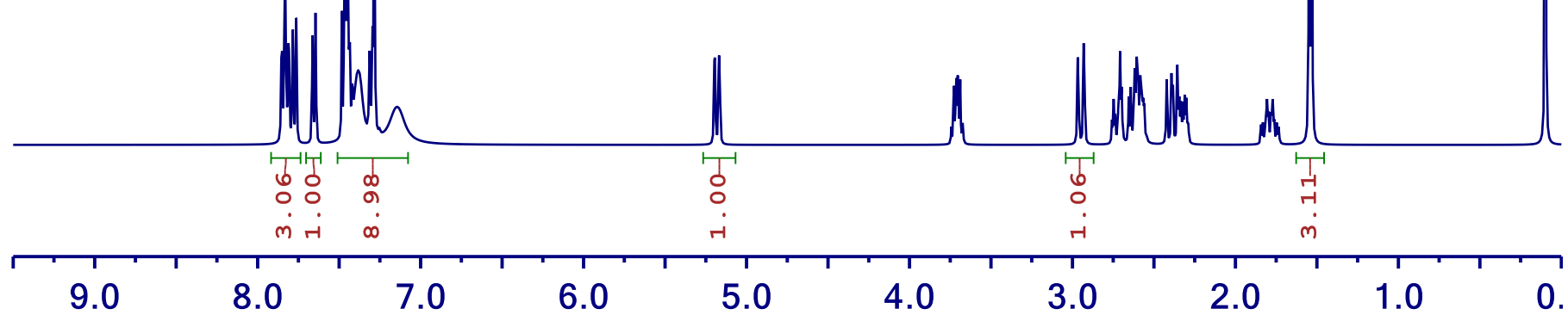

in momr haGm

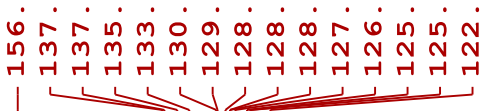

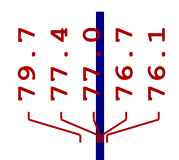

$m$ in $\mathrm{r}$

$\infty \infty$

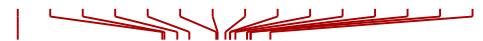

$\rightarrow \stackrel{m}{m}$

$m a$<smiles>CI</smiles><smiles>C[C@@H]1OC(c2cccc3ccccc23)CC2=C(c3ccccc3)C(=O)CC[C@H]21</smiles>

13C $\{1 \mathrm{H}\} \mathrm{NMR}$ (100 $\mathrm{MHz}, \mathrm{CDCh}_{3}$ )

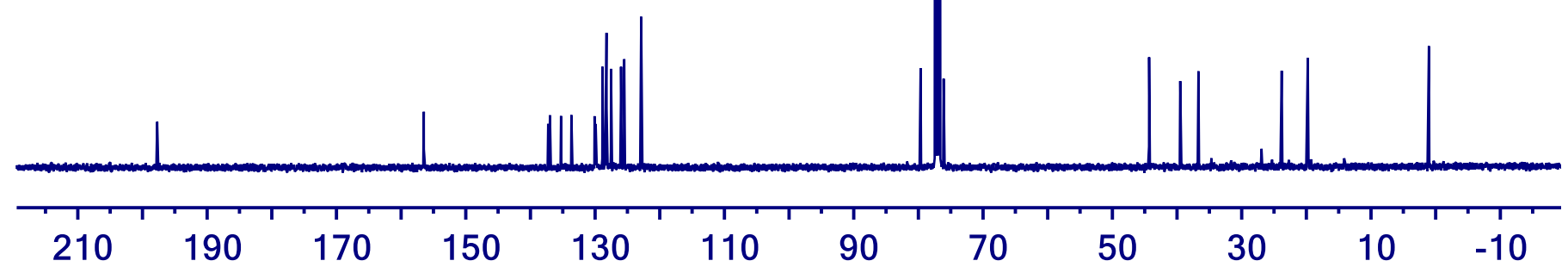


(1 $S, 3 S, 8 a S)$-3-benzyl-1-(4-methoxyphenyl)-5-phenyl-1,3,4,7,8,8a-hexahydro-6H-isochromen-6-one

$7 d$

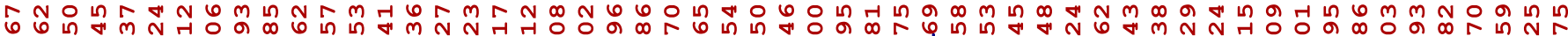

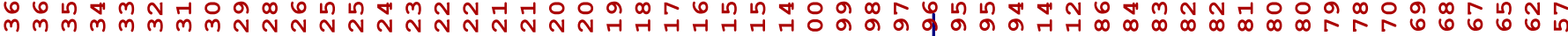

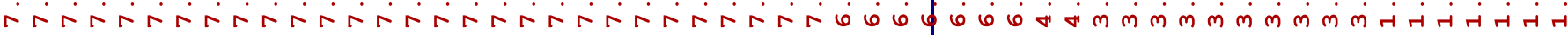

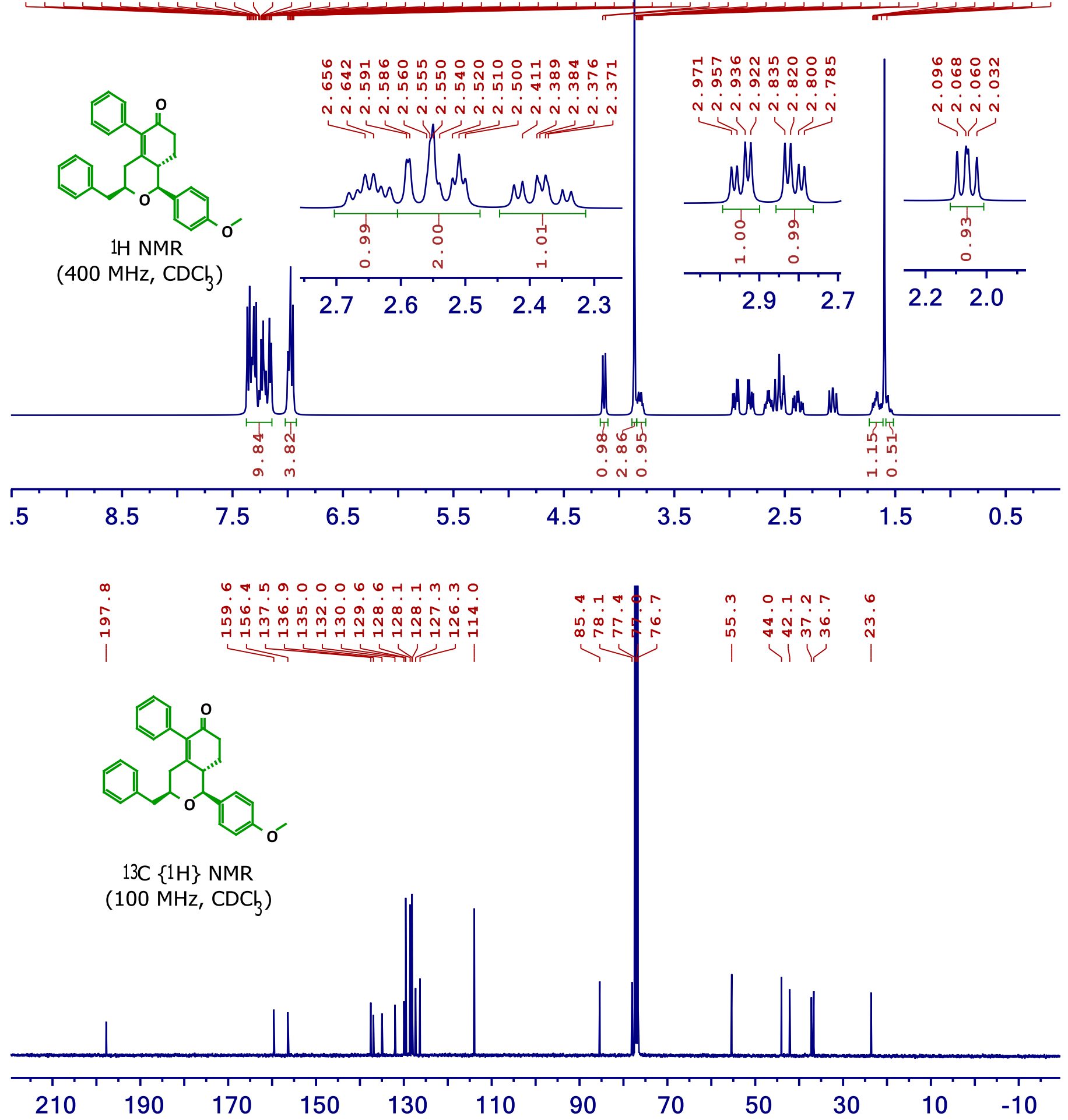


$(1 R, 3 R, 8 a S)-5$-benzyl-1-isobutyl-3-phenyl-1,3,4,7,8,8a-hexahydro-6H-isochromen-6-one

$7 \mathrm{e}$

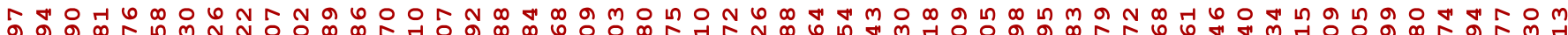

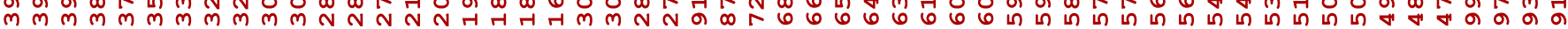

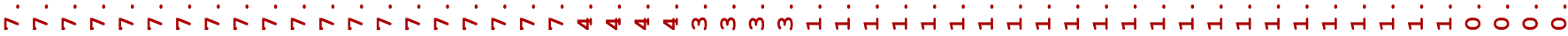

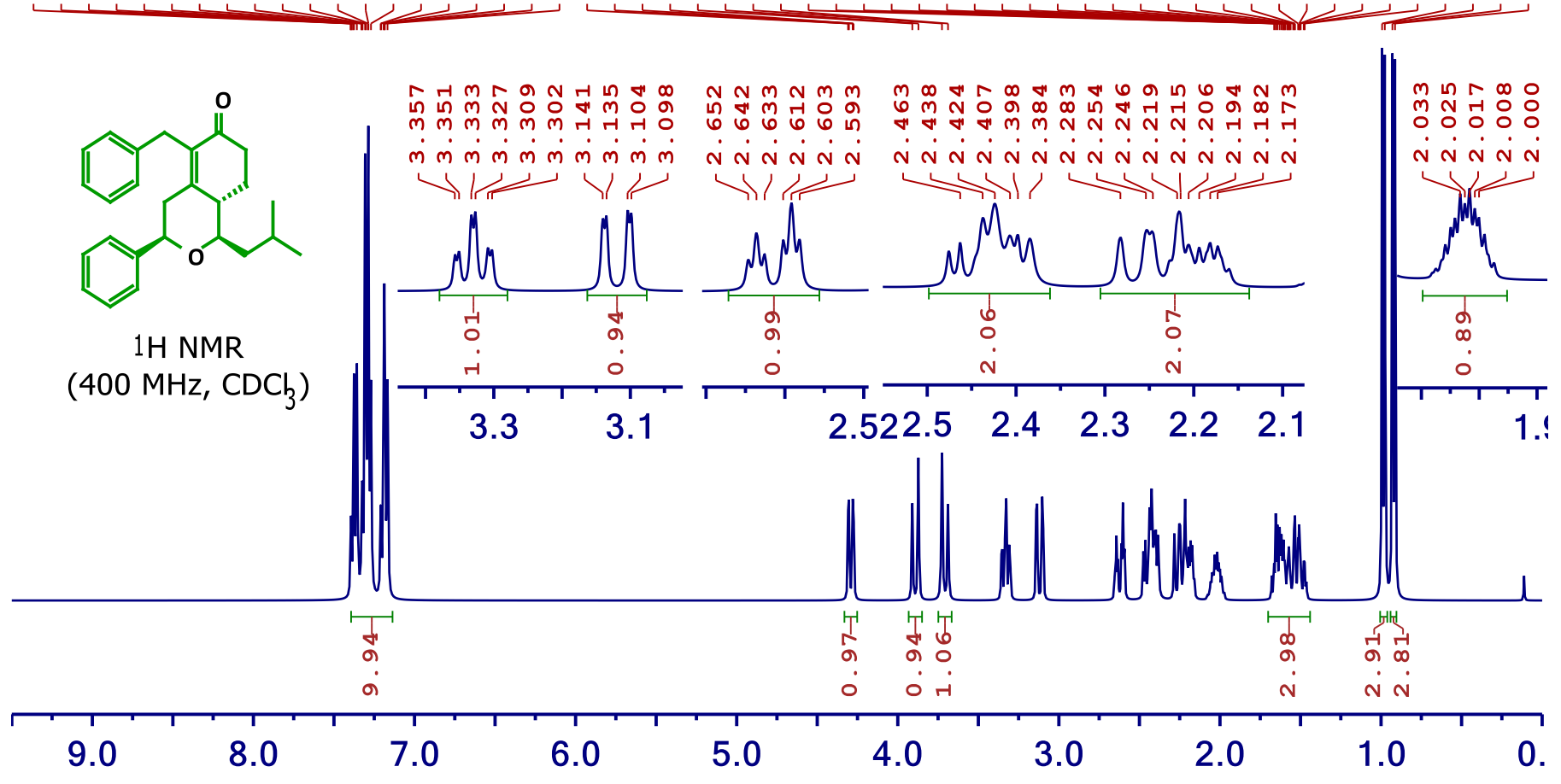

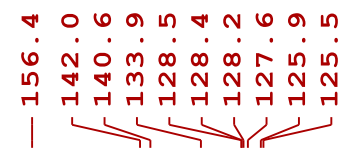<smiles>CC(C)CC1OC(c2ccccc2)CC2=C(Cc3ccccc3)C(=O)CCC21</smiles>

$13 \mathrm{C}\{1 \mathrm{H}\} \mathrm{NMR}$ (100 MHz, $\mathrm{CDCl}_{3}$ )

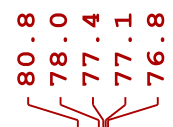

$\infty m$ ก กู่

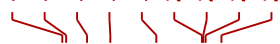

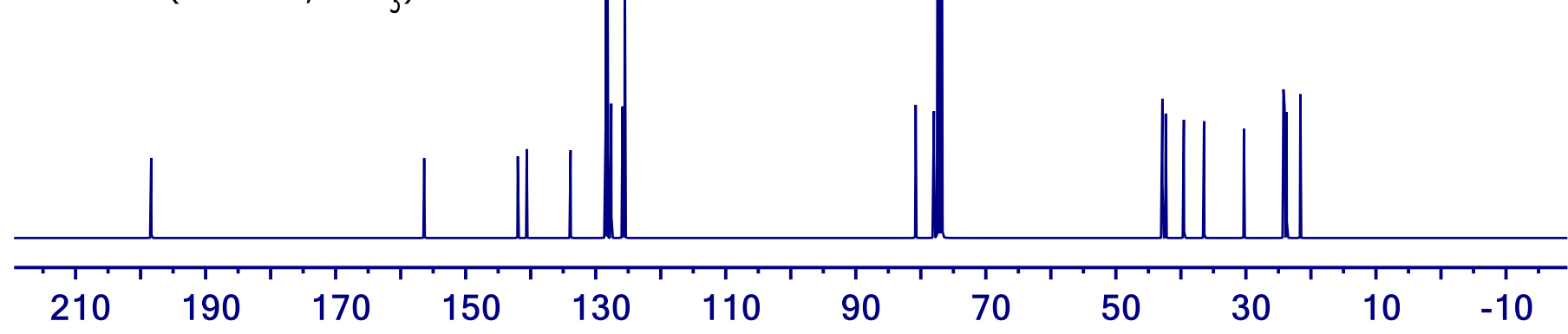


(1R,3R,8 aS)-5-benzyl-1-cyclopropyl-3-phenyl-1,3,4,7,8,8a-hexahydro-6H-isochromen-6-one

7f

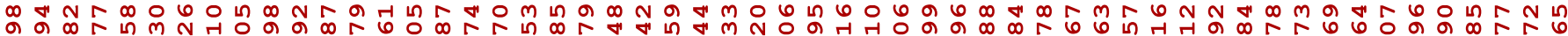

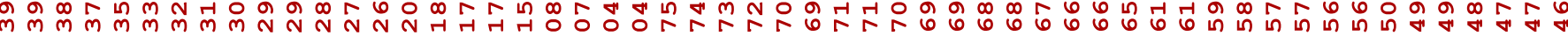

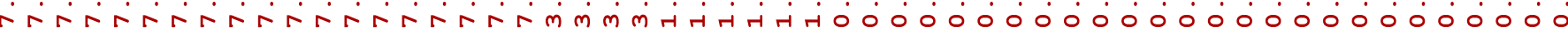
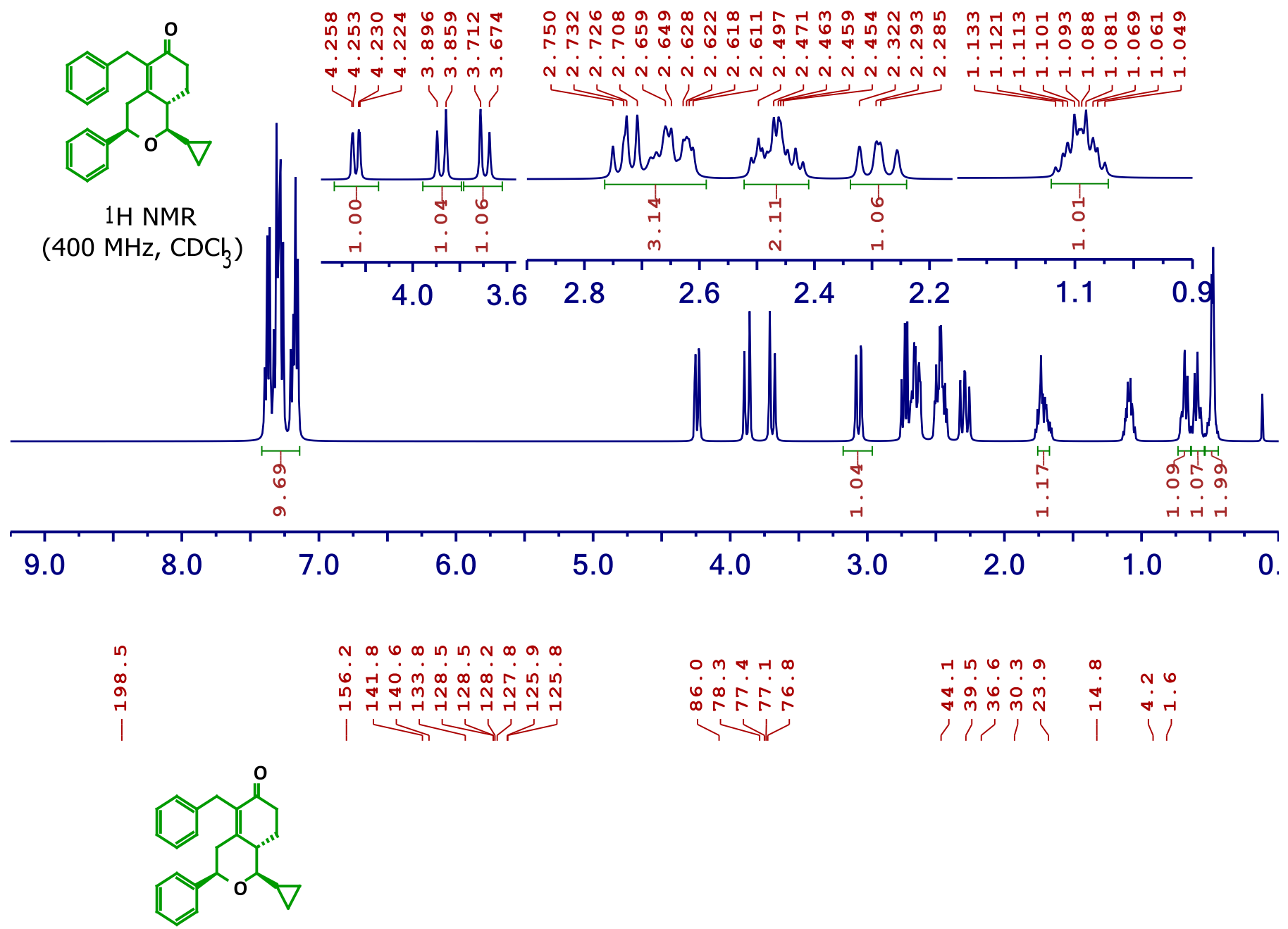

$13 \mathrm{C}\{1 \mathrm{H}\} \mathrm{NMR}$

(100 MHz, CDCb)

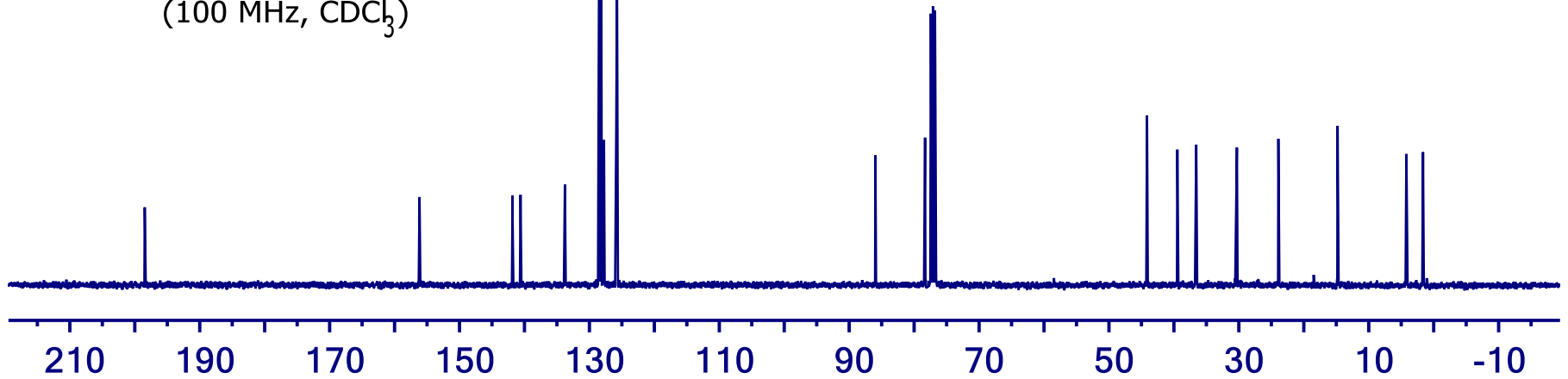


(1 R,3 R,8 aS)-5-benzyl-1-ethyl-3-phenyl-1,3,4,7,8,8a-hexahydro-6H-isochromen-6-one

$7 \mathrm{~g}$

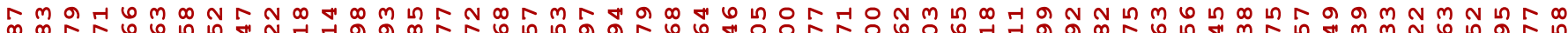

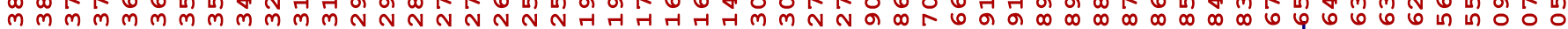

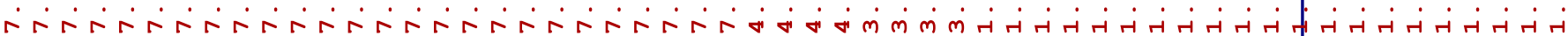

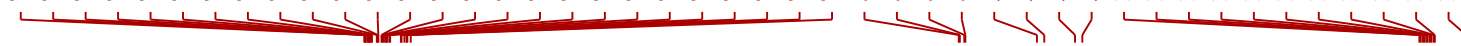<smiles>CC[C@H]1OC(c2ccccc2)CC2=C(Cc3ccccc3)C(=O)CC[C@H]21</smiles>

1H NMR

(400 $\left.\mathrm{MHz}, \mathrm{CDCl}_{3}\right)$

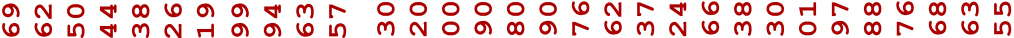

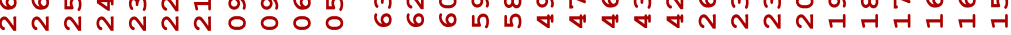

$\dot{m} \dot{m} \dot{m} \dot{m} \dot{m} \dot{m} \dot{m} \dot{m} \mathrm{~N} N \mathrm{~N} N \mathrm{~N} N \mathrm{~N} N \mathrm{~N} N \mathrm{~N} N \mathrm{~N}$

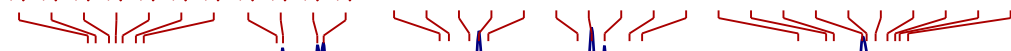

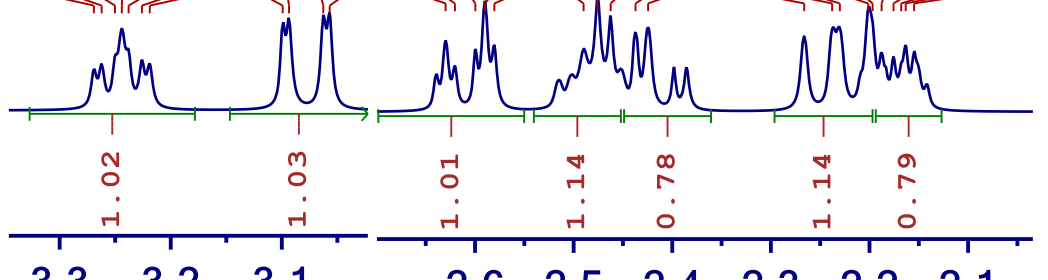

\section{$\begin{array}{lll}3.3 & 3.2 & 3.1\end{array}$}

$\begin{array}{llllll}2.6 & 2.5 & 2.4 & 2.3 & 2.2 & 2.1\end{array}$
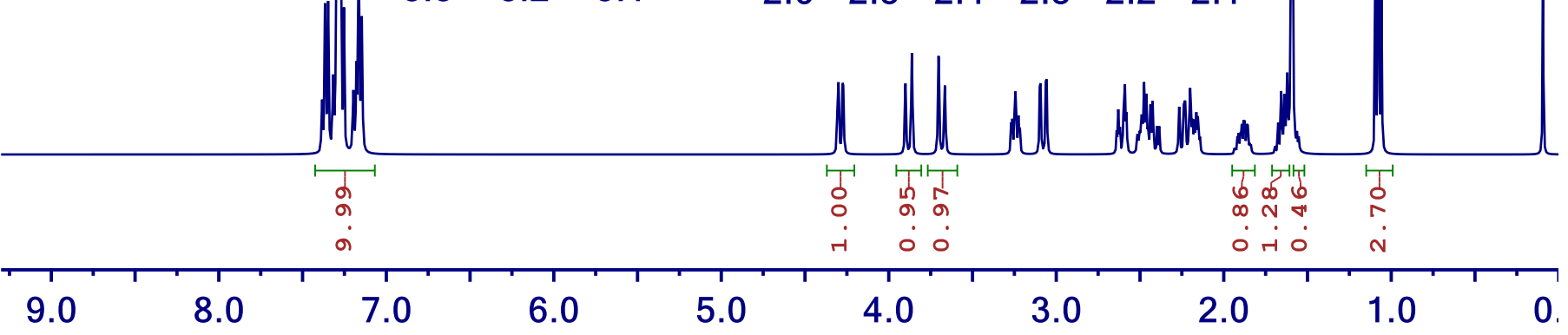

$m$
$\dot{\infty}$
0
+1
+1

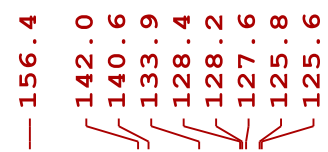

(1)

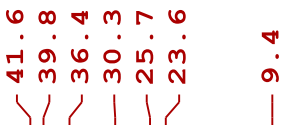<smiles>CCC1OC(c2ccccc2)CC2=C(Cc3ccccc3)C(=O)CC[C@H]21</smiles>

$13 \mathrm{C}\{1 \mathrm{H}\} \mathrm{NMR}$ (100 MHz, $\mathrm{CDC}_{3}$ )

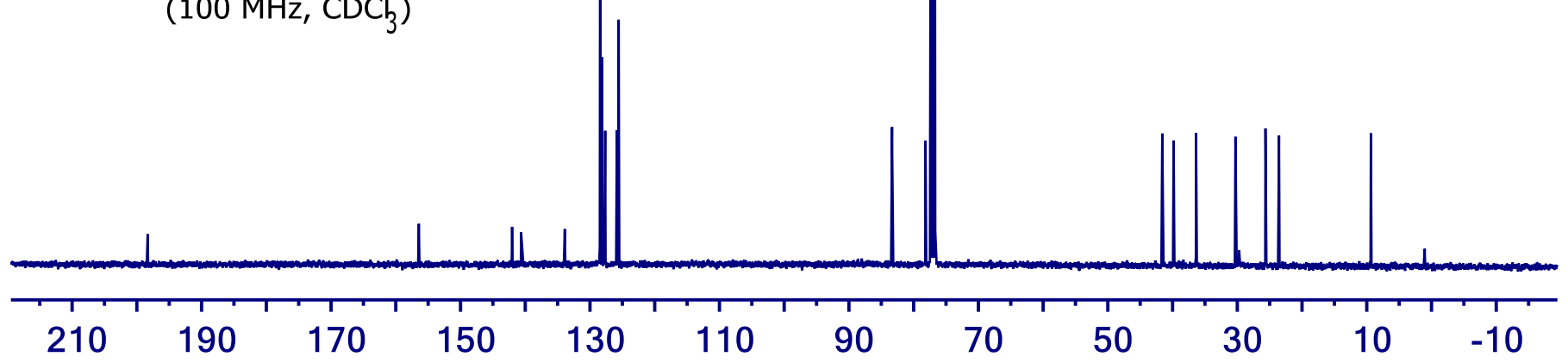


(1 $S, 3 R, 8 a S)$-5-benzyl-1-(4-methoxyphenyl)-3-phenyl-1,3,4,7,8,8a-hexahydro-6H-isochromen-6-one

$7 \mathrm{~h}$

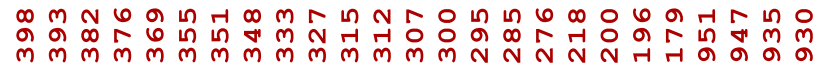

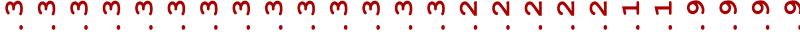

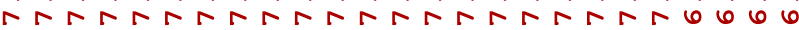

นn

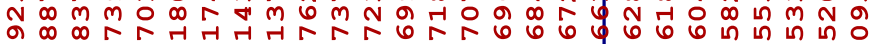

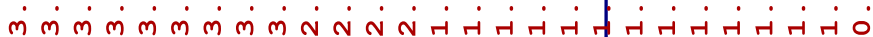<smiles>COc1ccc(C2OC(c3ccccc3)CC3=C(Cc4ccccc4)C(=O)CC[C@H]32)cc1</smiles>

$1 \mathrm{H} N \mathrm{NMR}$ (400 $\mathrm{MHz}, \mathrm{CDCl}_{3}$ )

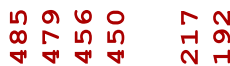

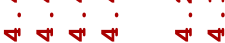
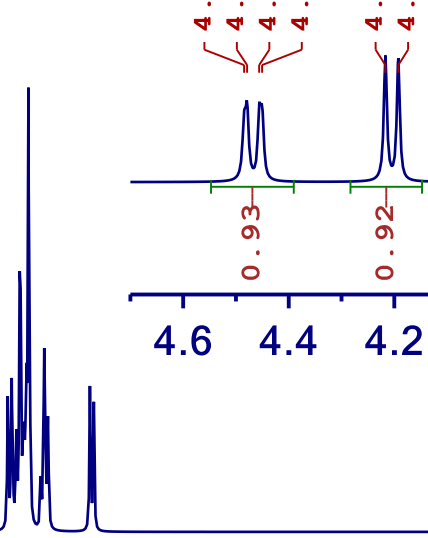

ग्ञात्त ग

ำ ถ⿱

a $\mathrm{n}$ -

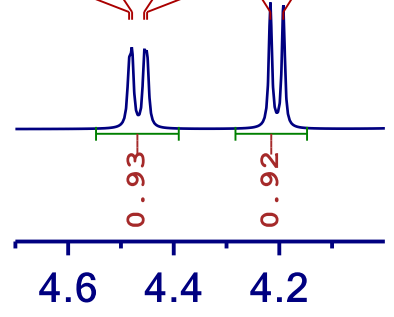

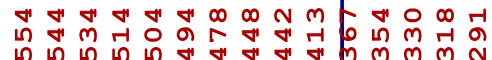

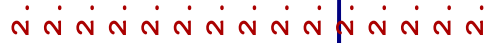

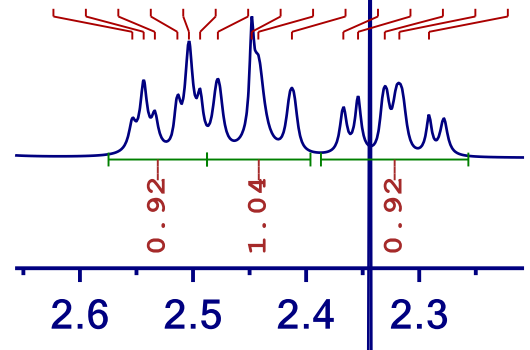

$\begin{array}{llllllllll} & & & & & & & & & \\ & 8.0 & 7.0 & 6.0 & 5.0 & 4.0 & 3.0 & 2.0 & 1.0 & 0 .\end{array}$

$m$
0
0
$a$
$r$

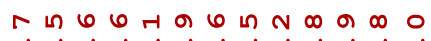

की मों

in

$\longrightarrow$<smiles>COc1ccc([C@H]2OC(c3ccccc3)CC3=C(Cc4ccccc4)C(=O)CC[C@H]32)cc1</smiles>

13C $\{1 \mathrm{H}\}$ NMR

(100 $\left.\mathrm{MHz}, \mathrm{CDCh}_{3}\right)$

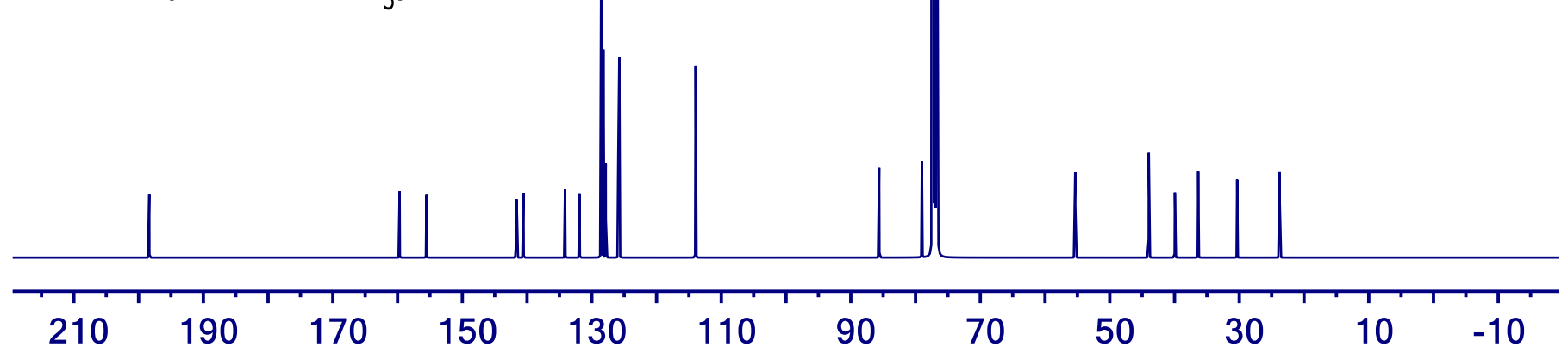


(1 $R, 3 R, 8 a S)$-5-benzyl-1-cyclohexyl-3-phenyl-1,3,4,7,8,8a-hexahydro-k-isochromen-6-one

$7 \mathrm{i}$

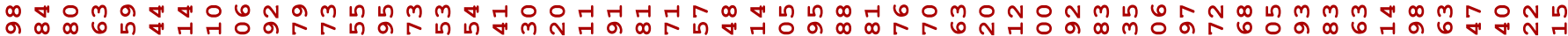
N N N N N N N N N

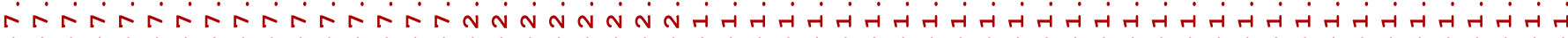
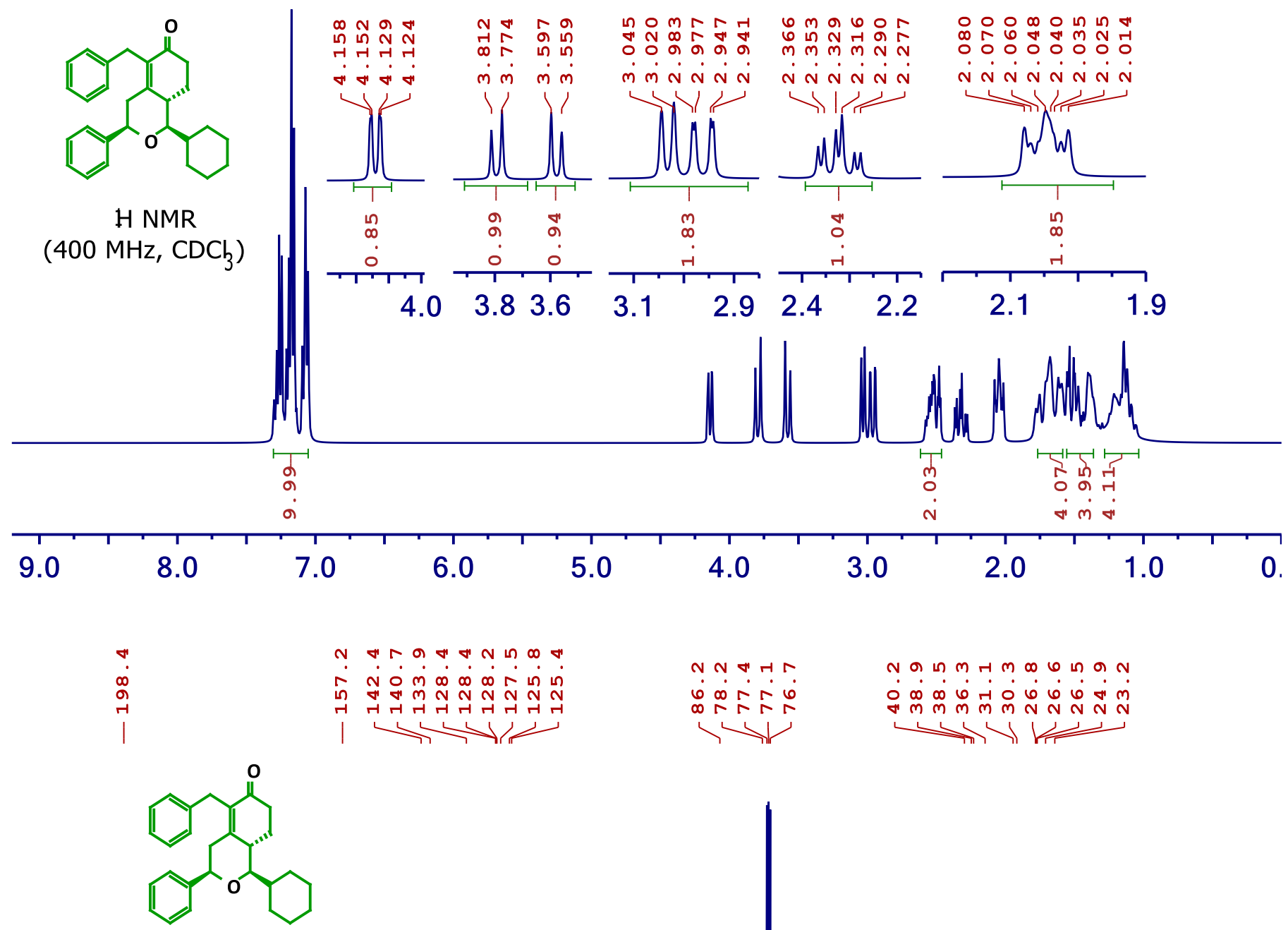

$13 \mathrm{C}\{1 \mathrm{H}\} \mathrm{NMR}$

(100 MHz, CDCb)

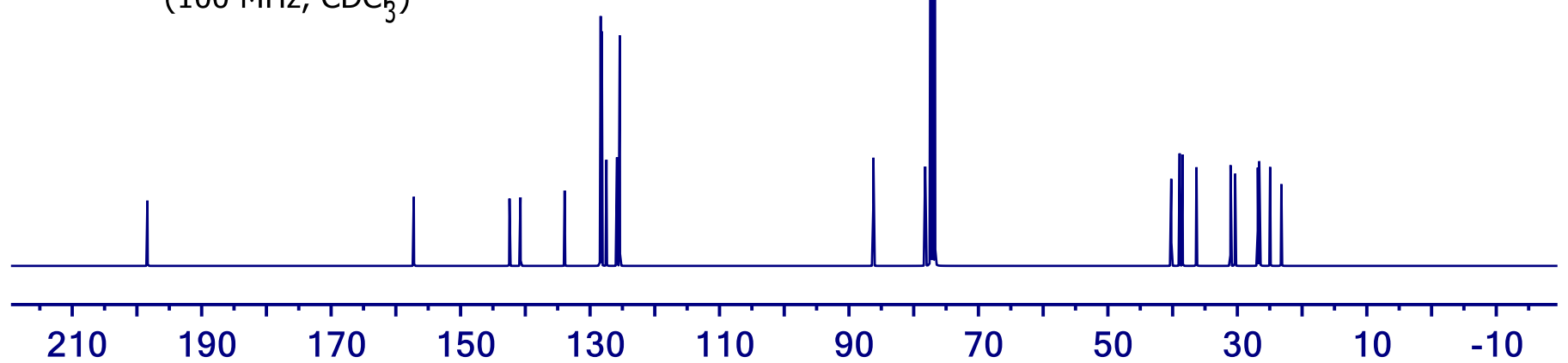


(1 $R, 3 R, 8 a S)$-5-benzyl-1-methyl-3-phenyl-1,3,4,7,8,8a-hexahydro-6H-isochromen-6-one

$7 \mathbf{j}$

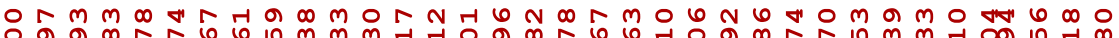

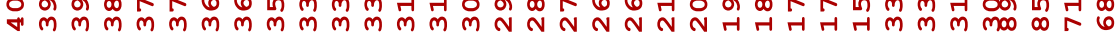

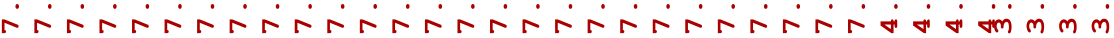

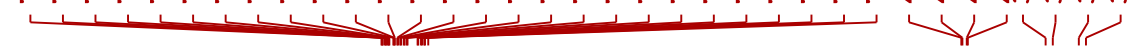

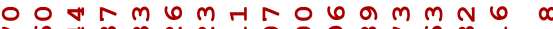

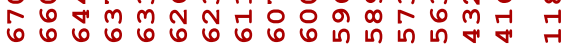

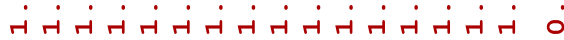<smiles>C[C@H]1O[C@H](c2ccccc2)CC2=C(Cc3ccccc3)C(=O)CC[C@H]21</smiles>

$1 \mathrm{H} N \mathrm{NR}$ (400 $\mathrm{MHz}, \mathrm{CDC}_{3}$ )

$m \infty a N+\pi m \infty m N 6$ -

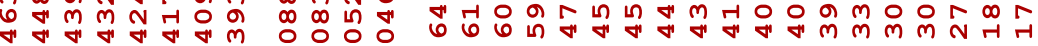

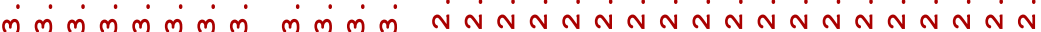

N

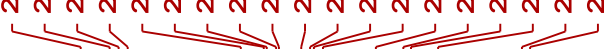
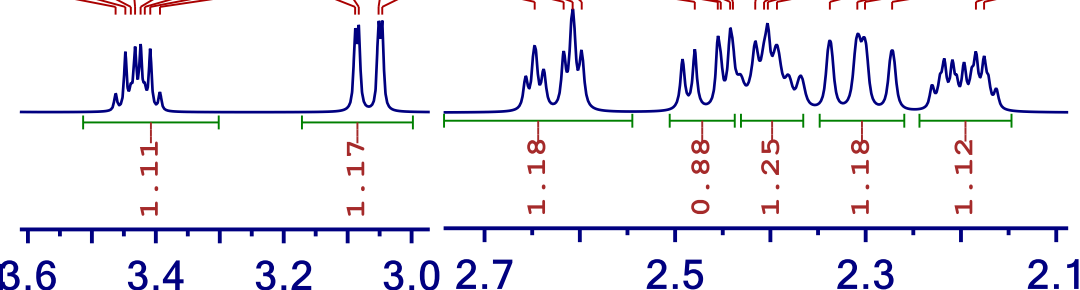

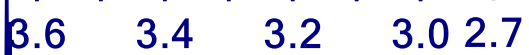
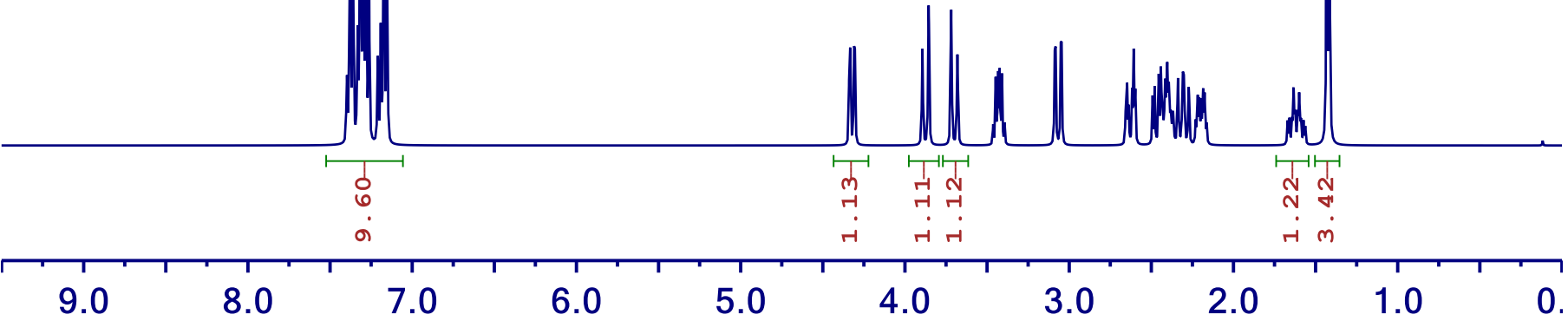

$m$
0
$\infty$
0
$r$
$\mid$
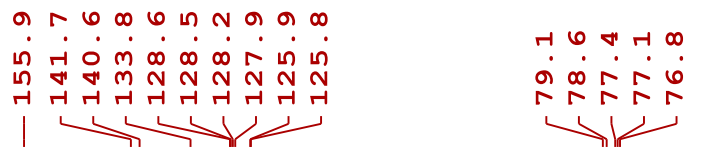

$m$
$\infty$
0
0

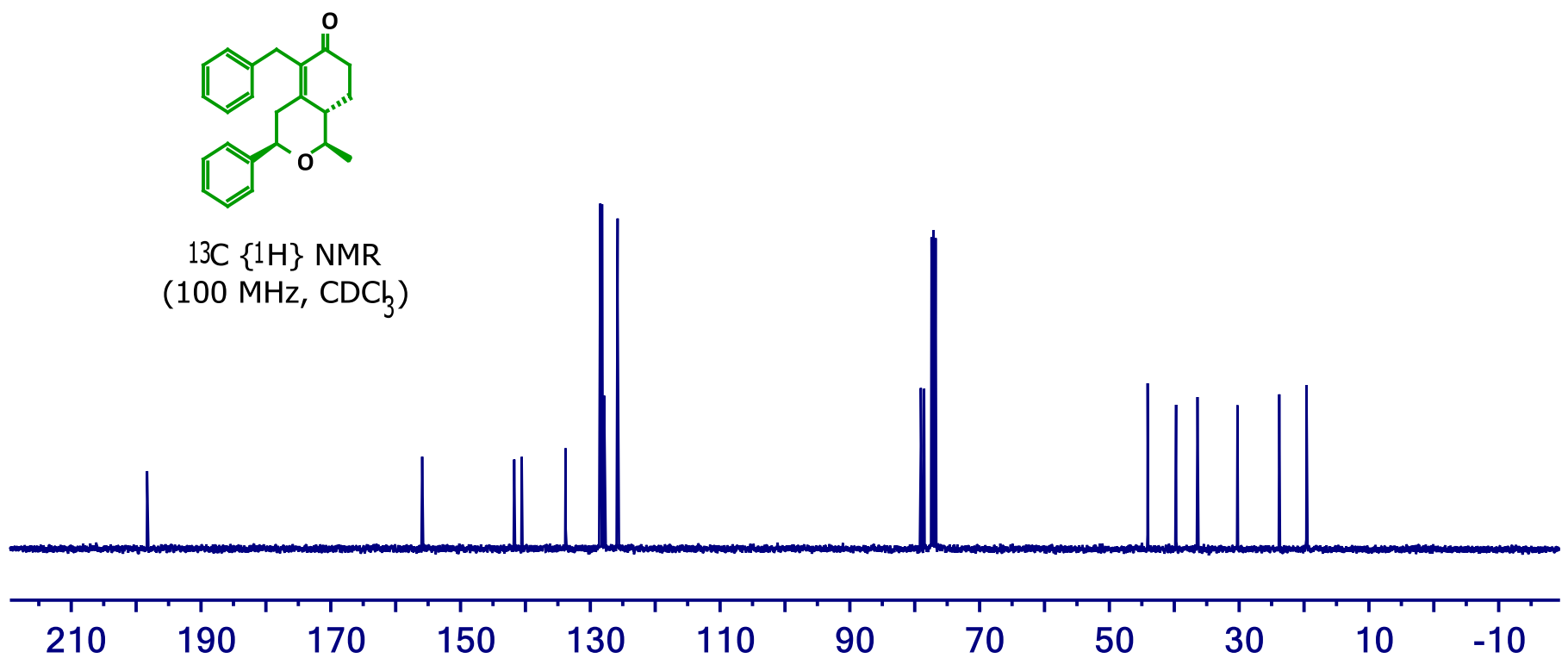

$13 \mathrm{C}\{1 \mathrm{H}\} \mathrm{NMR}$ $\left(100 \mathrm{MHz}, \mathrm{CDC}_{3}\right)$
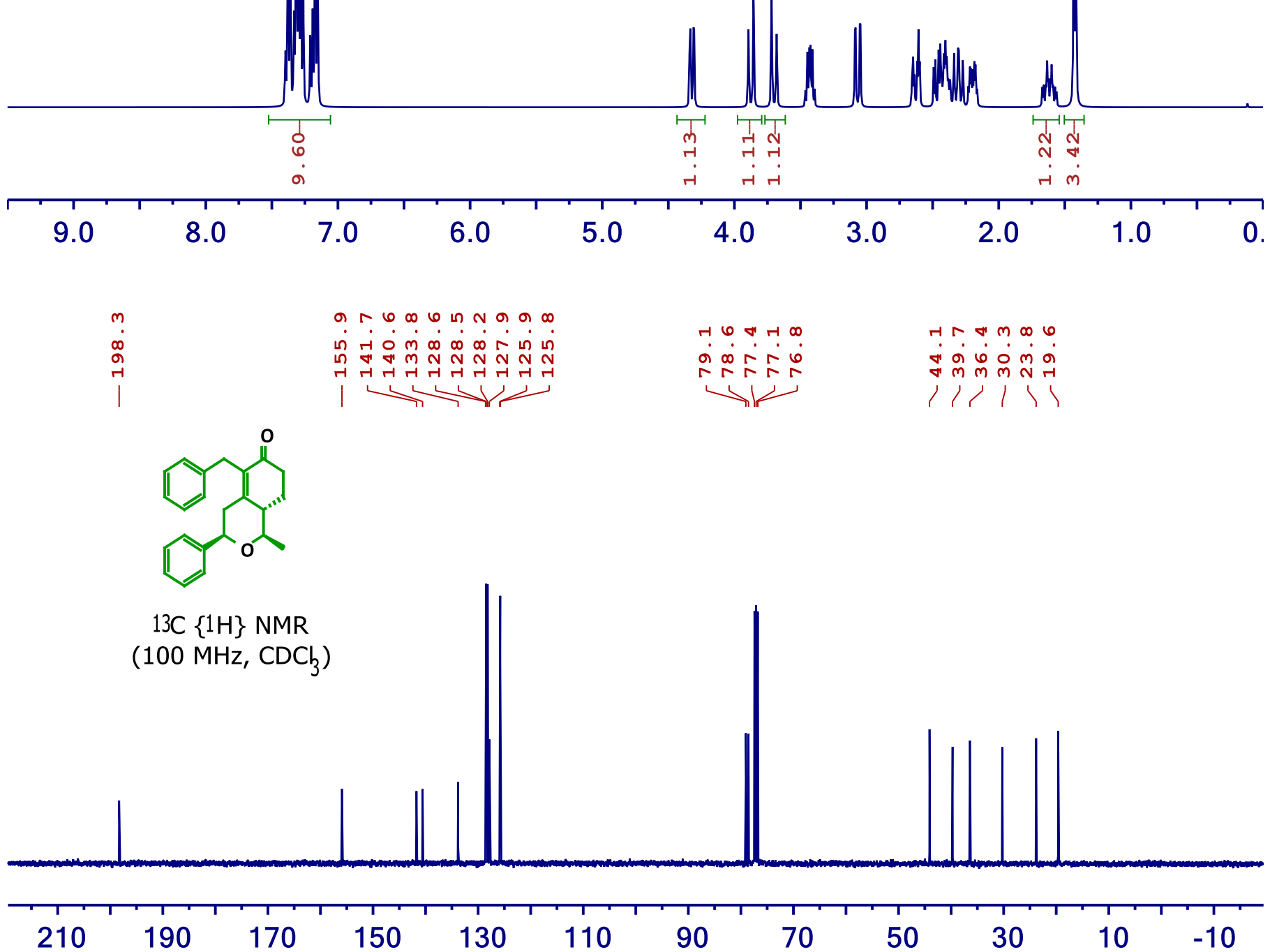

$\stackrel{4}{2}$

$\stackrel{\text { N }}{\mathfrak{N}}$

-i m 
( $1 S, 3 R, 8 a S)$-5-benzyl-3-phenyl-1-(p-tolyl)-1,3,4,7,8,8a-hexahydro- $H$-isochromen-6-one

$7 \mathbf{k}$

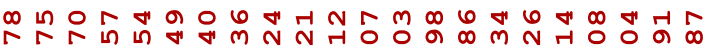

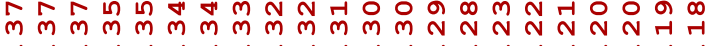

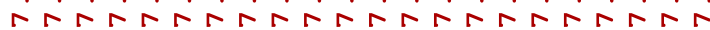

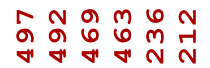

$\nabla \dot{\nabla} \nabla \dot{\nabla}$

a $\infty$ in

4

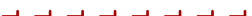

$\rightarrow$

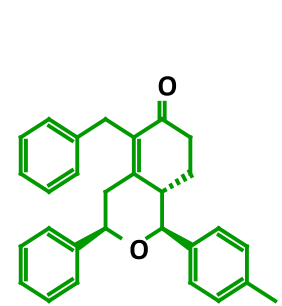

$1 \mathrm{H}$ NMR

(400 $\left.\mathrm{MHz}, \mathrm{CDCb}_{3}\right)$

mån

a d

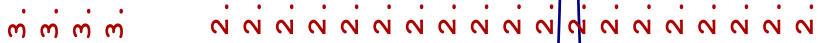

11

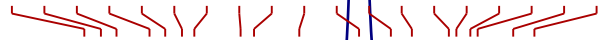
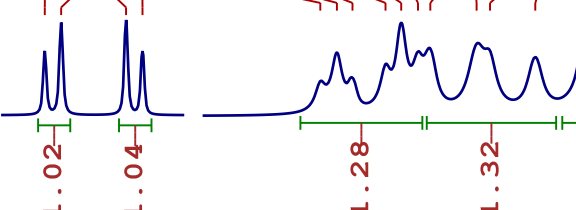

W

M

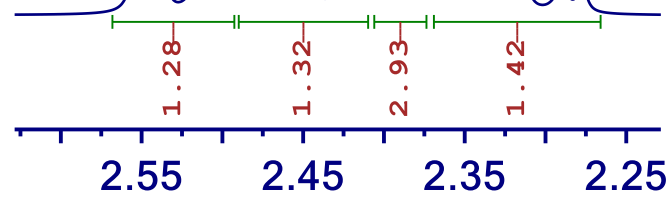

0

$5 \sqrt{5} \frac{1}{2}$

nin $\operatorname{nin}$

$\rightarrow$
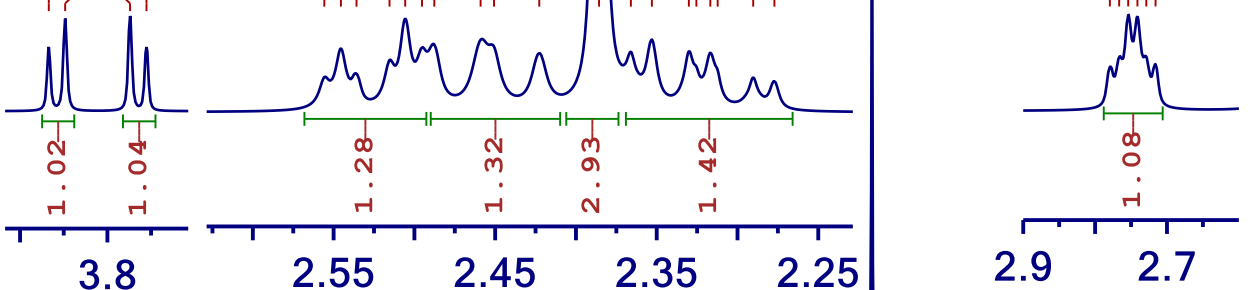

2.55

2.45

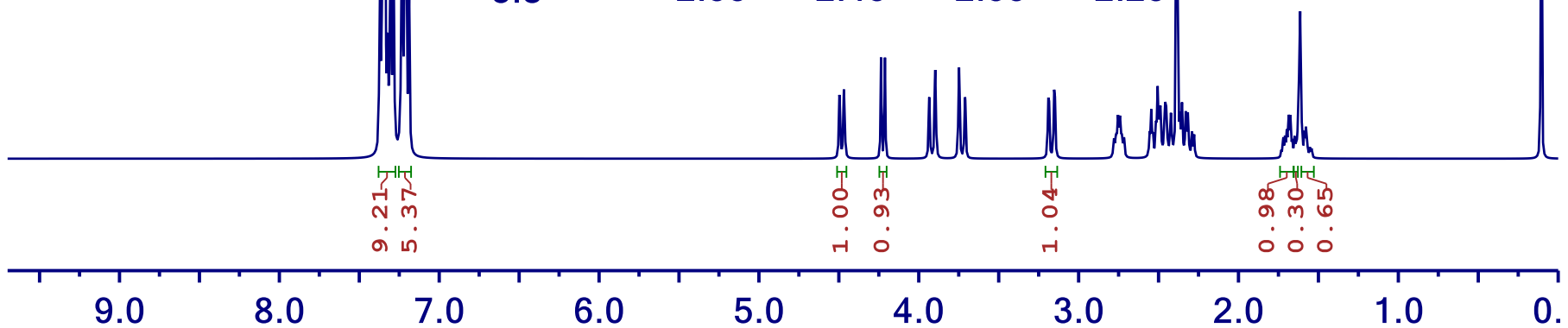

29

2.7

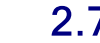

+
$\infty$
0

$\mid$

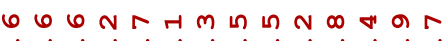

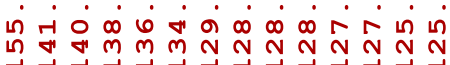

a o thm hr

a O $m m \infty N$

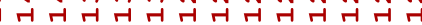

mokisin

$\stackrel{m}{m} \circ \underset{m}{\infty} \stackrel{0}{m} \stackrel{m}{N} \stackrel{-1}{N}$

| ।

11111

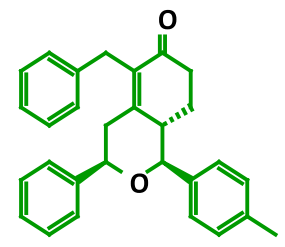

13C $\{1 \mathrm{H}\}$ NMR

(100 $\mathrm{MHz}, \mathrm{CDC}_{3}$ )

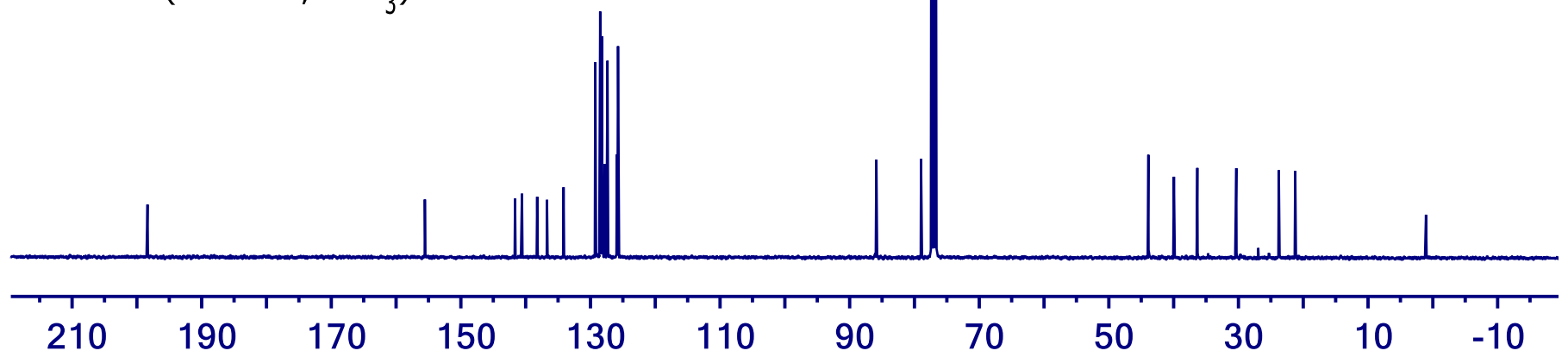


(1 $R, 3 R, 8 a S)$-5-benzyl-1-butyl-3-phenyl-1,3,4,7,8,8a-hexahydro-6H-isochromen-6-one

71

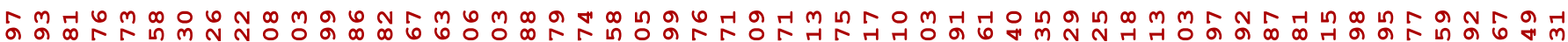

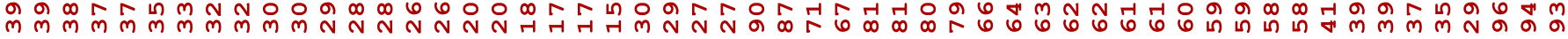

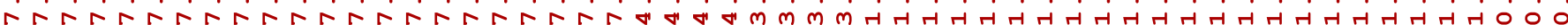
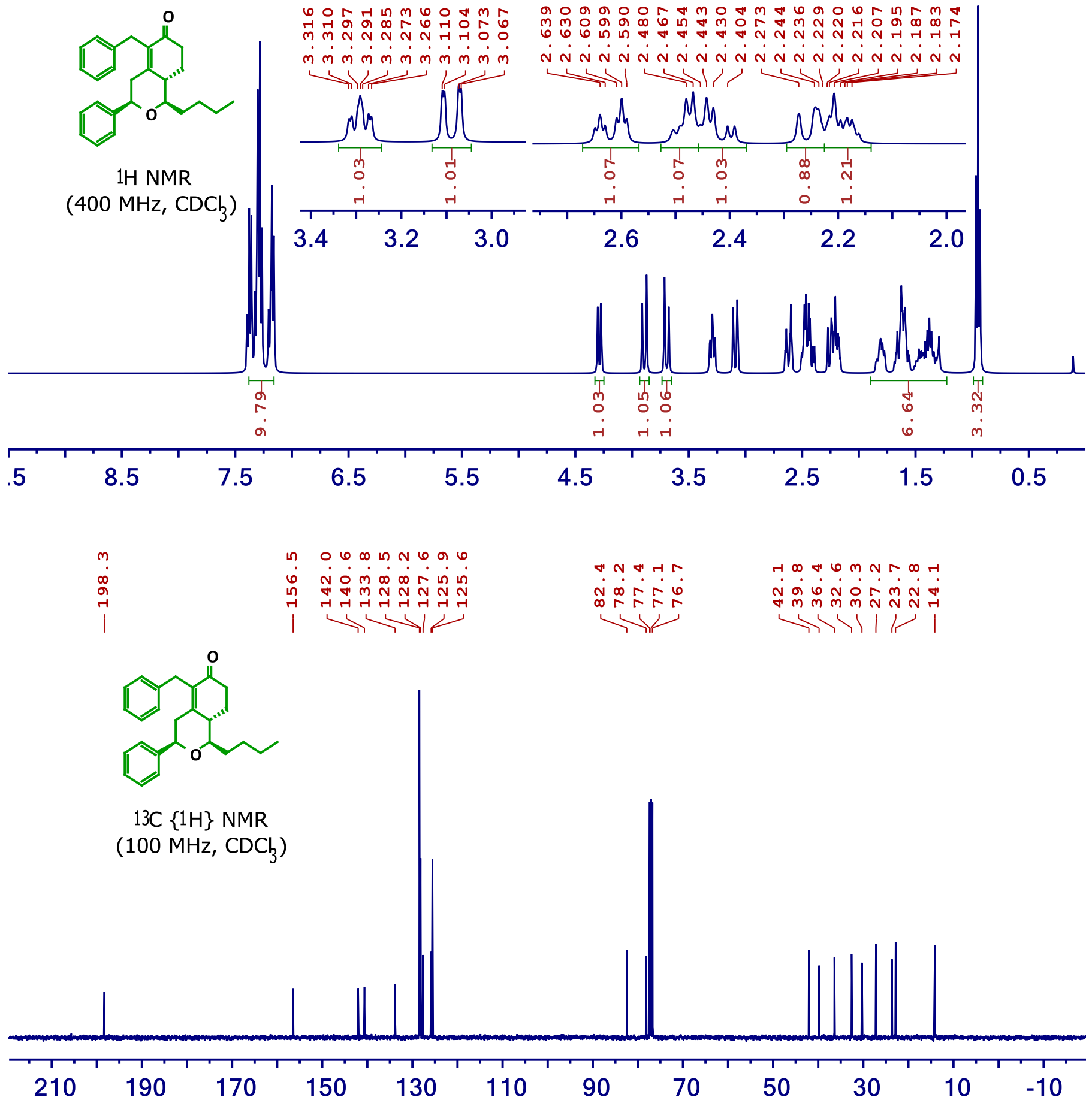
(1 R,3R,8 $a S)$-1,5-dibenzyl-3-phenyl-1,3,4,7,8,8a-hexahydro-6H-isochromen-6-one

$7 \mathrm{~m}$

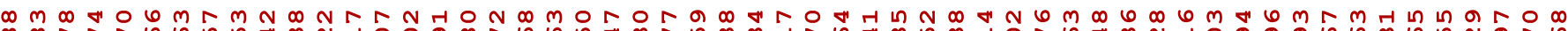

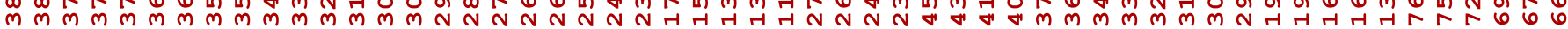

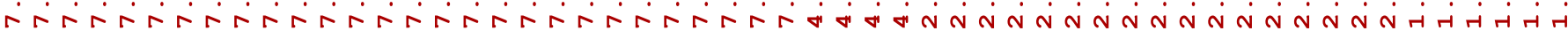

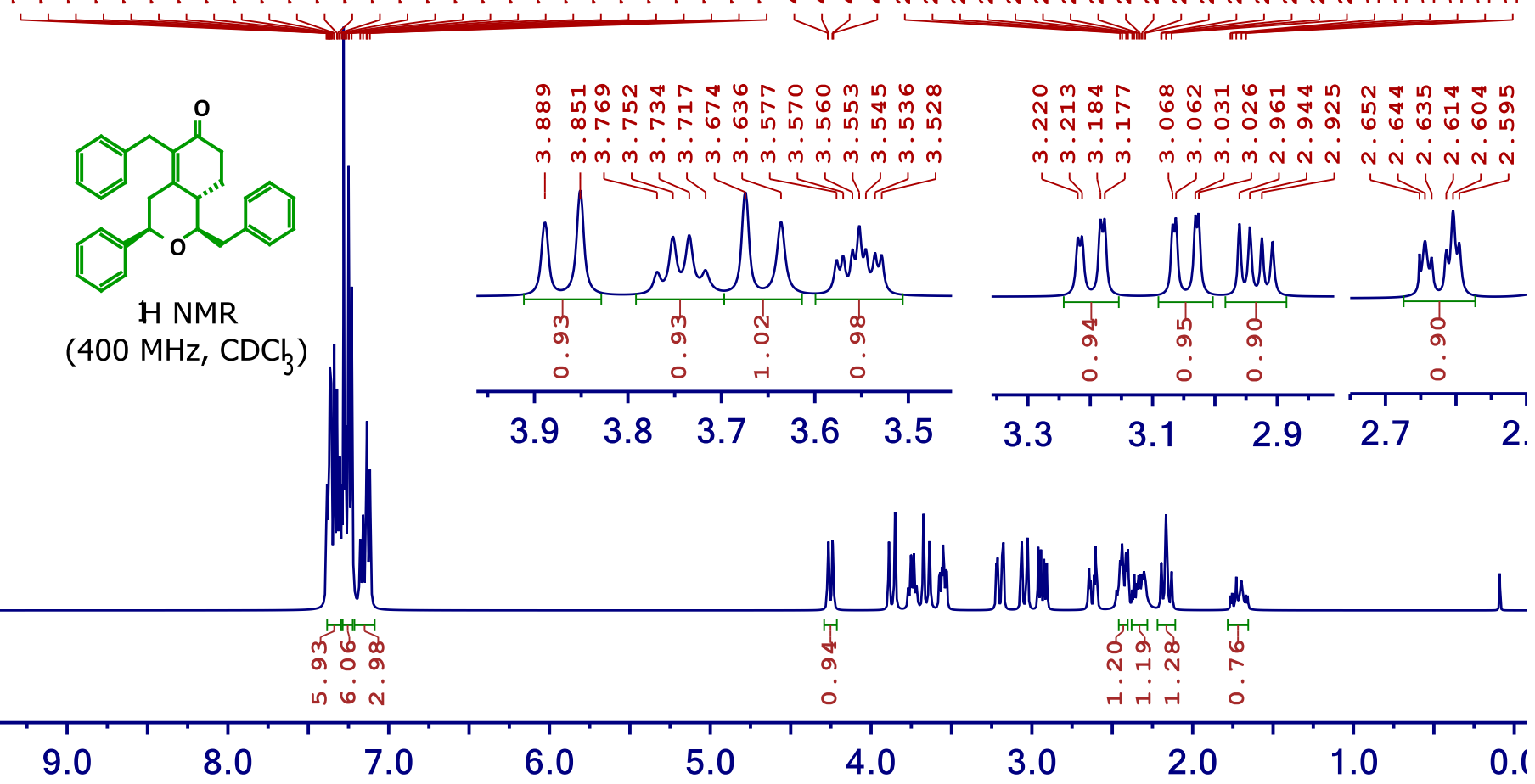

$r$
$\infty$
$\infty$
$a$
$r$
$\mid$
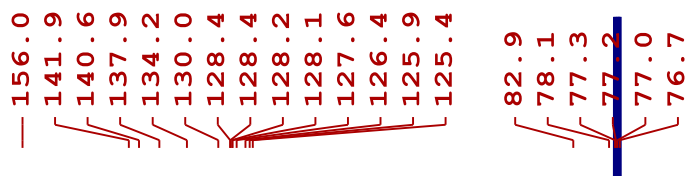

$0 \infty a m m a$

Ho $\infty \circ \circ m$

।<smiles></smiles>

13C $\{1 \mathrm{H}\}$ NMR

$\left(100 \mathrm{MHz}, \mathrm{CDCl}_{3}\right)$

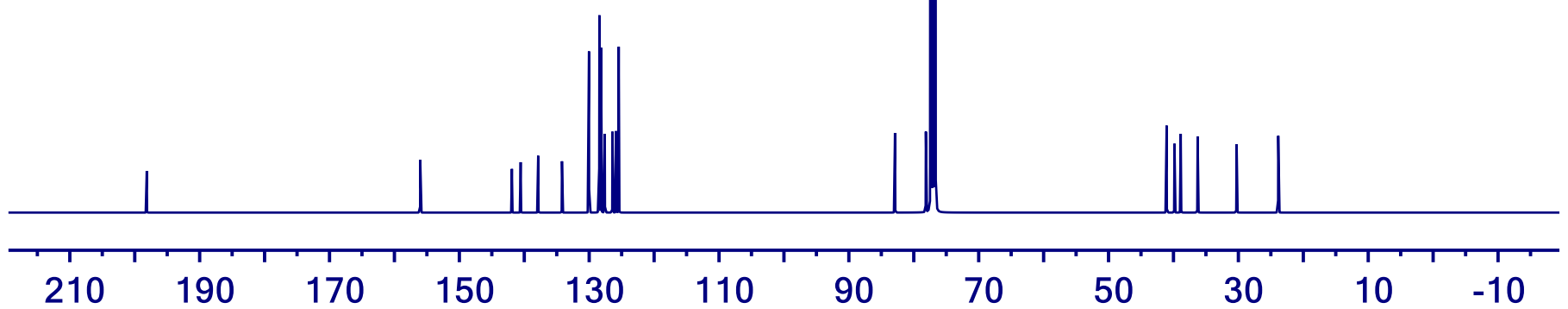


(1R,3R,8aS)-5-benzyl-3-(2-chlorophenyl)-1-methyl-1,3,4,7,8,8a-hexahydro-6H-isochromen-6-one

7n

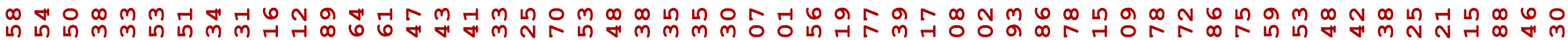

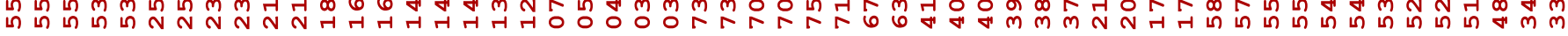

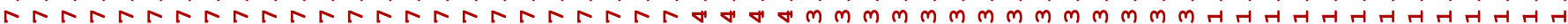<smiles>C[C@H]1OC(c2ccccc2Cl)CC2=C(Cc3ccccc3)C(=O)CC[C@H]21</smiles>

$1 \mathrm{H}$ NMR $\left(400 \mathrm{MHz}, \mathrm{CDC}_{3}\right)$
Nำ

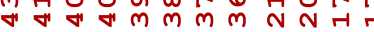
$\dot{m} \dot{m} \dot{m} \dot{m} \dot{m} \dot{m} \dot{m} \dot{m} \dot{m} \dot{m}$

ill

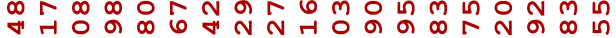

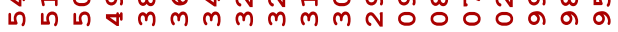

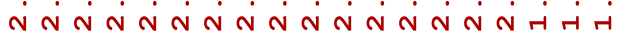

)

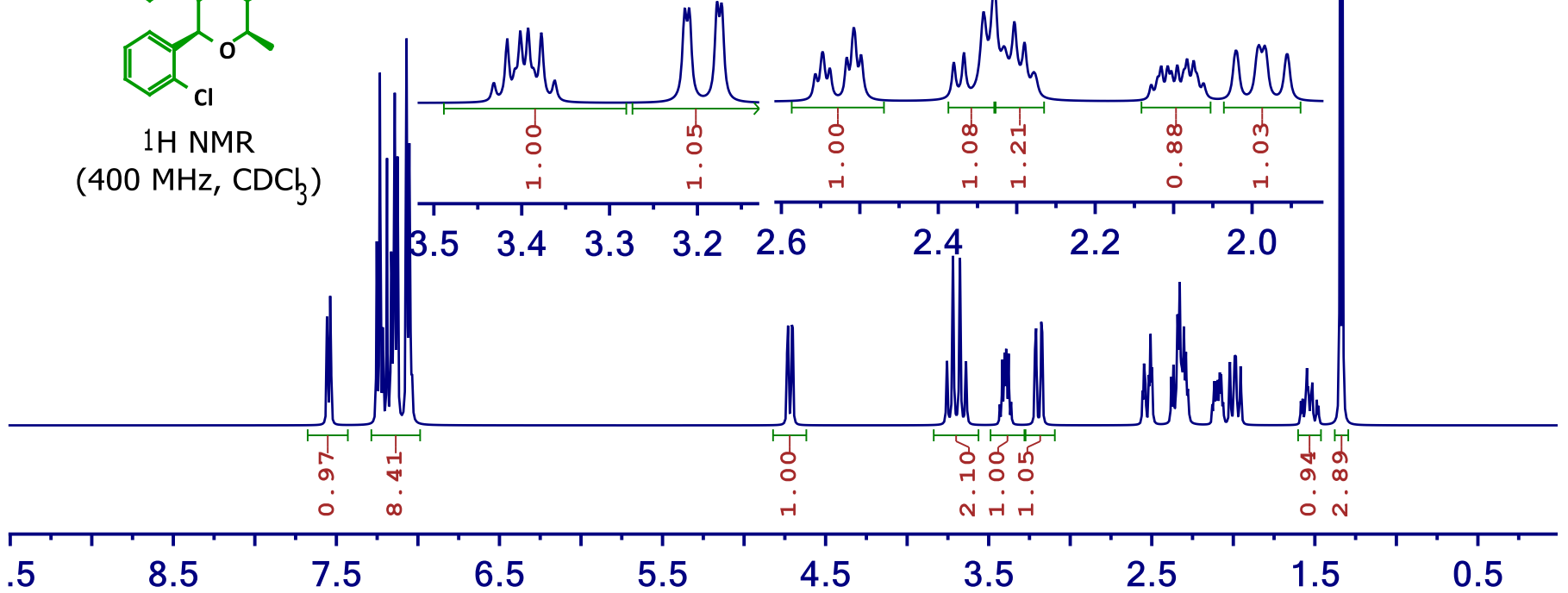

$m$
$\infty$
0
$a$
$r$

n H L

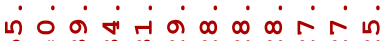

תִ

11<smiles>C[C@@H]1OC(c2ccccc2Cl)CC2=C(Cc3ccccc3)C(=O)CC[C@H]21</smiles>

Ho n $m a b$

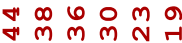

$1 / 111$

$13 \mathrm{C}\{1 \mathrm{H}\} \mathrm{NMR}$

(100 $\left.\mathrm{MHz}, \mathrm{CDCl}_{3}\right)$
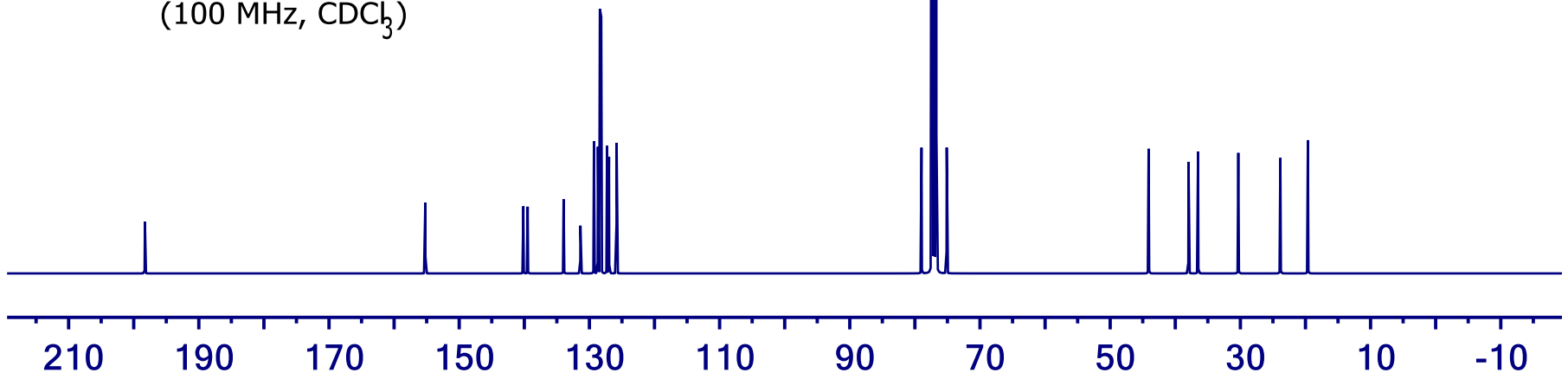
(1 $R, 3 R, 8 a S)$-5-benzyl-3-(4-isopropylphenyl)-1-methyl-1,3,4,7,8,8a-hexahydro-6H-isochromen-6-one

70
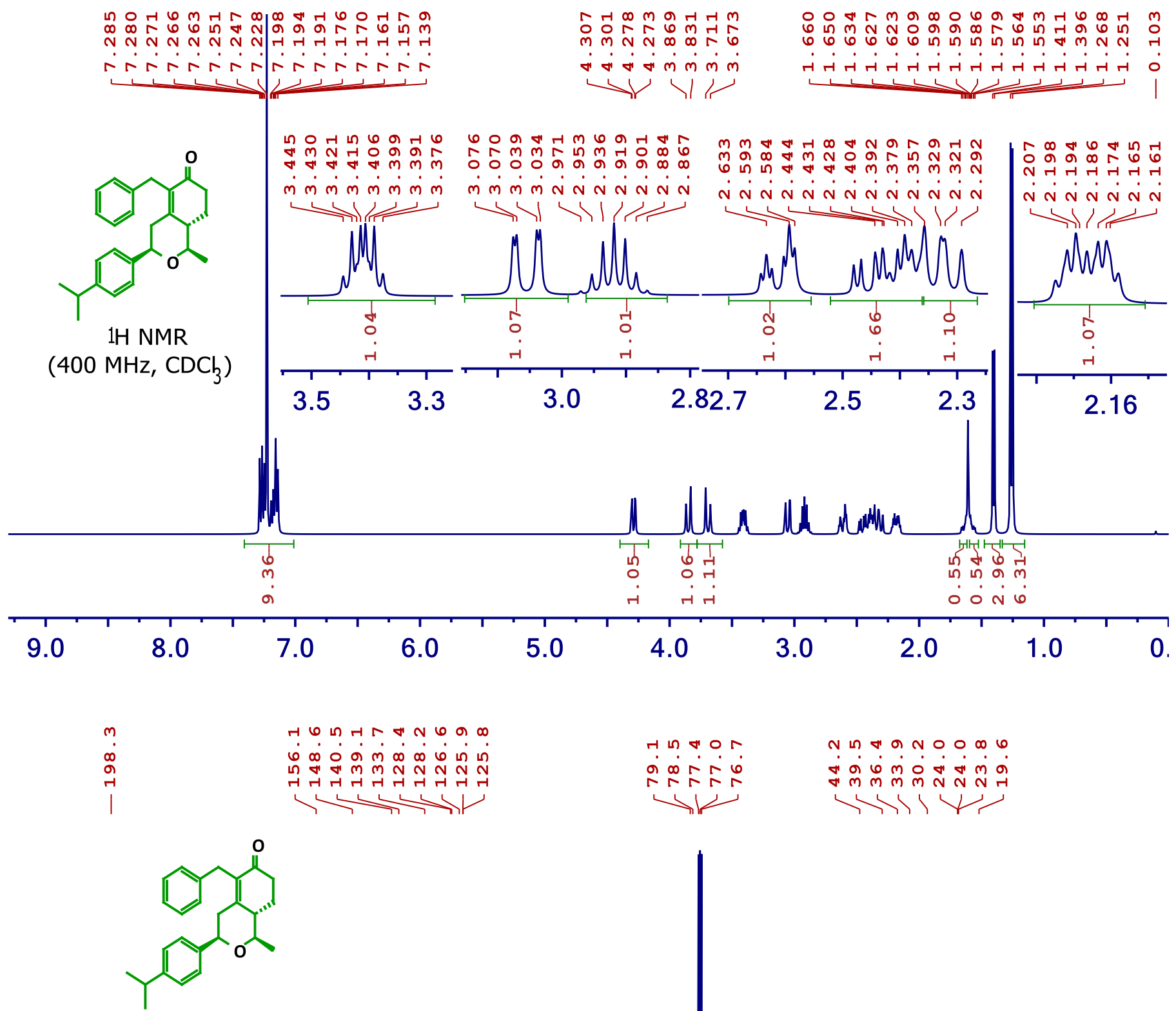

$13 \mathrm{C}\{1 \mathrm{H}\}$ NMR

$\left(100 \mathrm{MHz}, \mathrm{CDCl}_{3}\right)$
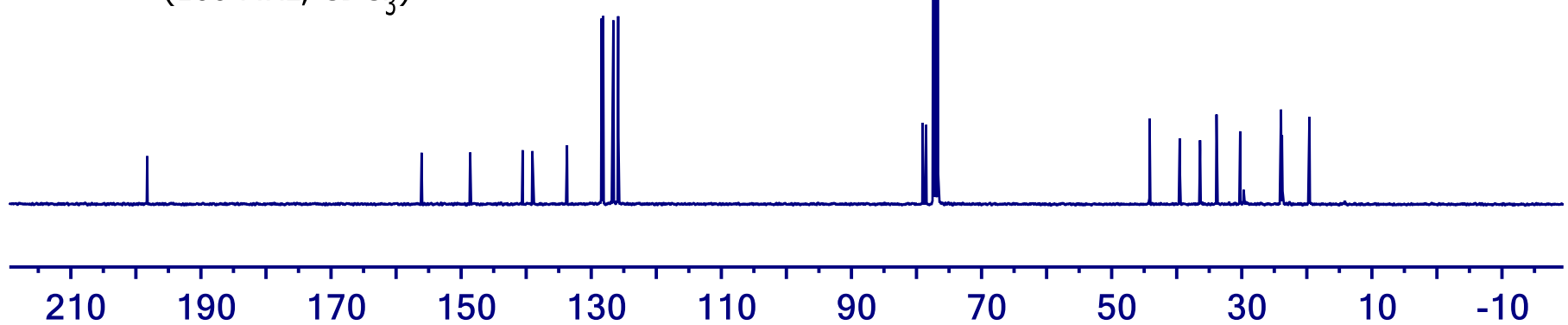
(1 $R, 3 R, 8 a S)$-5-benzyl-3-cyclohexyl-1-methyl-1,3,4,7,8,8a-hexahydro-6H-isochromen-6-one

$7 p$

صm N $N$ N

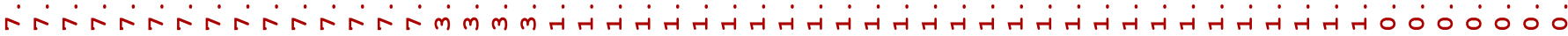
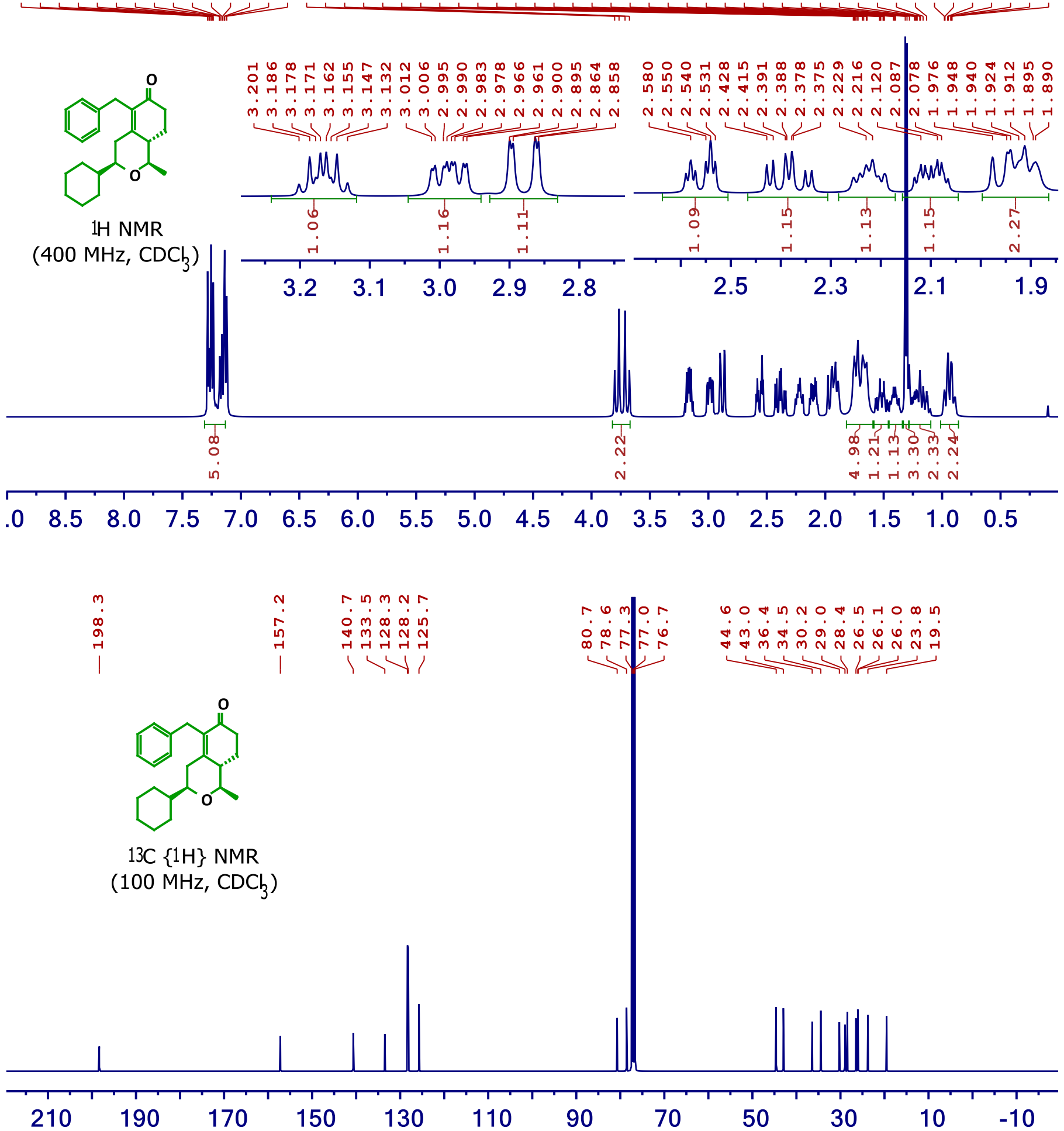
(1 $R, 3 S, 8 a S)-3,5$-dibenzyl-1-methyl-1,3,4,7,8,8a-hexahydro-6H-isochromen-6-one

$7 \mathrm{q}$

6 N 6 N $m$ a

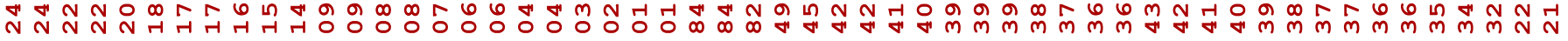

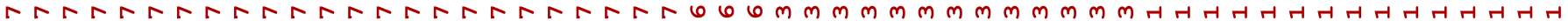<smiles>C[C@H]1OC(Cc2ccccc2)CC2=C(Cc3ccccc3)C(=O)CC[C@@H]21</smiles>

$1 \mathrm{H} N M R$ (400 $\left.\mathrm{MHz}, \mathrm{CDCh}_{3}\right)$

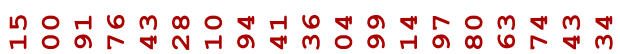
H H 0 o g a

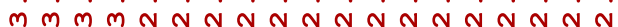
II,

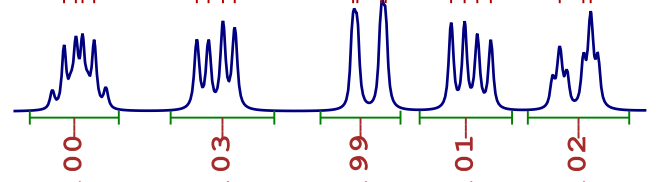

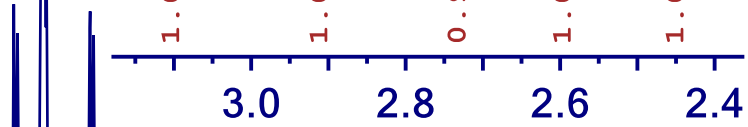

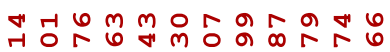
m

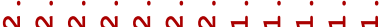

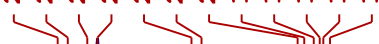

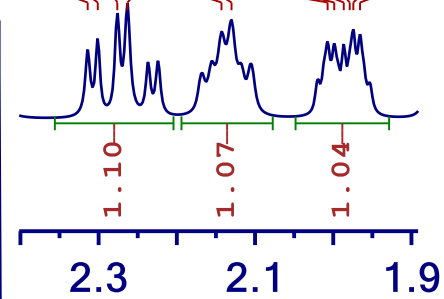

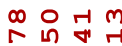

$\infty \quad \infty \quad \infty$

4 म

1/

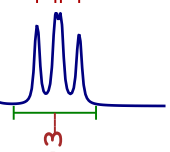

m

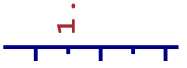

1.7

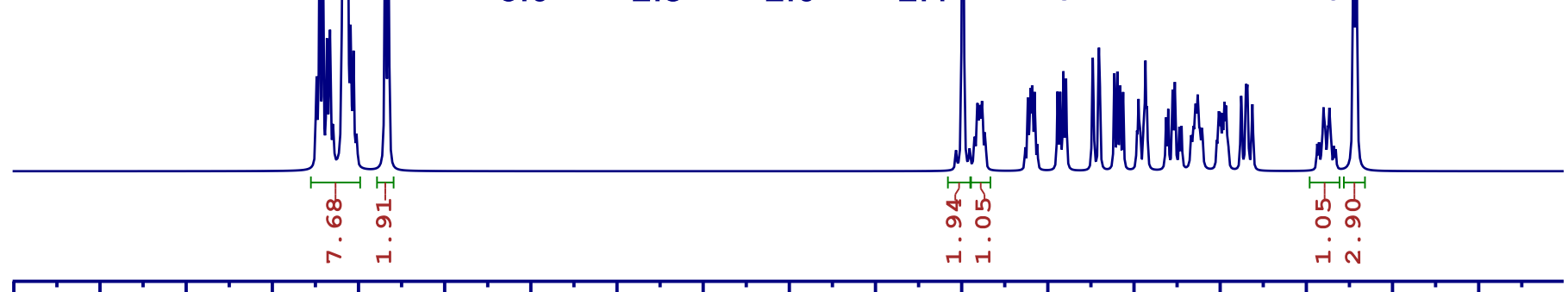

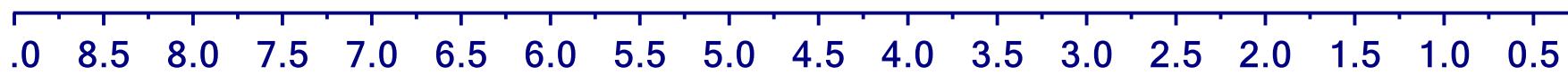

$m$
$\infty$
0
0
+1
$\mid$

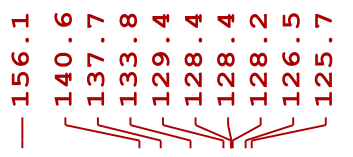

$13 \mathrm{C}\{1 \mathrm{H}\} \mathrm{NMR}$ (100 MHz, CDCb)

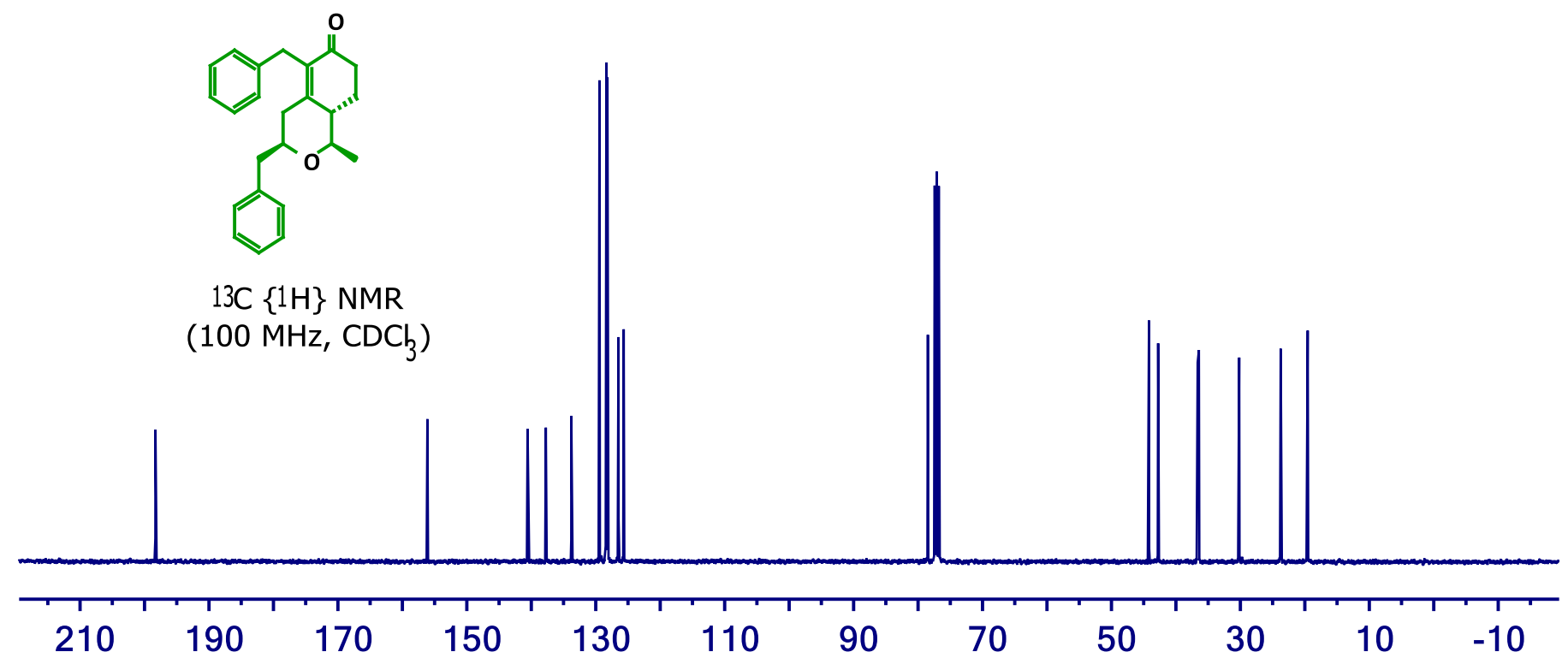

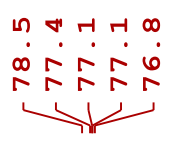

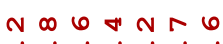
* $N$ $6 \circ m a$ i) 
(1 $R, 3 S, 8 a S)$-5-benzyl-3-butyl-1-methyl-1,3,4,7,8,8a-hexahydro-6H-isochromen-6-one

$7 \mathrm{r}$

ம

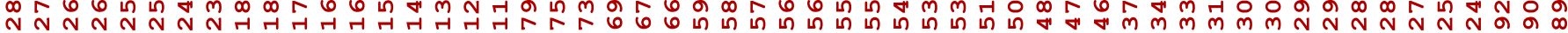

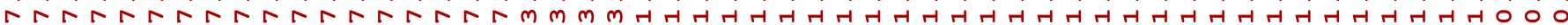

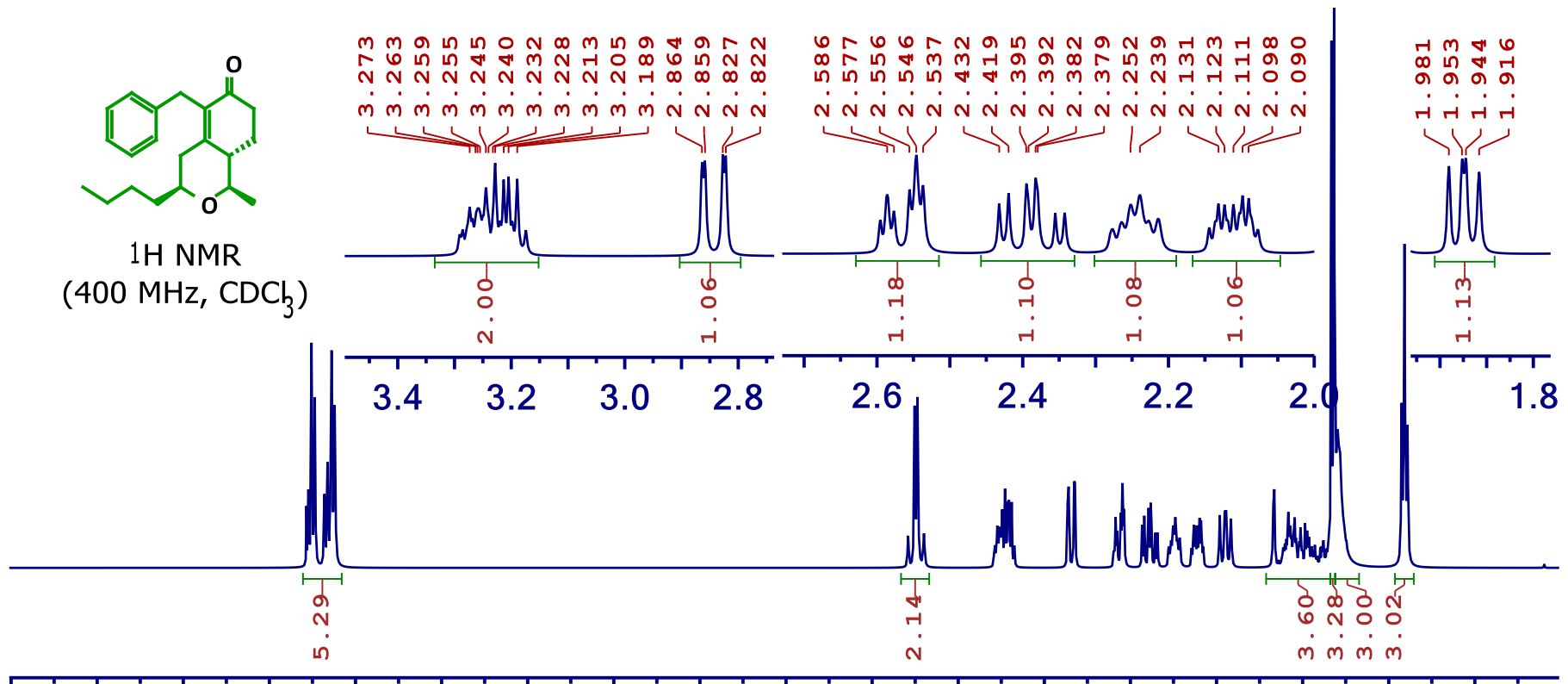

$\begin{array}{llllllllllllllllll}.0 & 8.5 & 8.0 & 7.5 & 7.0 & 6.5 & 6.0 & 5.5 & 5.0 & 4.5 & 4.0 & 3.5 & 3.0 & 2.5 & 2.0 & 1.5 & 1.0 & 0.5\end{array}$

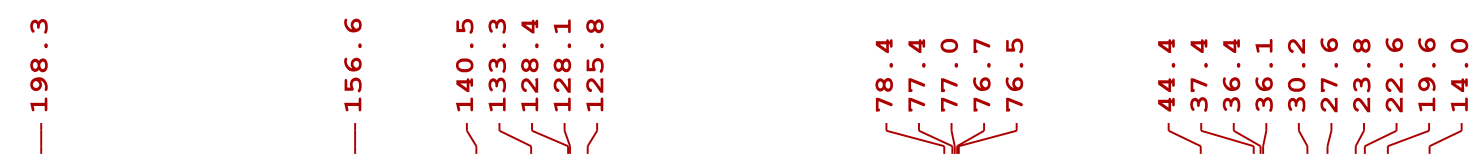<smiles>CCCC[C@H]1CC2=C(Cc3ccccc3)C(=O)CC[C@H]2[C@H](C)O1</smiles>

mising

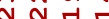
$\left(100 \mathrm{MHz}, \mathrm{CDC}_{3}\right)$

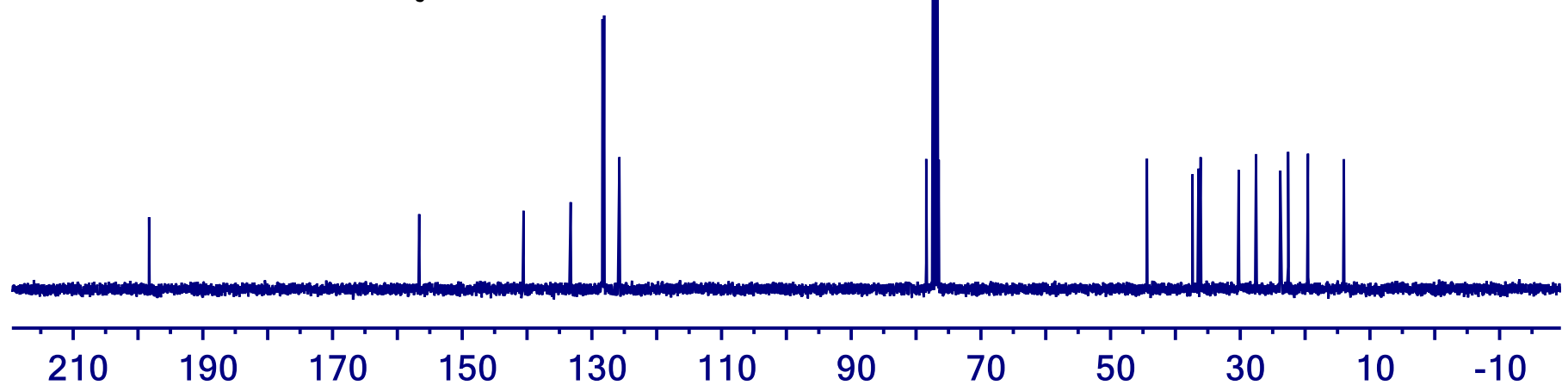


(1 $R, 3 R, 8 a S)$-5-benzyl-1,3-dimethyl-3-phenyl-1,3,4, 7,8,8a-hexahydro- 6 -isochromen-6-one

$7 \mathrm{~s} 1$

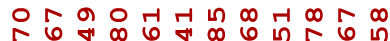

จ"

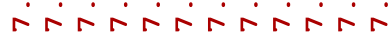

0

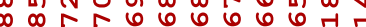

$\dot{m} \dot{m} \dot{m} \dot{m} \dot{m} \dot{m} \dot{m} \dot{m} \dot{m} \dot{m} \dot{m}$

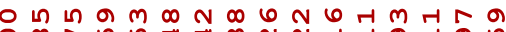

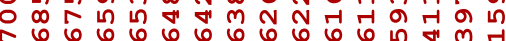

L

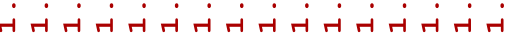

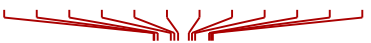<smiles>C[C@@H]1O[C@](C)(c2ccccc2)CC2=C(Cc3ccccc3)C(=O)CC[C@H]21</smiles>

$1 \mathrm{H} N M R$

$\left(400 \mathrm{MHz}, \mathrm{CDC}_{3}\right)$

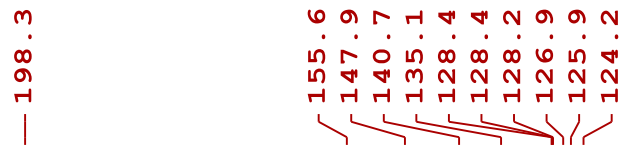

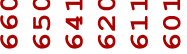

in $\operatorname{cin} 4 \sin$

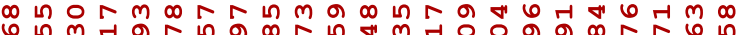

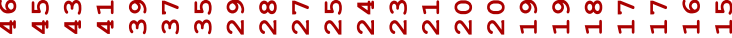

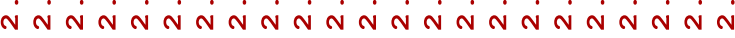

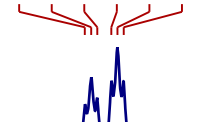

is
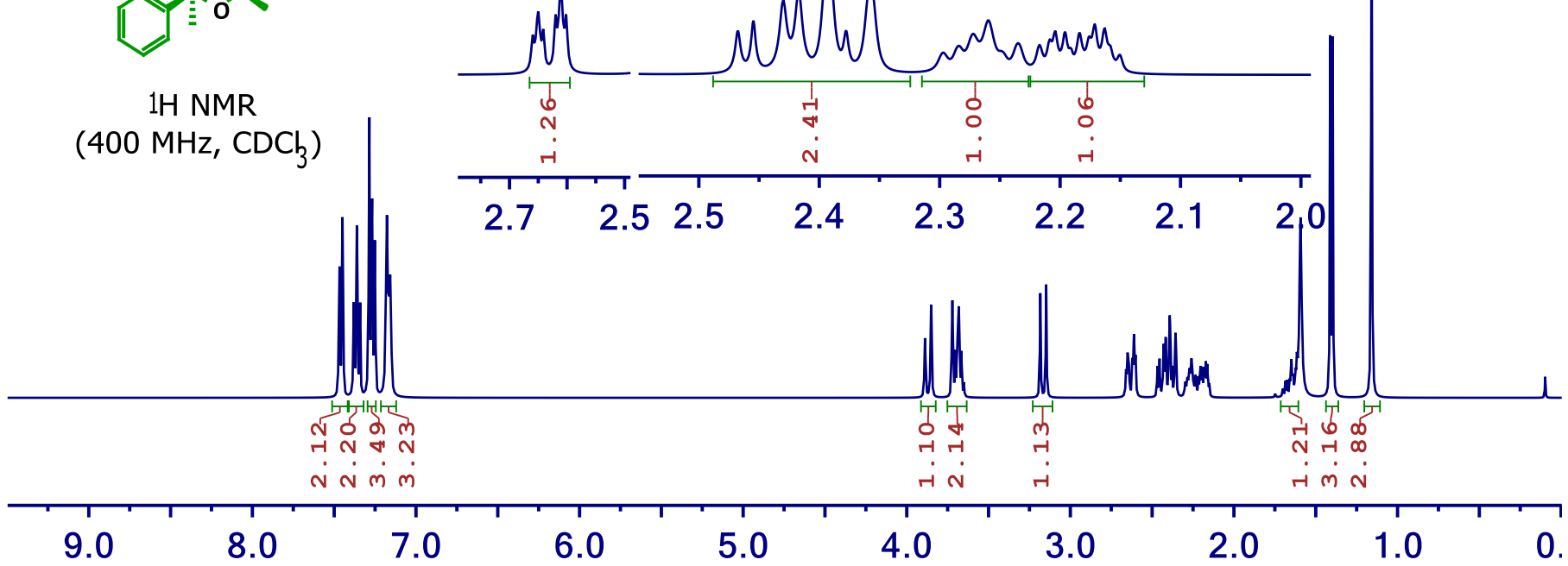<smiles>CC1OC(c2ccccc2)CC2=C(Cc3ccccc3)C(=O)CCC21</smiles>

13C $\{1 \mathrm{H}\}$ NMR (100 MHz, $\mathrm{CDC}_{3}$ )

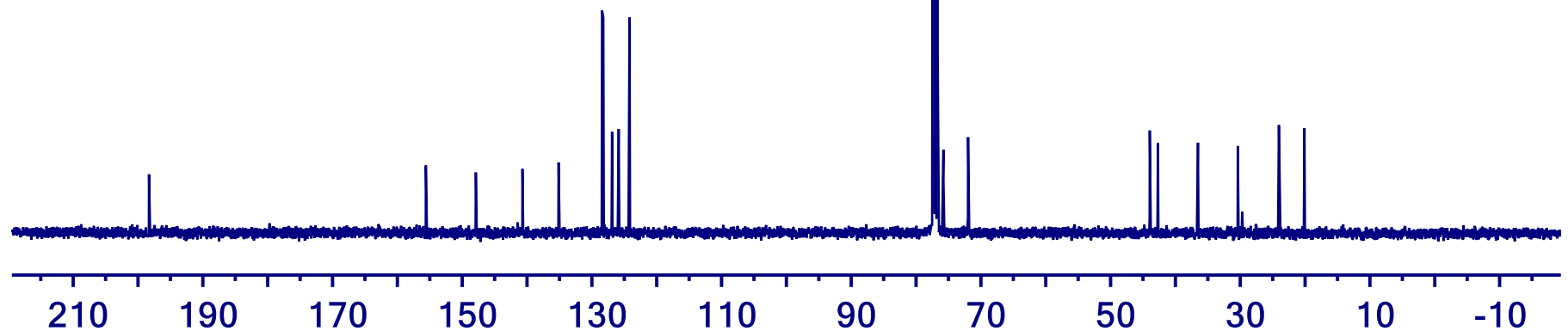


(1 R,3 $S, 8 a S)$-5-benzyl-1,3-dimethyl-3-phenyl-1,3,4,7,8,8a-hexahydro- 6 -isochromen-6-one

$7 \mathrm{~s} 2$

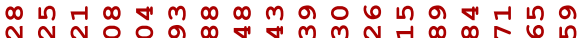
N N N N

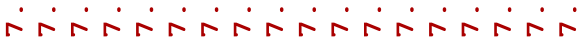

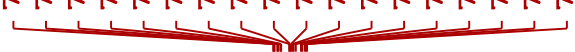

1H NMR

$3.1 \quad 2.9$

nิ

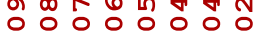

$\dot{m} \dot{m} \dot{m} \dot{m} \dot{m} \dot{m} \dot{m} \dot{m}$

$\longrightarrow$

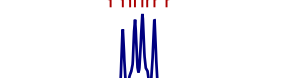

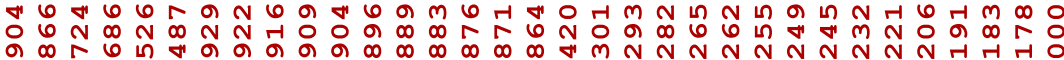
m m m m m m

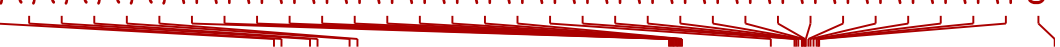

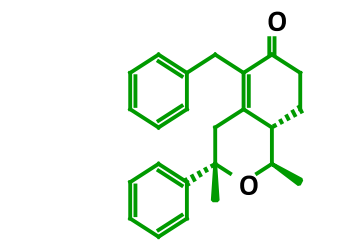

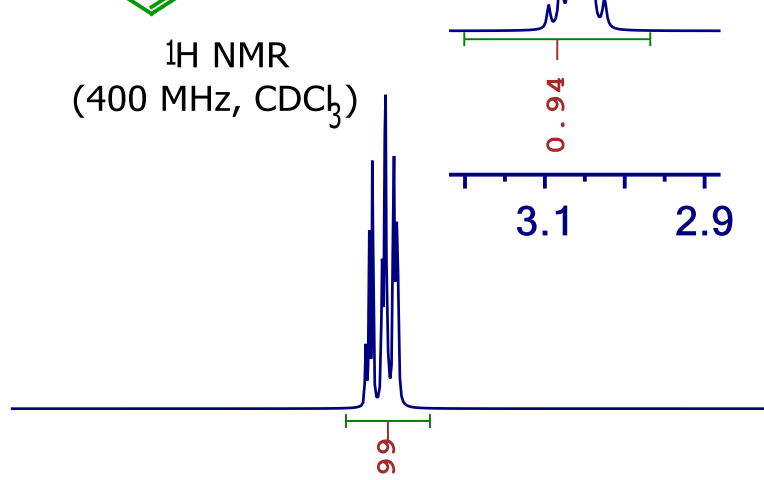

9

⿰丿⿱丄𠃍冂卄八

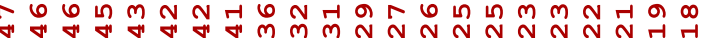

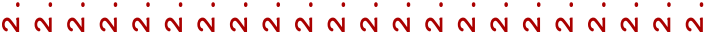

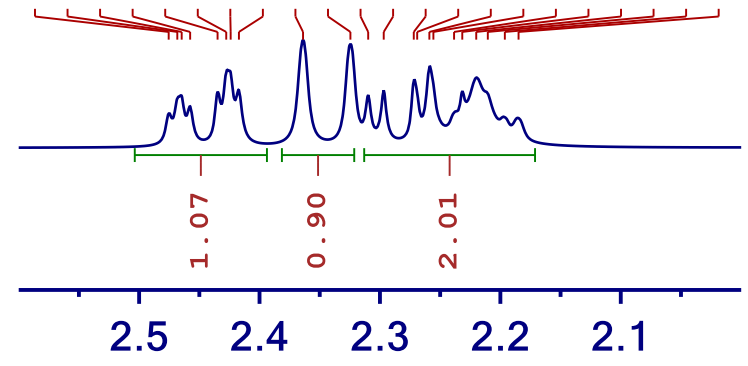

.0

8.0

7.0

6.0

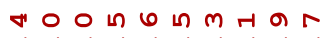

i न 0 m $\infty \dot{\infty} \infty \dot{n}$ in

นึ

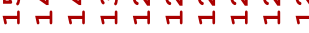

次<smiles>C[C@@H]1O[C@](C)(c2ccccc2)CC2=C(Cc3ccccc3)C(=O)CC[C@H]21</smiles>

13C $\{1 \mathrm{H}\}$ NMR

(100 $\mathrm{MHz}, \mathrm{CDCh}_{3}$ )

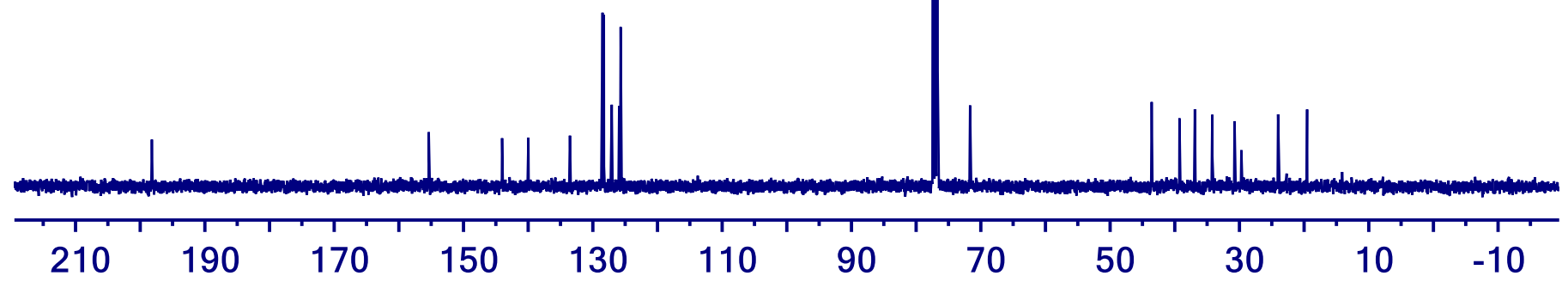




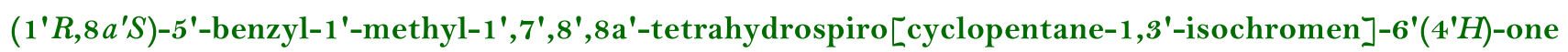

$7 \mathrm{t}$

几

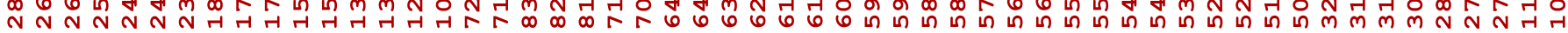

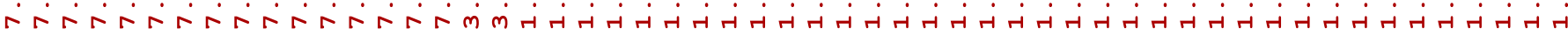
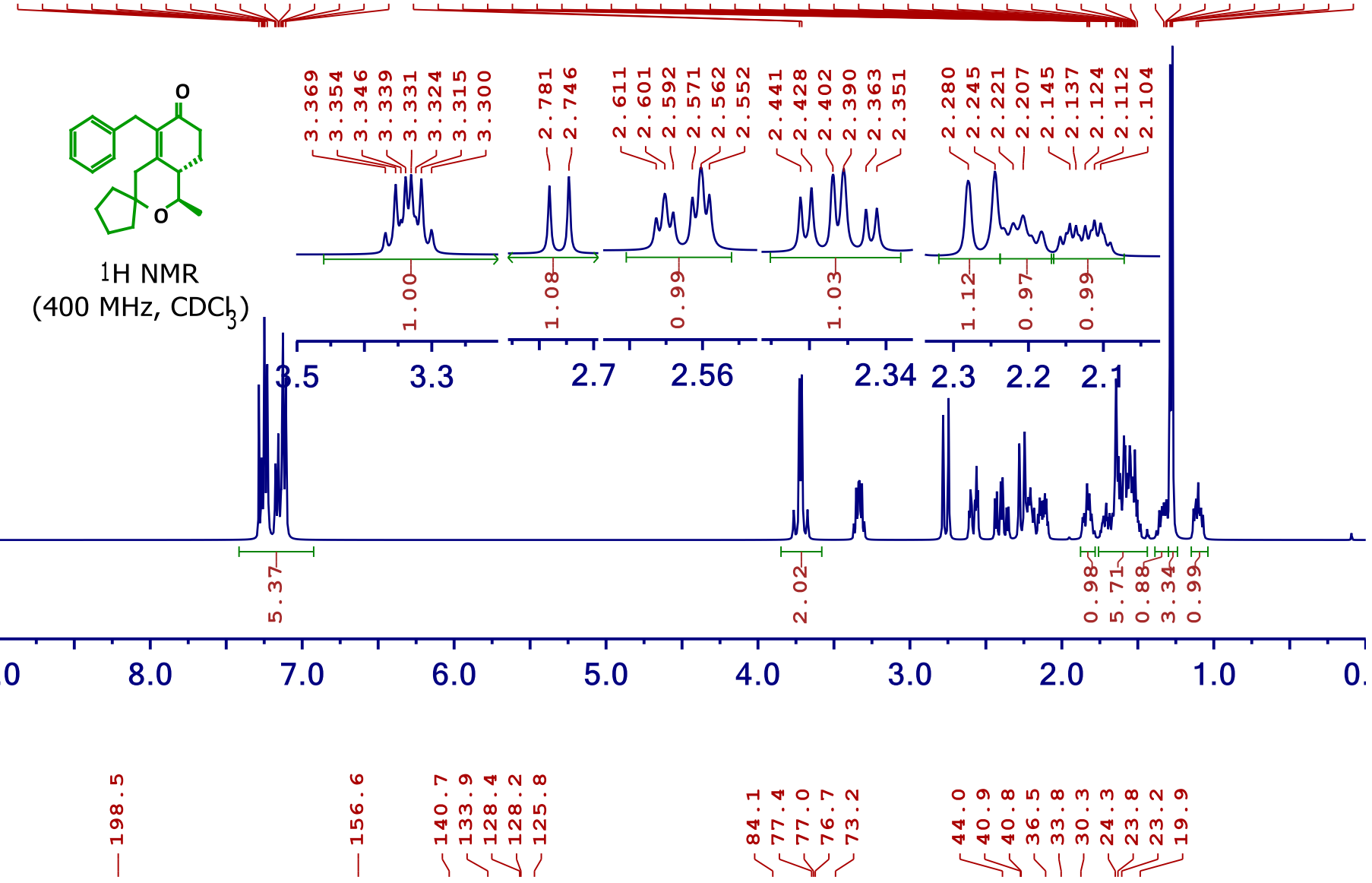<smiles>C[C@H]1CCC(=O)C(Cc2ccccc2)=C1C1CCCC1</smiles>

$13 \mathrm{C}\{1 \mathrm{H}\} \mathrm{NMR}$

$\left(100 \mathrm{MHz}, \mathrm{CDCb}_{3}\right)$

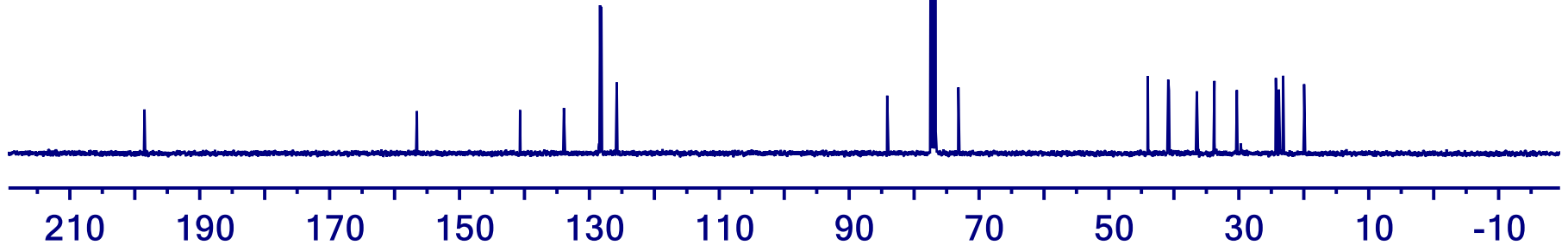


(1 $R, 3 R, 8 a S)$-1-methyl-3,5-diphenyl-1,3,4,7,8,8a-hexahydro-6H-isochromen-6-one

7b

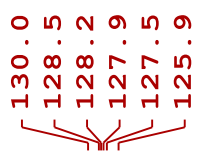

$r o$
ro
ro

\ْ

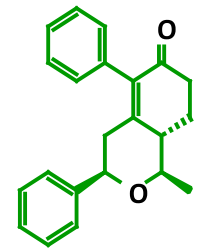

DEPT 90

$100 \mathrm{MHz}, \mathrm{CDCl}_{3}$

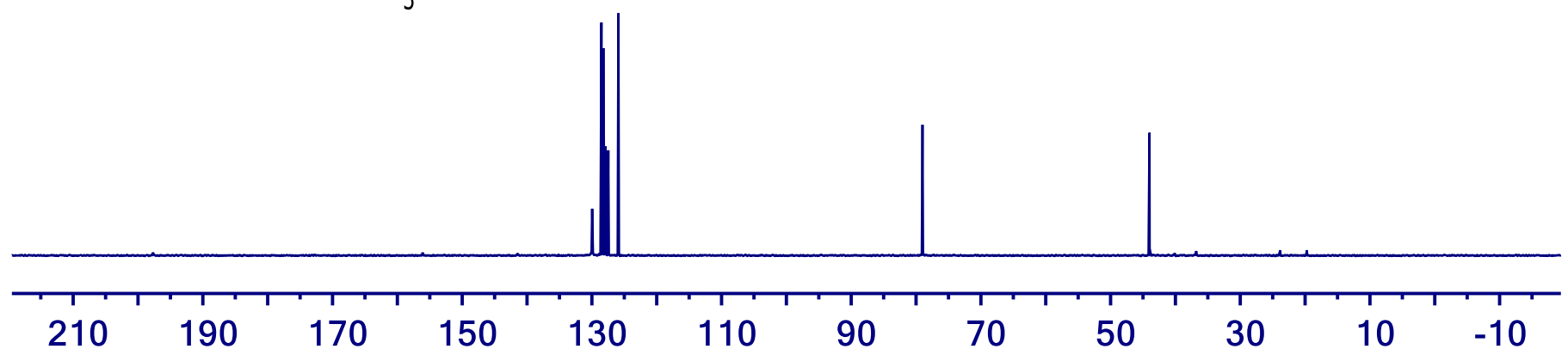

- in $N$ a $\ln$ a

i $\infty \dot{\infty} \dot{\sim} \dot{\sim}$ in

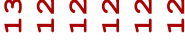

$\rightarrow$ $r o$
ro
20

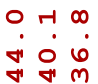

111 $\infty$ r

m 9

|

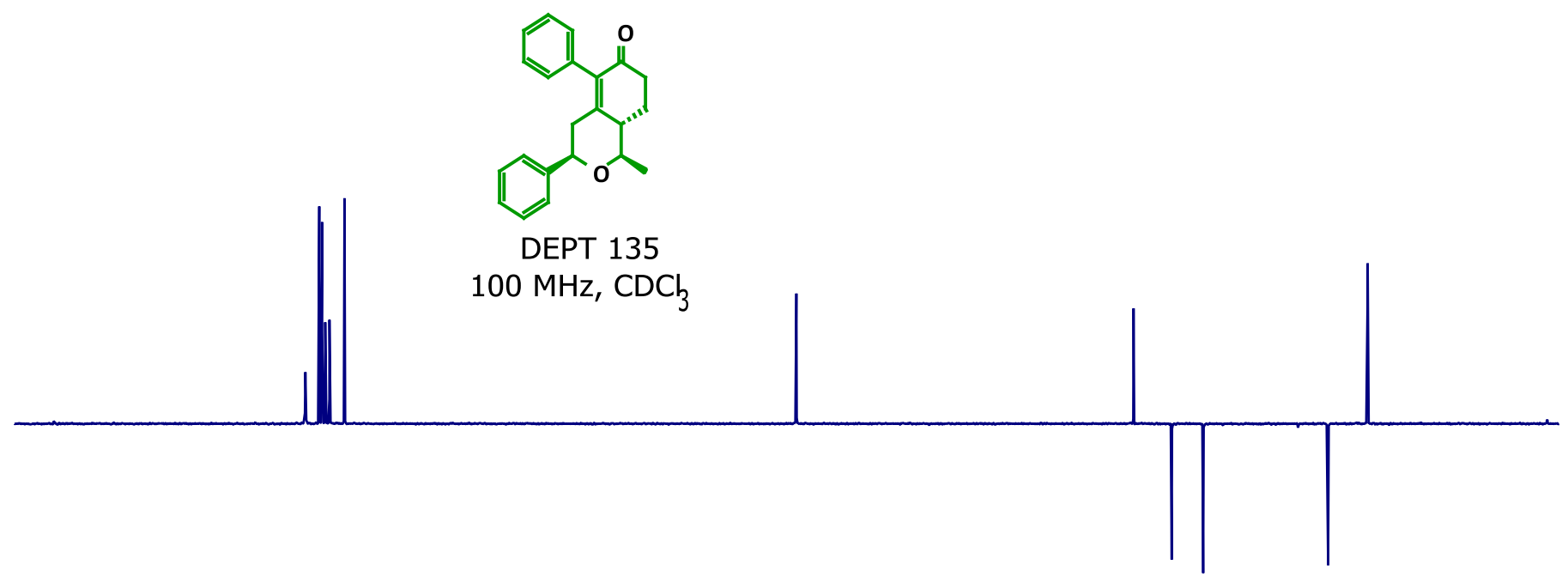

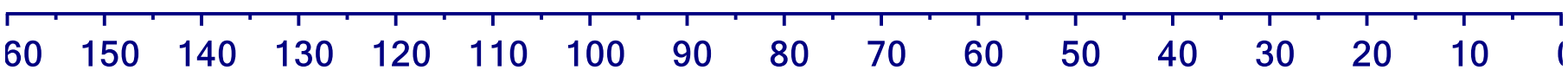


(1 $R, 3 R, 8 a S)$-5-benzyl-1-isobutyl-3-phenyl-1,3,4,7,8,8a-hexahydro- $6 H$-isochromen-6-one

7 e
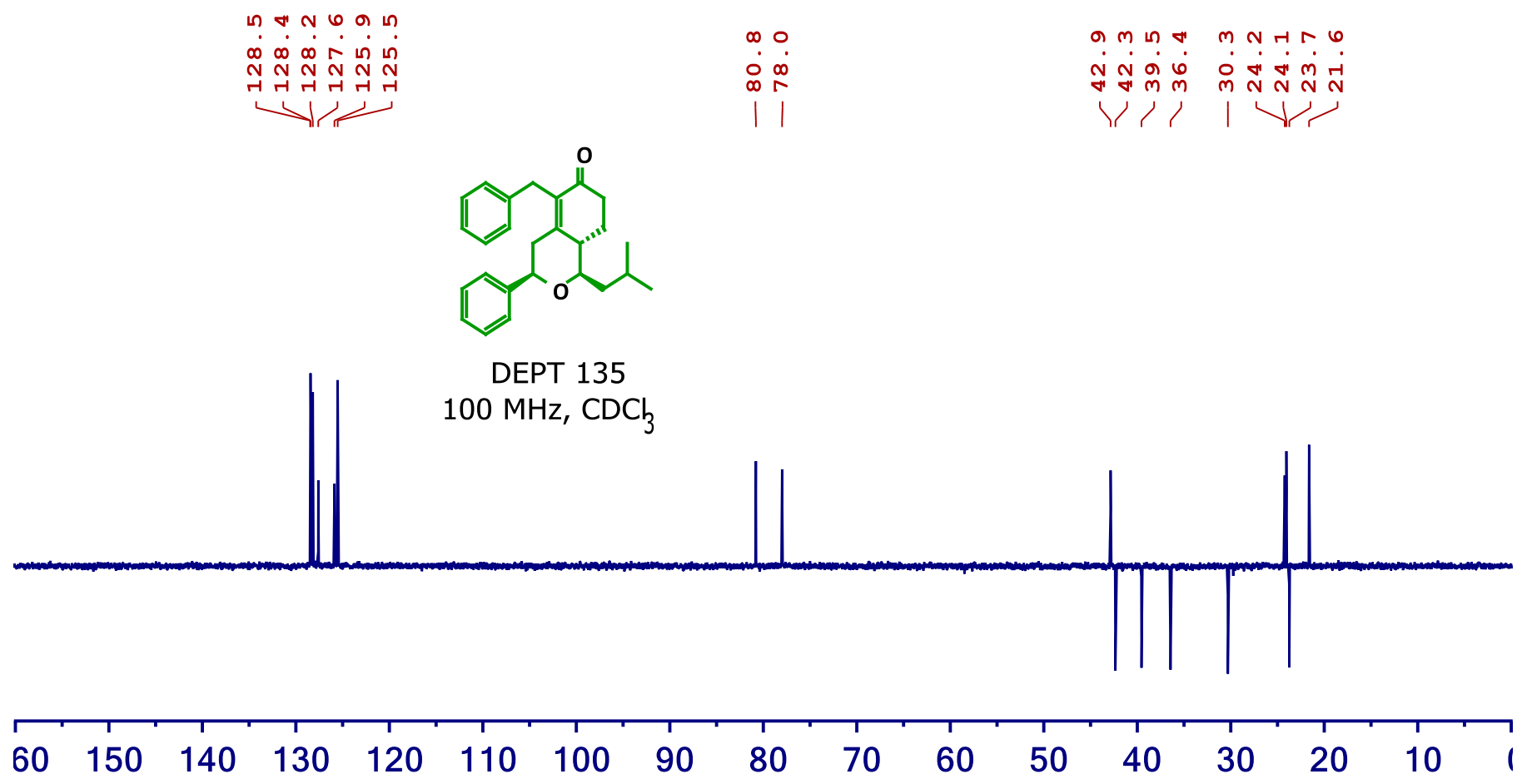

(1 $R, 3 R, 8 a S)$-5-benzyl-1-cyclopropyl-3-phenyl-1,3,4,7,8,8a-hexahydro-6H-isochromen-6-one

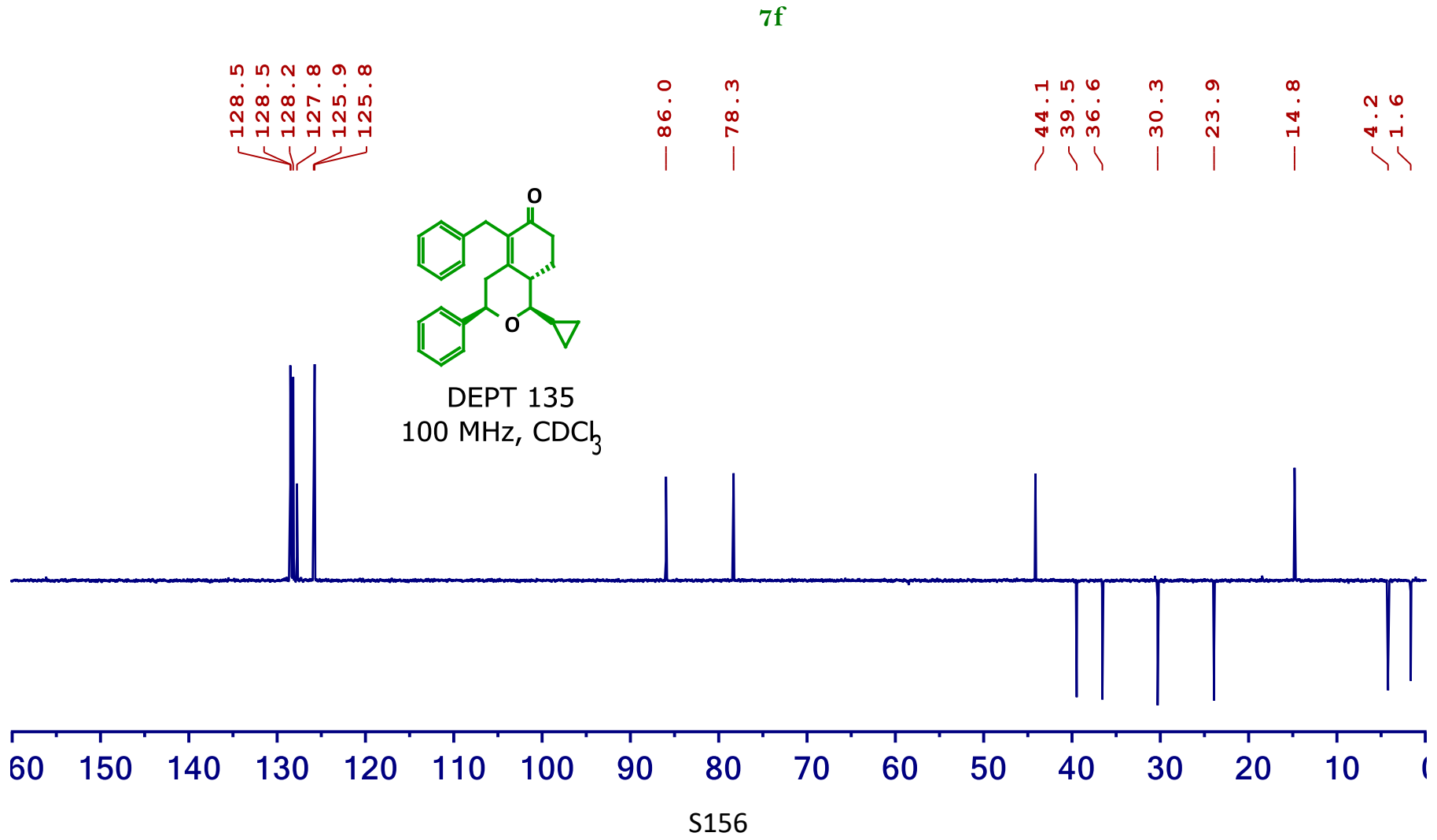


(1R,3R,8aS)-5-benzyl-1-ethyl-3-phenyl-1,3,4,7,8,8a-hexahydro-6H-isochromen-6-one

$7 \mathrm{~g}$

ก

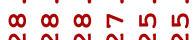

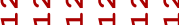

पा/

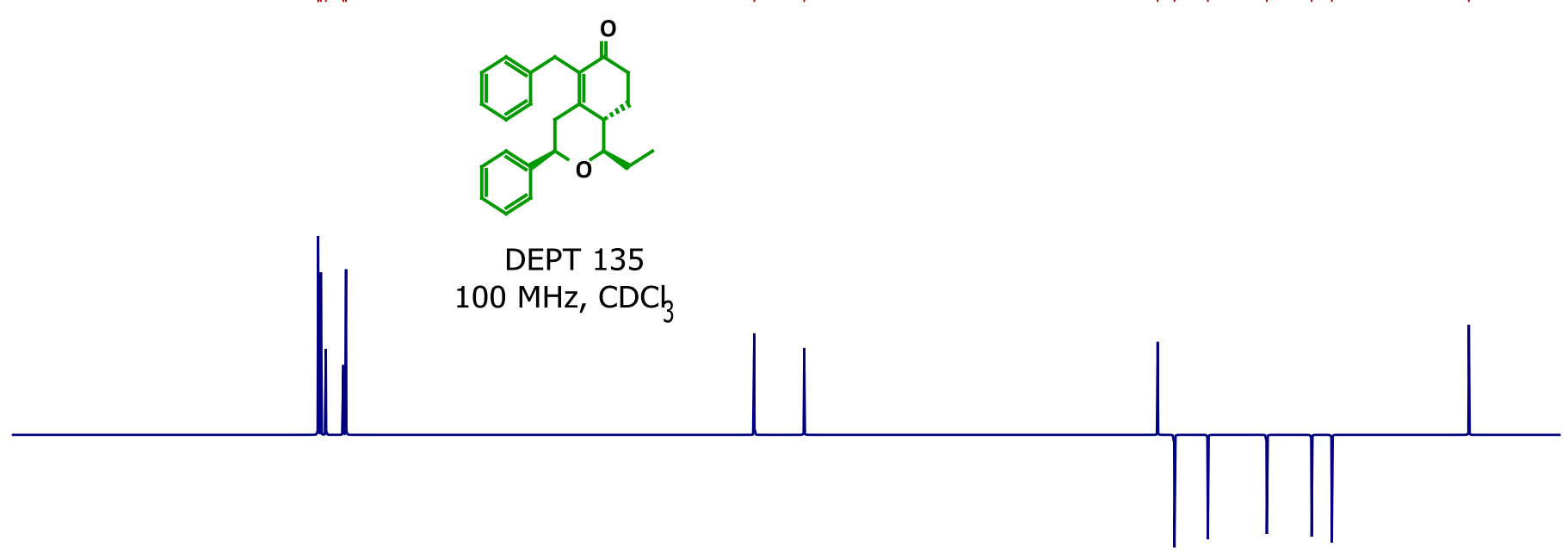

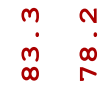

$60 \% \quad m 60$

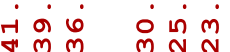

1) 1 1 1

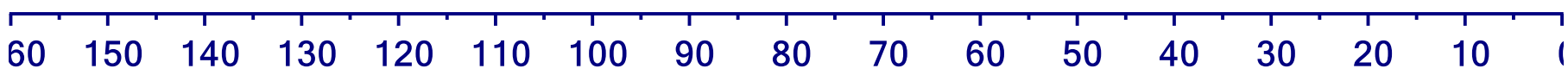

(1 $S, 3 R, 8 a S)$-5-benzyl-1-(4-methoxyphenyl)-3-phenyl-1,3,4,7,8,8a-hexahydro- $6 H$-isochromen-6-one
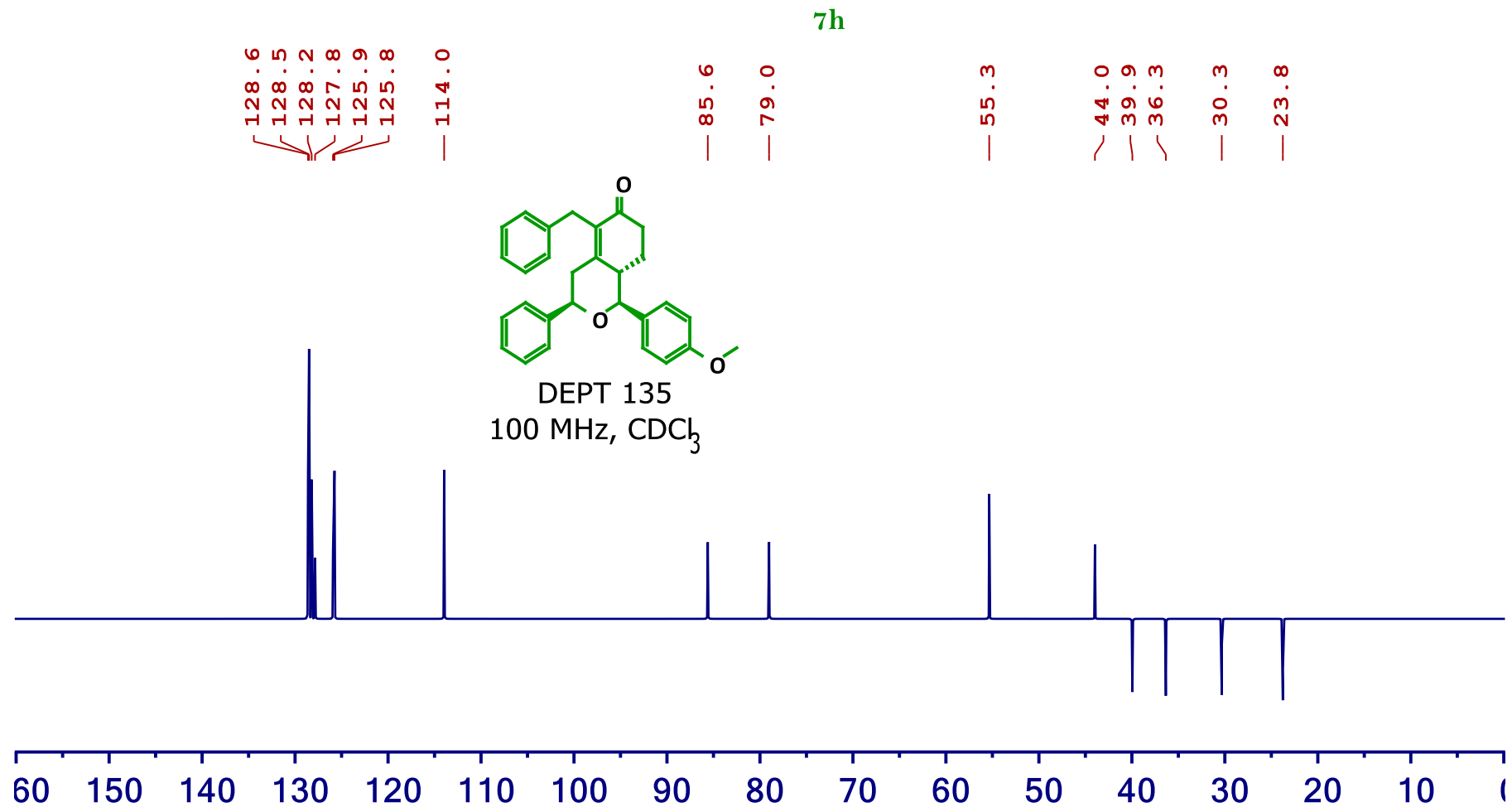
(1R,3R,8aS)-5-benzyl-1-butyl-3-phenyl-1,3,4,7,8,8a-hexahydro-6H-isochromen-6-one

71

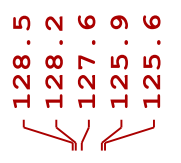

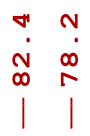

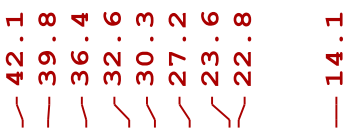
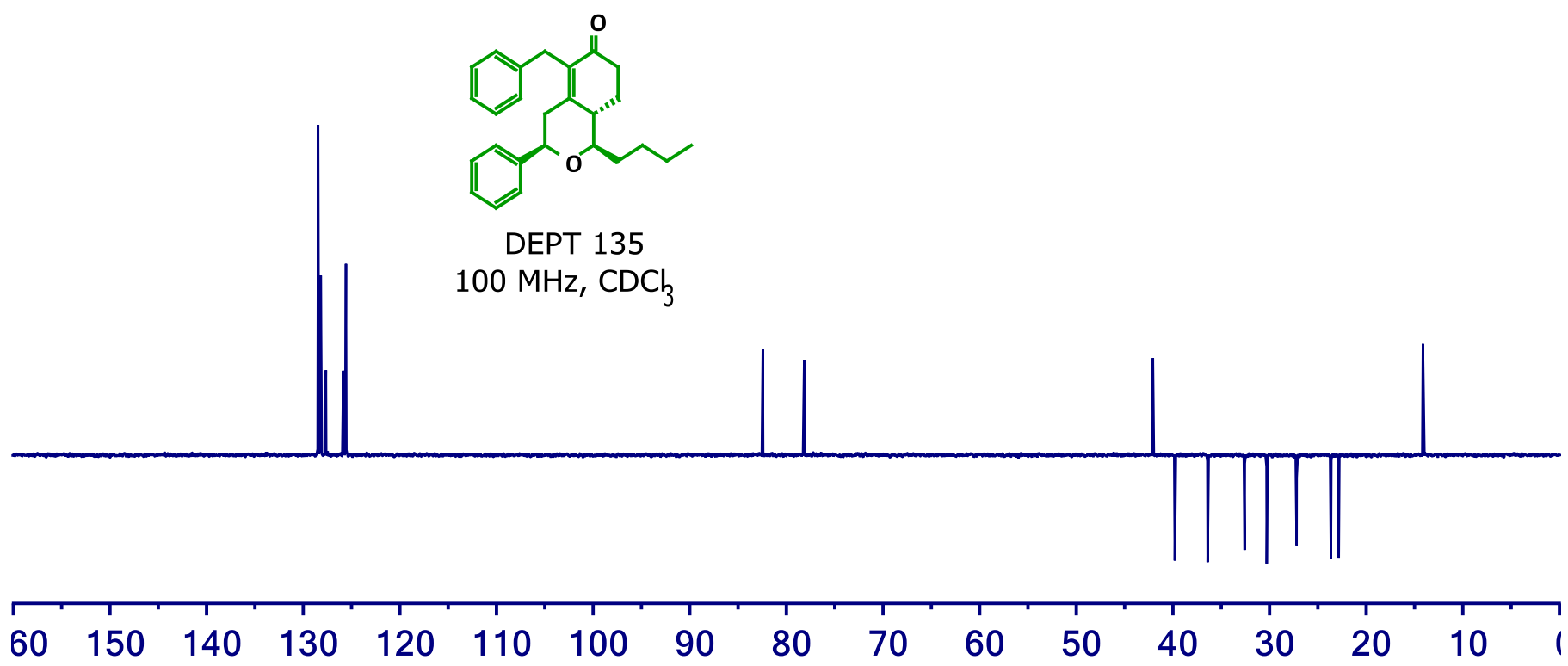

(1R,3R,8aS)-1,5-dibenzyl-3-phenyl-1,3,4,7,8,8a-hexahydro-6H-isochromen-6-one

$7 \mathrm{~m}$

ก

N N N N N N N N N

每

$\stackrel{0}{\infty} \stackrel{0}{i}$

$\stackrel{\infty}{m} \stackrel{m}{m} \stackrel{\infty}{m} \stackrel{m}{m} \stackrel{\infty}{N} \stackrel{-1}{N}$<smiles>O=C1CCC2=C(Cc3ccccc3)C(c3ccccc3)OC(Cc3ccccc3)C2C1</smiles>

DEPT 135

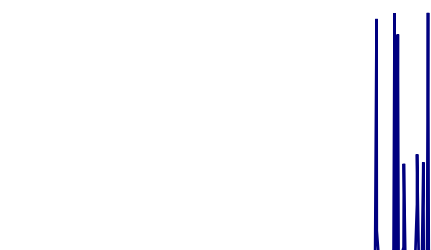

$100 \mathrm{MHz}, \mathrm{CDCl}_{3}$

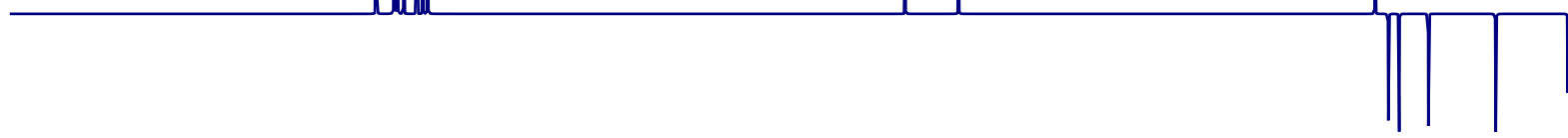

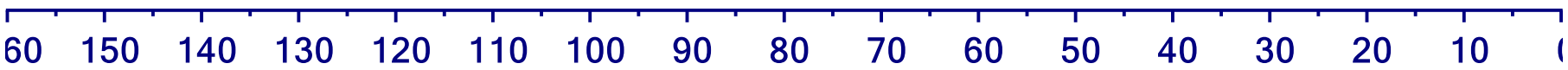


(1 $R, 3 R, 8 a S)$-5-benzyl-1,3-dimethyl-3-phenyl-1,3,4, 7,8,8a-hexahydro- $6 H$-isochromen-6-one

$7 \mathrm{~s} 1$
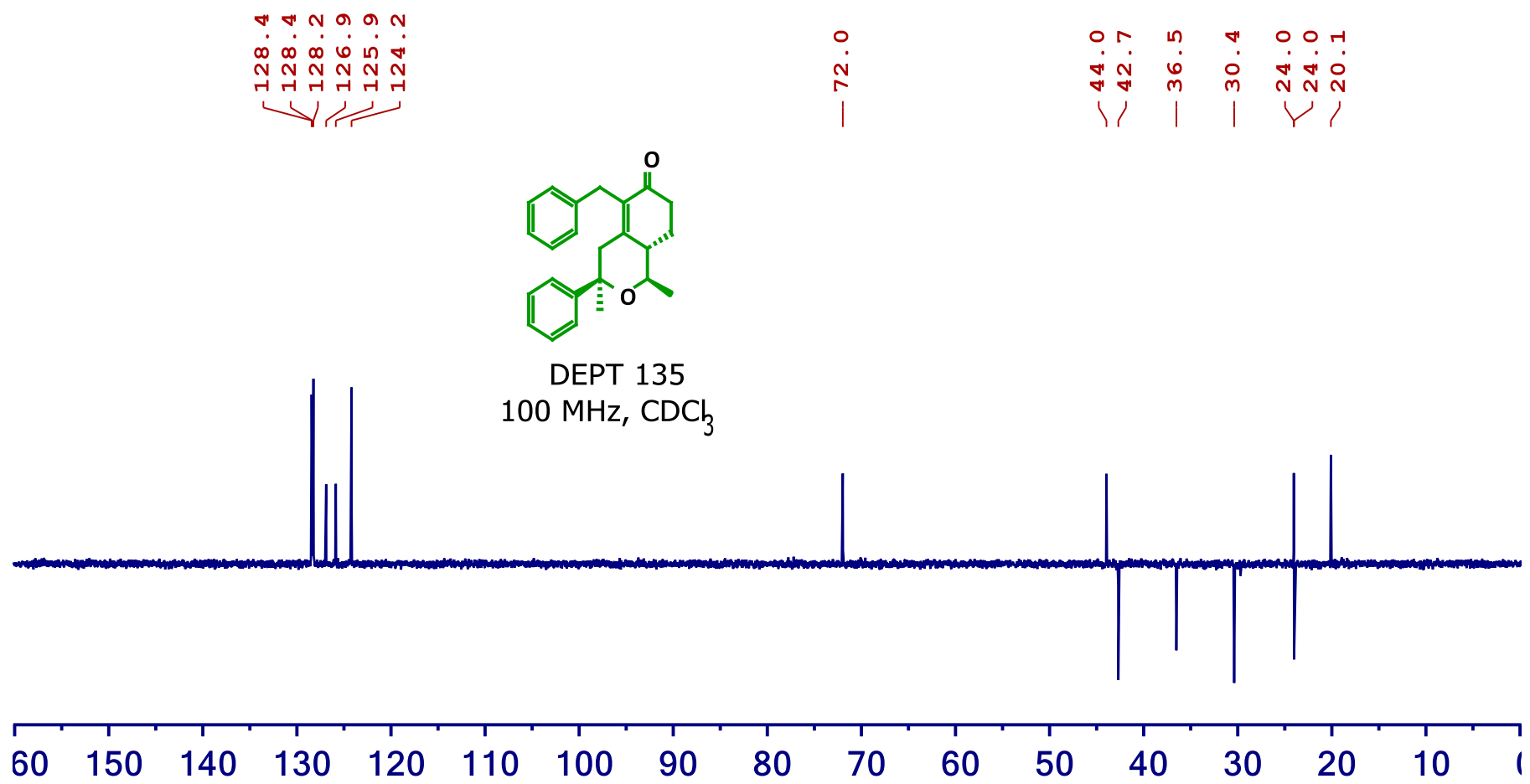

$\left(1^{\prime} R, 8 a^{\prime} S\right)$-5'-benzyl-1'-methyl-1',7',8',8a'-tetrahydrospiro[cyclopentane-1,3'-isochromen]-6'(4'H)-one

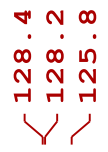

N

$\circ a \infty$ in $\infty m m \infty N o$

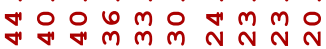

$\stackrel{m}{n}$

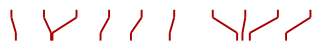

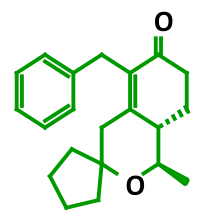

DEPT 135
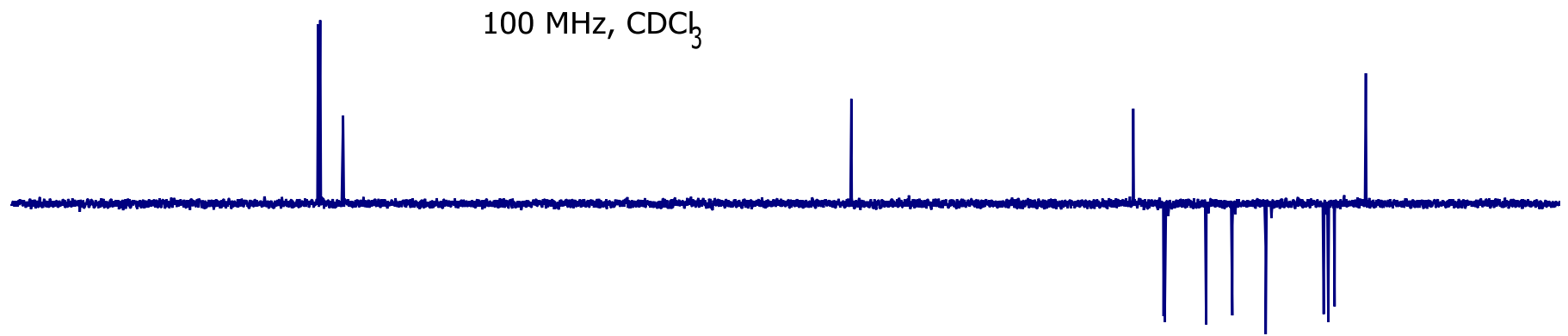

$\begin{array}{llllllllllllllll}60 & 150 & 140 & 130 & 120 & 110 & 100 & 90 & 80 & 70 & 60 & 50 & 40 & 30 & 20 & 10\end{array}$


(1R,3R,8aS)-1-methyl-3,5-diphenyl-1,3,4,7,8,8a-hexahydro-6H-isochromen-6-one

$\mathbf{7 b}$

a 아의

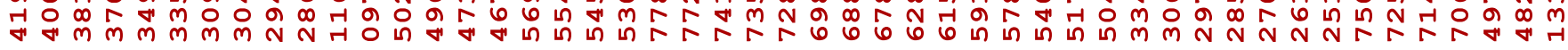

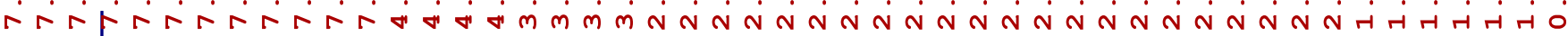
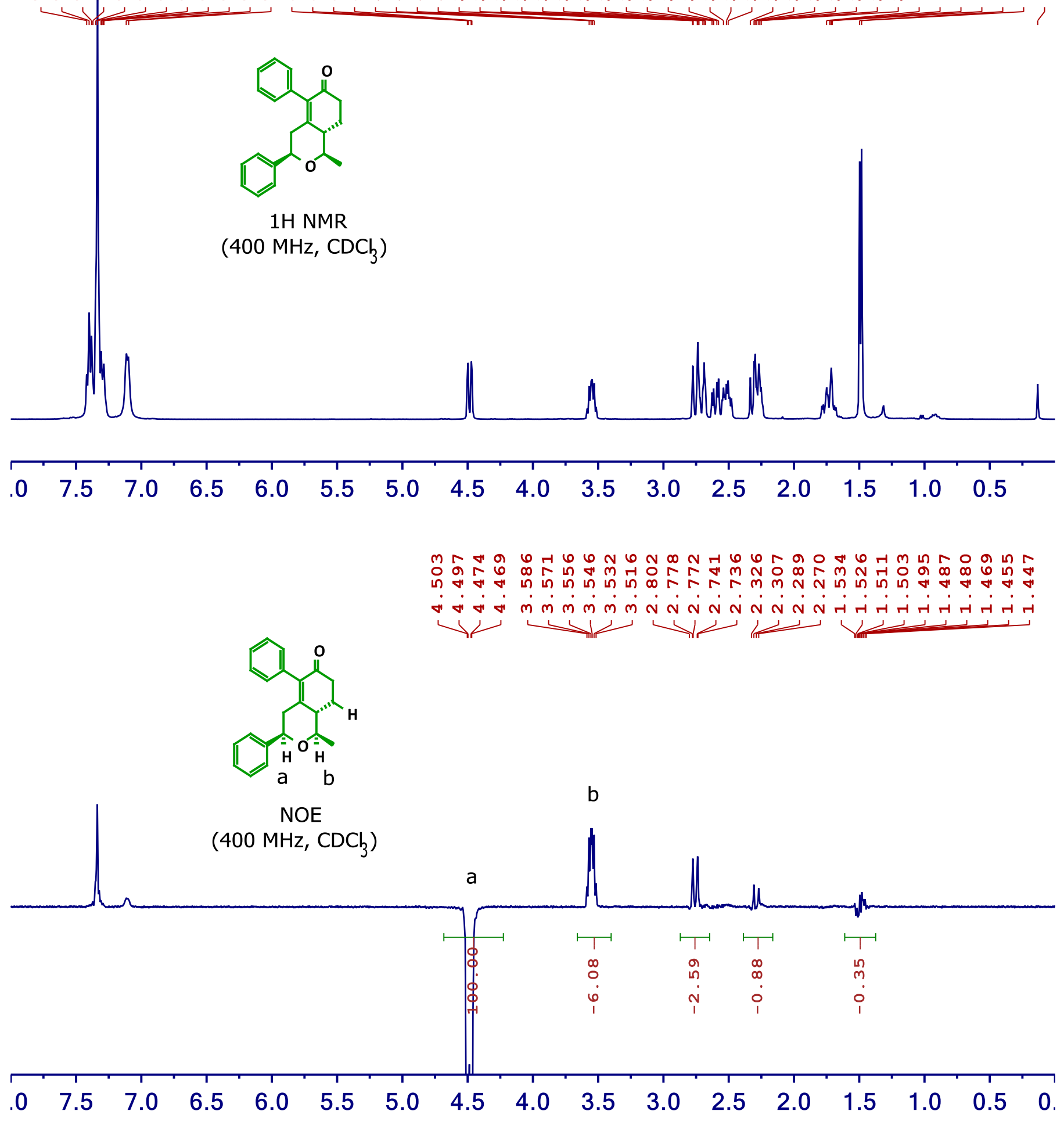
(1 $R, 3 R, 8 a S)$-1-methyl-3,5-diphenyl-1,3,4,7,8,8a-hexahydro-6H-isochromen-6-one

$7 \mathbf{b}$

m人テr ㅇํㄱㅇㅛ

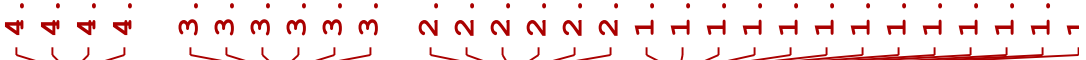

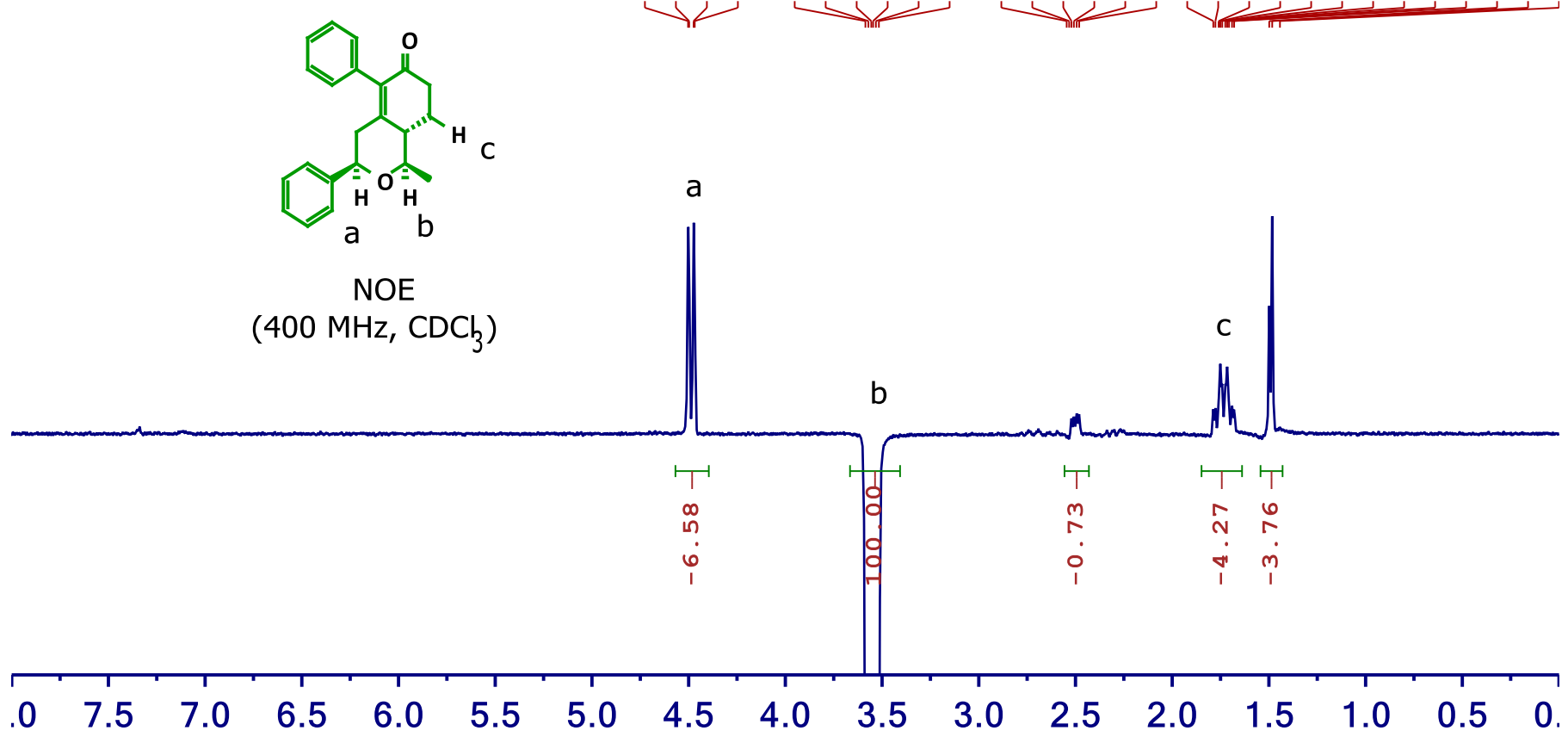

$\underbrace{m m m m}$

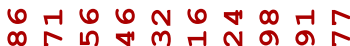

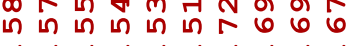

ம n $⿻ 上 丨$

$m M M M M N N N$

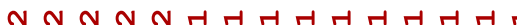

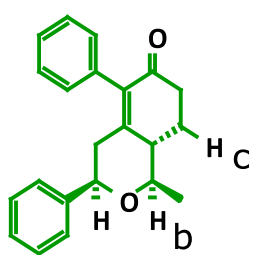

NOE (400 MHz, $\mathrm{CDC}_{3}$ )
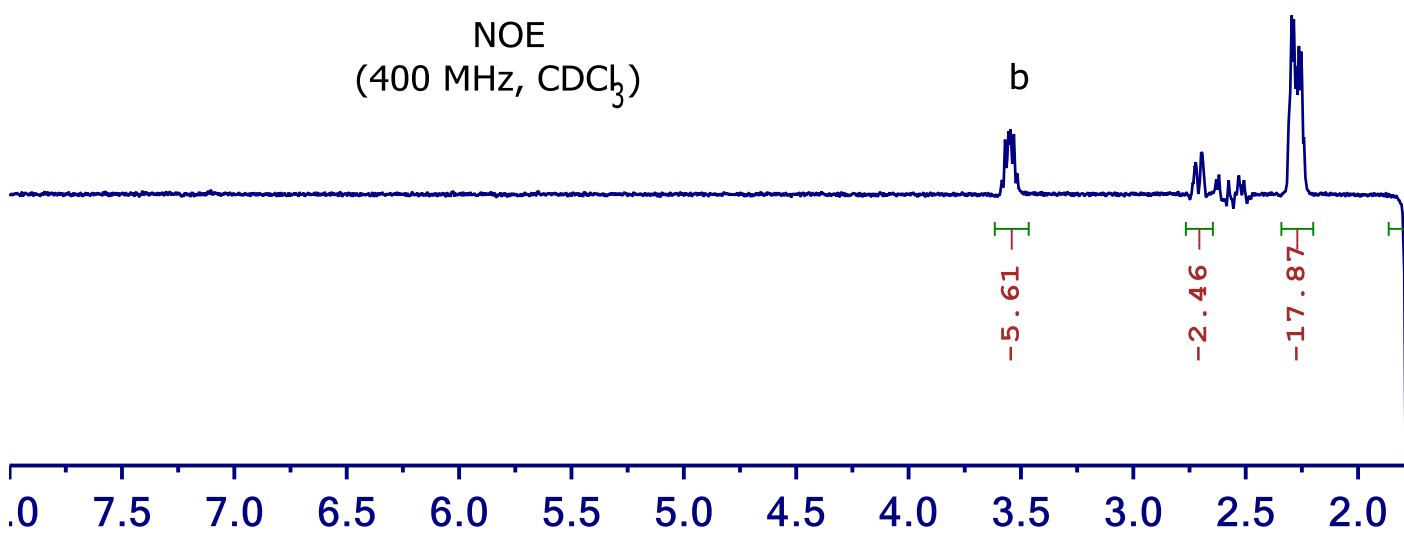

$\begin{array}{lll}1.5 & 1.0 & 0.5\end{array}$ 


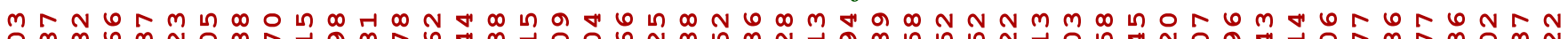

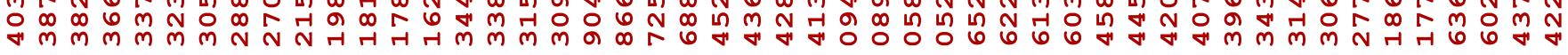

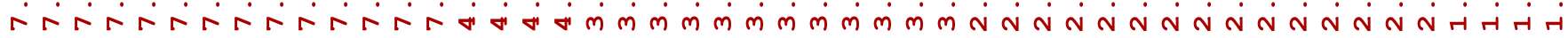

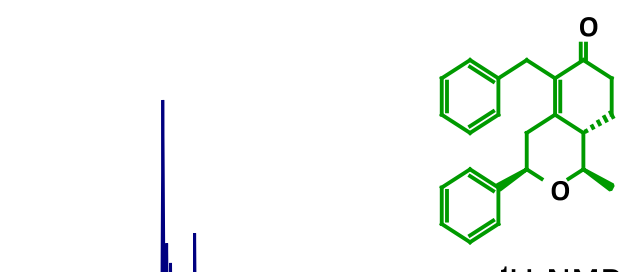

$1 \mathrm{H}$ NMR

$\left(400 \mathrm{MHz}, \mathrm{CDCl}_{3}\right)$

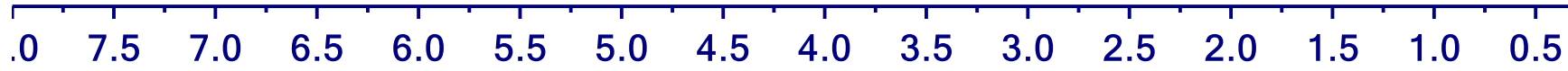
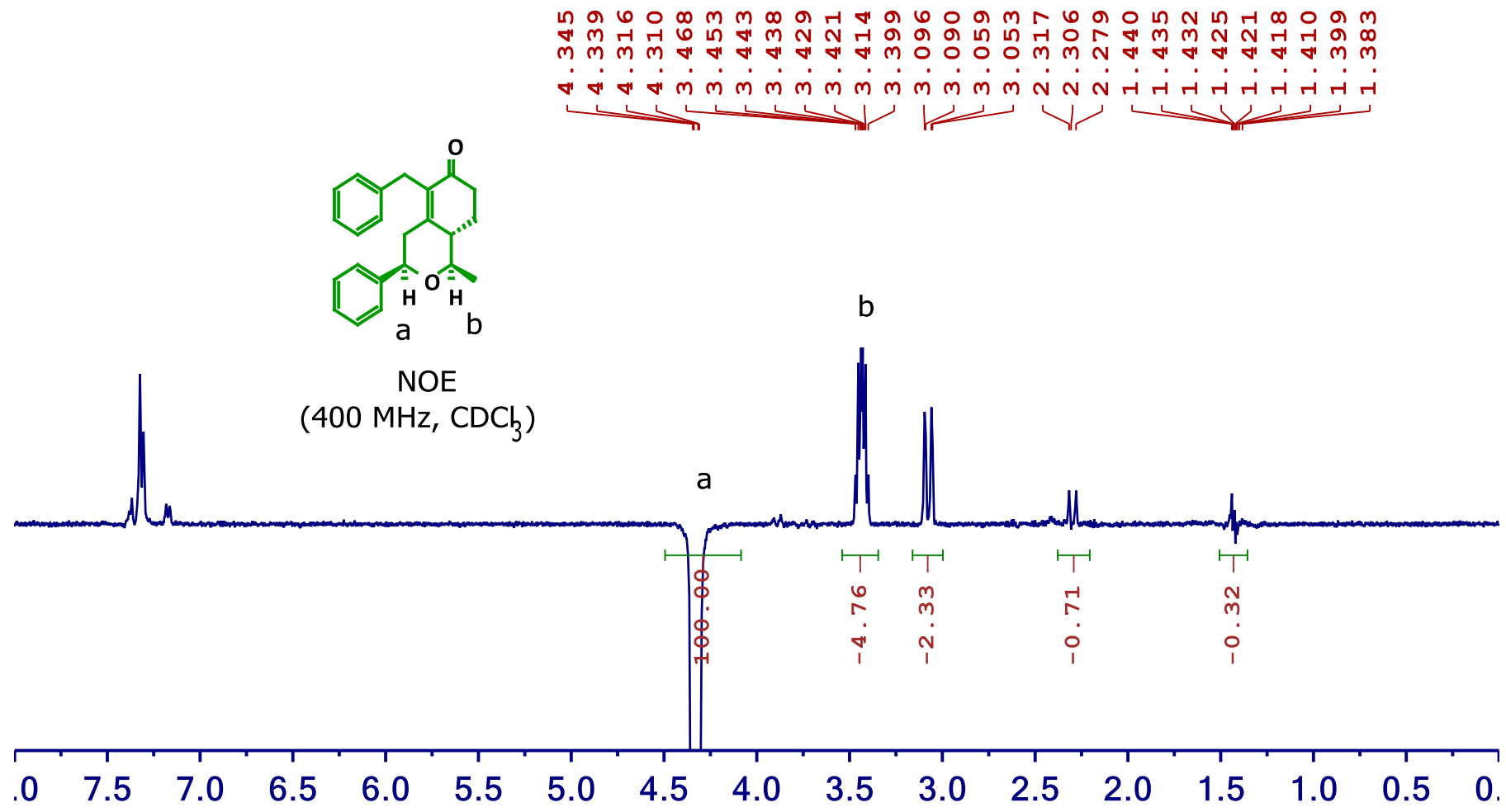
(1 R,3 R,8aS)-5-benzyl-1-methyl-3-phenyl-1,3,4,7,8,8a-hexahydro-6H-isochromen-6-one

$7 \mathbf{j}$

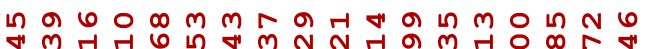

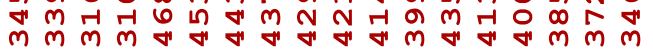

$\dot{\nabla} \dot{\nabla} \dot{m} \dot{m} \dot{m} \dot{m} \dot{m} \dot{m} \dot{m} \dot{N} \dot{N} \dot{N} \dot{N} \mathbf{N}$

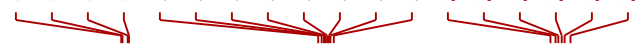

1)

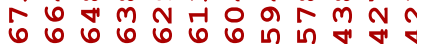
(1)

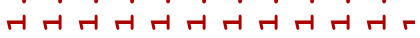
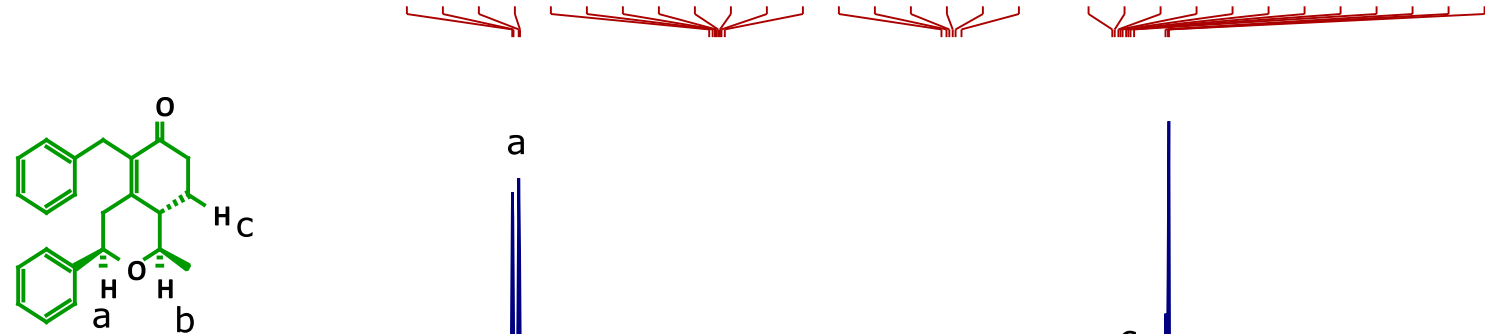

NOE

(400 $\left.\mathrm{MHz}, \mathrm{CDC}_{3}\right)$
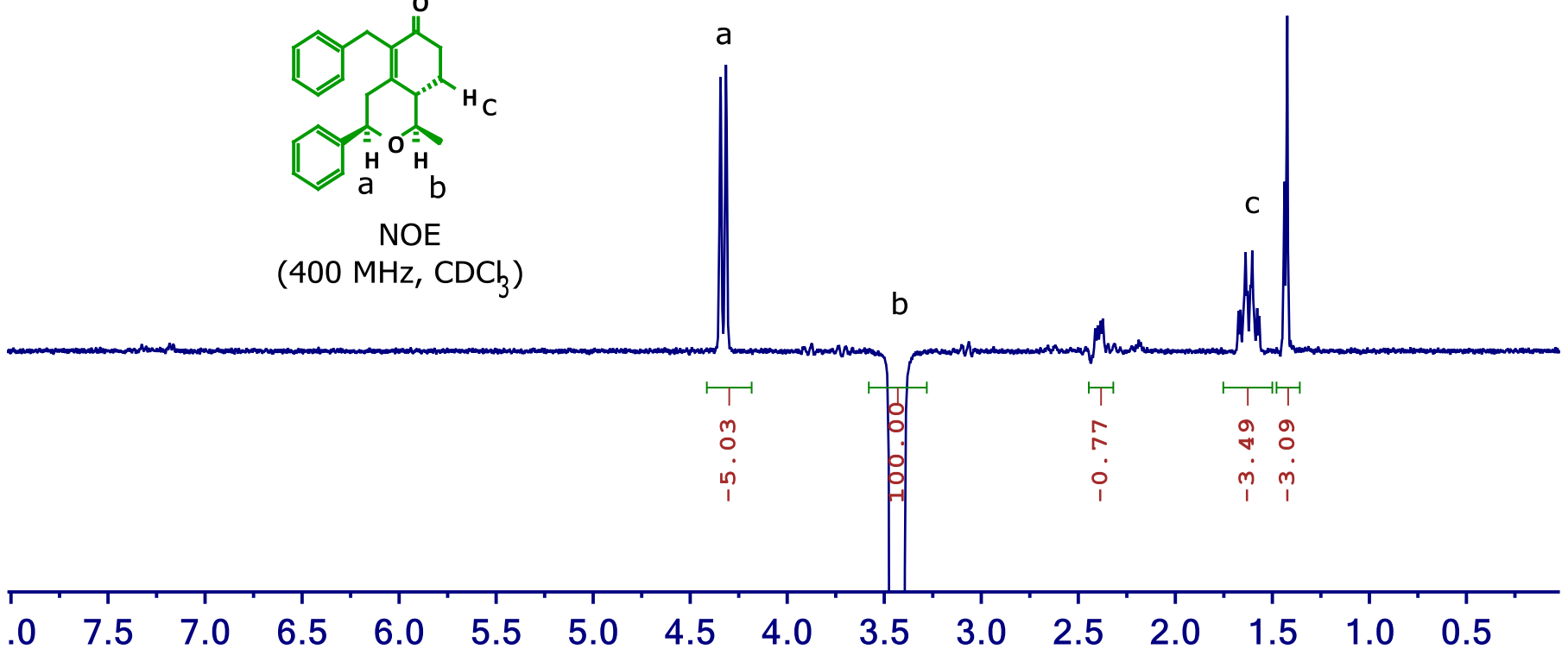

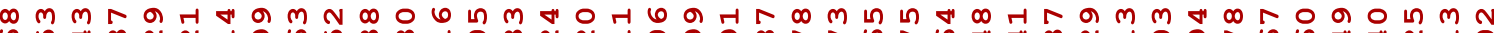

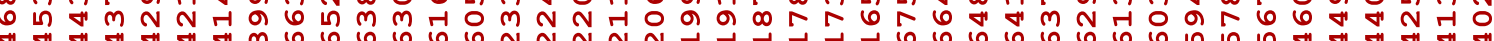

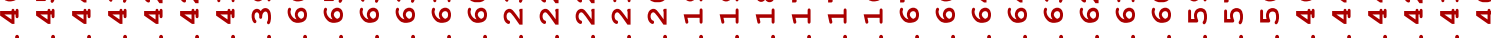

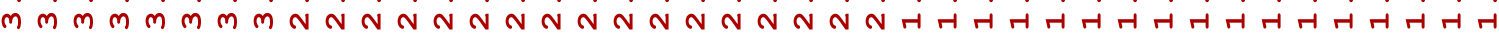

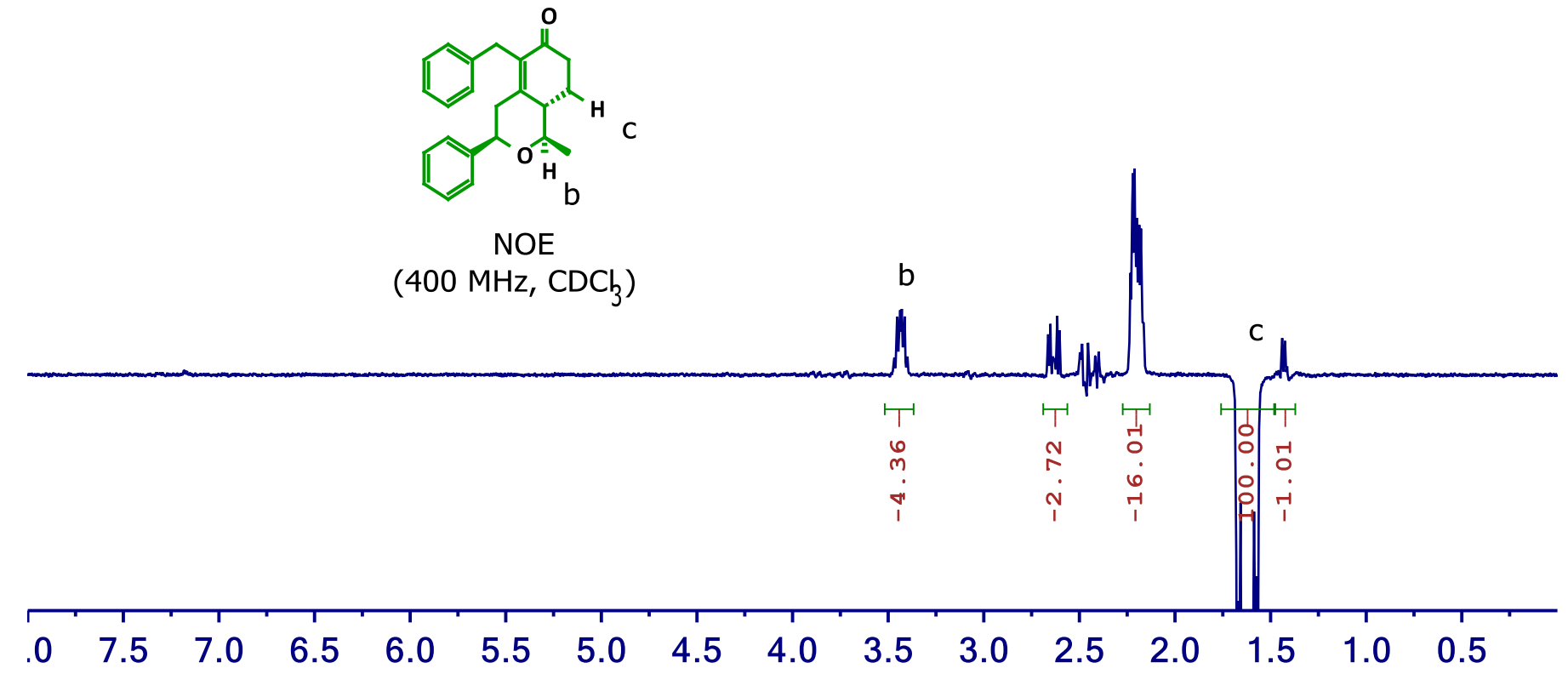


(1 R,3R,8aS)-5-benzyl-3-(2-chlorophenyl)-1-methyl-1,3,4,7,8,8a-hexahydro-6H-isochromen-6-one

$7 n$

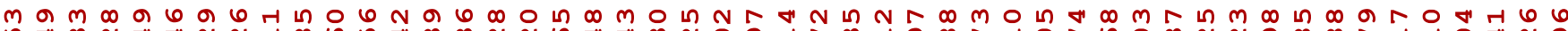
గn

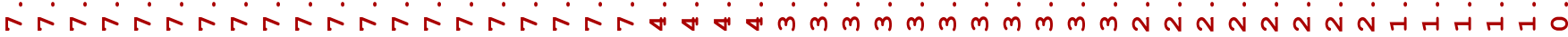
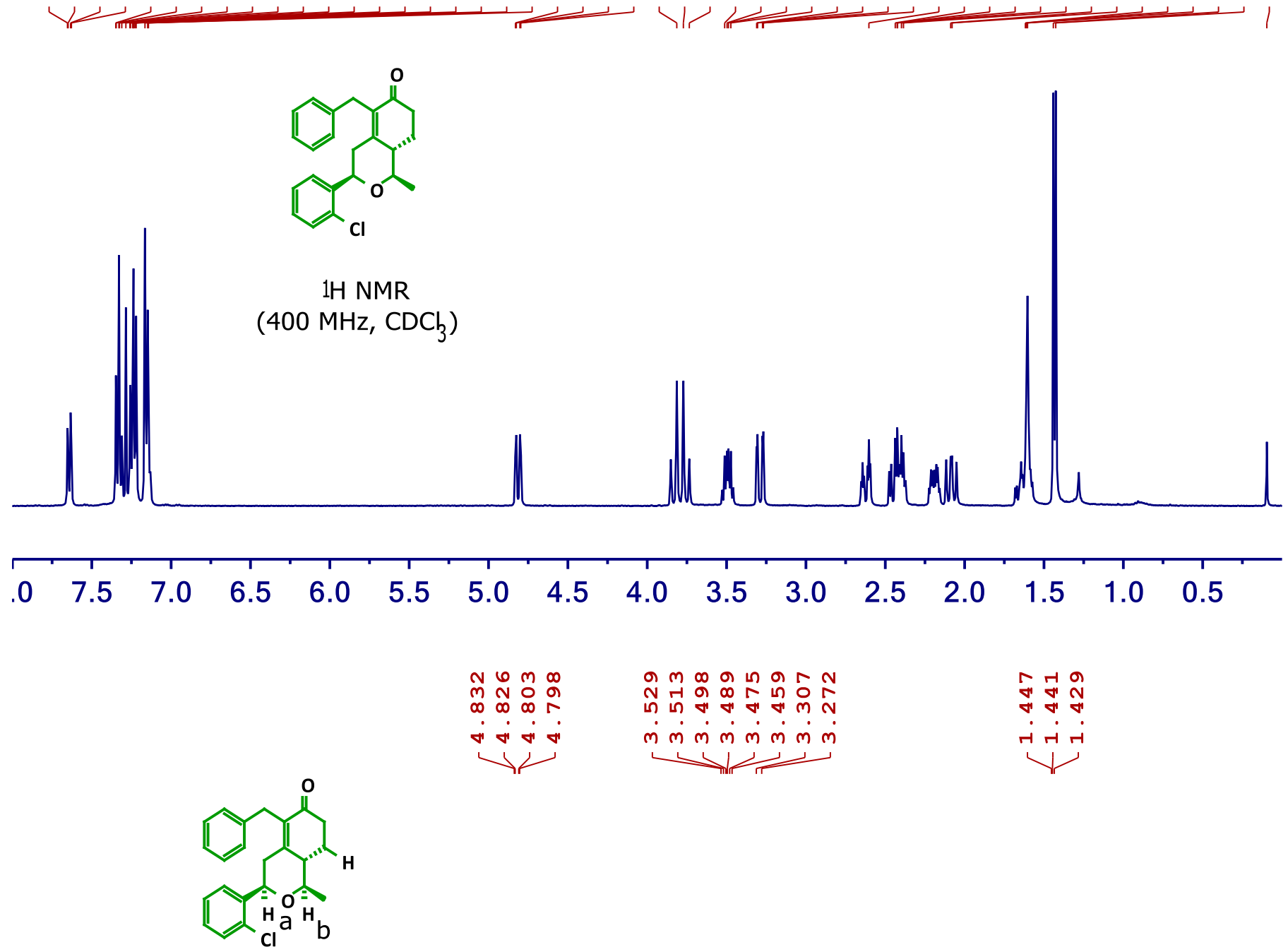

NOE

$\left(400 \mathrm{MHz}, \mathrm{CDC}_{3}\right)$

b

a

lllin

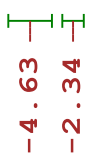

'T

N

이

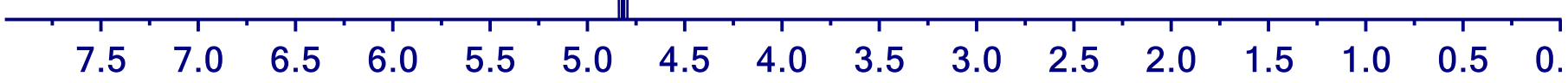


(1 R,3R,8aS)-5-benzyl-3-(2-chlorophenyl)-1-methyl-1,3,4,7,8,8a-hexahydro-6H-isochromen-6-one

$7 n$

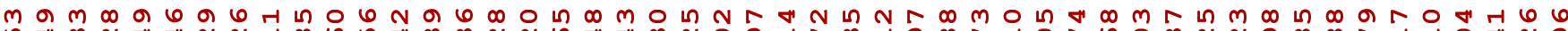
గn

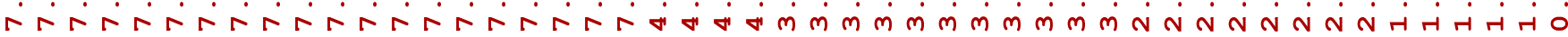
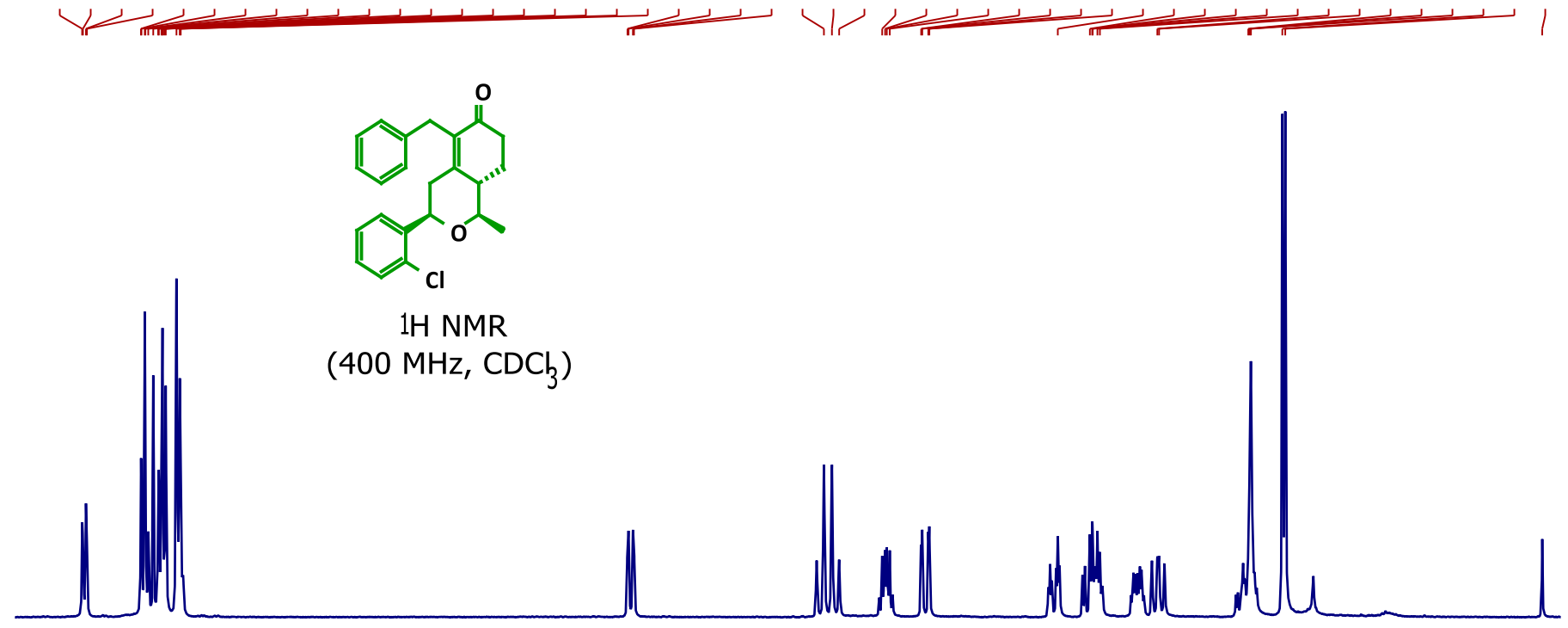

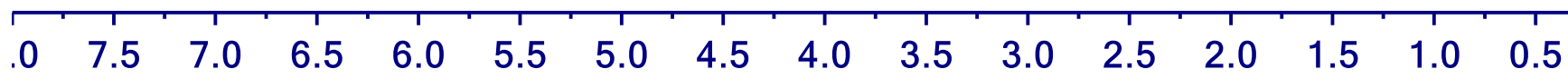
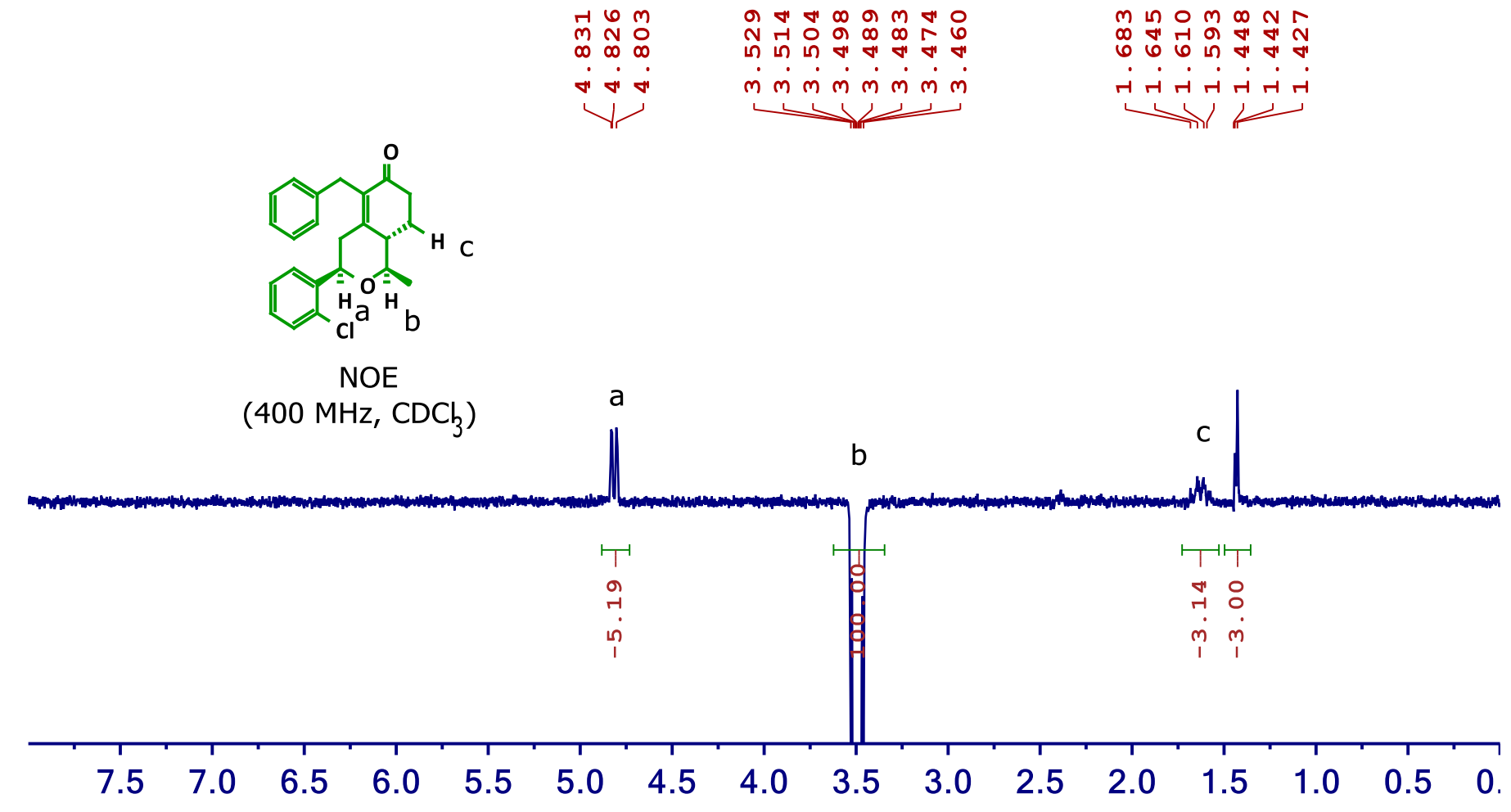
$7 \mathbf{q}$

ᄃㅇำ น̂

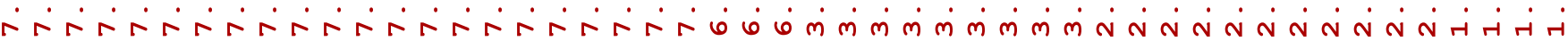
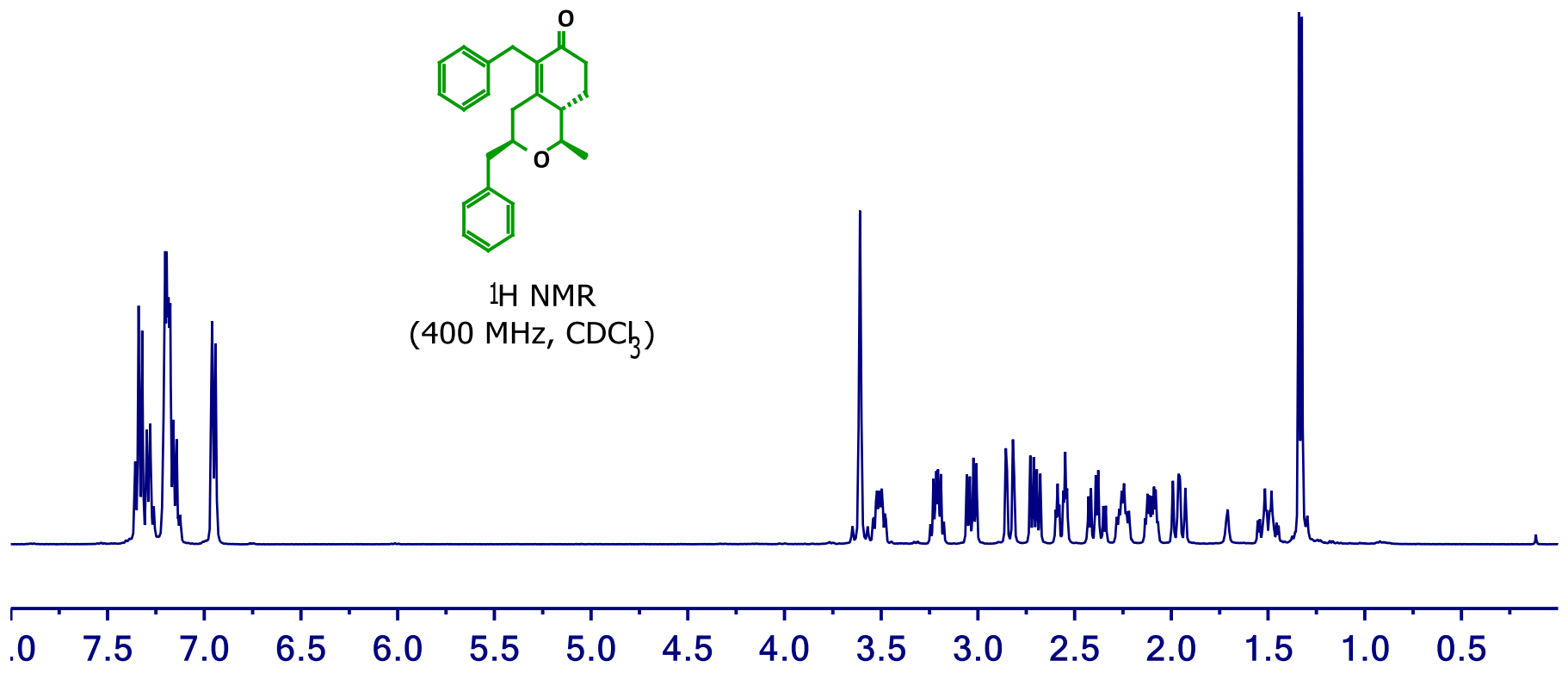

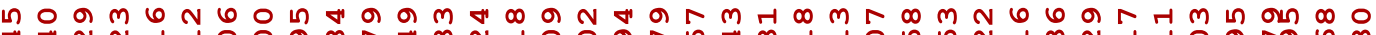

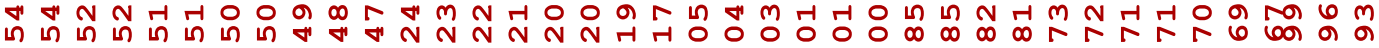

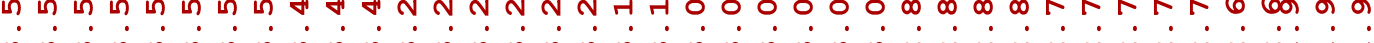

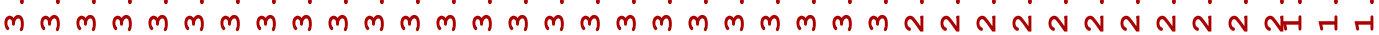

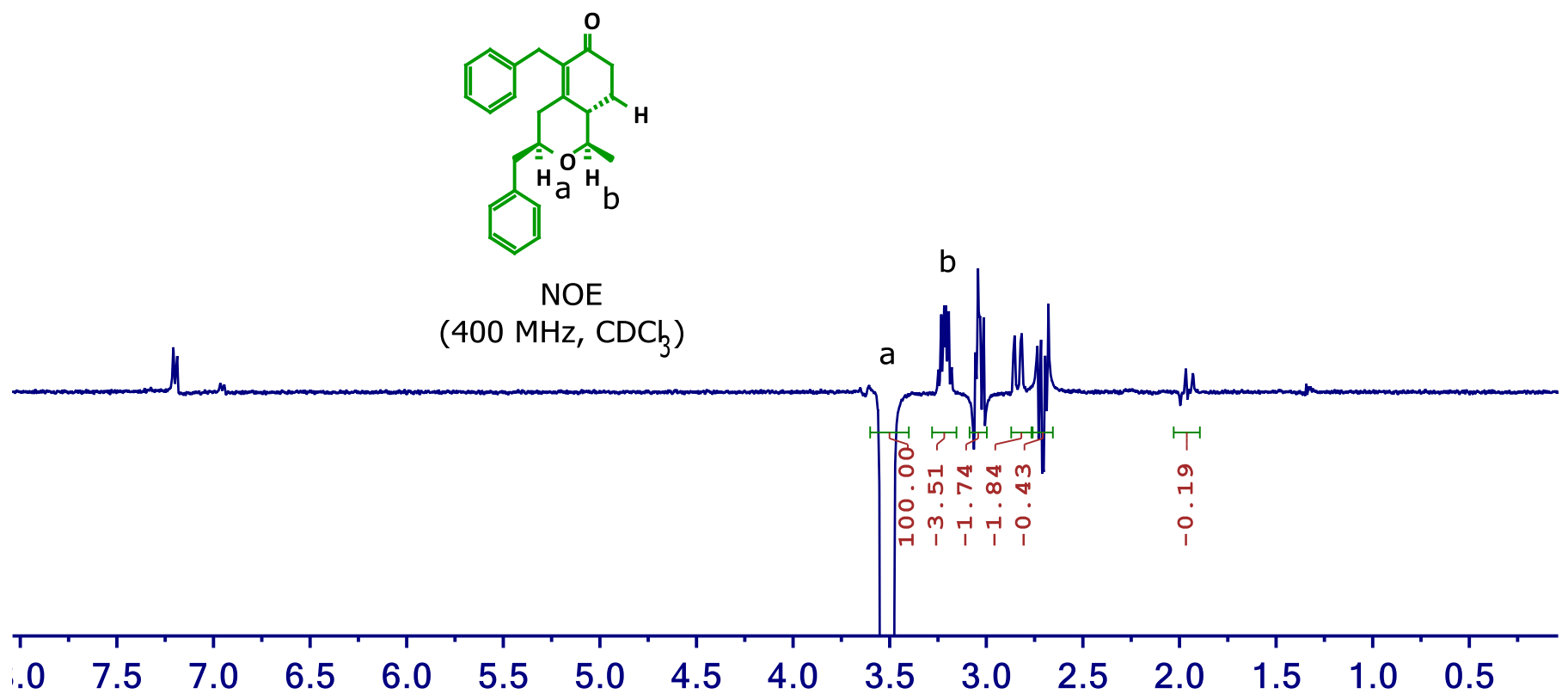


(1 R,3 $S, 8 a S)$-3,5-dibenzyl-1-methyl-1,3,4,7,8,8a-hexahydro-6H-isochromen-6-one

\section{$7 q$}

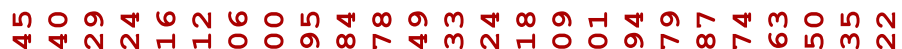

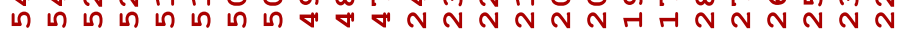
$\dot{m} \dot{m} \dot{m} \dot{m} \dot{m} \dot{m} \dot{m} \dot{m} \dot{m} \dot{m} \dot{m} \dot{m} \dot{m} \dot{N} \dot{N} \dot{N} \dot{N}$

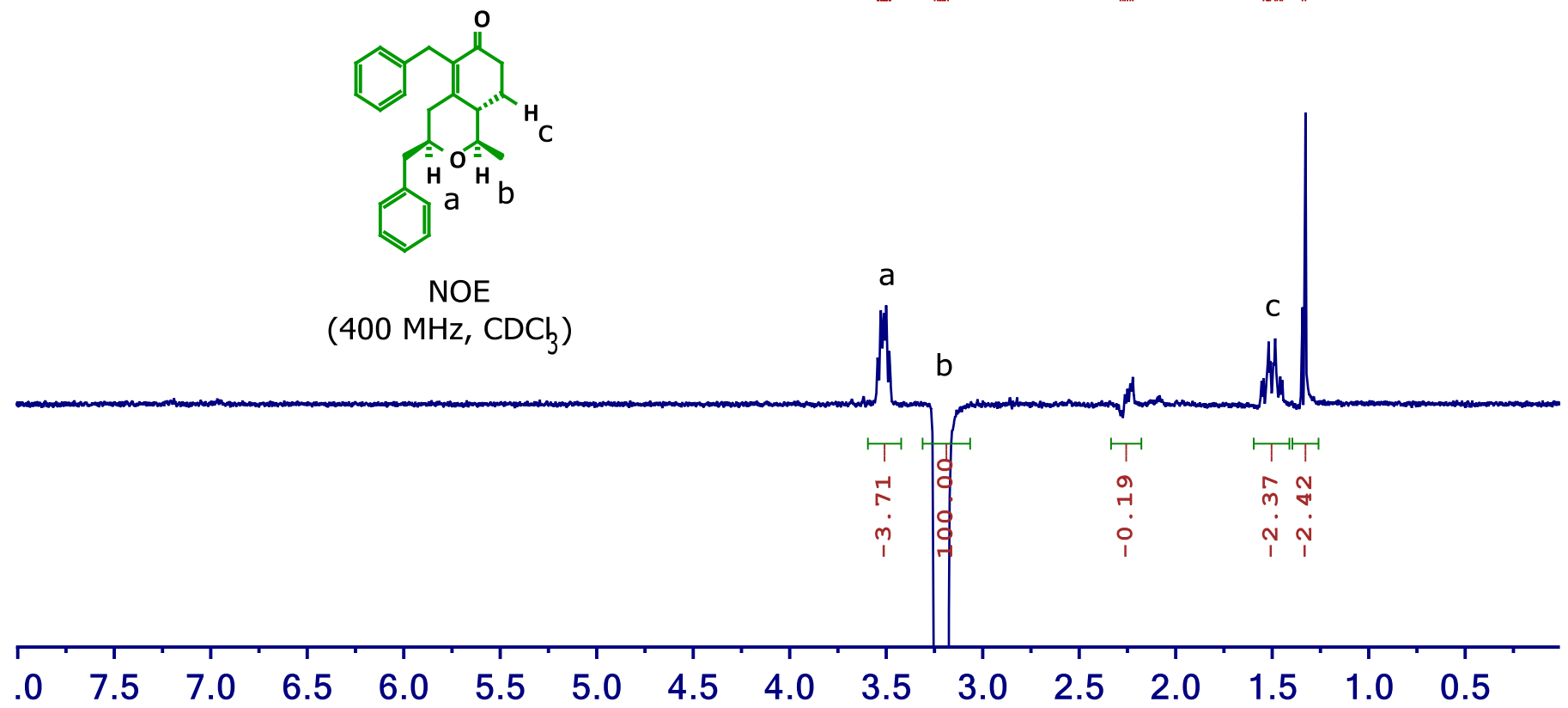

$\sqrt{4}+4 \sin$

nO

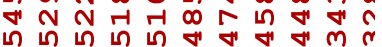
iririritriritr

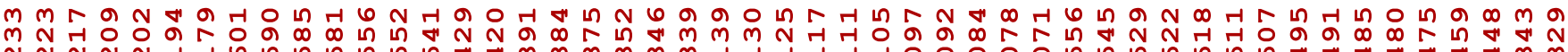

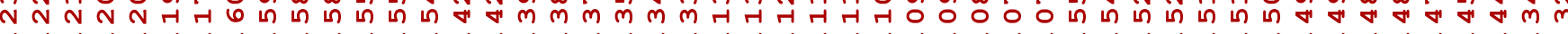

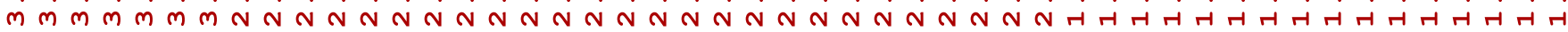

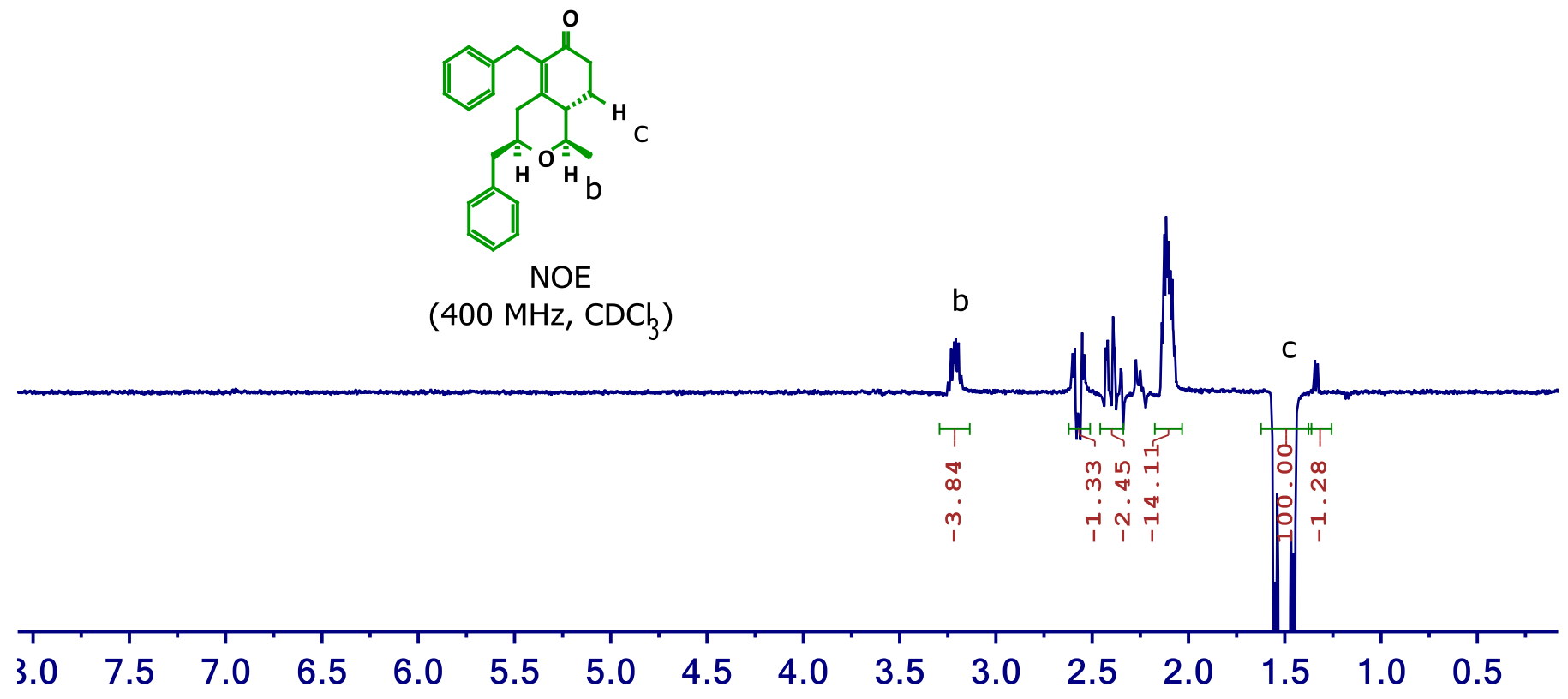


동ํำ

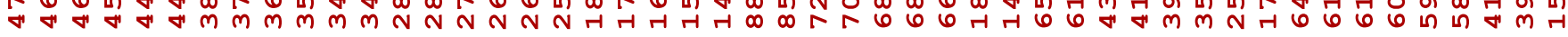

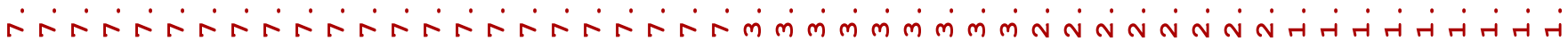

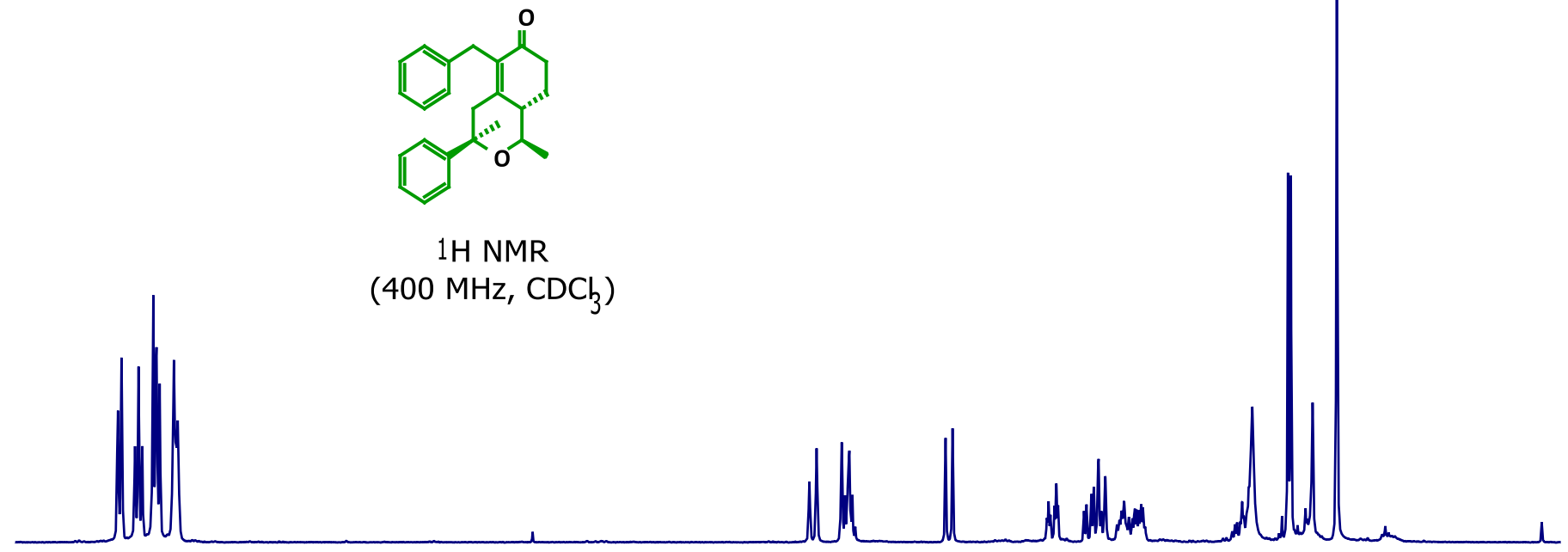

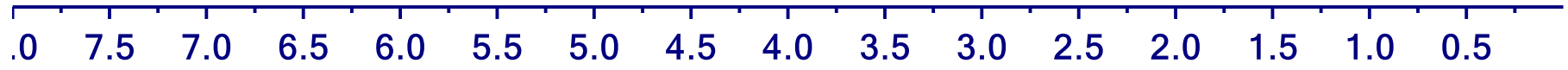

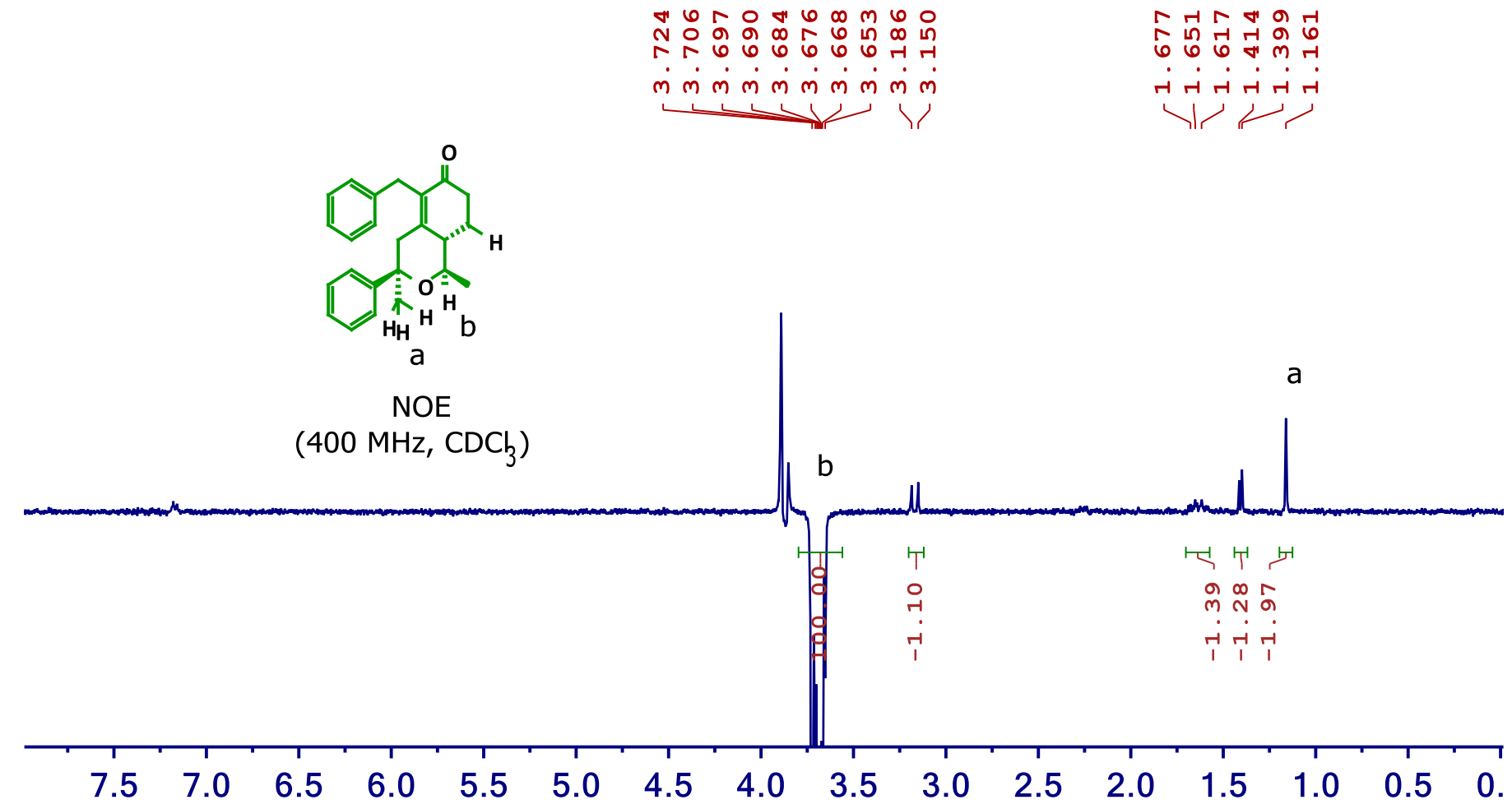


(1 R,3 $S, 8 a S)$-5-benzyl-1,3-dimethyl-3-phenyl-1,3,4,7,8,8a-hexahydro-6H-isochromen-6-one

$7 \mathrm{~s} 2$

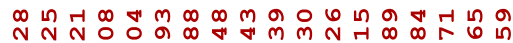

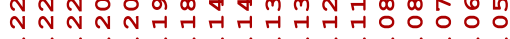

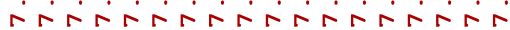

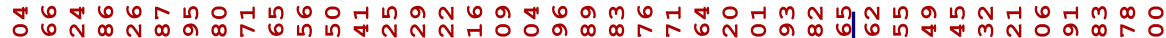
ด mmmmmmmmmmmmmm

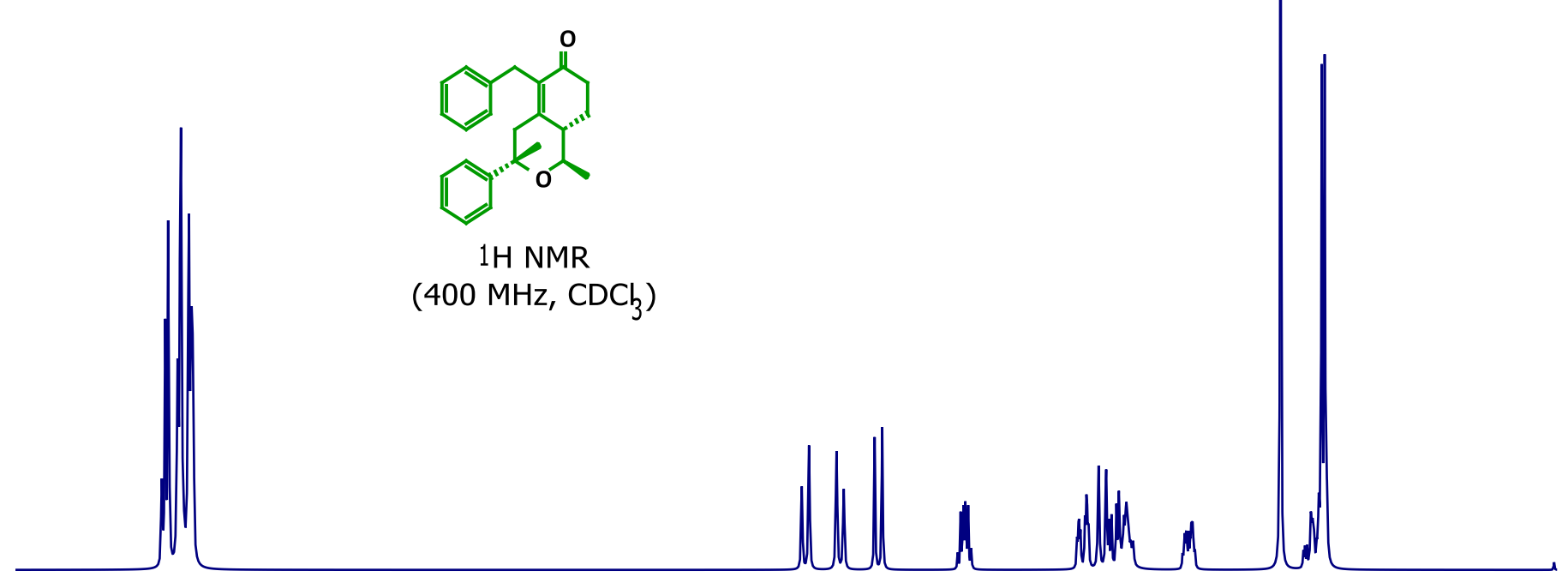

$1 \mathrm{H} N \mathrm{NMR}$

(400 $\mathrm{MHz}, \mathrm{CDC}_{3}$ )
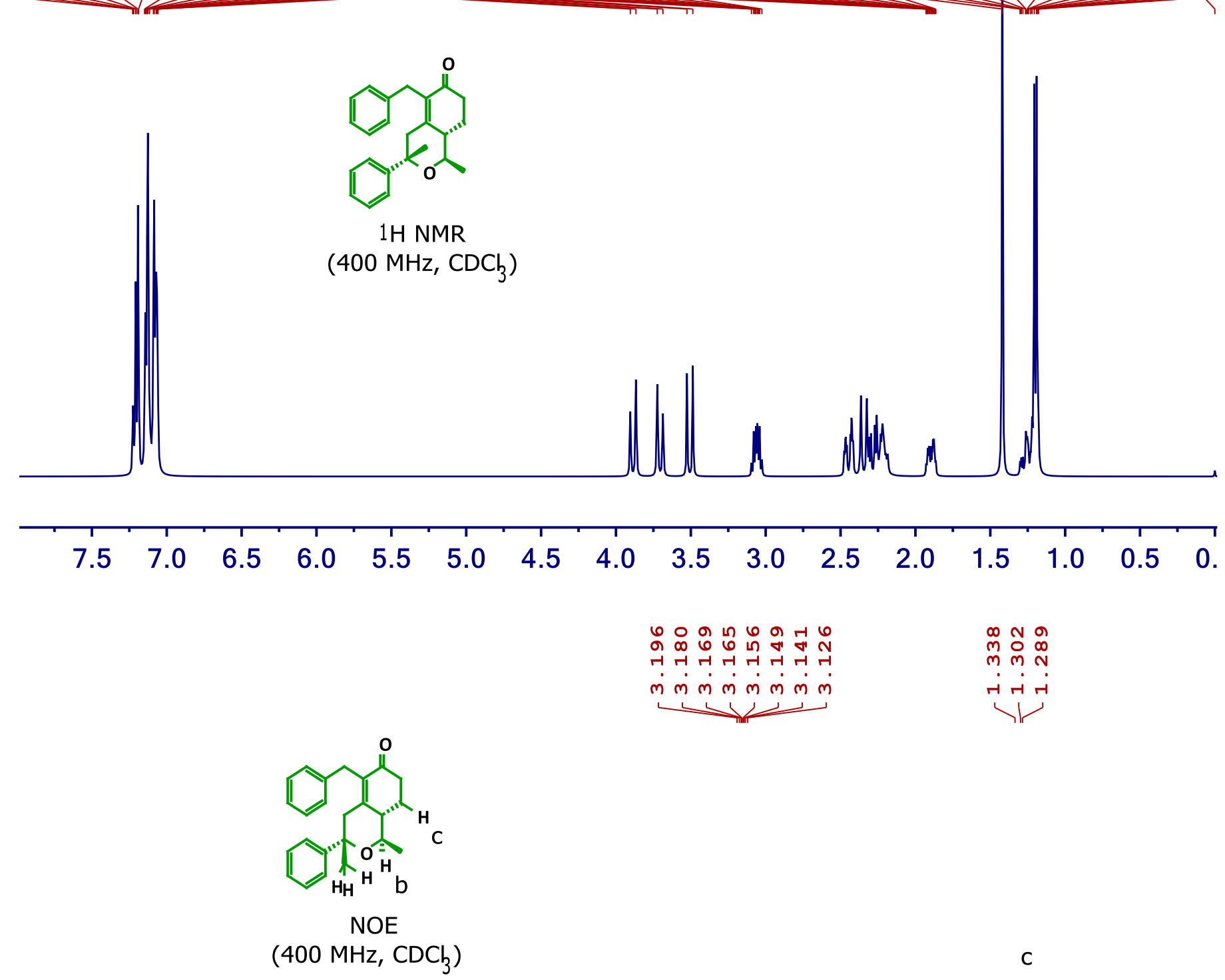

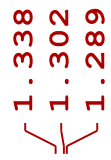

C

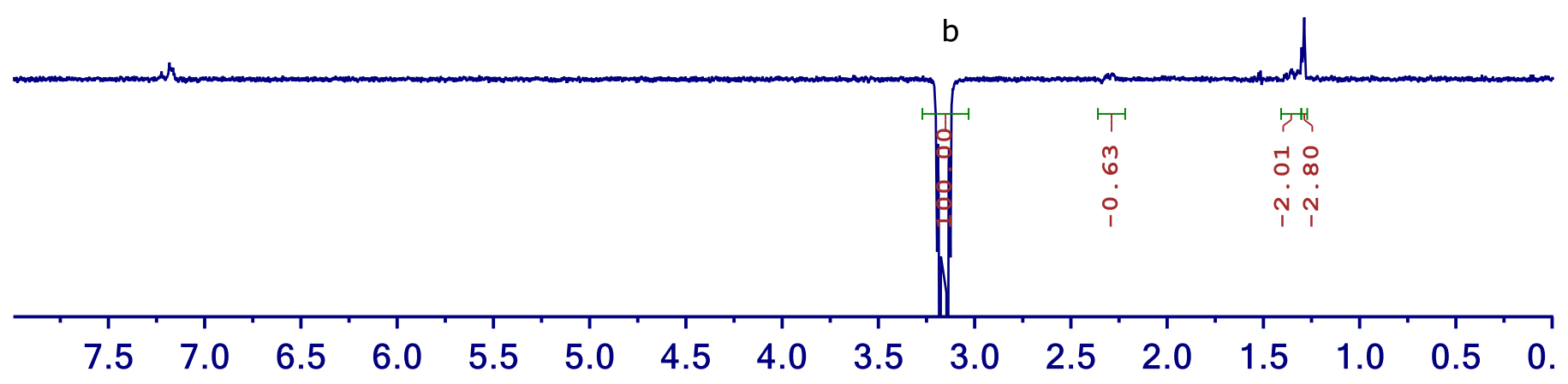


(1R,3R)-1-methyl-3,5-diphenylisochroman-6-ol

$8 \mathbf{a}$

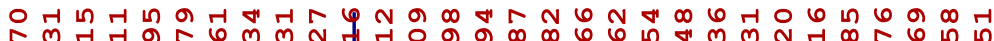

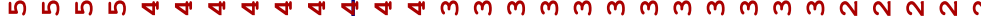

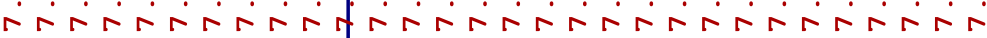

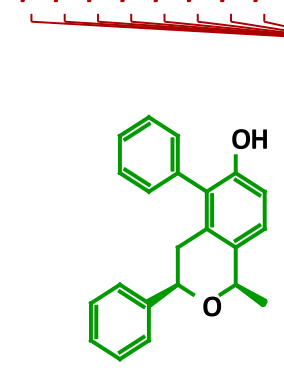

$1 \mathrm{H}$ NMR

$\left(400 \mathrm{MHz}, \mathrm{CDC}_{3}\right)$

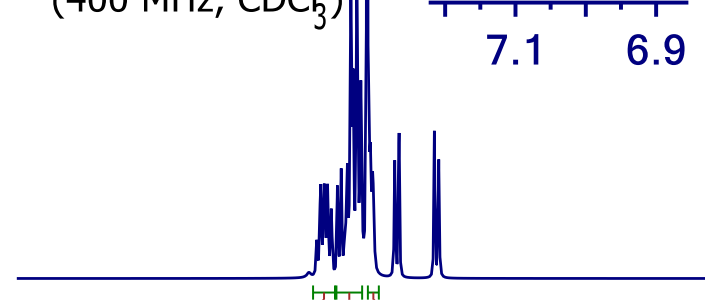

1.

$0 \cos ^{-1}$

i $\dot{0} \dot{4}$

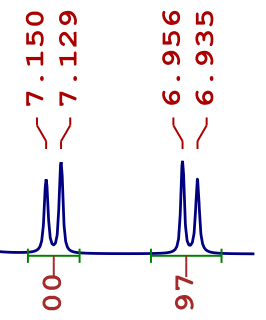

$\begin{array}{llll}\pi & 0 & 0 \\ 0 & \infty & N & 0 \\ 0 & 0 & 0 & 0\end{array}$

10.0.

>1

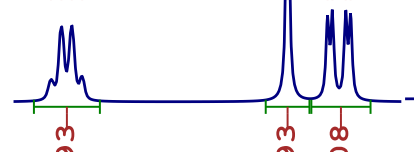

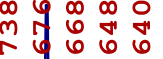

$\sin \sin _{\infty}$

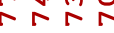

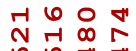

$\sin \sin$

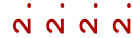

$>1 /$

$\begin{array}{ll}\nabla & \infty \\ \infty & 0 \\ 0 & 0\end{array}$

i

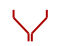

il
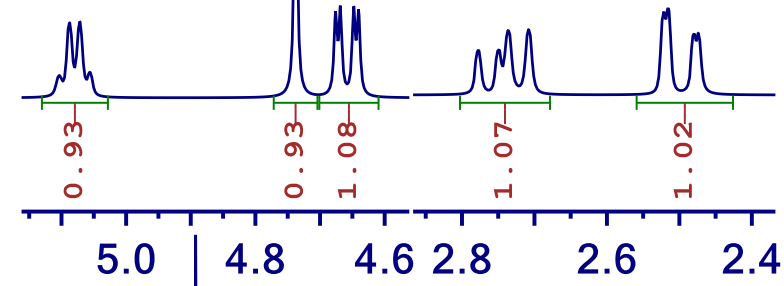

.0

8.0

7.0

6.0

5.0

4.0

3.0

2.0

1.0

0.

N In man - $\dot{7}$ ก

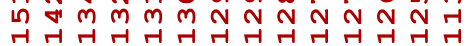
Lᄂ?<smiles>C[C@@H]1O[C@@H](c2ccccc2)Cc2c1ccc(O)c2-c1ccccc1</smiles>

$13 \mathrm{C}\{1 \mathrm{H}\} \mathrm{NMR}$

(100 MHz, $\mathrm{CDC}_{3}$ )

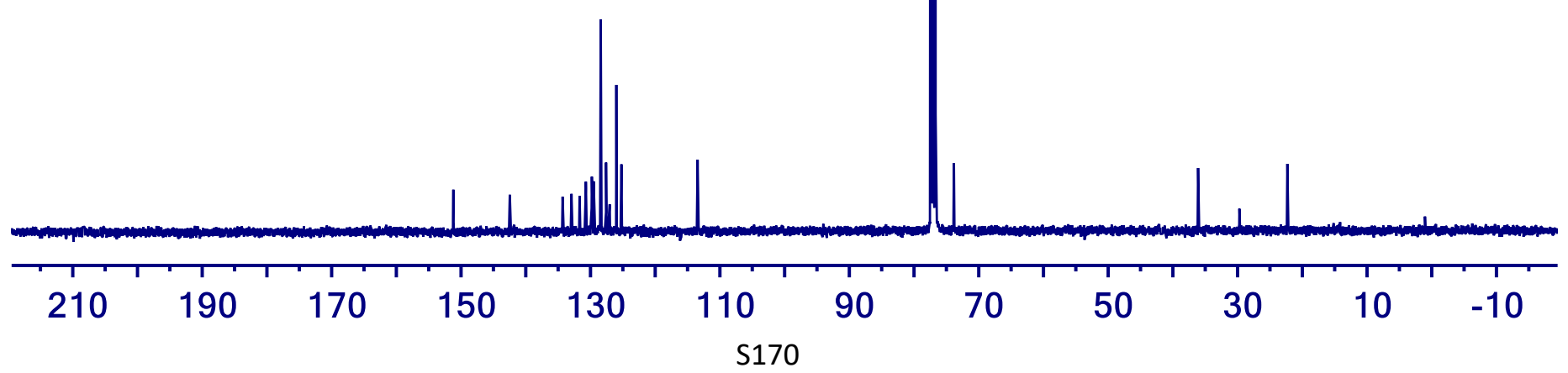


$(1 R, 3 R)$-1-methyl-3,5-diphenylisochroman-6-ol

$8 \mathbf{a}$

$\sim \infty$ ก

ó $\dot{0} \dot{0} \dot{0} \dot{0}$ in $\dot{m}$

m N N N N N

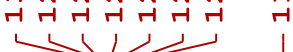

ता?

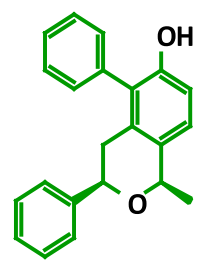

DEPT 135

$100 \mathrm{MHz}, \mathrm{CDCl}_{3}$

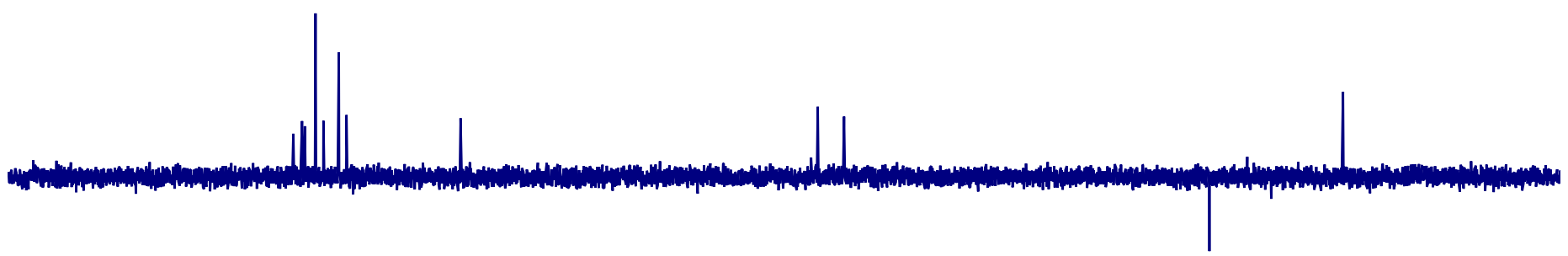

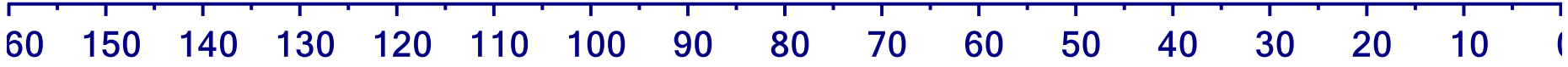


(1R,3R)-1-methyl-5-phenyl-3-(o-tolyl)isochroman-6-ol

$8 b$

ก 근유

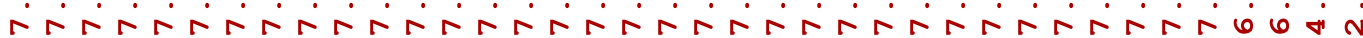

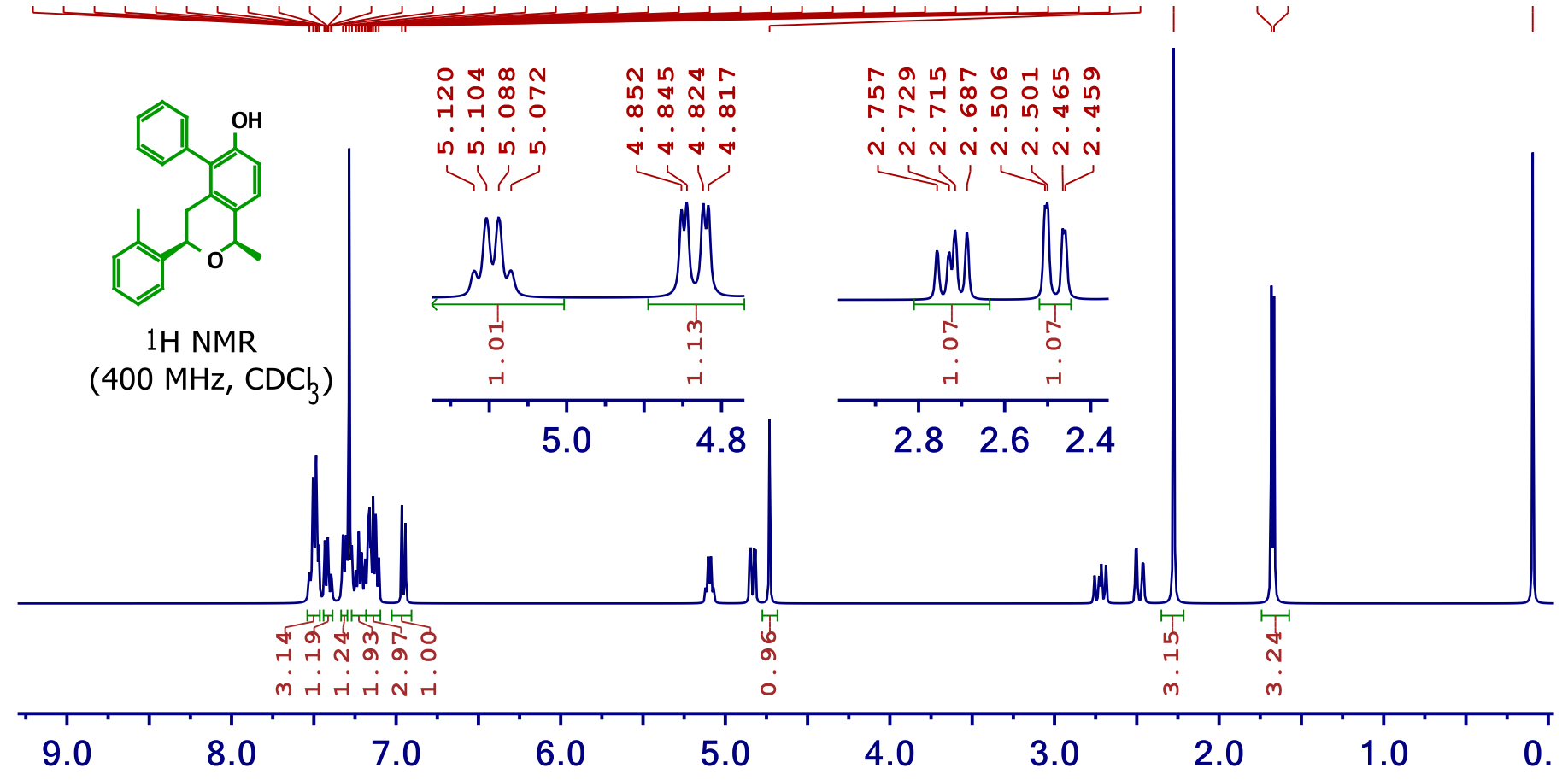

n

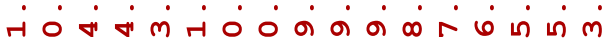

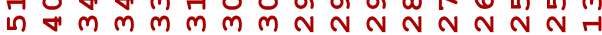

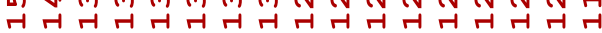

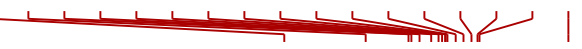<smiles>Cc1ccccc1C1Cc2c(ccc(O)c2-c2ccccc2)C(C)O1</smiles>

13C $\{1 \mathrm{H}\}$ NMR

$\left(100 \mathrm{MHz}, \mathrm{CDCl}_{3}\right)$
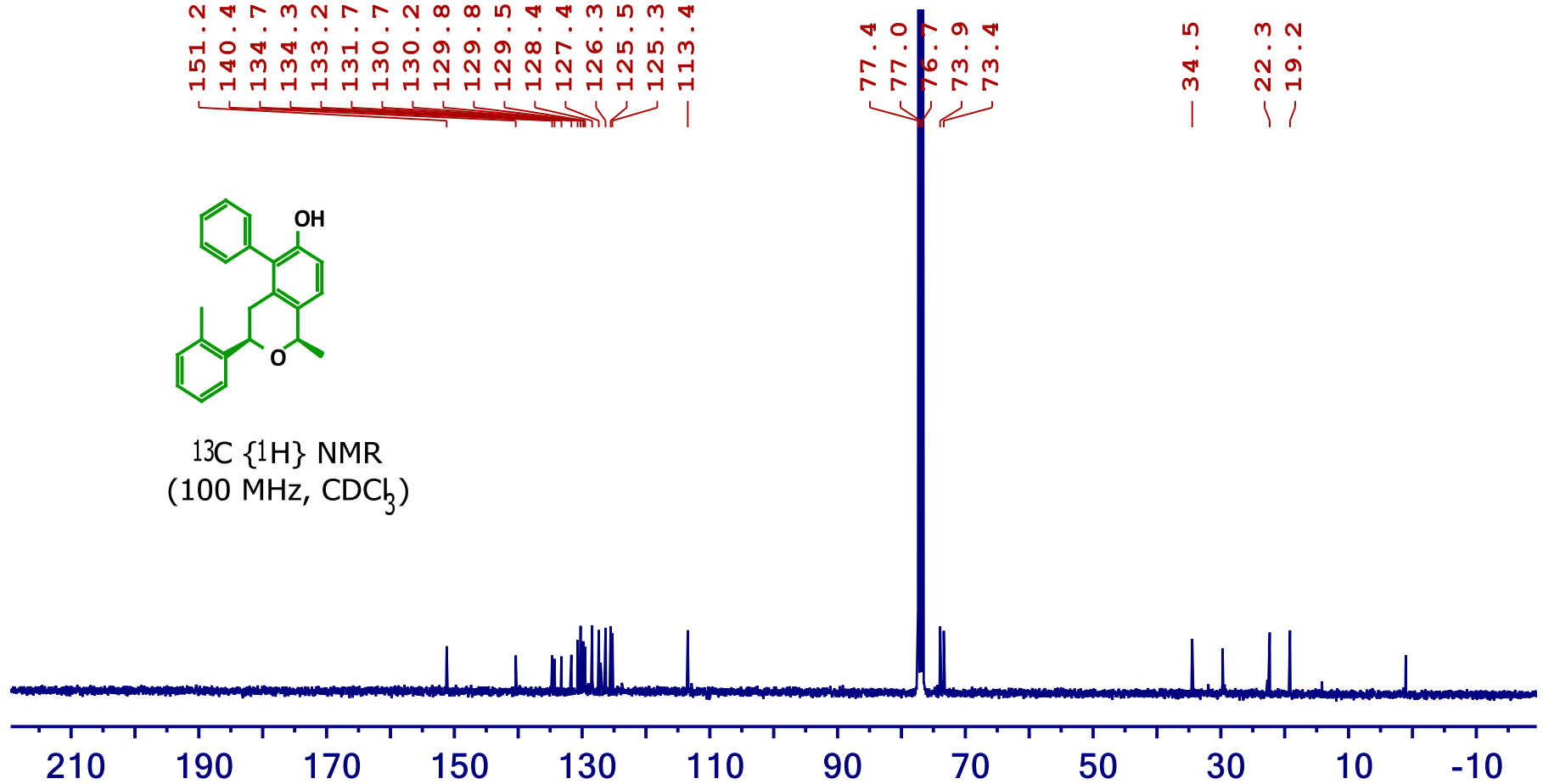
$(1 R, 3 S)-3,5-d i b e n z y l-1-m e t h y l i s o c h r o m a n-6-o l$

$8 \mathrm{c}$

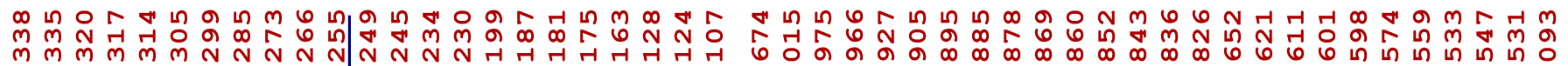

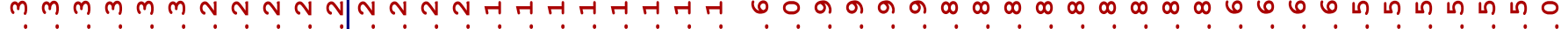

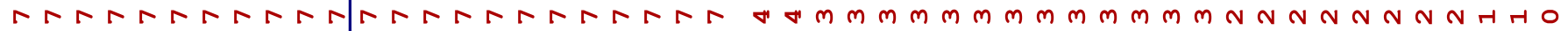

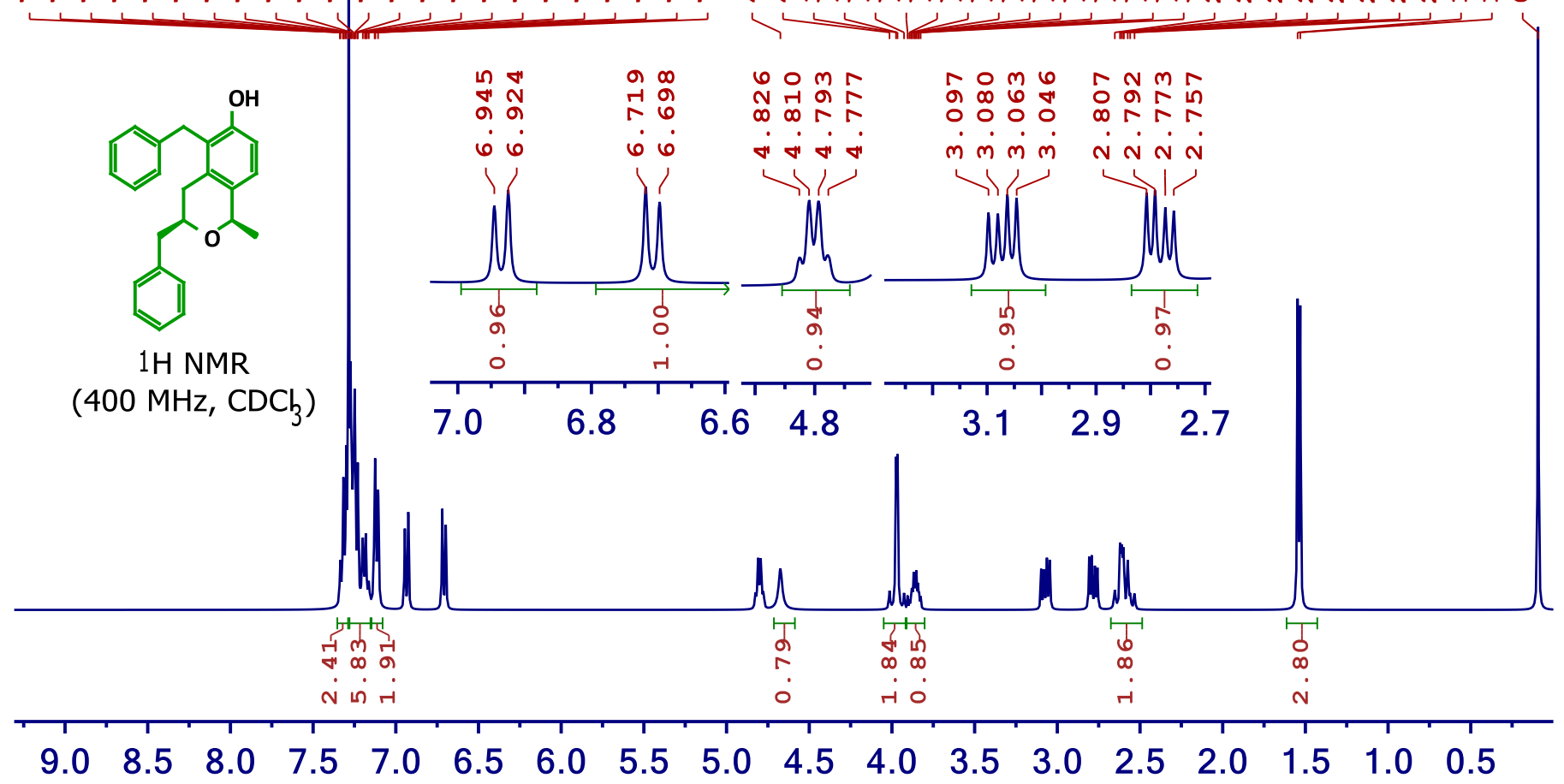

N

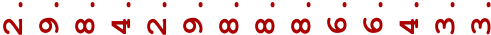

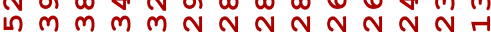

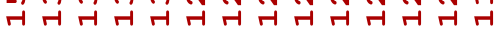<smiles>C[C@H]1OC(Cc2ccccc2)Cc2c(Cc3ccccc3)ccc(O)c21</smiles>

$13 \mathrm{C}\{1 \mathrm{H}\} \mathrm{NMR}$ (100 $\mathrm{MHz}, \mathrm{CDCl}_{3}$ )
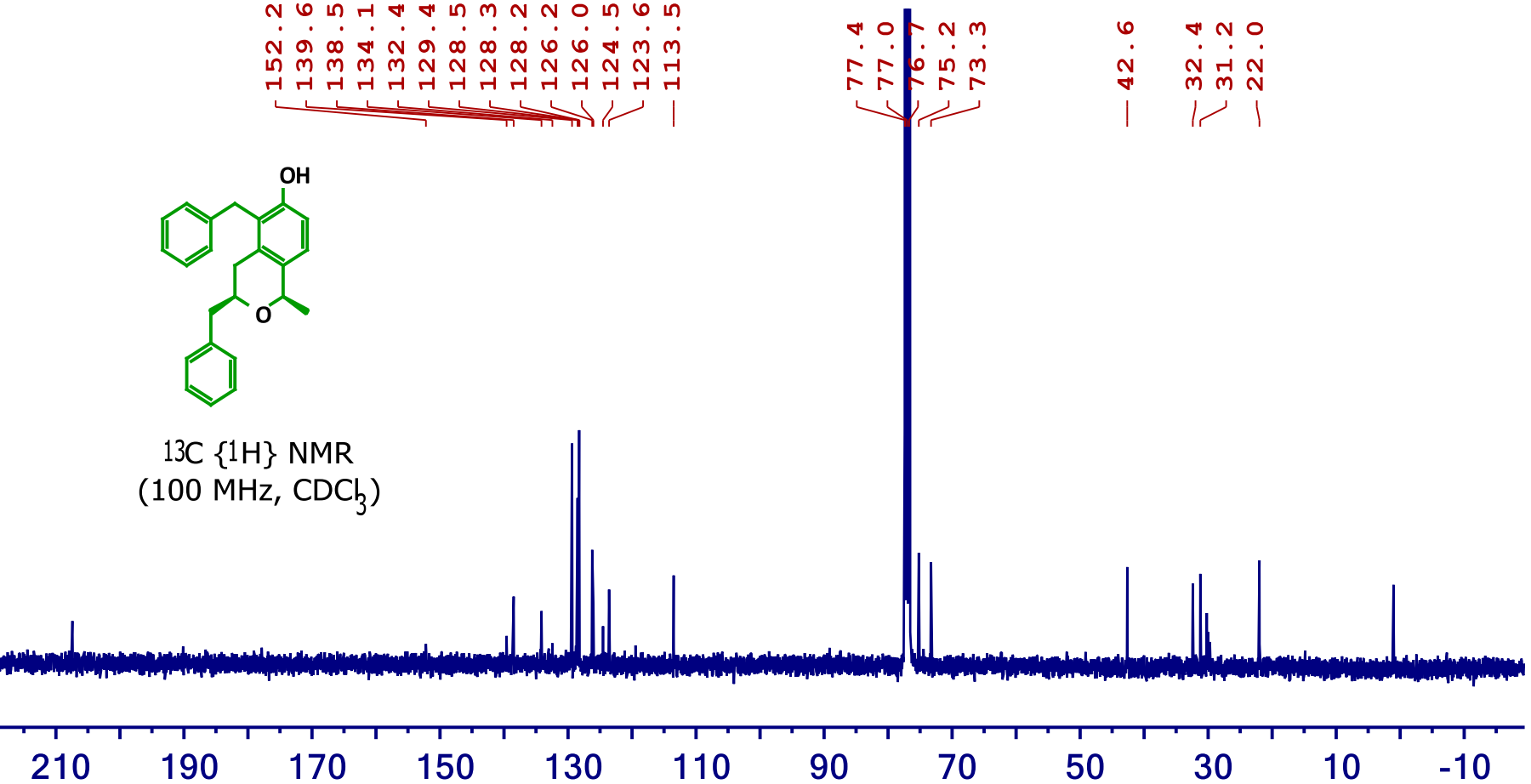


\section{$(R)-5$ '-benzyl-1'-methylspiro [cyclopentane-1,3'-isochroman]-6'-ol}

$8 \mathrm{~d}$

サ ก 6 N N O N N N N N H H H h G

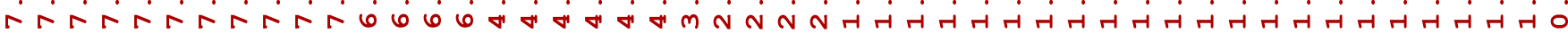<smiles></smiles>

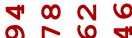

$\infty \infty \infty$

षण खं

पil

$1 \mathrm{H} N \mathrm{NR}$

$\left(400 \mathrm{MHz}, \mathrm{CDC}_{3}\right)$
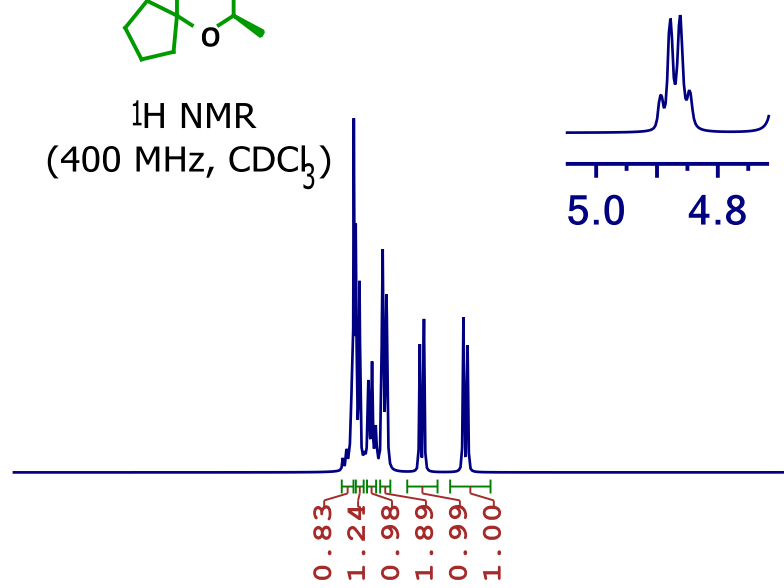

\begin{tabular}{llllllllllllllllll}
\hline 8.5 & 8.0 & 7.5 & 7.0 & 6.5 & 6.0 & 5.5 & 5.0 & 4.5 & 4.0 & 3.5 & 3.0 & 2.5 & 2.0 & 1.5 & 1.0 & 0.5 & 0.
\end{tabular}

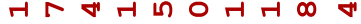
Nं $\dot{m} \dot{N} \infty \dot{\omega} \nabla \dot{m} m$

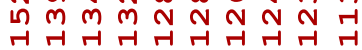<smiles>C[C@H]1OC2(CCCC2)Cc2c1ccc(O)c2Cc1ccccc1</smiles>

$13 \mathrm{C}\{1 \mathrm{H}\} \mathrm{NMR}$ $\left(100 \mathrm{MHz}, \mathrm{CDCh}_{3}\right)$

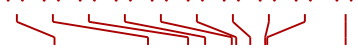

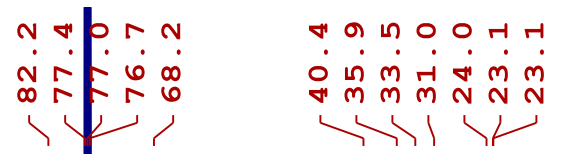

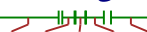
की 60 0 n⿺辶一 N Ho n

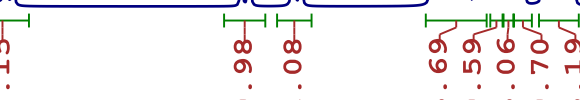

(0 